\title{
Synthesis of Chiral 2-Substituted 1,4-Benzoxazin-3-ones via Iridium-Catalyzed Enantioselective Hydrogenation of \\ Benzoxazinones
}

\author{
Yu Nie, ${ }^{\dagger}$ Jing Li, ${ }^{\dagger}$ Jun Yan, ${ }^{\dagger}$ Qianjia Yuan, ${ }^{\dagger, *}$ and Wanbin Zhang ${ }^{\dagger,+, *}$ \\ ${ }^{\dagger}$ Shanghai Key Laboratory of Molecular Engineering of Chiral Drugs, Frontiers Science Center \\ for Transformative Molecules, School of Chemistry and Chemical Engineering, Shanghai Jiao \\ Tong University, 800 Dongchuan Road, Shanghai 200240, P. R. China \\ ${ }^{*}$ College of Chemistry, Zhengzhou University, 75 Daxue Road, Zhengzhou 450052, P. R. China
}

\section{Contents}

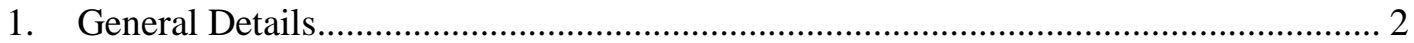

2. General Procedure for the Synthesis of the Substrates................................................ 3

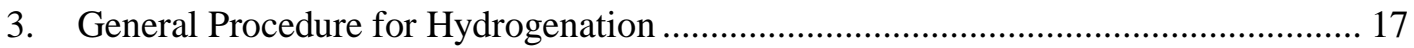

4. Transformations of the Reduced Products .................................................................... 33

5. Control Experiments and the Stereochemical Model................................................... 37

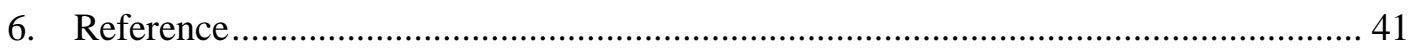

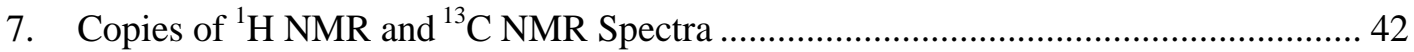

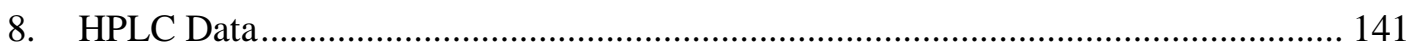

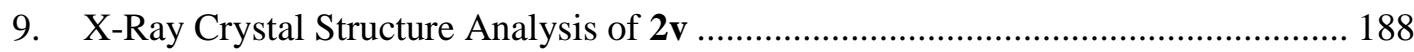




\section{General Details}

All reagents were commercially available and were used without further purification unless otherwise indicated. All solvents were dried and distilled before use according to the standard methods. Analytical thin layer chromatography (TLC) was performed on precoated silica gel F254 plates. Visualization on TLC was achieved with UV light $(254 \mathrm{~nm})$ and potassium permanganate as visualization methods. ${ }^{1} \mathrm{H}$ NMR spectra were recorded on Bruker (400 or $500 \mathrm{MHz}$ ). Chemical shifts were quoted in parts per million ( $\mathrm{ppm}$ ) referenced to $0.00 \mathrm{ppm}$ for tetramethylsilane. Data for ${ }^{1} \mathrm{H}$ NMR spectra are reported as follows: chemical shift ( $\delta$ shift), multiplicity ( $\mathrm{s}=$ singlet, $\mathrm{d}=$ doublet, $\mathrm{t}=$ triplet, $\mathrm{q}=$ quartet, $\mathrm{m}=$ multiplet, $\mathrm{dd}=$ double of doublet, $\mathrm{ddd}=$ double of $\mathrm{dd}, \mathrm{dt}=$ double of triplet, $\mathrm{td}=$ triple of doublet), coupling constant and integration $(\mathrm{Hz}) .{ }^{13} \mathrm{C}$ NMR spectra were recorded on Bruker (101 or $126 \mathrm{MHz}$ ). Chemical shifts were reported in ppm referenced to the center line of a triplet at $77.0 \mathrm{ppm}$ of $\mathrm{CDCl}_{3}$. High resolution mass spectra (HRMS) were obtained with ACQUITYTM UPLC \& Q-TOF MS Premier Spectrometer at the Instrumental Analysis Center of Shanghai Jiao Tong University. Melting points were measured with SGW X-4 micro melting point apparatus. Optical rotations were measured on a Rudolph Research Analytical Autopol VI automatic polarimeter using a $50 \mathrm{~mm}$ path-length cell at $589 \mathrm{~nm}$. Chiral HPLC analyses were performed using a Shimadzu LC-10Avp system using isopropanol-hexane mobile phase and UV detection. 


\section{General Procedure for the Synthesis of the Substrates ${ }^{[1]}$}

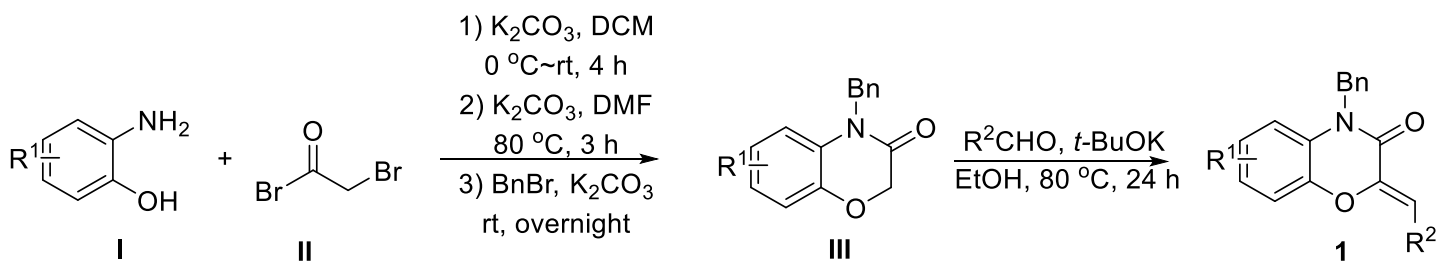

Aminophenol I (10 mmol) and an equimolar amount of $\mathrm{K}_{2} \mathrm{CO}_{3}$ were dissolved in a $100 \mathrm{~mL}$ of $\mathrm{CH}_{2} \mathrm{Cl}_{2}$ solution and the mixture was maintained at $0{ }^{\circ} \mathrm{C}$ in an ice-bath for 30 minutes. 2-Bromoacetyl bromide II (15 mmol) were slowly added, in a dropwise manner and the reaction was left to stir at room temperature for 4 hours for a full conversion. Then, the organic phase was washed with water and brine $(3 \times 100 \mathrm{~mL})$, dried over $\mathrm{Na}_{2} \mathrm{SO}_{4}$, filtered and evaporated under reduced pressure. The residue was dissolved in $100 \mathrm{~mL}$ of DMF, $\mathrm{K}_{2} \mathrm{CO}_{3}(12 \mathrm{mmol})$ was added and the reaction was heated at $80^{\circ} \mathrm{C}$ for 3 hours. When the reaction is completed, cooling to room temperature, $\mathrm{K}_{2} \mathrm{CO}_{3}(30 \mathrm{mmol})$ and $\mathrm{BnBr}(15 \mathrm{mmol})$ were added, and the mixture was stirred at room temperature overnight for a full conversion. Then, the mixture was diluted with EtOAc $(3 \times 50 \mathrm{~mL})$ and washed with water and brine $(3 \times 100 \mathrm{~mL})$. The organic layer was dried over $\mathrm{Na}_{2} \mathrm{SO}_{4}$ and the solvent was removed under reduced pressure. The residue was purified by flash chromatography on silica gel to give III.

To a stirred solution of III $(10 \mathrm{mmol})$ and aldehyde $(15 \mathrm{mmol})$ in $100 \mathrm{~mL}$ EtOH, $15 \mathrm{~mL} t$-BuOK ( $1 \mathrm{M}$ in THF) were slowly added and the reaction was refluxed at $80{ }^{\circ} \mathrm{C}$ for $24 \mathrm{~h}$. After $24 \mathrm{~h}$, if III was not full conversed, another $15 \mathrm{mmol}$ aldehyde was added and the mixture was refluxed for another $12 \mathrm{~h}$. After cooling to $0{ }^{\circ} \mathrm{C}$, the reaction was quenched with saturated $\mathrm{NH}_{4} \mathrm{Cl}$ and diluted with EtOAc $(3 \times 50 \mathrm{~mL})$ and washed with water and brine $(3 \times 100 \mathrm{~mL})$, dried over anhydrous $\mathrm{Na}_{2} \mathrm{SO}_{4}$ and evaporated under reduced pressure. The residue was purified by flash silica gel chromatography to give substrates $\mathbf{1}$.<smiles>O=C1/C(=C\c2ccccc2)Oc2ccccc2N1Cc1ccccc1</smiles>

1a

(Z)-4-Benzyl-2-benzylidene-2H-benzo[b][1,4] oxazin-3(4H)-one (1a). Yellow solid, 78\% yield, 2.55 g, m.p. $=142.1-142.5{ }^{\circ} \mathrm{C} .{ }^{1} \mathrm{H}$ NMR $\left(500 \mathrm{MHz}, \mathrm{CDCl}_{3}\right) \delta 7.93-7.89(\mathrm{~m}, 2 \mathrm{H}), 7.48-7.43$ (m, 2H), $7.40-7.32(\mathrm{~m}, 6 \mathrm{H}), 7.22(\mathrm{dd}, J=8.0,1.5 \mathrm{~Hz}, 1 \mathrm{H}), 7.08(\mathrm{~s}, 1 \mathrm{H}), 7.07-7.03(\mathrm{~m}, 1 \mathrm{H})$, $6.99-6.92(\mathrm{~m}, 2 \mathrm{H}), 5.32(\mathrm{~s}, 2 \mathrm{H}) .{ }^{13} \mathrm{C}$ NMR $\left(126 \mathrm{MHz}, \mathrm{CDCl}_{3}\right) \delta 157.6,141.9,140.9,135.7$, 133.7, 130.1, 128.9, 128.6, 128.3, 127.6, 126.7, 126.5, 123.9, 123.2, 116.1, 115.3, 113.5, 45.5. HRMS (ESI) calcd for $\mathrm{C}_{22} \mathrm{H}_{17} \mathrm{NO}_{2}(\mathrm{M}+\mathrm{H})^{+}: 328.1332$; found: 328.1331 . 
<smiles>Cc1ccc(/C=C2\Oc3ccccc3N(Cc3ccccc3)C2=O)cc1</smiles>

(Z)-4-Benzyl-2-(4-methylbenzylidene)-2 $H$-benzo[b][1,4]oxazin-3(4H)-one (1e). Yellow solid, $80 \%$ yield, 2.73 g, m.p. $=146.7-147.0{ }^{\circ} \mathrm{C} .{ }^{1} \mathrm{H}$ NMR $\left(400 \mathrm{MHz}, \mathrm{CDCl}_{3}\right) \delta 7.80-7.74(\mathrm{~m}, 2 \mathrm{H})$, $7.34-7.27(\mathrm{~m}, 4 \mathrm{H}), 7.27-7.19(\mathrm{~m}, 3 \mathrm{H}), 7.19-7.14(\mathrm{~m}, 1 \mathrm{H}), 7.04-6.96(\mathrm{~m}, 2 \mathrm{H}), 6.94-6.84(\mathrm{~m}$, 2H), 5.26 (s, 2H), 2.38 (s, 3H). ${ }^{13} \mathrm{C} \mathrm{NMR}\left(101 \mathrm{MHz}, \mathrm{CDCl}_{3}\right) \delta$ 157.7, 142.0, 140.3, 138.4, 135.7, 130.9, 130.1, 129.3, 128.9, 127.5, 126.7, 126.6, 123.8, 123.1, 116.1, 115.2, 113.6, 45.5, 21.5. HRMS (ESI) calcd for $\mathrm{C}_{23} \mathrm{H}_{19} \mathrm{NO}_{2}(\mathrm{M}+\mathrm{H})^{+}: 342.1489$; found: 342.1493 .<smiles>COc1ccc(/C=C2/Oc3ccccc3N(Cc3ccccc3)C2=O)cc1</smiles>

(Z)-4-Benzyl-2-(4-methoxybenzylidene)-2H-benzo[b][1,4] oxazin-3(4H)-one (1f). Yellow solid, $68 \%$ yield, 2.43 g, m.p. $=206.7-207.6{ }^{\circ} \mathrm{C} .{ }^{1} \mathrm{H}$ NMR $\left(400 \mathrm{MHz}, \mathrm{CDCl}_{3}\right) \delta 7.87-7.80(\mathrm{~m}, 2 \mathrm{H})$, $7.36-7.28(\mathrm{~m}, 4 \mathrm{H}), 7.28-7.22(\mathrm{~m}, 2 \mathrm{H}), 7.16(\mathrm{dd}, J=8.0,1.2 \mathrm{~Hz}, 1 \mathrm{H}), 7.03-6.98(\mathrm{~m}, 2 \mathrm{H}), 6.98$ - $6.94(\mathrm{~m}, 2 \mathrm{H}), 6.94-6.85(\mathrm{~m}, 2 \mathrm{H}), 5.27(\mathrm{~s}, 2 \mathrm{H}), 3.85(\mathrm{~s}, 3 \mathrm{H}) .{ }^{13} \mathrm{C} \mathrm{NMR}\left(101 \mathrm{MHz}, \mathrm{CDCl}_{3}\right) \delta$ 159.6, 157.9, 139.4, 135.8, 131.6, 128.9, 127.5, 126.66, 126.64, 126.5, 123.7, 123.0, 116.0, 115.2, 114.1, 113.4, 55.3, 45.4. HRMS (ESI) calcd for $\mathrm{C}_{23} \mathrm{H}_{19} \mathrm{NO}_{3}(\mathrm{M}+\mathrm{Na})^{+}: 380.1257$; found: 380.1254 .<smiles>CC(C)(C)c1ccc(/C=C2/Oc3ccccc3N(Cc3ccccc3)C2=O)cc1</smiles>

(Z)-4-Benzyl-2-(4-(tert-butyl)benzylidene)-2H-benzo[b][1,4]oxazin-3(4H)-one $\quad(1 \mathrm{~g})$. Yellow solid, 50\% yield, 1.72 g, m.p. $=168.3-169.4{ }^{\circ} \mathrm{C} .{ }^{1} \mathrm{H}$ NMR $\left(400 \mathrm{MHz}, \mathrm{CDCl}_{3}\right) \delta 7.86-7.75(\mathrm{~m}$, 2H), $7.50-7.40(\mathrm{~m}, 2 \mathrm{H}), 7.34-7.26(\mathrm{~m}, 4 \mathrm{H}), 7.26-7.21(\mathrm{~m}, 1 \mathrm{H}), 7.15(\mathrm{dd}, J=8.0,1.2 \mathrm{~Hz}, 1 \mathrm{H})$, $7.03(\mathrm{~s}, 1 \mathrm{H}), 7.01-6.94(\mathrm{~m}, 1 \mathrm{H}), 6.92-6.81(\mathrm{~m}, 2 \mathrm{H}), 5.23(\mathrm{~s}, 2 \mathrm{H}), 1.35(\mathrm{~s}, 9 \mathrm{H}) .{ }^{13} \mathrm{C}$ NMR $(101$ $\left.\mathrm{MHz}, \mathrm{CDCl}_{3}\right) \delta 157.7,151.6,142.0,140.5,135.9,130.9,129.9,128.9,127.5,126.7,126.6,125.6$, 123.8, 123.1, 116.1, 115.3, 113.5, 45.5, 34.8, 31.3. HRMS (ESI) calcd for $\mathrm{C}_{26} \mathrm{H}_{25} \mathrm{NO}_{2}(\mathrm{M}+\mathrm{H})^{+}$: 384.1958; found: 384.1950 . 
<smiles>O=C1/C(=C/c2ccc(F)cc2)Oc2ccccc2N1Cc1ccccc1</smiles>

(Z)-4-Benzyl-2-(4-fluorobenzylidene)-2H-benzo[b][1,4]oxazin-3(4H)-one (1h). Pale yellow solid, 69\% yield, 2.38 g, m.p. $=176.1-177.3{ }^{\circ} \mathrm{C} .{ }^{1} \mathrm{H}$ NMR $\left(400 \mathrm{MHz}, \mathrm{CDCl}_{3}\right) \delta 7.90-7.82(\mathrm{~m}$, 2H), $7.37-7.26(\mathrm{~m}, 5 \mathrm{H}), 7.17(\mathrm{dd}, J=8.0,1.2 \mathrm{~Hz}, 1 \mathrm{H}), 7.15-7.07(\mathrm{~m}, 2 \mathrm{H}), 7.05-6.99(\mathrm{~m}, 2 \mathrm{H})$, $6.97-6.88(\mathrm{~m}, 2 \mathrm{H}), 5.28(\mathrm{~s}, 2 \mathrm{H}) .{ }^{13} \mathrm{C} \mathrm{NMR}\left(101 \mathrm{MHz}, \mathrm{CDCl}_{3}\right) \delta 163.6,161.1,157.5,157.5$, 141.8, 140.5 (d, $J=2.6 \mathrm{~Hz}), 135.6,131.8(\mathrm{~d}, J=8.1 \mathrm{~Hz}), 129.9$ (d, $J=3.3 \mathrm{~Hz}), 128.9,127.6$, 126.7, 126.5, 123.9, 123.3, 116.0, $115.5(\mathrm{t}, J=21.5 \mathrm{~Hz}), 112.3,45.5 .{ }^{19} \mathrm{~F}$ NMR $\left(376 \mathrm{MHz}, \mathrm{CDCl}_{3}\right)$ $\delta$-111.94. HRMS (ESI) calcd for $\mathrm{C}_{22} \mathrm{H}_{16} \mathrm{FNO}_{2}(\mathrm{M}+\mathrm{H})^{+}: 346.1238$; found: 346.1249 .<smiles>O=C1/C(=C/c2ccc(Cl)cc2)Oc2ccccc2N1Cc1ccccc1</smiles>

(Z)-4-Benzyl-2-(4-chlorobenzylidene)-2H-benzo[b][1,4]oxazin-3(4H)-one (1i). Yellow solid, 76\% yield, 2.75 g, m.p. $=166.8-167.9{ }^{\circ} \mathrm{C} .{ }^{1} \mathrm{H}$ NMR $\left(400 \mathrm{MHz}, \mathrm{CDCl}_{3}\right) \delta 7.82-7.75(\mathrm{~m}, 2 \mathrm{H}), 7.41-$ $7.36(\mathrm{~m}, 2 \mathrm{H}), 7.36-7.26(\mathrm{~m}, 5 \mathrm{H}), 7.17(\mathrm{dd}, J=8.0,1.2 \mathrm{~Hz}, 1 \mathrm{H}), 7.05-7.00(\mathrm{~m}, 1 \mathrm{H}), 6.99(\mathrm{~s}$, $1 \mathrm{H}), 6.97-6.88(\mathrm{~m}, 2 \mathrm{H}), 5.28(\mathrm{~s}, 2 \mathrm{H}) .{ }^{13} \mathrm{C} \mathrm{NMR}\left(101 \mathrm{MHz}, \mathrm{CDCl}_{3}\right) \delta 157.3,141.7,141.2,135.5$, 133.9, 132.2, 131.2, 128.9, 128.8, 127.6, 126.7, 126.4, 123.9, 123.4, 116.1, 115.3, 112.1, 45.6. HRMS (ESI) calcd for $\mathrm{C}_{22} \mathrm{H}_{16} \mathrm{ClNO}_{2}(\mathrm{M}+\mathrm{H})^{+}: 362.0942$; found: 362.0943 .<smiles>O=C1/C(=C/c2ccc(Br)cc2)Oc2ccccc2N1Cc1ccccc1</smiles>

(Z)-4-Benzyl-2-(4-bromobenzylidene)-2H-benzo[b][1,4]oxazin-3(4H)-one (1j). Yellow solid, $79 \%$ yield, 3.21 g, m.p. $=166.7-168.2{ }^{\circ} \mathrm{C} .{ }^{1} \mathrm{H}$ NMR $\left(400 \mathrm{MHz}, \mathrm{CDCl}_{3}\right) \delta 7.76-7.69(\mathrm{~m}, 2 \mathrm{H})$, $7.57-7.51(\mathrm{~m}, 2 \mathrm{H}), 7.36-7.25(\mathrm{~m}, 5 \mathrm{H}), 7.17(\mathrm{dd}, J=8.0,1.2 \mathrm{~Hz}, 1 \mathrm{H}), 7.06-7.00(\mathrm{~m}, 1 \mathrm{H}), 6.97$ (s, 1H), $6.95-6.88(\mathrm{~m}, 2 \mathrm{H}), 5.28(\mathrm{~s}, 2 \mathrm{H}) .{ }^{13} \mathrm{C} \mathrm{NMR}\left(101 \mathrm{MHz}, \mathrm{CDCl}_{3}\right) \delta$ 157.3, 141.7, 141.3, 135.5, 132.6, 131.7, 131.4, 128.9, 127.6, 126.7, 126.4, 123.9, 123.4, 122.2, 116.1, 115.3, 112.1, 45.6. HRMS (ESI) calcd for $\mathrm{C}_{22} \mathrm{H}_{16} \mathrm{BrNO}_{2}(\mathrm{M}+\mathrm{Na})^{+}: 428.0257$; found: 428.0256 . 
<smiles>O=C1/C(=C/c2ccc(C(F)(F)F)cc2)Oc2ccccc2N1Cc1ccccc1</smiles>

(Z)-4-Benzyl-2-(4-(trifluoromethyl)benzylidene)-2H-benzo[b][1,4]oxazin-3(4H)-one

(1k). Yellow solid, 74\% yield, 2.92 g, m.p. $=177.6-179.4{ }^{\circ} \mathrm{C} .{ }^{1} \mathrm{H}$ NMR $\left(500 \mathrm{MHz}, \mathrm{CDCl}_{3}\right) \delta 7.95(\mathrm{~d}, J$ $=8.0 \mathrm{~Hz}, 2 \mathrm{H}), 7.66(\mathrm{~d}, J=8.5 \mathrm{~Hz}, 2 \mathrm{H}), 7.37-7.25(\mathrm{~m}, 5 \mathrm{H}), 7.20(\mathrm{dd}, J=8.0,1.0 \mathrm{~Hz}, 1 \mathrm{H}), 7.08-$ $7.02(\mathrm{~m}, 2 \mathrm{H}), 7.00-6.91(\mathrm{~m}, 2 \mathrm{H}), 5.29(\mathrm{~s}, 2 \mathrm{H}) .{ }^{13} \mathrm{C} \mathrm{NMR}\left(126 \mathrm{MHz}, \mathrm{CDCl}_{3}\right) \delta 157.0,142.3$, $141.5,137.1,135.4,130.0,129.6(\mathrm{q}, J=32.4 \mathrm{~Hz}), 129.0,127.7,126.7,126.3,125.4(\mathrm{q}, J=3.9$ $\mathrm{Hz}), 124.1(\mathrm{q}, J=272.4 \mathrm{~Hz}), 124.08,123.6,116.1,115.4,111.5,45.7 .{ }^{19} \mathrm{~F}$ NMR $(471 \mathrm{MHz}$, $\left.\mathrm{CDCl}_{3}\right) \delta$-62.62. HRMS (ESI) calcd for $\mathrm{C}_{23} \mathrm{H}_{16} \mathrm{~F}_{3} \mathrm{NO}_{2}(\mathrm{M}+\mathrm{H})^{+}$: 396.1206; found: 396.1207 .<smiles>O=C1/C(=C/c2ccc(OC(F)(F)F)cc2)Oc2ccccc2N1Cc1ccccc1</smiles>

(Z)-4-Benzyl-2-(4-(trifluoromethyl)benzylidene)-2H-benzo[b][1,4] oxazin-3(4H)-one (11). Pale yellow solid, $56 \%$ yield, 2.30 g, m.p. $=168.5-170.3{ }^{\circ} \mathrm{C} .{ }^{1} \mathrm{H}$ NMR $\left(500 \mathrm{MHz}, \mathrm{CDCl}_{3}\right) \delta 7.92-$ $7.87(\mathrm{~m}, 2 \mathrm{H}), 7.37-7.24(\mathrm{~m}, 7 \mathrm{H}), 7.18(\mathrm{dd}, J=8.0,1.5 \mathrm{~Hz}, 1 \mathrm{H}), 7.06-7.00(\mathrm{~m}, 2 \mathrm{H}), 6.98-6.90$ (m, 2H), 5.29 (s, 2H). ${ }^{13} \mathrm{C}$ NMR $\left(126 \mathrm{MHz}, \mathrm{CDCl}_{3}\right) \delta 157.3,148.7,141.7,141.2,135.5,132.4$, $131.4,129.0,127.6,126.7,126.4,124.0,123.4,120.9,116.1,115.4,111.7,45.6 .{ }^{19} \mathrm{~F}$ NMR $(376$ $\mathrm{MHz}, \mathrm{CDCl}_{3}$ ) $\delta$-57.69. HRMS (ESI) calcd for $\mathrm{C}_{23} \mathrm{H}_{16} \mathrm{~F}_{3} \mathrm{NO}_{3}(\mathrm{M}+\mathrm{H})^{+}$: 412.1155; found: 412.1151 .<smiles>CCOC(=O)c1ccc(/C=C2\Oc3ccccc3N(Cc3ccccc3)C2=O)cc1</smiles>

Ethyl(Z)-4-((4-benzyl-3-oxo-3,4-dihydro-2H-benzo[b][1,4]oxazin-2-ylidene)methyl)benzoate (1m). Yellow solid, 77\% yield, 3.07 g, m.p. $=145.2-146.7{ }^{\circ} \mathrm{C} .{ }^{1} \mathrm{H}$ NMR $\left(400 \mathrm{MHz}, \mathrm{CDCl}_{3}\right) \delta$ $8.14-8.03(\mathrm{~m}, 2 \mathrm{H}), 7.94-7.86(\mathrm{~m}, 2 \mathrm{H}), 7.36-7.25(\mathrm{~m}, 5 \mathrm{H}), 7.19(\mathrm{dd}, J=8.0,1.2 \mathrm{~Hz}, 1 \mathrm{H}), 7.07$ $-7.00(\mathrm{~m}, 2 \mathrm{H}), 6.98-6.88(\mathrm{~m}, 2 \mathrm{H}), 5.28(\mathrm{~s}, 2 \mathrm{H}), 4.39(\mathrm{q}, J=7.2 \mathrm{~Hz}, 2 \mathrm{H}), 1.41(\mathrm{t}, J=7.2 \mathrm{~Hz}, 3 \mathrm{H})$ ${ }^{13} \mathrm{C}$ NMR $\left(101 \mathrm{MHz}, \mathrm{CDCl}_{3}\right) \delta 166.3,157.0,142.2,141.6,138.0,135.5,129.7,129.6,129.0$, 127.6, 126.7, 126.3, 124.0, 123.5, 116.2, 115.4, 112.0, 61.0, 45.6, 14.4. HRMS (ESI) calcd for $\mathrm{C}_{25} \mathrm{H}_{21} \mathrm{NO}_{4}(\mathrm{M}+\mathrm{Na})^{+}$: 422.1363; found: 422.1364 . 
<smiles>O=C1/C(=C/c2cccc(Cl)c2)Oc2ccccc2N1Cc1ccccc1</smiles>

(Z)-4-Benzyl-2-(3-chlorobenzylidene)-2H-benzo[b][1,4]oxazin-3(4H)-one (1n). Yellow solid, $80 \%$ yield, 2.89 g, m.p. $=129.7-130.5{ }^{\circ} \mathrm{C} .{ }^{1} \mathrm{H}$ NMR $\left(400 \mathrm{MHz}, \mathrm{CDCl}_{3}\right) \delta 7.97-7.90(\mathrm{~m}, 1 \mathrm{H})$, $7.71-7.64(\mathrm{~m}, 1 \mathrm{H}), 7.37-7.26(\mathrm{~m}, 7 \mathrm{H}), 7.23-7.18(\mathrm{~m}, 1 \mathrm{H}), 7.07-7.01(\mathrm{~m}, 1 \mathrm{H}), 6.99-6.90(\mathrm{~m}$, $3 \mathrm{H}), 5.29(\mathrm{~s}, 2 \mathrm{H}) .{ }^{13} \mathrm{C}$ NMR $\left(101 \mathrm{MHz}, \mathrm{CDCl}_{3}\right) \delta 157.1,141.7,141.6,135.5,135.4,134.4,129.7$, 129.6, 129.0, 128.2, 128.2, 127.6, 126.7, 126.3, 124.0, 123.5, 116.2, 115.3, 111.8, 45.6. HRMS (ESI) calcd for $\mathrm{C}_{22} \mathrm{H}_{16} \mathrm{ClNO}_{2}(\mathrm{M}+\mathrm{H})^{+}: 362.0942$; found: 362.0941 .<smiles>COc1cccc(/C=C2\Oc3ccccc3N(Cc3ccccc3)C2=O)c1</smiles>

(Z)-4-Benzyl-2-(3-methoxybenzylidene)-2H-benzo[b][1,4] oxazin-3(4H)-one (10). Yellow solid, $86 \%$ yield, 3.07 g, m.p. $=117.7-118.5{ }^{\circ} \mathrm{C} .{ }^{1} \mathrm{H}$ NMR $\left(400 \mathrm{MHz}, \mathrm{CDCl}_{3}\right) \delta 7.51-7.48(\mathrm{~m}, 1 \mathrm{H})$, $7.45-7.40(\mathrm{~m}, 1 \mathrm{H}), 7.36-7.28(\mathrm{~m}, 5 \mathrm{H}), 7.18-7.14(\mathrm{~m}, 1 \mathrm{H}), 7.04-6.98(\mathrm{~m}, 2 \mathrm{H}), 6.96-6.86(\mathrm{~m}$, $3 \mathrm{H}), 5.28(\mathrm{~s}, 2 \mathrm{H}), 3.87(\mathrm{~s}, 3 \mathrm{H}) .{ }^{13} \mathrm{C} \mathrm{NMR}\left(101 \mathrm{MHz}, \mathrm{CDCl}_{3}\right) \delta 159.5,157.5,141.8,141.0,135.6$, 134.9, 129.4, 128.9, 127.6, 126.7, 126.5, 123.8, 123.2, 122.9, 116.1, 115.3, 115.0, 114.3, 113.3, 55.2, 45.5. HRMS (ESI) calcd for $\mathrm{C}_{23} \mathrm{H}_{19} \mathrm{NO}_{3}(\mathrm{M}+\mathrm{Na})^{+}: 380.1257$; found: 380.1266 .<smiles>O=C1/C(=C\c2ccccc2Cl)Oc2ccccc2N1Cc1ccccc1</smiles>

(Z)-4-Benzyl-2-(2-chlorobenzylidene)-2H-benzo[b][1,4]oxazin-3(4H)-one (1p). Yellow solid, $53 \%$ yield, 1.92 g, m.p. $=152.1-152.4{ }^{\circ} \mathrm{C} .{ }^{1} \mathrm{H}$ NMR $\left(400 \mathrm{MHz}, \mathrm{CDCl}_{3}\right) \delta 8.26(\mathrm{dd}, J=7.6,1.6$ $\mathrm{Hz}, 1 \mathrm{H}), 7.48-7.42(\mathrm{~m}, 2 \mathrm{H}), 7.38-7.30(\mathrm{~m}, 5 \mathrm{H}), 7.29-7.21(\mathrm{~m}, 2 \mathrm{H}), 7.16-7.10(\mathrm{~m}, 1 \mathrm{H}), 7.04$ - $6.98(\mathrm{~m}, 1 \mathrm{H}), 6.97-6.90(\mathrm{~m}, 2 \mathrm{H}), 5.29(\mathrm{~s}, 2 \mathrm{H}) .{ }^{13} \mathrm{C} \mathrm{NMR}\left(101 \mathrm{MHz}, \mathrm{CDCl}_{3}\right) \delta 157.2,141.9$, 141.6, 135.6, 134.5, 131.6, 130.6, 129.7, 129.1, 128.9, 127.6, 126.8, 126.6, 126.5, 123.9, 123.4, 116.1, 115.3, 108.9, 45.6. HRMS (ESI) calcd for $\mathrm{C}_{22} \mathrm{H}_{16} \mathrm{ClNO}_{2}(\mathrm{M}+\mathrm{Na})^{+}$: 384.0762; found: 384.0763 .<smiles>Cc1ccccc1/C=C1\Oc2ccccc2N(Cc2ccccc2)C1=O</smiles> 
(Z)-4-Benzyl-2-(2-methoxybenzylidene)-2H-benzo[b][1,4]oxazin-3(4H)-one (1q). Yellow solid, $41 \%$ yield, 1.40 g, m.p. $=98.2-98.7{ }^{\circ} \mathrm{C} .{ }^{1} \mathrm{H}$ NMR $\left(400 \mathrm{MHz}, \mathrm{CDCl}_{3}\right) \delta 8.15-8.10(\mathrm{~m}, 1 \mathrm{H}), 7.35$ $-7.26(\mathrm{~m}, 6 \mathrm{H}), 7.24-7.22(\mathrm{~m}, 2 \mathrm{H}), 7.14-7.10(\mathrm{~m}, 1 \mathrm{H}), 7.03-6.97(\mathrm{~m}, 1 \mathrm{H}), 6.95-6.88(\mathrm{~m}$, 2H), $5.28(\mathrm{~s}, 2 \mathrm{H}), 2.46(\mathrm{~s}, 3 \mathrm{H}) .{ }^{13} \mathrm{C} \mathrm{NMR}\left(101 \mathrm{MHz}, \mathrm{CDCl}_{3}\right) \delta 157.7,141.8,140.8,137.6,135.7$, 132.1, 130.3, 129.4, 128.9, 128.2, 127.6, 126.7, 126.6, 125.9, 123.9, 123.1, 116.1, 115.2, 110.8, 45.6, 20.5. HRMS (ESI) calcd for $\mathrm{C}_{23} \mathrm{H}_{19} \mathrm{NO}_{2}(\mathrm{M}+\mathrm{H})^{+}: 342.1489$; found: 342.1490 .

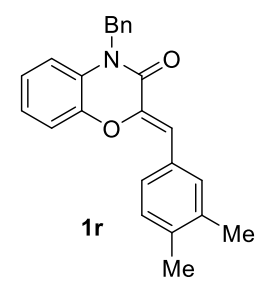

(Z)-4-Benzyl-2-(3,4-dimethylbenzylidene)-2H-benzo[b][1,4]oxazin-3(4H)-one (1r). Yellow solid, $82 \%$ yield, $2.91 \mathrm{~g}$, m.p. $=137.3-138.8^{\circ} \mathrm{C} .{ }^{1} \mathrm{H}$ NMR $\left(400 \mathrm{MHz}, \mathrm{CDCl}_{3}\right) \delta 7.69-7.63(\mathrm{~m}$, $1 \mathrm{H}), 7.61(\mathrm{~s}, 1 \mathrm{H}), 7.35-7.24(\mathrm{~m}, 5 \mathrm{H}), 7.21-7.13(\mathrm{~m}, 2 \mathrm{H}), 7.02-6.96(\mathrm{~m}, 2 \mathrm{H}), 6.94-6.84(\mathrm{~m}$, 2H), 5.26 (s, 2H), 2.31 (s, 3H), 2.29 (s, 3H). $\left.{ }^{13} \mathrm{C} \mathrm{NMR} \mathrm{(101} \mathrm{MHz,} \mathrm{CDCl}_{3}\right) \delta 157.8,142.1,140.2$, 137.3, 136.6, 135.8, 131.5, 131.3, 129.9, 128.9, 127.6, 127.5, 126.7, 126.6, 123.7, 123.1, 116.0, 115.2, 113.8, 45.5, 20.0, 19.8. HRMS (ESI) calcd for $\mathrm{C}_{24} \mathrm{H}_{21} \mathrm{NO}_{2}(\mathrm{M}+\mathrm{Na})^{+}$: 378.1465; found: 378.1472 .

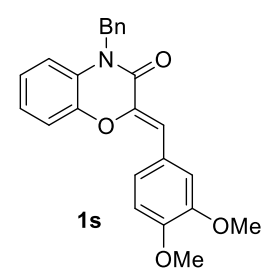

(Z)-4-Benzyl-2-(3,4-dimethoxybenzylidene)-2H-benzo[b][1,4]oxazin-3(4H)-one (1s). Yellow solid, 85\% yield, 3.29 g, m.p. $=142.1-143.9{ }^{\circ} \mathrm{C} .{ }^{1} \mathrm{H}$ NMR $\left(500 \mathrm{MHz}, \mathrm{CDCl}_{3}\right) \delta 7.57(\mathrm{~d}, J=2.0$ $\mathrm{Hz}, 1 \mathrm{H}), 7.41(\mathrm{dd}, J=8.5,2.0 \mathrm{~Hz}, 1 \mathrm{H}), 7.36-7.26(\mathrm{~m}, 5 \mathrm{H}), 7.10(\mathrm{dd}, J=8.0,1.5 \mathrm{~Hz}, 1 \mathrm{H}), 7.03-$ 6.97 (m, 2H), $6.94-6.87$ (m, 3H), 5.27 (s, 2H), 3.97 (s, 3H), 3.93 (s, 3H). ${ }^{13} \mathrm{C}$ NMR (126 MHz, $\left.\mathrm{CDCl}_{3}\right) \delta 157.8,149.3,148.7,142.0,139.6,135.7,128.9,127.5,126.7,126.7,123.8,123.7,123.1$, 115.8, 115.3, 113.6, 112.7, 111.1, 55.9, 55.8, 45.4. HRMS (ESI) calcd for $\mathrm{C}_{24} \mathrm{H}_{21} \mathrm{NO}_{4}(\mathrm{M}+\mathrm{Na})^{+}$: 410.1363; found: 410.1360 .

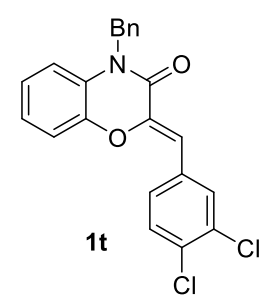

(Z)-4-Benzyl-2-(3,4-dichlorobenzylidene)-2H-benzo[b][1,4]oxazin-3(4H)-one $\quad(1 \mathrm{t}) . \quad$ Yellow solid, 78\% yield, 3.09 g, m.p. $=174.1-175.4{ }^{\circ} \mathrm{C} .{ }^{1} \mathrm{H}$ NMR $\left(400 \mathrm{MHz}, \mathrm{CDCl}_{3}\right) \delta 8.01(\mathrm{~d}, J=2.0$ $\mathrm{Hz}, 1 \mathrm{H}), 7.63(\mathrm{dd}, J=8.4,2.4 \mathrm{~Hz}, 1 \mathrm{H}), 7.46(\mathrm{~d}, J=8.4 \mathrm{~Hz}, 1 \mathrm{H}), 7.37-7.25(\mathrm{~m}, 5 \mathrm{H}), 7.19(\mathrm{dd}, J$ $=8.0,1.2 \mathrm{~Hz}, 1 \mathrm{H}), 7.08-7.02(\mathrm{~m}, 1 \mathrm{H}), 7.00-6.90(\mathrm{~m}, 3 \mathrm{H}), 5.28(\mathrm{~s}, 2 \mathrm{H}) .{ }^{13} \mathrm{C} \mathrm{NMR}(101 \mathrm{MHz}$, 
$\left.\mathrm{CDCl}_{3}\right) \delta 156.9,141.9,141.5,135.4,133.7,132.6,131.9,131.3,130.4,129.1,129.0,127.6,126.7$, 126.3, 124.0, 123.6, 116.1, 115.4, 110.7, 45.6. HRMS (ESI) calcd for $\mathrm{C}_{22} \mathrm{H}_{15} \mathrm{Cl}_{2} \mathrm{NO}_{2}(\mathrm{M}+\mathrm{H})^{+}$: 396.0553; found: 396.0554 .<smiles>O=C1/C(=C/c2ccc3c(c2)OCO3)Oc2ccccc2N1Cc1ccccc1</smiles>

(Z)-2-(Benzo $[d][1,3]$ dioxol-5-ylmethylene)-4-benzyl-2H-benzo[b][1,4]oxazin-3(4H)-one $\quad(1 \mathrm{u})$. Yellow solid, $67 \%$ yield, $2.48 \mathrm{~g}$, m.p. $=161.5-162.4{ }^{\circ} \mathrm{C} .{ }^{1} \mathrm{H}$ NMR $\left(500 \mathrm{MHz}, \mathrm{CDCl}_{3}\right) \delta 7.58-$ $7.51(\mathrm{~m}, 1 \mathrm{H}), 7.36-7.25(\mathrm{~m}, 5 \mathrm{H}), 7.18-7.13(\mathrm{~m}, 1 \mathrm{H}), 7.01-7.00(\mathrm{~m}, 1 \mathrm{H}), 6.95(\mathrm{~s}, 1 \mathrm{H}), 6.93-$ $6.82(\mathrm{~m}, 3 \mathrm{H}), 5.99$ (s, 2H), 5.25 (s, 2H). ${ }^{13} \mathrm{C}$ NMR $\left(126 \mathrm{MHz}, \mathrm{CDCl}_{3}\right) \delta$ 157.7, 147.8, 147.7, 142.0, 139.6, 135.7, 128.9, 128.0, 127.5, 126.7, 126.6, 125.3, 123.8, 123.1, 116.0, 115.2, 113.5, 109.6, 108.5, 101.3, 45.5. HRMS (ESI) calcd for $\mathrm{C}_{23} \mathrm{H}_{17} \mathrm{NO}_{4}(\mathrm{M}+\mathrm{H})^{+}$: 372.1230; found: 372.1233 .<smiles>O=C1/C(=C/c2ccc3ccccc3c2)Oc2ccccc2N1Cc1ccccc1</smiles>

(Z)-4-Benzyl-2-(naphthalen-2-ylmethylene)-2H-benzo[b][1,4]oxazin-3(4H)-one (1v). Yellow solid, $82 \%$ yield, 3.09 g, m.p. $=137.4-139.9{ }^{\circ} \mathrm{C} .{ }^{1} \mathrm{H}$ NMR $\left(500 \mathrm{MHz}, \mathrm{CDCl}_{3}\right) \delta 8.28(\mathrm{br}, 1 \mathrm{H})$, $7.99(\mathrm{dd}, J=8.5,2.0 \mathrm{~Hz}, 1 \mathrm{H}), 7.89-7.77(\mathrm{~m}, 3 \mathrm{H}), 7.51-7.43(\mathrm{~m}, 2 \mathrm{H}), 7.34-7.27$ (m, 4H), 7.26 $-7.22(\mathrm{~m}, 1 \mathrm{H}), 7.22-7.16(\mathrm{~m}, 2 \mathrm{H}), 7.04-6.97(\mathrm{~m}, 1 \mathrm{H}), 6.94-6.83(\mathrm{~m}, 2 \mathrm{H}), 5.23(\mathrm{~s}, 2 \mathrm{H}) .{ }^{13} \mathrm{C}$ NMR $\left(126 \mathrm{MHz}, \mathrm{CDCl}_{3}\right) \delta 157.5,141.9,141.1,135.7,133.4,133.0,131.3,129.9,129.0,128.6$, 128.01, 127.7, 127.6, 127.4, 126.7, 126.6, 126.5, 126.3, 123.9, 123.3, 116.1, 115.3, 113.5, 45.5. HRMS (ESI) calcd for $\mathrm{C}_{26} \mathrm{H}_{19} \mathrm{NO}_{2}(\mathrm{M}+\mathrm{Na})^{+}$: 400.1308; found: 400.1309 .<smiles>O=C1/C(=C/c2cccc3ccccc23)Oc2ccccc2N1Cc1ccccc1</smiles>

(Z)-4-Benzyl-2-(naphthalen-1-ylmethylene)-2H-benzo[b][1,4]oxazin-3(4H)-one (1w). Yellow solid, $80 \%$ yield, 3.02 g, m.p. $=135.7-136.8{ }^{\circ} \mathrm{C} .{ }^{1} \mathrm{H}$ NMR $\left(500 \mathrm{MHz}, \mathrm{CDCl}_{3}\right) \delta 8.30-8.23(\mathrm{~m}$, 2H), $7.90-7.82(\mathrm{~m}, 2 \mathrm{H}), 7.79(\mathrm{~s}, 1 \mathrm{H}), 7.60-7.48(\mathrm{~m}, 3 \mathrm{H}), 7.37-7.32(\mathrm{~m}, 4 \mathrm{H}), 7.30-7.24(\mathrm{~m}$, 1H), $7.11-7.07(\mathrm{~m}, 1 \mathrm{H}), 7.01-6.96(\mathrm{~m}, 1 \mathrm{H}), 6.94-6.89(\mathrm{~m}, 2 \mathrm{H}), 5.31(\mathrm{~s}, 2 \mathrm{H}) .{ }^{13} \mathrm{C}$ NMR $(126$ $\left.\mathrm{MHz}, \mathrm{CDCl}_{3}\right) \delta 157.7,141.9,141.6,135.7,133.7,131.9,129.7,129.0,128.8,128.7,127.7,127.6$, 126.7, 126.6, 126.5, 125.9, 125.4, 124.1, 123.9, 123.2, 116.2, 115.2, 109.7, 45.6. HRMS (ESI) calcd for $\mathrm{C}_{26} \mathrm{H}_{19} \mathrm{NO}_{2}(\mathrm{M}+\mathrm{H})^{+}$: 378.1489 ; found: 378.1498 . 
<smiles>O=C1/C(=C\c2ccco2)Oc2ccccc2N1Cc1ccccc1</smiles>

(Z)-4-Benzyl-2-(furan-2-ylmethylene)-2H-benzo[b][1,4]oxazin-3(4H)-one (1x). Yellow solid, $70 \%$ yield, 2.22 g, m.p. $=175.4-176.0{ }^{\circ} \mathrm{C} .{ }^{1} \mathrm{H}$ NMR $\left(500 \mathrm{MHz}, \mathrm{CDCl}_{3}\right) \delta 7.50(\mathrm{~d}, J=1.5 \mathrm{~Hz}$, $1 \mathrm{H}), 7.35-7.22(\mathrm{~m}, 5 \mathrm{H}), 7.16(\mathrm{dd}, J=8.0,1.5 \mathrm{~Hz}, 1 \mathrm{H}), 7.07-7.04(\mathrm{~m}, 2 \mathrm{H}), 7.03-6.99(\mathrm{~m}, 1 \mathrm{H})$, $6.96-6.87(\mathrm{~m}, 2 \mathrm{H}), 6.55(\mathrm{dd}, J=3.5,2.0 \mathrm{~Hz}, 1 \mathrm{H}), 5.27(\mathrm{~s}, 2 \mathrm{H}) .{ }^{13} \mathrm{C} \mathrm{NMR}\left(126 \mathrm{MHz}, \mathrm{CDCl}_{3}\right) \delta$ 156.9, 149.7, 142.9, 141.9, 139.0, 135.6, 128.9, 127.6, 126.7, 126.5, 123.8, 123.3, 116.1, 115.3, 112.9, 112.3, 103.0, 45.4. HRMS (ESI) calcd for $\mathrm{C}_{20} \mathrm{H}_{15} \mathrm{NO}_{3}(\mathrm{M}+\mathrm{Na})^{+}$: 340.0944; found: 340.0952 .<smiles>O=C1/C(=C\c2cccs2)Oc2ccccc2N1Cc1ccccc1</smiles>

(Z)-4-Benzyl-2-(thiophen-2-ylmethylene)-2H-benzo $[b][1,4]$ oxazin-3(4H)-one $\quad(1 y)$. Yellow solid, 63\% yield, 2.10 g, m.p. $=134.0-135.2{ }^{\circ} \mathrm{C} .{ }^{1} \mathrm{H}$ NMR $\left(500 \mathrm{MHz}, \mathrm{CDCl}_{3}\right) \delta 7.50-7.43(\mathrm{~m}, 1 \mathrm{H})$, $7.38-7.22(\mathrm{~m}, 8 \mathrm{H}), 7.11(\mathrm{dd}, J=5.0,3.5 \mathrm{~Hz}, 1 \mathrm{H}), 7.06-6.99(\mathrm{~m}, 1 \mathrm{H}), 6.96-6.85(\mathrm{~m}, 2 \mathrm{H}), 5.27(\mathrm{~s}$, 2H). ${ }^{13} \mathrm{C}$ NMR $\left(126 \mathrm{MHz}, \mathrm{CDCl}_{3}\right) \delta 157.3,142.0,138.7,136.3,135.6,129.8,128.9,128.4,127.5$, 127.1, 126.7, 126.5, 123.9, 123.3, 116.2, 115.3, 108.2, 45.3. HRMS (ESI) calcd for $\mathrm{C}_{20} \mathrm{H}_{15} \mathrm{NO}_{2} \mathrm{~S}$ $(\mathrm{M}+\mathrm{H})^{+}:$334.0896; found: 334.0894 .<smiles>O=C1/C(=C\c2ccsc2)Oc2ccccc2N1Cc1ccccc1</smiles>

(Z)-4-Benzyl-2-(thiophen-3-ylmethylene)-2H-benzo[b][1,4]oxazin-3(4H)-one (1z). Pale yellow solid, 59\% yield, 1.96 g, m.p. $=151.5-152.9^{\circ} \mathrm{C} .{ }^{1} \mathrm{H} \mathrm{NMR}\left(500 \mathrm{MHz}, \mathrm{CDCl}_{3}\right) \delta 7.86-7.81(\mathrm{~m}$, $1 \mathrm{H}), 7.53(\mathrm{dd}, J=5.0,1.0 \mathrm{~Hz}, 1 \mathrm{H}), 7.36-7.23(\mathrm{~m}, 6 \mathrm{H}), 7.15(\mathrm{dd}, J=8.0,1.5 \mathrm{~Hz}, 1 \mathrm{H}), 7.10(\mathrm{~s}$, 1H), $7.03-6.98(\mathrm{~m}, 1 \mathrm{H}), 6.94-6.86(\mathrm{~m}, 2 \mathrm{H}), 5.26(\mathrm{~s}, 2 \mathrm{H}) .{ }^{13} \mathrm{C} \mathrm{NMR}\left(126 \mathrm{MHz}, \mathrm{CDCl}_{3}\right) \delta 157.6$, 142.0, 139.9, 135.7, 134.6, 129.0, 128.9, 127.5, 126.7, 126.6, 126.5, 125.3, 123.8, 123.2, 116.0, 115.3, 108.1, 45.4. HRMS (ESI) calcd for $\mathrm{C}_{20} \mathrm{H}_{15} \mathrm{NO}_{2} \mathrm{~S}(\mathrm{M}+\mathrm{H})^{+}$: 334.0896; found: 334.0898 . 
<smiles>O=C1/C(=C/C2CCCCC2)Oc2ccccc2N1Cc1ccccc1</smiles>

(Z)-4-Benzyl-2-(cyclohexylmethylene)-2 $H$-benzo[b][1,4]oxazin-3(4H)-one (1aa). Yellow solid, $57 \%$ yield, 1.89 g, m.p. $=99.5-101.8{ }^{\circ} \mathrm{C} .{ }^{1} \mathrm{H}$ NMR $\left(500 \mathrm{MHz}, \mathrm{CDCl}_{3}\right) \delta 7.34-7.22(\mathrm{~m}, 5 \mathrm{H})$, $7.03(\mathrm{dd}, J=8.0,1.5 \mathrm{~Hz}, 1 \mathrm{H}), 6.98-6.93(\mathrm{~m}, 1 \mathrm{H}), 6.89-6.81(\mathrm{~m}, 2 \mathrm{H}), 6.06(\mathrm{~d}, J=9.5 \mathrm{~Hz}, 1 \mathrm{H})$, $5.20(\mathrm{~s}, 2 \mathrm{H}), 2.77-2.64(\mathrm{~m}, 1 \mathrm{H}), 1.83-1.73(\mathrm{~m}, 4 \mathrm{H}), 1.44-1.32(\mathrm{~m}, 2 \mathrm{H}), 1.31-1.17(\mathrm{~m}, 4 \mathrm{H})$. ${ }^{13} \mathrm{C}$ NMR $\left(126 \mathrm{MHz}, \mathrm{CDCl}_{3}\right) \delta 157.9,142.7,140.0,135.9,128.89,127.49,126.7,126.6,123.6$, $122.8,122.5,115.8,115.1,45.2,34.2,32.3,26.0,25.7$. HRMS (ESI) calcd for $\mathrm{C}_{22} \mathrm{H}_{23} \mathrm{NO}_{2}(\mathrm{M}+\mathrm{H})^{+}$: 334.1802; found: 334.1802 .<smiles>O=C1/C(=C/C2CCCC2)Oc2ccccc2N1Cc1ccccc1</smiles>

(Z)-4-Benzyl-2-(cyclopentylmethylene)-2H-benzo[b][1,4]oxazin-3(4H)-one (1ab). Yellow oil, 24\% yield, 0.76 g. ${ }^{1} \mathrm{H}$ NMR (500 MHz, $\left.\mathrm{CDCl}_{3}\right) \delta 7.35-7.20(\mathrm{~m}, 5 \mathrm{H}), 7.05-6.99(\mathrm{~m}, 1 \mathrm{H}), 6.98-$ $6.92(\mathrm{~m}, 1 \mathrm{H}), 6.89-6.80(\mathrm{~m}, 2 \mathrm{H}), 6.15(\mathrm{~d}, J=9.5 \mathrm{~Hz}, 1 \mathrm{H}), 5.21(\mathrm{~s}, 2 \mathrm{H}), 3.16-3.02(\mathrm{~m}, 1 \mathrm{H})$, $2.00-1.88(\mathrm{~m}, 2 \mathrm{H}), 1.82-1.70(\mathrm{~m}, 2 \mathrm{H}), 1.68-1.61(\mathrm{~m}, 2 \mathrm{H}), 1.49-1.34(\mathrm{~m}, 2 \mathrm{H}) .{ }^{13} \mathrm{C} \mathrm{NMR}$ $\left(126 \mathrm{MHz}, \mathrm{CDCl}_{3}\right) \delta 157.8,142.7,140.4,135.8,128.9,127.4,126.64,126.59,123.7,122.8,122.5$, 115.8, 115.1, 45.2, 35.9, 33.2, 25.4. HRMS (ESI) calcd for $\mathrm{C}_{21} \mathrm{H}_{21} \mathrm{NO}_{2}(\mathrm{M}+\mathrm{Na})^{+}: 342.1465$; found: 342.1457 .<smiles>O=C1/C(=C/C2CC2)Oc2ccccc2N1Cc1ccccc1</smiles>

(Z)-4-Benzyl-2-(cyclopropylmethylene)-2H-benzo[b][1,4]oxazin-3(4H)-one (1ac). White solid, $79 \%$ yield, 2.30 g, m.p. $=105.8-106.7{ }^{\circ} \mathrm{C} .{ }^{1} \mathrm{H}$ NMR $\left(500 \mathrm{MHz}, \mathrm{CDCl}_{3}\right) \delta 7.38-7.24(\mathrm{~m}, 5 \mathrm{H})$, $7.06(\mathrm{dd}, J=8.0,1.5 \mathrm{~Hz}, 1 \mathrm{H}), 7.01-6.96(\mathrm{~m}, 1 \mathrm{H}), 6.92-6.83(\mathrm{~m}, 2 \mathrm{H}), 5.69(\mathrm{~d}, J=10.5 \mathrm{~Hz}, 1 \mathrm{H})$, $5.23(\mathrm{~s}, 2 \mathrm{H}), 2.11-2.00(\mathrm{~m}, 1 \mathrm{H}), 1.05-0.98(\mathrm{~m}, 2 \mathrm{H}), 0.73-0.65(\mathrm{~m}, 2 \mathrm{H}) .{ }^{13} \mathrm{C}$ NMR $(126 \mathrm{MHz}$, $\left.\mathrm{CDCl}_{3}\right) \delta 157.6,142.7,141.2,135.9,128.9,127.4,126.7,126.6,123.7,122.6,122.5,115.8,115.1$, 45.1, 8.6, 8.2. HRMS (ESI) calcd for $\mathrm{C}_{19} \mathrm{H}_{17} \mathrm{NO}_{2}(\mathrm{M}+\mathrm{H})^{+}: 292.1332$; found: 292.1332 . 


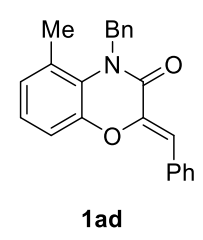

(Z)-4-Benzyl-2-benzylidene-5-methyl-2H-benzo[b][1,4]oxazin-3(4H)-one (1ad). Yellow solid, $83 \%$ yield, 2.83 g, m.p. $=146.5-148.1{ }^{\circ} \mathrm{C} .{ }^{1} \mathrm{H}_{\mathrm{NMR}}\left(500 \mathrm{MHz}, \mathrm{CDCl}_{3}\right) \delta 7.89-7.81(\mathrm{~m}, 2 \mathrm{H})$, $7.44-7.38(\mathrm{~m}, 2 \mathrm{H}), 7.34-7.26(\mathrm{~m}, 3 \mathrm{H}), 7.24-7.16(\mathrm{~m}, 3 \mathrm{H}), 7.09$ (dd, J=8.0, $1.5 \mathrm{~Hz}, 1 \mathrm{H}), 6.96$ $(\mathrm{t}, J=8.0 \mathrm{~Hz}, 1 \mathrm{H}), 6.91(\mathrm{~s}, 1 \mathrm{H}), 6.83-6.78(\mathrm{~m}, 1 \mathrm{H}), 5.37(\mathrm{~s}, 2 \mathrm{H}), 2.36(\mathrm{~s}, 3 \mathrm{H}) .{ }^{13} \mathrm{C} \mathrm{NMR}(126$ $\left.\mathrm{MHz}_{,} \mathrm{CDCl}_{3}\right) \delta 161.9,145.1,141.7,137.2,133.4,130.1,128.7,128.6,128.4,127.9,127.2,127.1$, 127.0, 126.1, 124.4, 114.7, 114.6, 49.7, 21.9. HRMS (ESI) calcd for $\mathrm{C}_{23} \mathrm{H}_{19} \mathrm{NO}_{2}(\mathrm{M}+\mathrm{H})^{+}$: 342.1489; found: 342.1491 .

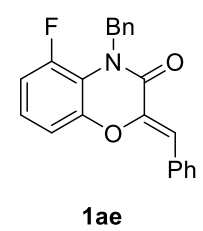

(Z)-4-Benzyl-2-benzylidene-5-fluoro-2H-benzo $[b][1,4]$ oxazin-3(4H)-one (1ae). Yellow solid, $47 \%$ yield, 1.62 g, m.p. $=169.2-170.9{ }^{\circ} \mathrm{C} .{ }^{1} \mathrm{H}$ NMR $\left(500 \mathrm{MHz}, \mathrm{CDCl}_{3}\right) \delta 7.84(\mathrm{~d}, J=7.0 \mathrm{~Hz}$, 2H), $7.45-7.39(\mathrm{~m}, 2 \mathrm{H}), 7.36-7.27(\mathrm{~m}, 6 \mathrm{H}), 7.03(\mathrm{~s}, 1 \mathrm{H}), 7.02-6.92(\mathrm{~m}, 2 \mathrm{H}), 6.74(\mathrm{ddd}, J=$ 13.0, 7.5, $1.5 \mathrm{~Hz}, 1 \mathrm{H}), 5.44(\mathrm{~s}, 2 \mathrm{H}) .{ }^{13} \mathrm{C} \mathrm{NMR}\left(126 \mathrm{MHz}, \mathrm{CDCl}_{3}\right) \delta 158.5,140.3,136.9(\mathrm{~d}, J=$ $12.7 \mathrm{~Hz}), 133.3,130.1,128.8,128.6(\mathrm{~d}, J=7.2 \mathrm{~Hz}), 127.3,127.0,126.7$ (d, $J=1.8 \mathrm{~Hz}), 123.9(\mathrm{~d}$, $J=10.2 \mathrm{~Hz}), 120.5,116.0,114.6,112.4(\mathrm{~d}, J=3.2 \mathrm{~Hz}), 112.0,111.8,48.0(\mathrm{~d}, J=12.5 \mathrm{~Hz}) .{ }^{19} \mathrm{~F}$ NMR (471 MHz, $\left.\mathrm{CDCl}_{3}\right) \delta$-121.71. HRMS (ESI) calcd for $\mathrm{C}_{22} \mathrm{H}_{16} \mathrm{FNO}_{2}(\mathrm{M}+\mathrm{H})^{+}:$346.1238; found: 346.1237 .<smiles>Cc1ccc2c(c1)N(Cc1ccccc1)C(=O)/C(=C/c1ccccc1)O2</smiles>

(Z)-4-Benzyl-2-benzylidene-6-methyl-2H-benzo[b][1,4]oxazin-3(4H)-one (1af). Yellow solid, $30 \%$ yield, 1.02 g, m.p. $=184.8-185.7{ }^{\circ} \mathrm{C} .{ }^{1} \mathrm{H}$ NMR $\left(500 \mathrm{MHz}, \mathrm{CDCl}_{3}\right) \delta 7.87(\mathrm{~d}, J=8.0 \mathrm{~Hz}$, $2 \mathrm{H}), 7.45-7.38(\mathrm{~m}, 2 \mathrm{H}), 7.37-7.29(\mathrm{~m}, 5 \mathrm{H}), 7.28-7.23(\mathrm{~m}, 1 \mathrm{H}), 7.07(\mathrm{~d}, J=8.0 \mathrm{~Hz}, 1 \mathrm{H}), 7.02$ (s, 1H), $6.82(\mathrm{dd}, J=8.0,1.5 \mathrm{~Hz}, 1 \mathrm{H}), 6.71(\mathrm{~d}, J=1.5 \mathrm{~Hz}, 1 \mathrm{H}), 5.26(\mathrm{~s}, 2 \mathrm{H}), 2.22(\mathrm{~s}, 3 \mathrm{H}) .{ }^{13} \mathrm{C}$ NMR $\left(126 \mathrm{MHz}, \mathrm{CDCl}_{3}\right) \delta 157.7,141.0,139.8,135.8$ 133.8, 132.8, 130.0, 128.9, 128.5, 128.2, 127.5, 126.7, 126.1, 124.3, 115.8, 115.6, 113.0, 45.5, 21.1. HRMS (ESI) calcd for $\mathrm{C}_{23} \mathrm{H}_{19} \mathrm{NO}_{2}$ $(\mathrm{M}+\mathrm{H})^{+}:$342.1489; found: 342.1490 . 
<smiles>O=C1/C(=C/c2ccccc2)Oc2ccc(Cl)cc2N1Cc1ccccc1</smiles>

1 ag

(Z)-4-Benzyl-2-benzylidene-6-chloro-2H-benzo[b][1,4]oxazin-3(4H)-one (1ag). Pale yellow solid, $52 \%$ yield, 1.88 g, m.p. $=163.8-165.3{ }^{\circ} \mathrm{C} .{ }^{1} \mathrm{H}$ NMR $\left(500 \mathrm{MHz}, \mathrm{CDCl}_{3}\right) \delta 7.84(\mathrm{~d}, J=7.5$ $\mathrm{Hz}, 2 \mathrm{H}), 7.46-7.39(\mathrm{~m}, 2 \mathrm{H}), 7.38-7.32(\mathrm{~m}, 3 \mathrm{H}), 7.32-7.26(\mathrm{~m}, 3 \mathrm{H}), 7.10(\mathrm{~d}, J=8.5 \mathrm{~Hz}, 1 \mathrm{H})$, $7.05(\mathrm{~s}, 1 \mathrm{H}), 6.97(\mathrm{dd}, J=8.5,2.0 \mathrm{~Hz}, 1 \mathrm{H}), 6.88(\mathrm{~d}, J=2.5 \mathrm{~Hz}, 1 \mathrm{H}), 5.23(\mathrm{~s}, 2 \mathrm{H}) .{ }^{13} \mathrm{C}$ NMR $(126$ $\left.\mathrm{MHz}, \mathrm{CDCl}_{3}\right) \delta 157.3,140.5,140.3,135.0,133.3,130.1,129.1,128.6,128.6,128.2,127.8,127.7$, 126.7, 123.5, 117.1, 115.3, 114.2, 45.6. HRMS (ESI) calcd for $\mathrm{C}_{22} \mathrm{H}_{16} \mathrm{ClNO}_{2}(\mathrm{M}+\mathrm{Na})^{+}$: 384.0762; found: 384.0761 .<smiles>O=C1Nc2cc(Br)ccc2O/C1=C/c1ccccc1</smiles>

1ah

(Z)-4-Benzyl-2-benzylidene-6-bromo-2 $H$-benzo[b][1,4]oxazin-3(4H)-one (1ah). Yellow solid, $82 \%$ yield, 3.32 g, m.p. $=164.5-165.9{ }^{\circ} \mathrm{C} .{ }^{1} \mathrm{H}$ NMR $\left(500 \mathrm{MHz}, \mathrm{CDCl}_{3}\right) \delta 7.83(\mathrm{~d}, J=7.5 \mathrm{~Hz}$, 2H), $7.45-7.39(\mathrm{~m}, 2 \mathrm{H}), 7.39-7.26(\mathrm{~m}, 6 \mathrm{H}), 7.11(\mathrm{dd}, J=8.5,2.0 \mathrm{~Hz}, 1 \mathrm{H}), 7.07-7.00(\mathrm{~m}, 3 \mathrm{H})$, $5.22(\mathrm{~s}, 2 \mathrm{H}) .{ }^{13} \mathrm{C}$ NMR $\left(126 \mathrm{MHz}, \mathrm{CDCl}_{3}\right) \delta 157.3,141.0,140.2,135.0,133.3,130.1,129.1$, 128.61, 128.57, 128.0, 127.8, 126.7, 126.5, 118.0, 117.5, 115.4, 114.2, 45.6. HRMS (ESI) calcd for $\mathrm{C}_{22} \mathrm{H}_{16} \mathrm{BrNO}_{2}(\mathrm{M}+\mathrm{Na})^{+}$: 428.0257; found: 428.0249 .<smiles>O=C1/C(=C/c2ccccc2)Oc2ccc(C(F)(F)F)cc2N1Cc1ccccc1</smiles>

1 ai

(Z)-4-benzyl-2-benzylidene-6-(trifluoromethyl)-2H-benzo[b][1,4]oxazin-3(4H)-one (1ai). Pale yellow solid, $94 \%$ yield, $3.71 \mathrm{~g}$, m.p. $=151.1-152.7{ }^{\circ} \mathrm{C} .{ }^{1} \mathrm{H}$ NMR $\left(500 \mathrm{MHz}, \mathrm{CDCl}_{3}\right) \delta 7.85(\mathrm{~d}, J$ $=7.5 \mathrm{~Hz}, 2 \mathrm{H}), 7.47-7.40(\mathrm{~m}, 2 \mathrm{H}), 7.39-7.22(\mathrm{~m}, 8 \mathrm{H}), 7.17(\mathrm{~d}, J=2.0 \mathrm{~Hz}, 1 \mathrm{H}), 7.10(\mathrm{~s}, 1 \mathrm{H})$, $5.28(\mathrm{~s}, 2 \mathrm{H}) .{ }^{13} \mathrm{C} \mathrm{NMR}\left(126 \mathrm{MHz}, \mathrm{CDCl}_{3}\right) \delta 157.2,144.1,140.0,134.9,133.1,130.2,129.1,128.8$, 128.7, 127.9, 127.0, 126.9, 125.2 (q, $J=58.8 \mathrm{~Hz}), 122.6,121.0(\mathrm{q}, J=3.5 \mathrm{~Hz}), 116.4,114.9$, $112.4(\mathrm{q}, J=3.9 \mathrm{~Hz}), 45.64 .{ }^{19} \mathrm{~F}$ NMR $\left(376 \mathrm{MHz}, \mathrm{CDCl}_{3}\right) \delta-62.06$. HRMS (ESI) calcd for $\mathrm{C}_{23} \mathrm{H}_{16} \mathrm{~F}_{3} \mathrm{NO}_{2}(\mathrm{M}+\mathrm{Na})^{+}$: 418.1025; found: 418.1026 .<smiles>Cc1ccc2c(c1)O/C(=C\c1ccccc1)C(=O)N2Cc1ccccc1</smiles>

1aj 
(Z)-4-Benzyl-2-benzylidene-7-methyl-2H-benzo[b][1,4]oxazin-3(4H)-one (1aj). Yellow solid, $87 \%$ yield, 2.96 g, m.p. $=156.7-157.8{ }^{\circ} \mathrm{C} .{ }^{1} \mathrm{H}$ NMR $\left(500 \mathrm{MHz}, \mathrm{CDCl}_{3}\right) \delta 7.86(\mathrm{~d}, J=7.5 \mathrm{~Hz}$, 2H), $7.46-7.38(\mathrm{~m}, 2 \mathrm{H}), 7.34-7.21(\mathrm{~m}, 6 \mathrm{H}), 7.02(\mathrm{~s}, 1 \mathrm{H}), 7.00(\mathrm{~d}, J=1.5 \mathrm{~Hz}, 1 \mathrm{H}), 6.76(\mathrm{~d}, J=$ $8.5 \mathrm{~Hz}, 1 \mathrm{H}), 6.72(\mathrm{dd}, J=8.5,2.0 \mathrm{~Hz}, 1 \mathrm{H}), 5.24(\mathrm{~s}, 2 \mathrm{H}), 2.28(\mathrm{~s}, 3 \mathrm{H}) .{ }^{13} \mathrm{C} \mathrm{NMR}\left(126 \mathrm{MHz}, \mathrm{CDCl}_{3}\right)$ $\delta 157.4,141.7,141.0,135.8,134.0,133.8,130.0,128.9,128.5,128.2,127.5,126.7,124.90,123.7$, 116.6, 115.0, 113.2, 45.5, 20.7. HRMS (ESI) calcd for $\mathrm{C}_{23} \mathrm{H}_{19} \mathrm{NO}_{2}(\mathrm{M}+\mathrm{Na})^{+}$: 364.1308; found: 364.1304 .<smiles>O=C1/C(=C/c2ccccc2)Oc2cc(Cl)ccc2N1Cc1ccccc1</smiles>

1 ak

(Z)-4-Benzyl-2-benzylidene-7-chloro-2 $H$-benzo[b][1,4]oxazin-3(4H)-one (1ak). Yellow solid, $85 \%$ yield, 3.07 g, m.p. $=183.4-184.7{ }^{\circ} \mathrm{C} .{ }^{1} \mathrm{H}$ NMR $\left(500 \mathrm{MHz}, \mathrm{CDCl}_{3}\right) \delta 7.84(\mathrm{~d}, J=7.0 \mathrm{~Hz}$, 2H), $7.46-7.41(\mathrm{~m}, 2 \mathrm{H}), 7.38-7.31(\mathrm{~m}, 3 \mathrm{H}), 7.30-7.26(\mathrm{~m}, 3 \mathrm{H}), 7.20(\mathrm{~d}, J=2.5 \mathrm{~Hz}, 1 \mathrm{H}), 7.06$ (s, $1 \mathrm{H}), 6.90(\mathrm{dd}, J=8.5,2.5 \mathrm{~Hz}, 1 \mathrm{H}), 6.80(\mathrm{~d}, J=8.5 \mathrm{~Hz}, 1 \mathrm{H}), 5.26(\mathrm{~s}, 2 \mathrm{H}) .{ }^{13} \mathrm{C} \mathrm{NMR}(126 \mathrm{MHz}$, $\left.\mathrm{CDCl}_{3}\right) \delta 157.2,142.2,140.2,135.2,133.3,130.1,129.0,128.9,128.63,128.6,127.7,126.6$, 125.4, 123.2, 116.4, 116.0, 114.4, 45.6. HRMS (ESI) calcd for $\mathrm{C}_{22} \mathrm{H}_{16} \mathrm{ClNO}_{2}(\mathrm{M}+\mathrm{H})^{+}$: 362.0942 ; found: 362.0940 .<smiles>O=C1/C(=C/c2ccccc2)Oc2cc(Br)ccc2N1Cc1ccccc1</smiles>

1al

(Z)-4-Benzyl-2-benzylidene-7-bromo-2H-benzo[b][1,4] oxazin-3(4H)-one (1al). Yellow solid, $71 \%$ yield, 2.87 g, m.p. $=194.4-195.5{ }^{\circ} \mathrm{C} .{ }^{1} \mathrm{H}$ NMR $\left(500 \mathrm{MHz}, \mathrm{CDCl}_{3}\right) \delta 7.46(\mathrm{~d}, J=5.0 \mathrm{~Hz}$, $1 \mathrm{H}), 7.39-7.21(\mathrm{~m}, 9 \mathrm{H}), 7.13-7.08(\mathrm{~m}, 1 \mathrm{H}), 7.05-6.99(\mathrm{~m}, 1 \mathrm{H}), 6.96-6.86(\mathrm{~m}, 2 \mathrm{H}), 5.27(\mathrm{~s}$, 2H). ${ }^{13} \mathrm{C}$ NMR $\left(126 \mathrm{MHz}, \mathrm{CDCl}_{3}\right) \delta 157.3,142.0,138.7,136.3,135.6,129.8,128.9,128.4,127.5$, 127.1, 126.7, 126.5, 123.9, 123.3, 116.2, 115.3, 108.2, 45.3. HRMS (ESI) calcd for $\mathrm{C}_{22} \mathrm{H}_{16} \mathrm{BrNO}_{2}$ $(\mathrm{M}+\mathrm{Na})^{+}:$428.0257; found: 428.0254 .

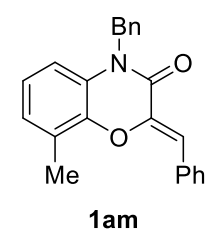

(Z)-4-Benzyl-2-benzylidene-8-methyl-2H-benzo[b][1,4]oxazin-3(4H)-one (1am). Yellow solid, $76 \%$ yield, 2.59 g, m.p. $=165.4-166.9^{\circ} \mathrm{C} .{ }^{1} \mathrm{H}$ NMR $\left(500 \mathrm{MHz}, \mathrm{CDCl}_{3}\right) \delta 7.88(\mathrm{~d}, J=7.5 \mathrm{~Hz}$, 2H), $7.46-7.40(\mathrm{~m}, 2 \mathrm{H}), 7.35-7.28(\mathrm{~m}, 5 \mathrm{H}), 7.27-7.23(\mathrm{~m}, 1 \mathrm{H}), 7.08(\mathrm{~s}, 1 \mathrm{H}), 6.92-6.87(\mathrm{~m}$, $1 \mathrm{H}), 6.86-6.81(\mathrm{~m}, 1 \mathrm{H}), 6.76(\mathrm{dd}, J=8.0,1.5 \mathrm{~Hz}, 1 \mathrm{H}), 5.28(\mathrm{~s}, 2 \mathrm{H}), 2.46(\mathrm{~s}, 3 \mathrm{H}) .{ }^{13} \mathrm{C}$ NMR $(126$ $\left.\mathrm{MHz}, \mathrm{CDCl}_{3}\right) \delta 157.5,140.9,140.3,135.8,133.7,130.0,128.9,128.5,128.2,127.5,126.6,126.2$, 
125.7, 125.4, 122.6, 113.4, 113.1, 45.7, 16.6. HRMS (ESI) calcd for $\mathrm{C}_{23} \mathrm{H}_{19} \mathrm{NO}_{2}(\mathrm{M}+\mathrm{H})^{+}$: 342.1489; found: 342.1489 .<smiles>O=C1/C(=C/c2ccccc2)Oc2c(F)cccc2N1Cc1ccccc1</smiles>

(Z)-4-Benzyl-2-benzylidene-8-fluoro-2H-benzo[b][1,4]oxazin-3(4H)-one (1an). Pale yellow solid, 53\% yield, $1.83 \mathrm{~g}$, m.p. $=158.1-159.4{ }^{\circ} \mathrm{C} .{ }^{1} \mathrm{H}$ NMR $\left(500 \mathrm{MHz}, \mathrm{CDCl}_{3}\right) \delta 7.92(\mathrm{~d}, J=7.5$ $\mathrm{Hz}, 2 \mathrm{H}), 7.47-7.39(\mathrm{~m}, 2 \mathrm{H}), 7.36-7.22(\mathrm{~m}, 6 \mathrm{H}), 7.09(\mathrm{~s}, 1 \mathrm{H}), 6.87-6.77(\mathrm{~m}, 2 \mathrm{H}), 6.71-6.62$ (m, 1H), $5.25(\mathrm{~s}, 2 \mathrm{H}) .{ }^{13} \mathrm{C}$ NMR $\left(126 \mathrm{MHz}, \mathrm{CDCl}_{3}\right) \delta$ 157.4, 151.6, 149.6, 139.9, 135.4, 133.2, $130.9(\mathrm{~d}, J=14.6 \mathrm{~Hz}), 130.4,129.0,128.7,128.5$ (d, $J=2.8 \mathrm{~Hz}), 127.7,126.7,122.6$ (d, $J=7.9$ $\mathrm{Hz}), 114.9,111.2(\mathrm{~d}, J=17.5 \mathrm{~Hz}), 110.4(\mathrm{~d}, J=3.4 \mathrm{~Hz}), 45.8 .{ }^{19} \mathrm{~F}$ NMR $\left(471 \mathrm{MHz}, \mathrm{CDCl}_{3}\right) \delta$ -134.82. HRMS (ESI) calcd for $\mathrm{C}_{22} \mathrm{H}_{16} \mathrm{FNO}_{2}(\mathrm{M}+\mathrm{H})^{+}$: 346.1238; found: 346.1237 .<smiles>CCCCN1C(=O)/C(=C\C2CC2)Oc2cc(Br)ccc21</smiles>

(Z)-4-benzyl-7-bromo-2-(cyclopropylmethylene)-2H-benzo[b][1,4]oxazin-3(4H)-one (1ao). White solid, 81\% yield, 2.99 g, m.p. $=140.3-141.9{ }^{\circ} \mathrm{C} .{ }^{1} \mathrm{H}$ NMR $\left(500 \mathrm{MHz}, \mathrm{CDCl}_{3}\right) \delta 7.35-$ $7.29(\mathrm{~m}, 2 \mathrm{H}), 7.28-7.21(\mathrm{~m}, 3 \mathrm{H}), 7.19(\mathrm{~d}, J=2.0 \mathrm{~Hz}, 1 \mathrm{H}), 6.96(\mathrm{dd}, J=9.0,2.5 \mathrm{~Hz}, 1 \mathrm{H}), 6.66(\mathrm{~d}$, $J=8.5 \mathrm{~Hz}, 1 \mathrm{H}), 5.67(\mathrm{~d}, J=10.5 \mathrm{~Hz}, 1 \mathrm{H}), 5.17(\mathrm{~s}, 2 \mathrm{H}), 2.04-1.91(\mathrm{~m}, 1 \mathrm{H}), 0.99(\mathrm{dt}, J=7.0,4.5$ $\mathrm{Hz}, 2 \mathrm{H}), 0.66$ (dt, $J=7.0,4.5 \mathrm{~Hz}, 2 \mathrm{H}) .{ }^{13} \mathrm{C}$ NMR $\left(126 \mathrm{MHz}, \mathrm{CDCl}_{3}\right) \delta 157.2,143.3,140.6,135.4$, 128.9, 127.6, 126.5, 126.0, 125.3, 123.7, 119.0, 116.3, 115.7, 45.1, 8.6, 8.3. HRMS (ESI) calcd for $\mathrm{C}_{19} \mathrm{H}_{16} \mathrm{BrNO}_{2}(\mathrm{M}+\mathrm{Na})^{+}: 392.0257$; found: 392.0258 .<smiles>O=C1COc2ccccc2N1</smiles>

X-I<smiles>O[PbH2]</smiles>
X-II

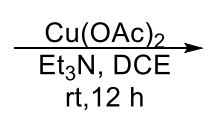

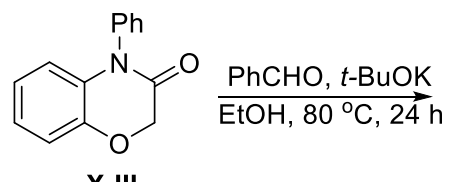

X-III<smiles>O=C1/C(=C/c2ccccc2)Oc2ccccc2N1c1ccccc1</smiles>

A mixture of the $2 H$-benzo[b][1,4]oxazin-3(4H)-one $\mathbf{X}$-I $(10 \mathrm{mmol})$, phenylboronic acid (15 mmol), anhydrous $\mathrm{Cu}(\mathrm{OAc})_{2}(10 \mathrm{mmol})$ and $\mathrm{Et}_{3} \mathrm{~N}(2.0 \mathrm{mmol})$ in 1,2-dichloroethane $(15 \mathrm{~mL})$ was stirred at room temperature for $12 \mathrm{~h}$ (the progress of the reaction was monitored by TLC). After completion of the reaction the mixture was filtered through celite. The filtrate was collected, concentrated in vacuo, and the residue was purified by column chromatography on silica gel to afford the desired product $\mathbf{X}$-III.

To a stirred solution of X-III $(10 \mathrm{mmol})$ and aldehyde $(15 \mathrm{mmol})$ in $100 \mathrm{~mL}$ EtOH, $15 \mathrm{~mL}$ $t$-BuOK (1 M in THF) were slowly added and the reaction was refluxed at $80{ }^{\circ} \mathrm{C}$ for $24 \mathrm{~h}$. After 24 $\mathrm{h}$, if X-III was not full conversed, another $15 \mathrm{mmol}$ aldehyde was added and the mixture was refluxed for another $12 \mathrm{~h}$. After cooling to $0{ }^{\circ} \mathrm{C}$, the reaction was quenched with saturated $\mathrm{NH}_{4} \mathrm{Cl}$ and diluted with EtOAc $(3 \times 50 \mathrm{~mL})$ and washed with water and brine $(3 \times 100 \mathrm{~mL})$, dried over 
anhydrous $\mathrm{Na}_{2} \mathrm{SO}_{4}$ and evaporated under reduced pressure. The residue was purified by flash silica gel chromatography to give substrate $\mathbf{1 b}$.<smiles>O=C1/C(=C/c2ccccc2)Oc2ccccc2N1c1ccccc1</smiles>

$1 \mathrm{~b}$

(Z)-2-Benzylidene-4-phenyl-2H-benzo[b][1,4]oxazin-3(4H)-one (1b). White solid, 87\% yield, 2.72 g, m.p. $=156.3-157.6{ }^{\circ} \mathrm{C} .{ }^{1} \mathrm{H}$ NMR $\left(500 \mathrm{MHz}, \mathrm{CDCl}_{3}\right) \delta 7.89(\mathrm{~d}, J=7.5 \mathrm{~Hz}, 2 \mathrm{H}), 7.58(\mathrm{t}, J$ $=7.5 \mathrm{~Hz}, 2 \mathrm{H}), 7.53-7.48(\mathrm{~m}, 1 \mathrm{H}), 7.46-7.39(\mathrm{~m}, 2 \mathrm{H}), 7.36-7.30(\mathrm{~m}, 3 \mathrm{H}), 7.23(\mathrm{dd}, J=8.0$, $1.5 \mathrm{~Hz}, 1 \mathrm{H}), 7.08-6.99(\mathrm{~m}, 2 \mathrm{H}), 6.87(\mathrm{td}, J=8.0,1.5 \mathrm{~Hz}, 1 \mathrm{H}), 6.37(\mathrm{dd}, J=8.0,1.5 \mathrm{~Hz}, 1 \mathrm{H}) .{ }^{13} \mathrm{C}$ NMR $\left(126 \mathrm{MHz}, \mathrm{CDCl}_{3}\right) \delta 157.1,141.6,141.3,135.9,133.6,130.2,130.1,129.1,128.9,128.6$, 128.5, 128.3, 123.9, 122.9, 116.2, 116.1, 113.5. HRMS (ESI) calcd for $\mathrm{C}_{21} \mathrm{H}_{15} \mathrm{NO}_{2}(\mathrm{M}+\mathrm{H})^{+}$: 314.1176; found: 314.1187 .

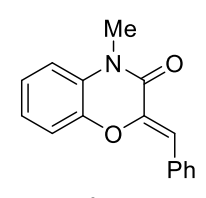

$1 \mathrm{c}$

(Z)-2-Benzylidene-4-methyl-2H-benzo[b][1,4]oxazin-3(4H)-one (1c) was synthesized according to a reported literature ${ }^{[1]}$, white solid, ${ }^{1} \mathrm{H}$ NMR $\left(500 \mathrm{MHz}, \mathrm{CDCl}_{3}\right) \delta 7.86(\mathrm{~d}, J=7.5 \mathrm{~Hz}, 2 \mathrm{H}), 7.43$ (t, $J=7.5 \mathrm{~Hz}, 2 \mathrm{H}), 7.36-7.32(\mathrm{~m}, 1 \mathrm{H}), 7.20-7.16(\mathrm{~m}, 1 \mathrm{H}), 7.11-7.05(\mathrm{tdm}, 2 \mathrm{H}), 7.00-6.95$ (m, 2H), $3.46(\mathrm{~s}, 3 \mathrm{H}) .{ }^{13} \mathrm{C}$ NMR $\left(126 \mathrm{MHz}, \mathrm{CDCl}_{3}\right) \delta 157.1,141.7,141.0,133.7,130.0,128.5$, 128.2, 127.4, 123.7, 123.2, 115.9, 114.3, 112.7, 28.7. HRMS (ESI) calcd for $\mathrm{C}_{16} \mathrm{H}_{13} \mathrm{NO}_{2}(\mathrm{M}+\mathrm{H})^{+}$: 252.1019; found: 252.1021. Characterization data matches those reported in the literature ${ }^{[1]}$.<smiles>O=C1Nc2ccccc2O/C1=C\c1ccccc1</smiles>

$1 \mathrm{~d}$

(Z)-2-Benzylidene-2H-benzo[b][1,4] oxazin-3(4H)-one (1d) was synthesized according to a reported literature ${ }^{[1]}$, yellow solid, ${ }^{1} \mathrm{H}$ NMR $\left(500 \mathrm{MHz}, \mathrm{DMSO}-d_{6}\right) \delta 11.16(\mathrm{~s}, 1 \mathrm{H}), 7.89(\mathrm{~d}, J=$ $7.5 \mathrm{~Hz}, 2 \mathrm{H}), 7.45(\mathrm{t}, J=7.5 \mathrm{~Hz}, 2 \mathrm{H}), 7.35(\mathrm{t}, J=7.5 \mathrm{~Hz}, 1 \mathrm{H}), 7.29(\mathrm{dd}, J=7.0,1.5 \mathrm{~Hz}, 1 \mathrm{H}), 7.08$ $-6.97(\mathrm{~m}, 3 \mathrm{H}), 6.79(\mathrm{~s}, 1 \mathrm{H}) .{ }^{13} \mathrm{C}$ NMR $\left(126 \mathrm{MHz}, \mathrm{DMSO}-d_{6}\right) \delta 156.7,142.2,140.9,133.7,130.2$, 129.1, 128.6, 125.8, 123.9, 123.7, 116.0, 116.0, 111.0. HRMS (ESI) calcd for $\mathrm{C}_{15} \mathrm{H}_{11} \mathrm{NO}_{2}(\mathrm{M}+\mathrm{H})^{+}$: 238.0863; found: 238.0880 . Characterization data matches those reported in the literature ${ }^{[1][2]}$. 


\section{General Procedure for Hydrogenation}

The catalyst ( $3.2 \mathrm{mg}, 0.002 \mathrm{mmol}, 0.01$ equiv.) and substrate ( $0.2 \mathrm{mmol}, 1.0$ equiv.) were placed in a $5 \mathrm{~mL}$ tube equipped with a magnetic stirrer bar. This tube was then put into an nitrogen-filled autoclave. Solvent $(2 \mathrm{~mL})$ was added to the mixture under nitrogen atmosphere. The autoclave was then closed, purged three times with hydrogen (less than the pressure needed), and finally pressurized to 30 bar. The reaction mixture was stirred at room temperature for $24 \mathrm{~h}$. Then, the hydrogen was slowly released, and the solvent was evaporated under reduced pressure. The conversion of the reaction was determined by ${ }^{1} \mathrm{H}$ NMR spectroscopic analysis of the crude reaction mixture and isolated by chromatography. The ee was determined by chiral HPLC.

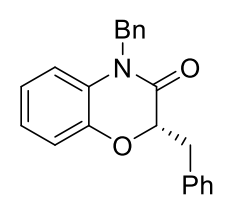

$2 \mathbf{a}$

(S)-2,4-Dibenzyl-2H-benzo[b][1,4] oxazin-3(4H)-one (2a). White solid, 99\% yield, $65.1 \mathrm{mg}$, m.p. $=86.8-87.5^{\circ} \mathrm{C} .{ }^{1} \mathrm{H}$ NMR $\left(500 \mathrm{MHz}, \mathrm{CDCl}_{3}\right) \delta 7.36-7.16(\mathrm{~m}, 10 \mathrm{H}), 6.98-6.92(\mathrm{~m}, 2 \mathrm{H}), 6.90-$ $6.80(\mathrm{~m}, 2 \mathrm{H}), 5.23(\mathrm{~d}, J=16.0 \mathrm{~Hz}, 1 \mathrm{H}), 5.04(\mathrm{~d}, J=16.0 \mathrm{~Hz}, 1 \mathrm{H}), 4.90(\mathrm{dd}, J=10.0,3.5 \mathrm{~Hz}, 1 \mathrm{H})$, $3.30(\mathrm{dd}, J=14.5,3.0 \mathrm{~Hz}, 1 \mathrm{H}), 3.16(\mathrm{dd}, J=14.5,9.5 \mathrm{~Hz}, 1 \mathrm{H}) .{ }^{13} \mathrm{C} \mathrm{NMR}\left(126 \mathrm{MHz}, \mathrm{CDCl}_{3}\right) \delta$ 166.0, 143.8, 136.7, 136.1, 129.7, 128.9, 128.8, 128.5, 127.5, 126.9, 126.6, 124.1, 122.6, 117.7, 115.5, 78.1, 45.3, 36.7. HPLC [DAICEL CHIRALPAK OD-H, hexane $/ \mathrm{PrOH}=95 / 5,220 \mathrm{~nm}, 0.5$ $\mathrm{mL} / \mathrm{min}, 25{ }^{\circ} \mathrm{C} . \mathrm{t}_{\mathrm{R} 1}=19.7 \min$ (major), $\mathrm{t}_{\mathrm{R} 2}=22.5 \mathrm{~min}$ (minor)]; $e e=97 \% .[\alpha]_{\mathrm{D}}^{25}=-64.04(c 0.49$, $\mathrm{CH}_{2} \mathrm{Cl}_{2}$ ). HRMS (ESI) calcd for $\mathrm{C}_{22} \mathrm{H}_{19} \mathrm{NO}_{2}(\mathrm{M}+\mathrm{H})^{+}: 330.1489$; found: 330.1495 .<smiles>O=C1C(Cc2ccccc2)Oc2ccccc2N1c1ccccc1</smiles>

2b

(S)-2-Benzyl-4-phenyl-2H-benzo[b][1,4]oxazin-3(4H)-one (2b). Yellow solid, 99\% conv., (99\% yield), $62.3 \mathrm{mg}$, m.p. $=95.8-96.9^{\circ} \mathrm{C} .{ }^{1} \mathrm{H}$ NMR $\left(500 \mathrm{MHz}, \mathrm{CDCl}_{3}\right) \delta 7.57-7.50(\mathrm{~m}, 2 \mathrm{H}), 7.49-$ $7.43(\mathrm{~m}, 1 \mathrm{H}), 7.34-7.28(\mathrm{~m}, J 4 \mathrm{H}), 7.28-7.22(\mathrm{~m}, 3 \mathrm{H}), 7.02-6.95(\mathrm{~m}, 2 \mathrm{H}), 6.85-6.80(\mathrm{~m}, 1 \mathrm{H})$, $6.36(\mathrm{dd}, J=7.5,1.5 \mathrm{~Hz}, 1 \mathrm{H}), 4.96(\mathrm{dd}, J=9.0,3.5 \mathrm{~Hz}, 1 \mathrm{H}), 3.34(\mathrm{dd}, J=14.0,3.5 \mathrm{~Hz}, 1 \mathrm{H}), 3.21$ $(\mathrm{dd}, J=14.5,9.5 \mathrm{~Hz}, 1 \mathrm{H}) .{ }^{13} \mathrm{C} \mathrm{NMR}\left(126 \mathrm{MHz}, \mathrm{CDCl}_{3}\right) \delta 165.5,143.4,136.5,136.2,130.5,130.0$, 129.7, 128.8, 128.8, 128.4, 126.9, 124.2, 122.4, 117.6, 116.6, 78.4, 36.8. HPLC [DAICEL CHIRALPAK OD-H, hexane $/ \mathrm{iPrOH}=95 / 5,220 \mathrm{~nm}, 0.5 \mathrm{~mL} / \mathrm{min}, 25^{\circ} \mathrm{C} \cdot \mathrm{t}_{\mathrm{R} 1}=28.2 \mathrm{~min}$ (major), $\mathrm{t}_{\mathrm{R} 2}=37.6$ min (minor)]; ee $=89 \%$. [ $\left.\alpha\right]_{\mathrm{D}}^{25}=-57.20\left(c 0.30, \mathrm{CH}_{2} \mathrm{Cl}_{2}\right)$. HRMS (ESI) calcd for $\mathrm{C}_{21} \mathrm{H}_{17} \mathrm{NO}_{2}(\mathrm{M}+\mathrm{H})^{+}:$316.1332; found: 316.1335 .<smiles>CN1C(=O)[C@H](Cc2ccccc2)Oc2ccccc21</smiles>

2c 
(S)-2-Benzyl-4-methyl-2H-benzo[b][1,4]oxazin-3(4H)-one (2c). Yellow wax, 99\% conv., (98\% yield), $49.6 \mathrm{mg} .{ }^{1} \mathrm{H}$ NMR $\left(500 \mathrm{MHz}, \mathrm{CDCl}_{3}\right) \delta 7.32-7.26(\mathrm{~m}, 2 \mathrm{H}), 7.26-7.20(\mathrm{~m}, 4 \mathrm{H}), 7.04-$ $6.97(\mathrm{~m}, 2 \mathrm{H}), 6.95-6.90(\mathrm{~m}, 2 \mathrm{H}), 4.78(\mathrm{dd}, J=9.5,3.5 \mathrm{~Hz}, 1 \mathrm{H}), 3.35(\mathrm{~s}, 3 \mathrm{H}), 3.22(\mathrm{dd}, J=14.5$, $3.5 \mathrm{~Hz}, 1 \mathrm{H}), 3.06(\mathrm{dd}, J=14.5,9.5 \mathrm{~Hz}, 1 \mathrm{H}) .{ }^{13} \mathrm{C} \mathrm{NMR}\left(126 \mathrm{MHz}, \mathrm{CDCl}_{3}\right) \delta 165.8,143.7,136.7$, $129.6,129.5,128.4,126.8,124.0,122.6,117.5,114.5,78.0,36.8,28.4$. HPLC [DAICEL CHIRALPAK OJ-H, hexane $/ \mathrm{iPrOH}=90 / 10,220 \mathrm{~nm}, 1.0 \mathrm{~mL} / \mathrm{min}, 25^{\circ} \mathrm{C} \cdot \mathrm{t}_{\mathrm{R} 1}=19.1 \mathrm{~min}$ (minor), $\mathrm{t}_{\mathrm{R} 2}=21.9 \min$ (major)]; $e e=78 \%$. $[\alpha]_{\mathrm{D}}^{25}=-274.80\left(c 0.21, \mathrm{CH}_{2} \mathrm{Cl}_{2}\right)$. HRMS (ESI) calcd for $\mathrm{C}_{16} \mathrm{H}_{15} \mathrm{NO}_{2}(\mathrm{M}+\mathrm{H})^{+}:$254.1176; found: 254.1179.<smiles>Cc1ccc(CC2Oc3ccccc3N(Cc3ccccc3)C2=O)cc1</smiles>

(S)-4-Benzyl-2-(4-methylbenzyl)-2H-benzo[b][1,4]oxazin-3(4H)-one (2e). White solid, 99\% yield, $67.9 \mathrm{mg}$, m.p. $=83.2-84.5{ }^{\circ} \mathrm{C} .{ }^{1} \mathrm{H}$ NMR $\left(500 \mathrm{MHz}, \mathrm{CDCl}_{3}\right) \delta 7.33-7.28(\mathrm{~m}, 2 \mathrm{H}), 7.27-$ $7.20(\mathrm{~m}, 3 \mathrm{H}), 7.17(\mathrm{~d}, J=8.0 \mathrm{~Hz}, 2 \mathrm{H}), 7.12(\mathrm{~d}, J=8.0 \mathrm{~Hz}, 2 \mathrm{H}), 6.99-6.93(\mathrm{~m}, 2 \mathrm{H}), 6.91-6.82$ $(\mathrm{m}, 2 \mathrm{H}), 5.25(\mathrm{~d}, J=16.0 \mathrm{~Hz}, 1 \mathrm{H}), 5.04(\mathrm{~d}, J=16.0 \mathrm{~Hz}, 1 \mathrm{H}), 4.88(\mathrm{dd}, J=9.5,3.5 \mathrm{~Hz}, 1 \mathrm{H}), 3.25$ $(\mathrm{dd}, J=14.5,3.5 \mathrm{~Hz}, 1 \mathrm{H}), 3.12(\mathrm{dd}, J=14.5,9.5 \mathrm{~Hz}, 1 \mathrm{H}), 2.34(\mathrm{~s}, 3 \mathrm{H}) .{ }^{13} \mathrm{C}$ NMR $(126 \mathrm{MHz}$, $\left.\mathrm{CDCl}_{3}\right) \delta 166.1,143.7,136.4,136.1,133.5,129.5,129.2,128.9,128.8,127.4,126.6,124.1,122.6$, 117.7, 115.4, 78.3, 45.2, 36.3, 21.1. HPLC [DAICEL CHIRALPAK OD-H, hexane/iPrOH $=95 / 5$, $220 \mathrm{~nm}, 0.5 \mathrm{~mL} / \mathrm{min}, 25^{\circ} \mathrm{C} \cdot \mathrm{t}_{\mathrm{R} 1}=16.3 \mathrm{~min}$ (major), $\mathrm{t}_{\mathrm{R} 2}=21.9 \mathrm{~min}$ (minor) $] ; e e=97 \% .[\alpha]_{\mathrm{D}}^{25}=$ -50.92 ( $c$ 0.51, $\mathrm{CH}_{2} \mathrm{Cl}_{2}$ ). HRMS (ESI) calcd for $\mathrm{C}_{23} \mathrm{H}_{21} \mathrm{NO}_{2}(\mathrm{M}+\mathrm{H})^{+}:$: 344.1645; found: 344.1646 .<smiles>CCCCCc1ccc(OC)cc1</smiles>

(S)-4-Benzyl-2-(4-methoxybenzyl)-2H-benzo[b][1,4]oxazin-3(4H)-one (2f). White solid, 99\% yield, $71.1 \mathrm{mg}$, m.p. $=74.0-75.6{ }^{\circ} \mathrm{C} .{ }^{1} \mathrm{H}$ NMR $\left(500 \mathrm{MHz}, \mathrm{CDCl}_{3}\right) \delta 7.33-7.28(\mathrm{~m}, 2 \mathrm{H}), 7.27-$ $7.17(\mathrm{~m}, 5 \mathrm{H}), 6.99-6.93(\mathrm{~m}, 2 \mathrm{H}), 6.91-6.82(\mathrm{~m}, 4 \mathrm{H}), 5.24(\mathrm{~d}, J=16.0 \mathrm{~Hz}, 1 \mathrm{H}), 5.04(\mathrm{~d}, J=$ $16.0 \mathrm{~Hz}, 1 \mathrm{H}), 4.87(\mathrm{dd}, J=9.0,3.5 \mathrm{~Hz}, 1 \mathrm{H}), 3.79(\mathrm{~s}, 3 \mathrm{H}), 3.23(\mathrm{dd}, J=14.5,3.5 \mathrm{~Hz}, 1 \mathrm{H}), 3.12(\mathrm{dd}$ $J=14.5,9.5 \mathrm{~Hz}, 1 \mathrm{H}) .{ }^{13} \mathrm{C} \mathrm{NMR}\left(126 \mathrm{MHz}, \mathrm{CDCl}_{3}\right) \delta 166.1,158.6,143.8,136.1,130.7,128.9$, 128.7, 128.6, 127.4, 126.5, 124.1, 122.5, 117.6, 115.4, 113.9, 78.3, 55.3, 45.2, 35.9. HPLC [DAICEL CHIRALPAK OD-H, hexane $/ \mathrm{iPrOH}=95 / 5,220 \mathrm{~nm}, 0.5 \mathrm{~mL} / \mathrm{min}, 25^{\circ} \mathrm{C} . \mathrm{t}_{\mathrm{R} 1}=23.3 \mathrm{~min}$ (major), $\mathrm{t}_{\mathrm{R} 2}=30.3 \min$ (minor)]; $e e=97 \%$. [ $\left.\alpha\right]_{\mathrm{D}}^{25}=-42.50\left(c 0.54, \mathrm{CH}_{2} \mathrm{Cl}_{2}\right.$ ). HRMS (ESI) calcd for $\mathrm{C}_{23} \mathrm{H}_{21} \mathrm{NO}_{3}(\mathrm{M}+\mathrm{H})^{+}$: 360.1594; found: 360.1589 . 
<smiles>CC(C)(C)c1ccc(CC2Oc3ccccc3N(Cc3ccccc3)C2=O)cc1</smiles>

(S)-4-Benzyl-2-(4-(tert-butyl)benzyl)-2H-benzo[b][1,4]oxazin-3(4H)-one (2g). White solid, 98\% yield, $75.5 \mathrm{mg}$, m.p. $=86.1-87.4{ }^{\circ} \mathrm{C} .{ }^{1} \mathrm{H}$ NMR $\left(500 \mathrm{MHz}, \mathrm{CDCl}_{3}\right) \delta 7.35-7.29(\mathrm{~m}, 4 \mathrm{H}), 7.28-$ $7.19(\mathrm{~m}, 5 \mathrm{H}), 7.01-6.93(\mathrm{~m}, 2 \mathrm{H}), 6.91-6.82(\mathrm{~m}, 2 \mathrm{H}), 5.24(\mathrm{~d}, J=16.0 \mathrm{~Hz}, 1 \mathrm{H}), 5.06(\mathrm{~d}, J=$ $16.0 \mathrm{~Hz}, 1 \mathrm{H}), 4.90(\mathrm{dd}, J=9.5,3.5 \mathrm{~Hz}, 1 \mathrm{H}), 3.26(\mathrm{dd}, J=14.5,3.0 \mathrm{~Hz}, 1 \mathrm{H}), 3.13$ (dd, $J=14.5$, $9.5 \mathrm{~Hz}, 1 \mathrm{H}), 1.32$ (s, 9H). ${ }^{13} \mathrm{C}$ NMR $\left(126 \mathrm{MHz}, \mathrm{CDCl}_{3}\right) \delta 166.1,149.6,143.8,136.1,133.5,129.3$, 128.9, 128.8, 127.4, 126.6, 125.3, 124.1, 122.6, 117.7, 115.4, 78.2, 45.3, 36.1, 34.5, 31.4, 31.4. HPLC [DAICEL CHIRALPAK OD-H, hexane $/ \mathrm{PrOH}=95 / 5,220 \mathrm{~nm}, 0.5 \mathrm{~mL} / \mathrm{min}, 25^{\circ} \mathrm{C} . \mathrm{t}_{\mathrm{R} 1}=$ $14.5 \min$ (major), $\mathrm{t}_{\mathrm{R} 2}=17.3 \min$ (minor)]; $e e=97 \%$. $[\alpha]_{\mathrm{D}}^{25}=-54.73\left(c 0.58, \mathrm{CH}_{2} \mathrm{Cl}_{2}\right) . \mathrm{HRMS}(\mathrm{ESI})$ calcd for $\mathrm{C}_{26} \mathrm{H}_{27} \mathrm{NO}_{2}(\mathrm{M}+\mathrm{Na})^{+}$: 408.1934; found: 408.1936 .<smiles>O=C1C(Cc2ccc(F)cc2)Oc2ccccc2N1Cc1ccccc1</smiles>

(S)-4-Benzyl-2-(4-fluorobenzyl)-2H-benzo[b][1,4]oxazin-3(4H)-one (2h). White solid, $98 \%$ yield, $68.0 \mathrm{mg}$, m.p. $=78.5-79.4{ }^{\circ} \mathrm{C} .{ }^{1} \mathrm{H}$ NMR $\left(500 \mathrm{MHz}, \mathrm{CDCl}_{3}\right) \delta 7.33-7.28(\mathrm{~m}, 2 \mathrm{H}), 7.28-$ $7.22(\mathrm{~m}, 3 \mathrm{H}), 7.22-7.16(\mathrm{~m}, 2 \mathrm{H}), 7.03-6.92(\mathrm{~m}, 4 \mathrm{H}), 6.92-6.82(\mathrm{~m}, 2 \mathrm{H}), 5.22(\mathrm{~d}, J=16.0 \mathrm{~Hz}$, $1 \mathrm{H}), 5.06(\mathrm{~d}, J=16.0 \mathrm{~Hz}, 1 \mathrm{H}), 4.86(\mathrm{dd}, J=9.5,4.0 \mathrm{~Hz}, 1 \mathrm{H}), 3.29(\mathrm{dd}, J=14.5,3.5 \mathrm{~Hz}, 1 \mathrm{H}), 3.17$ $(\mathrm{dd}, J=14.5,9.0 \mathrm{~Hz}, 1 \mathrm{H}) .{ }^{13} \mathrm{C}$ NMR $\left(126 \mathrm{MHz}, \mathrm{CDCl}_{3}\right) \delta 165.8,162.9,161.0,143.8,136.0,132.2$ $(\mathrm{d}, J=3.4 \mathrm{~Hz}), 131.2(\mathrm{~d}, J=7.8 \mathrm{~Hz}), 128.9,128.7,127.5,126.5,124.1,122.7,117.5,115.3(\mathrm{t}, J=$ $21.5 \mathrm{~Hz}), 77.9,45.2,35.9 .{ }^{19} \mathrm{~F}$ NMR $\left(471 \mathrm{MHz}, \mathrm{CDCl}_{3}\right) \delta$-116.17. HPLC [DAICEL CHIRALPAK OD-H, hexane $/ \mathrm{PrOH}=95 / 5,220 \mathrm{~nm}, 0.5 \mathrm{~mL} / \mathrm{min}, 25^{\circ} \mathrm{C} \cdot \mathrm{t}_{\mathrm{R} 1}=20.3 \mathrm{~min}$ (major), $\mathrm{t}_{\mathrm{R} 2}=25.6 \min$ (minor)]; $e e=97 \% .[\alpha]_{\mathrm{D}}^{25}=-24.81\left(c 0.52, \mathrm{CH}_{2} \mathrm{Cl}_{2}\right)$. HRMS (ESI) calcd for $\mathrm{C}_{22} \mathrm{H}_{18} \mathrm{FNO}_{2}(\mathrm{M}+\mathrm{H})^{+}:$348.1394; found: 348.1391 .<smiles>O=C1C(Cc2ccc(Cl)cc2)Oc2ccccc2N1Cc1ccccc1</smiles>

(S)-4-Benzyl-2-(4-chlorobenzyl)-2H-benzo[b][1,4]oxazin-3(4H)-one (2i). White solid, 99\% yield, $71.9 \mathrm{mg}$, m.p. $=49.4-51.2{ }^{\circ} \mathrm{C} .{ }^{1} \mathrm{H}$ NMR $\left(500 \mathrm{MHz}, \mathrm{CDCl}_{3}\right) \delta 7.34-7.21(\mathrm{~m}, 7 \mathrm{H}), 7.21-$ $7.16(\mathrm{~m}, 2 \mathrm{H}), 6.99-6.92(\mathrm{~m}, 2 \mathrm{H}), 6.92-6.83(\mathrm{~m}, 2 \mathrm{H}), 5.21(\mathrm{~d}, J=16.0 \mathrm{~Hz}, 1 \mathrm{H}), 5.06(\mathrm{~d}, J=$ $16.0 \mathrm{~Hz}, 1 \mathrm{H}), 4.86(\mathrm{dd}, J=9.0,3.5 \mathrm{~Hz}, 1 \mathrm{H}), 3.29$ (dd, $J=14.5,3.5 \mathrm{~Hz}, 1 \mathrm{H}), 3.17$ (dd, $J=14.5$, 
$9.0 \mathrm{~Hz}, 1 \mathrm{H}) .{ }^{13} \mathrm{C} \mathrm{NMR}\left(126 \mathrm{MHz}, \mathrm{CDCl}_{3}\right) \delta 165.7,143.7,135.9,135.0,132.8,131.1,128.9,128.6$, 128.5, 127.5, 126.5, 124.2, 122.7, 117.5, 115.5, 77.7, 45.2, 36.1. HPLC [DAICEL CHIRALPAK OD-H, hexane $/ \mathrm{iPrOH}=95 / 5,220 \mathrm{~nm}, 0.5 \mathrm{~mL} / \mathrm{min}, 25^{\circ} \mathrm{C} . \mathrm{t}_{\mathrm{R} 1}=21.8 \mathrm{~min}$ (major), $\mathrm{t}_{\mathrm{R} 2}=28.4 \mathrm{~min}$ (minor)]; $e e=97 \%$. $[\alpha]_{\mathrm{D}}^{25}=-25.73\left(c 0.53, \mathrm{CH}_{2} \mathrm{Cl}_{2}\right)$. HRMS (ESI) calcd for $\mathrm{C}_{22} \mathrm{H}_{18} \mathrm{ClNO}_{2}(\mathrm{M}+\mathrm{H})^{+}$: 364.1099; found: 364.1107 .<smiles>O=C1Cc2ccccc2N(Cc2ccc(Br)cc2)O1</smiles>

(S)-4-Benzyl-2-(4-bromobenzyl)-2H-benzo[b][1,4] oxazin-3(4H)-one (2j). White solid, 99\% yield, $80.6 \mathrm{mg}$, m.p. $=82.4-83.9{ }^{\circ} \mathrm{C} .{ }^{1} \mathrm{H}$ NMR $\left(500 \mathrm{MHz}, \mathrm{CDCl}_{3}\right) \delta 7.46-7.40(\mathrm{~m}, 2 \mathrm{H}), 7.35-$ $7.28(\mathrm{~m}, 2 \mathrm{H}), 7.28-7.22(\mathrm{~m}, 1 \mathrm{H}), 7.22-7.12(\mathrm{~m}, 4 \mathrm{H}), 6.99-6.92(\mathrm{~m}, 2 \mathrm{H}), 6.92-6.82(\mathrm{~m}, 2 \mathrm{H})$, $5.21(\mathrm{~d}, J=16.0 \mathrm{~Hz}, 1 \mathrm{H}), 5.06(\mathrm{~d}, J=16.0 \mathrm{~Hz}, 1 \mathrm{H}), 4.86(\mathrm{dd}, J=9.0,3.5 \mathrm{~Hz}, 1 \mathrm{H}), 3.27(\mathrm{dd}, J=$ 14.5, 3.5 Hz, 1H), $3.15(\mathrm{dd}, J=14.5,9.0 \mathrm{~Hz}, 1 \mathrm{H}) .{ }^{13} \mathrm{C} \mathrm{NMR}\left(126 \mathrm{MHz}, \mathrm{CDCl}_{3}\right) \delta 165.7,143.7$, 135.9, 135.6, 131.48, 131.46, 128.9, 128.6, 127.5, 126.5, 124.2, 122.7, 120.9, 117.5, 115.5, 77.7, 45.2, 36.2. HPLC [DAICEL CHIRALPAK AD-H, hexane $/ \mathrm{PPOH}=95 / 5,220 \mathrm{~nm}, 0.5 \mathrm{~mL} / \mathrm{min}, 25$ ${ }^{\circ} \mathrm{C} . \mathrm{t}_{\mathrm{R} 1}=43.2 \mathrm{~min}$ (minor), $\mathrm{t}_{\mathrm{R} 2}=62.4 \mathrm{~min}$ (major) $]$; $e e=97 \%$. $[\alpha]_{\mathrm{D}}^{25}=-32.17\left(c 0.61, \mathrm{CH}_{2} \mathrm{Cl}_{2}\right.$ ). HRMS (ESI) calcd for $\mathrm{C}_{22} \mathrm{H}_{18} \mathrm{BrNO}_{2}(\mathrm{M}+\mathrm{H})^{+}$: 408.0594; found: 408.0600 .<smiles>O=C1C(Cc2ccc(C(F)(F)F)cc2)Oc2ccccc2N1Cc1ccccc1</smiles>

(S)-4-Benzyl-2-(4-(trifluoromethyl)benzyl)-2H-benzo[b][1,4] bxazin-3(4H)-one (2k). White solid, 99\% yield, $78.6 \mathrm{mg}$, m.p. $=73.1-74.4{ }^{\circ} \mathrm{C} .{ }^{1} \mathrm{H}$ NMR $\left(500 \mathrm{MHz}, \mathrm{CDCl}_{3}\right) \delta 7.57(\mathrm{~d}, J=8.0$ $\mathrm{Hz}, 2 \mathrm{H}), 7.42(\mathrm{~d}, J=8.0 \mathrm{~Hz}, 2 \mathrm{H}), 7.33-7.27(\mathrm{~m}, 2 \mathrm{H}), 7.27-7.22(\mathrm{~m}, 1 \mathrm{H}), 7.19(\mathrm{~d}, J=7.0 \mathrm{~Hz}$, $2 \mathrm{H}), 6.99-6.93(\mathrm{~m}, 2 \mathrm{H}), 6.92-6.85(\mathrm{~m}, 2 \mathrm{H}), 5.20(\mathrm{~d}, J=16.0 \mathrm{~Hz}, 1 \mathrm{H}), 5.08(\mathrm{~d}, J=16.0 \mathrm{~Hz}, 1 \mathrm{H})$, $4.90(\mathrm{dd}, J=9.0,4.0 \mathrm{~Hz}, 1 \mathrm{H}), 3.39(\mathrm{dd}, J=14.5,4.0 \mathrm{~Hz}, 1 \mathrm{H}), 3.26(\mathrm{dd}, J=14.5,9.0 \mathrm{~Hz}, 1 \mathrm{H}) .{ }^{13} \mathrm{C}$ $\operatorname{NMR}\left(126 \mathrm{MHz}, \mathrm{CDCl}_{3}\right) \delta 165.6,143.7,140.8,135.9,130.1,129.2$ (q, $\left.J=32.4 \mathrm{~Hz}\right), 128.9,128.7$, 127.5, 126.5, $125.3(\mathrm{q}, J=3.8 \mathrm{~Hz}), 124.2,122.9,119.8(\mathrm{q}, J=313.4 \mathrm{~Hz}), 117.5$ 115.6, 77.5, 45.3, 36.5. ${ }^{19} \mathrm{~F}$ NMR (471 MHz, $\left.\mathrm{CDCl}_{3}\right) \delta$-62.36. HPLC [DAICEL CHIRALPAK OD-H, hexane $/ \mathrm{PrOH}=95 / 5,220 \mathrm{~nm}, 0.5 \mathrm{~mL} / \mathrm{min}, 25{ }^{\circ} \mathrm{C} . \mathrm{t}_{\mathrm{R} 1}=25.7 \mathrm{~min}$ (major), $\mathrm{t}_{\mathrm{R} 2}=27.2 \mathrm{~min}$ (minor)]; $e e=98 \% .[\alpha]_{\mathrm{D}}^{25}=-37.25\left(c 0.16, \mathrm{CH}_{2} \mathrm{Cl}_{2}\right)$. HRMS (ESI) calcd for $\mathrm{C}_{23} \mathrm{H}_{18} \mathrm{~F}_{3} \mathrm{NO}_{2}(\mathrm{M}+\mathrm{Na})^{+}$: 398.1362; found: 398.1364 . 


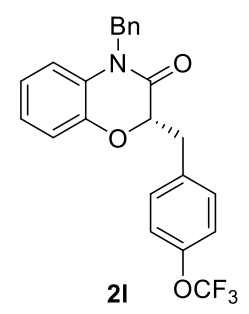

(S)-4-Benzyl-2-(4-(trifluoromethoxy)benzyl)-2H-benzo[b][1,4]oxazin-3(4H)-one (2l). White solid, 99\% yield, $81.7 \mathrm{mg}$, m.p. $=77.1-78.8^{\circ} \mathrm{C} .{ }^{1} \mathrm{H}$ NMR $\left(500 \mathrm{MHz}, \mathrm{CDCl}_{3}\right) \delta 7.36-7.28(\mathrm{~m}$, $4 \mathrm{H}), 7.27-7.22(\mathrm{~m}, 1 \mathrm{H}), 7.22-7.18(\mathrm{~m}, 2 \mathrm{H}), 7.17-7.12(\mathrm{~m}, 2 \mathrm{H}), 6.99-6.93(\mathrm{~m}, 2 \mathrm{H}), 6.92-$ $6.83(\mathrm{~m}, 2 \mathrm{H}), 5.20(\mathrm{~d}, J=16.0 \mathrm{~Hz}, 1 \mathrm{H}), 5.08(\mathrm{~d}, J=16.0 \mathrm{~Hz}, 1 \mathrm{H}), 4.88(\mathrm{dd}, J=9.0,3.5 \mathrm{~Hz}, 1 \mathrm{H})$, $3.33(\mathrm{dd}, J=14.5,3.5 \mathrm{~Hz}, 1 \mathrm{H}), 3.21(\mathrm{dd}, J=14.5,9.0 \mathrm{~Hz}, 1 \mathrm{H}) .{ }^{13} \mathrm{C}$ NMR $\left(126 \mathrm{MHz}, \mathrm{CDCl}_{3}\right) \delta$ 165.7, 148.2, 143.8, 135.9, 135.3, 131.1, 128.9, 128.7, 127.5, 126.6, 124.2, 122.8, 120.8, 117.5, 115.5, 77.7, 45.3, 36.0. ${ }^{19} \mathrm{~F}$ NMR $\left(471 \mathrm{MHz}, \mathrm{CDCl}_{3}\right) \delta-57.82$. HPLC [DAICEL CHIRALPAK OD-H, hexane $/ \mathrm{iPrOH}=95 / 5,220 \mathrm{~nm}, 0.5 \mathrm{~mL} / \mathrm{min}, 25^{\circ} \mathrm{C} . \mathrm{t}_{\mathrm{R} 1}=19.5 \mathrm{~min}$ (major), $\mathrm{t}_{\mathrm{R} 2}=22.3 \mathrm{~min}$ (minor)]; ee $=97 \%$. $[\alpha]_{\mathrm{D}}^{25}=-18.48\left(c \quad 0.59, \mathrm{CH}_{2} \mathrm{Cl}_{2}\right.$ ). HRMS (ESI) calcd for $\mathrm{C}_{23} \mathrm{H}_{18} \mathrm{~F}_{3} \mathrm{NO}_{3}$ $(\mathrm{M}+\mathrm{Na})^{+}:$436.1131; found: 436.1130 .<smiles>CCOc1ccc(CC2Oc3ccccc3N(Cc3ccccc3)C2=O)cc1</smiles>

Ethyl (S)-4-((4-benzyl-3-oxo-3,4-dihydro-2H-benzo[ $b][1,4]$ oxazin-2-yl)methyl)benzoate (2m). White solid, 99\% yield, $79.4 \mathrm{mg}$, m.p. $=60.7-61.6{ }^{\circ} \mathrm{C} .{ }^{1} \mathrm{H}$ NMR $\left(500 \mathrm{MHz}, \mathrm{CDCl}_{3}\right) \delta 8.00(\mathrm{~d}, J$ $=8.0 \mathrm{~Hz}, 2 \mathrm{H}), 7.37(\mathrm{~d}, J=8.0 \mathrm{~Hz}, 2 \mathrm{H}), 7.34-7.28(\mathrm{~m}, 2 \mathrm{H}), 7.28-7.17(\mathrm{~m}, 3 \mathrm{H}), 7.01-6.82(\mathrm{~m}$, 4H), $5.21(\mathrm{~d}, J=16.0 \mathrm{~Hz}, 1 \mathrm{H}), 5.08(\mathrm{~d}, J=16.0 \mathrm{~Hz}, 1 \mathrm{H}), 4.91(\mathrm{dd}, J=9.0,3.5 \mathrm{~Hz}, 1 \mathrm{H}), 4.38(\mathrm{q}, J$ $=7.5 \mathrm{~Hz}, 2 \mathrm{H}), 3.38(\mathrm{dd}, J=14.5,3.5 \mathrm{~Hz}, 1 \mathrm{H}), 3.24(\mathrm{dd}, J=14.5,9.5 \mathrm{~Hz}, 1 \mathrm{H}), 1.39(\mathrm{t}, J=7.0 \mathrm{~Hz}$, $3 \mathrm{H}) .{ }^{13} \mathrm{C}$ NMR $\left(126 \mathrm{MHz}, \mathrm{CDCl}_{3}\right) \delta 166.5,165.6,143.7,141.9,136.0,129.69,129.66,129.2$, 128.9, 128.7, 127.5, 126.5, 124.2, 122.8, 117.5, 115.5, 77.6, 60.9, 45.3, 36.7, 14.4. HPLC [DAICEL CHIRALPAK OD-H, hexane $/ \mathrm{iPrOH}=95 / 5,220 \mathrm{~nm}, 0.5 \mathrm{~mL} / \mathrm{min}, 25^{\circ} \mathrm{C} . \mathrm{t}_{\mathrm{R} 1}=31.3 \mathrm{~min}$ (major), $\mathrm{t}_{\mathrm{R} 2}=33.8 \mathrm{~min}$ (minor)]; $e e=98 \%$. $[\alpha]_{\mathrm{D}}^{25}=-42.56\left(c 0.60, \mathrm{CH}_{2} \mathrm{Cl}_{2}\right.$ ). HRMS (ESI) calcd for $\mathrm{C}_{25} \mathrm{H}_{23} \mathrm{NO}_{4}(\mathrm{M}+\mathrm{Na})^{+}$: 424.1519; found: 424.1522 .<smiles>O=C1Nc2ccccc2O[C@H]1Cc1cccc(Cl)c1</smiles>

$2 n$

(S)-4-Benzyl-2-(3-chlorobenzyl)-2H-benzo[b][1,4]oxazin-3(4H)-one (2n). White solid, 99\% yield, $71.8 \mathrm{mg}$, m.p. $=79.8-81.6{ }^{\circ} \mathrm{C} .{ }^{1} \mathrm{H}$ NMR $\left(500 \mathrm{MHz}, \mathrm{CDCl}_{3}\right) \delta 7.36-7.29(\mathrm{~m}, 3 \mathrm{H}), 7.28-$ $7.15(\mathrm{~m}, 6 \mathrm{H}), 7.01-6.94(\mathrm{~m}, 2 \mathrm{H}), 6.92-6.82(\mathrm{~m}, 2 \mathrm{H}), 5.21(\mathrm{~d}, J=16.5 \mathrm{~Hz}, 1 \mathrm{H}), 5.08(\mathrm{~d}, J=$ 
$16.0 \mathrm{~Hz}, 1 \mathrm{H}), 4.87(\mathrm{dd}, J=9.0,3.5 \mathrm{~Hz}, 1 \mathrm{H}), 3.30(\mathrm{dd}, J=14.5,3.4 \mathrm{~Hz}, 1 \mathrm{H}), 3.17$ (dd, $J=14.5$, $9.5 \mathrm{~Hz}, 1 \mathrm{H}) .{ }^{13} \mathrm{C} \mathrm{NMR}\left(126 \mathrm{MHz}, \mathrm{CDCl}_{3}\right) \delta 165.7,143.7,138.6,136.0,134.2,129.8,129.6,128.9$, 128.7, 127.9, 127.5, 127.1, 126.5, 124.2, 122.8, 117.6, 115.5, 77.6, 45.3, 36.3. HPLC [DAICEL CHIRALPAK OD-H, hexane $/ \mathrm{iPrOH}=95 / 5,220 \mathrm{~nm}, 0.5 \mathrm{~mL} / \mathrm{min}, 25^{\circ} \mathrm{C} \cdot \mathrm{t}_{\mathrm{R} 1}=20.6 \mathrm{~min}$ (major), $\mathrm{t}_{\mathrm{R} 2}=24.3 \min$ (minor)]; $e e=97 \%$. [ $\left.\alpha\right]_{\mathrm{D}}^{25}=-24.82\left(c 0.54, \mathrm{CH}_{2} \mathrm{Cl}_{2}\right)$. HRMS (ESI) calcd for $\mathrm{C}_{22} \mathrm{H}_{18} \mathrm{ClNO}_{2}(\mathrm{M}+\mathrm{H})^{+}$: 364.1099; found: 364.1100 .

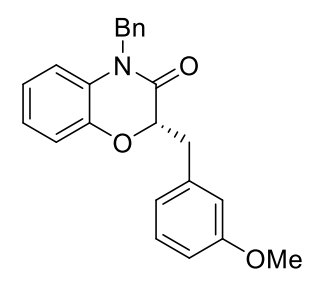

20

(S)-4-Benzyl-2-(3-methoxybenzyl)-2H-benzo[b][1,4]oxazin-3(4H)-one (2o). White solid, $98 \%$ yield, $70.4 \mathrm{mg}$, m.p. $=105.7-106.4{ }^{\circ} \mathrm{C} .{ }^{1} \mathrm{H}$ NMR $\left(500 \mathrm{MHz}, \mathrm{CDCl}_{3}\right) \delta 7.35-7.29(\mathrm{~m}, 2 \mathrm{H}), 7.28$ $-7.20(\mathrm{~m}, 4 \mathrm{H}), 6.99-6.94(\mathrm{~m}, 2 \mathrm{H}), 6.91-6.83(\mathrm{~m}, 4 \mathrm{H}), 6.81(\mathrm{dd}, J=8.5,2.5 \mathrm{~Hz}, 1 \mathrm{H}), 5.25(\mathrm{~d}, J$ $=16.5 \mathrm{~Hz}, 1 \mathrm{H}), 5.06(\mathrm{~d}, J=16.0 \mathrm{~Hz}, 1 \mathrm{H}), 4.91(\mathrm{dd}, J=9.5,3.5 \mathrm{~Hz}, 1 \mathrm{H}), 3.79(\mathrm{~s}, 3 \mathrm{H}), 3.28(\mathrm{dd}, J$ $=14.5,3.5 \mathrm{~Hz}, 1 \mathrm{H}), 3.14(\mathrm{dd}, J=14.0,9.5 \mathrm{~Hz}, 1 \mathrm{H}) .{ }^{13} \mathrm{C} \mathrm{NMR}\left(126 \mathrm{MHz}, \mathrm{CDCl}_{3}\right) \delta 166.0,159.6$, 143.7, 138.2, 136.1, 129.4, 128.9, 128.7, 127.4, 126.5, 124.1, 122.6, 122.0, 117.6, 115.4, 115.1, 112.5, 78.1, 55.2, 45.3, 36.7. HPLC [DAICEL CHIRALPAK AD-H, hexane/iPrOH = 90/10, 220 $\mathrm{nm}, 1.0 \mathrm{~mL} / \mathrm{min}, 25^{\circ} \mathrm{C} \cdot \mathrm{t}_{\mathrm{R} 1}=16.8 \mathrm{~min}$ (minor), $\mathrm{t}_{\mathrm{R} 2}=22.7 \min$ (major) $] ; e e=93 \% .[\alpha]_{\mathrm{D}}^{25}=-32.84$ (c $0.53, \mathrm{CH}_{2} \mathrm{Cl}_{2}$ ). HRMS (ESI) calcd for $\mathrm{C}_{23} \mathrm{H}_{21} \mathrm{NO}_{3}(\mathrm{M}+\mathrm{Na})^{+}:$382.1414; found: 382.1422 .<smiles>O=C1Nc2ccccc2OC1Cc1ccccc1Cl</smiles>

$2 p$

(S)-4-Benzyl-2-(2-chlorobenzyl)-2H-benzo[b][1,4]oxazin-3(4H)-one (2p). White solid, 99\% yield, $71.9 \mathrm{mg}$, m.p. $=73.4-74.2{ }^{\circ} \mathrm{C} .{ }^{1} \mathrm{H}$ NMR $\left(500 \mathrm{MHz}, \mathrm{CDCl}_{3}\right) \delta 7.41-7.36(\mathrm{~m}, 1 \mathrm{H}), 7.36-$ $7.30(\mathrm{~m}, 2 \mathrm{H}), 7.29-7.23(\mathrm{~m}, 4 \mathrm{H}), 7.23-7.18(\mathrm{~m}, 2 \mathrm{H}), 7.00-6.86(\mathrm{~m}, 4 \mathrm{H}), 5.23(\mathrm{~d}, J=16.0 \mathrm{~Hz}$, $1 \mathrm{H}), 5.11(\mathrm{~d}, J=16.0 \mathrm{~Hz}, 1 \mathrm{H}), 5.02(\mathrm{dd}, J=10.0,3.5 \mathrm{~Hz}, 1 \mathrm{H}), 3.58(\mathrm{dd}, J=14.5,3.5 \mathrm{~Hz}, 1 \mathrm{H})$, $3.19(\mathrm{dd}, J=14.5,10.0 \mathrm{~Hz}, 1 \mathrm{H}) .{ }^{13} \mathrm{C} \mathrm{NMR}\left(126 \mathrm{MHz}, \mathrm{CDCl}_{3}\right) \delta 165.7,143.7,136.1,134.4,134.3$, 132.0, 129.6, 128.9, 128.8, 128.4, 127.5, 126.7, 124.1, 122.7, 117.8, 115.5, 76.3, 45.3, 34.5. HPLC [DAICEL CHIRALPAK OD-H, hexane $/ \mathrm{PrOH}=95 / 5,220 \mathrm{~nm}, 0.5 \mathrm{~mL} / \mathrm{min}, 25^{\circ} \mathrm{C} . \mathrm{t}_{\mathrm{R} 1}=$ $16.9 \min$ (major), $\mathrm{t}_{\mathrm{R} 2}=18.7 \min$ (minor)]; $e e=83 \% .[\alpha]_{\mathrm{D}}^{25}=-28.80\left(c 0.55, \mathrm{CH}_{2} \mathrm{Cl}_{2}\right) . \mathrm{HRMS}$ (ESI) calcd for $\mathrm{C}_{22} \mathrm{H}_{18} \mathrm{ClNO}_{2}(\mathrm{M}+\mathrm{Na})^{+}$: 386.0918; found: 386.0923 .<smiles>Cc1ccccc1CC1Oc2ccccc2N(Cc2ccccc2)C1=O</smiles> 
(S)-4-Benzyl-2-(2-methoxybenzyl)-2H-benzo[b][1,4]oxazin-3(4H)-one (2q). Yellow wax, 99\% yield, $67.9 \mathrm{mg} .{ }^{1} \mathrm{H}$ NMR $\left(500 \mathrm{MHz}, \mathrm{CDCl}_{3}\right) \delta 7.36-7.29(\mathrm{~m}, 2 \mathrm{H}), 7.29-7.22(\mathrm{~m}, 3 \mathrm{H}), 7.22-$ $7.12(\mathrm{~m}, 4 \mathrm{H}), 6.99-6.92(\mathrm{~m}, 2 \mathrm{H}), 6.92-6.86(\mathrm{~m}, 2 \mathrm{H}), 5.24(\mathrm{~d}, J=16.0 \mathrm{~Hz}, 1 \mathrm{H}), 5.10(\mathrm{~d}, J=$ $16.0 \mathrm{~Hz}, 1 \mathrm{H}), 4.91(\mathrm{dd}, J=10.0,3.0 \mathrm{~Hz}, 1 \mathrm{H}), 3.37(\mathrm{dd}, J=14.5,3.0 \mathrm{~Hz}, 1 \mathrm{H}), 3.12(\mathrm{dd}, J=15.0$, $10.0 \mathrm{~Hz}, 1 \mathrm{H}), 2.37$ (s, 3H). ${ }^{13} \mathrm{C}$ NMR $\left(126 \mathrm{MHz}, \mathrm{CDCl}_{3}\right) \delta 166.1,143.8,136.7,136.1,135.0$, 130.4, 130.3, 128.9, 128.8, 127.5, 127.0, 126.6, 125.9, 124.1, 122.6, 117.7, 115.4, 77.4, 45.3, 34.0, 19.6. HPLC [DAICEL CHIRALPAK OD-H, hexane $/ \mathrm{PrOH}=95 / 5,220 \mathrm{~nm}, 0.5 \mathrm{~mL} / \mathrm{min}, 25^{\circ} \mathrm{C}$. $\mathrm{t}_{\mathrm{R} 1}=15.9 \min$ (major), $\mathrm{t}_{\mathrm{R} 2}=17.2 \min$ (minor)]; $e e=82 \%$. $[\alpha]_{\mathrm{D}}^{25}=-33.06\left(c 0.54, \mathrm{CH}_{2} \mathrm{Cl}_{2}\right) . \mathrm{HRMS}$ (ESI) calcd for $\mathrm{C}_{23} \mathrm{H}_{21} \mathrm{NO}_{2}(\mathrm{M}+\mathrm{Na})^{+}$: 366.1465; found: 366.1466 .

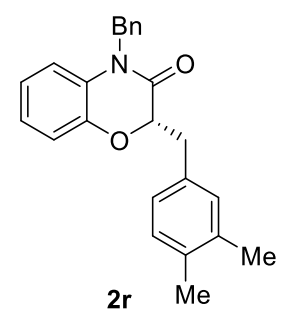

(S)-4-Benzyl-2-(3,4-dimethylbenzyl)-2H-benzo[b][1,4]oxazin-3(4H)-one (2r). White solid, 99\% yield, $70.7 \mathrm{mg}$, m.p. $=62.7-63.9{ }^{\circ} \mathrm{C} .{ }^{1} \mathrm{H} \mathrm{NMR}\left(500 \mathrm{MHz}, \mathrm{CDCl}_{3}\right) \delta 7.35-7.28(\mathrm{~m}, 2 \mathrm{H}), 7.27-$ $7.20(\mathrm{~m}, 3 \mathrm{H}), 7.10-7.05(\mathrm{~m}, 2 \mathrm{H}), 7.02-6.99(\mathrm{~m}, 1 \mathrm{H}), 6.98-6.94(\mathrm{~m}, 2 \mathrm{H}), 6.91-6.82(\mathrm{~m}, 2 \mathrm{H})$, $5.26(\mathrm{~d}, J=16.0 \mathrm{~Hz}, 1 \mathrm{H}), 5.03(\mathrm{~d}, J=16.0 \mathrm{~Hz}, 1 \mathrm{H}), 4.89(\mathrm{dd}, J=10.0,3.5 \mathrm{~Hz}, 1 \mathrm{H}), 3.22(\mathrm{dd}, J=$ 14.5, 3.5 Hz, 1H), $3.08(\mathrm{dd}, J=14.5,10.0 \mathrm{~Hz}, 1 \mathrm{H}), 2.25(\mathrm{~s}, 6 \mathrm{H}) .{ }^{13} \mathrm{C} \mathrm{NMR}\left(126 \mathrm{MHz}, \mathrm{CDCl}_{3}\right) \delta$ 166.1, 143.7, 136.6, 136.1, 135.0, 133.9, 130.8, 129.7, 128.9, 128.8, 127.4, 126.9, 126.5, 124.1, 122.5, 117.7, 115.4, 78.3, 45.2, 36.2, 19.8, 19.4. HPLC [DAICEL CHIRALPAK AD-H, hexane $/ \mathrm{PrOH}=95 / 5,220 \mathrm{~nm}, 0.5 \mathrm{~mL} / \mathrm{min}, 25{ }^{\circ} \mathrm{C} . \mathrm{t}_{\mathrm{R} 1}=30.9 \min$ (minor), $\mathrm{t}_{\mathrm{R} 2}=42.7 \mathrm{~min}$ (major)]; $e e=97 \% .[\alpha]_{\mathrm{D}}^{25}=-36.97\left(c 0.53, \mathrm{CH}_{2} \mathrm{Cl}_{2}\right)$. HRMS (ESI) calcd for $\mathrm{C}_{24} \mathrm{H}_{23} \mathrm{NO}_{2}(\mathrm{M}+\mathrm{Na})^{+}: 380.1621$; found: 380.1619 .

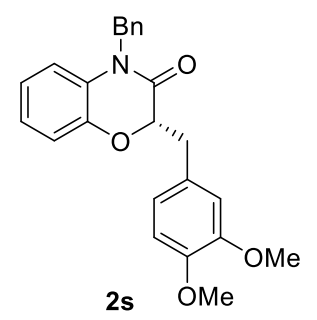

(S)-4-Benzyl-2-(3,4-dimethoxybenzyl)-2H-benzo[b][1,4] oxazin-3(4H)-one (2s). White solid, 92\% yield, $71.6 \mathrm{mg}$, m.p. $=57.7-59.4{ }^{\circ} \mathrm{C} .{ }^{1} \mathrm{H}$ NMR $\left(500 \mathrm{MHz}, \mathrm{CDCl}_{3}\right) \delta 7.35-7.27(\mathrm{~m}, 3 \mathrm{H}), 7.26-$ $7.22(\mathrm{~m}, 1 \mathrm{H}), 7.20(\mathrm{~d}, J=8.0 \mathrm{~Hz}, 2 \mathrm{H}), 6.98-6.94(\mathrm{~m}, 2 \mathrm{H}), 6.90-6.83(\mathrm{~m}, 2 \mathrm{H}), 6.82(\mathrm{~s}, 2 \mathrm{H})$, $5.26(\mathrm{~d}, J=16.0 \mathrm{~Hz}, 1 \mathrm{H}), 5.03(\mathrm{~d}, J=16.0 \mathrm{~Hz}, 1 \mathrm{H}), 4.90(\mathrm{dd}, J=9.5,3.5 \mathrm{~Hz}, 1 \mathrm{H}), 3.87(\mathrm{~s}, 3 \mathrm{H})$, $3.84(\mathrm{~s}, 3 \mathrm{H}), 3.24(\mathrm{dd}, J=14.5,3.5 \mathrm{~Hz}, 1 \mathrm{H}), 3.12(\mathrm{dd}, J=14.5,9.5 \mathrm{~Hz}, 1 \mathrm{H}) .{ }^{13} \mathrm{C} \mathrm{NMR}(126 \mathrm{MHz}$, $\left.\mathrm{CDCl}_{3}\right) \delta 166.0,148.8,148.0,143.7,136.0,129.0,128.9,128.7,127.4,126.5,124.1,122.6,121.8$, 117.5, 115.5, 112.9, 111.2, 78.2, 55.9, 55.8, 45.2, 36.5. HPLC [DAICEL CHIRALPAK OD-H, hexane $/ \mathrm{PrOH}=95 / 5,220 \mathrm{~nm}, 0.5 \mathrm{~mL} / \mathrm{min}, 25{ }^{\circ} \mathrm{C} . \mathrm{t}_{\mathrm{R} 1}=50.9 \mathrm{~min}$ (major), $\mathrm{t}_{\mathrm{R} 2}=56.5 \mathrm{~min}$ (minor)]; $e e=97 \%$. $[\alpha]_{\mathrm{D}}^{25}=-40.78\left(c 0.58, \mathrm{CH}_{2} \mathrm{Cl}_{2}\right)$. HRMS (ESI) calcd for $\mathrm{C}_{24} \mathrm{H}_{23} \mathrm{NO}_{4}(\mathrm{M}+\mathrm{H})^{+}: 390.1700$; found: 390.1699 . 


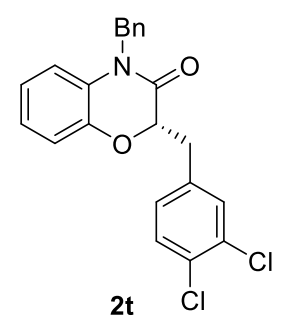

(S)-4-Benzyl-2-(3,4-dichlorobenzyl)-2H-benzo[b][1,4] oxazin-3(4H)-one (2t). White solid, 99\% yield, $78.6 \mathrm{mg}$, m.p. $=88.7-90.1{ }^{\circ} \mathrm{C} .{ }^{1} \mathrm{H}$ NMR $\left(500 \mathrm{MHz}, \mathrm{CDCl}_{3}\right) \delta 7.41(\mathrm{~s}, 1 \mathrm{H}), 7.37(\mathrm{~d}, J=8.0$ $\mathrm{Hz}, 1 \mathrm{H}), 7.34-7.28(\mathrm{~m}, 2 \mathrm{H}), 7.28-7.22(\mathrm{~m}, 1 \mathrm{H}), 7.22-7.17(\mathrm{~m}, 2 \mathrm{H}), 7.14(\mathrm{~d}, J=8.0 \mathrm{~Hz}$, $1 \mathrm{H}), 7.01-6.93(\mathrm{~m}, 2 \mathrm{H}), 6.93-6.82(\mathrm{~m}, 2 \mathrm{H}), 5.19(\mathrm{~d}, J=16.0 \mathrm{~Hz}, 1 \mathrm{H}), 5.09(\mathrm{~d}, J=16.0 \mathrm{~Hz}, 1 \mathrm{H})$, $4.84(\mathrm{dd}, J=9.5,3.5 \mathrm{~Hz}, 1 \mathrm{H}), 3.29(\mathrm{dd}, J=14.5,3.5 \mathrm{~Hz}, 1 \mathrm{H}), 3.18(\mathrm{dd}, J=14.5,8.5 \mathrm{~Hz}, 1 \mathrm{H}) .{ }^{13} \mathrm{C}$ NMR $\left(126 \mathrm{MHz}, \mathrm{CDCl}_{3}\right) \delta 165.4,143.7,136.8,135.9,132.3,131.7,131.0,130.3,129.2,128.9$, 128.6, 127.5, 126.5, 124.2, 122.9, 117.4, 115.5, 77.4, 45.3, 35.8. HPLC [DAICEL CHIRALPAK $\mathrm{OD}-\mathrm{H}$, hexane $/ \mathrm{iPrOH}=95 / 5,220 \mathrm{~nm}, 0.5 \mathrm{~mL} / \mathrm{min}, 25^{\circ} \mathrm{C} . \mathrm{t}_{\mathrm{R} 1}=26.4 \mathrm{~min}$ (major), $\mathrm{t}_{\mathrm{R} 2}=30.3 \mathrm{~min}$ (minor)]; $e e=97 \%$. $[\alpha]_{\mathrm{D}}^{25}=-18.93\left(c \quad 0.59, \mathrm{CH}_{2} \mathrm{Cl}_{2}\right.$ ). HRMS (ESI) calcd for $\mathrm{C}_{22} \mathrm{H}_{17} \mathrm{Cl}_{2} \mathrm{NO}_{2}$ $(\mathrm{M}+\mathrm{H})^{+}:$398.0709; found: 398.0713 .<smiles>O=C1C(Cc2ccc3c(c2)OCO3)Oc2ccccc2N1Cc1ccccc1</smiles>

(S)-2-(Benzo[d][1,3]dioxol-5-ylmethyl)-4-benzyl-2H-benzo[b][1,4]oxazin-3(4H)-one White wax, 98\% yield, $73.1 \mathrm{mg} .{ }^{1} \mathrm{H}$ NMR $\left(500 \mathrm{MHz}, \mathrm{CDCl}_{3}\right) \delta 7.33-7.27(\mathrm{~m}, 2 \mathrm{H}), 7.26-7.18$ (m, 3H), $6.99-6.92(\mathrm{~m}, 2 \mathrm{H}), 6.89-6.82(\mathrm{~m}, 2 \mathrm{H}), 6.78(\mathrm{~s}, 1 \mathrm{H}), 6.76-6.70(\mathrm{~m}, 2 \mathrm{H}), 5.92-5.88$ $(\mathrm{m}, 2 \mathrm{H}), 5.22(\mathrm{~d}, J=16.0 \mathrm{~Hz}, 1 \mathrm{H}), 5.03(\mathrm{~d}, J=16.0 \mathrm{~Hz}, 1 \mathrm{H}), 4.84(\mathrm{dd}, J=9.0,3.5 \mathrm{~Hz}, 1 \mathrm{H}), 3.20$ $(\mathrm{dd}, J=14.5,3.5 \mathrm{~Hz}, 1 \mathrm{H}), 3.08(\mathrm{dd}, J=14.5,9.5 \mathrm{~Hz}, 1 \mathrm{H}) .{ }^{13} \mathrm{C} \mathrm{NMR}\left(126 \mathrm{MHz}, \mathrm{CDCl}_{3}\right) \delta 165.92$, $147.7,146.5,143.7,136.1,130.2,128.9,128.7,127.5,126.6,124.1,122.8,122.6,117.6,115.4$, 110.1, 108.2, 100.9, 78.2, 45.2, 36.4. HPLC [DAICEL CHIRALPAK AD-H, hexane $/ i \operatorname{PrOH}=$ $95 / 5,220 \mathrm{~nm}, 0.5 \mathrm{~mL} / \mathrm{min}, 25^{\circ} \mathrm{C} \cdot \mathrm{t}_{\mathrm{R} 1}=68.8 \mathrm{~min}$ (minor), $\mathrm{t}_{\mathrm{R} 2}=82.8 \min$ (major) $] ; e e=97 \% .[\alpha]_{\mathrm{D}}^{25}$ $=-127.62$ (c $0.55, \mathrm{CH}_{2} \mathrm{Cl}_{2}$ ). HRMS (ESI) calcd for $\mathrm{C}_{23} \mathrm{H}_{19} \mathrm{NO}_{4}(\mathrm{M}+\mathrm{H})^{+}$: 374.1387; found: 374.1387 .<smiles>O=C1Nc2ccccc2O[C@H]1Cc1ccc2ccccc2c1</smiles>

(S)-4-Benzyl-2-(naphthalen-2-ylmethyl)-2H-benzo[b][1,4]oxazin-3(4H)-one (2v). White solid, 99\% yield, $75.0 \mathrm{mg}$, m.p. $=131.2-133.4{ }^{\circ} \mathrm{C} .{ }^{1} \mathrm{H}$ NMR $\left(500 \mathrm{MHz}, \mathrm{CDCl}_{3}\right) \delta 7.84-7.74(\mathrm{~m}, 3 \mathrm{H})$, $7.72(\mathrm{~s}, 1 \mathrm{H}), 7.49-7.39$ (m, 3H), $7.27-7.14(\mathrm{~m}, 5 \mathrm{H}), 6.96-6.90$ (m, 2H), $6.88-6.79(\mathrm{~m}, 2 \mathrm{H})$, 
$5.21(\mathrm{~d}, J=16.0 \mathrm{~Hz}, 1 \mathrm{H}), 5.04-4.95(\mathrm{~m}, 2 \mathrm{H}), 3.47(\mathrm{dd}, J=14.5,3.5 \mathrm{~Hz}, 1 \mathrm{H}), 3.33(\mathrm{dd}, J=14.5$, $9.5 \mathrm{~Hz}, 1 \mathrm{H}) .{ }^{13} \mathrm{C} \mathrm{NMR}\left(126 \mathrm{MHz}, \mathrm{CDCl}_{3}\right) \delta 166.0,143.9,136.1,134.2,133.6,132.5,128.9,128.8$, 128.4, 128.1, 127.9, 127.8, 127.7, 127.5, 126.6, 126.1, 125.7, 124.2, 122.7, 117.7, 115.5, 78.1, 45.3, 37.0. HPLC [DAICEL CHIRALPAK OD-H, hexane $/ \mathrm{PrOH}=95 / 5,220 \mathrm{~nm}, 0.5 \mathrm{~mL} / \mathrm{min}, 25$ ${ }^{\circ} \mathrm{C} . \mathrm{t}_{\mathrm{R} 1}=25.9 \min$ (major), $\mathrm{t}_{\mathrm{R} 2}=34.7 \mathrm{~min}$ (minor) $] ; e e=93 \%$. $[\alpha]_{\mathrm{D}}^{25}=-19.01\left(c 0.57, \mathrm{CH}_{2} \mathrm{Cl}_{2}\right.$ ). HRMS (ESI) calcd for $\mathrm{C}_{26} \mathrm{H}_{21} \mathrm{NO}_{2}(\mathrm{M}+\mathrm{H})^{+}: 380.1645$; found: 380.1648 .

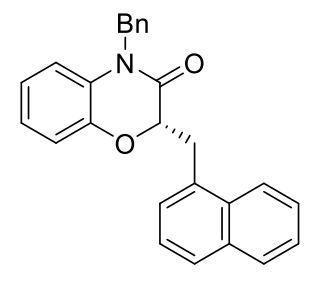

$2 w$

(S)-4-Benzyl-2-(naphthalen-1-ylmethyl)-2H-benzo[b][1,4]oxazin-3(4H)-one (2w). White solid, 99\% yield, $75.0 \mathrm{mg}$, m.p. $=109.8-111.7{ }^{\circ} \mathrm{C} .{ }^{1} \mathrm{H}$ NMR $\left(500 \mathrm{MHz}, \mathrm{CDCl}_{3}\right) \delta 8.12(\mathrm{~d}, J=8.5 \mathrm{~Hz}$, $1 \mathrm{H}), 7.85(\mathrm{~d}, J=8.0 \mathrm{~Hz}, 1 \mathrm{H}), 7.76(\mathrm{~d}, J=8.0 \mathrm{~Hz}, 1 \mathrm{H}), 7.55-7.49(\mathrm{~m}, 1 \mathrm{H}), 7.49-7.44(\mathrm{~m}, 1 \mathrm{H})$, $7.43-7.38(\mathrm{~m}, 1 \mathrm{H}), 7.37-7.33(\mathrm{~m}, 1 \mathrm{H}), 7.33-7.28(\mathrm{~m}, 2 \mathrm{H}), 7.28-7.19(\mathrm{~m}, 3 \mathrm{H}), 6.94-6.89(\mathrm{~m}$, $1 \mathrm{H}), 6.89-6.82(\mathrm{~m}, 3 \mathrm{H}), 5.24(\mathrm{~d}, J=16.0 \mathrm{~Hz}, 1 \mathrm{H}), 5.11-5.01(\mathrm{~m}, 2 \mathrm{H}), 3.90(\mathrm{dd}, J=14.5,3.0$ $\mathrm{Hz}, 1 \mathrm{H}), 3.45(\mathrm{dd}, J=15.0,10.0 \mathrm{~Hz}, 1 \mathrm{H}) .{ }^{13} \mathrm{C} \mathrm{NMR}\left(126 \mathrm{MHz}, \mathrm{CDCl}_{3}\right) \delta 166.3,143.6,136.2$, 134.0, 132.7, 132.0, 128.99, 128.97, 128.8, 128.1, 127.9, 127.6, 126.7, 126.4, 125.8, 125.4, 124.2, 123.7, 122.7, 118.0, 115.5, 77.5, 45.4, 34.0. HPLC [DAICEL CHIRALPAK OD-H, hexane/iPrOH $=95 / 5,220 \mathrm{~nm}, 0.5 \mathrm{~mL} / \mathrm{min}, 25{ }^{\circ} \mathrm{C} . \mathrm{t}_{\mathrm{R} 1}=26.9 \min$ (major), $\mathrm{t}_{\mathrm{R} 2}=31.7 \mathrm{~min}$ (minor)]; $e e=77 \%$. $[\alpha]_{\mathrm{D}}^{25}=-78.47\left(c 0.57, \mathrm{CH}_{2} \mathrm{Cl}_{2}\right)$. HRMS (ESI) calcd for $\mathrm{C}_{26} \mathrm{H}_{21} \mathrm{NO}_{2}(\mathrm{M}+\mathrm{Na})^{+}: 402.1465$; found: 402.1474 .

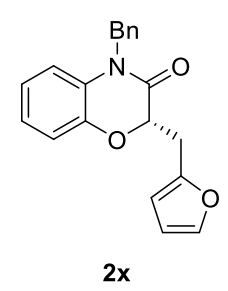

(S)-4-Benzyl-2-(furan-2-ylmethyl)-2H-benzo[b][1,4]oxazin-3(4H)-one (2x). White solid, $91 \%$ yield, $58.0 \mathrm{mg}$, m.p. $=140.3-141.9{ }^{\circ} \mathrm{C}$. Brown solid, m.p. $=61.5-62.3{ }^{\circ} \mathrm{C} .{ }^{1} \mathrm{H}$ NMR $(500 \mathrm{MHz}$, $\left.\mathrm{CDCl}_{3}\right) \delta 7.36-7.29(\mathrm{~m}, 3 \mathrm{H}), 7.27-7.21(\mathrm{~m}, 3 \mathrm{H}), 7.00-6.92(\mathrm{~m}, 2 \mathrm{H}), 6.92-6.83(\mathrm{~m}, 2 \mathrm{H}), 6.30$ $(\mathrm{dd}, J=3.0,2.0 \mathrm{~Hz}, 1 \mathrm{H}), 6.18(\mathrm{~d}, J=3.5 \mathrm{~Hz}, 1 \mathrm{H}), 5.21(\mathrm{~d}, J=16.0 \mathrm{~Hz}, 1 \mathrm{H}), 5.09(\mathrm{~d}, J=16.0 \mathrm{~Hz}$, $1 \mathrm{H}), 4.97(\mathrm{dd}, J=9.0,4.0 \mathrm{~Hz}, 1 \mathrm{H}), 3.39(\mathrm{dd}, J=15.5,4.0 \mathrm{~Hz}, 1 \mathrm{H}), 3.26(\mathrm{dd}, J=15.5,8.5 \mathrm{~Hz}, 1 \mathrm{H})$. ${ }^{13} \mathrm{C}$ NMR $\left(126 \mathrm{MHz}, \mathrm{CDCl}_{3}\right) \delta 165.6,150.5,143.9,141.7,136.1,128.9,128.7,127.5,126.6$, 124.1, 122.7, 117.6, 115.4, 110.4, 107.8, 75.8, 45.3, 29.6. HPLC [DAICEL CHIRALPAK OD-H, hexane $/ \mathrm{PrOH}=95 / 5,220 \mathrm{~nm}, 0.5 \mathrm{~mL} / \mathrm{min}, 25^{\circ} \mathrm{C} . \mathrm{t}_{\mathrm{R} 1}=19.1 \mathrm{~min}$ (major), $\mathrm{t}_{\mathrm{R} 2}=20.9 \min$ (minor)]; $e e=96 \% .[\alpha]_{\mathrm{D}}^{25}=-38.80\left(c 0.48, \mathrm{CH}_{2} \mathrm{Cl}_{2}\right) \cdot \mathrm{C}_{20} \mathrm{H}_{17} \mathrm{NO}_{3}(\mathrm{M}+\mathrm{H})^{+}: 320.1281$; found: 320.1282 . 


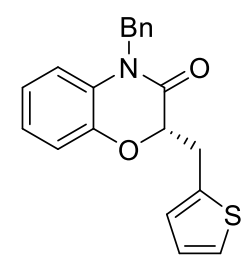

$2 y$

(S)-4-Benzyl-2-(thiophen-2-ylmethyl)-2H-benzo[b][1,4]oxazin-3(4H)-one (2y). White solid, 96\% yield, $64.3 \mathrm{mg}$, m.p. $=96.7-97.6{ }^{\circ} \mathrm{C} .{ }^{1} \mathrm{H}$ NMR $\left(500 \mathrm{MHz}, \mathrm{CDCl}_{3}\right) \delta 7.33-7.28(\mathrm{~m}, 1 \mathrm{H}), 7.27-$ $7.22(\mathrm{~m}, 2 \mathrm{H}), 7.21-7.16(\mathrm{~m}, 3 \mathrm{H}), 7.04(\mathrm{dd}, J=8.0,1.5 \mathrm{~Hz}, 1 \mathrm{H}), 7.00-6.93(\mathrm{~m}, 3 \mathrm{H}), 6.92-6.82$ $(\mathrm{m}, 2 \mathrm{H}), 5.21(\mathrm{~d}, J=16.0 \mathrm{~Hz}, 1 \mathrm{H}), 5.07(\mathrm{~d}, J=16.0 \mathrm{~Hz}, 1 \mathrm{H}), 4.88(\mathrm{dd}, J=8.5,3.5 \mathrm{~Hz}, 1 \mathrm{H}), 3.57$ $(\mathrm{dd}, J=15.5,3.5 \mathrm{~Hz}, 1 \mathrm{H}), 3.45(\mathrm{dd}, J=15.5,8.5 \mathrm{~Hz}, 1 \mathrm{H}) .{ }^{13} \mathrm{C} \mathrm{NMR}\left(126 \mathrm{MHz}, \mathrm{CDCl}_{3}\right) \delta 165.6$, 143.8, 138.2, 136.0, 128.9, 128.7, 127.5, 126.9, 126.8, 126.5, 124.7, 124.1, 122.7, 117.7, 115.5, 77.5, 45.2, 31.1. HPLC [DAICEL CHIRALPAK OD-H, hexane $/ \mathrm{PrOH}=95 / 5,220 \mathrm{~nm}, 0.5$ $\mathrm{mL} / \mathrm{min}, 25{ }^{\circ} \mathrm{C} . \mathrm{t}_{\mathrm{R} 1}=20.5 \min$ (major), $\mathrm{t}_{\mathrm{R} 2}=23.1 \mathrm{~min}$ (minor)]; $e e=95 \% .[\alpha]_{\mathrm{D}}^{25}=-17.83(c 0.51$, $\left.\mathrm{CH}_{2} \mathrm{Cl}_{2}\right) . \mathrm{C}_{20} \mathrm{H}_{17} \mathrm{NO}_{2} \mathrm{~S}(\mathrm{M}+\mathrm{H})^{+}$: 336.1053 ; found: 336.1052 .<smiles>O=C1C(Cc2ccsc2)Oc2ccccc2N1Cc1ccccc1</smiles>

(S)-4-Benzyl-2-(thiophen-3-ylmethyl)-2H-benzo[b][1,4]oxazin-3(4H)-one (2z). White solid, 99\% yield, $66.3 \mathrm{mg}$, m.p. $=74.3-76 .^{\circ} \mathrm{C} .{ }^{1} \mathrm{H}$ NMR $\left(500 \mathrm{MHz}, \mathrm{CDCl}_{3}\right) \delta 7.34-7.27(\mathrm{~m}, 2 \mathrm{H}), 7.26-$ $7.21(\mathrm{~m}, 2 \mathrm{H}), 7.18(\mathrm{~d}, J=7.5 \mathrm{~Hz}, 2 \mathrm{H}), 7.13(\mathrm{~d}, J=3.0 \mathrm{~Hz}, 1 \mathrm{H}), 7.05(\mathrm{~d}, J=4.5 \mathrm{~Hz}, 1 \mathrm{H}), 7.00-$ $6.91(\mathrm{~m}, 2 \mathrm{H}), 6.90-6.80(\mathrm{~m}, 2 \mathrm{H}), 5.21(\mathrm{~d}, J=16.0 \mathrm{~Hz}, 1 \mathrm{H}), 5.05(\mathrm{~d}, J=16.0 \mathrm{~Hz}, 1 \mathrm{H}), 4.88(\mathrm{dd}, J$ $=8.5,3.5 \mathrm{~Hz}, 1 \mathrm{H}), 3.34(\mathrm{dd}, J=15.0,3.5 \mathrm{~Hz}, 1 \mathrm{H}), 3.26(\mathrm{dd}, J=15.0,9.0 \mathrm{~Hz}, 1 \mathrm{H}) .{ }^{13} \mathrm{C}$ NMR $(126$ $\left.\mathrm{MHz}, \mathrm{CDCl}_{3}\right) \delta 165.9,143.9,136.6,136.0,129.0,128.9,128.7,127.5,126.5,125.4,124.1,123.0$, 122.6, 117.5, 115.5, 77.5, 45.2, 31.3. HPLC [DAICEL CHIRALPAK OD-H, hexane $/ \mathrm{iPrOH}=$ $95 / 5,220 \mathrm{~nm}, 0.5 \mathrm{~mL} / \mathrm{min}, 25{ }^{\circ} \mathrm{C} . \mathrm{t}_{\mathrm{R} 1}=21.2 \min$ (major), $\mathrm{t}_{\mathrm{R} 2}=25.1 \mathrm{~min}$ (minor)]; $e e=98 \%$. $[\alpha]_{\mathrm{D}}^{25}$ $=-52.85\left(c 0.50, \mathrm{CH}_{2} \mathrm{Cl}_{2}\right) \cdot \mathrm{C}_{20} \mathrm{H}_{17} \mathrm{NO}_{2} \mathrm{~S}(\mathrm{M}+\mathrm{H})^{+}: 336.1053$; found: 336.1056 .<smiles>O=C1C(Cc2ccccc2)Oc2ccccc2N1Cc1ccccc1</smiles>

(S)-4-Benzyl-2-(cyclohexylmethyl)-2H-benzo[b][1,4] oxazin-3(4H)-one (2aa). Yellow wax, 99\% yield, $66.3 \mathrm{mg} .{ }^{1} \mathrm{H}$ NMR $\left(500 \mathrm{MHz}, \mathrm{CDCl}_{3}\right) \delta 7.34-7.28(\mathrm{~m}, 2 \mathrm{H}), 7.27-7.20(\mathrm{~m}, 3 \mathrm{H}), 7.01-$ $6.92(\mathrm{~m}, 2 \mathrm{H}), 6.91-6.82(\mathrm{~m}, 2 \mathrm{H}), 5.24(\mathrm{~d}, J=16.0 \mathrm{~Hz}, 1 \mathrm{H}), 5.02(\mathrm{~d}, J=16.0 \mathrm{~Hz}, 1 \mathrm{H}), 4.78(\mathrm{dd}, J$ $=9.0,4.5 \mathrm{~Hz}, 1 \mathrm{H}), 1.93-1.56(\mathrm{~m}, 10 \mathrm{H}), 1.22-1.13(\mathrm{~m}, 1 \mathrm{H}), 1.06-0.93(\mathrm{~m}, 2 \mathrm{H}) .{ }^{13} \mathrm{C} \mathrm{NMR}(126$ $\left.\mathrm{MHz}, \mathrm{CDCl}_{3}\right) \delta 167.1,143.9,136.3,129.0,128.9,127.4,126.5,123.9,122.5,117.5,115.3,75.4$, 45.2, 37.4, 33.8, 33.8, 32.3, 26.5, 26.3, 26.1. HPLC [DAICEL CHIRALPAK AD-H, 
hexane $/ \mathrm{PrOH}=90 / 10,220 \mathrm{~nm}, 1.0 \mathrm{~mL} / \mathrm{min}, 25^{\circ} \mathrm{C} . \mathrm{t}_{\mathrm{R} 1}=8.6 \mathrm{~min}$ (minor), $\mathrm{t}_{\mathrm{R} 2}=10.8 \mathrm{~min}$ (major) $]$; $e e=64 \% .[\alpha]_{\mathrm{D}}^{25}=-19.17\left(c 0.50, \mathrm{CH}_{2} \mathrm{Cl}_{2}\right)$. HRMS (ESI) calcd for $\mathrm{C}_{22} \mathrm{H}_{25} \mathrm{NO}_{2}(\mathrm{M}+\mathrm{H})^{+}: 336.1958$; found: 336.1956 .

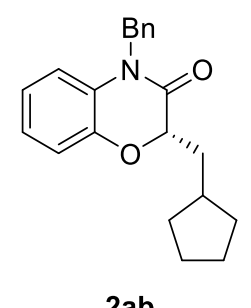

(S)-4-Benzyl-2-(cyclopentylmethyl)-2H-benzo[b][1,4]oxazin-3(4H)-one (2ab). Yellow wax, 98\% yield, $62.9 \mathrm{mg} .{ }^{1} \mathrm{H}$ NMR $\left(500 \mathrm{MHz}, \mathrm{CDCl}_{3}\right) \delta 7.35-7.29(\mathrm{~m}, 2 \mathrm{H}), 7.27-7.20(\mathrm{~m}, 3 \mathrm{H}), 7.01-$ $6.92(\mathrm{~m}, 2 \mathrm{H}), 6.91-6.81(\mathrm{~m}, 2 \mathrm{H}), 5.25(\mathrm{~d}, J=16.0 \mathrm{~Hz}, 1 \mathrm{H}), 5.01(\mathrm{~d}, J=16.0 \mathrm{~Hz}, 1 \mathrm{H}), 4.71(\mathrm{dd}, J$ $=9.5,4.0 \mathrm{~Hz}, 1 \mathrm{H}), 2.18-2.08(\mathrm{~m}, 1 \mathrm{H}), 1.99-1.83(\mathrm{~m}, 4 \mathrm{H}), 1.70-1.50(\mathrm{~m}, 4 \mathrm{H}), 1.24-1.12(\mathrm{~m}$, 2H). ${ }^{13} \mathrm{C}$ NMR $\left(126 \mathrm{MHz}, \mathrm{CDCl}_{3}\right) \delta 166.9,143.9,136.3,128.94,128.87,127.4,126.5,124.0$, 122.5, 117.5, 115.3, 77.1, 45.2, 36.4, 36.2, 33.1, 32.1, 25.2, 24.9. HPLC [DAICEL CHIRALPAK $\mathrm{AD}-\mathrm{H}$, hexane $/ \mathrm{iPrOH}=95 / 5,220 \mathrm{~nm}, 0.5 \mathrm{~mL} / \mathrm{min}, 25^{\circ} \mathrm{C} . \mathrm{t}_{\mathrm{R} 1}=18.5 \mathrm{~min}$ (minor), $\mathrm{t}_{\mathrm{R} 2}=22.8 \mathrm{~min}$ (major)]; ee $=78 \%$. $[\alpha]_{\mathrm{D}}^{25}=-24.84\left(c \quad 0.48, \mathrm{CH}_{2} \mathrm{Cl}_{2}\right) . \mathrm{C}_{21} \mathrm{H}_{23} \mathrm{NO}_{2}(\mathrm{M}+\mathrm{H})^{+}$: 322.1808; found: 322.1799 .<smiles>O=C1C(CC2CC2)Oc2ccccc2N1Cc1ccccc1</smiles>

2ac

(S)-4-benzyl-2-(cyclopropylmethyl)-2H-benzo[b][1,4]oxazin-3(4H)-one (2ac). Yellow solid, 98\% yield, $57.4 \mathrm{mg}, \mathrm{m} . \mathrm{p} .=38.6-40.9{ }^{\circ} \mathrm{C} .{ }^{1} \mathrm{H}$ NMR $\left(500 \mathrm{MHz}, \mathrm{CDCl}_{3}\right) \delta 7.34-7.28(\mathrm{~m}, 2 \mathrm{H}), 7.27-$ $7.20(\mathrm{~m}, 3 \mathrm{H}), 7.01(\mathrm{dd}, J=7.5,1.5 \mathrm{~Hz}, 1 \mathrm{H}), 6.96(\mathrm{td}, J=7.5,2.0 \mathrm{~Hz}, 1 \mathrm{H}), 6.90-6.82(\mathrm{~m}, 2 \mathrm{H})$, $5.22(\mathrm{~d}, J=16.0 \mathrm{~Hz}, 1 \mathrm{H}), 5.04(\mathrm{~d}, J=16.0 \mathrm{~Hz}, 1 \mathrm{H}), 4.78(\mathrm{dd}, J=9.0,4.0 \mathrm{~Hz}, 1 \mathrm{H}), 1.96(\mathrm{ddd}, J=$ 15.0, 9.5, 6.5 Hz, 1H), 1.70 (ddd, $J=12.0,8.0,4.0 \mathrm{~Hz}, 1 \mathrm{H}), 1.05-0.93(\mathrm{~m}, 1 \mathrm{H}), 0.59-0.45(\mathrm{~m}$, $2 \mathrm{H}), 0.23-0.07(\mathrm{~m}, 2 \mathrm{H}) .{ }^{13} \mathrm{C} \mathrm{NMR}\left(126 \mathrm{MHz}, \mathrm{CDCl}_{3}\right) \delta 166.6,144.1,136.2,128.9,127.4,126.5$, 124.0, 122.5, 117.4, 115.4, 77.8, 45.2, 35.6, 7.3, 5.0, 4.2. HPLC [DAICEL CHIRALPAK OJ-H, hexane $/ \mathrm{PrOH}=95 / 5,220 \mathrm{~nm}, 0.5 \mathrm{~mL} / \mathrm{min}, 25{ }^{\circ} \mathrm{C} . \mathrm{t}_{\mathrm{R} 1}=33.4 \min$ (minor), $\mathrm{t}_{\mathrm{R} 2}=40.9 \min$ (major)]; $e e=97 \% .[\alpha]_{\mathrm{D}}^{25}=-22.59\left(c 0.44, \mathrm{CH}_{2} \mathrm{Cl}_{2}\right) \cdot \mathrm{C}_{19} \mathrm{H}_{19} \mathrm{NO}_{2}(\mathrm{M}+\mathrm{H})^{+}: 294.1489$; found: 294.1494.<smiles>Cc1cccc2c1N(Cc1ccccc1)C(=O)C(Cc1ccccc1)O2</smiles>

(S)-2,4-Dibenzyl-5-methyl-2H-benzo[b][1,4]oxazin-3(4H)-one (2ad). Yellow wax, 99\% yield, $67.9 \mathrm{mg} .{ }^{1} \mathrm{H}$ NMR $\left(500 \mathrm{MHz}, \mathrm{CDCl}_{3}\right) \delta 7.33-7.26(\mathrm{~m}, 4 \mathrm{H}), 7.26-7.20(\mathrm{~m}, 3 \mathrm{H}), 7.19-7.14(\mathrm{~m}$, $1 \mathrm{H}), 7.08(\mathrm{~d}, J=7.5 \mathrm{~Hz}, 2 \mathrm{H}), 6.90(\mathrm{t}, J=7.5 \mathrm{~Hz}, 1 \mathrm{H}), 6.82(\mathrm{~d}, J=7.5 \mathrm{~Hz}, 1 \mathrm{H}), 6.76(\mathrm{~d}, J=7.5 \mathrm{~Hz}$, 
$1 \mathrm{H}), 5.31(\mathrm{~d}, J=16.0 \mathrm{~Hz}, 1 \mathrm{H}), 5.11(\mathrm{~d}, J=16.0 \mathrm{~Hz}, 1 \mathrm{H}), 4.62(\mathrm{dd}, J=9.5,3.5 \mathrm{~Hz}, 1 \mathrm{H}), 3.36(\mathrm{dd}$, $J=15.0,4.0 \mathrm{~Hz}, 1 \mathrm{H}), 3.11(\mathrm{dd}, J=14.5,9.5 \mathrm{~Hz}, 1 \mathrm{H}), 2.31(\mathrm{~s}, 3 \mathrm{H}) .{ }^{13} \mathrm{C} \mathrm{NMR}\left(126 \mathrm{MHz}, \mathrm{CDCl}_{3}\right) \delta$ 169.1, 148.1, 137.0, 136.8, 129.5, 129.4, 128.5, 128.4, 128.0, 127.1, 126.7, 126.7, 126.3, 124.9, 115.3, 78.9, 48.5, 35.6, 21.0. HPLC [DAICEL CHIRALPAK OD-H, hexane/iPrOH $=95 / 5,220$ $\mathrm{nm}, 0.5 \mathrm{~mL} / \mathrm{min}, 25^{\circ} \mathrm{C} . \mathrm{t}_{\mathrm{R} 1}=18.4 \min$ (minor), $\mathrm{t}_{\mathrm{R} 2}=19.5 \min$ (major)]; $e e=98 \% .[\alpha]_{\mathrm{D}}^{25}=+6.22$ (c 0.51, $\mathrm{CH}_{2} \mathrm{Cl}_{2}$ ). HRMS (ESI) calcd for $\mathrm{C}_{23} \mathrm{H}_{21} \mathrm{NO}_{2}(\mathrm{M}+\mathrm{H})^{+}$: 344.1645 ; found: 344.1645 .<smiles>O=C1C(Cc2ccccc2)Oc2cccc(F)c2N1Cc1ccccc1</smiles>

(S)-2,4-Dibenzyl-5-fluoro-2H-benzo[b][1,4]oxazin-3(4H)-one (2ae). White solid, 98\% yield, $68.0 \mathrm{mg}, \mathrm{m} . \mathrm{p} .=53.2-54.7{ }^{\circ} \mathrm{C} .{ }^{1} \mathrm{H}$ NMR $\left(500 \mathrm{MHz}, \mathrm{CDCl}_{3}\right) \delta 7.34-7.22(\mathrm{~m}, 7 \mathrm{H}), 7.22-7.16$ (m, 3H), $6.90(\mathrm{td}, J=8.5,5.5 \mathrm{~Hz}, 1 \mathrm{H}), 6.74(\mathrm{dt}, J=8.0,1.5 \mathrm{~Hz}, 1 \mathrm{H}), 6.69(\mathrm{ddd}, J=12.5,8.5,1.5$ $\mathrm{Hz}, 1 \mathrm{H}), 5.32(\mathrm{~d}, J=15.5 \mathrm{~Hz}, 1 \mathrm{H}), 5.26(\mathrm{~d}, J=15.5 \mathrm{~Hz}, 1 \mathrm{H}), 4.78(\mathrm{dd}, J=9.5,3.5 \mathrm{~Hz}, 1 \mathrm{H}), 3.33$ $(\mathrm{dd}, J=14.5,3.5 \mathrm{~Hz}, 1 \mathrm{H}), 3.13(\mathrm{dd}, J=15.0,9.5 \mathrm{~Hz}, 1 \mathrm{H}) .{ }^{13} \mathrm{C} \mathrm{NMR}\left(126 \mathrm{MHz}, \mathrm{CDCl}_{3}\right) \delta 166.5$, 152.6, 150.6, $147.2(\mathrm{~d}, J=4.5 \mathrm{~Hz}), 136.5(\mathrm{~d}, J=14.9 \mathrm{~Hz}), 129.5,128.48,128.45,127.3,127.0$, $126.9,124.5(\mathrm{~d}, J=10.0 \mathrm{~Hz}), 117.8(\mathrm{~d}, J=9.6 \mathrm{~Hz}), 113.6(\mathrm{~d}, J=3.0 \mathrm{~Hz}), 111.2(\mathrm{~d}, J=22.2 \mathrm{~Hz})$, 78.5, 47.1(d, $J=11.0 \mathrm{~Hz}), 47.0,36.0 .{ }^{19} \mathrm{~F}$ NMR $\left(471 \mathrm{MHz}, \mathrm{CDCl}_{3}\right) \delta$-121.42. HPLC [DAICEL CHIRALPAK OD-H, hexane $/ \mathrm{iPrOH}=95 / 5,220 \mathrm{~nm}, 0.5 \mathrm{~mL} / \mathrm{min}, 25^{\circ} \mathrm{C} \cdot \mathrm{t}_{\mathrm{R} 1}=17.5 \mathrm{~min}$ (major), $\mathrm{t}_{\mathrm{R} 2}=20.0 \min$ (minor)]; $e e=99 \%$. $[\alpha]_{\mathrm{D}}^{25}=-27.35\left(c 0.52, \mathrm{CH}_{2} \mathrm{Cl}_{2}\right)$. HRMS (ESI) calcd for $\mathrm{C}_{22} \mathrm{H}_{18} \mathrm{FNO}_{2}(\mathrm{M}+\mathrm{H})^{+}$: 348.1394; found: 348.1399 .<smiles>Cc1ccc2c(c1)N(Cc1ccccc1)C(=O)[C@H](Cc1ccccc1)O2</smiles>

(S)-2,4-Dibenzyl-6-methyl-2H-benzo[b][1,4]oxazin-3(4H)-one (2af). White solid, 99\% yield, $67.9 \mathrm{mg}, \mathrm{m} . \mathrm{p} .=73.2-75.8{ }^{\circ} \mathrm{C} .{ }^{1} \mathrm{H}$ NMR $\left(500 \mathrm{MHz}, \mathrm{CDCl}_{3}\right) \delta 7.35-7.20(\mathrm{~m}, 10 \mathrm{H}), 6.84(\mathrm{~d}, J=$ $8.0 \mathrm{~Hz}, 1 \mathrm{H}), 6.76(\mathrm{~d}, J=8.0 \mathrm{~Hz}, 1 \mathrm{H}), 6.67(\mathrm{~s}, 1 \mathrm{H}), 5.22(\mathrm{~d}, J=16.0 \mathrm{~Hz}, 1 \mathrm{H}), 5.04(\mathrm{~d}, J=16.5 \mathrm{~Hz}$, $1 \mathrm{H}), 4.86(\mathrm{dd}, J=10.0,3.5 \mathrm{~Hz}, 1 \mathrm{H}), 3.28(\mathrm{dd}, J=14.5,3.5 \mathrm{~Hz}, 1 \mathrm{H}), 3.14(\mathrm{dd}, J=14.5,9.5 \mathrm{~Hz}$, $1 \mathrm{H}), 2.20(\mathrm{~s}, 3 \mathrm{H}) .{ }^{13} \mathrm{C} \mathrm{NMR}\left(126 \mathrm{MHz}, \mathrm{CDCl}_{3}\right) \delta 166.2,141.5,136.8,136.2,132.2,129.6,128.9$, 128.6, 128.4 127.4, 126.8, 126.5, 124.5, 117.4, 115.9, 78.1, 45.2, 36.5, 21.1. HPLC [DAICEL CHIRALPAK OD-H, hexane $/ \mathrm{iPrOH}=95 / 5,220 \mathrm{~nm}, 0.5 \mathrm{~mL} / \mathrm{min}, 25^{\circ} \mathrm{C} \cdot \mathrm{t}_{\mathrm{R} 1}=16.1 \mathrm{~min}$ (major), $\mathrm{t}_{\mathrm{R} 2}=21.7 \mathrm{~min}$ (minor)]; ee $=97 \%$. $[\alpha]_{\mathrm{D}}^{25}=-51.17\left(c\right.$ 0.51, $\left.\mathrm{CH}_{2} \mathrm{Cl}_{2}\right)$. HRMS (ESI) calcd for $\mathrm{C}_{23} \mathrm{H}_{21} \mathrm{NO}_{2}(\mathrm{M}+\mathrm{H})^{+}:$344.1645; found: 344.1648 . 
<smiles>O=C1C(Cc2ccccc2)Oc2ccc(Cl)cc2N1Cc1ccccc1</smiles>

(S)-2,4-Dibenzyl-6-chloro-2H-benzo[b][1,4]oxazin-3(4H)-one (2ag). White solid, 99\% yield, $71.9 \mathrm{mg}, \mathrm{m} . \mathrm{p} .=113.9-115.4^{\circ} \mathrm{C} .{ }^{1} \mathrm{H}$ NMR $\left(500 \mathrm{MHz}, \mathrm{CDCl}_{3}\right) \delta 7.37-7.23(\mathrm{~m}, 8 \mathrm{H}), 7.20(\mathrm{~d}, J=$ $7.5 \mathrm{~Hz}, 2 \mathrm{H}), 6.92(\mathrm{dd}, J=8.5,2.5 \mathrm{~Hz}, 1 \mathrm{H}), 6.89-6.82(\mathrm{~m}, 2 \mathrm{H}), 5.18(\mathrm{~d}, J=16.5 \mathrm{~Hz}, 1 \mathrm{H}), 5.02(\mathrm{~d}$, $J=16.5 \mathrm{~Hz}, 1 \mathrm{H}), 4.90(\mathrm{dd}, J=9.5,3.5 \mathrm{~Hz}, 1 \mathrm{H}), 3.30(\mathrm{dd}, J=14.5,3.5 \mathrm{~Hz}, 1 \mathrm{H}), 3.15(\mathrm{dd}, J=14.5$, $9.5 \mathrm{~Hz}, 1 \mathrm{H}) .{ }^{13} \mathrm{C} \mathrm{NMR}\left(126 \mathrm{MHz}, \mathrm{CDCl}_{3}\right) \delta 165.7,142.4,136.3,135.4,129.8,129.6,129.0,128.5$, 127.7, 127.6, 127.0, 126.6, 123.8, 118.6, 115.5, 78.1, 45.3, 36.7. HPLC [DAICEL CHIRALPAK OJ-H, hexane $/ \mathrm{PrOH}=90 / 10,220 \mathrm{~nm}, 1.0 \mathrm{~mL} / \mathrm{min}, 25^{\circ} \mathrm{C} . \mathrm{t}_{\mathrm{R} 1}=16.7 \mathrm{~min}$ (major), $\mathrm{t}_{\mathrm{R} 2}=26.4 \mathrm{~min}$ (minor)]; $e e=97 \%$. $[\alpha]_{\mathrm{D}}^{25}=-49.90\left(c 0.54, \mathrm{CH}_{2} \mathrm{Cl}_{2}\right)$. HRMS (ESI) calcd for $\mathrm{C}_{22} \mathrm{H}_{18} \mathrm{ClNO}_{2}(\mathrm{M}+\mathrm{H})^{+}$: 364.1099; found: 364.1112 .<smiles>O=C1C(Cc2ccccc2)Oc2ccc(Br)cc2N1Cc1ccccc1</smiles>

(S)-2,4-Dibenzyl-6-bromo-2H-benzo[b][1,4] oxazin-3(4H)-one (2ah). White solid, 99\% yield, $80.6 \mathrm{mg}$, m.p. $=91.2-93.1{ }^{\circ} \mathrm{C} .{ }^{1} \mathrm{H}$ NMR $\left(500 \mathrm{MHz}, \mathrm{CDCl}_{3}\right) \delta 7.37-7.23(\mathrm{~m}, 8 \mathrm{H}), 7.20(\mathrm{~d}, J=$ $7.5 \mathrm{~Hz}, 2 \mathrm{H}), 7.06(\mathrm{~d}, J=8.5 \mathrm{~Hz}, 1 \mathrm{H}), 6.98(\mathrm{~s}, 1 \mathrm{H}), 6.81(\mathrm{~d}, J=8.5 \mathrm{~Hz}, 1 \mathrm{H}), 5.18(\mathrm{~d}, J=16.0 \mathrm{~Hz}$, $1 \mathrm{H}), 5.01(\mathrm{~d}, J=16.0 \mathrm{~Hz}, 1 \mathrm{H}), 4.90(\mathrm{dd}, J=9.5,3.0 \mathrm{~Hz}, 1 \mathrm{H}), 3.30(\mathrm{dd}, J=14.5,3.5 \mathrm{~Hz}, 1 \mathrm{H}), 3.15$ $(\mathrm{dd}, J=14.5,9.5 \mathrm{~Hz}, 1 \mathrm{H}) .{ }^{13} \mathrm{C} \mathrm{NMR}\left(126 \mathrm{MHz}, \mathrm{CDCl}_{3}\right) \delta 165.6,142.9,136.2,135.4,130.1,129.6$, 129.0, 128.5, 127.7, 127.0, 126.8, 126.6, 119.0, 118.3, 114.7, 78.0, 45.3, 36.7. HPLC [DAICEL CHIRALPAK OJ-H, hexane $/ \mathrm{PrOH}=90 / 10,220 \mathrm{~nm}, 1.0 \mathrm{~mL} / \mathrm{min}, 25^{\circ} \mathrm{C} . \mathrm{t}_{\mathrm{R} 1}=18.3 \mathrm{~min}$ (major), $\mathrm{t}_{\mathrm{R} 2}=27.7 \min ($ minor) $] ; e e=97 \% .[\alpha]_{\mathrm{D}}^{25}=-39.69\left(c 0.61, \mathrm{CH}_{2} \mathrm{Cl}_{2}\right)$. HRMS (ESI) calcd for $\mathrm{C}_{22} \mathrm{H}_{18} \mathrm{BrNO}_{2}(\mathrm{M}+\mathrm{H})^{+}:$408.0594; found: 408.0601 .<smiles>O=C1C(Cc2ccccc2)Oc2ccc(C(F)(F)F)cc2N1Cc1ccccc1</smiles>

(S)-2,4-Dibenzyl-6-(trifluoromethyl)-2H-benzo[b][1,4]oxazin-3(4H)-one (2ai). White solid, $99 \%$ yield, $78.6 \mathrm{mg}$, m.p. $=74.3-75.2{ }^{\circ} \mathrm{C} .{ }^{1} \mathrm{H}$ NMR $\left(500 \mathrm{MHz}, \mathrm{CDCl}_{3}\right) \delta 7.35-7.24(\mathrm{~m}, 8 \mathrm{H}), 7.24-$ $7.19(\mathrm{~m}, 3 \mathrm{H}), 7.10(\mathrm{~s}, 1 \mathrm{H}), 7.01(\mathrm{~d}, J=8.5 \mathrm{~Hz}, 1 \mathrm{H}), 5.23(\mathrm{~d}, J=16.0 \mathrm{~Hz}, 1 \mathrm{H}), 5.07(\mathrm{~d}, J=16.0$ $\mathrm{Hz}, 1 \mathrm{H}), 4.98(\mathrm{dd}, J=9.5,3.5 \mathrm{~Hz}, 1 \mathrm{H}), 3.33(\mathrm{dd}, J=14.5,3.5 \mathrm{~Hz}, 1 \mathrm{H}), 3.17(\mathrm{dd}, J=14.5,9.5 \mathrm{~Hz}$, 1H). ${ }^{13} \mathrm{C}$ NMR $\left(126 \mathrm{MHz}, \mathrm{CDCl}_{3}\right) \delta 165.3,146.4,135.9,135.3,129.6,129.0,128.8,128.5,127.8$, 127.1, 126.8, 124.8 (q, $J=33.3 \mathrm{~Hz}), 123.8(\mathrm{q}, J=272.4 \mathrm{~Hz}), 121.3(\mathrm{q}, J=3.8 \mathrm{~Hz}), 117.8,112.5$ $(\mathrm{q}, J=3.8 \mathrm{~Hz}), 78.1,45.3,37.1 .{ }^{19} \mathrm{~F}$ NMR $\left(471 \mathrm{MHz}, \mathrm{CDCl}_{3}\right) \delta-61.96$. HPLC [DAICEL CHIRALPAK OD-H, hexane $/ \mathrm{iPrOH}=95 / 5,220 \mathrm{~nm}, 0.5 \mathrm{~mL} / \mathrm{min}, 25^{\circ} \mathrm{C} \cdot \mathrm{t}_{\mathrm{R} 1}=16.7 \mathrm{~min}$ (major), 
$\mathrm{t}_{\mathrm{R} 2}=24.0 \min$ (minor)]; $e e=97 \% .[\alpha]_{\mathrm{D}}^{25}=-36.60\left(c 0.59, \mathrm{CH}_{2} \mathrm{Cl}_{2}\right)$. HRMS (ESI) calcd for $\mathrm{C}_{23} \mathrm{H}_{18} \mathrm{~F}_{3} \mathrm{NO}_{2}(\mathrm{M}+\mathrm{H})^{+}$: 398.1362; found: 398.1370 .<smiles>Cc1ccc2c(c1)OC(Cc1ccccc1)C(=O)N2Cc1ccccc1</smiles>

2aj

(S)-2,4-Dibenzyl-7-methyl-2H-benzo[b][1,4]oxazin-3(4H)-one (2aj). White solid, 99\% yield, $67.9 \mathrm{mg}, \mathrm{m} . \mathrm{p} .=78.2-79 .{ }^{\circ} \mathrm{C} .{ }^{1} \mathrm{H} \mathrm{NMR}\left(500 \mathrm{MHz}, \mathrm{CDCl}_{3}\right) \delta 7.34-7.18(\mathrm{~m}, 10 \mathrm{H}), 6.77(\mathrm{~s}, 1 \mathrm{H})$, $6.72(\mathrm{~d}, J=8.0 \mathrm{~Hz}, 1 \mathrm{H}), 6.67(\mathrm{~d}, J=7.5 \mathrm{~Hz}, 1 \mathrm{H}), 5.20(\mathrm{~d}, J=16.0 \mathrm{~Hz}, 1 \mathrm{H}), 5.03(\mathrm{~d}, J=16.0 \mathrm{~Hz}$, $1 \mathrm{H}), 4.87(\mathrm{dd}, J=9.5,3.5 \mathrm{~Hz}, 1 \mathrm{H}), 3.30(\mathrm{dd}, J=14.5,3.5 \mathrm{~Hz}, 1 \mathrm{H}), 3.16(\mathrm{dd}, J=14.5,9.5 \mathrm{~Hz}, 1 \mathrm{H})$, $2.24(\mathrm{~s}, 3 \mathrm{H}) .{ }^{13} \mathrm{C}$ NMR $\left(126 \mathrm{MHz}, \mathrm{CDCl}_{3}\right) \delta 165.8,143.6,136.8,136.2,134.2,129.6,128.9,128.4$, 127.4, 126.8, 126.6, 126.3, 123.1, 118.2, 115.2, 78.1, 45.2, 36.7, 20.8. HPLC [DAICEL CHIRALPAK OD-H, hexane $/ \mathrm{iPrOH}=95 / 5,220 \mathrm{~nm}, 0.5 \mathrm{~mL} / \mathrm{min}, 25^{\circ} \mathrm{C} \cdot \mathrm{t}_{\mathrm{R} 1}=17.3 \mathrm{~min}$ (major), $\mathrm{t}_{\mathrm{R} 2}=18.9 \min$ (minor) $] ; e e=97 \% .[\alpha]_{\mathrm{D}}^{25}=-61.79\left(c\right.$ 0.51, $\left.\mathrm{CH}_{2} \mathrm{Cl}_{2}\right)$. HRMS (ESI) calcd for $\mathrm{C}_{23} \mathrm{H}_{21} \mathrm{NO}_{2}(\mathrm{M}+\mathrm{H})^{+}:$344.1645; found: 344.1643 .<smiles>O=C1C(Cc2ccccc2)Oc2cc(Cl)ccc2N1Cc1ccccc1</smiles>

2ak

(S)-2,4-Dibenzyl-7-chloro-2H-benzo[b][1,4]oxazin-3(4H)-one (2ak). White solid, 99\% yield, $71.8 \mathrm{mg}$, m.p. $=66.7-68.2{ }^{\circ} \mathrm{C} .{ }^{1} \mathrm{H}$ NMR $\left(500 \mathrm{MHz}, \mathrm{CDCl}_{3}\right) \delta 7.36-7.22(\mathrm{~m}, 8 \mathrm{H}), 7.20-7.15$ $(\mathrm{m}, 2 \mathrm{H}), 6.95(\mathrm{~d}, J=2.0 \mathrm{~Hz}, 1 \mathrm{H}), 6.83(\mathrm{dd}, J=8.5,2.5 \mathrm{~Hz}, 1 \mathrm{H}), 6.73(\mathrm{~d}, J=8.5 \mathrm{~Hz}, 1 \mathrm{H}), 5.19(\mathrm{~d}$, $J=16.0 \mathrm{~Hz}, 1 \mathrm{H}), 5.03(\mathrm{~d}, J=16.0 \mathrm{~Hz}, 1 \mathrm{H}), 4.91(\mathrm{dd}, J=9.5,3.5 \mathrm{~Hz}, 1 \mathrm{H}), 3.32(\mathrm{dd}, J=14.5,3.5$ $\mathrm{Hz}, 1 \mathrm{H}), 3.16(\mathrm{dd}, J=14.5,9.5 \mathrm{~Hz}, 1 \mathrm{H}) .{ }^{13} \mathrm{C} \mathrm{NMR}\left(126 \mathrm{MHz}, \mathrm{CDCl}_{3}\right) \delta 165.4,144.5,136.2$, 135.6, 129.6, 129.0, 128.5, 127.6, 127.4, 127.0, 126.52, 126.47, 122.5, 117.9, 116.2, 78.2, 45.3, 36.9. HPLC [DAICEL CHIRALPAK OJ-H, hexane/iPrOH $=90 / 10,220 \mathrm{~nm}, 1.0 \mathrm{~mL} / \mathrm{min}, 2{ }^{\circ} \mathrm{C}$. $\mathrm{t}_{\mathrm{R} 1}=21.1 \min$ (major), $\mathrm{t}_{\mathrm{R} 2}=28.5 \min$ (minor) $] ; e e=97 \%$. $[\alpha]_{\mathrm{D}}^{25}=-49.43\left(c 0.54, \mathrm{CH}_{2} \mathrm{Cl}_{2}\right)$. HRMS (ESI) calcd for $\mathrm{C}_{22} \mathrm{H}_{18} \mathrm{ClNO}_{2}(\mathrm{M}+\mathrm{H})^{+}: 364.1099$; found: 364.1106 .<smiles>O=C1[C@H](Cc2ccccc2)Oc2cc(Br)ccc2N1Cc1ccccc1</smiles>

2al

(S)-2,4-Dibenzyl-7-bromo-2H-benzo[b][1,4]oxazin-3(4H)-one (2al). White solid, 99\% yield, $80.5 \mathrm{mg}$, m.p. $=79.4-81.2{ }^{\circ} \mathrm{C} .{ }^{1} \mathrm{H}$ NMR $\left(500 \mathrm{MHz}, \mathrm{CDCl}_{3}\right) \delta 7.34-7.21(\mathrm{~m}, 8 \mathrm{H}), 7.18-7.14$ $(\mathrm{m}, 2 \mathrm{H}), 7.09(\mathrm{~d}, J=2.0 \mathrm{~Hz}, 1 \mathrm{H}), 6.97(\mathrm{dd}, J=9.0,2.0 \mathrm{~Hz}, 1 \mathrm{H}), 6.67(\mathrm{~d}, J=9.0 \mathrm{~Hz}, 1 \mathrm{H}), 5.18(\mathrm{~d}$, $J=16.5 \mathrm{~Hz}, 1 \mathrm{H}), 5.03(\mathrm{~d}, J=16.0 \mathrm{~Hz}, 1 \mathrm{H}), 4.90(\mathrm{dd}, J=9.5,3.5 \mathrm{~Hz}, 1 \mathrm{H}), 3.32(\mathrm{dd}, J=14.5,3.5$ $\mathrm{Hz}, 1 \mathrm{H}), 3.16(\mathrm{dd}, J=14.5,9.5 \mathrm{~Hz}, 1 \mathrm{H}) .{ }^{13} \mathrm{C} \mathrm{NMR}\left(126 \mathrm{MHz}, \mathrm{CDCl}_{3}\right) \delta 165.4,144.6,136.2$, 
135.6, 129.6, 129.0, 128.5, 127.9, 127.6, 127.0, 126.5, 125.5, 120.7, 116.6, 116.2, 78.2, 45.2, 36.9. HPLC [DAICEL CHIRALPAK OJ-H, hexane $/ \mathrm{PrOH}=90 / 10,220 \mathrm{~nm}, 1.0 \mathrm{~mL} / \mathrm{min}, 25^{\circ} \mathrm{C} \cdot \mathrm{t}_{\mathrm{R} 1}=$ $22.9 \min$ (major), $\mathrm{t}_{\mathrm{R} 2}=31.3 \min$ (minor)]; $e e=97 \%$. $[\alpha]_{\mathrm{D}}^{25}=-41.07\left(c 0.61, \mathrm{CH}_{2} \mathrm{Cl}_{2}\right) . \mathrm{HRMS}(\mathrm{ESI})$ calcd for $\mathrm{C}_{22} \mathrm{H}_{18} \mathrm{BrNO}_{2}(\mathrm{M}+\mathrm{H})^{+}$: 408.0594 ; found: 408.0597 .<smiles>O=C1C(Cc2ccccc2)Oc2c(cccc2[N+](=O)[O-])N1Cc1ccccc1</smiles>

(S)-2,4-dibenzyl-8-methyl-2H-benzo[b][1,4]oxazin-3(4H)-one (2am). White wax, 99\% yield, $67.8 \mathrm{mg} .{ }^{1} \mathrm{H}$ NMR $\left(500 \mathrm{MHz}, \mathrm{CDCl}_{3}\right) \delta 7.35-7.19(\mathrm{~m}, 10 \mathrm{H}), 6.81(\mathrm{~d}, J=7.0 \mathrm{~Hz}, 1 \mathrm{H}), 6.79-$ $6.74(\mathrm{~m}, 1 \mathrm{H}), 6.70(\mathrm{~d}, J=8.0 \mathrm{~Hz}, 1 \mathrm{H}), 5.20(\mathrm{~d}, J=16.0 \mathrm{~Hz}, 1 \mathrm{H}), 5.07(\mathrm{~d}, J=16.0 \mathrm{~Hz}, 1 \mathrm{H}), 4.89$ $(\mathrm{dd}, J=10.0,3.5 \mathrm{~Hz}, 1 \mathrm{H}), 3.36(\mathrm{dd}, J=14.5,3.5 \mathrm{~Hz}, 1 \mathrm{H}), 3.13(\mathrm{dd}, J=14.5,10.0 \mathrm{~Hz}, 1 \mathrm{H}), 2.06$ (s, 3H). ${ }^{13} \mathrm{C}$ NMR $\left(126 \mathrm{MHz}, \mathrm{CDCl}_{3}\right) \delta 166.1,142.0,136.8,136.3,129.6,128.9,128.6,128.4$, 127.4, 127.1, 126.8, 126.6, 125.8, 122.0, 113.2, 78.0, 45.4, 36.7, 15.5. HPLC [DAICEL CHIRALPAK OJ-H, hexane $/ \mathrm{PrOH}=90 / 10,220 \mathrm{~nm}, 1.0 \mathrm{~mL} / \mathrm{min}, 25^{\circ} \mathrm{C} \cdot \mathrm{t}_{\mathrm{R} 1}=15.6 \mathrm{~min}$ (minor), $\mathrm{t}_{\mathrm{R} 2}=18.6 \min$ (major)]; $e e=96 \%$. $[\alpha]_{\mathrm{D}}^{25}=-6.13\left(c 0.52, \mathrm{CH}_{2} \mathrm{Cl}_{2}\right)$. HRMS (ESI) calcd for $\mathrm{C}_{23} \mathrm{H}_{21} \mathrm{NO}_{2}(\mathrm{M}+\mathrm{H})^{+}:$344.1645; found: 344.1654 .

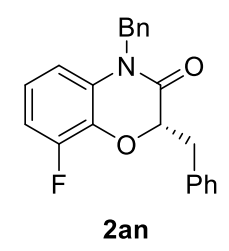

(S)-2,4-Dibenzyl-8-fluoro-2H-benzo[b][1,4]oxazin-3(4H)-one (2an). Pale yellow solid, 98\% yield, $68.0 \mathrm{mg}$, m.p. $=84.9-86.7{ }^{\circ} \mathrm{C} .{ }^{1} \mathrm{H}$ NMR $\left(500 \mathrm{MHz}, \mathrm{CDCl}_{3}\right) \delta 7.30(\mathrm{dd}, J=8.4,5.8 \mathrm{~Hz}$, $6 \mathrm{H}), 7.27-7.22(\mathrm{~m}, 2 \mathrm{H}), 7.20-7.15(\mathrm{~m}, 2 \mathrm{H}), 6.81-6.75(\mathrm{~m}, 2 \mathrm{H}), 6.63-6.58(\mathrm{~m}, 1 \mathrm{H}), 5.21(\mathrm{~d}, J$ $=16.0 \mathrm{~Hz}, 1 \mathrm{H}), 5.03(\mathrm{~d}, J=16.0 \mathrm{~Hz}, 1 \mathrm{H}), 4.97(\mathrm{dd}, J=8.5,3.5 \mathrm{~Hz}, 1 \mathrm{H}), 3.31(\mathrm{dd}, J=14.5,3.5$ $\mathrm{Hz}, 1 \mathrm{H}), 3.21(\mathrm{dd}, J=14.5,8.5 \mathrm{~Hz}, 1 \mathrm{H}) .{ }^{13} \mathrm{C} \mathrm{NMR}\left(126 \mathrm{MHz}, \mathrm{CDCl}_{3}\right) \delta 165.6,153.0,151.0$, 136.9 (d, $J=32.8 \mathrm{~Hz}), 132.3(\mathrm{~d}, J=7.1 \mathrm{~Hz}), 130.6(\mathrm{~d}, J=3.3 \mathrm{~Hz}), 129.7,128.9,128.5,127.6$, 127.0, 126.6, 122.1 (d, $J=8.2 \mathrm{~Hz}), 111.6$ (d, $J=18.1 \mathrm{~Hz}), 110.7$ (d, $J=3.3 \mathrm{~Hz}), 78.4,45.5,36.9$. ${ }^{19} \mathrm{~F}$ NMR $\left(471 \mathrm{MHz}, \mathrm{CDCl}_{3}\right) \delta-134.44$. HPLC [DAICEL CHIRALPAK OD-H, hexane $/ \mathrm{PPOH}=$ $95 / 5,220 \mathrm{~nm}, 0.5 \mathrm{~mL} / \mathrm{min}, 25^{\circ} \mathrm{C} . \mathrm{t}_{\mathrm{R} 1}=21.0 \min$ (major), $\mathrm{t}_{\mathrm{R} 2}=24.1 \mathrm{~min}($ minor) $] ; e e=97 \%$. $[\alpha]_{\mathrm{D}}^{25}$ $=-89.13\left(c\right.$ 0.52, $\mathrm{CH}_{2} \mathrm{Cl}_{2}$ ). HRMS (ESI) calcd for $\mathrm{C}_{22} \mathrm{H}_{18} \mathrm{FNO}_{2}(\mathrm{M}+\mathrm{H})^{+}$: 348.1394; found: 348.1388 .<smiles>O=C1C(CC2CC2)Oc2cc(Br)ccc2N1Br</smiles>

(S)-4-Benzyl-7-bromo-2-(cyclopropylmethyl)-2H-benzo[b][1,4]oxazin-3(4H)-one (2ao). White wax, 98\% yield, $72.7 \mathrm{mg} .{ }^{1} \mathrm{H} \mathrm{NMR}\left(500 \mathrm{MHz}, \mathrm{CDCl}_{3}\right) \delta 7.38-7.32(\mathrm{~m}, 2 \mathrm{H}), 7.31-7.26(\mathrm{~m}$, 
1H), $7.26-7.21(\mathrm{~m}, 2 \mathrm{H}), 7.19(\mathrm{~d}, J=2.0 \mathrm{~Hz}, 1 \mathrm{H}), 7.02(\mathrm{dd}, J=9.0,2.5 \mathrm{~Hz}, 1 \mathrm{H}), 6.73(\mathrm{~d}, J=8.5$ $\mathrm{Hz}, 1 \mathrm{H}), 5.22(\mathrm{~d}, J=16.0 \mathrm{~Hz}, 1 \mathrm{H}), 5.06(\mathrm{~d}, J=16.0 \mathrm{~Hz}, 1 \mathrm{H}), 4.82(\mathrm{dd}, J=9.5,4.0 \mathrm{~Hz}, 1 \mathrm{H}), 1.99$ (ddd, $J=14.5,9.0,6.0 \mathrm{~Hz}, 1 \mathrm{H}), 1.73$ (ddd, $J=14.5,8.0,4.0 \mathrm{~Hz}, 1 \mathrm{H}), 1.06-0.95$ (m, 1H), $0.63-$ $0.48(\mathrm{~m}, 2 \mathrm{H}), 0.27-0.14(\mathrm{~m}, 2 \mathrm{H}) .{ }^{13} \mathrm{C}$ NMR $\left(126 \mathrm{MHz}, \mathrm{CDCl}_{3}\right) \delta 166.1,144.9,135.7,129.0$, 128.1, 127.6, 126.5, 125.3, 120.6, 116.5, 116.1, 77.9, 45.1, 35.7, 7.2, 5.0, 4.2. HPLC [DAICEL CHIRALPAK OD-H, hexane $/ \mathrm{PrOH}=95 / 5,220 \mathrm{~nm}, 0.5 \mathrm{~mL} / \mathrm{min}, 25{ }^{\circ} \mathrm{C} . \mathrm{t}_{\mathrm{R} 1}=15.8 \mathrm{~min}$ (minor), $\mathrm{t}_{\mathrm{R} 2}=17.0 \min$ (major) $]$; $e e=97 \%$. $[\alpha]_{\mathrm{D}}^{25}=+2.80\left(c 0.55, \mathrm{CH}_{2} \mathrm{Cl}_{2}\right)$. HRMS (ESI) calcd for $\mathrm{C}_{19} \mathrm{H}_{18} \mathrm{BrNO}_{2}(\mathrm{M}+\mathrm{H})^{+}:$372.0594; found: 372.0596 . 


\section{Transformations of the Reduced Products}

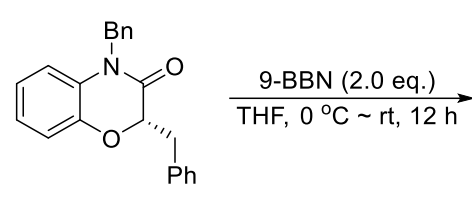

$2 a$

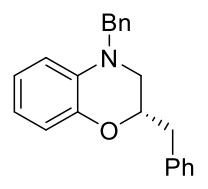

3

To a solution of $\mathbf{2 a}(66 \mathrm{mg}, 0.2 \mathrm{mmol})$ in THF $(2.0 \mathrm{~mL})$ was added 9-BBN $(0.8 \mathrm{~mL}, 0.4 \mathrm{mmol}$, $0.5 \mathrm{M}$ in THF) slowly under $\mathrm{N}_{2}$ atmosphere at $0{ }^{\circ} \mathrm{C}$, and the mixture was stirred at room temperature for $12 \mathrm{~h}$. Then, the reaction mixture was quenched with water, extracted with ethyl acetate, washed with saturated aqueous sodium chloride, and the combined aqueous layers extracted again with ethyl acetate. The combined organic fractions were dried over anhydrous sodium sulfate, filtered, and concentrated in vасио. The crude residue was purified by silica gel column chromatography eluting to give the desired product 3 .

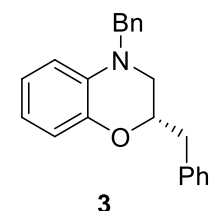

(S)-2,4-Dibenzyl-3,4-dihydro-2H-benzo[b][1,4] oxazine (3). Brown wax, 88\% yield, $55.4 \mathrm{mg}$. ${ }^{1} \mathrm{H}$ $\operatorname{NMR}\left(500 \mathrm{MHz}, \mathrm{CDCl}_{3}\right) \delta 7.34-7.19(\mathrm{~m}, 10 \mathrm{H}), 6.84(\mathrm{~d}, J=8.0 \mathrm{~Hz}, 1 \mathrm{H}), 6.79-6.74(\mathrm{~m}, 1 \mathrm{H})$, $6.68-6.61(\mathrm{~m}, 2 \mathrm{H}), 4.46(\mathrm{~d}, J=16.0 \mathrm{~Hz}, 1 \mathrm{H}), 4.36(\mathrm{~d}, J=16.0 \mathrm{~Hz}, 1 \mathrm{H}), 3.25(\mathrm{dd}, J=12.0,2.5$ $\mathrm{Hz}, 1 \mathrm{H}), 3.20-3.08(\mathrm{~m}, 2 \mathrm{H}), 2.85(\mathrm{dd}, J=14.0,7.5 \mathrm{~Hz}, 1 \mathrm{H}) .{ }^{13} \mathrm{C} \mathrm{NMR}\left(126 \mathrm{MHz}, \mathrm{CDCl}_{3}\right) \delta$ $143.5,138.1,137.3,135.1,129.4,128.7,128.5,127.1,127.0,126.6,121.5,117.9,116.5,112.4$, 74.1, 55.0, 51.1, 39.4. HPLC [DAICEL CHIRALPAK OD-H, hexane/iPrOH = 95/5, $220 \mathrm{~nm}, 0.5$ $\mathrm{mL} / \mathrm{min}, 25{ }^{\circ} \mathrm{C} . \mathrm{t}_{\mathrm{R} 1}=17.7 \min$ (minor), $\mathrm{t}_{\mathrm{R} 2}=21.1 \min$ (major) $] ;$ ee $=97 \% .[\alpha]_{\mathrm{D}}^{25}=-16.15(c 0.47$, $\mathrm{CH}_{2} \mathrm{Cl}_{2}$ ). HRMS (ESI) calcd for $\mathrm{C}_{22} \mathrm{H}_{21} \mathrm{ClNO}(\mathrm{M}+\mathrm{H})^{+}:$316.1696; found: 316.1696.<smiles>O=C1[C@H](Cc2ccccc2)Oc2ccccc2N1Cc1ccccc1</smiles>

$2 \mathbf{a}$

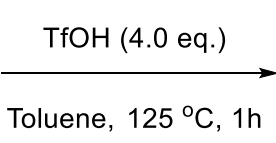

Toluene, $125^{\circ} \mathrm{C}, 1 \mathrm{~h}$<smiles>O=C1Nc2ccccc2O[C@H]1CPc1ccccc1</smiles>

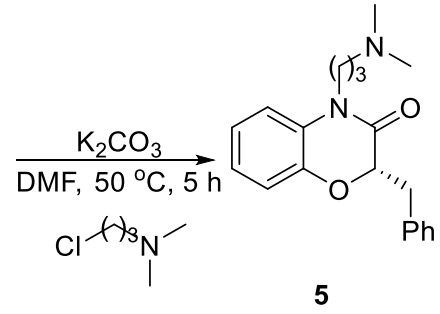

Trifluoromethanesulfonic acid (0.36 mL, $4.08 \mathrm{mmol}, 4.0$ equiv.) was added to a solution of $\mathbf{2 a}$ (198 mg, $0.6 \mathrm{mmol}, 1.0$ equiv.) in toluene $(6 \mathrm{~mL}, 0.1 \mathrm{M})$. The mixture was heated at $125{ }^{\circ} \mathrm{C}$ for one hour. Then, the mixture was poured into a small amount of saturated aqueous sodium bicarbonate, extracted with ethyl acetate, washed with saturated aqueous sodium chloride, and the combined aqueous layers extracted again with ethyl acetate. The combined organic fractions were dried over anhydrous sodium sulfate, filtered, and concentrated in vacuo. The crude residue was purified by silica gel column chromatography eluting to give the desired product 4 .

To a stirred solution of 4 (96 mg, $0.4 \mathrm{mmol}, 1.0$ equiv.) in DMF was added $\mathrm{K}_{2} \mathrm{CO}_{3}(126 \mathrm{mg}, 1.2$ mmol, 3.0 equiv.), and the mixture was stirred at $50{ }^{\circ} \mathrm{C}$ for $1 \mathrm{~h}$. Then 3-chloro-N,N-dimethylpropa 
n-1-amine ( $96 \mathrm{mg}, 0.6 \mathrm{mmol}, 1.5$ equiv.) was added to the reaction miture and stirred for another 5 $\mathrm{h}$. Then, the reaction mixture was extracted with ethyl acetate, washed with saturated aqueous sodium chloride, and the combined aqueous layers extracted again with ethyl acetate. The combined organic fractions were dried over anhydrous sodium sulfate, filtered, and concentrated in vacuo. The crude residue was purified by silica gel column chromatography eluting to give the desired product 5 .

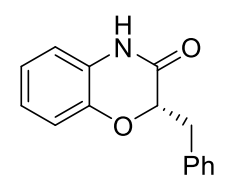

4

(S)-2-Benzyl-2H-benzo[b][1,4]oxazin-3(4H)-one (4). White solid, $88 \%$ yield, $126.2 \mathrm{mg}$, m.p. = $123.4-124.3{ }^{\circ} \mathrm{C} .{ }^{1} \mathrm{H}$ NMR $\left(500 \mathrm{MHz}, \mathrm{CDCl}_{3}\right) \delta 10.02(\mathrm{~s}, 1 \mathrm{H}), 7.33-7.13(\mathrm{~m}, 5 \mathrm{H}), 6.99-6.89$ $(\mathrm{m}, 3 \mathrm{H}), 6.85(\mathrm{~d}, J=7.0 \mathrm{~Hz}, 1 \mathrm{H}), 4.79(\mathrm{dd}, J=9.5,3.5 \mathrm{~Hz}, 1 \mathrm{H}), 3.25(\mathrm{dd}, J=14.5,3.5 \mathrm{~Hz}, 1 \mathrm{H})$, $3.11(\mathrm{dd}, J=14.5,9.5 \mathrm{~Hz}, 1 \mathrm{H}) .{ }^{13} \mathrm{C}$ NMR $\left(126 \mathrm{MHz}, \mathrm{CDCl}_{3}\right) \delta 168.1,142.6,136.6,129.7,128.5$, 126.9, 126.3, 124.4, 122.7, 117.4, 116.1, 77.8, 36.7. HPLC [DAICEL CHIRALPAK OJ-H, hexane $/ \mathrm{PrOH}=95 / 5,220 \mathrm{~nm}, 0.5 \mathrm{~mL} / \mathrm{min}, 25^{\circ} \mathrm{C} . \mathrm{t}_{\mathrm{R} 1}=25.5 \mathrm{~min}$ (major), $\mathrm{t}_{\mathrm{R} 2}=28.9 \min$ (minor)]; $e e=97 \% .[\alpha]_{\mathrm{D}}^{25}=-44.00\left(c 2.15, \mathrm{CH}_{2} \mathrm{Cl}_{2}\right)$. HRMS (ESI) calcd for $\mathrm{C}_{15} \mathrm{H}_{13} \mathrm{NO}_{2}(\mathrm{M}+\mathrm{H})^{+}: 240.1019$; found: 240.1022 .<smiles>CN(C)CN1C(=O)C(Cc2ccccc2)Oc2ccccc21</smiles>

5

(S)-2-Benzyl-4-(3-(dimethylamino)propyl)-2H-benzo[b][1,4]oxazin-3(4H)-one (5). Yellow wax, 81\% yield, $104.9 \mathrm{mg} .{ }^{1} \mathrm{H}$ NMR $\left(500 \mathrm{MHz}, \mathrm{CDCl}_{3}\right) \delta 7.32-7.15(\mathrm{~m}, 5 \mathrm{H}), 7.06-6.96(\mathrm{~m}$, $3 \mathrm{H}), 6.95-6.91(\mathrm{~m}, 1 \mathrm{H}), 4.76(\mathrm{dd}, J=9.5,3.5 \mathrm{~Hz}, 1 \mathrm{H}), 4.01-3.91(\mathrm{~m}, 2 \mathrm{H}), 3.21(\mathrm{dd}, J=14.5$, $3.5 \mathrm{~Hz}, 1 \mathrm{H}), 3.06(\mathrm{dd}, J=14.5,9.5 \mathrm{~Hz}, 1 \mathrm{H}), 2.33(\mathrm{t}, J=7.0 \mathrm{~Hz}, 2 \mathrm{H}), 2.23(\mathrm{~s}, 6 \mathrm{H}), 1.81(\mathrm{t}, J=7.0$ $\mathrm{Hz}, 2 \mathrm{H}) .{ }^{13} \mathrm{C}$ NMR $\left(126 \mathrm{MHz}, \mathrm{CDCl}_{3}\right) \delta 165.4,143.9,136.7,129.6,128.6,128.3,126.8,123.8$, 122.6, 117.7, 114.7, 77.9, 56.8, 45.5, 39.9, 36.7, 25.4. HPLC [DAICEL CHIRALPAK AS-H, hexane $/ \mathrm{PrOH}=97 / 3,220 \mathrm{~nm}, 0.3 \mathrm{~mL} / \mathrm{min}, 25^{\circ} \mathrm{C} . \mathrm{t}_{\mathrm{R} 1}=30.4 \mathrm{~min}$ (minor), $\mathrm{t}_{\mathrm{R} 2}=34.3 \mathrm{~min}$ (major)]; $e e=97 \% .[\alpha]_{\mathrm{D}}^{25}=-6.17\left(c 0.32, \mathrm{CH}_{2} \mathrm{Cl}_{2}\right)$. HRMS (ESI) calcd for $\mathrm{C}_{20} \mathrm{H}_{24} \mathrm{~N}_{2} \mathrm{O}_{2}(\mathrm{M}+\mathrm{H})^{+}: 325.1911$; found: 325.1920 .<smiles>O=C1[C@H](Cc2ccccc2)Oc2ccc(Cl)cc2N1Cc1ccccc1</smiles>

2ag<smiles>O=C1Nc2cc(Cl)ccc2O[C@@H]1Cc1ccccc1</smiles>

6

Trifluoromethanesulfonic acid $(0.12 \mathrm{~mL}, 1.36 \mathrm{mmol}, 4.0$ equiv.) was added to a solution of $2 \mathbf{2 a g}$ (72 mg, $0.2 \mathrm{mmol}, 1.0$ equiv.) in toluene $(2 \mathrm{~mL}, 0.1 \mathrm{M})$. The mixture was heated at $125^{\circ} \mathrm{C}$ for one hour. Then, the mixture was poured into a small amount of saturated aqueous sodium bicarbonate, 
extracted with ethyl acetate, washed with saturated aqueous sodium chloride, and the combined aqueous layers extracted again with ethyl acetate. The combined organic fractions were dried over anhydrous sodium sulfate, filtered, and concentrated in vасио. The crude residue was purified by silica gel column chromatography eluting to give the desired product $\mathbf{6}$.<smiles>O=C1Nc2cc(Cl)ccc2OC1Cc1ccccc1</smiles>

(S)-2-Benzyl-6-chloro-2H-benzo[b][1,4]oxazin-3(4H)-one (6). White solid, $88 \%$ yield, $48.0 \mathrm{mg}$, m.p. $=143.5-144.9^{\circ} \mathrm{C} .{ }^{1} \mathrm{H}$ NMR $\left(500 \mathrm{MHz}, \mathrm{CDCl}_{3}\right) \delta 9.88(\mathrm{~s}, 1 \mathrm{H}), 7.33-7.28(\mathrm{~m}, 2 \mathrm{H}), 7.28-$ $7.23(\mathrm{~m}, 3 \mathrm{H}), 6.93(\mathrm{dd}, J=8.5,2.5 \mathrm{~Hz}, 1 \mathrm{H}), 6.87-6.82(\mathrm{~m}, 2 \mathrm{H}), 4.80(\mathrm{dd}, J=9.0,3.5 \mathrm{~Hz}, 1 \mathrm{H})$, $3.26(\mathrm{dd}, J=14.5,3.5 \mathrm{~Hz}, 1 \mathrm{H}), 3.12(\mathrm{dd}, J=14.5,9.5 \mathrm{~Hz}, 1 \mathrm{H}) .{ }^{13} \mathrm{C} \mathrm{NMR}\left(126 \mathrm{MHz}, \mathrm{CDCl}_{3}\right) \delta$ 167.8, 141.2, 136.1, 129.6, 128.5, 127.5, 127.2, 127.0, 124.1, 118.4, 115.9, 77.8, 36.7. HPLC [DAICEL CHIRALPAK OJ-H, hexane $/ \mathrm{iPrOH}=90 / 10,220 \mathrm{~nm}, 1.0 \mathrm{~mL} / \mathrm{min}, 25{ }^{\circ} \mathrm{C}_{\mathrm{t}} \mathrm{t}_{\mathrm{R} 1}=14.4$ $\min$ (major), $\mathrm{t}_{\mathrm{R} 2}=22.3 \min$ (minor)]; $e e=96 \%$. $[\alpha]_{\mathrm{D}}^{25}=-19.74\left(c 2.46, \mathrm{CH}_{2} \mathrm{Cl}_{2}\right)$. HRMS (ESI) calcd for $\mathrm{C}_{15} \mathrm{H}_{12} \mathrm{ClNO}_{2}(\mathrm{M}+\mathrm{H})^{+}$: 274.0629; found: 274.0627 .<smiles>O=C1C(Cc2ccccc2)Oc2cc(Br)ccc2N1Cc1ccccc1</smiles>

2al<smiles>O=C1Nc2ccc(Br)cc2OC1Cc1ccccc1</smiles>

8

Trifluoromethanesulfonic acid $(0.12 \mathrm{~mL}, 1.36 \mathrm{mmol}, 4.0$ equiv. $)$ was added to a solution of $2 \mathrm{al}$ ( $82 \mathrm{mg}, 0.2 \mathrm{mmol}, 1.0$ equiv.) in toluene $(2 \mathrm{~mL}, 0.1 \mathrm{M})$. The mixture was heated at $125^{\circ} \mathrm{C}$ for one hour. Then, the mixture was poured into a small amount of saturated aqueous sodium bicarbonate, extracted with ethyl acetate, washed with saturated aqueous sodium chloride, and the combined aqueous layers extracted again with ethyl acetate. The combined organic fractions were dried over anhydrous sodium sulfate, filtered, and concentrated in vасио. The crude residue was purified by silica gel column chromatography eluting to give the desired product $\mathbf{8}$.<smiles>O=C1Nc2ccc(Br)cc2OC1Cc1ccccc1</smiles>

8

(S)-2-Benzyl-7-bromo-2H-benzo[b][1,4]oxazin-3(4H)-one (8). White solid, 89\% yield, $56.4 \mathrm{mg}$, m.p. $=154.9-155.6{ }^{\circ} \mathrm{C} .{ }^{1} \mathrm{H}$ NMR $\left(500 \mathrm{MHz}, \mathrm{CDCl}_{3}\right) \delta 9.86(\mathrm{~s}, 1 \mathrm{H}), 7.33-7.28(\mathrm{~m}, 2 \mathrm{H}), 7.28-$ $7.22(\mathrm{~m}, 3 \mathrm{H}), 7.10-7.04(\mathrm{~m}, 2 \mathrm{H}), 6.70(\mathrm{~d}, J=8.5 \mathrm{~Hz}, 1 \mathrm{H}), 4.79(\mathrm{dd}, J=9.5,4.0 \mathrm{~Hz}, 1 \mathrm{H}), 3.26$ $(\mathrm{dd}, J=14.5,3.5 \mathrm{~Hz}, 1 \mathrm{H}), 3.11(\mathrm{dd}, J=14.5,9.5 \mathrm{~Hz}, 1 \mathrm{H}) .{ }^{13} \mathrm{C} \mathrm{NMR}\left(126 \mathrm{MHz}, \mathrm{CDCl}_{3}\right) \delta 167.4$, 143.4, 136.0, 129.6, 128.5, 127.0, 125.5, 125.4, 120.5, 116.9, 116.2, 77.8, 36.8. HPLC [DAICEL CHIRALPAK AD-H, hexane $/ \mathrm{PrOH}=90 / 10,220 \mathrm{~nm}, 1.0 \mathrm{~mL} / \mathrm{min}, 25^{\circ} \mathrm{C} \cdot \mathrm{t}_{\mathrm{R} 1}=9.4 \mathrm{~min}$ (major), 
$\mathrm{t}_{\mathrm{R} 2}=10.9 \min$ (minor) $] ; e e=97 \% .[\alpha]_{\mathrm{D}}^{25}=-7.14\left(c\right.$ 2.83, $\left.\mathrm{CH}_{2} \mathrm{Cl}_{2}\right)$. HRMS (ESI) calcd for $\mathrm{C}_{15} \mathrm{H}_{12} \mathrm{BrNO}_{2}(\mathrm{M}+\mathrm{Na})^{+}$: 339.9944; found: 339.9943 . 


\section{Control Experiments and the Stereochemical Model}

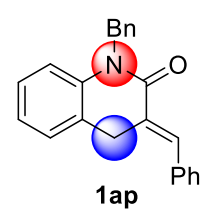

standard $\underset{\text { conditions }}{\longrightarrow}$

lap

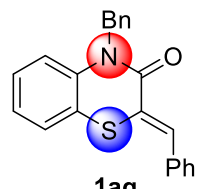

standard $\underset{\text { conditions }}{\stackrel{\text { standard }}{\longrightarrow}}$

$1 \mathrm{aq}$

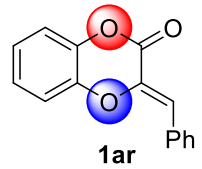
$\underset{\text { conditions }}{\stackrel{\text { standard }}{\longrightarrow}}$

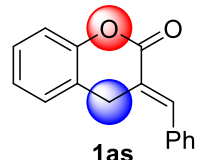

standard $\overrightarrow{\text { conditions }}$

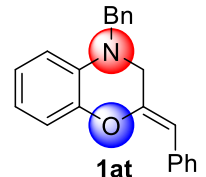
$\underset{\text { conditions }}{\stackrel{\text { standard }}{\longrightarrow}}$<smiles>O=C1CCc2ccccc2N1</smiles>

I $\underset{\mathrm{rt} \text {, overnight }}{\stackrel{\mathrm{BnBr}, \mathrm{K}_{2} \mathrm{CO}_{3}, \mathrm{DMF}}{\longrightarrow}}$<smiles>O=C1CCc2ccccc2N1Cc1ccccc1</smiles>

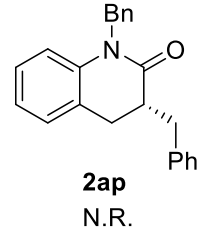<smiles>O=C(C[C@H]1Sc2ccccc2N(Cc2ccccc2)C1=O)OC(=O)OCc1ccccc1</smiles>
conv. $30 \%, 60 \%$ ee.<smiles>[R]N[14CH2]C[C@H]1Oc2ccccc2OC1=O</smiles><smiles>O=C1Oc2ccccc2C[C@H]1CPc1ccccc1</smiles>
conv. $78 \%, 68 \%$ ee<smiles>c1ccc(C[C@H]2CN(Cc3ccccc3)c3ccccc3O2)cc1</smiles>
conv. $93 \%, 27 \%$ ee

$\mathrm{PhCHO}, t$-BuOK $\overrightarrow{\mathrm{EtOH}, 80^{\circ} \mathrm{C}, 24 \mathrm{~h}}$<smiles>O=C1/C(=C/c2ccccc2)Cc2ccccc2N1Cc1ccccc1</smiles>

I $(10 \mathrm{mmol})$ was dissolved in $100 \mathrm{~mL}$ of DMF, $\mathrm{K}_{2} \mathrm{CO}_{3}(15 \mathrm{mmol})$ and $\mathrm{BnBr}(15 \mathrm{mmol})$ were added, and the mixture was stirred at room temperature overnight for a full conversion. Then, the mixture was diluted with EtOAc $(3 \times 50 \mathrm{~mL})$ and washed with water and brine $(3 \times 100 \mathrm{~mL})$. The organic layer was dried over anhydrous $\mathrm{Na} 2 \mathrm{SO} 4$ and the solvent was removed under reduced pressure. The residue was purified by flash chromatography on silica gel to give II.

To a stirred solution of III $(10 \mathrm{mmol})$ and aldehyde $(15 \mathrm{mmol})$ in $100 \mathrm{~mL} \mathrm{EtOH}, 15 \mathrm{~mL} t$-BuOK ( $1 \mathrm{M}$ in THF) were slowly added and the reaction was refluxed at $80{ }^{\circ} \mathrm{C}$ for $24 \mathrm{~h}$. After $24 \mathrm{~h}$, if II was not full conversed, another $15 \mathrm{mmol}$ aldehyde was added and the mixture was refluxed for another $12 \mathrm{~h}$. After cooling to $0{ }^{\circ} \mathrm{C}$, the reaction was quenched with saturated $\mathrm{NH}_{4} \mathrm{Cl}$ and diluted with EtOAc $(3 \times 50 \mathrm{~mL})$ and washed with water and brine $(3 \times 100 \mathrm{~mL})$, dried over anhydrous $\mathrm{Na}_{2} \mathrm{SO}_{4}$ and evaporated under reduced pressure. The residue was purified by flash silica gel chromatography to give substrate 1ap. 
<smiles>O=C1/C(=C/c2ccccc2)Cc2ccccc2N1Cc1ccccc1</smiles>

(E)-1-Benzyl-3-benzylidene-3,4-dihydroquinolin-2(1H)-one (1ap). Colorless oil, 34\% yield, 1.1 g. ${ }^{1} \mathrm{H}$ NMR $\left(500 \mathrm{MHz}, \mathrm{CDCl}_{3}\right) \delta 7.41(\mathrm{dd}, J=7.5,1.5 \mathrm{~Hz}, 1 \mathrm{H}), 7.37-7.30(\mathrm{~m}, 6 \mathrm{H}), 7.29-7.24$ (m, 3H), $7.24-7.18(\mathrm{~m}, 4 \mathrm{H}), 7.10(\mathrm{td}, J=7.5,1.0 \mathrm{~Hz}, 1 \mathrm{H}), 5.57$ (s, 2H), 4.04 (d, $J=1.0 \mathrm{~Hz}, 2 \mathrm{H})$. ${ }^{13} \mathrm{C}$ NMR $\left(126 \mathrm{MHz}, \mathrm{CDCl}_{3}\right) \delta 162.5,139.1,138.5,136.5,136.4,133.5,129.7,129.5,128.8$, 128.7, 128.4, 127.3, 126.7, 126.5, 122.1, 120.9, 114.8, 46.4, 37.0. HRMS (ESI) calcd for $\mathrm{C}_{23} \mathrm{H}_{19} \mathrm{NO}(\mathrm{M}+\mathrm{H})^{+}: 326.1539$; found: 326.1541 .<smiles>O=C1CSc2ccccc2N1</smiles>

I

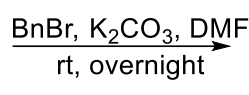<smiles>O=C1CSc2ccccc2N1Cc1ccccc1</smiles>

II<smiles>O=C1/C(=C\c2ccccc2)Sc2ccccc2N1Cc1ccccc1</smiles>

I $(10 \mathrm{mmol})$ was dissolved in $100 \mathrm{~mL}$ of $\mathrm{DMF}, \mathrm{K}_{2} \mathrm{CO}_{3}(15 \mathrm{mmol})$ and $\mathrm{BnBr}(15 \mathrm{mmol})$ were added, and the mixture was stirred at room temperature overnight for a full conversion. Then, the mixture was diluted with EtOAc $(3 \times 50 \mathrm{~mL})$ and washed with water and brine $(3 \times 100 \mathrm{~mL})$. The organic layer was dried over anhydrous $\mathrm{Na} 2 \mathrm{SO} 4$ and the solvent was removed under reduced pressure. The residue was purified by flash chromatography on silica gel to give II.

To a stirred solution of II $(10 \mathrm{mmol})$ and aldehyde $(15 \mathrm{mmol})$ in $100 \mathrm{mLEtOH}, 15 \mathrm{~mL} t$-BuOK $(1$ $\mathrm{M}$ in THF) were slowly added and the reaction was refluxed at $80^{\circ} \mathrm{C}$ for $24 \mathrm{~h}$. After $24 \mathrm{~h}$, if II was not full conversed, another $15 \mathrm{mmol}$ aldehyde was added and the mixture was refluxed for another $12 \mathrm{~h}$. After cooling to $0{ }^{\circ} \mathrm{C}$, the reaction was quenched with saturated $\mathrm{NH}_{4} \mathrm{Cl}$ and diluted with EtOAc $(3 \times 50 \mathrm{~mL})$ and washed with water and brine $(3 \times 100 \mathrm{~mL})$, dried over anhydrous $\mathrm{Na}_{2} \mathrm{SO}_{4}$ and evaporated under reduced pressure. The residue was purified by flash silica gel chromatography to give substrate 1aq.<smiles>O=C1/C(=C/c2ccccc2)Sc2ccccc2N1Cc1ccccc1</smiles>
1aq

(Z)-4-Benzyl-2-benzylidene-2H-benzo[b][1,4]thiazin-3(4H)-one (1aq). Yellow oil, 67\% yield, 2.3 g. ${ }^{1} \mathrm{H}$ NMR $\left(500 \mathrm{MHz}, \mathrm{CDCl}_{3}\right) \delta 7.96(\mathrm{~s}, 1 \mathrm{H}), 7.65(\mathrm{~d}, J=7.5 \mathrm{~Hz}, 2 \mathrm{H}), 7.45$ (t, $J=7.5 \mathrm{~Hz}$, $2 \mathrm{H}), 7.39-7.32(\mathrm{~m}, 3 \mathrm{H}), 7.29-7.22(\mathrm{~m}, 4 \mathrm{H}), 7.08-7.03(\mathrm{~m}, 1 \mathrm{H}), 6.97(\mathrm{td}, J=8.0,1.5 \mathrm{~Hz}, 1 \mathrm{H})$, $6.93(\mathrm{dd}, J=8.5,1.0 \mathrm{~Hz}, 1 \mathrm{H}), 5.36(\mathrm{~s}, 2 \mathrm{H}) .{ }^{13} \mathrm{C} \mathrm{NMR}\left(126 \mathrm{MHz}, \mathrm{CDCl}_{3}\right) \delta 162.1,136.8,136.4$, 135.2, 134.8, 130.3, 128.94, 128.89, 128.4, 127.2, 127.0, 126.3, 126.1, 123.6, 120.5, 119.4, 117.5, 49.4. HRMS (ESI) calcd for $\mathrm{C}_{22} \mathrm{H}_{17} \mathrm{NOS}(\mathrm{M}+\mathrm{H})^{+}: 344.1104$; found: 344.1118. Characterization data matches those reported in the literature ${ }^{[3]}$.<smiles>O=C1Oc2ccccc2O/C1=C/c1ccccc1</smiles>

1 ar 
(Z)-3-Benzylidenebenzo[b][1,4] dioxin-2(3H)-one (1ar) was synthesized according to a reported literature ${ }^{[3]}$, white solid, ${ }^{1} \mathrm{H}$ NMR $\left(500 \mathrm{MHz}, \mathrm{CDCl}_{3}\right) \delta 7.84(\mathrm{~d}, J=7.5 \mathrm{~Hz}, 2 \mathrm{H}), 7.47-7.42(\mathrm{~m}$, $2 \mathrm{H}), 7.41-7.36(\mathrm{~m}, 1 \mathrm{H}), 7.20(\mathrm{dd}, J=8.0,1.5 \mathrm{~Hz}, 1 \mathrm{H}), 7.17-7.04(\mathrm{~m}, 4 \mathrm{H}) .{ }^{13} \mathrm{C}$ NMR $(126 \mathrm{MHz}$, $\left.\mathrm{CDCl}_{3}\right) \delta 156.9,139.5,139.1,136.0,132.5,130.8,129.7,128.8,125.5,123.9,119.4,117.4,116.3$. HRMS (ESI) calcd for $\mathrm{C}_{15} \mathrm{H}_{10} \mathrm{NO}_{3}(\mathrm{M}+\mathrm{H})^{+}$: 239.0703; found: 239.0711. Characterization data matches those reported in the literature ${ }^{[4]}$.

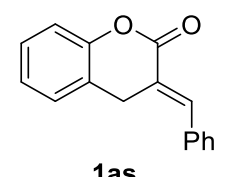

(E)-3-Benzylidenechroman-2-one (1as) was synthesized according to a reported literature ${ }^{[4]}$, white solid, ${ }^{1} \mathrm{H}$ NMR $\left(400 \mathrm{MHz}, \mathrm{CDCl}_{3}\right) \delta 7.98(\mathrm{t}, J=2.4 \mathrm{~Hz}, 1 \mathrm{H}), 7.52-7.40(\mathrm{~m}, 5 \mathrm{H}), 7.19-$ $7.04(\mathrm{~m}, 4 \mathrm{H}), 4.07(\mathrm{~d}, J=2.4 \mathrm{~Hz}, 2 \mathrm{H}) .{ }^{13} \mathrm{C} \mathrm{NMR}\left(126 \mathrm{MHz}, \mathrm{CDCl}_{3}\right) \delta 164.5,150.5,142.7,134.6$, $130.2,129.6,128.7,128.3,128.2,124.5,122.9,120.3,117.0,28.9$. HRMS (ESI) calcd for $\mathrm{C}_{16} \mathrm{H}_{12} \mathrm{NO}_{2}(\mathrm{M}+\mathrm{Na})^{+}: 259.0730$; found: 259.0742. Characterization data matches those reported in the literature ${ }^{[5][6]}$.

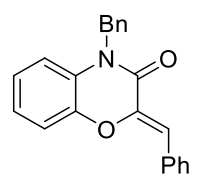

$1 \mathrm{a}$

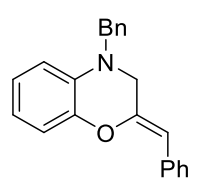

1 at

To a solution of 1a $(2 \mathrm{mmol})$ in THF $(10 \mathrm{~mL})$ was added 9 -BBN $(8 \mathrm{~mL}, 4 \mathrm{mmol}, 0.5 \mathrm{M}$ in THF) slowly under $\mathrm{N}_{2}$ atmosphere at $0{ }^{\circ} \mathrm{C}$, and the mixture was stirred at room temperature for $12 \mathrm{~h}$. Then, the reaction mixture was quenched with water, extracted with ethyl acetate, washed with saturated aqueous sodium chloride, and the combined aqueous layers extracted again with ethyl acetate. The combined organic fractions were dried over anhydrous sodium sulfate, filtered, and concentrated in vacuo. The crude residue was purified by silica gel column chromatography eluting to give the desired product 1at.<smiles>C(=Cc1ccccc1NCc1ccccc1)c1ccccc1</smiles>

(Z)-4-Benzyl-2-benzylidene-3,4-dihydro-2H-benzo[b][1,4]oxazine (1at). Yellow wax, $42 \%$ yield, $262.9 \mathrm{mg} .{ }^{1} \mathrm{H}$ NMR $\left(500 \mathrm{MHz}, \mathrm{CDCl}_{3}\right) \delta 7.68(\mathrm{~d}, J=7.0 \mathrm{~Hz}, 2 \mathrm{H}), 7.38-7.26(\mathrm{~m}, 7 \mathrm{H}), 7.20$ $-7.16(\mathrm{~m}, 1 \mathrm{H}), 7.12(\mathrm{dd}, J=7.5,1.5 \mathrm{~Hz}, 1 \mathrm{H}), 6.90(\mathrm{td}, J=7.5,1.5 \mathrm{~Hz}, 1 \mathrm{H}), 6.84-6.78(\mathrm{~m}, 2 \mathrm{H})$, 5.34 (s, 1H), 4.36 (s, 2H), 3.65 (s, 2H). ${ }^{13} \mathrm{C}$ NMR (126 MHz, $\left.\mathrm{CDCl}_{3}\right) \delta$ 145.6, 143.7, 137.3, 136.2, 135.2, 128.8, 128.6, 128.4, 127.8, 127.5, 126.2, 122.6, 119.7, 116.2, 114.1, 104.8, 54.8, 49.8. HRMS (ESI) calcd for $\mathrm{C}_{22} \mathrm{H}_{19} \mathrm{NO}(\mathrm{M}+\mathrm{H})^{+}: 314.1539$; found: 314.1544 . 
<smiles>O=C1C(Cc2ccccc2)Sc2ccccc2N1Cc1ccccc1</smiles>

2aq

(S)-2,4-Dibenzyl-2H-benzo[b][1,4]thiazin-3(4H)-one (2aq). Yellow wax, 30\% conv., (28\% yield), $19.3 \mathrm{mg} .{ }^{1} \mathrm{H}$ NMR (500 MHz, $\left.\mathrm{CDCl}_{3}\right) \delta 7.33-7.29(\mathrm{~m}, 5 \mathrm{H}), 7.27-7.25(\mathrm{~m}, 2 \mathrm{H}), 7.19(\mathrm{t}, J$ $=7.5 \mathrm{~Hz}, 4 \mathrm{H}), 7.09-7.07(\mathrm{~m}, 1 \mathrm{H}), 7.00(\mathrm{dd}, J=7.5,1.0 \mathrm{~Hz}, 1 \mathrm{H}), 6.94(\mathrm{td}, J=5.5,1.0 \mathrm{~Hz}, 1 \mathrm{H})$, $5.32(\mathrm{~d}, J=16.0 \mathrm{~Hz}, 1 \mathrm{H}), 5.13(\mathrm{~d}, J=16.0 \mathrm{~Hz}, 1 \mathrm{H}), 3.74(\mathrm{dd}, J=10.0,5.0 \mathrm{~Hz}, 1 \mathrm{H}), 3.36(\mathrm{dd}, J=$ 14.0, $5.0 \mathrm{~Hz}, 1 \mathrm{H}), 2.82(\mathrm{dd}, J=14.0,10.0 \mathrm{~Hz}, 1 \mathrm{H}) .{ }^{13} \mathrm{C} \mathrm{NMR}\left(126 \mathrm{MHz}, \mathrm{CDCl}_{3}\right) \delta 167.0,139.4$, 137.3, 136.9, 129.4, $128.9128 .8,128.5,127.3,127.2,127.0,126.3,123.9,121.8,117.9,48.9,45.3$, 34.9. Characterization data matches those reported in the literature ${ }^{[7]}$. HPLC [DAICEL CHIRALPAK AD-H, hexane $/ \mathrm{PrOH}=95 / 5,220 \mathrm{~nm}, 0.5 \mathrm{~mL} / \mathrm{min}, 25^{\circ} \mathrm{C} \cdot \mathrm{t}_{\mathrm{R} 1}=43.4 \mathrm{~min}$ (major), $\mathrm{t}_{\mathrm{R} 2}=51.4 \min$ (minor) $]$; $e e=60 \%$. $[\alpha]_{\mathrm{D}}^{25}=-176.00\left(c 0.10, \mathrm{CH}_{2} \mathrm{Cl}_{2}\right)$. HRMS (ESI) calcd for $\mathrm{C}_{22} \mathrm{H}_{19} \mathrm{NOS}(\mathrm{M}+\mathrm{H})^{+}:$346.1260; found: 346.1270 .<smiles>O=C1Oc2ccccc2C[C@H]1Cc1ccccc1</smiles>

2as

(R)-3-Benzylchroman-2-one (2as). Colorless oil, 78\% conv., (76\% yield), $36.2 \mathrm{mg} .{ }^{1} \mathrm{H}$ NMR $\left(500 \mathrm{MHz}, \mathrm{CDCl}_{3}\right) \delta 7.35(\mathrm{dd}, J=8.0,6.5 \mathrm{~Hz}, 2 \mathrm{H}), 7.31-7.25(\mathrm{~m}, 2 \mathrm{H}), 7.22-7.14(\mathrm{~m}, 2 \mathrm{H}), 7.13$ $-7.00(\mathrm{~m}, 3 \mathrm{H}), 3.45(\mathrm{dd}, J=14.0,4.5 \mathrm{~Hz}, 1 \mathrm{H}), 3.03-2.90(\mathrm{~m}, 1 \mathrm{H}), 2.83(\mathrm{dd}, J=16.0,6.0 \mathrm{~Hz}$, $1 \mathrm{H}), 2.82-2.69(\mathrm{~m}, 2 \mathrm{H}) .{ }^{13} \mathrm{C} \mathrm{NMR}\left(126 \mathrm{MHz}, \mathrm{CDCl}_{3}\right) \delta 170.6,151.6,138.0,129.2,128.7,128.3$, $128.2,126.9,124.4,122.4,116.6,40.8,35.6,28.2$. Characterization data matches those reported in the literature ${ }^{[8][9]}$. HPLC [DAICEL CHIRALPAK AD-H, hexane $/ i \operatorname{PrOH}=95 / 5,220 \mathrm{~nm}, 1.0$ $\mathrm{mL} / \mathrm{min}, 25{ }^{\circ} \mathrm{C} . \mathrm{t}_{\mathrm{R} 1}=9.8 \mathrm{~min}$ (minor), $\mathrm{t}_{\mathrm{R} 2}=12.3 \mathrm{~min}$ (major) $] ; e e=68 \% .[\alpha]_{\mathrm{D}}^{25}=-4.80(c 0.10$, $\mathrm{CH}_{2} \mathrm{Cl}_{2}$ ). HRMS (ESI) calcd for $\mathrm{C}_{16} \mathrm{H}_{14} \mathrm{O}_{2}(\mathrm{M}+\mathrm{H})^{+}: 239.1067$; found: 239.1070 .

\section{The Stereochemical Model}

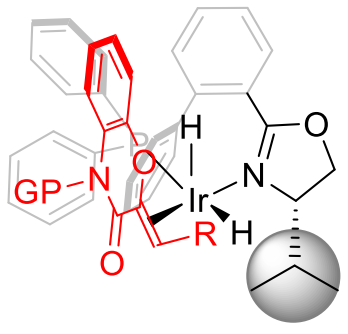

A favored

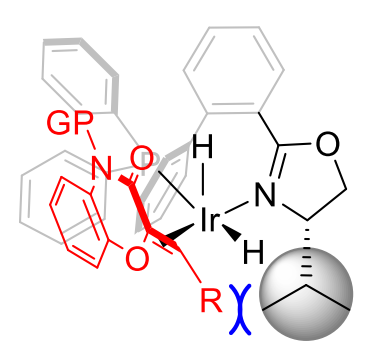

B disfavored 


\section{Reference}

[1] Zampieri, D.; Mamolo, M. G.; Filingeri, J.; Fortuna, S.; De Logu, A.; Sanna, A.; Zanon, D. Bioorg. Med. Chem. Lett. 2019, 29, 2468-2474.

[2] Chaudhari, M. B.; Chaudhary, A.; Kumar, V.; Gnanaprakasam, B. Org. Lett., 2019, 21, 1617-1621.

[3] Sharifi, A.; Ansari, M.; Darabi, H. R.; Abaee, M. S. J. Sulfur Chem. 2016, 37, 593-600.

[4] Gabillet, S.; Lecerclé, D.; Loreau, O.; Dézard, S.; Gomis, J.-M.; Taran, F., Synthesis 2007, 4, 515-522.

[5] Xu, X.; Li, X.; Yan, X.; Wang, H.; Deng, Y.; Shao, J. Synlett 2011, 20, 3026-3030.

[6] Liu, Y.; Qian, J.; Lou, S.; Xu, Z. Synlett 2009, 18, 2971-2976.

[7] Liu, W.; Min, H.; Zhu, X.; Deng, G.; Liang, Y., Org. Biomol. Chem., 2017, 15, 9804-9808.

[8] Martzel, T.; Annibaletto, J.; Levacher, V.; Brière, J.-F.; Oudeyer, S. Adv. Synth. Catal., 2019, 361, 995-1000.

[9] Lee, A.; Scheidt, K. A. Chem. Commun., 2015, 51, 3407-3410. 


\section{Copies of ${ }^{1} \mathrm{H}$ NMR and ${ }^{13} \mathrm{C}$ NMR Spectra}

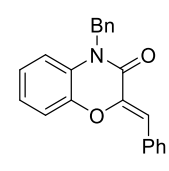

$1 \mathrm{a}$

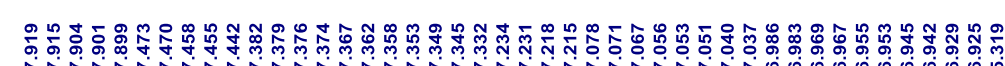

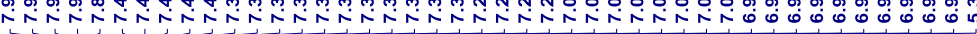

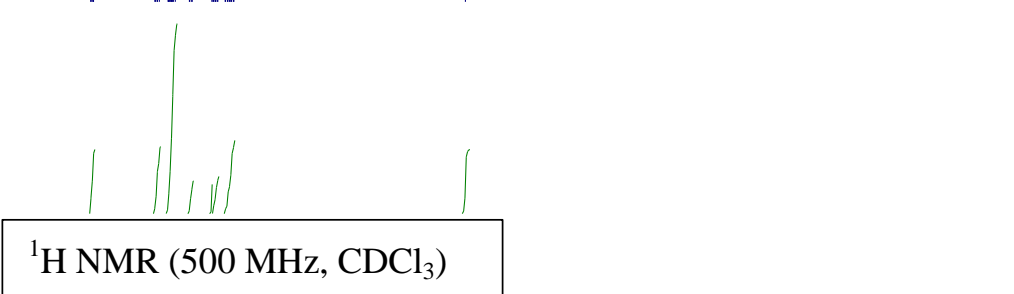

${ }^{1} \mathrm{H}$ NMR (500 MHz, $\mathrm{CDCl}_{3}$ )

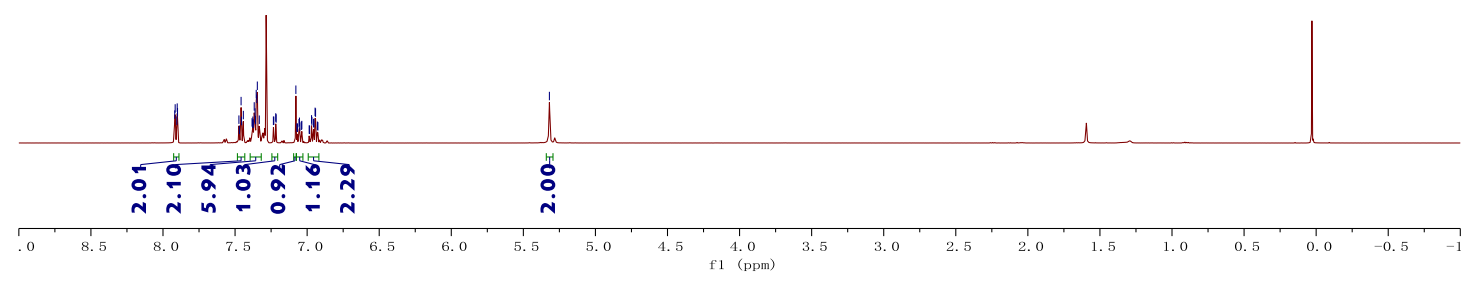

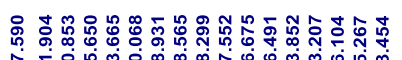

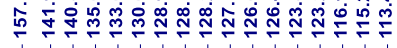

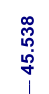

${ }^{13} \mathrm{C}$ NMR (126 MHz, $\left.\mathrm{CDCl}_{3}\right)$
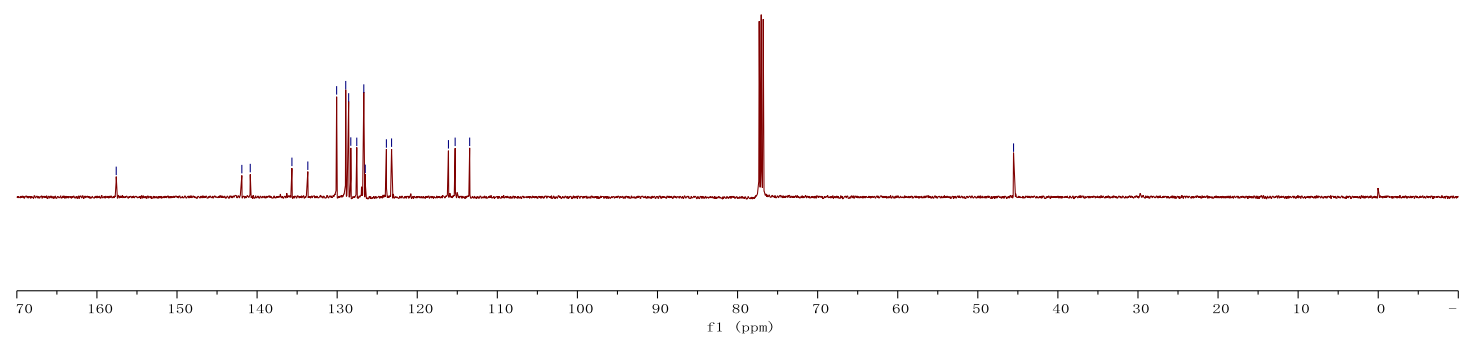


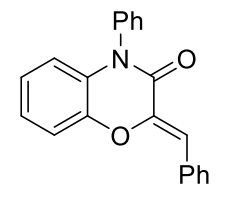

$1 \mathrm{~b}$

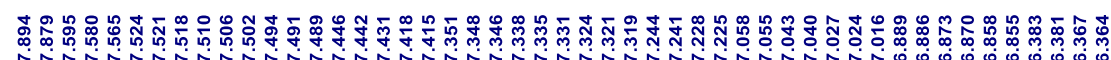

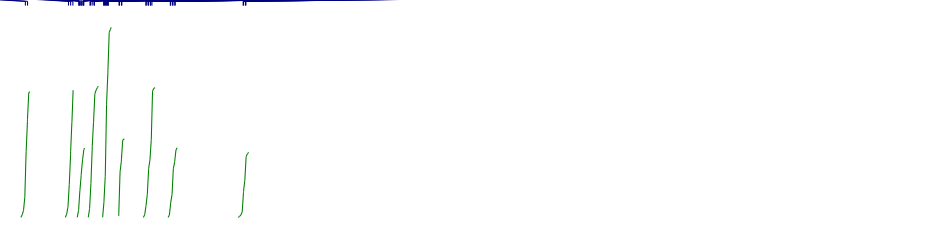

${ }^{1} \mathrm{H}$ NMR $\left(500 \mathrm{MHz}, \mathrm{CDCl}_{3}\right)$

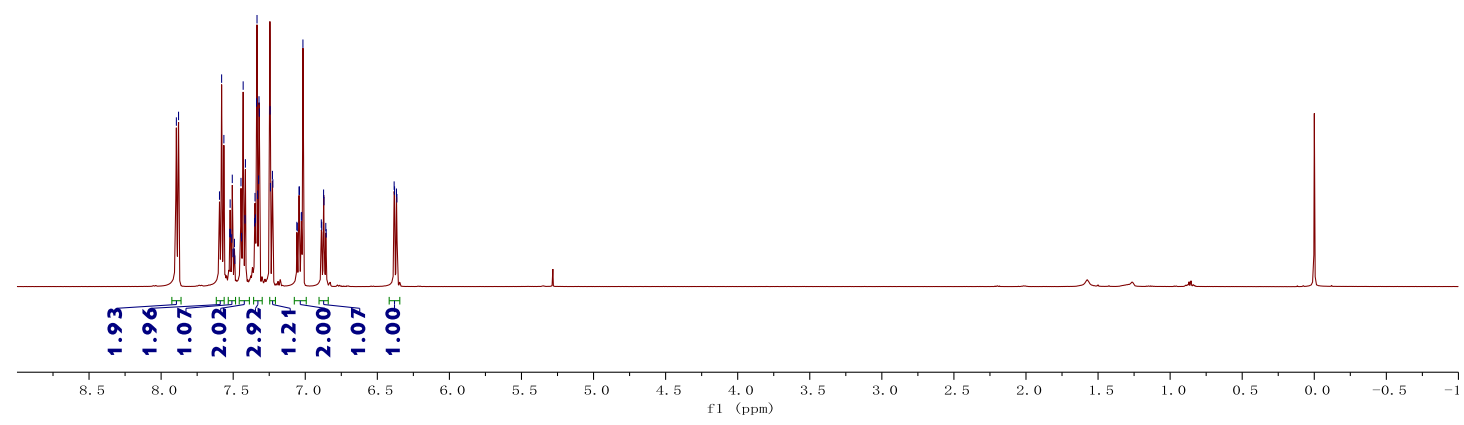

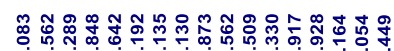

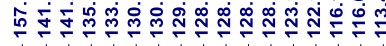

${ }^{13} \mathrm{C} \mathrm{NMR}\left(126 \mathrm{MHz}, \mathrm{CDCl}_{3}\right)$

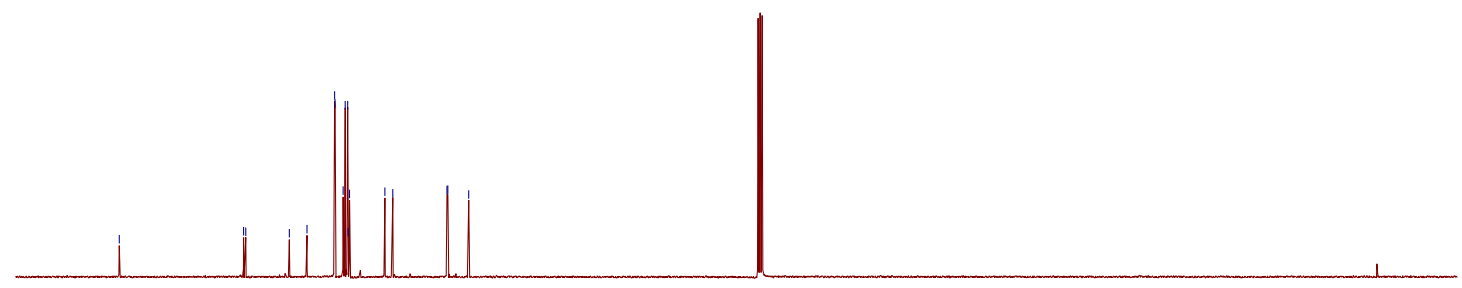

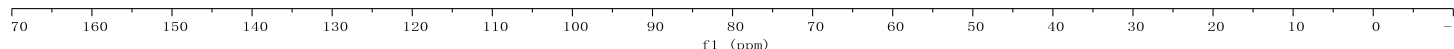




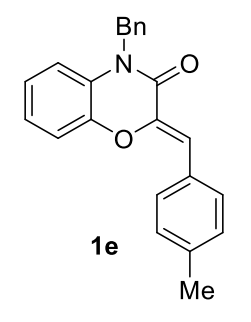

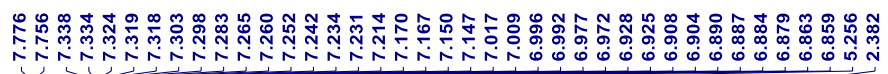

(

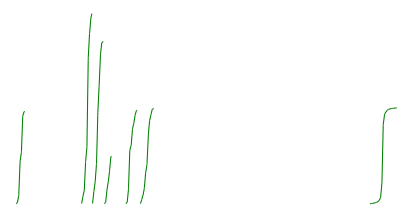

${ }^{1} \mathrm{H}$ NMR $\left(400 \mathrm{MHz}, \mathrm{CDCl}_{3}\right.$ )

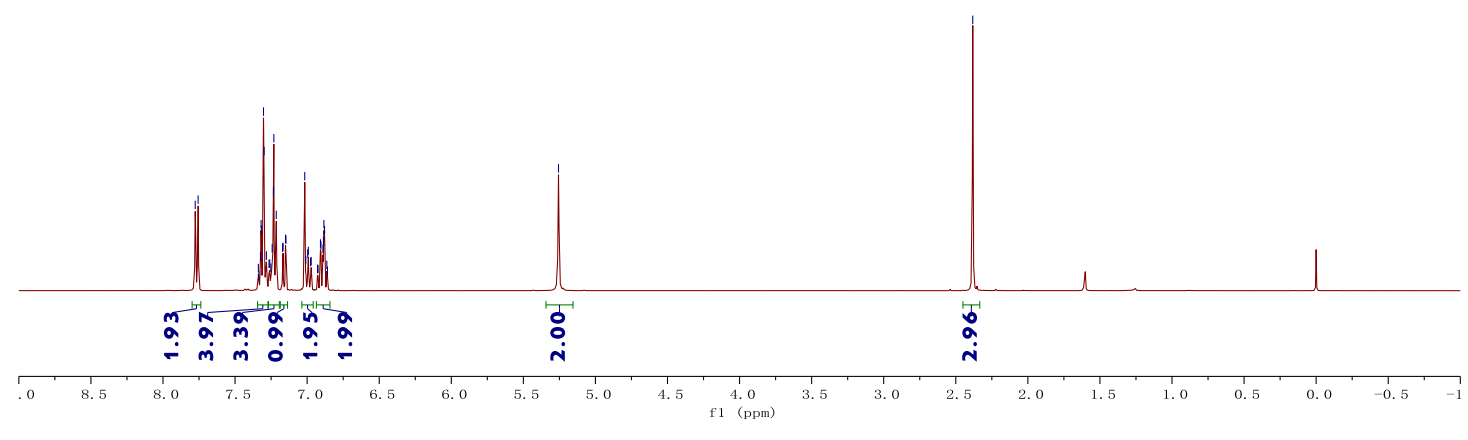

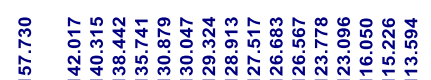

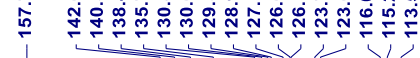

${ }^{13} \mathrm{C}$ NMR (101 MHz, $\left.\mathrm{CDCl}_{3}\right)$
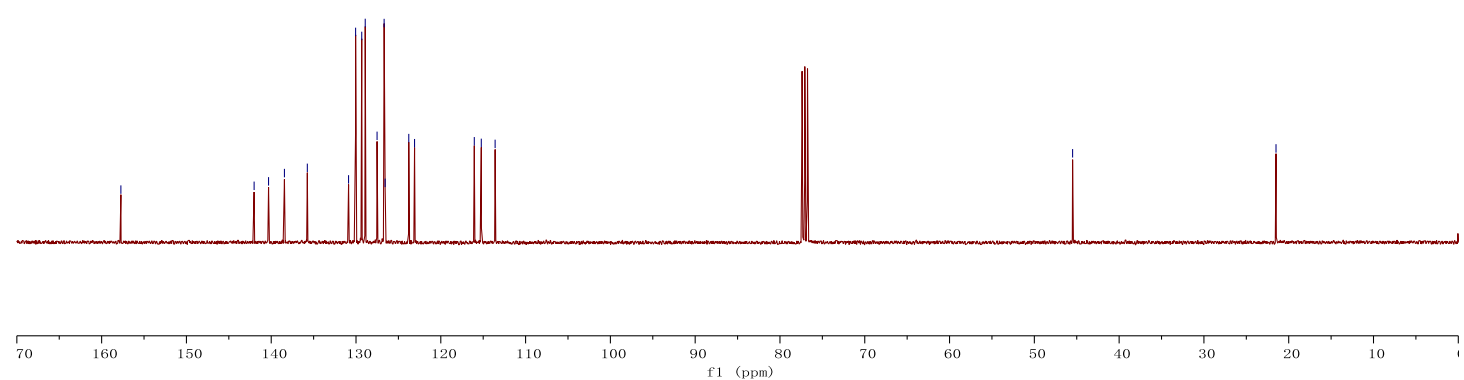

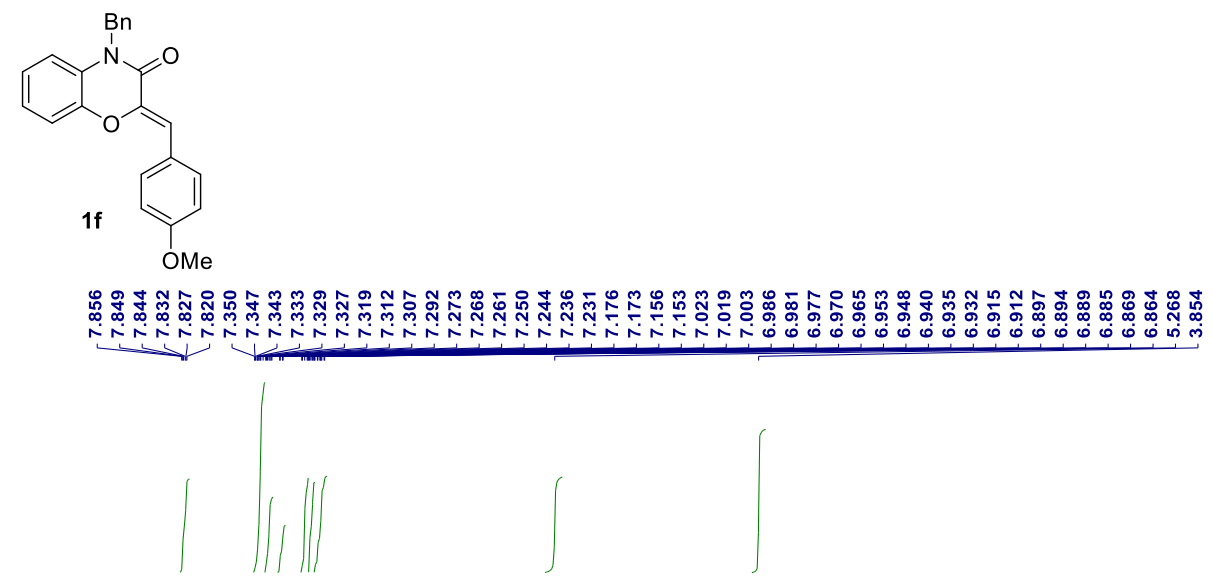

${ }^{1} \mathrm{H}$ NMR (400 MHz, $\mathrm{CDCl}_{3}$ )

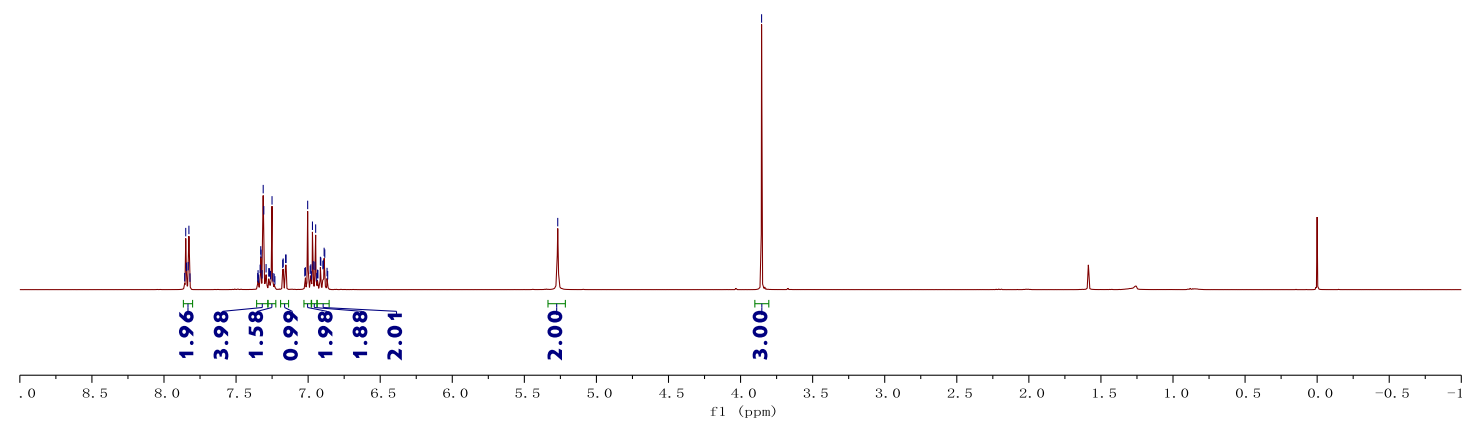

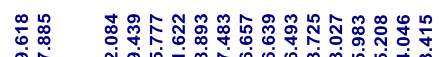

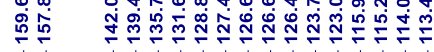

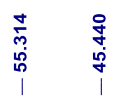

${ }^{13} \mathrm{C}$ NMR (101 MHz, $\left.\mathrm{CDCl}_{3}\right)$

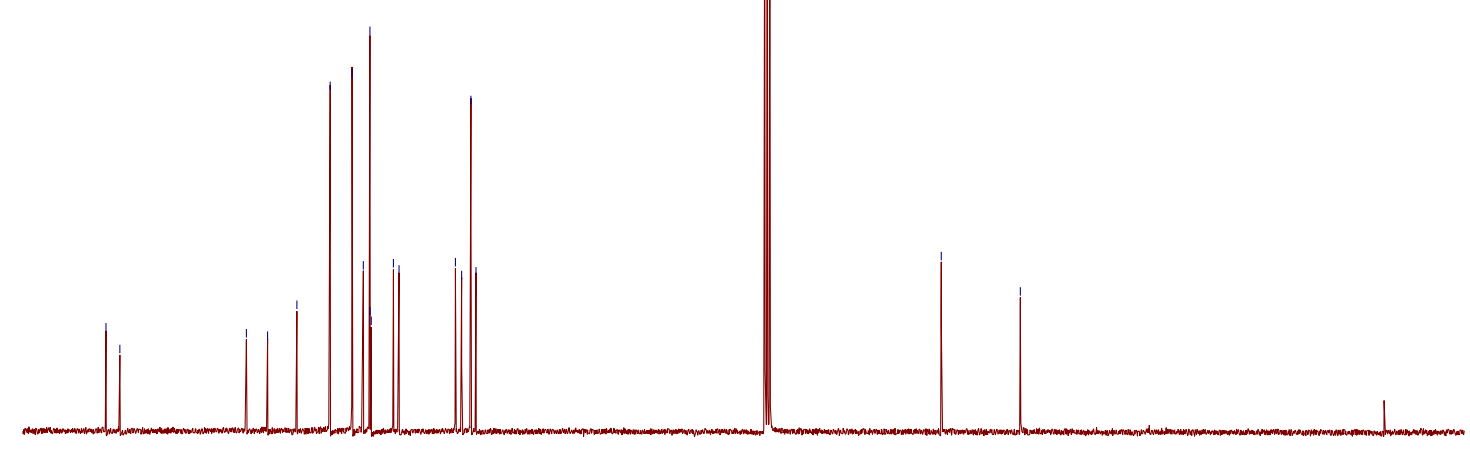




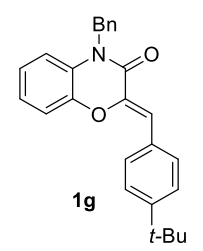

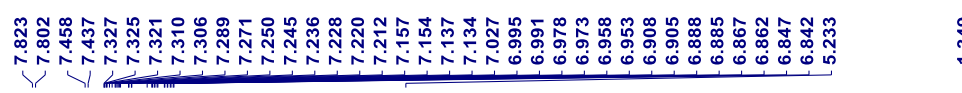

$\iiint_{1 / 1 /} \int$

${ }^{1} \mathrm{H}$ NMR (400 MHz, $\left.\mathrm{CDCl}_{3}\right)$

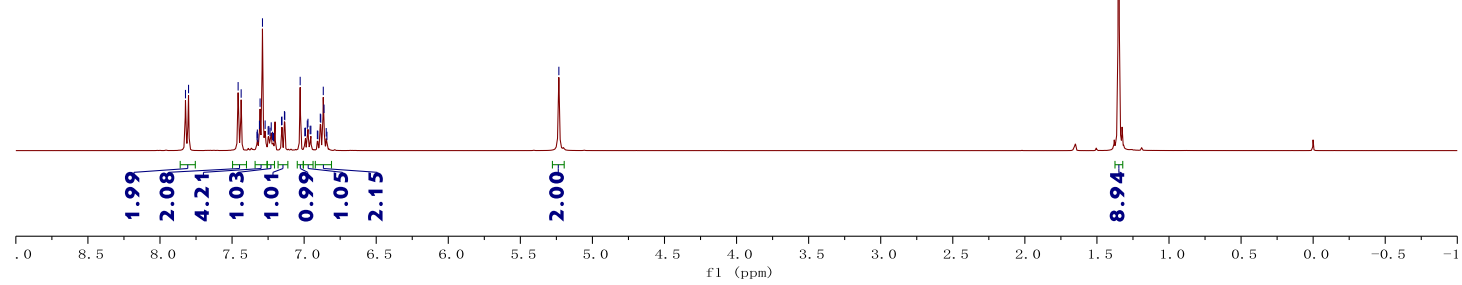

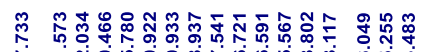

ํำ

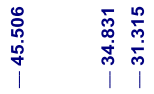

${ }^{13} \mathrm{C}$ NMR (101 MHz, $\left.\mathrm{CDCl}_{3}\right)$

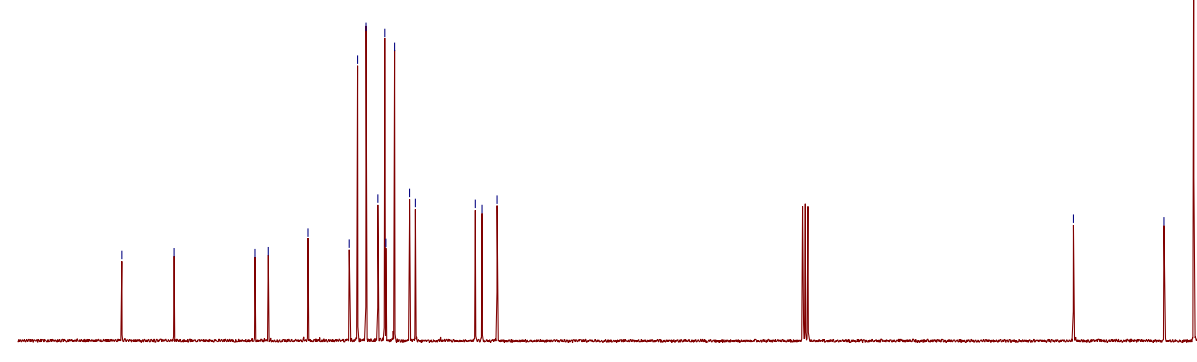



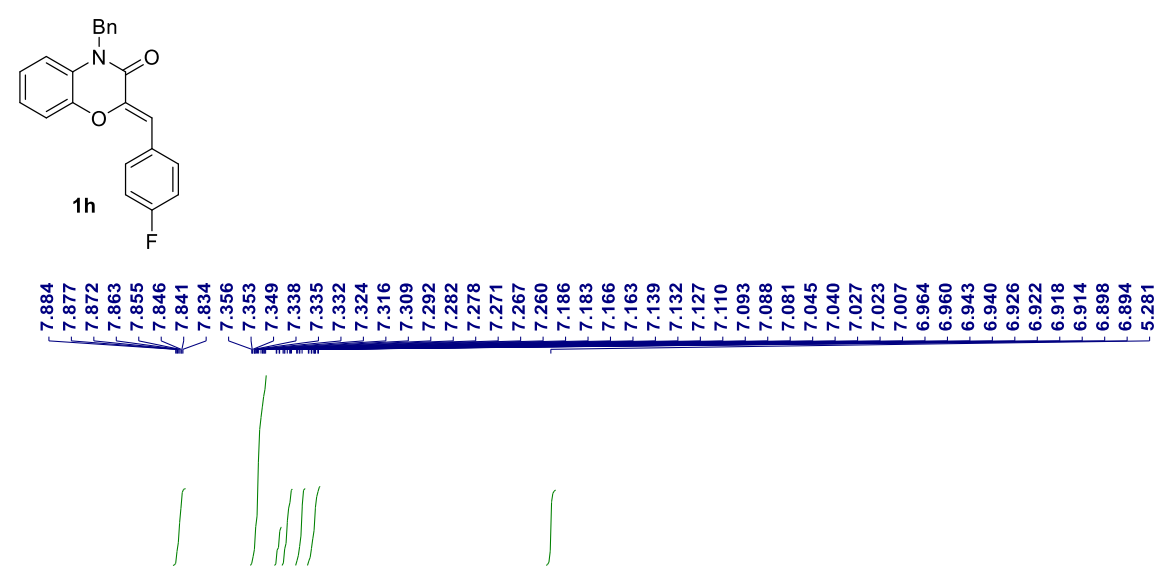

${ }^{1} \mathrm{H}$ NMR $\left(400 \mathrm{MHz}, \mathrm{CDCl}_{3}\right)$

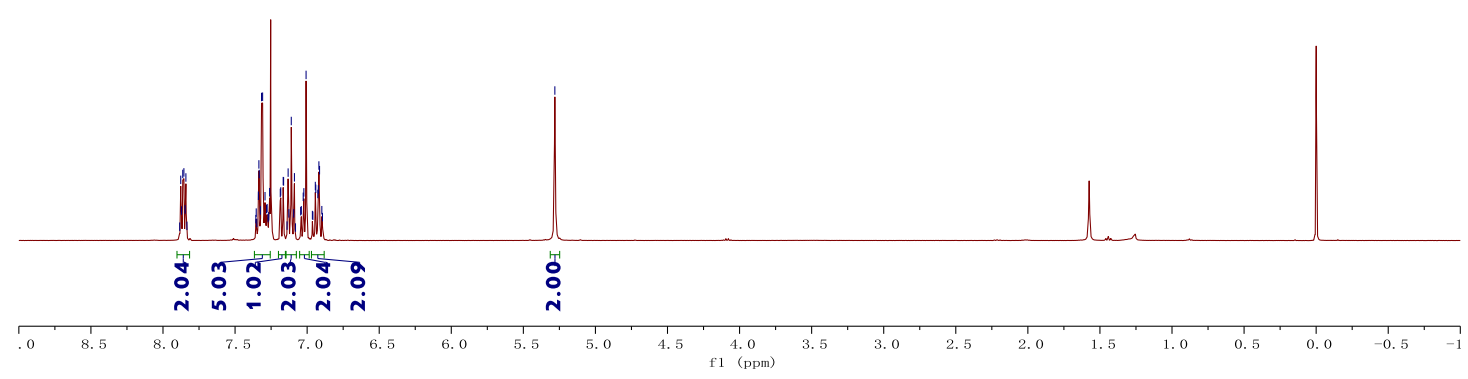

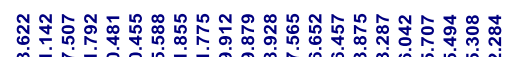

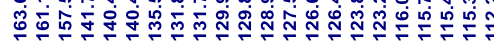

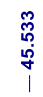

${ }^{13} \mathrm{C}$ NMR $\left(101 \mathrm{MHz}, \mathrm{CDCl}_{3}\right)$

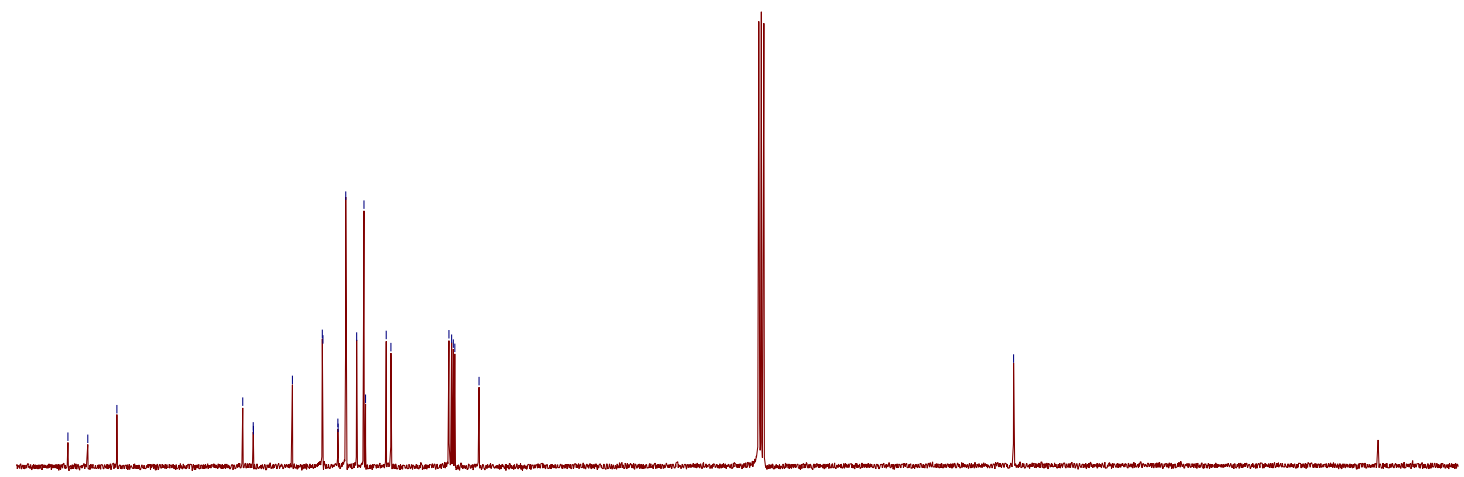


${ }^{19} \mathrm{~F}$ NMR $\left(376 \mathrm{MHz}, \mathrm{CDCl}_{3}\right)$

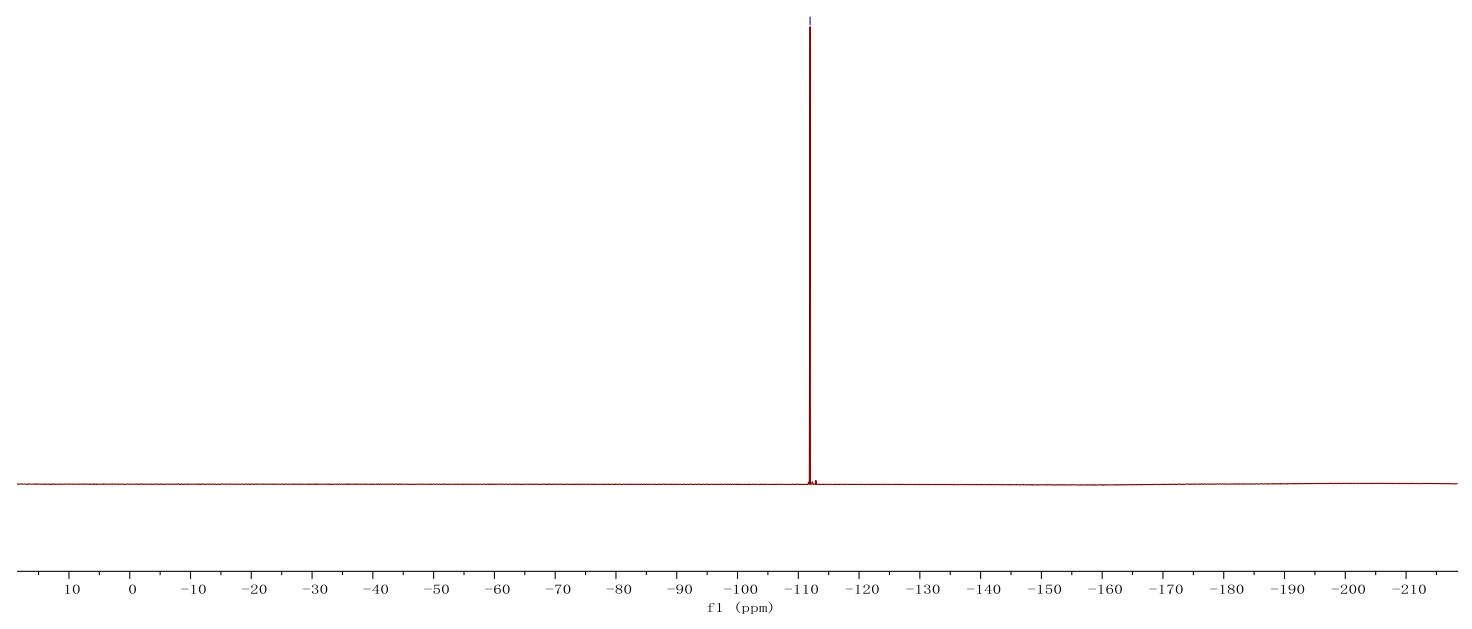




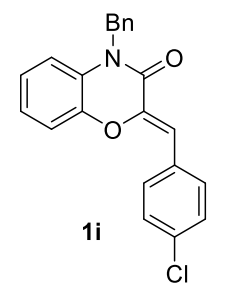

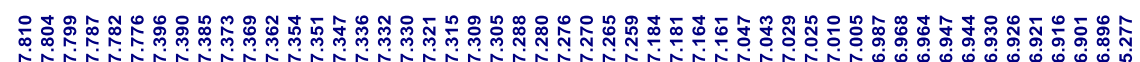

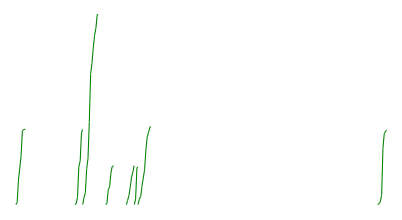

${ }^{1} \mathrm{H}$ NMR $\left(400 \mathrm{MHz}, \mathrm{CDCl}_{3}\right)$

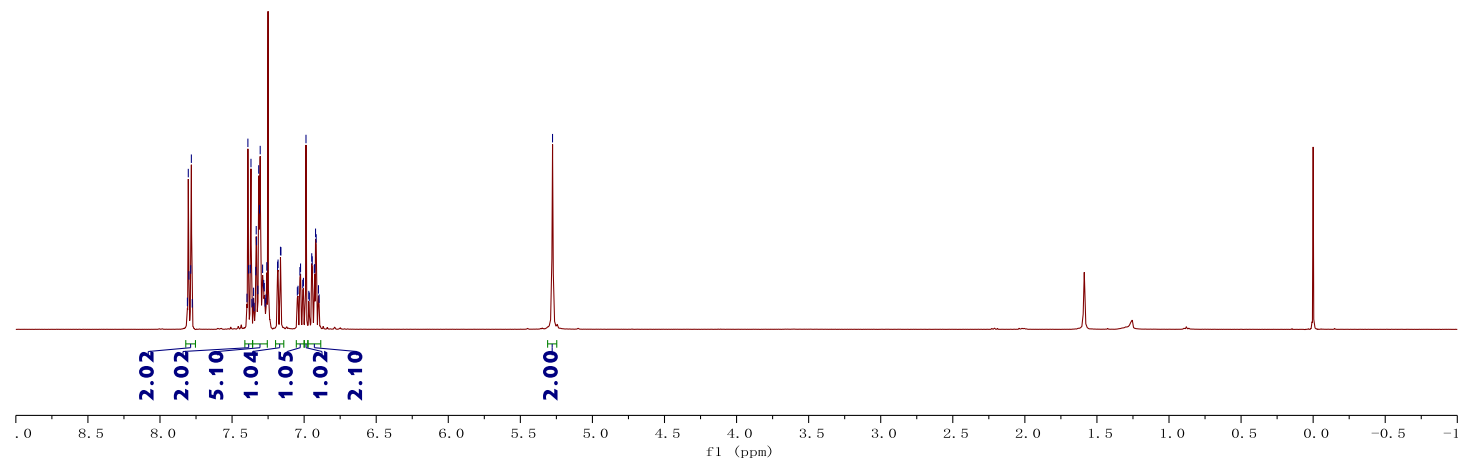

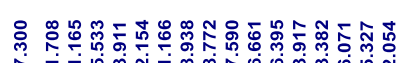

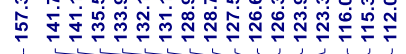

葛

${ }^{13} \mathrm{C}$ NMR $\left(101 \mathrm{MHz}, \mathrm{CDCl}_{3}\right)$

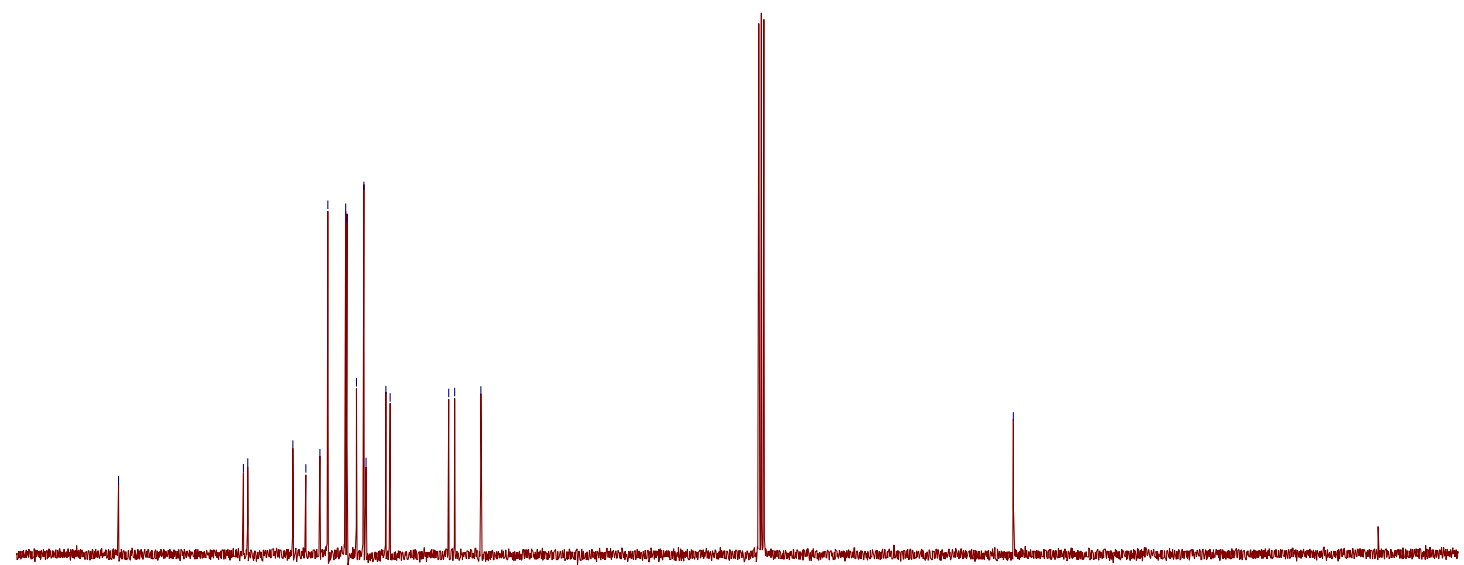

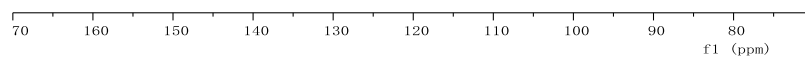




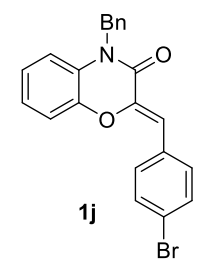

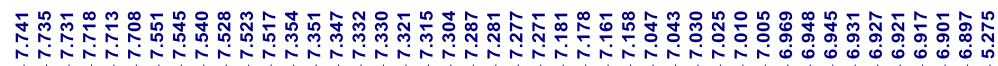

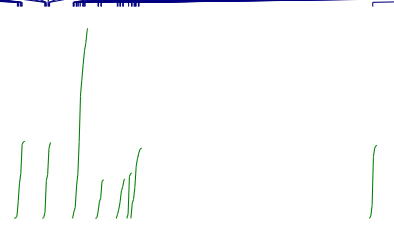

${ }^{1} \mathrm{H}$ NMR $\left(400 \mathrm{MHz}, \mathrm{CDCl}_{3}\right)$

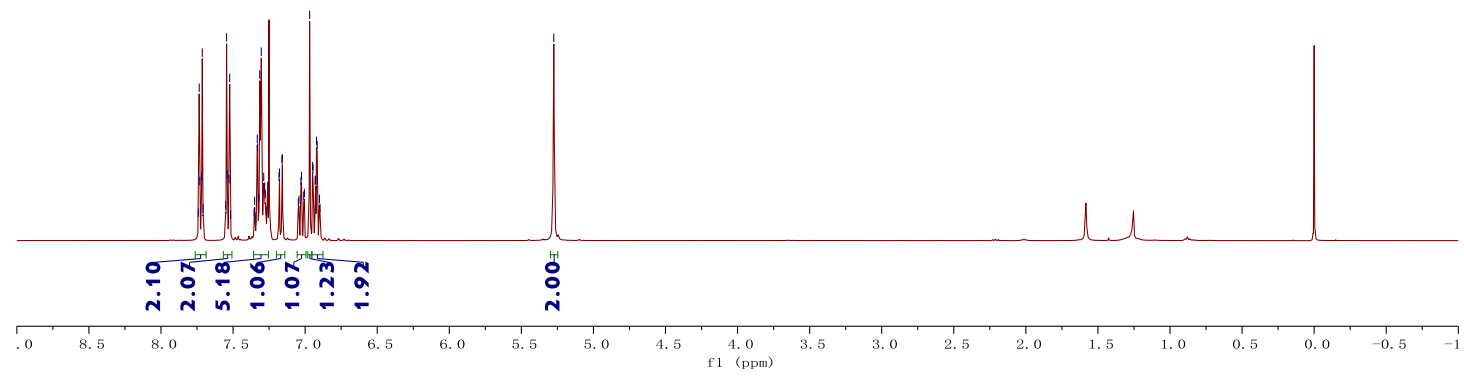

ํํำ

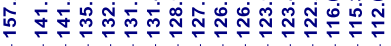

${ }^{13} \mathrm{C}$ NMR $\left(101 \mathrm{MHz}, \mathrm{CDCl}_{3}\right)$

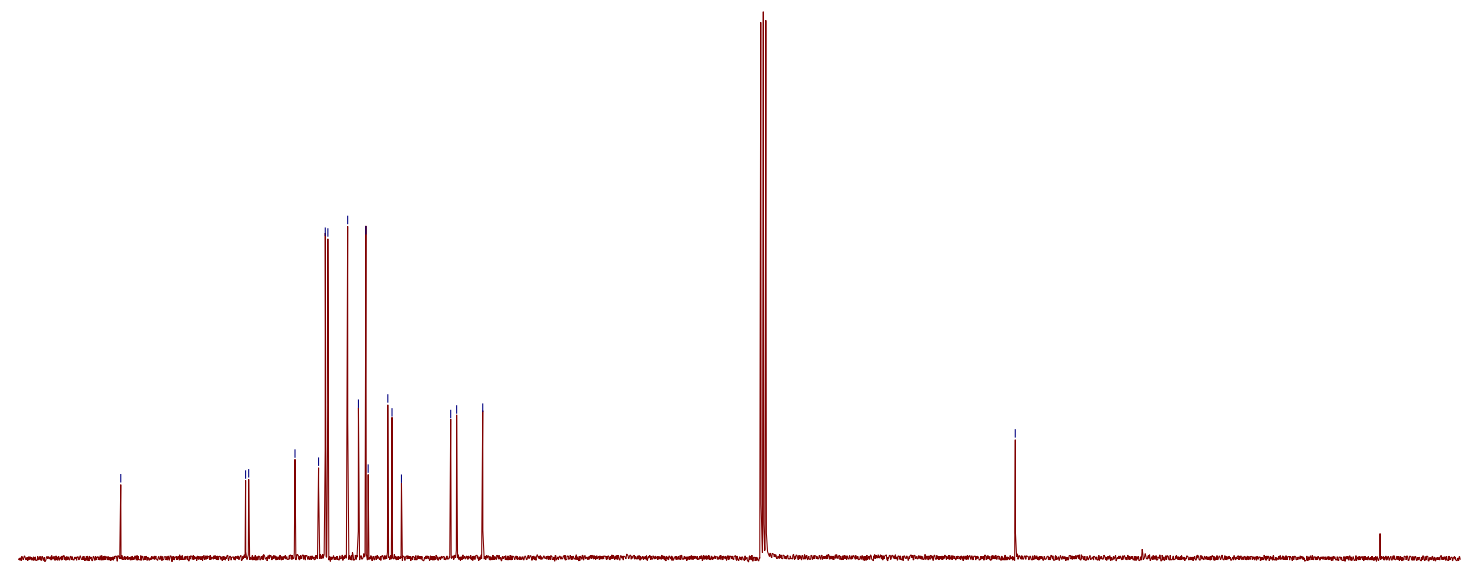

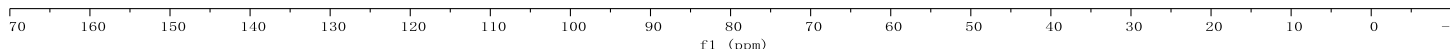


<smiles></smiles>

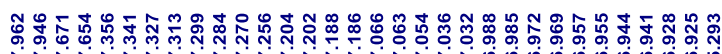

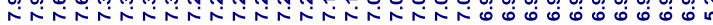

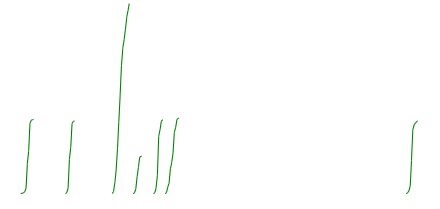

${ }^{1} \mathrm{H}$ NMR $\left(500 \mathrm{MHz}, \mathrm{CDCl}_{3}\right)$

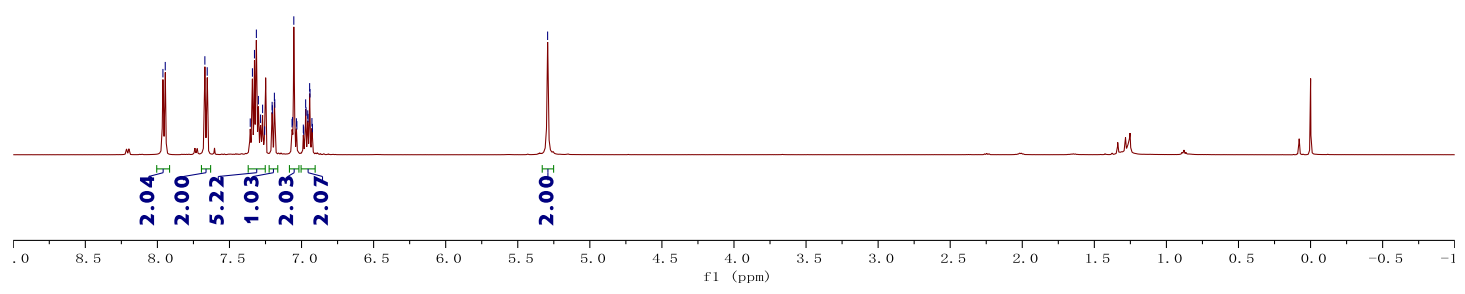

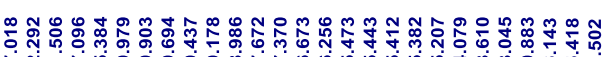

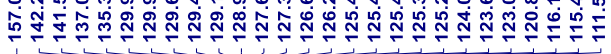

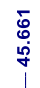

${ }^{13} \mathrm{C} \mathrm{NMR}\left(126 \mathrm{MHz}, \mathrm{CDCl}_{3}\right)$

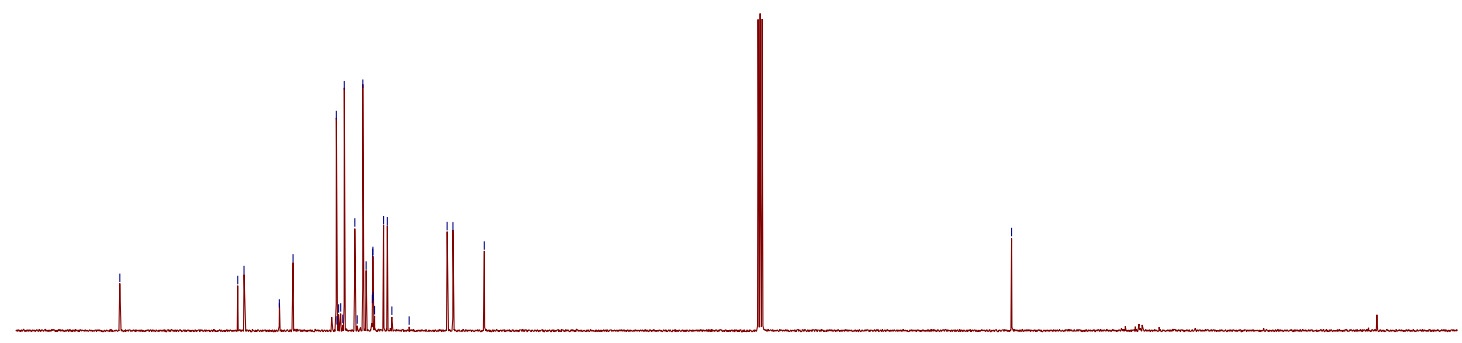

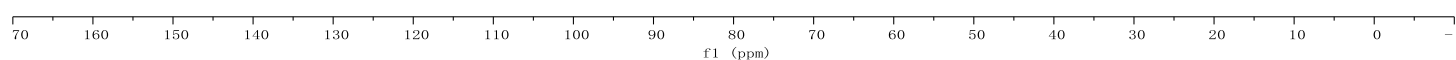


${ }^{19}$ F NMR (471 MHz, $\mathrm{CDCl}_{3}$ )

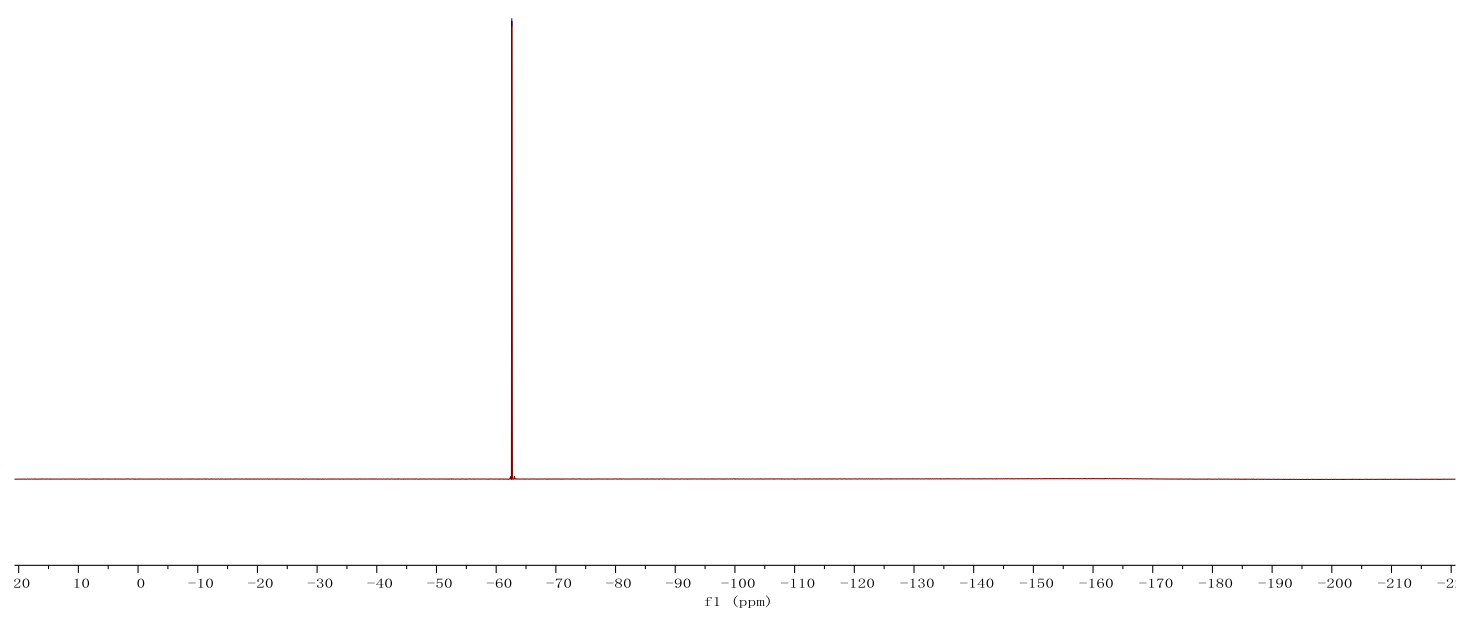




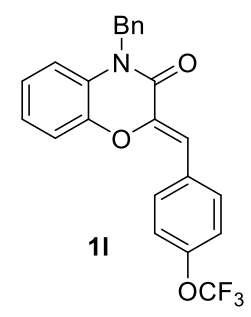

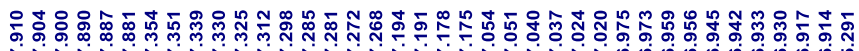

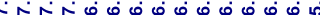

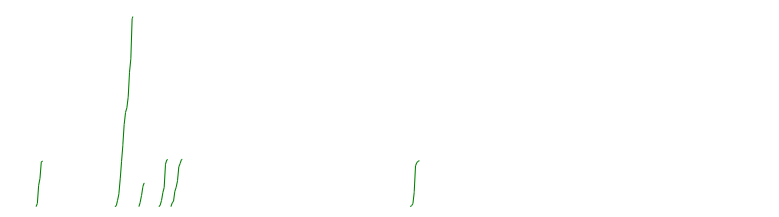

${ }^{1} \mathrm{H}$ NMR $\left(500 \mathrm{MHz}, \mathrm{CDCl}_{3}\right)$

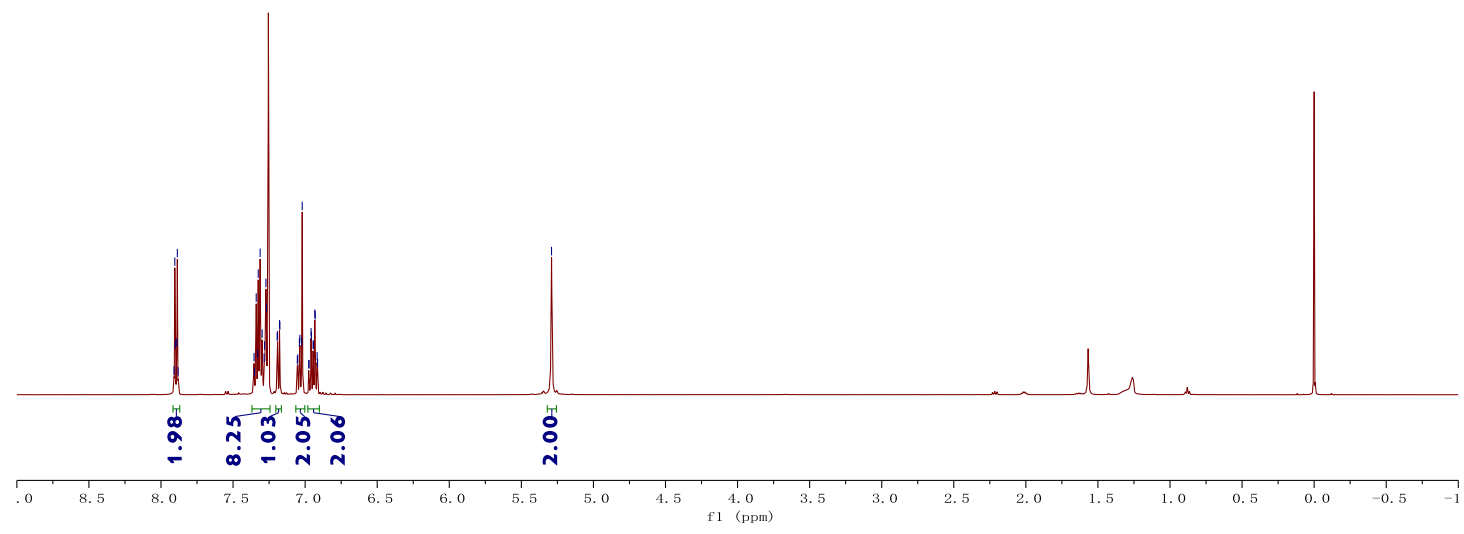

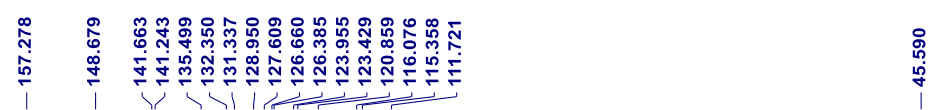

${ }^{13} \mathrm{C} \mathrm{NMR}\left(126 \mathrm{MHz}, \mathrm{CDCl}_{3}\right)$

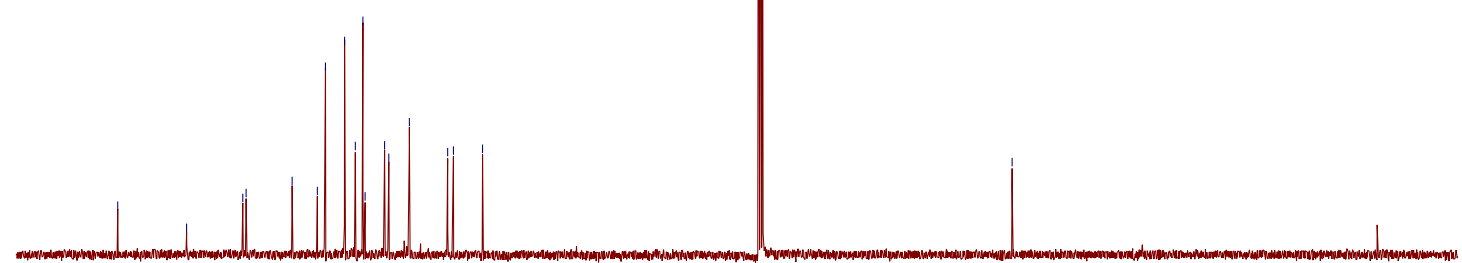

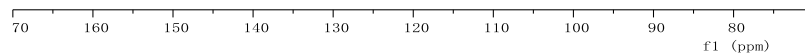


${ }^{19} \mathrm{~F}$ NMR $\left(376 \mathrm{MHz}, \mathrm{CDCl}_{3}\right)$

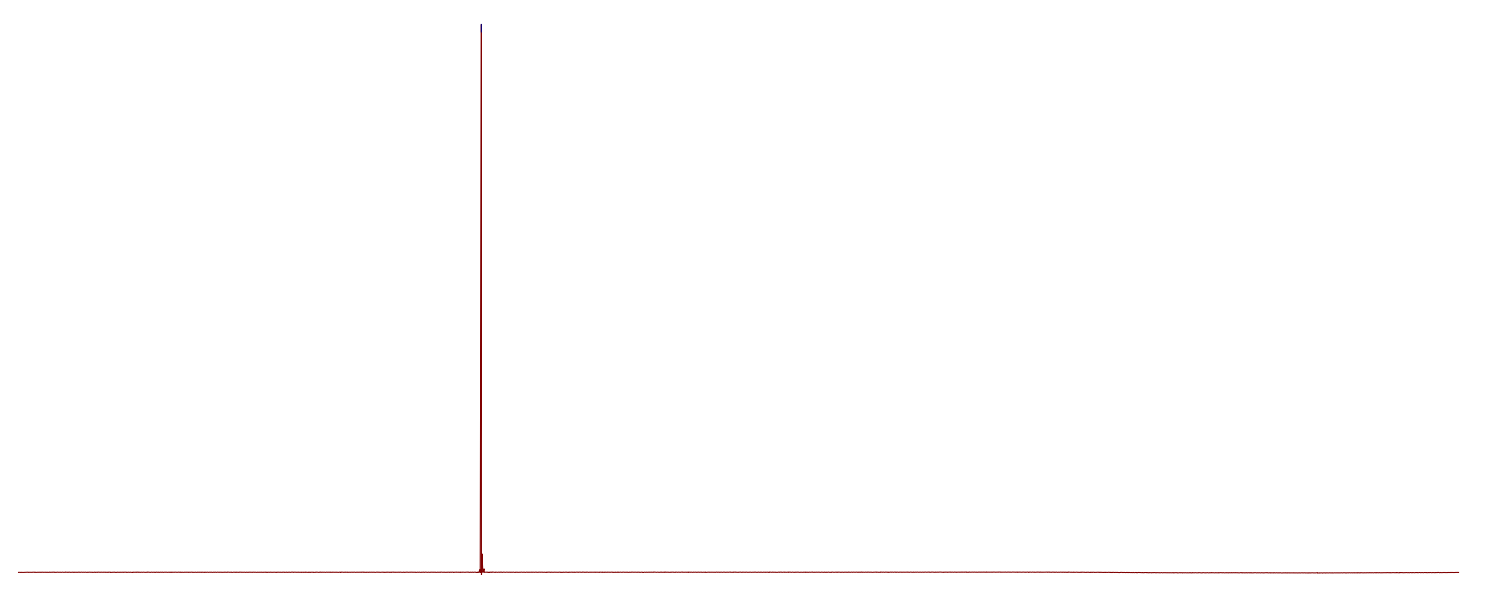

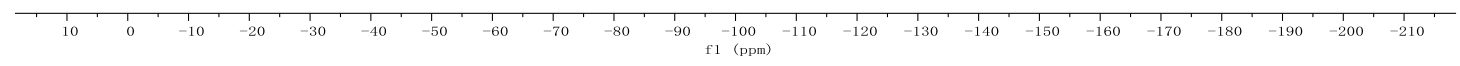



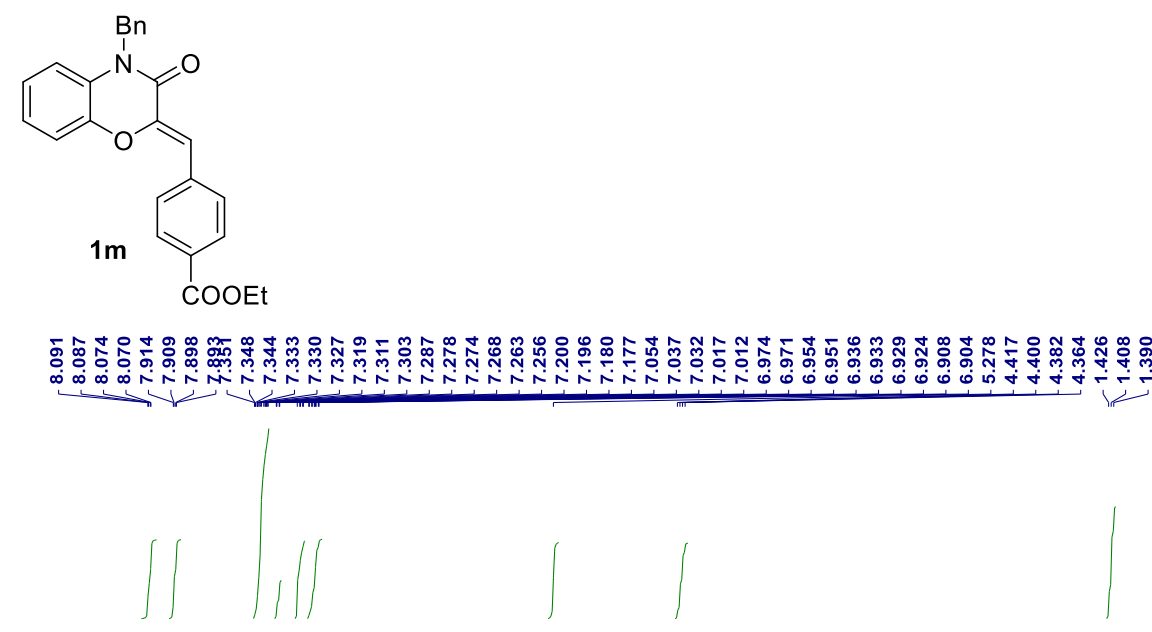

${ }^{1} \mathrm{H} \mathrm{NMR}\left(400 \mathrm{MHz}, \mathrm{CDCl}_{3}\right)$

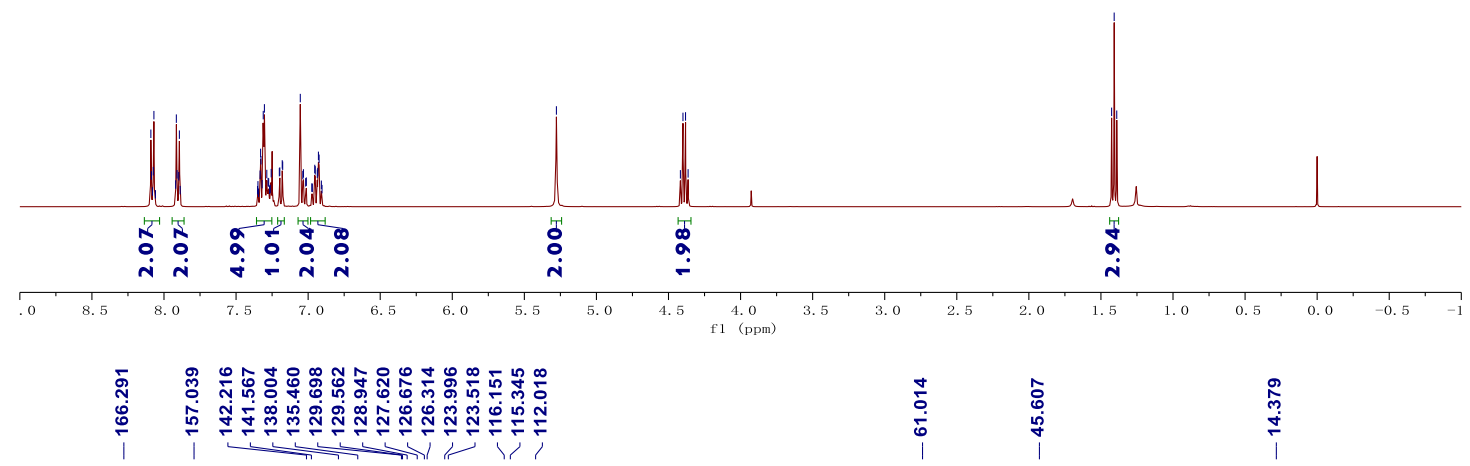

${ }^{13} \mathrm{C}$ NMR $\left(101 \mathrm{MHz}, \mathrm{CDCl}_{3}\right)$

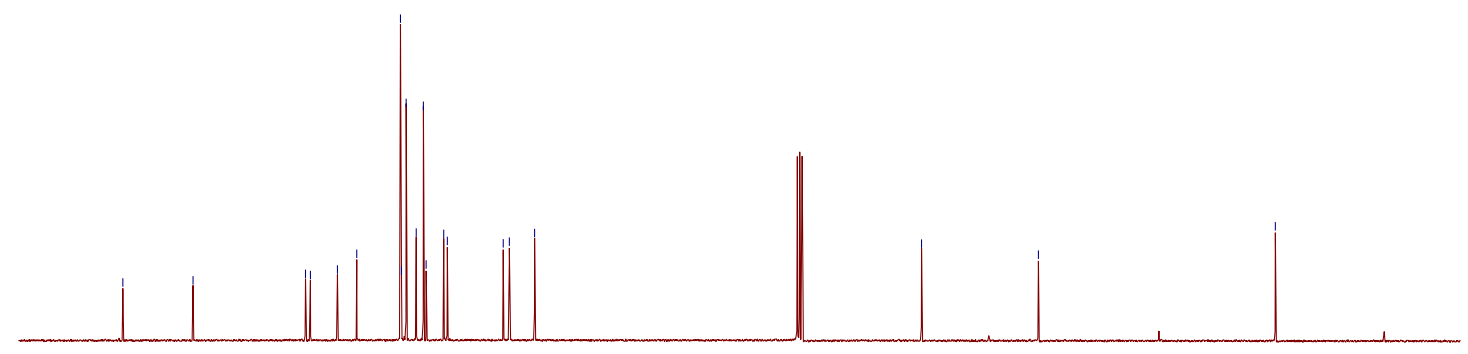

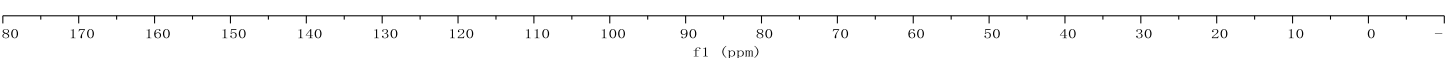




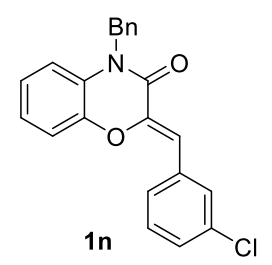

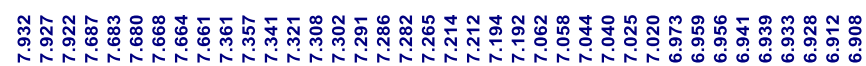

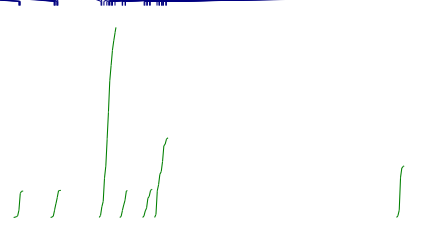

${ }^{1} \mathrm{H}$ NMR $\left(400 \mathrm{MHz}, \mathrm{CDCl}_{3}\right)$

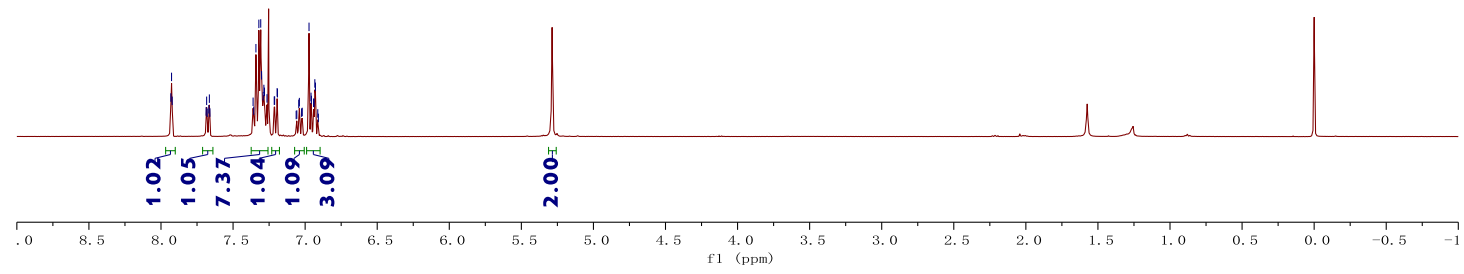

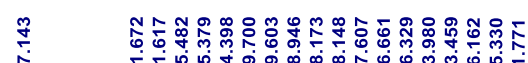

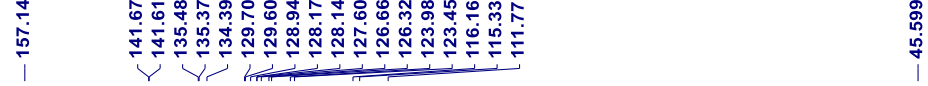

${ }^{13} \mathrm{C}$ NMR (101 MHz, $\left.\mathrm{CDCl}_{3}\right)$

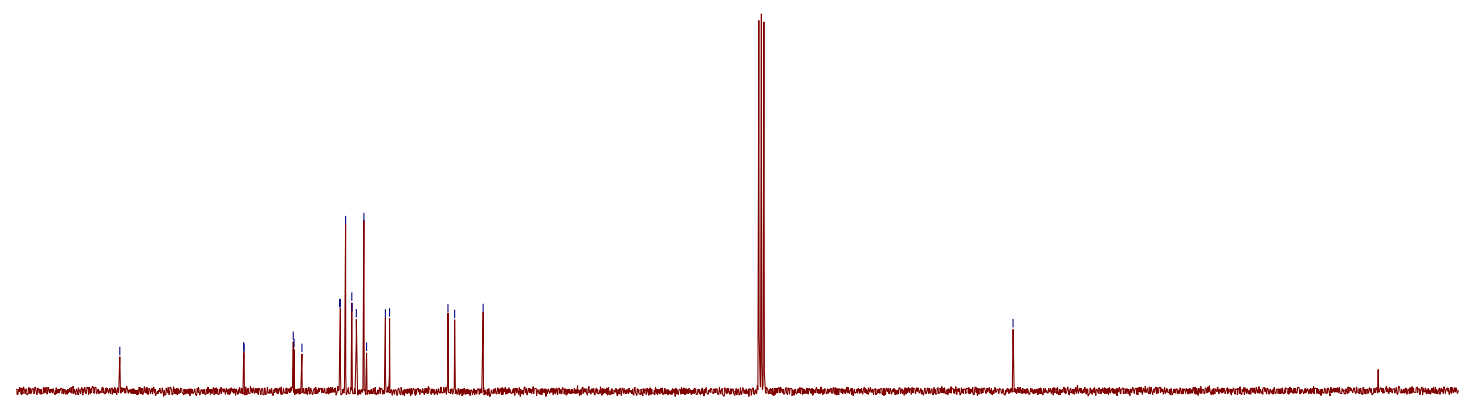




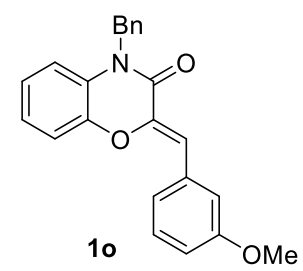

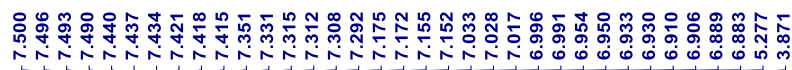

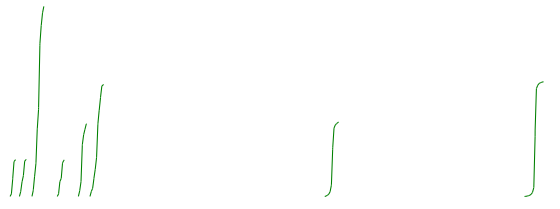

${ }^{1} \mathrm{H}$ NMR (400 MHz, $\left.\mathrm{CDCl}_{3}\right)$

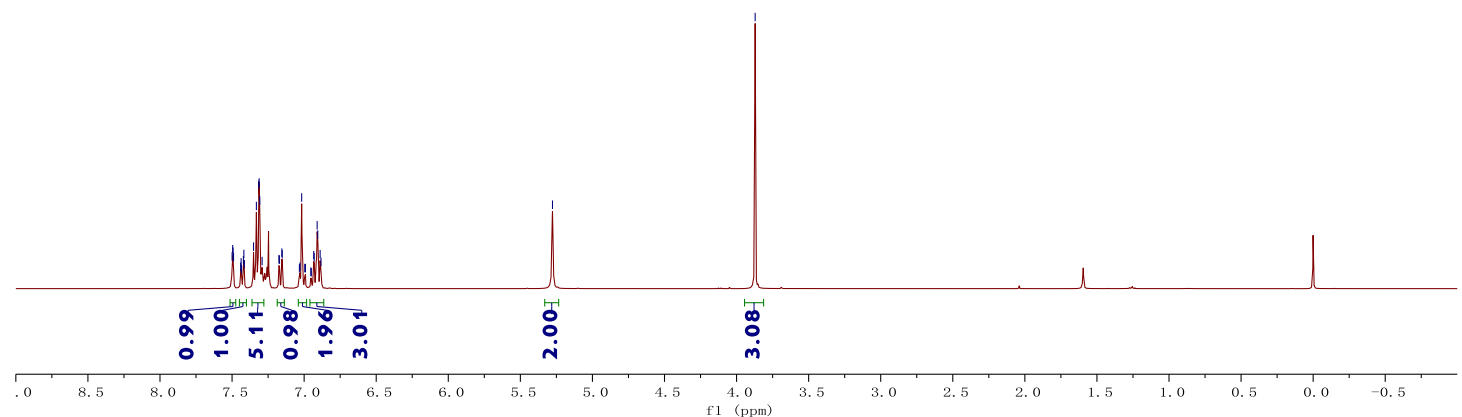

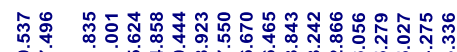

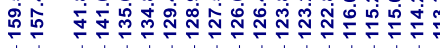

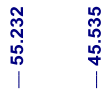

${ }^{13} \mathrm{C}$ NMR (101 MHz, $\left.\mathrm{CDCl}_{3}\right)$

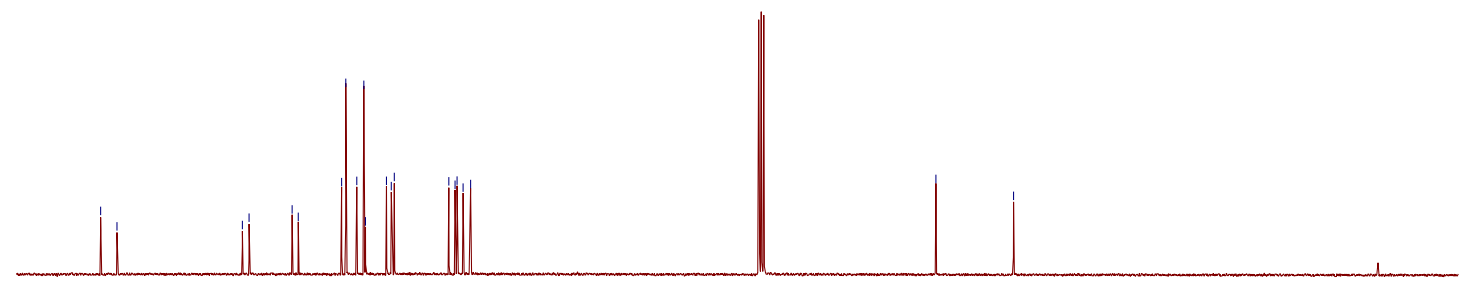

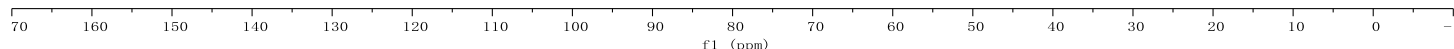




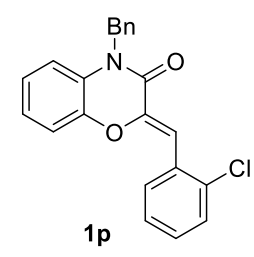

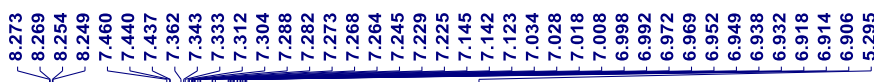

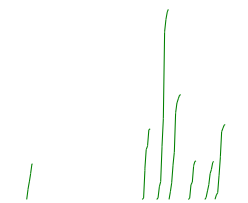

${ }^{1} \mathrm{H}$ NMR $\left(400 \mathrm{MHz}, \mathrm{CDCl}_{3}\right)$

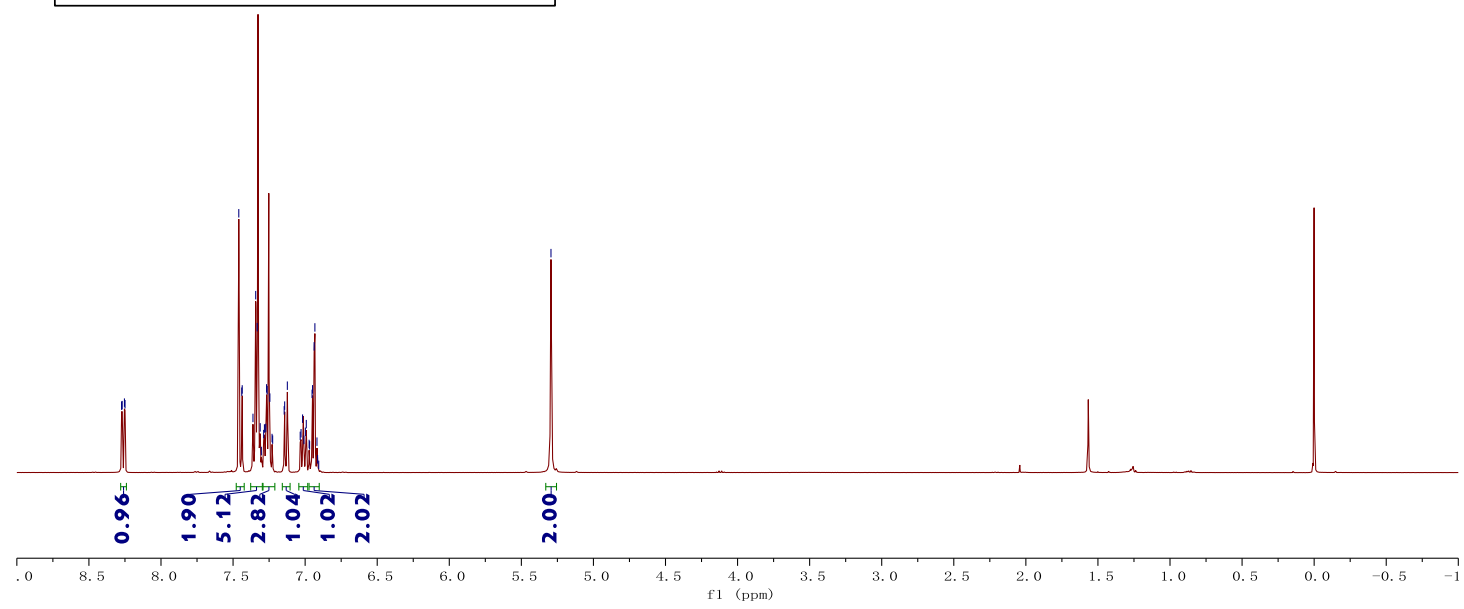

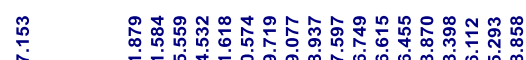

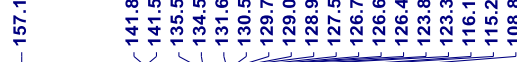

${ }^{13} \mathrm{C}$ NMR $\left(101 \mathrm{MHz}, \mathrm{CDCl}_{3}\right)$

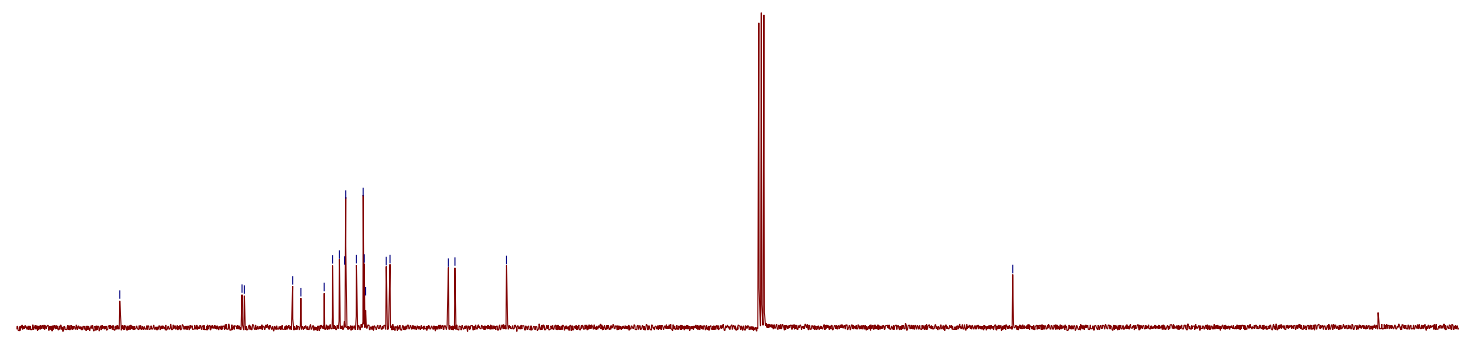


<smiles>COc1ccccc1/C=C1\Oc2ccccc2N(Cc2ccccc2)C1=O</smiles>

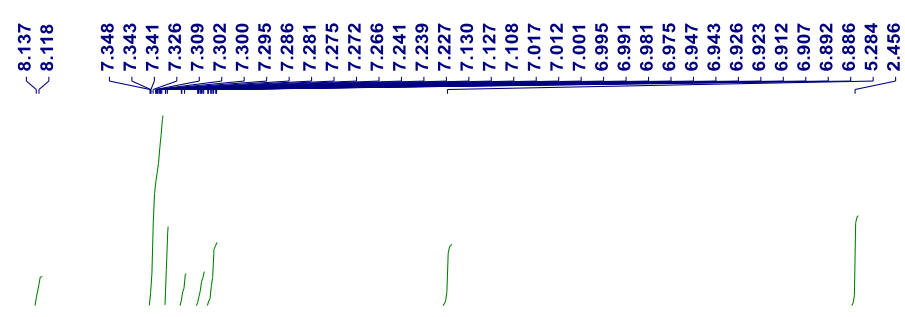

${ }^{1} \mathrm{H}$ NMR $\left(400 \mathrm{MHz}, \mathrm{CDCl}_{3}\right.$ )

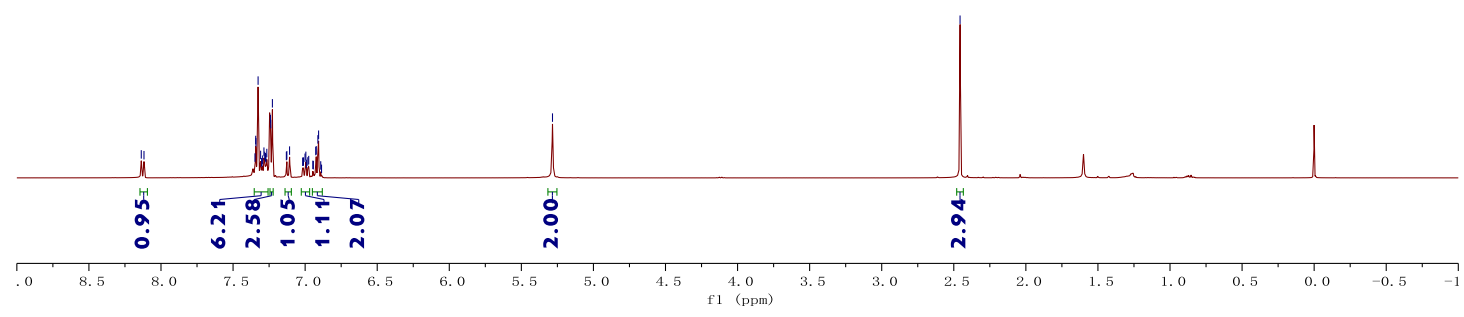

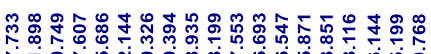

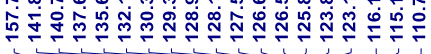

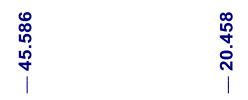

${ }^{13} \mathrm{C}$ NMR (101 MHz, $\mathrm{CDCl}_{3}$ )
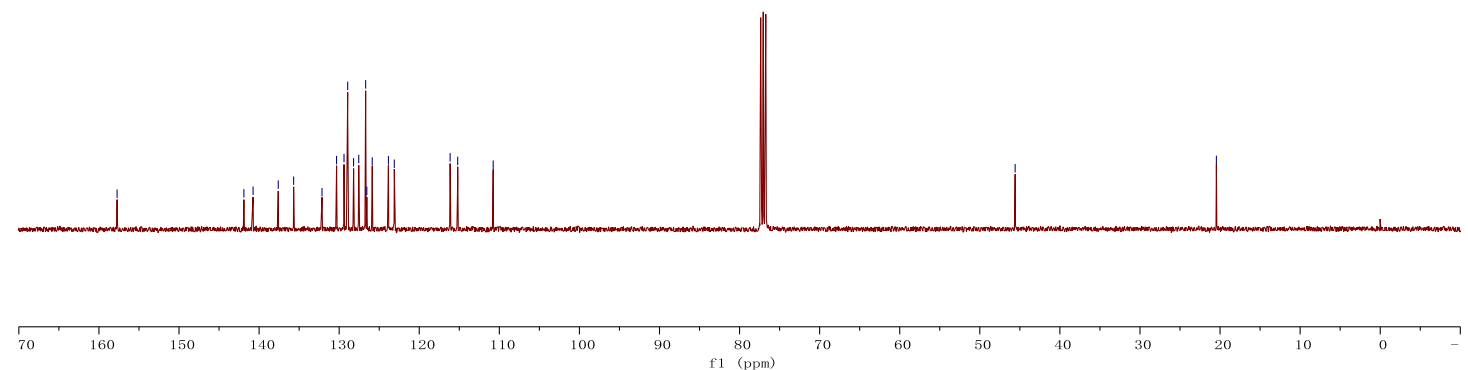

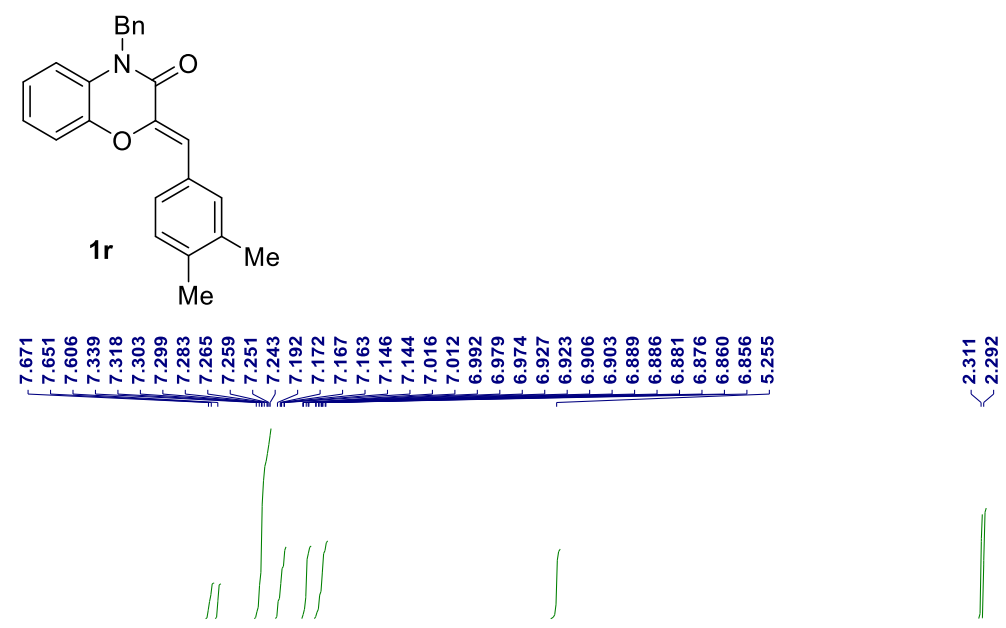

${ }^{1} \mathrm{H}$ NMR $\left(400 \mathrm{MHz}, \mathrm{CDCl}_{3}\right)$

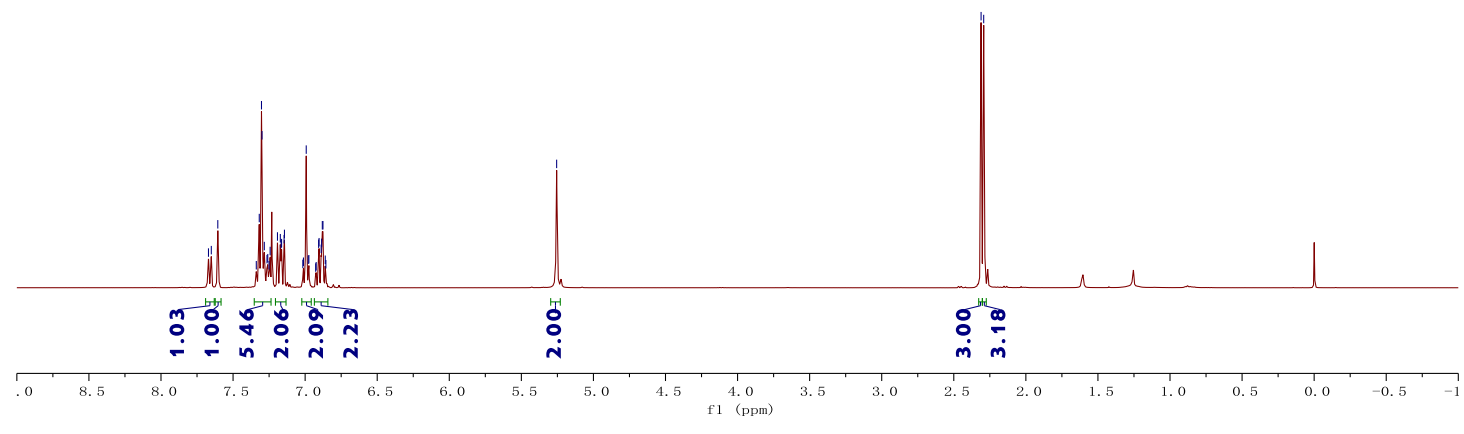

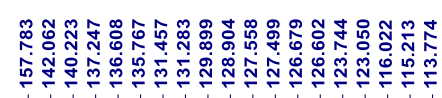

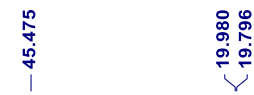

${ }^{13} \mathrm{C}$ NMR (101 MHz, $\mathrm{CDCl}_{3}$ )
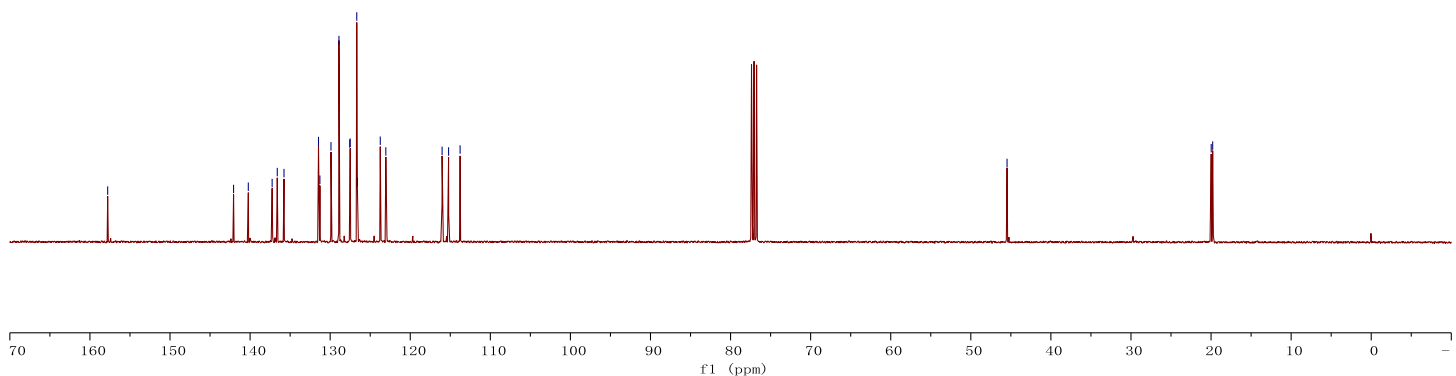


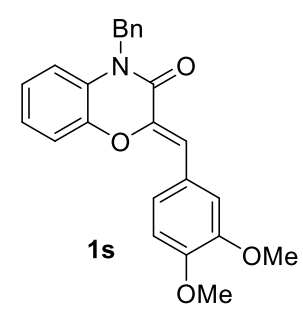

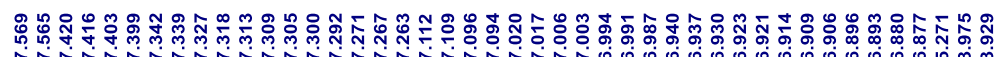



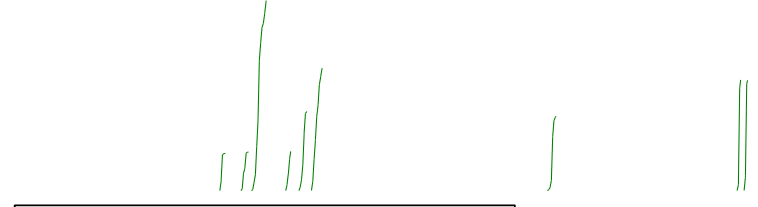

${ }^{1} \mathrm{H} \mathrm{NMR}\left(500 \mathrm{MHz}, \mathrm{CDCl}_{3}\right)$

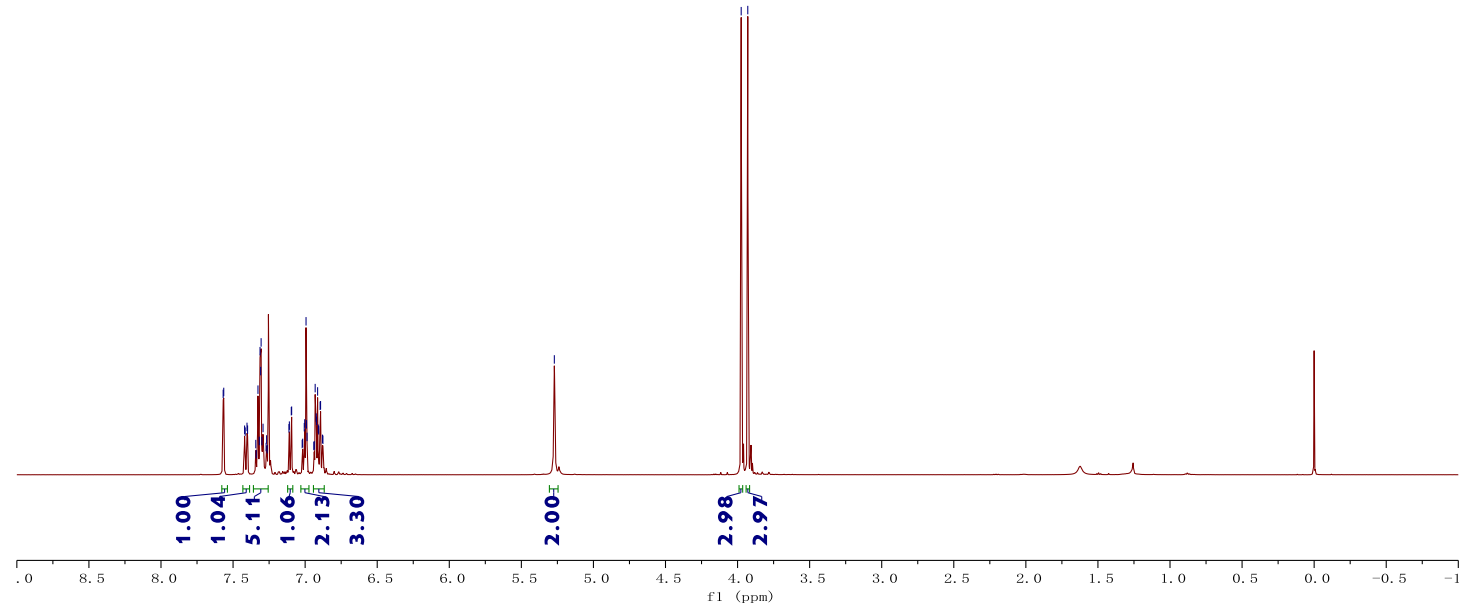

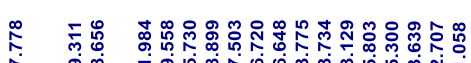

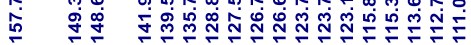

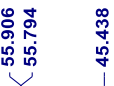

${ }^{13} \mathrm{C}$ NMR $\left(126 \mathrm{MHz}, \mathrm{CDCl}_{3}\right)$

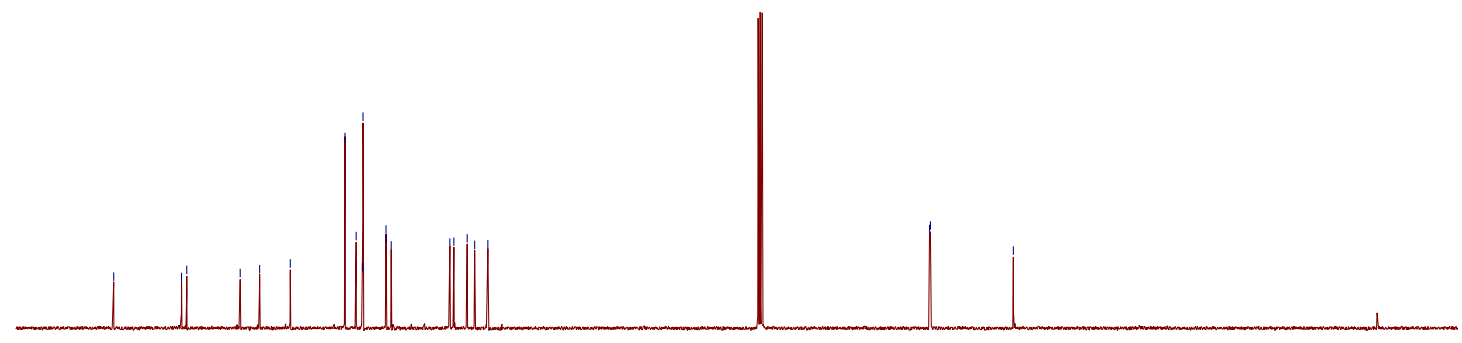

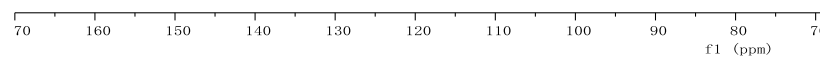




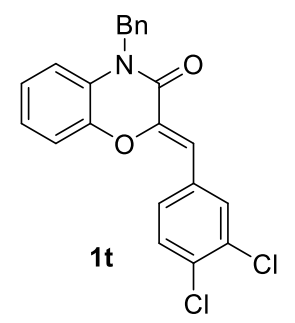

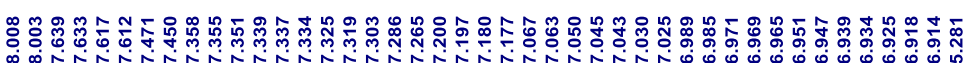

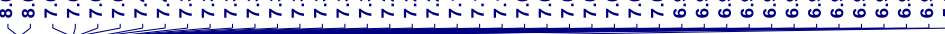

${ }^{1} \mathrm{H}$ NMR $\left(400 \mathrm{MHz}, \mathrm{CDCl}_{3}\right)$

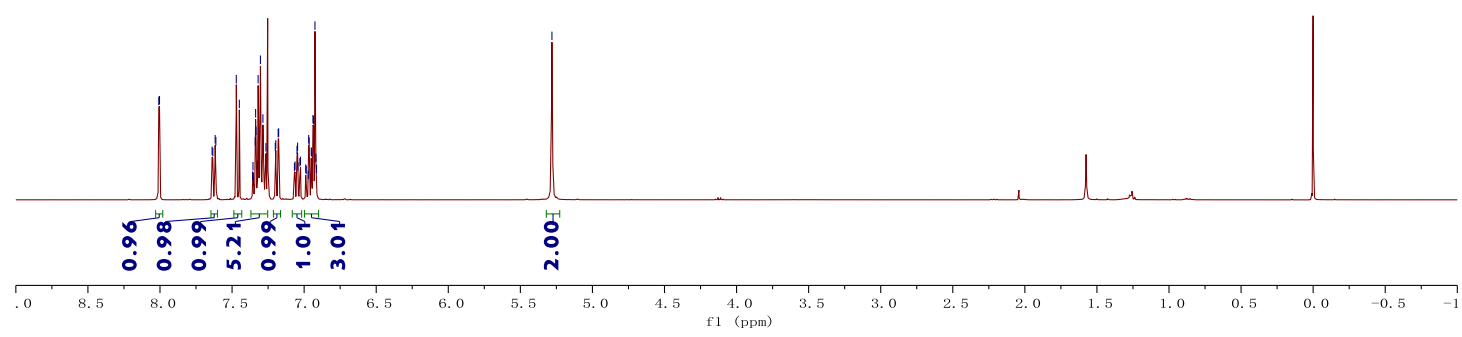

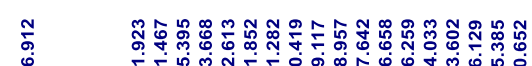

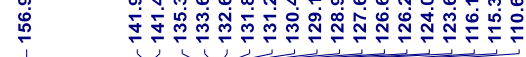

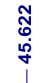

${ }^{13} \mathrm{C}$ NMR $\left(101 \mathrm{MHz}, \mathrm{CDCl}_{3}\right)$

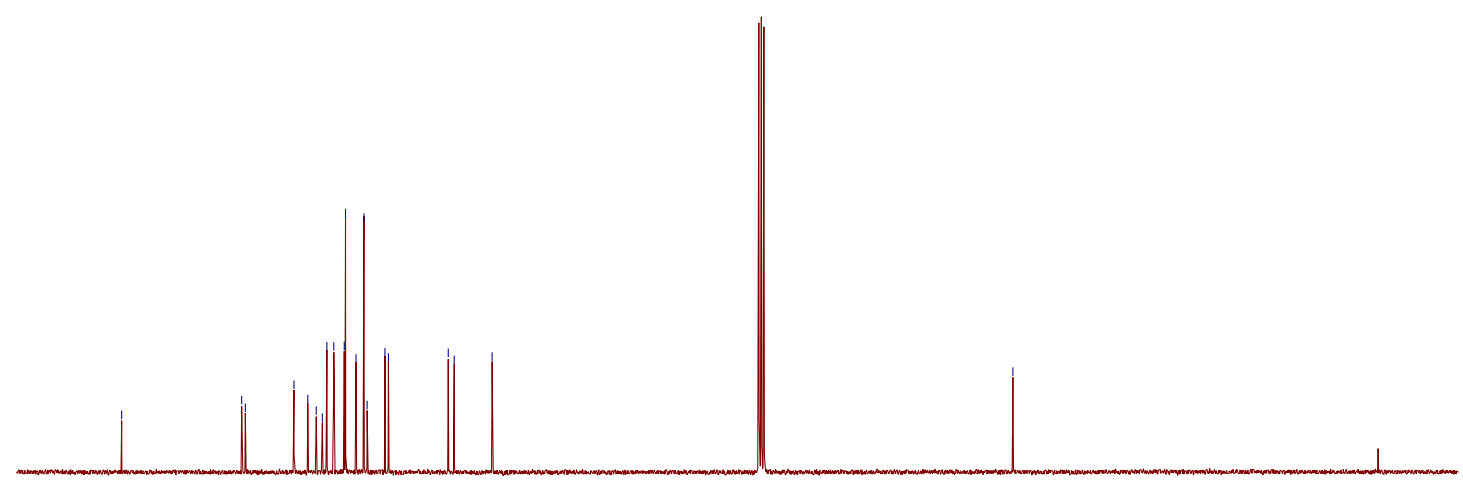

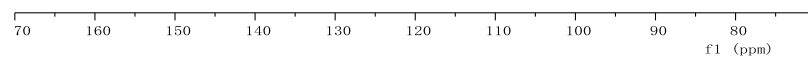




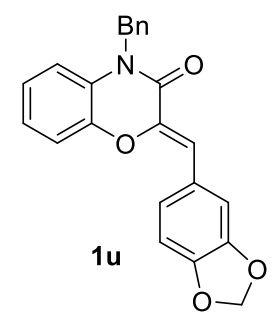

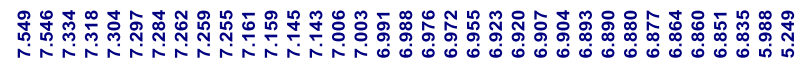

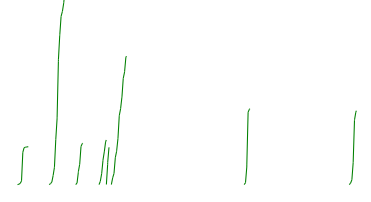

${ }^{1} \mathrm{H}$ NMR $\left(500 \mathrm{MHz}, \mathrm{CDCl}_{3}\right.$ )

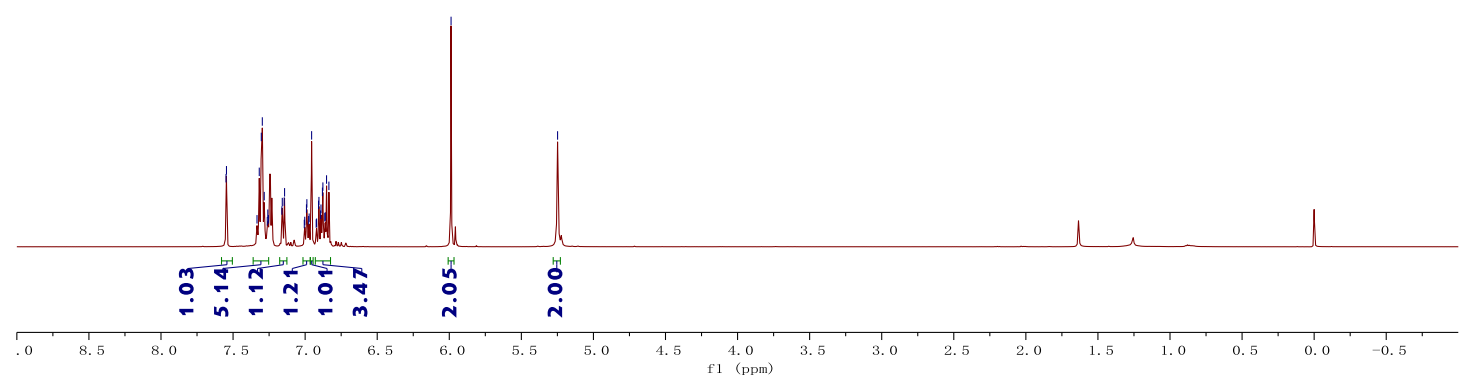

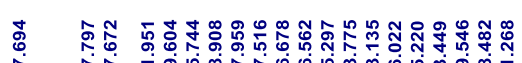

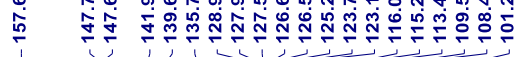

京

${ }^{13} \mathrm{C}$ NMR (126 MHz, $\left.\mathrm{CDCl}_{3}\right)$
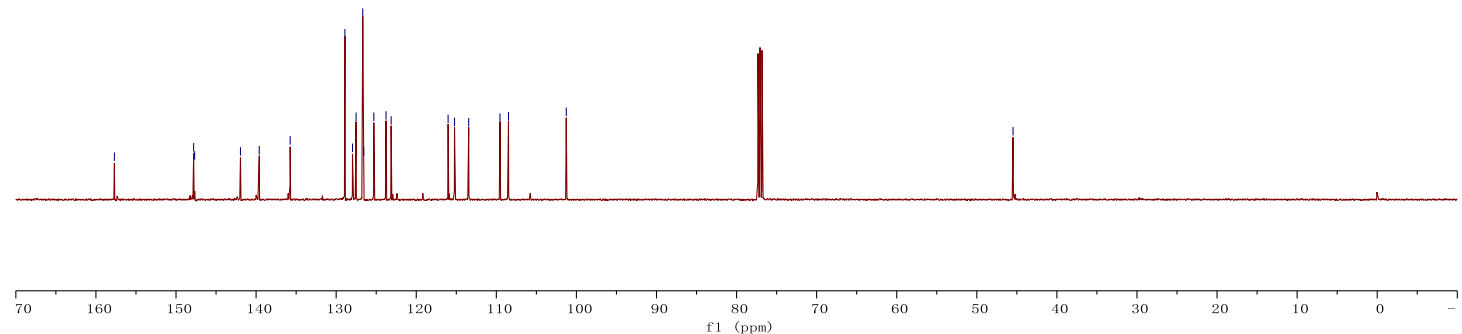


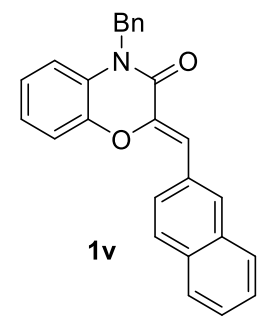

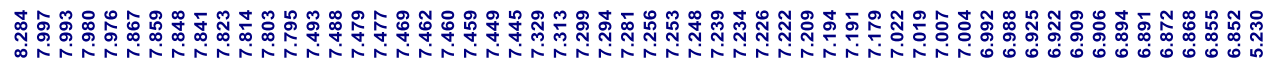

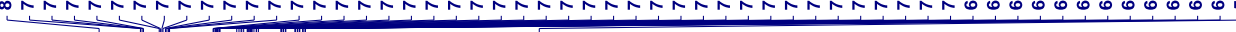

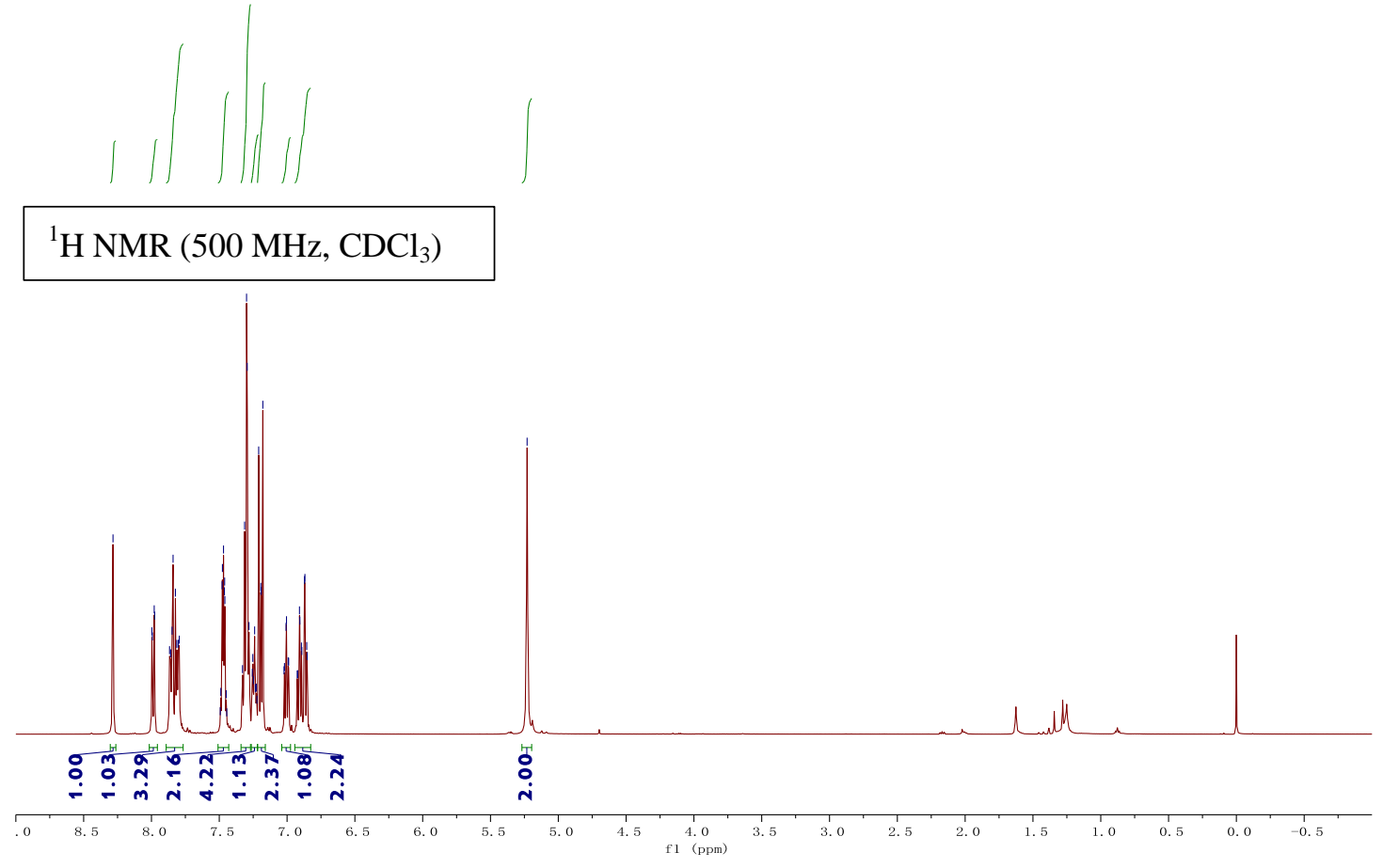

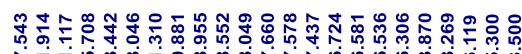

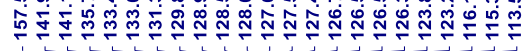

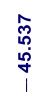

${ }^{13} \mathrm{C} \mathrm{NMR}\left(126 \mathrm{MHz}, \mathrm{CDCl}_{3}\right)$

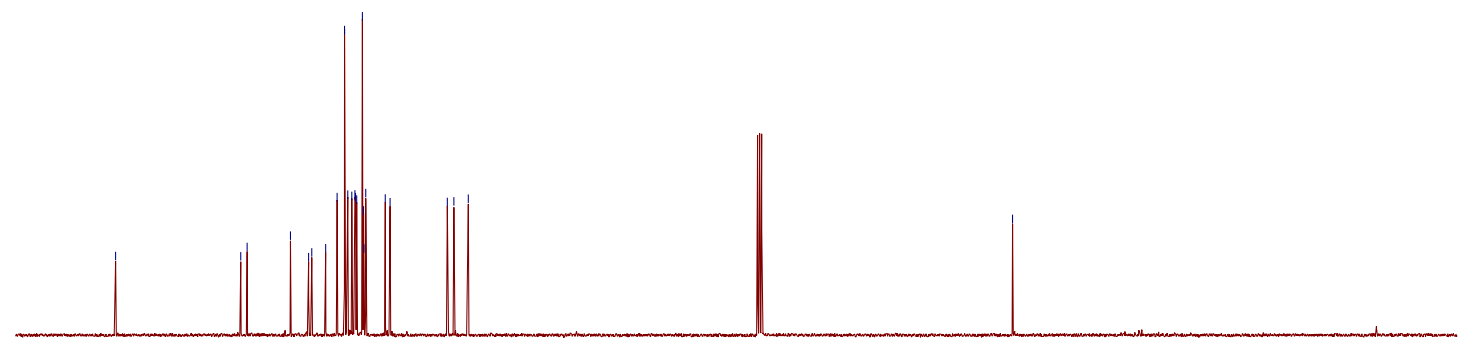

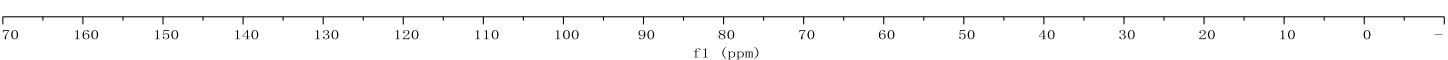




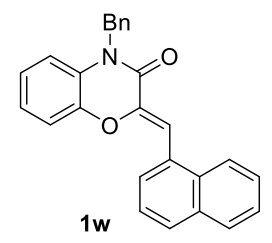

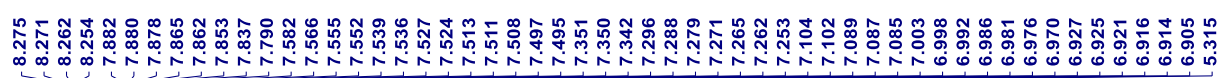

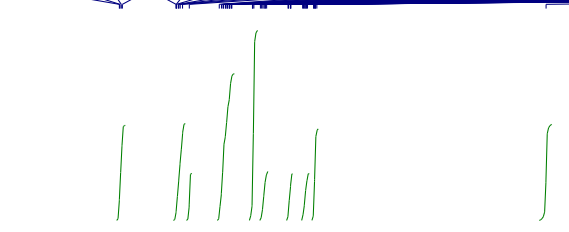

${ }^{1} \mathrm{H}$ NMR $\left(500 \mathrm{MHz}, \mathrm{CDCl}_{3}\right)$

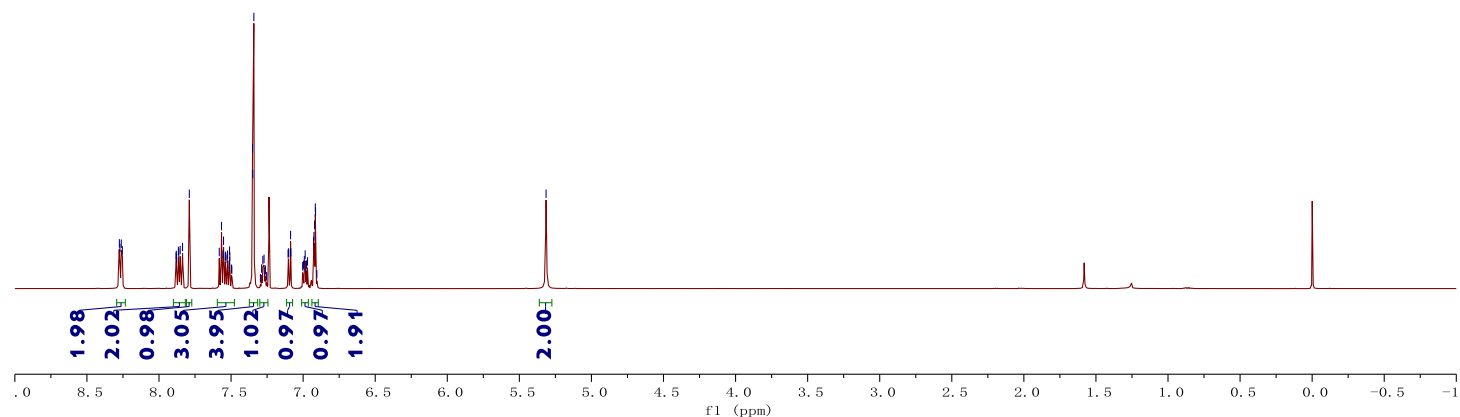

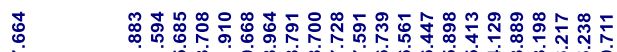

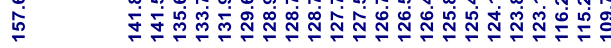

${ }^{13} \mathrm{C}$ NMR $\left(126 \mathrm{MHz}, \mathrm{CDCl}_{3}\right)$

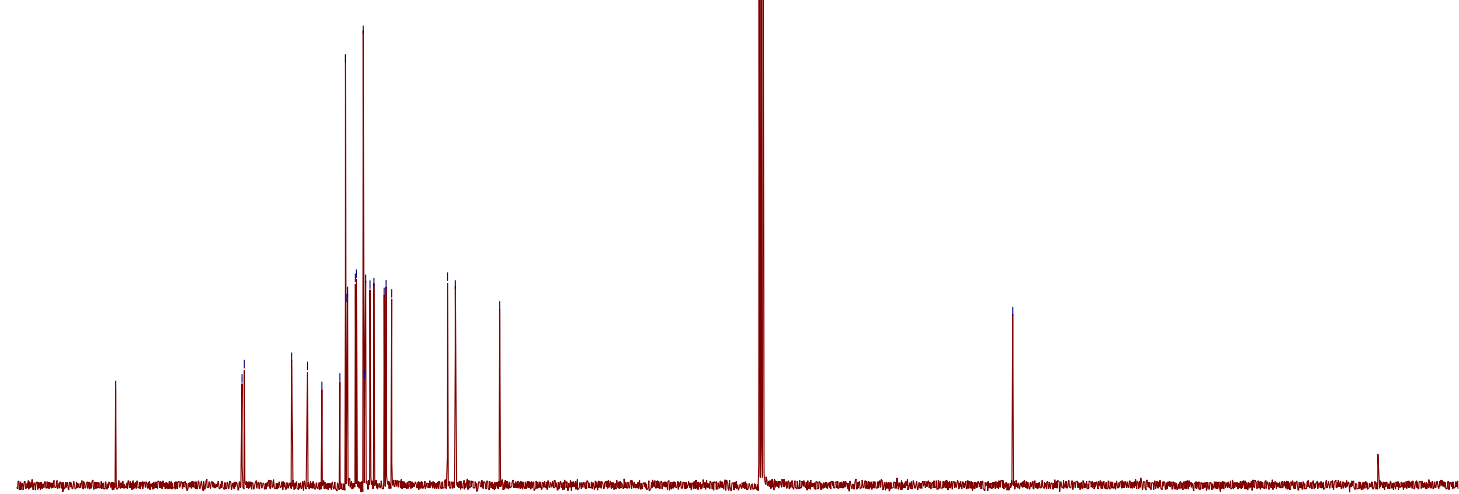




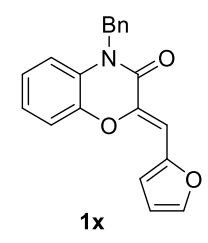

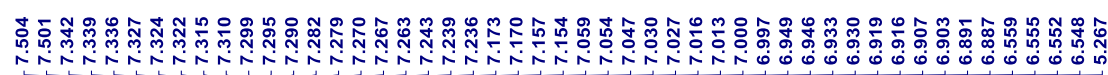

${ }^{1} \mathrm{H}$ NMR $\left(500 \mathrm{MHz}, \mathrm{CDCl}_{3}\right)$

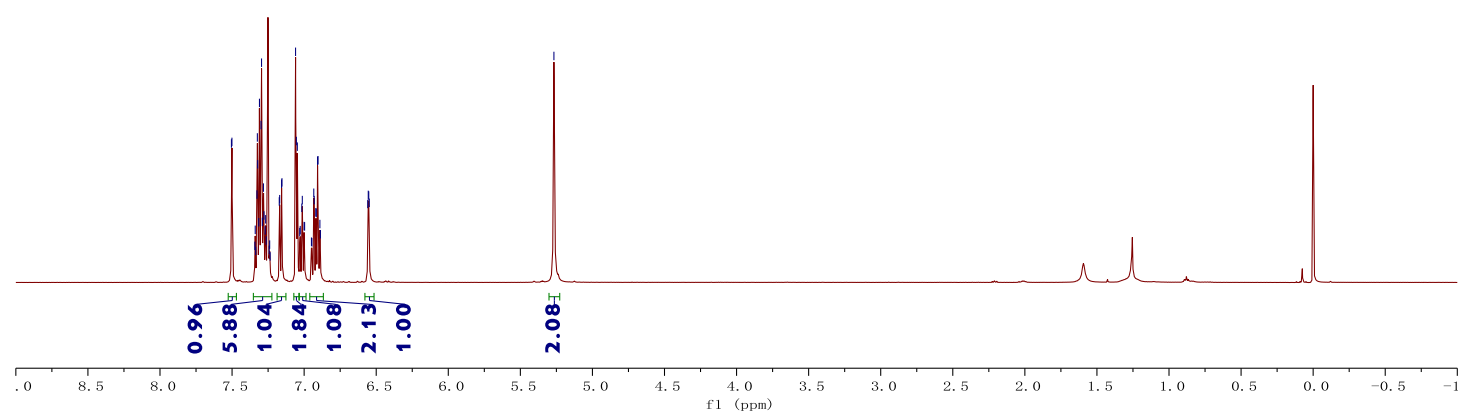

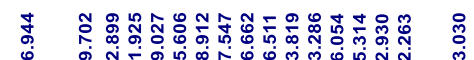

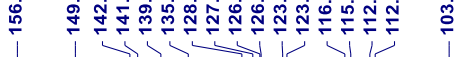

${ }^{13} \mathrm{C} \mathrm{NMR}\left(126 \mathrm{MHz}, \mathrm{CDCl}_{3}\right)$

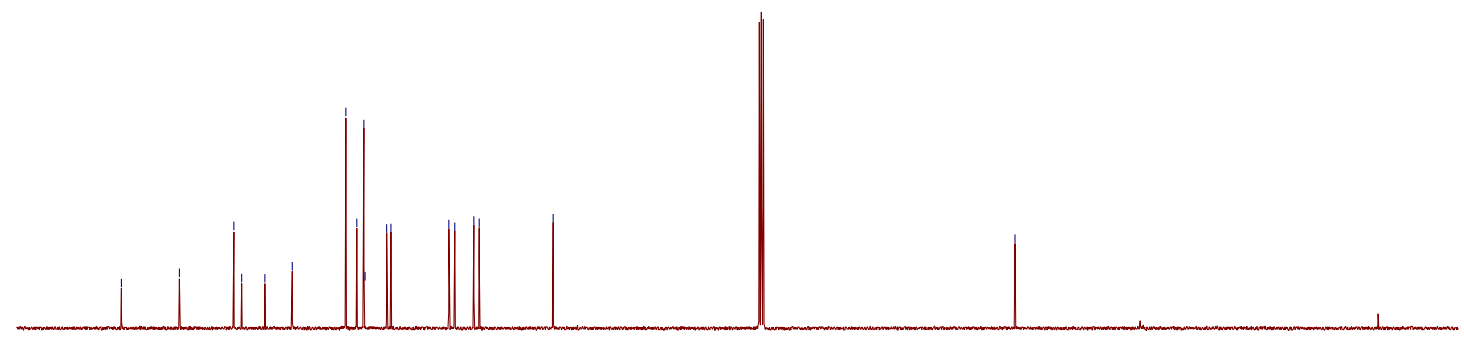

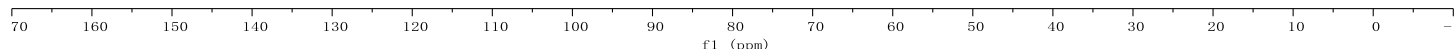




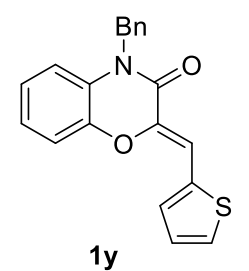

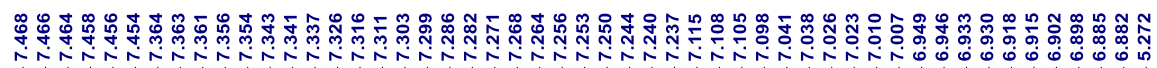

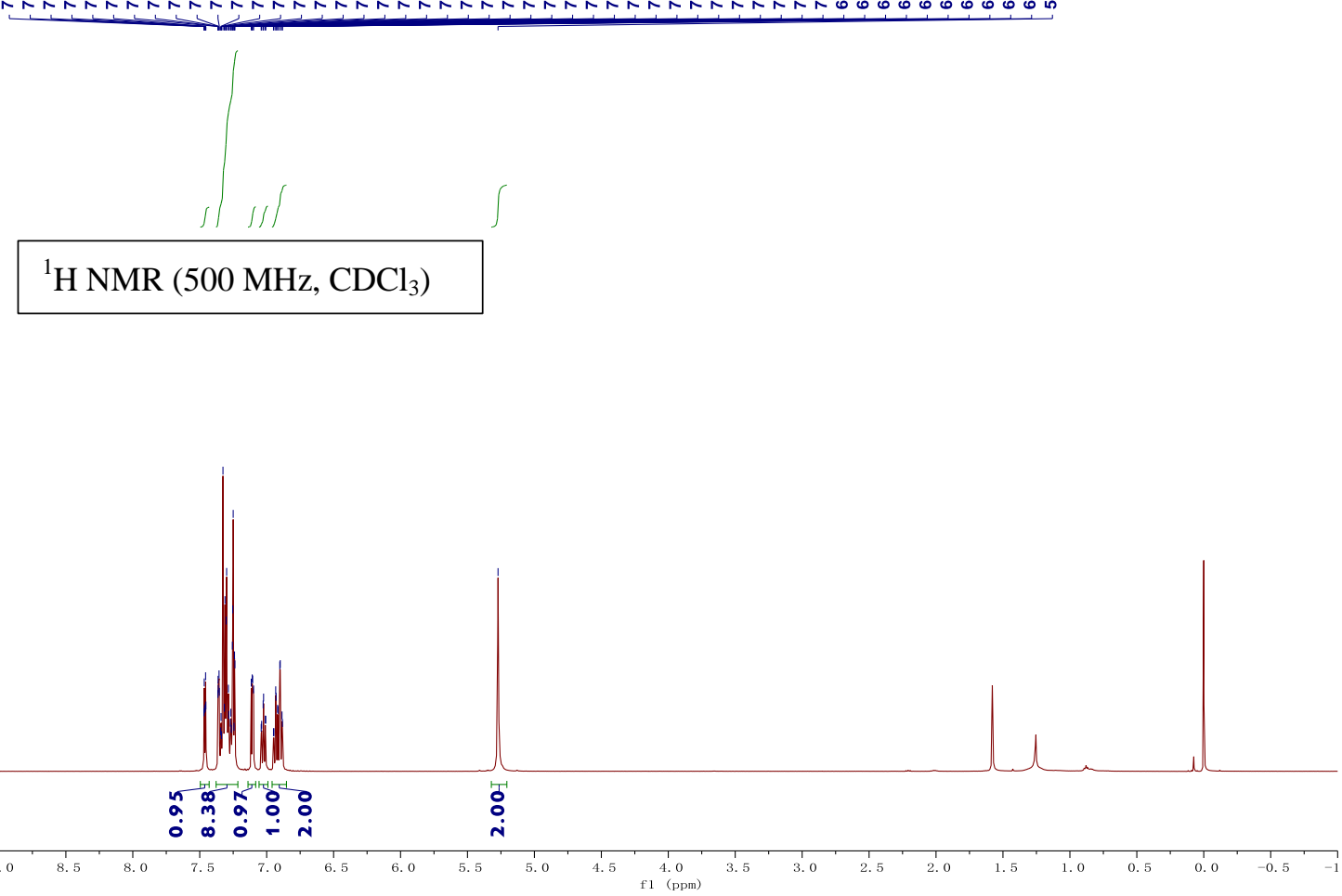

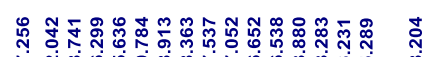

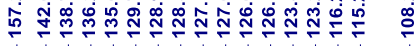

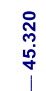

${ }^{13} \mathrm{C} \mathrm{NMR}\left(126 \mathrm{MHz}, \mathrm{CDCl}_{3}\right)$

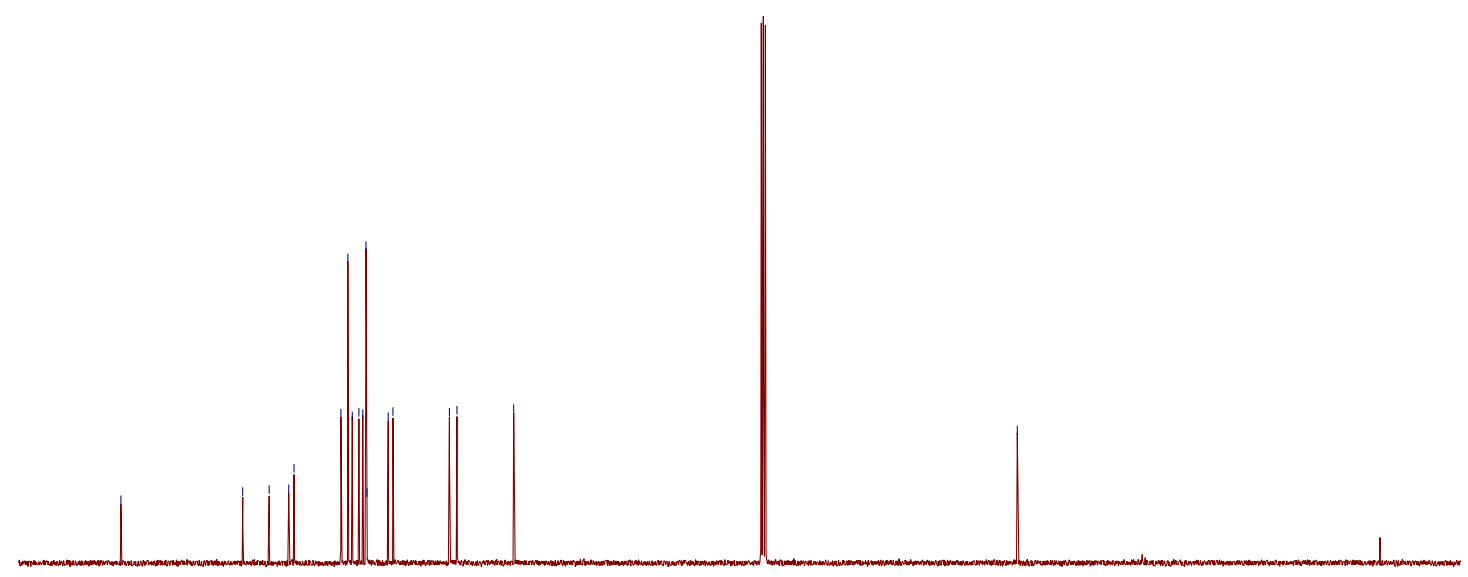

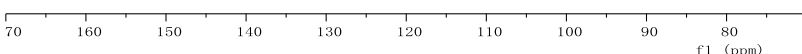




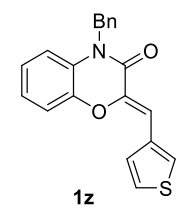

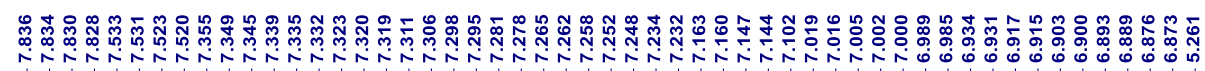

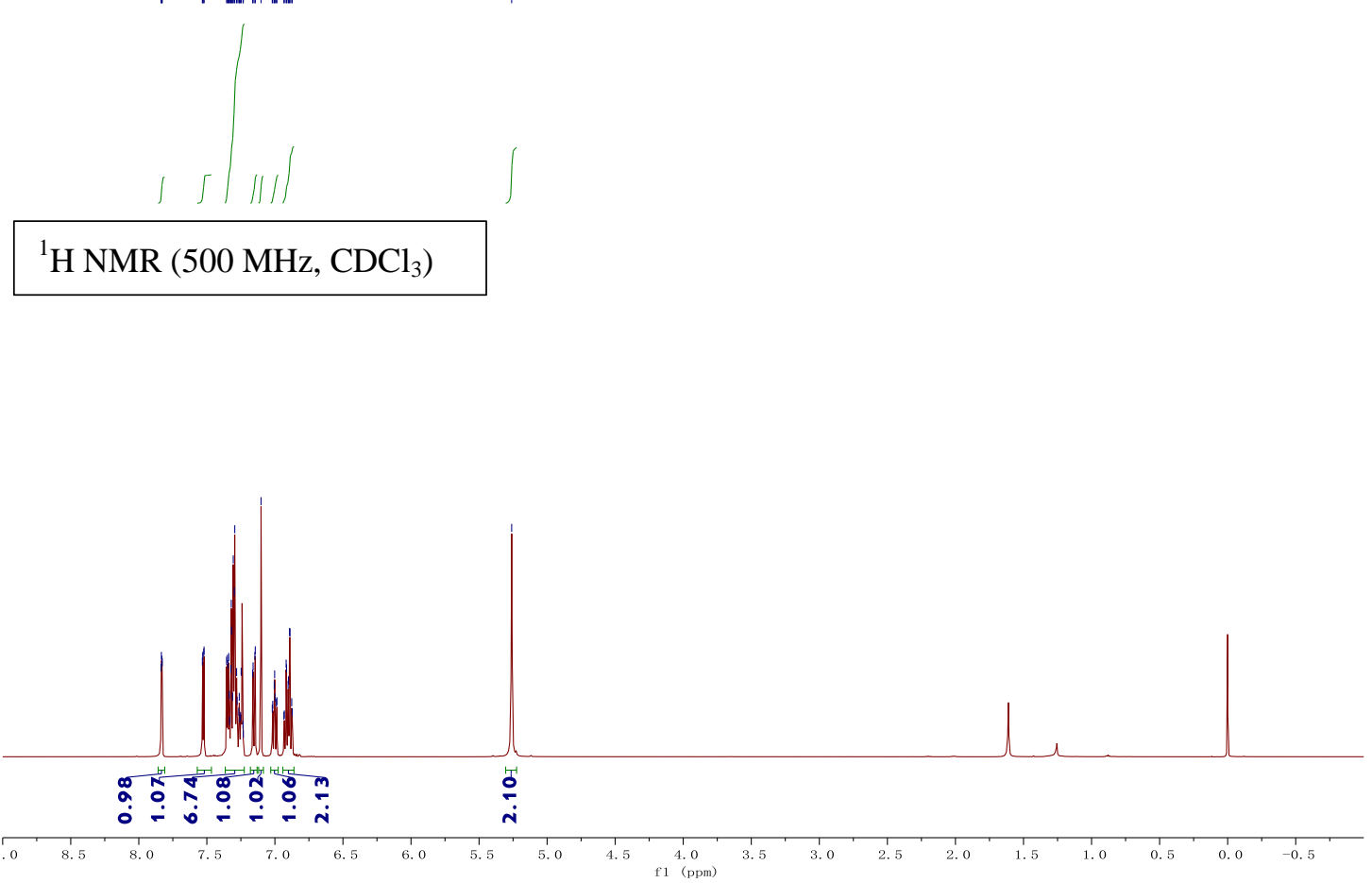

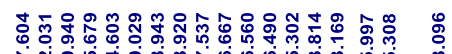

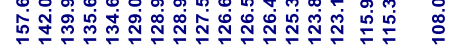

${ }^{13} \mathrm{C}$ NMR $\left(126 \mathrm{MHz}, \mathrm{CDCl}_{3}\right)$

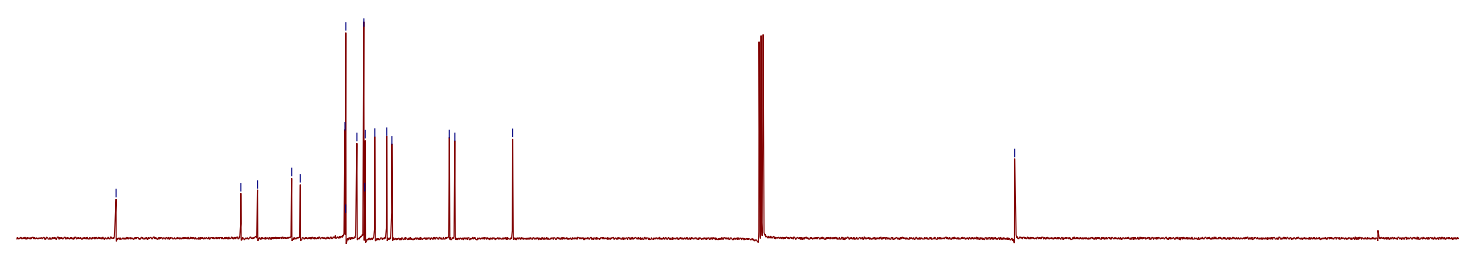




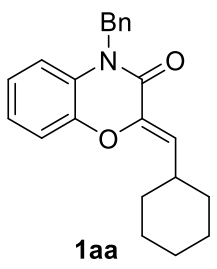

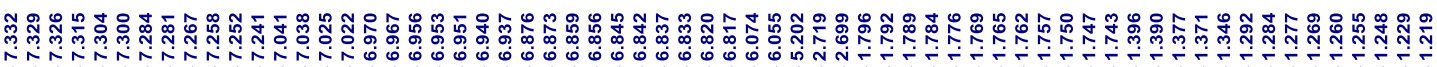

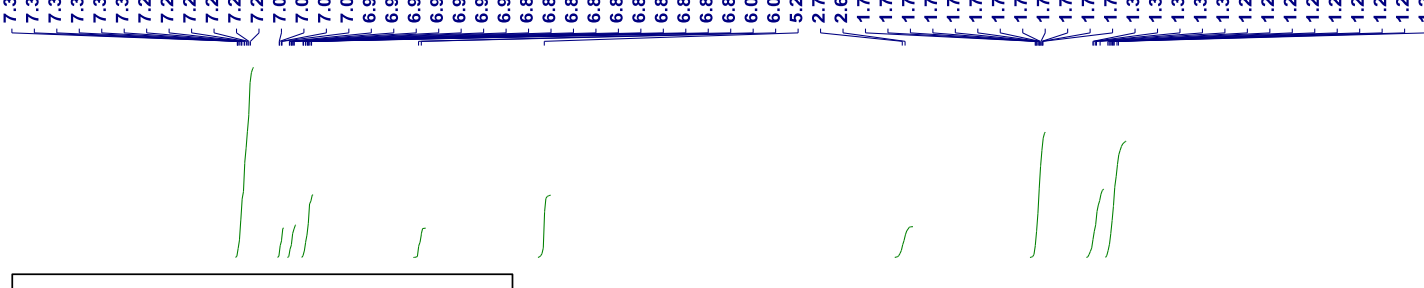

${ }^{1} \mathrm{H}$ NMR $\left(500 \mathrm{MHz}, \mathrm{CDCl}_{3}\right.$ )

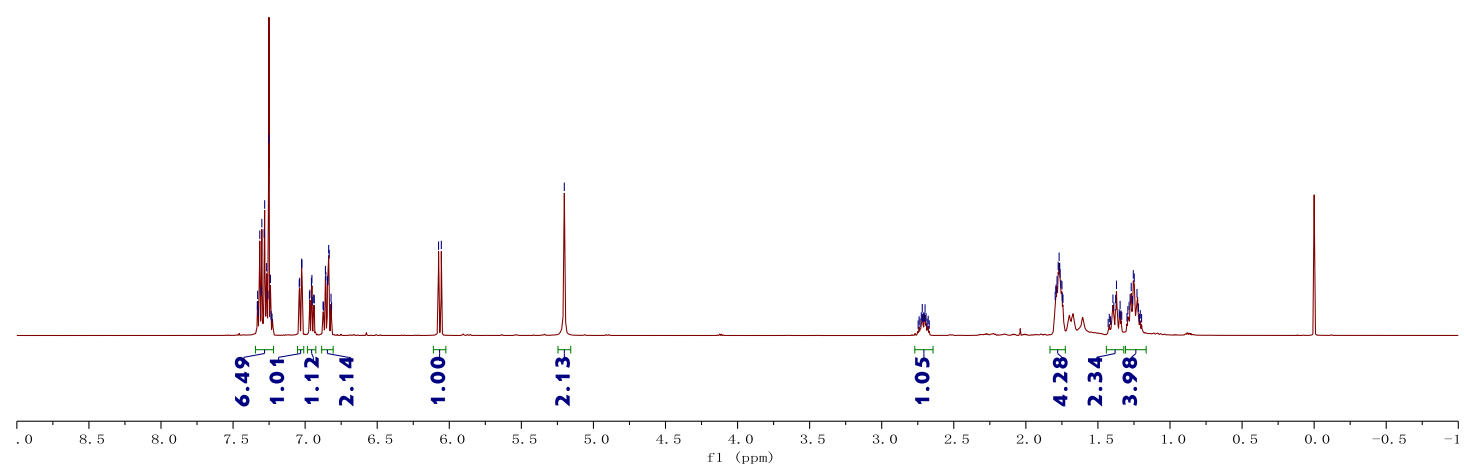

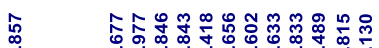

每

商

${ }^{13} \mathrm{C}$ NMR $\left(126 \mathrm{MHz}, \mathrm{CDCl}_{3}\right)$

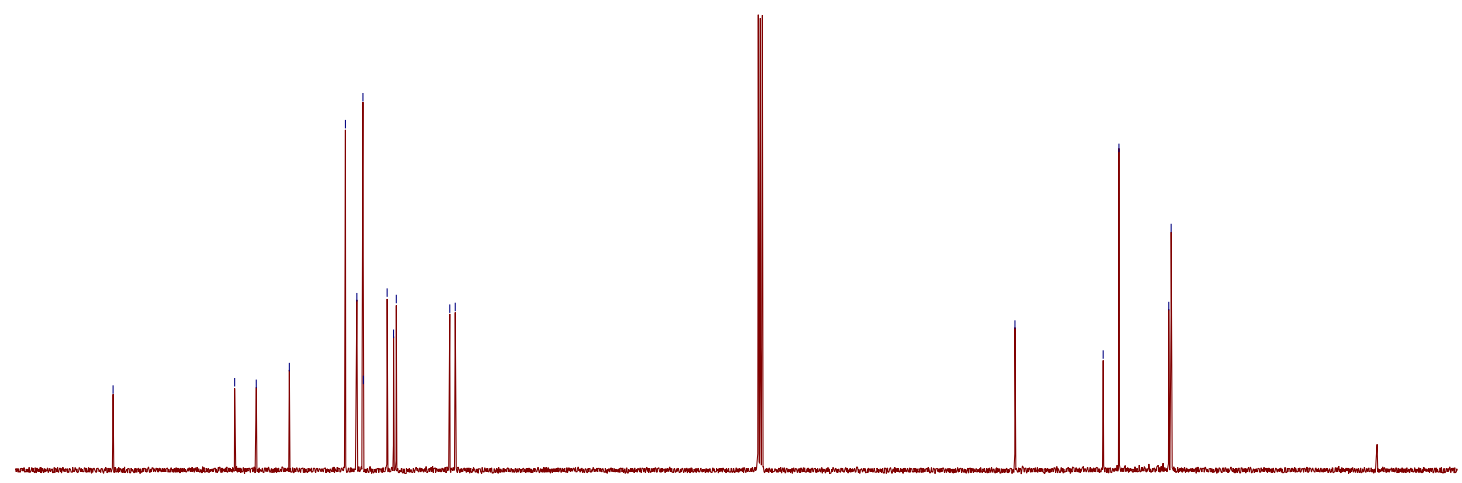

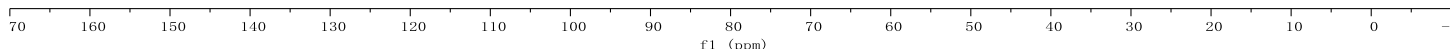




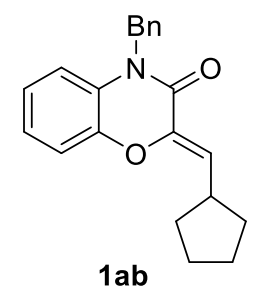

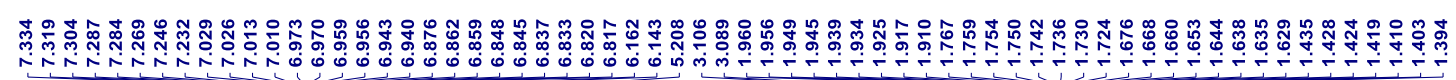

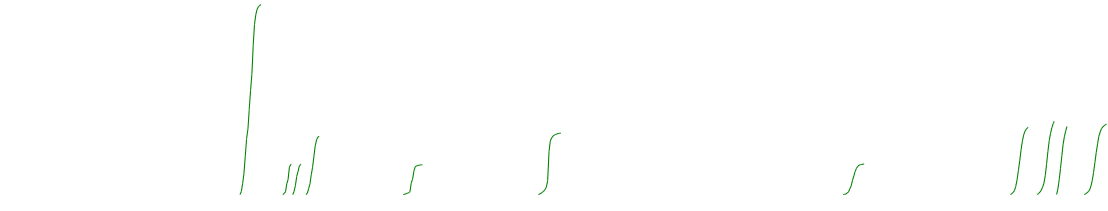

${ }^{1} \mathrm{H}$ NMR $\left(500 \mathrm{MHz}, \mathrm{CDCl}_{3}\right)$

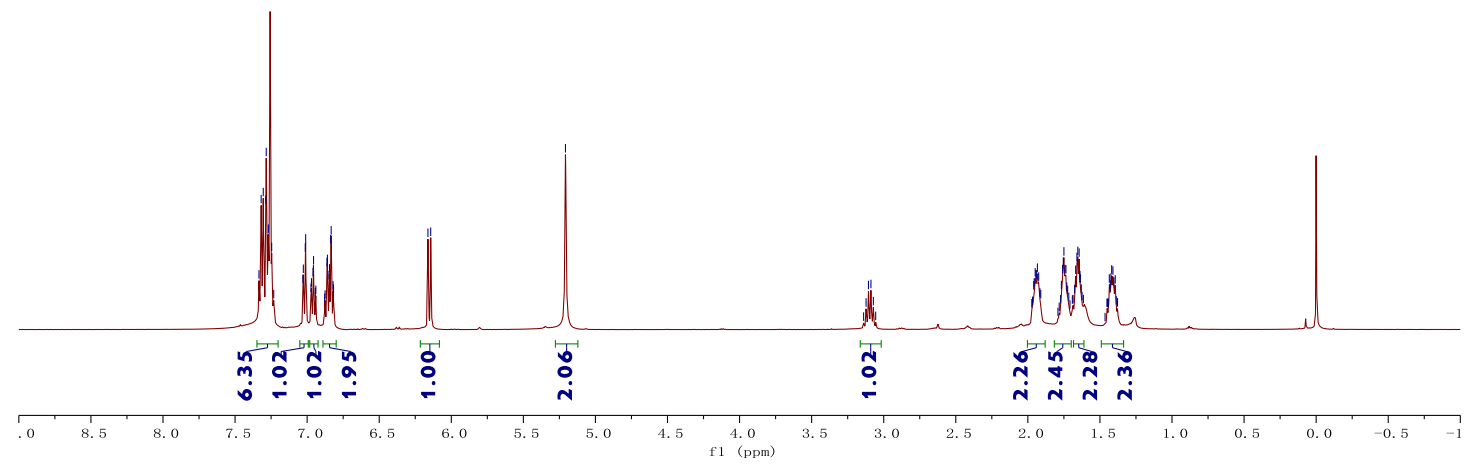

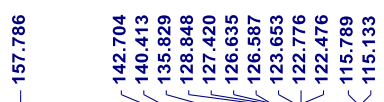

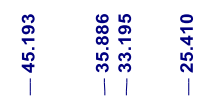

${ }^{13} \mathrm{C} \mathrm{NMR}\left(126 \mathrm{MHz}, \mathrm{CDCl}_{3}\right)$

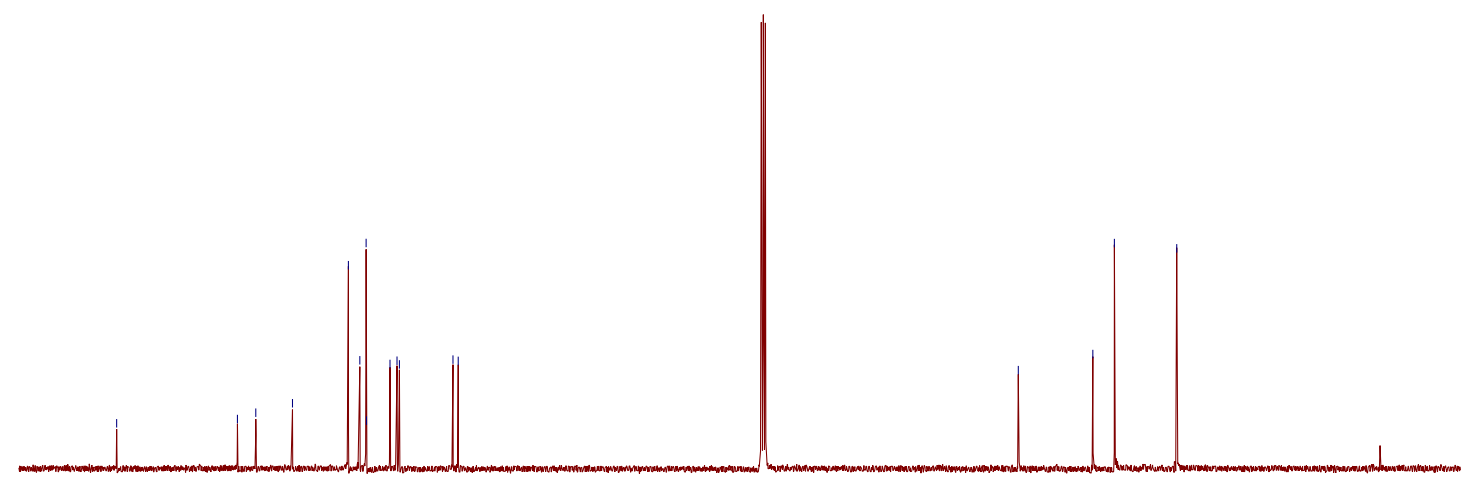

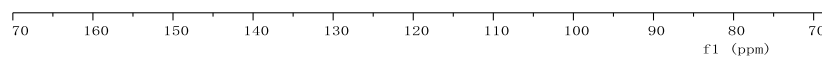




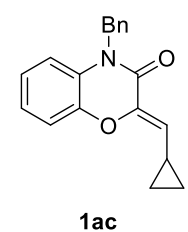

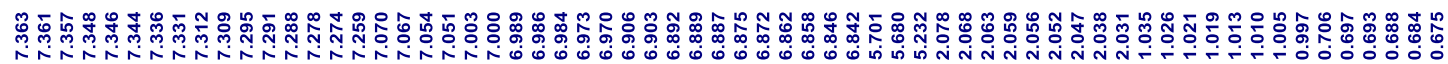

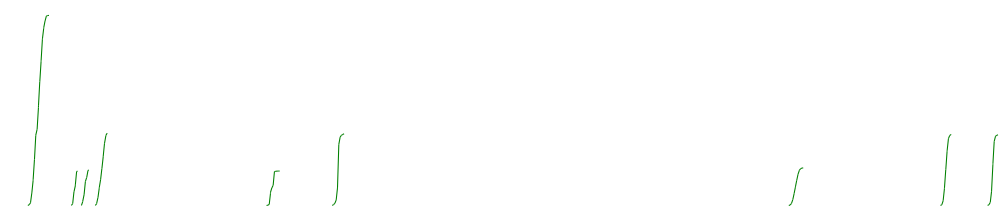

${ }^{1} \mathrm{H}$ NMR $\left(500 \mathrm{MHz}, \mathrm{CDCl}_{3}\right)$

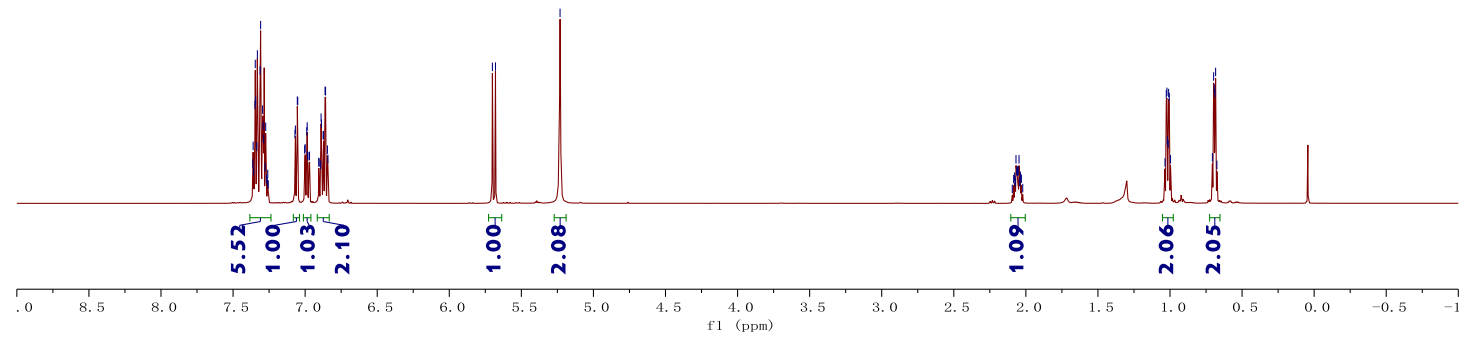

㫣

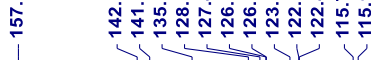

${ }^{13} \mathrm{C}$ NMR $\left(126 \mathrm{MHz}, \mathrm{CDCl}_{3}\right)$

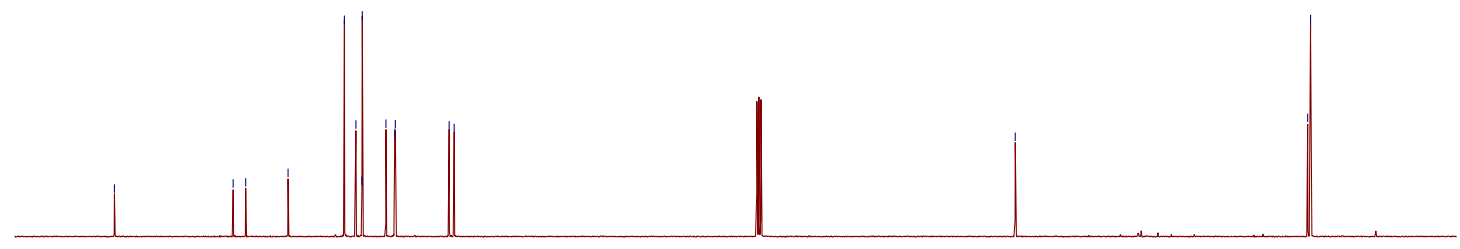

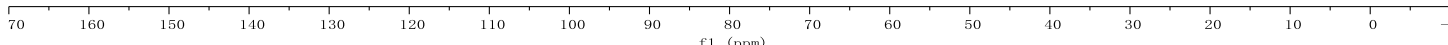




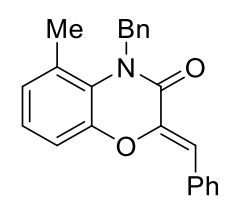

1 ad

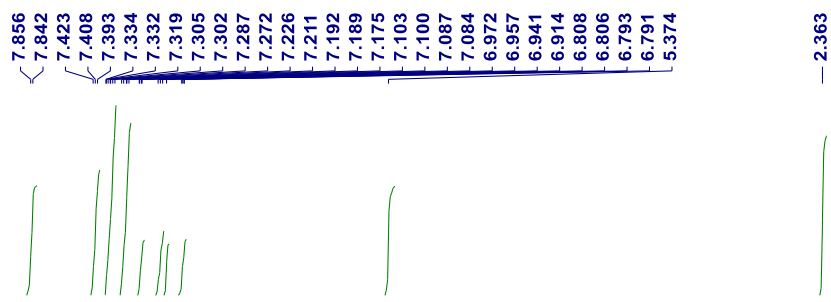

${ }^{1} \mathrm{H} \mathrm{NMR}\left(500 \mathrm{MHz}, \mathrm{CDCl}_{3}\right)$
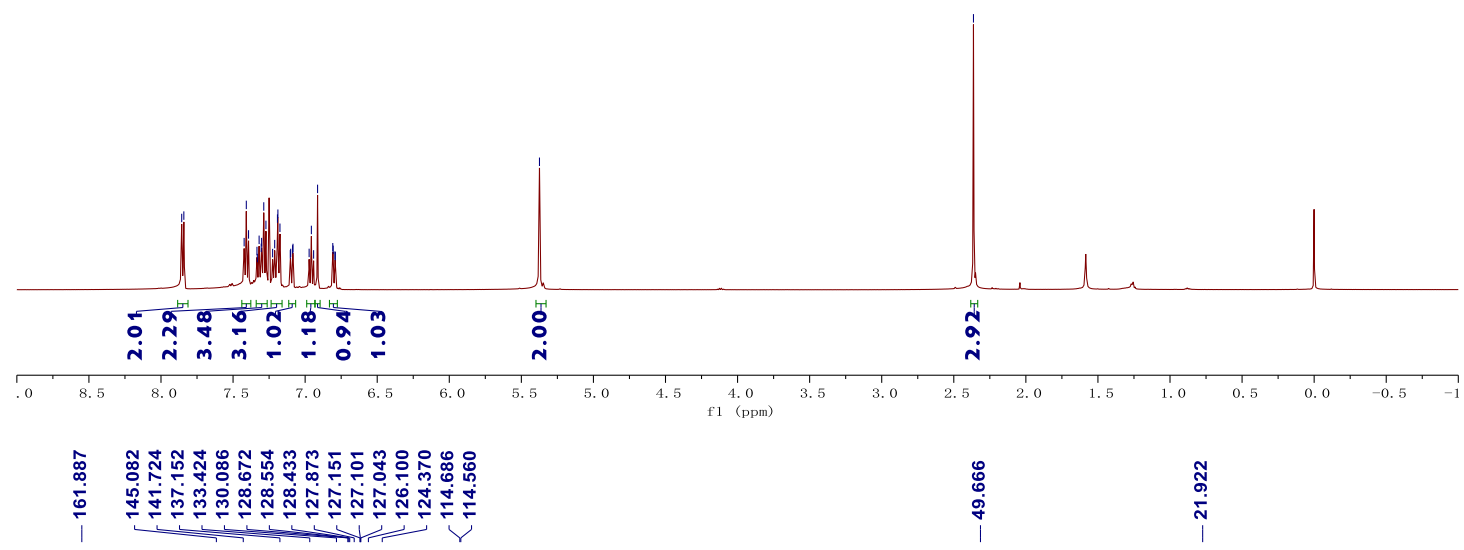

${ }^{13} \mathrm{C}$ NMR (126 MHz, $\left.\mathrm{CDCl}_{3}\right)$
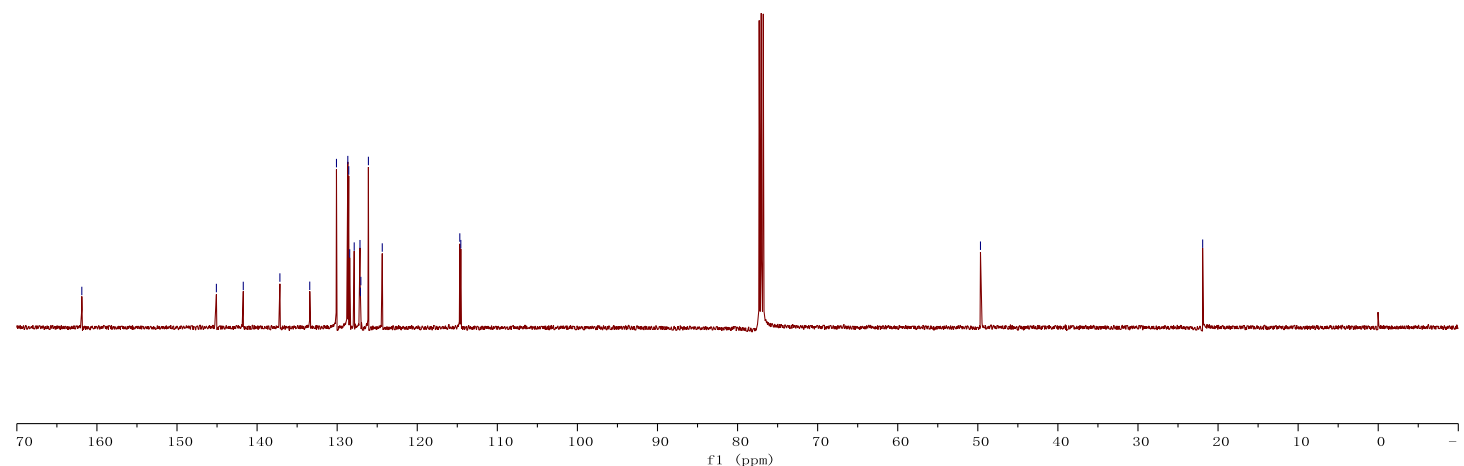


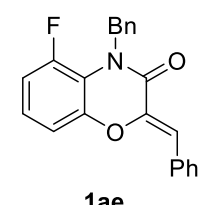

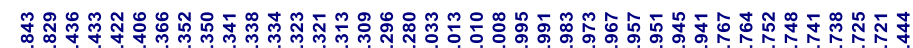

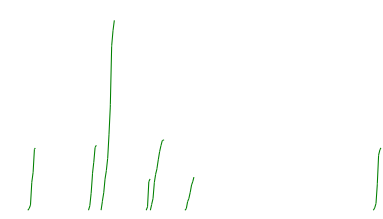

${ }^{1} \mathrm{H}$ NMR $\left(500 \mathrm{MHz}, \mathrm{CDCl}_{3}\right.$ )

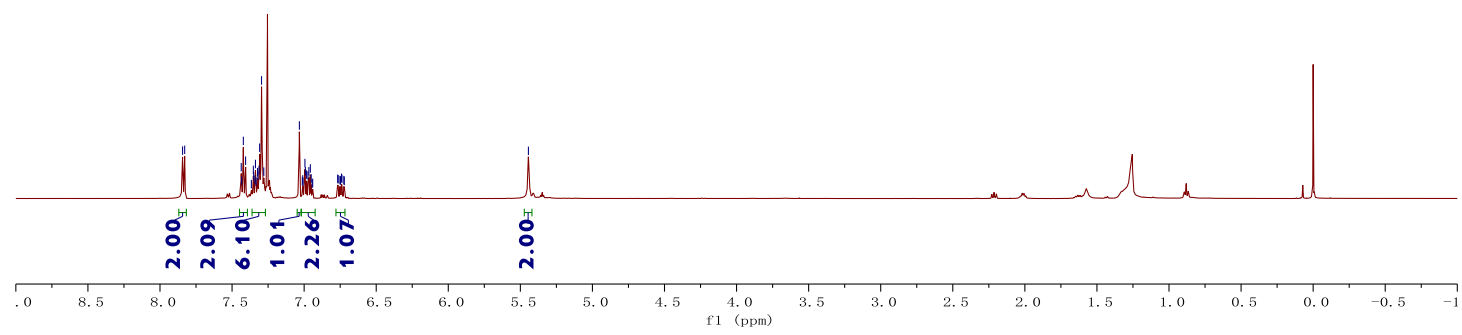

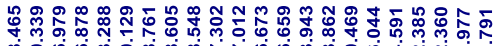

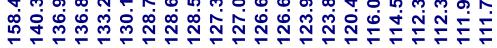

${ }^{13} \mathrm{C}$ NMR $\left(126 \mathrm{MHz}, \mathrm{CDCl}_{3}\right)$

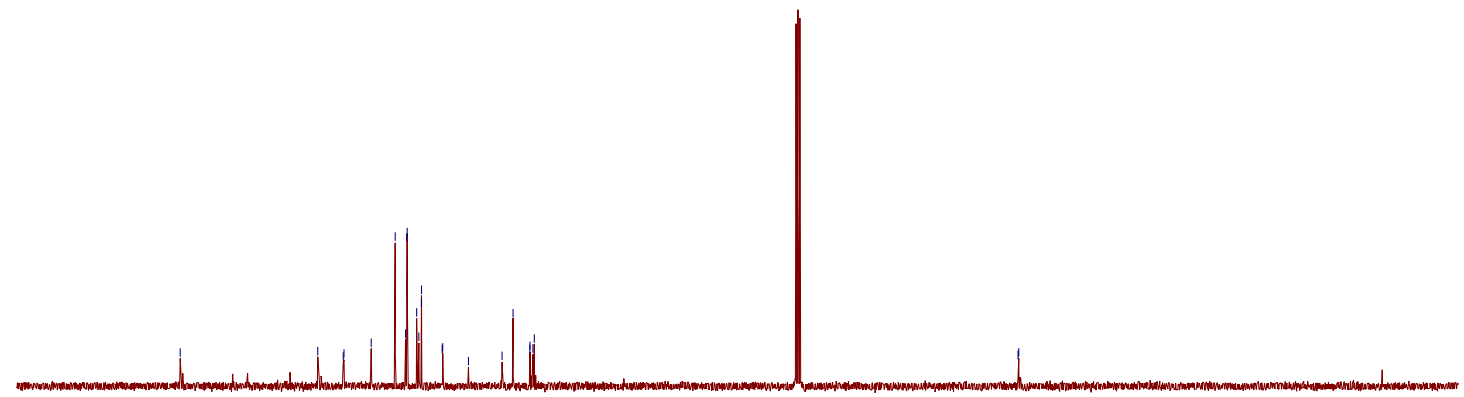


${ }^{19} \mathrm{~F}$ NMR $\left(471 \mathrm{MHz}, \mathrm{CDCl}_{3}\right)$

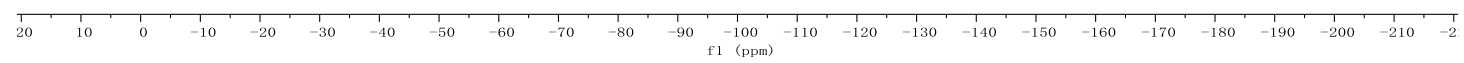




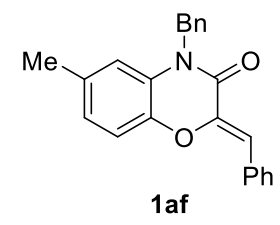

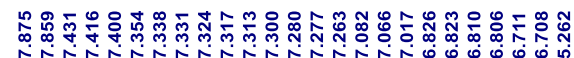

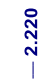

${ }^{1} \mathrm{H}$ NMR $\left(500 \mathrm{MHz}, \mathrm{CDCl}_{3}\right)$

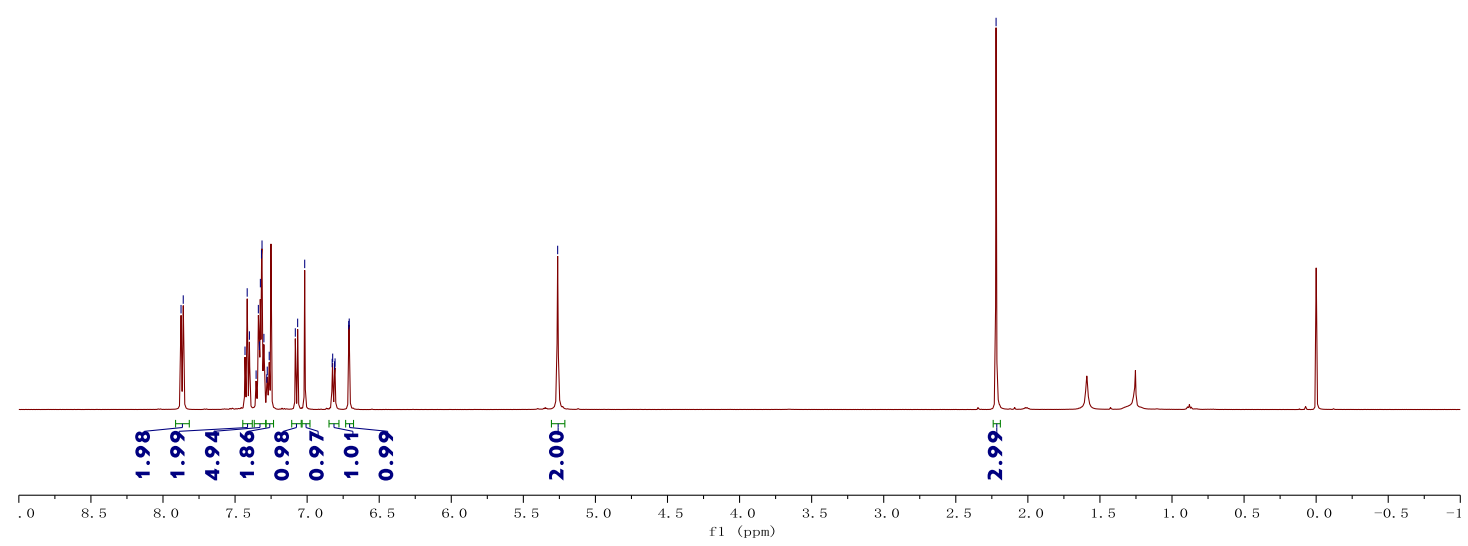

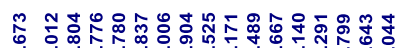

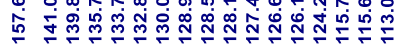

${ }^{13} \mathrm{C}$ NMR $\left(126 \mathrm{MHz}, \mathrm{CDCl}_{3}\right)$

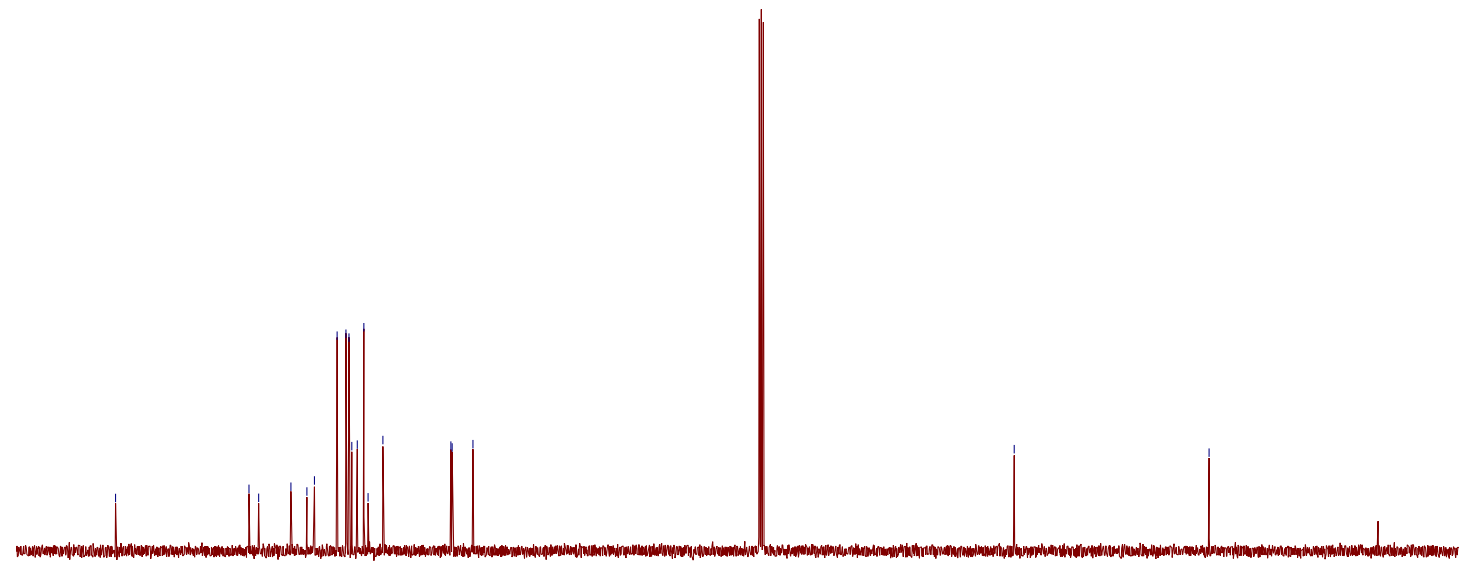

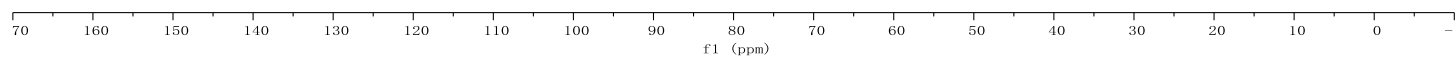




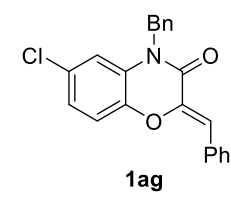

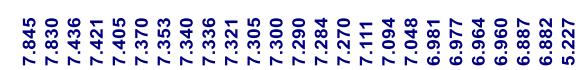

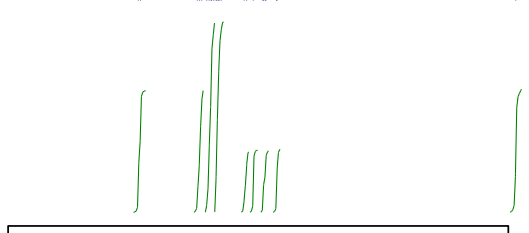

${ }^{1} \mathrm{H}$ NMR $\left(500 \mathrm{MHz}, \mathrm{CDCl}_{3}\right.$ )

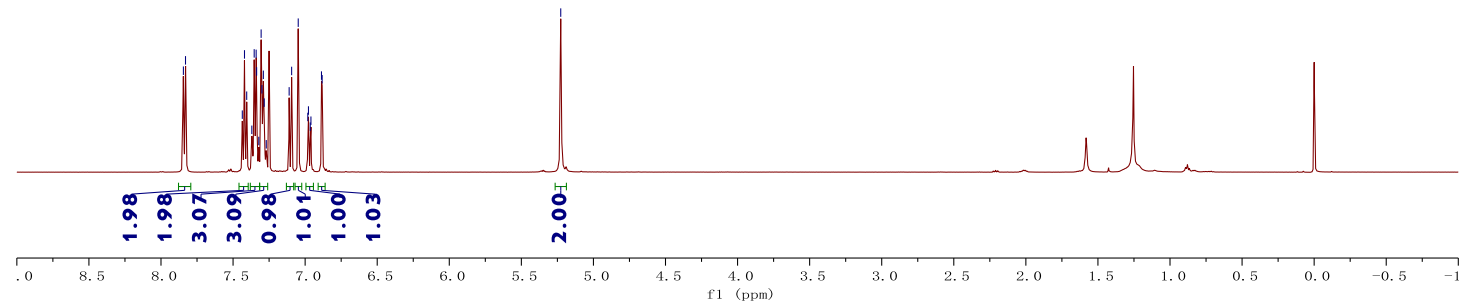

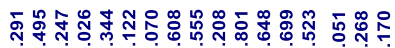

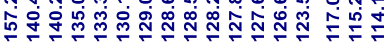

กิ
ڤุ

${ }^{13} \mathrm{C}$ NMR (126 MHz, $\mathrm{CDCl}_{3}$ )

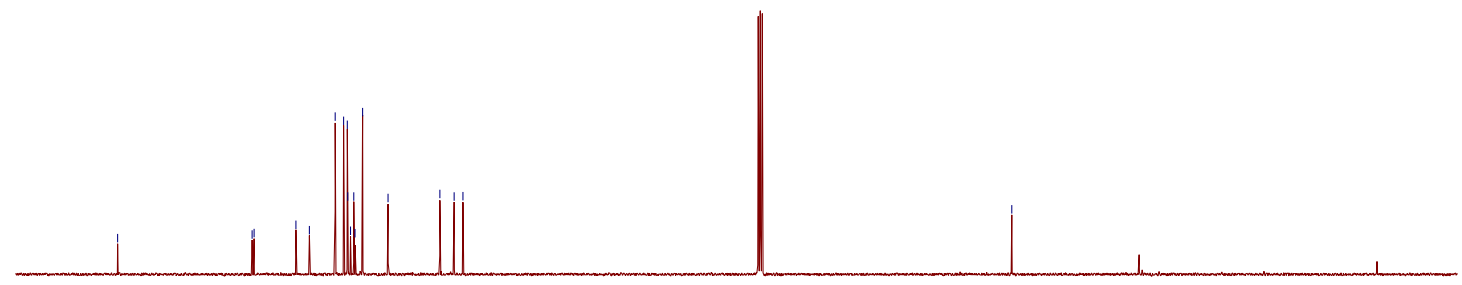




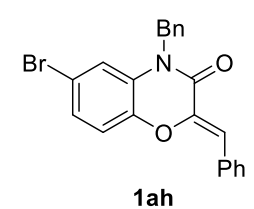

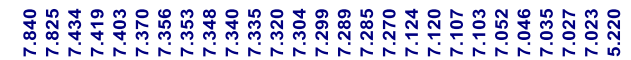

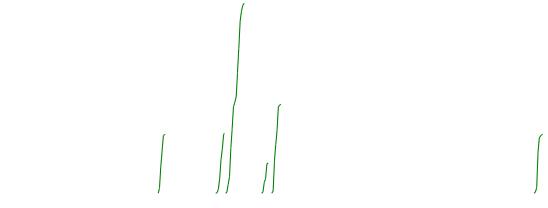

${ }^{1} \mathrm{H}$ NMR $\left(500 \mathrm{MHz}, \mathrm{CDCl}_{3}\right.$ )

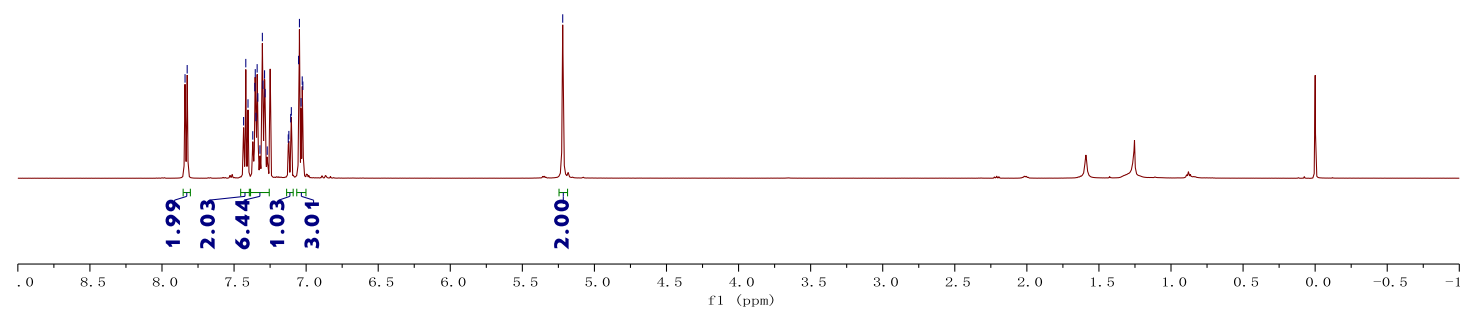

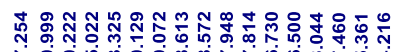

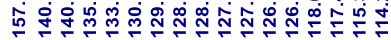

$\stackrel{\substack{0 \\ \hdashline}}{\substack{j \\ \text { I }}}$

${ }^{13} \mathrm{C} \mathrm{NMR}\left(126 \mathrm{MHz}, \mathrm{CDCl}_{3}\right)$

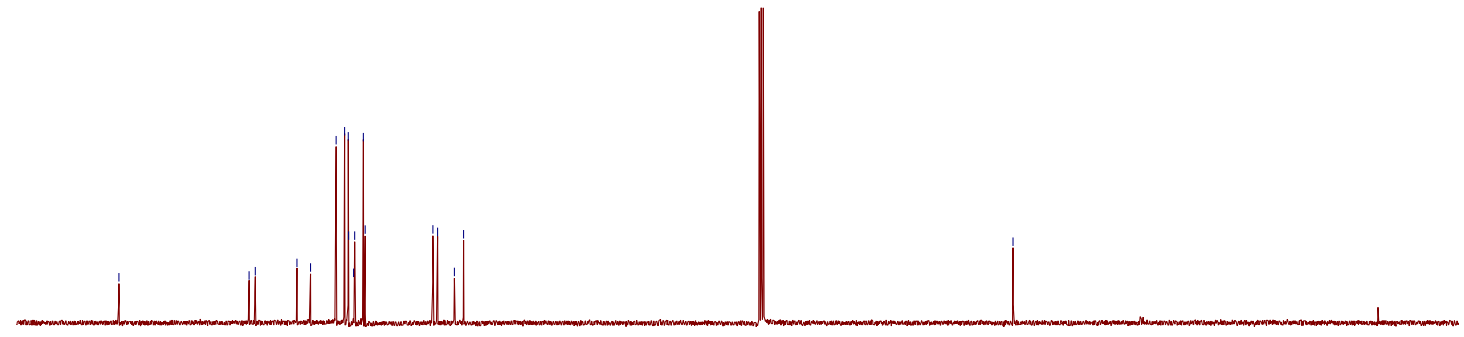

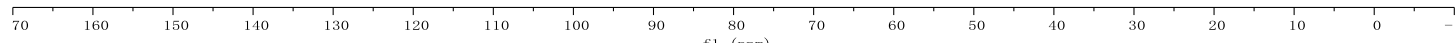




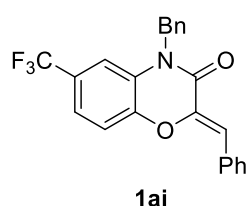

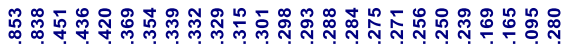

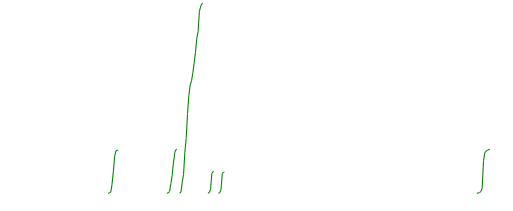

${ }^{1} \mathrm{H}$ NMR $\left(500 \mathrm{MHz}, \mathrm{CDCl}_{3}\right)$

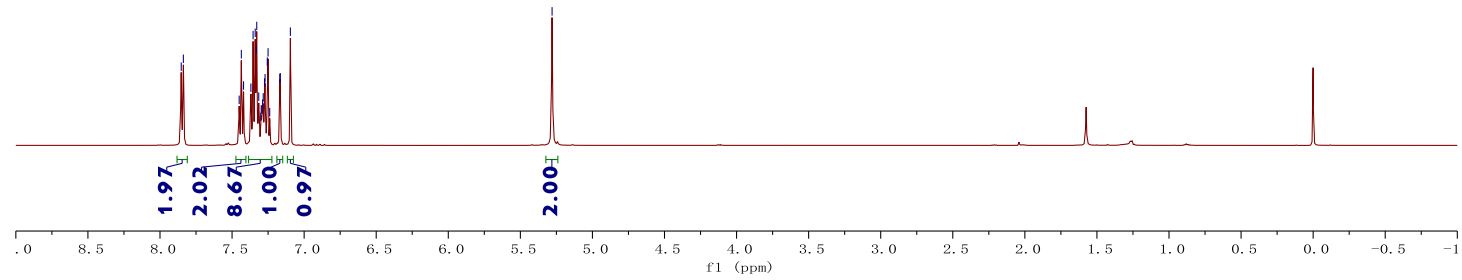

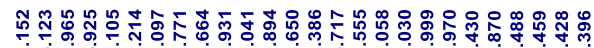

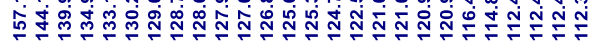

迹

${ }^{13} \mathrm{C}$ NMR $\left(126 \mathrm{MHz}, \mathrm{CDCl}_{3}\right)$

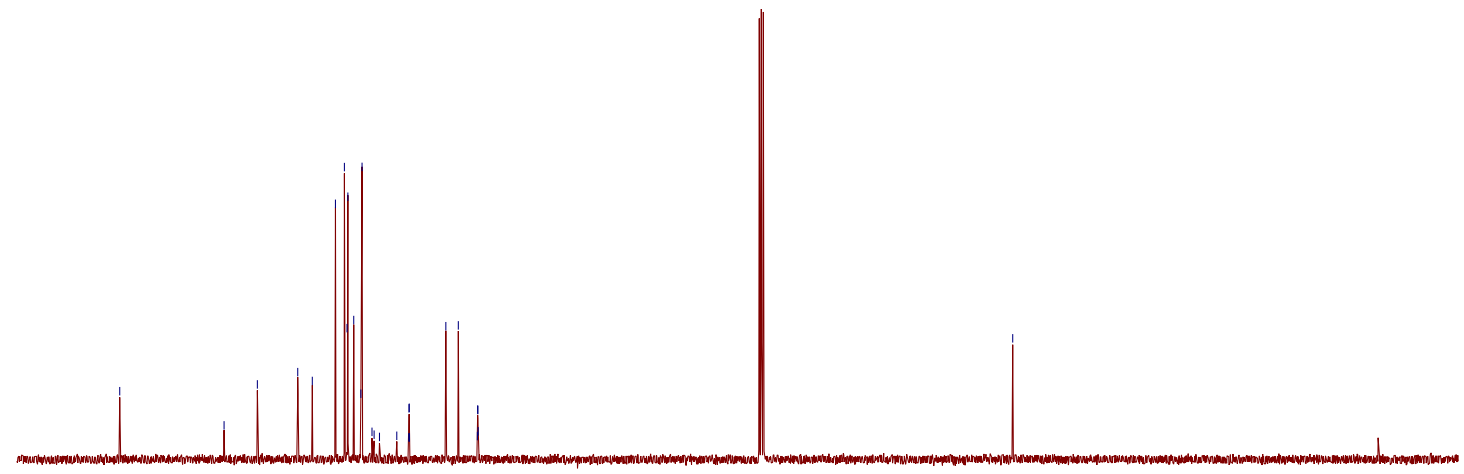


${ }^{19} \mathrm{~F}$ NMR $\left(376 \mathrm{MHz}, \mathrm{CDCl}_{3}\right)$

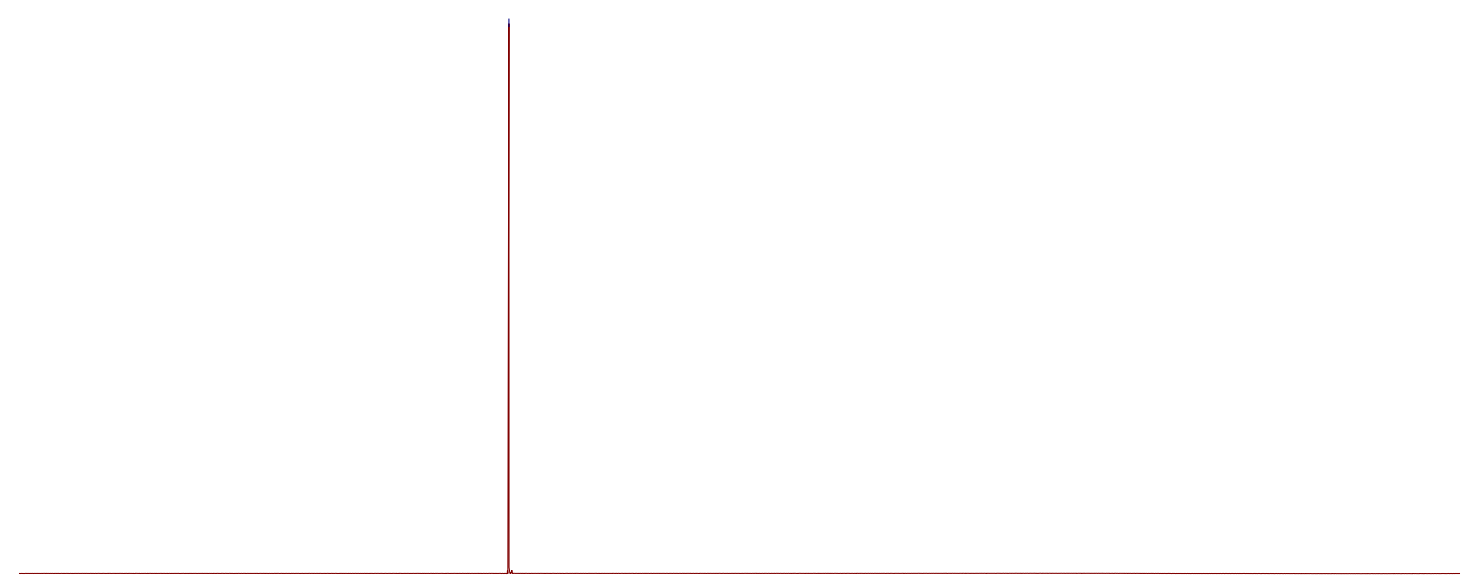

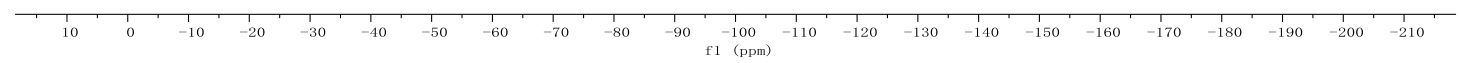


<smiles>Cc1ccc2c(c1)O/C(=C\c1ccccc1)C(=O)N2Cc1ccccc1</smiles>

1 aj

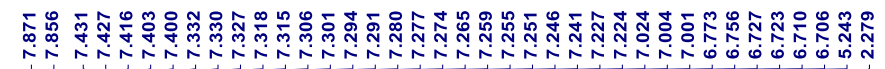

N

(

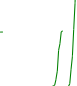

${ }^{1} \mathrm{H}$ NMR (500 MHz, $\left.\mathrm{CDCl}_{3}\right)$

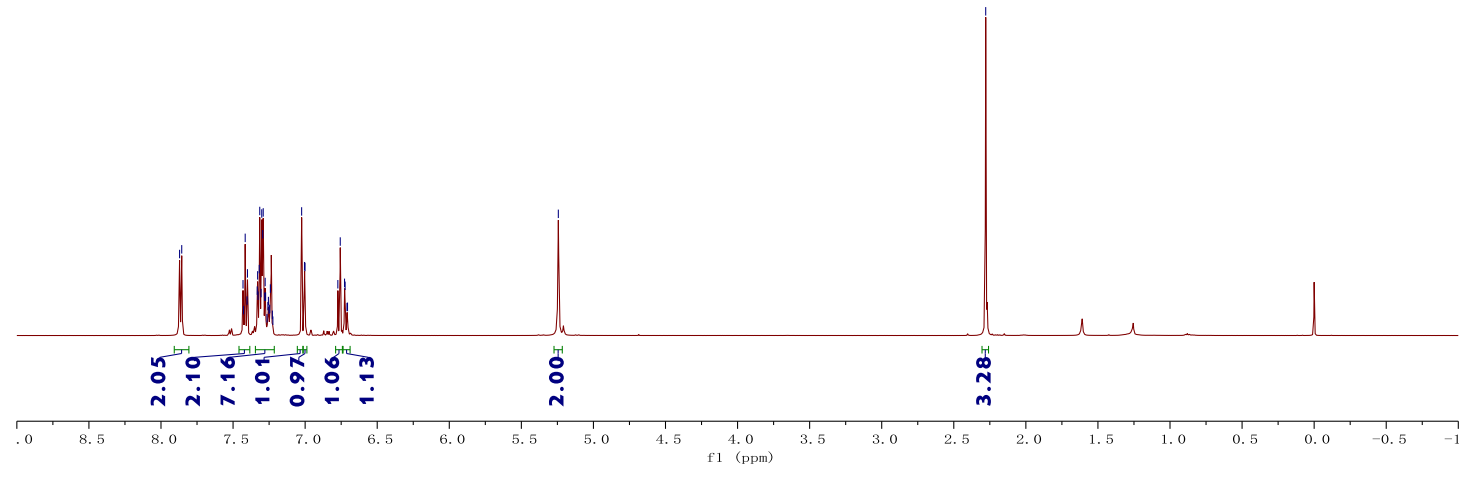

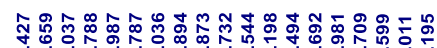

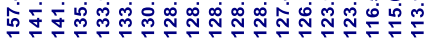

${ }^{13} \mathrm{C} \mathrm{NMR}\left(126 \mathrm{MHz}, \mathrm{CDCl}_{3}\right)$

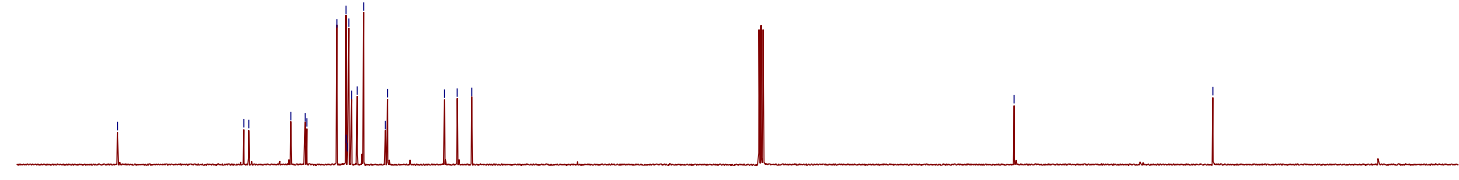

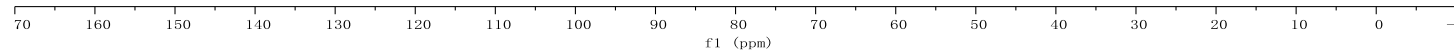




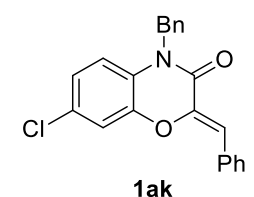

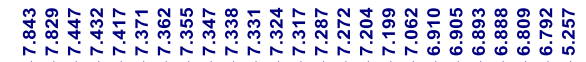

${ }^{1} \mathrm{H}$ NMR $\left(500 \mathrm{MHz}, \mathrm{CDCl}_{3}\right)$

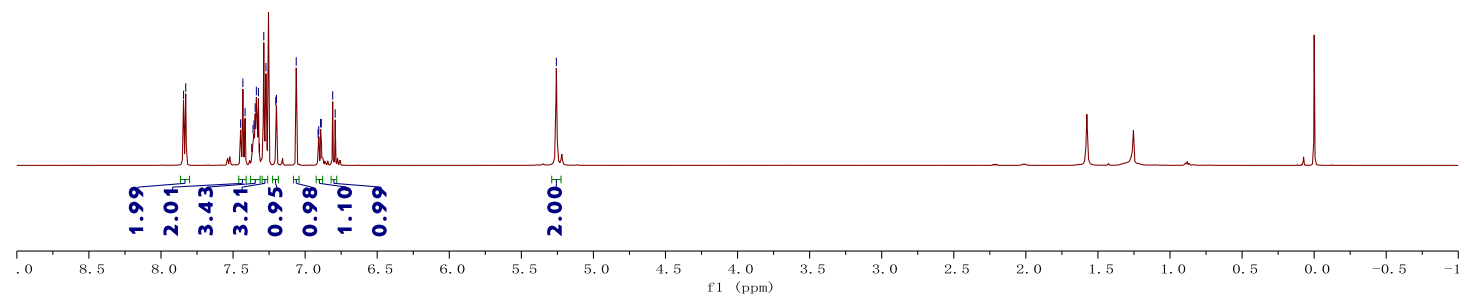

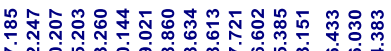

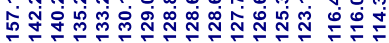

${ }^{13} \mathrm{C}$ NMR (126 MHz, $\mathrm{CDCl}_{3}$ )

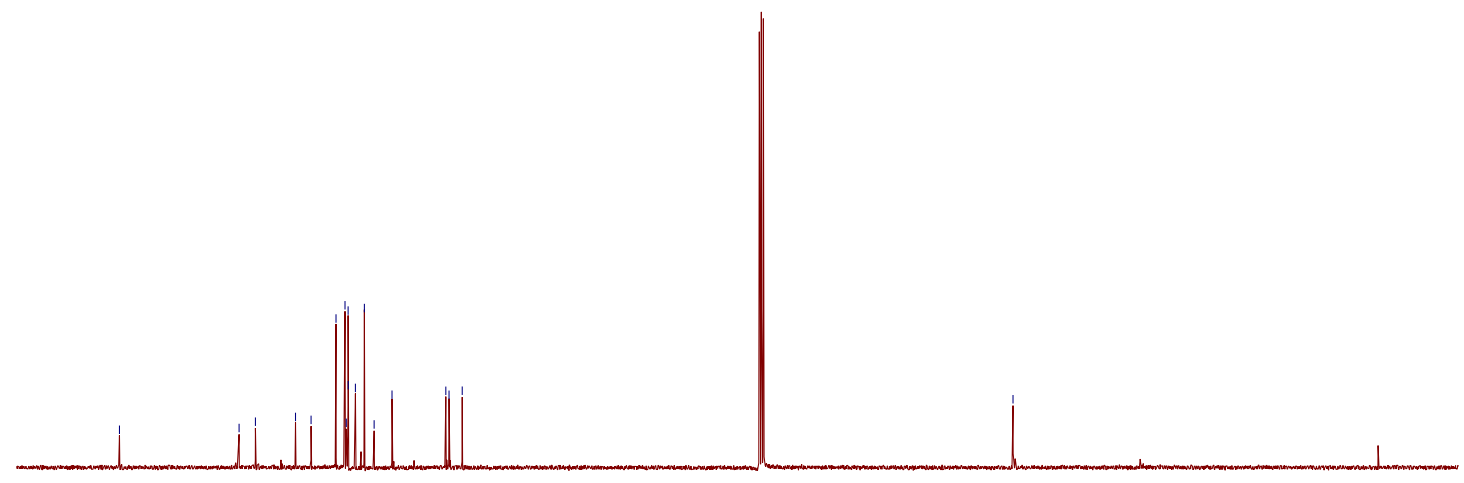


<smiles>O=C1C(=Cc2ccccc2)Oc2cc(Br)ccc2N1Cc1ccccc1</smiles>

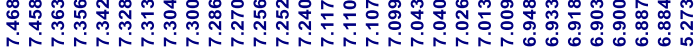

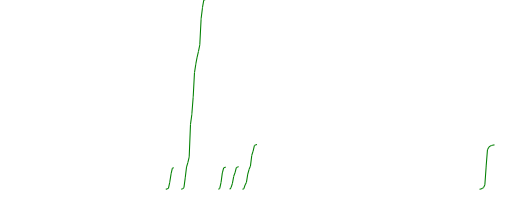

${ }^{1} \mathrm{H} \mathrm{NMR}\left(500 \mathrm{MHz}, \mathrm{CDCl}_{3}\right)$

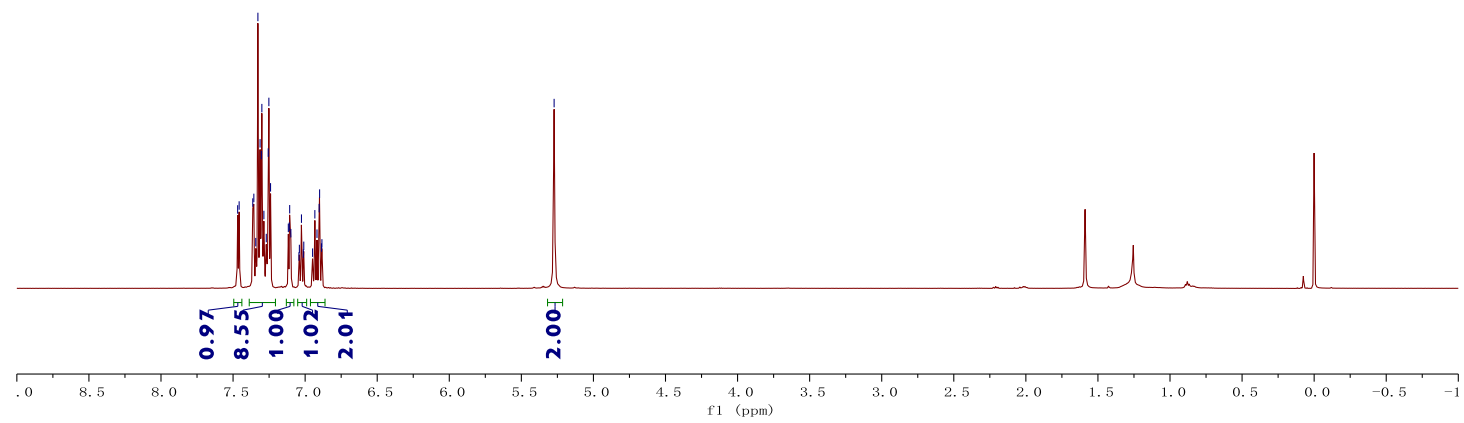

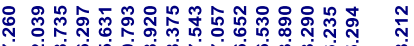

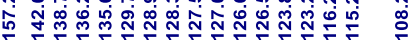

্ָল

${ }^{13} \mathrm{C}$ NMR $\left(126 \mathrm{MHz}, \mathrm{CDCl}_{3}\right)$

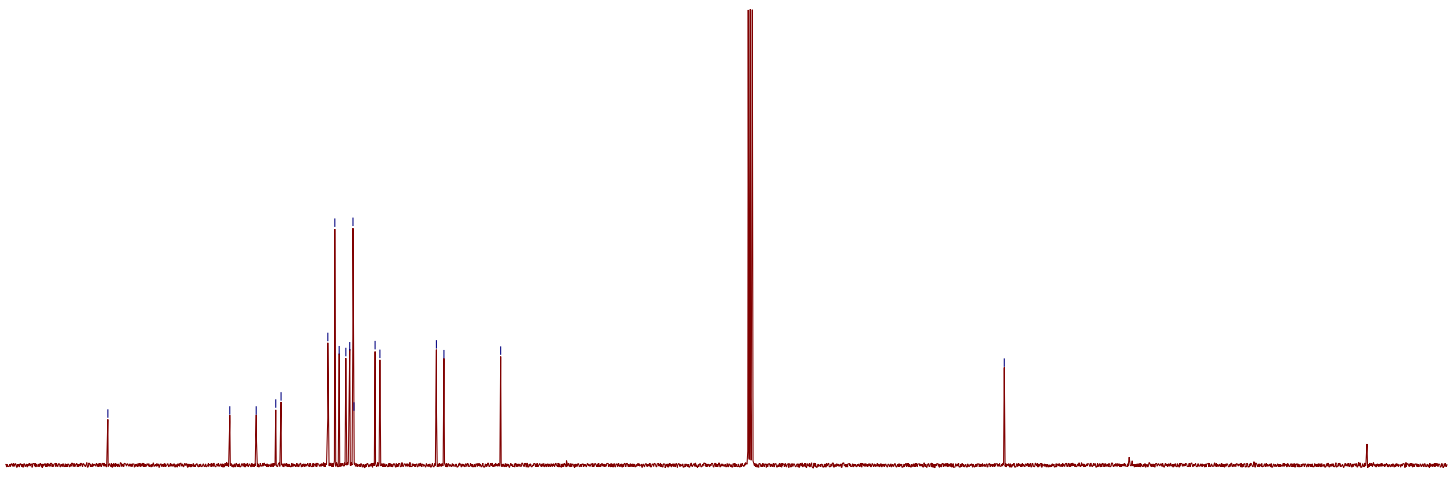




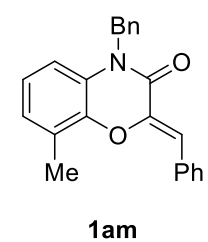

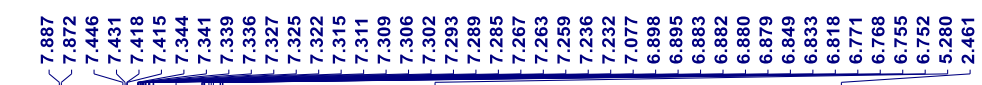

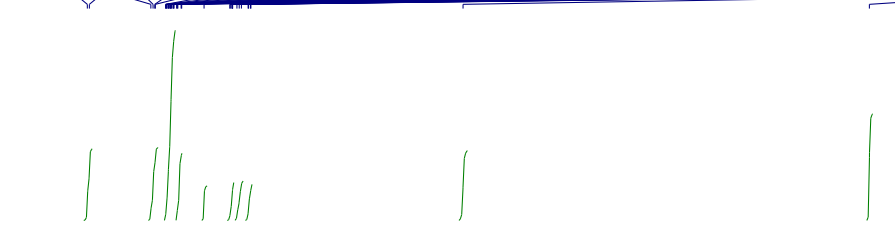

${ }^{1} \mathrm{H}$ NMR $\left(500 \mathrm{MHz}, \mathrm{CDCl}_{3}\right)$

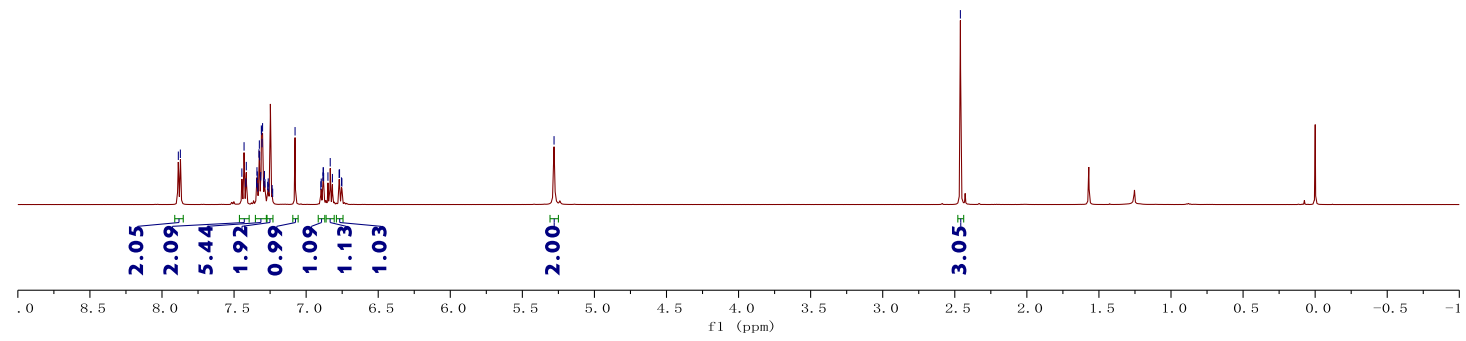

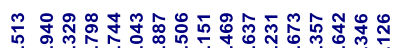

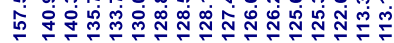

${ }^{13} \mathrm{C}$ NMR $\left(126 \mathrm{MHz}, \mathrm{CDCl}_{3}\right)$

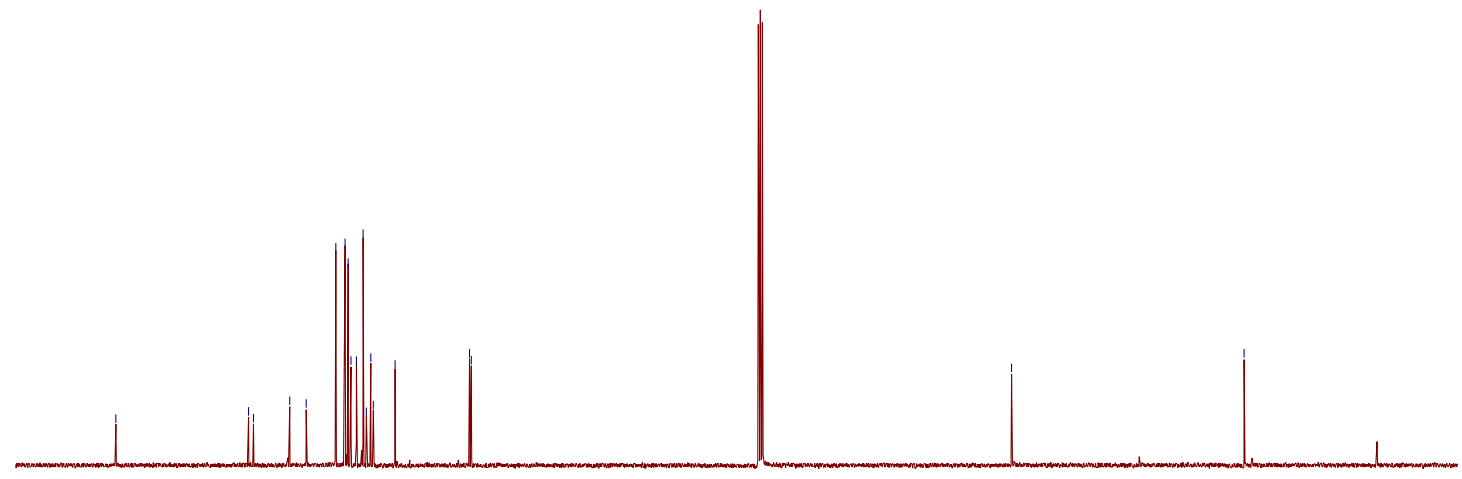

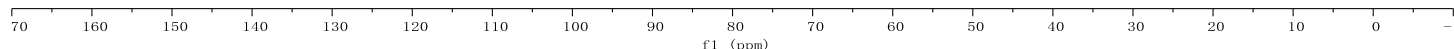



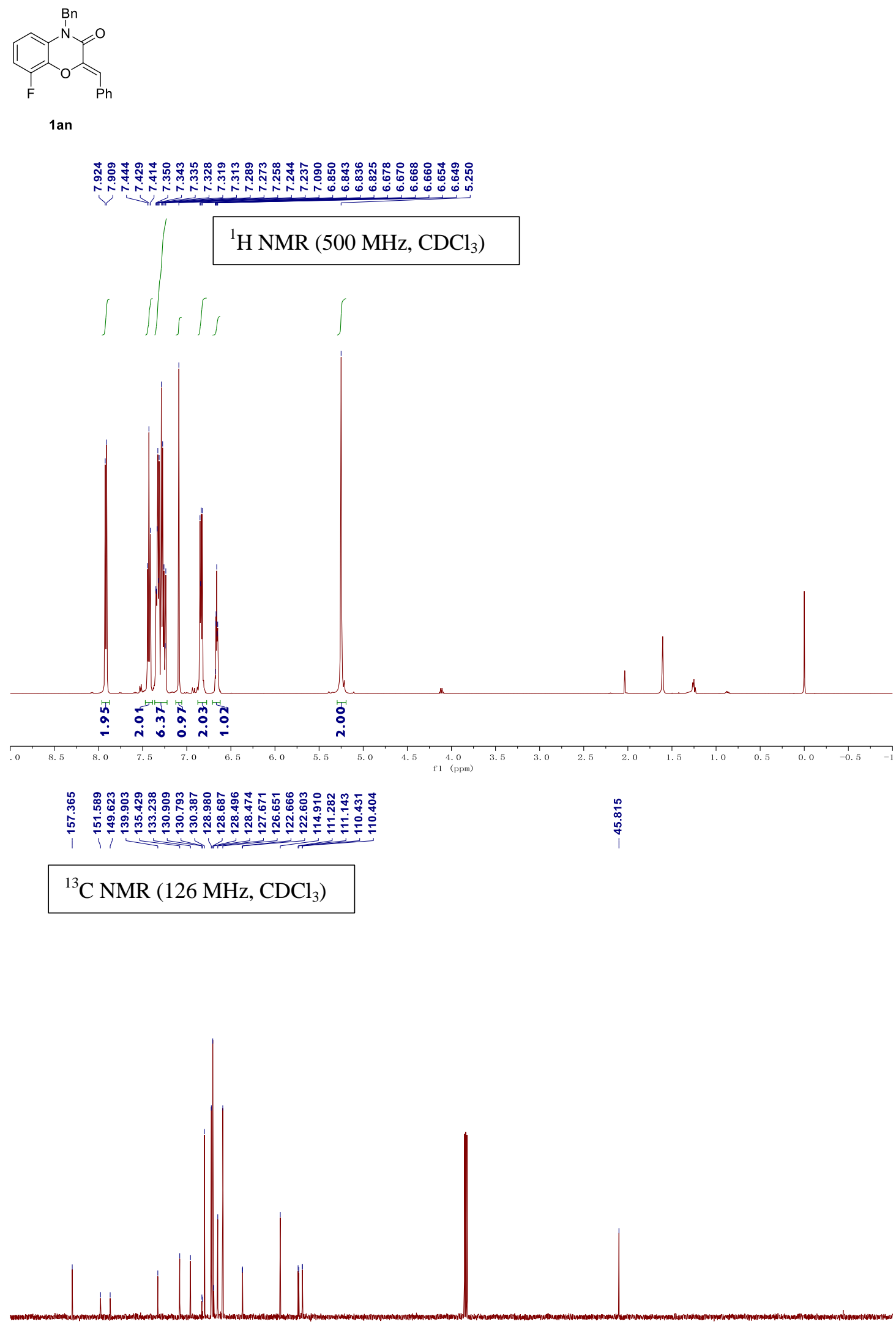
${ }^{19} \mathrm{~F}$ NMR (471 MHz, $\left.\mathrm{CDCl}_{3}\right)$

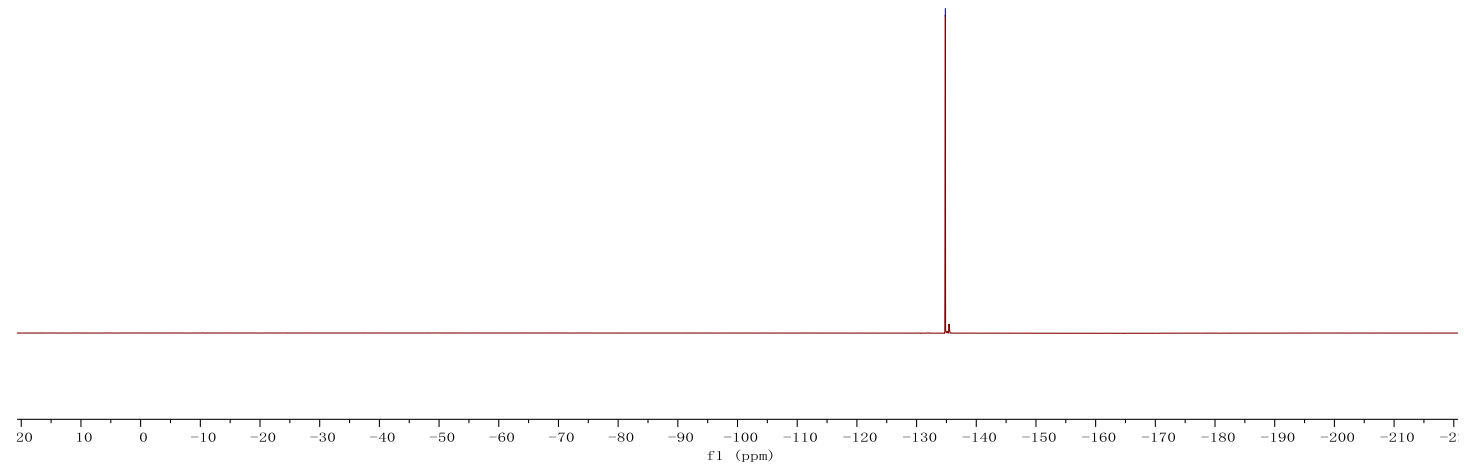


<smiles>O=C1/C(=C/C2CC2)Oc2cc(Br)ccc2N1Cc1ccccc1</smiles>

1 ao

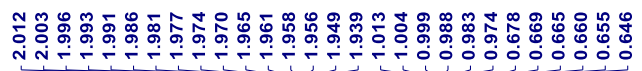

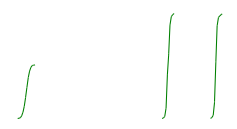

${ }^{1} \mathrm{H}$ NMR (500 MHz, $\mathrm{CDCl}_{3}$ )
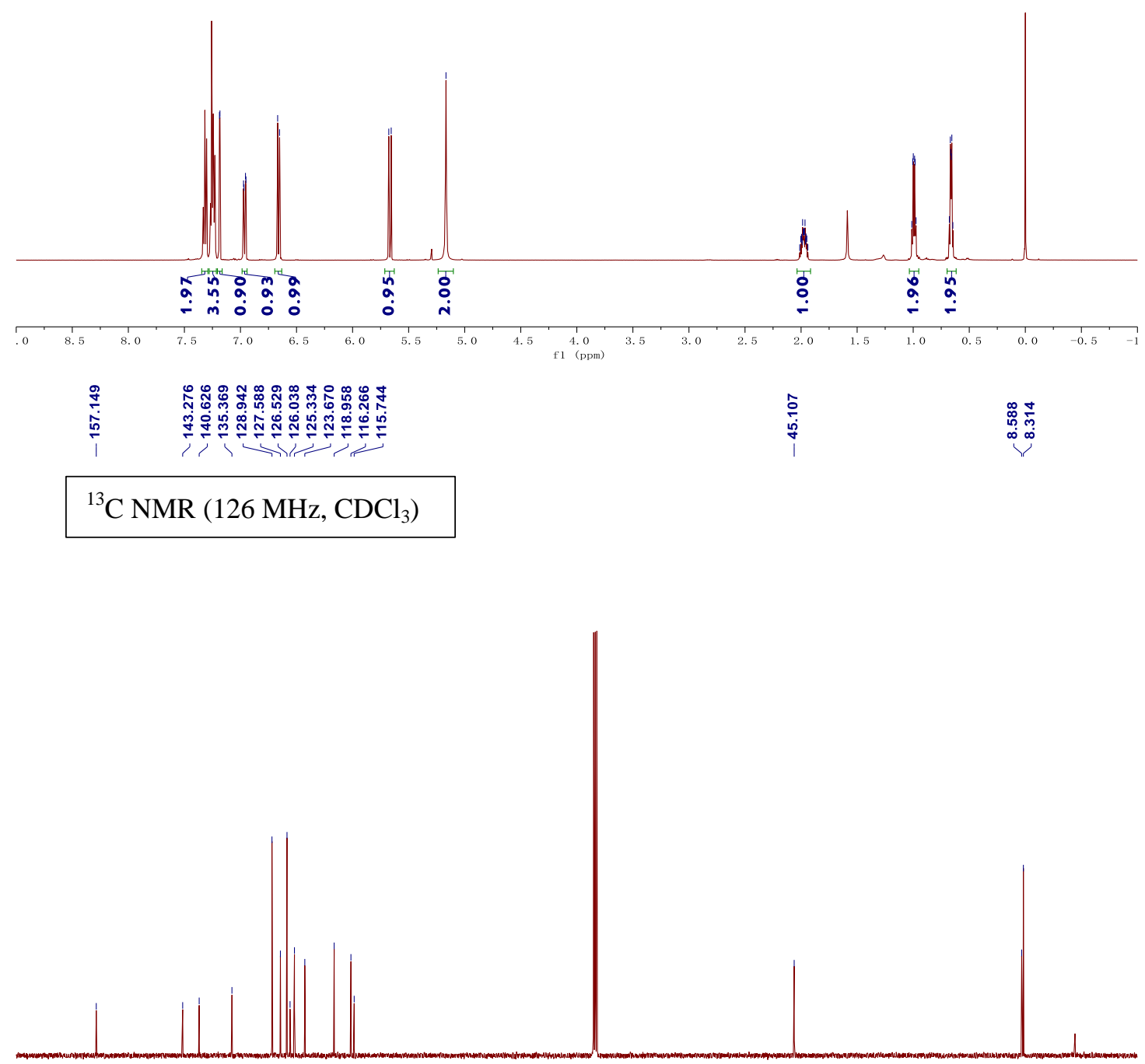

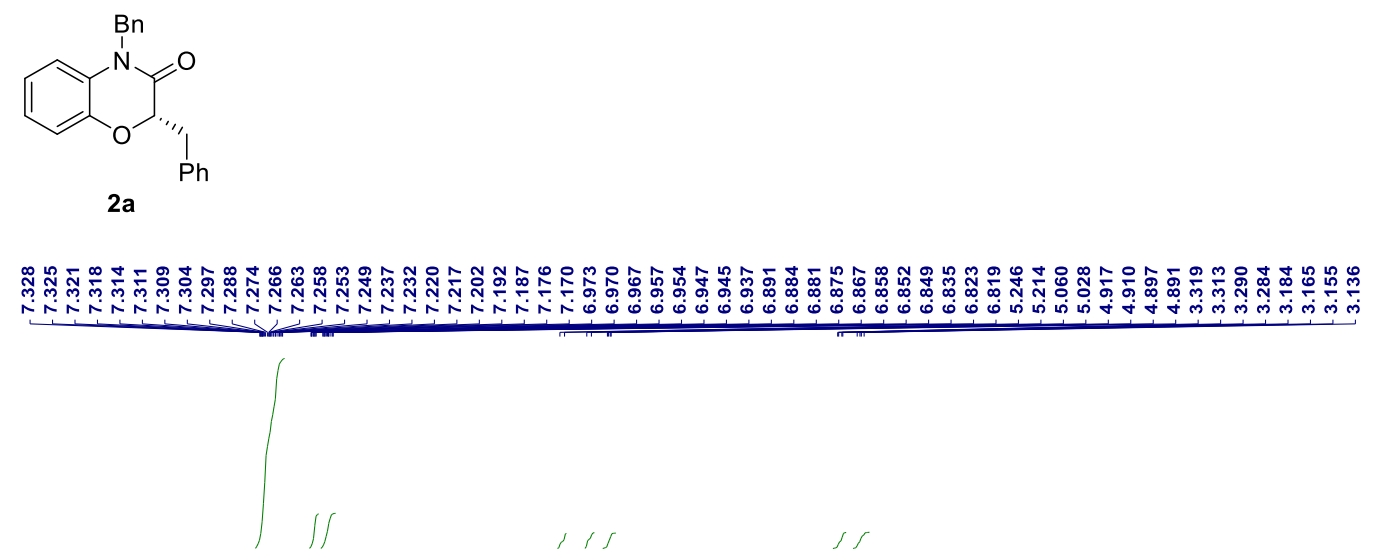

${ }^{1} \mathrm{H}$ NMR $\left(500 \mathrm{MHz}, \mathrm{CDCl}_{3}\right.$ )
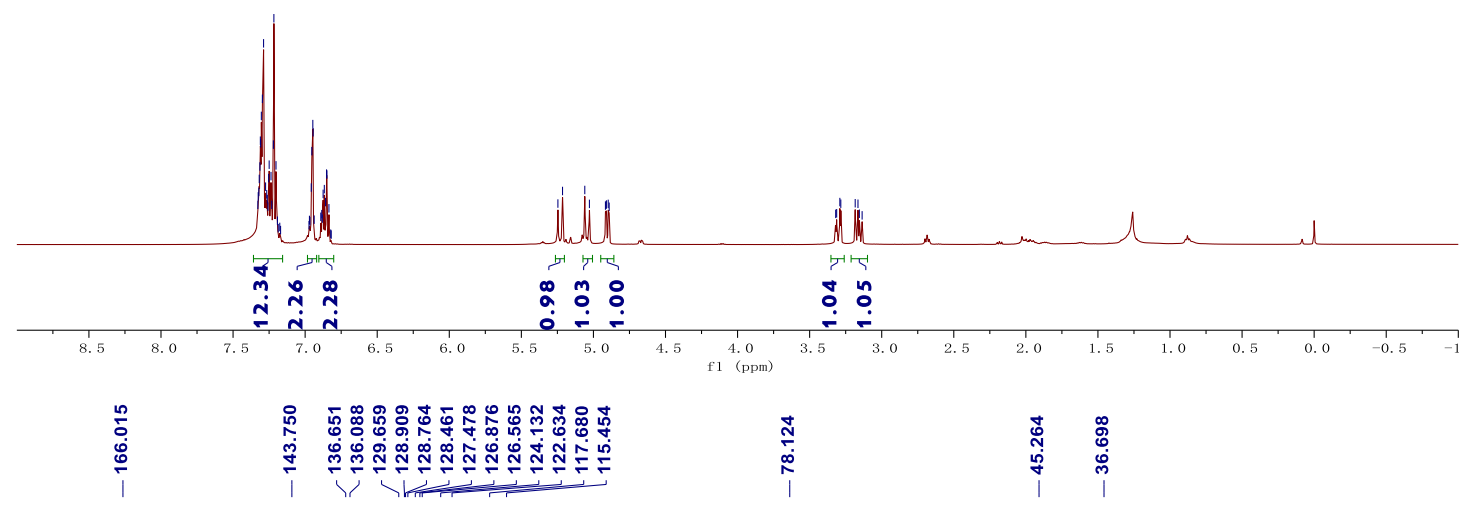

${ }^{13} \mathrm{C}$ NMR $\left(126 \mathrm{MHz}, \mathrm{CDCl}_{3}\right)$
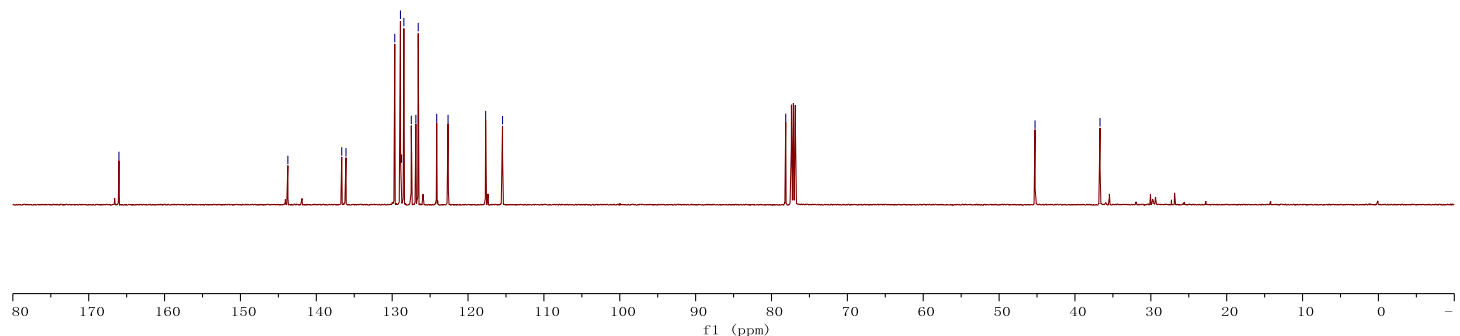


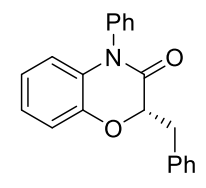

2b

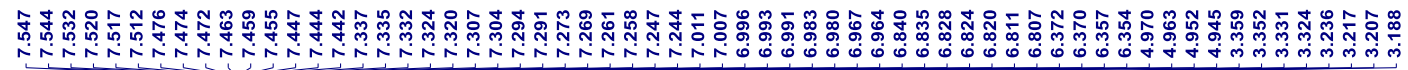

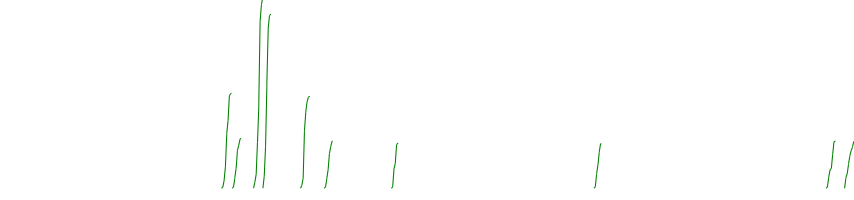

${ }^{1} \mathrm{H}$ NMR (500 MHz, $\left.\mathrm{CDCl}_{3}\right)$
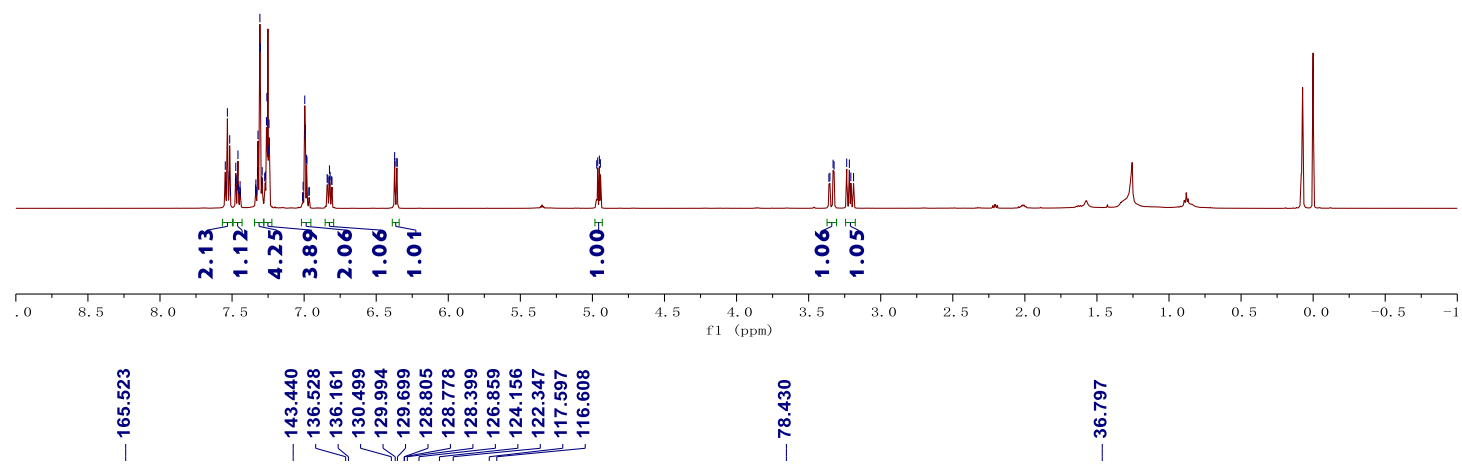

${ }^{13} \mathrm{C} \mathrm{NMR}\left(126 \mathrm{MHz}, \mathrm{CDCl}_{3}\right)$

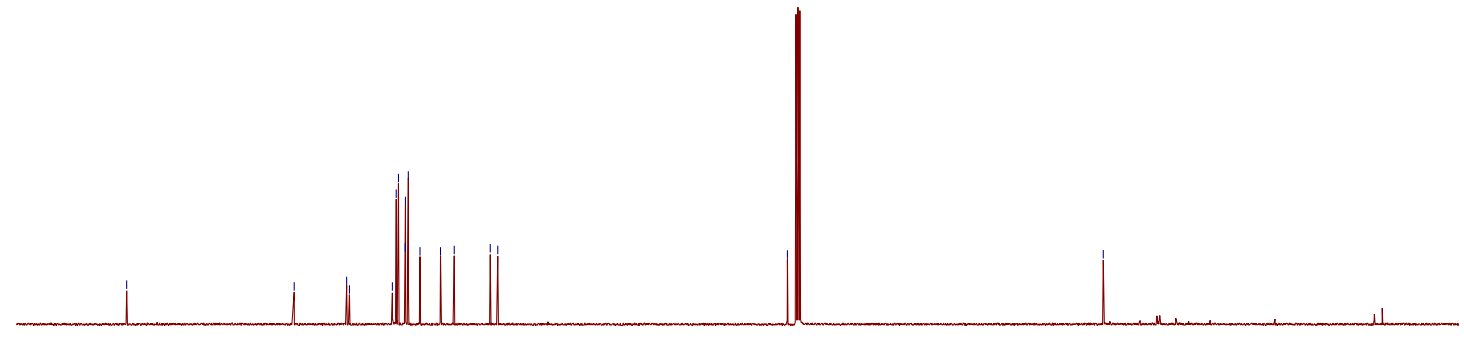




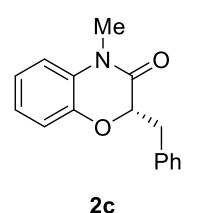

$2 c$

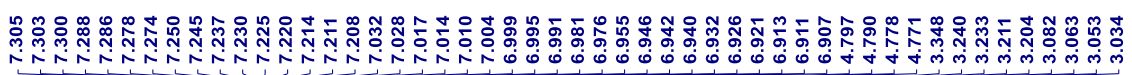

${ }^{1} \mathrm{H}$ NMR $\left(500 \mathrm{MHz}, \mathrm{CDCl}_{3}\right)$

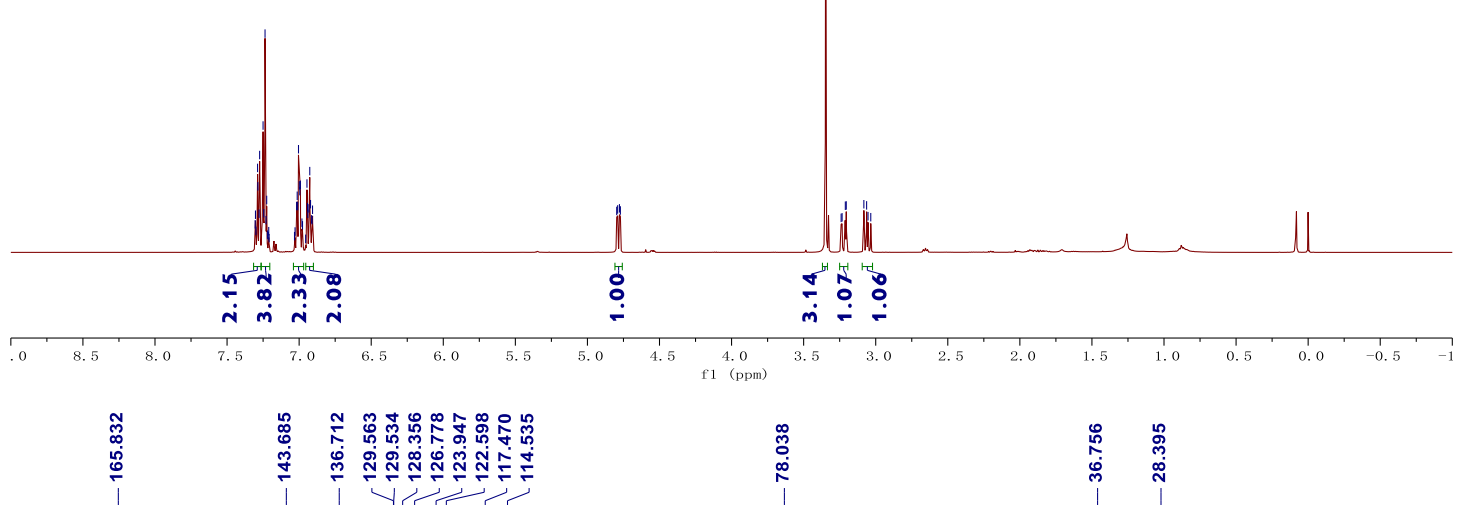

${ }^{13} \mathrm{C}$ NMR (126 MHz, $\left.\mathrm{CDCl}_{3}\right)$

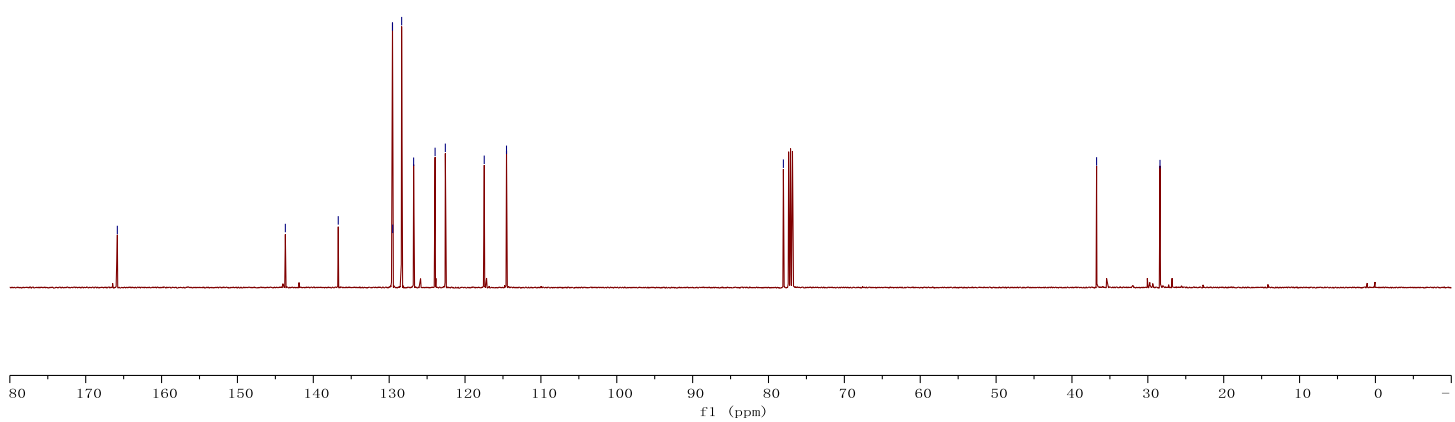

89 


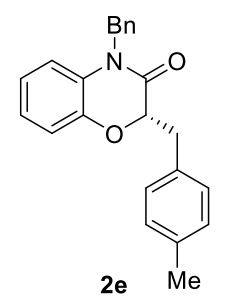

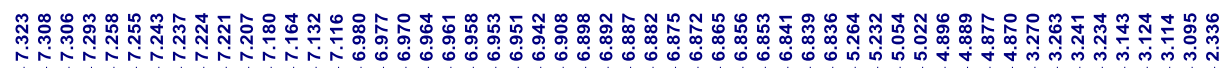

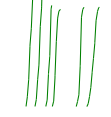

${ }^{1} \mathrm{H}$ NMR (500 MHz, $\mathrm{CDCl}_{3}$ )

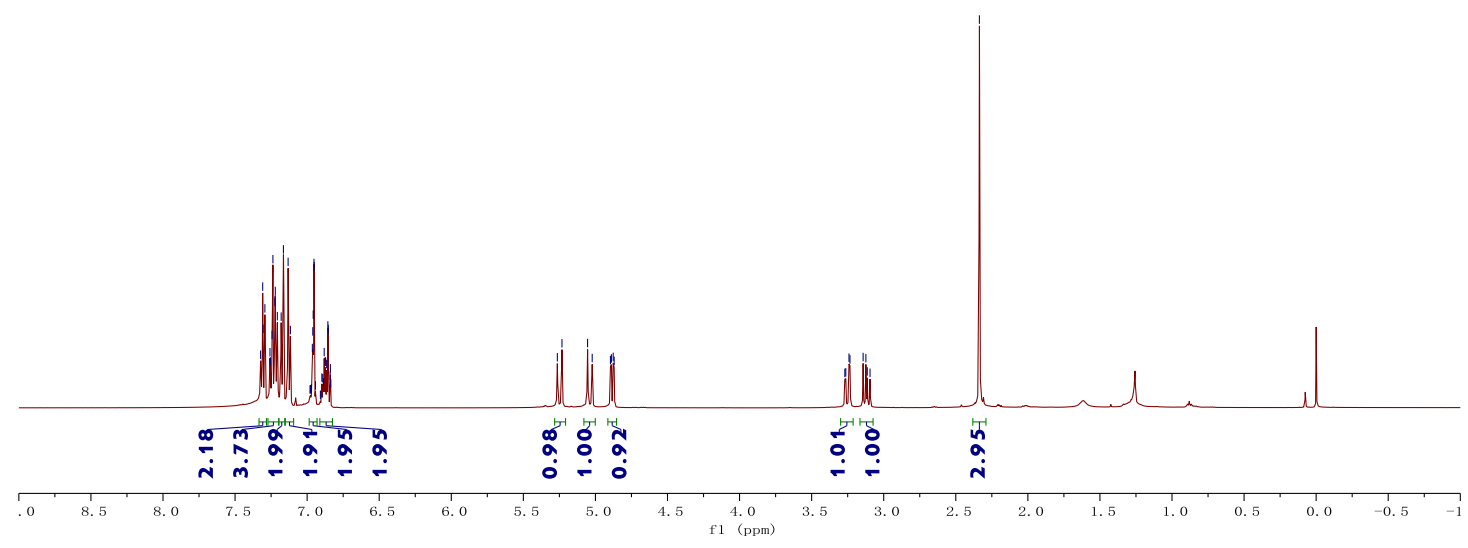
亩

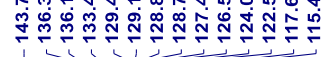
$\underset{\substack{0 \\ \infty}}{\substack{\infty \\ 1}}$

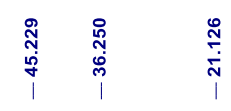

${ }^{13} \mathrm{C} \mathrm{NMR}\left(126 \mathrm{MHz}, \mathrm{CDCl}_{3}\right)$

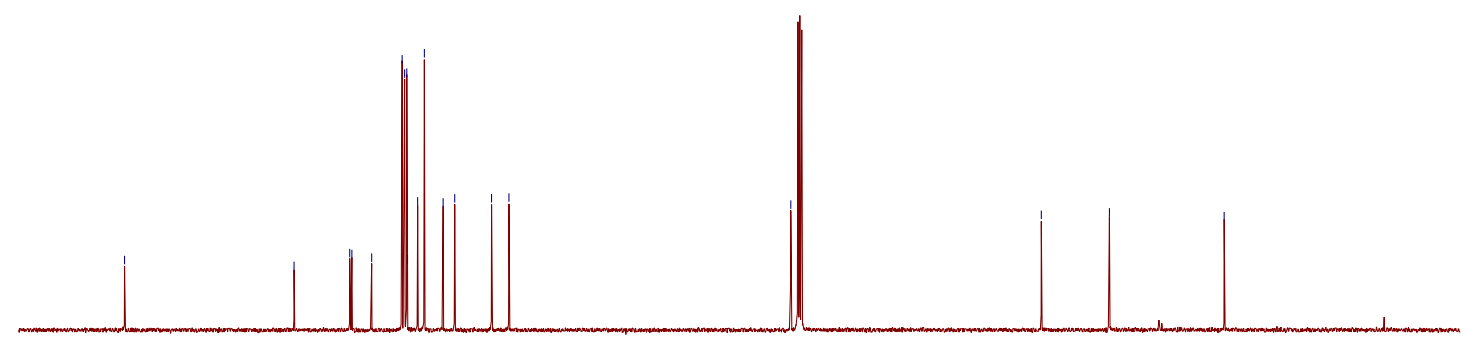

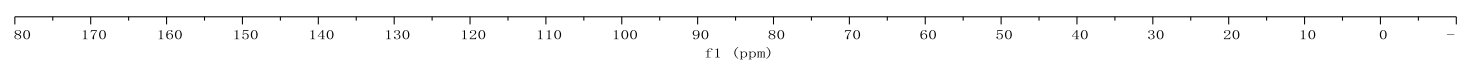



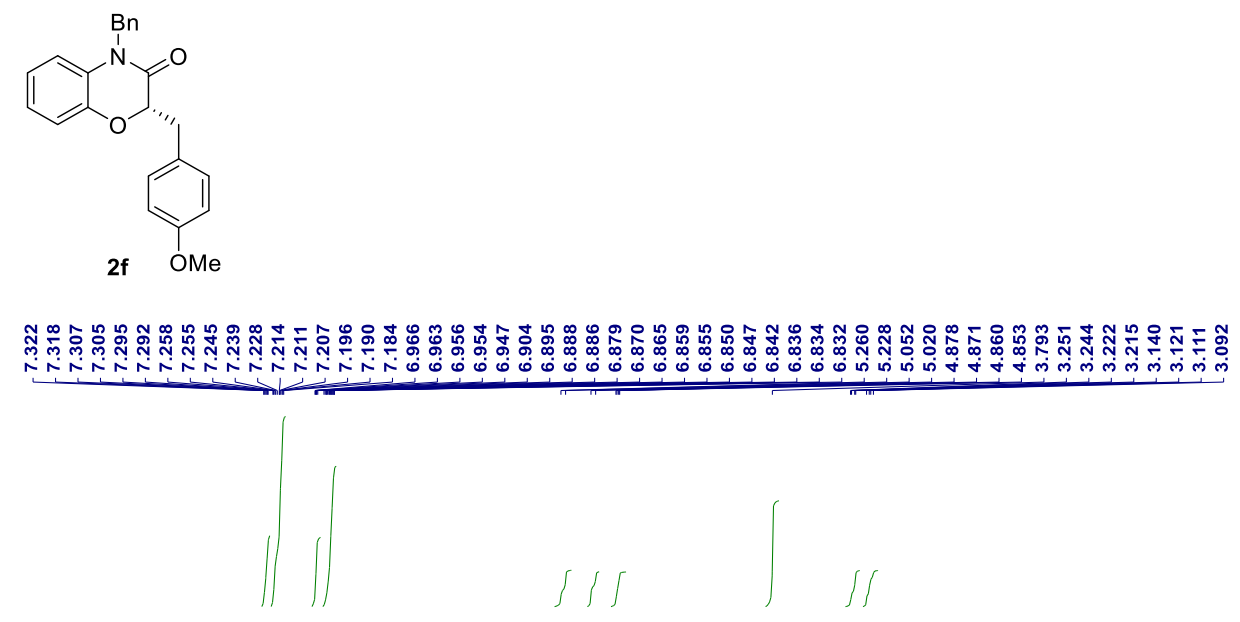

${ }^{1} \mathrm{H}$ NMR $\left(500 \mathrm{MHz}, \mathrm{CDCl}_{3}\right.$ )

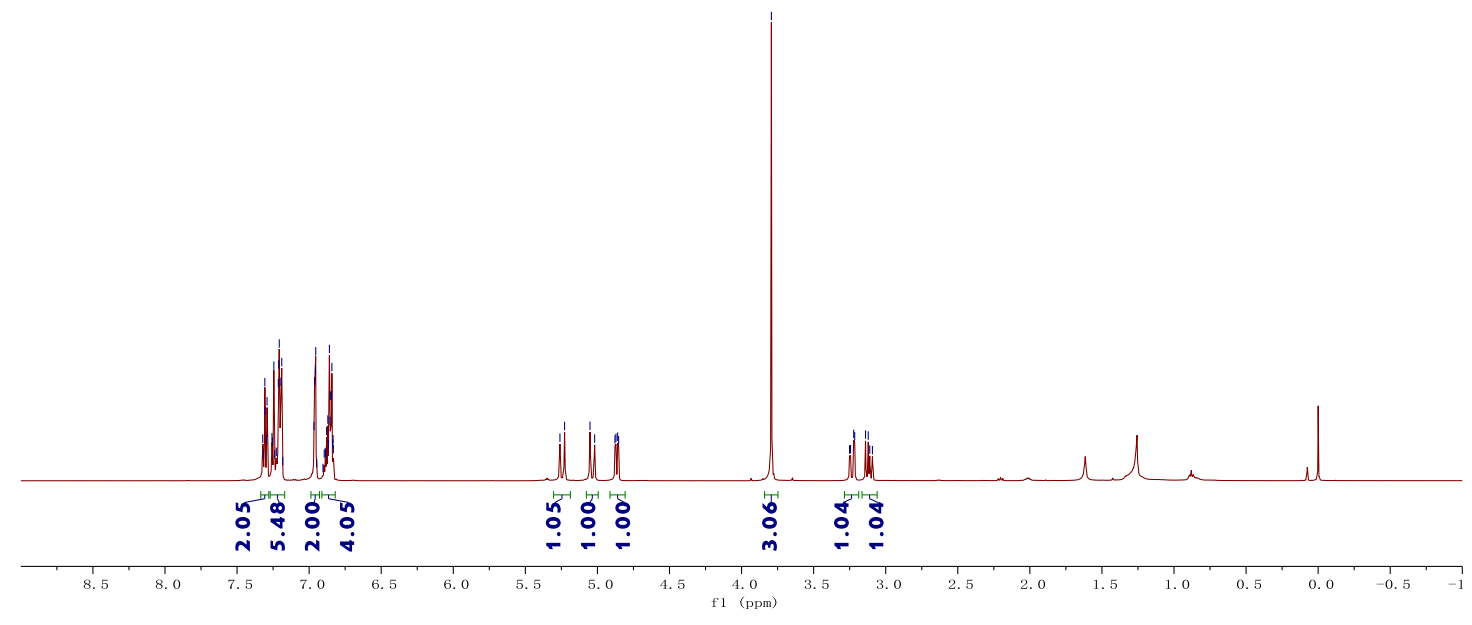

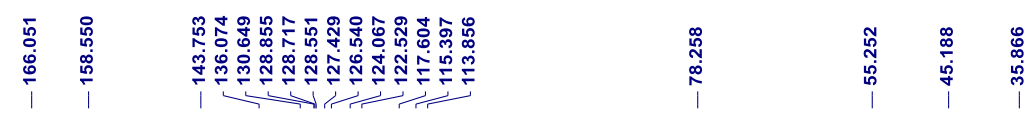

${ }^{13} \mathrm{C}$ NMR $\left(126 \mathrm{MHz}, \mathrm{CDCl}_{3}\right)$

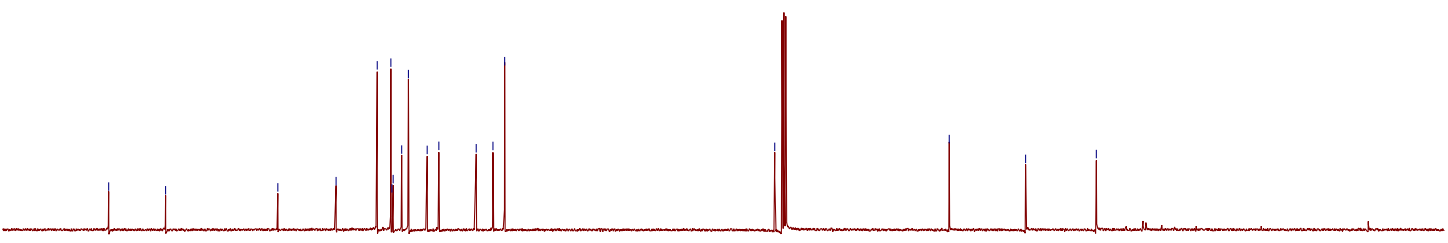

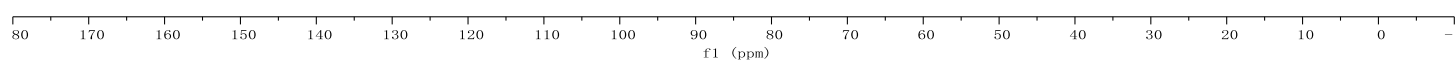




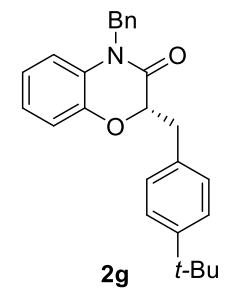

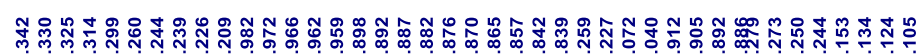

Nive

ind

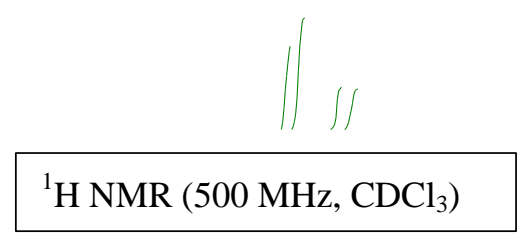

S I

${ }^{1} \mathrm{H}$ NMR $\left(500 \mathrm{MHz}, \mathrm{CDCl}_{3}\right)$
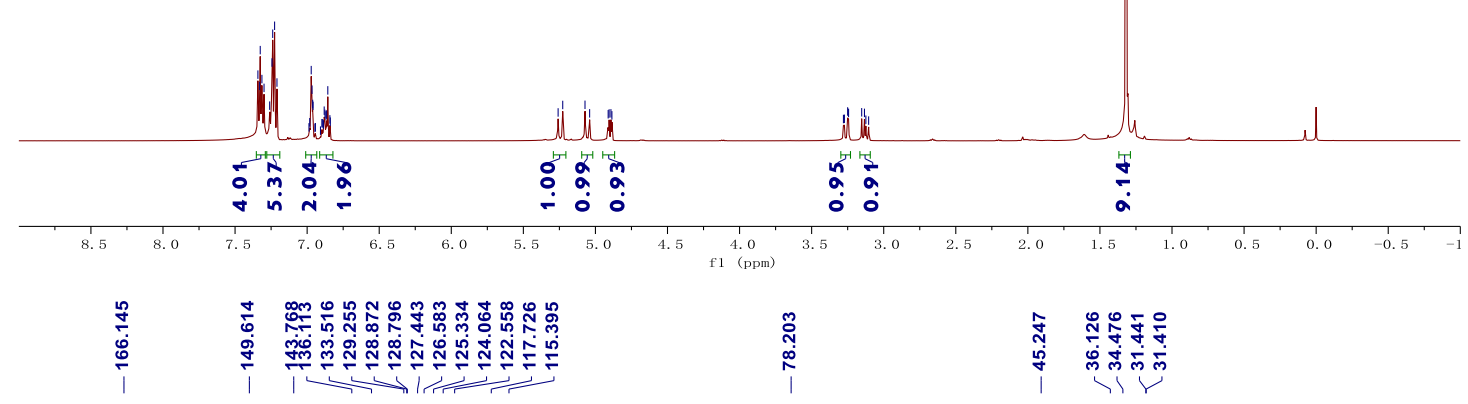

${ }^{13} \mathrm{C}$ NMR $\left(126 \mathrm{MHz}, \mathrm{CDCl}_{3}\right)$

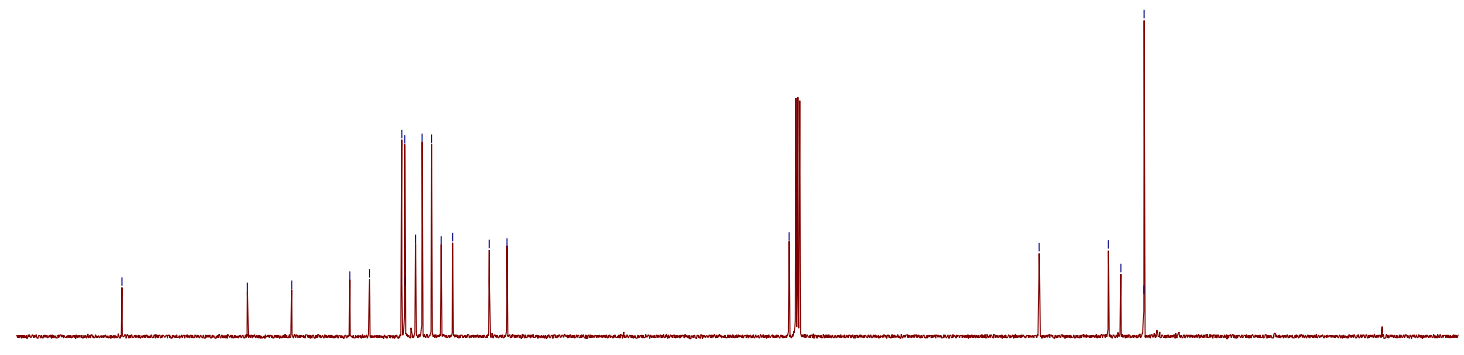

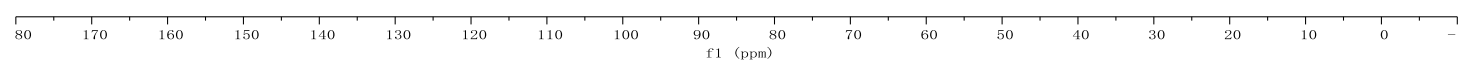


${ }^{19} \mathrm{~F}$ NMR (471 MHz, $\left.\mathrm{CDCl}_{3}\right)$

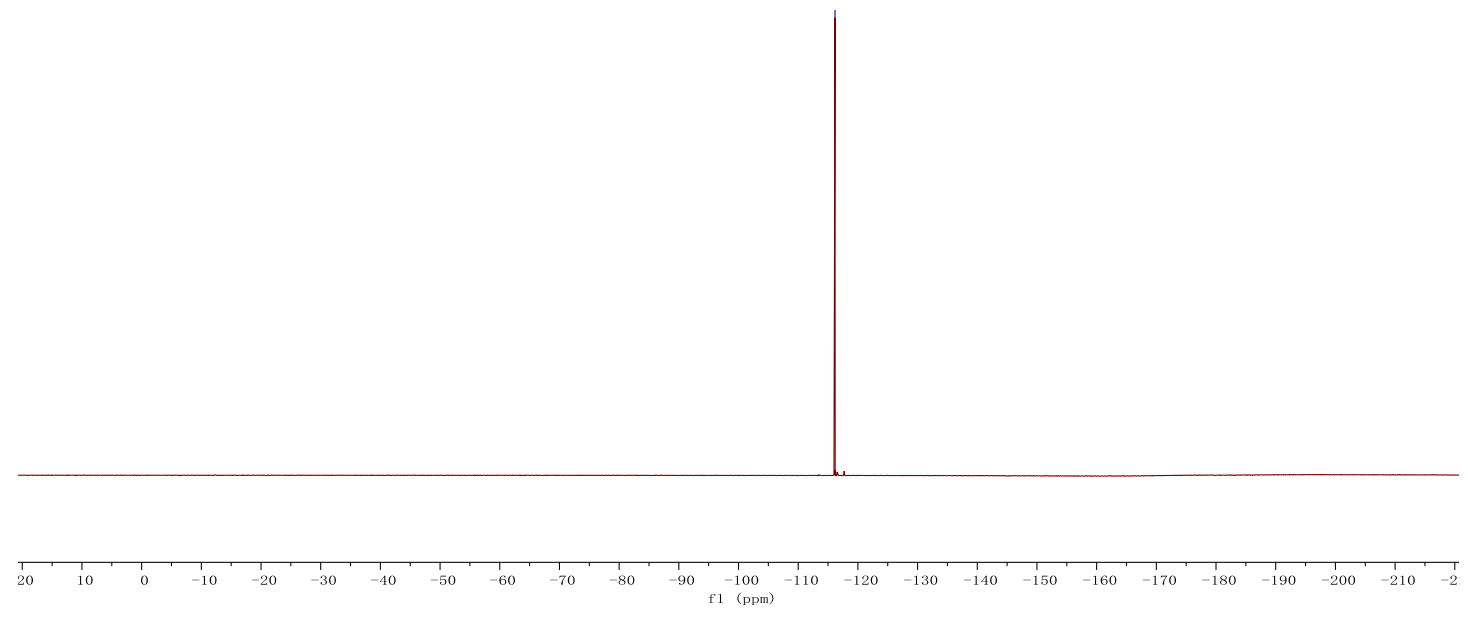




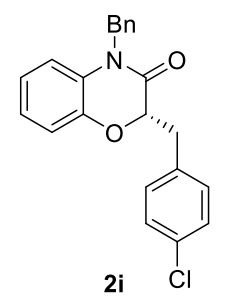

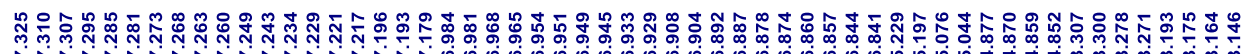

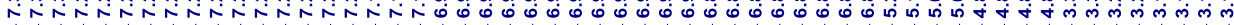

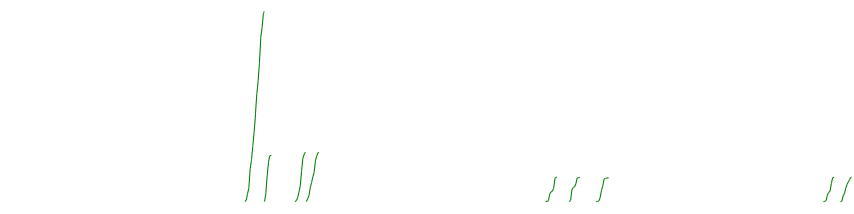

${ }^{1} \mathrm{H}$ NMR $\left(500 \mathrm{MHz}, \mathrm{CDCl}_{3}\right)$
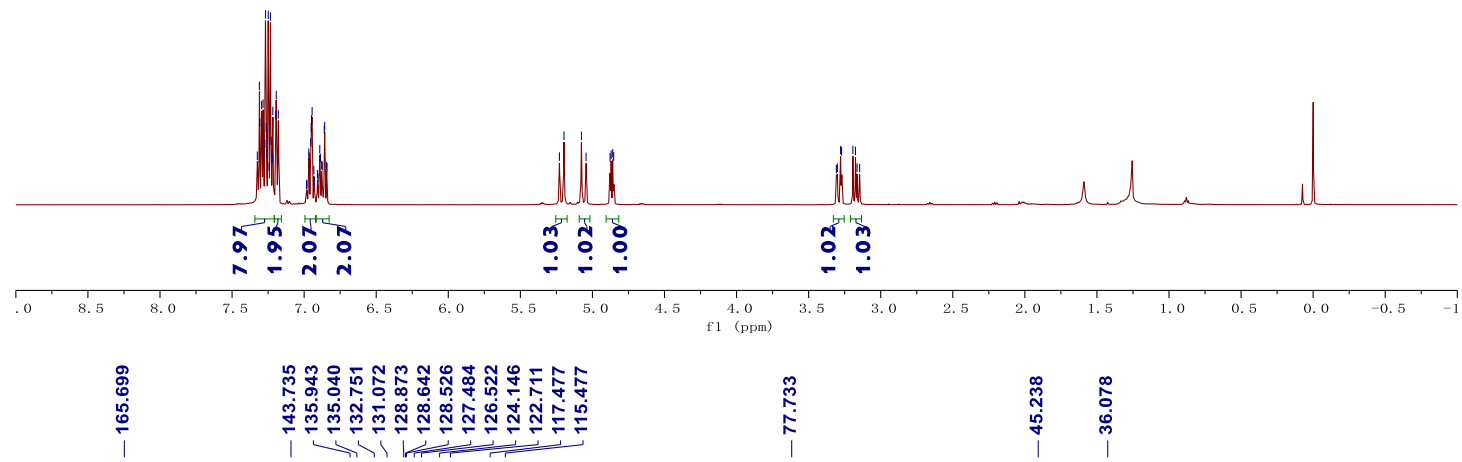

${ }^{13} \mathrm{C}$ NMR $\left(126 \mathrm{MHz}, \mathrm{CDCl}_{3}\right)$

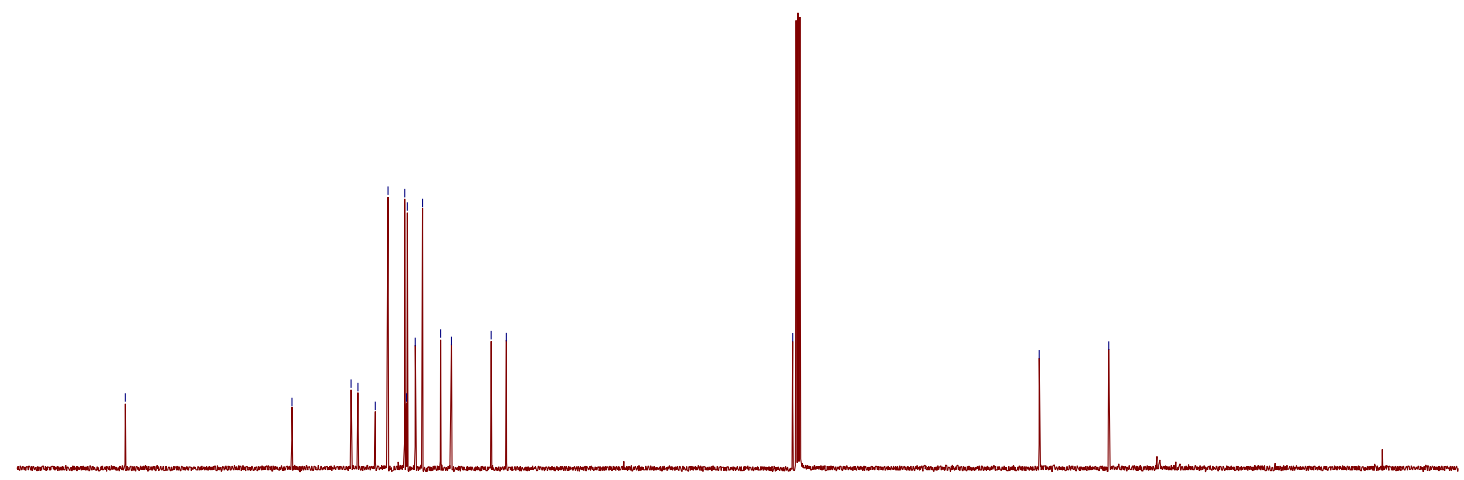

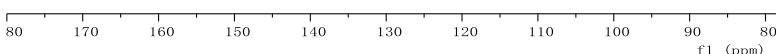




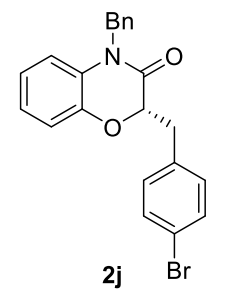

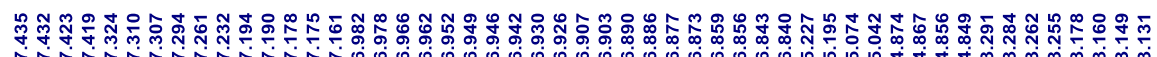

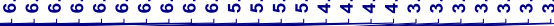
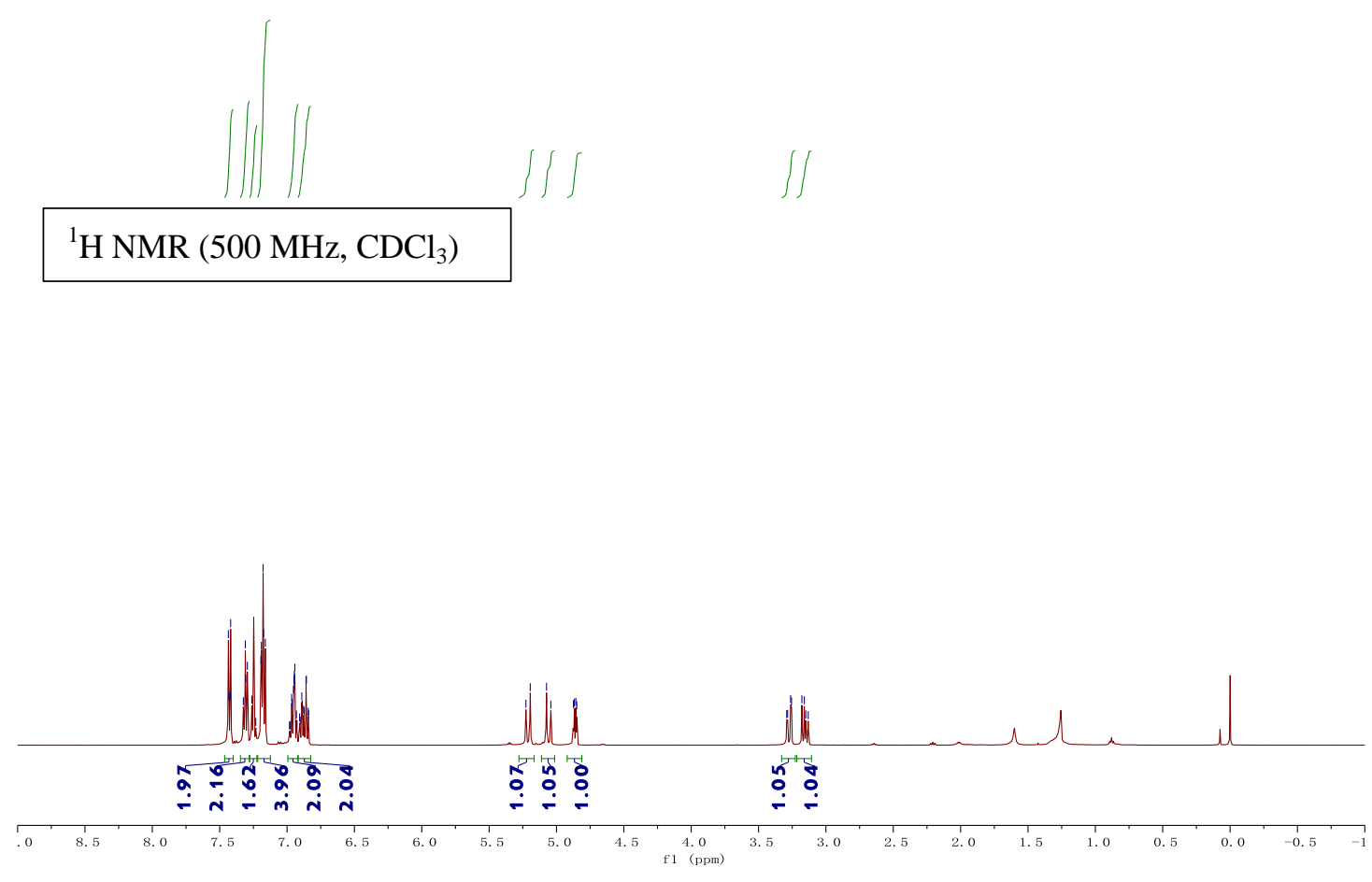

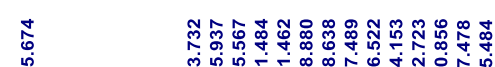

每

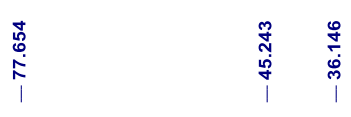

${ }^{13} \mathrm{C}$ NMR $\left(126 \mathrm{MHz}, \mathrm{CDCl}_{3}\right)$

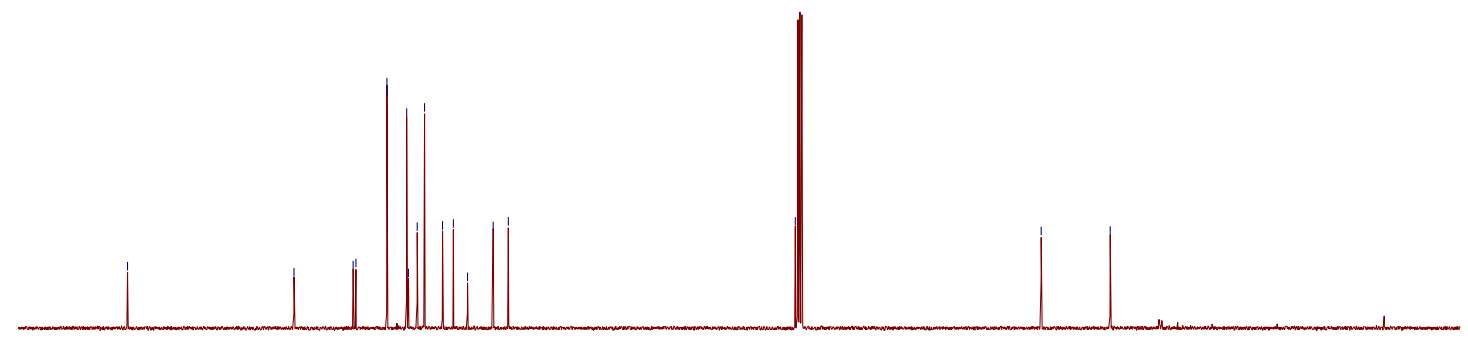

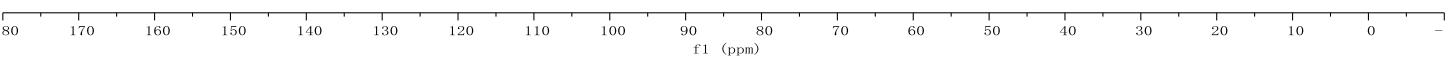




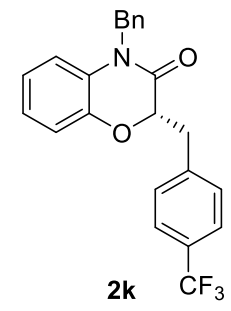

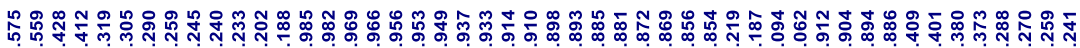

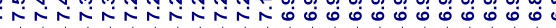

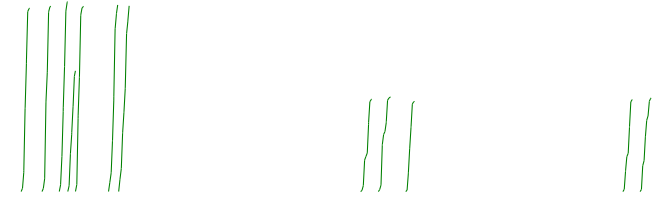

${ }^{1} \mathrm{H}$ NMR (500 MHz, $\mathrm{CDCl}_{3}$ )
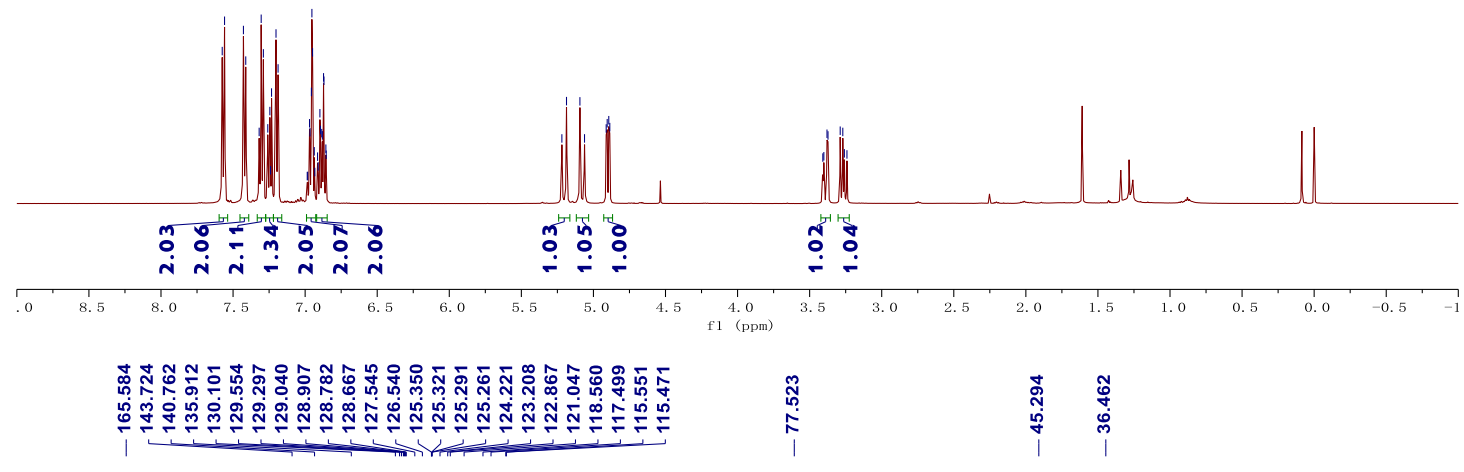

${ }^{13} \mathrm{C}$ NMR $\left(126 \mathrm{MHz}, \mathrm{CDCl}_{3}\right)$
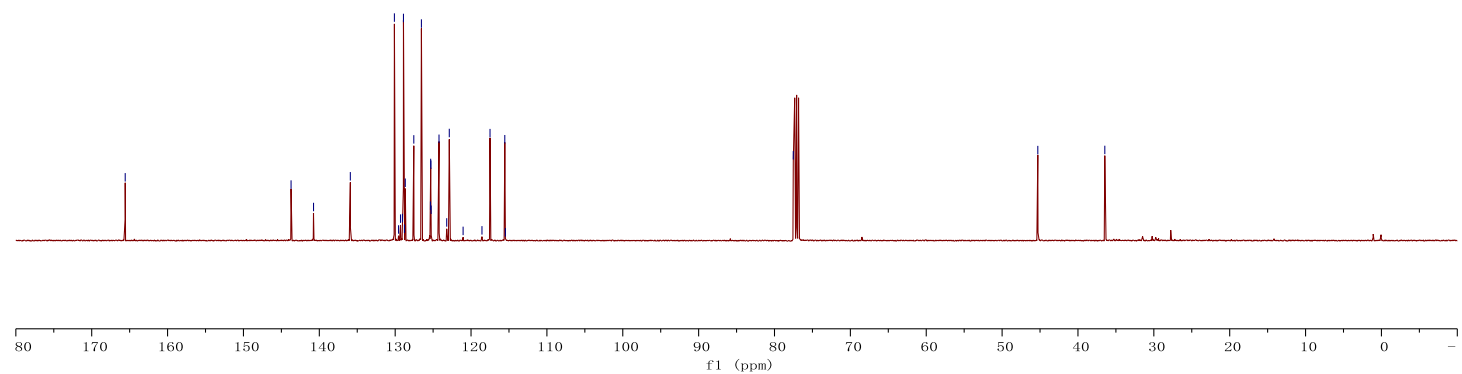
${ }^{19} \mathrm{~F}$ NMR $\left(471 \mathrm{MHz}, \mathrm{CDCl}_{3}\right)$

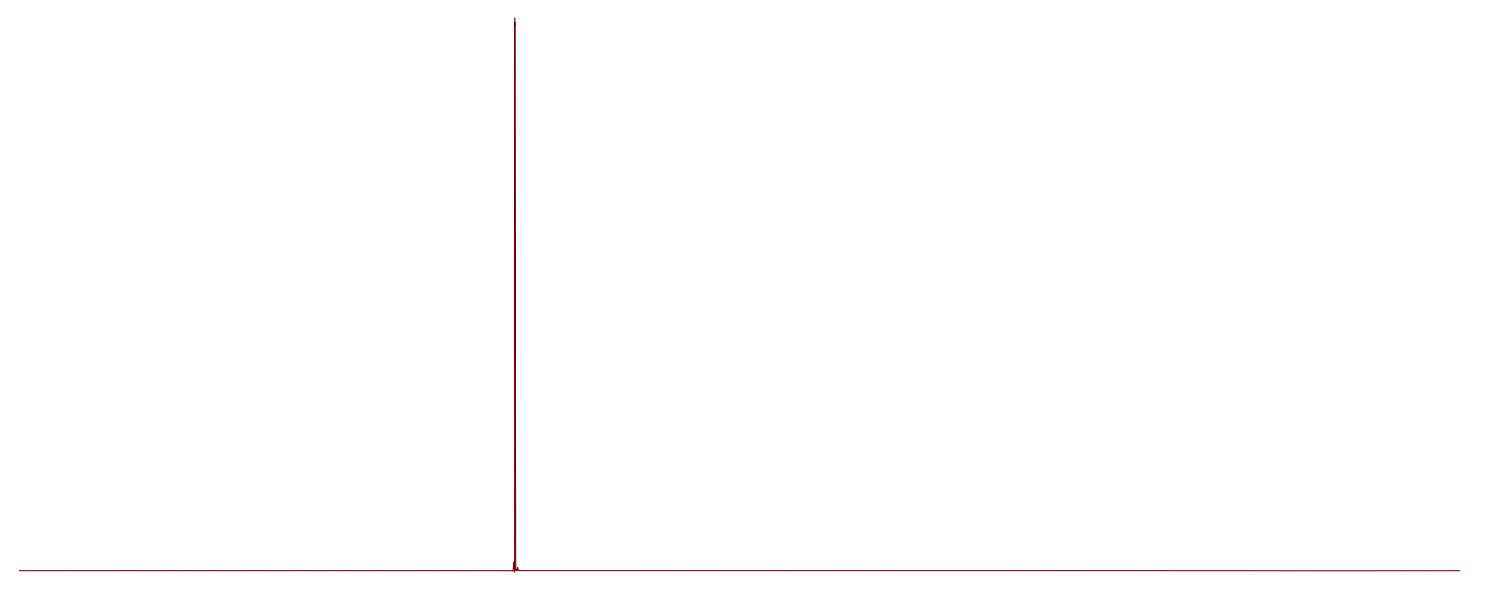

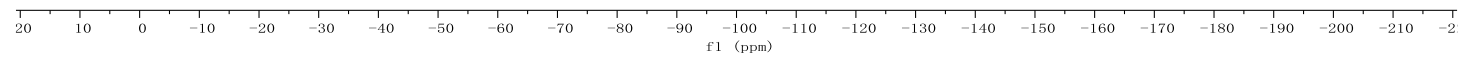



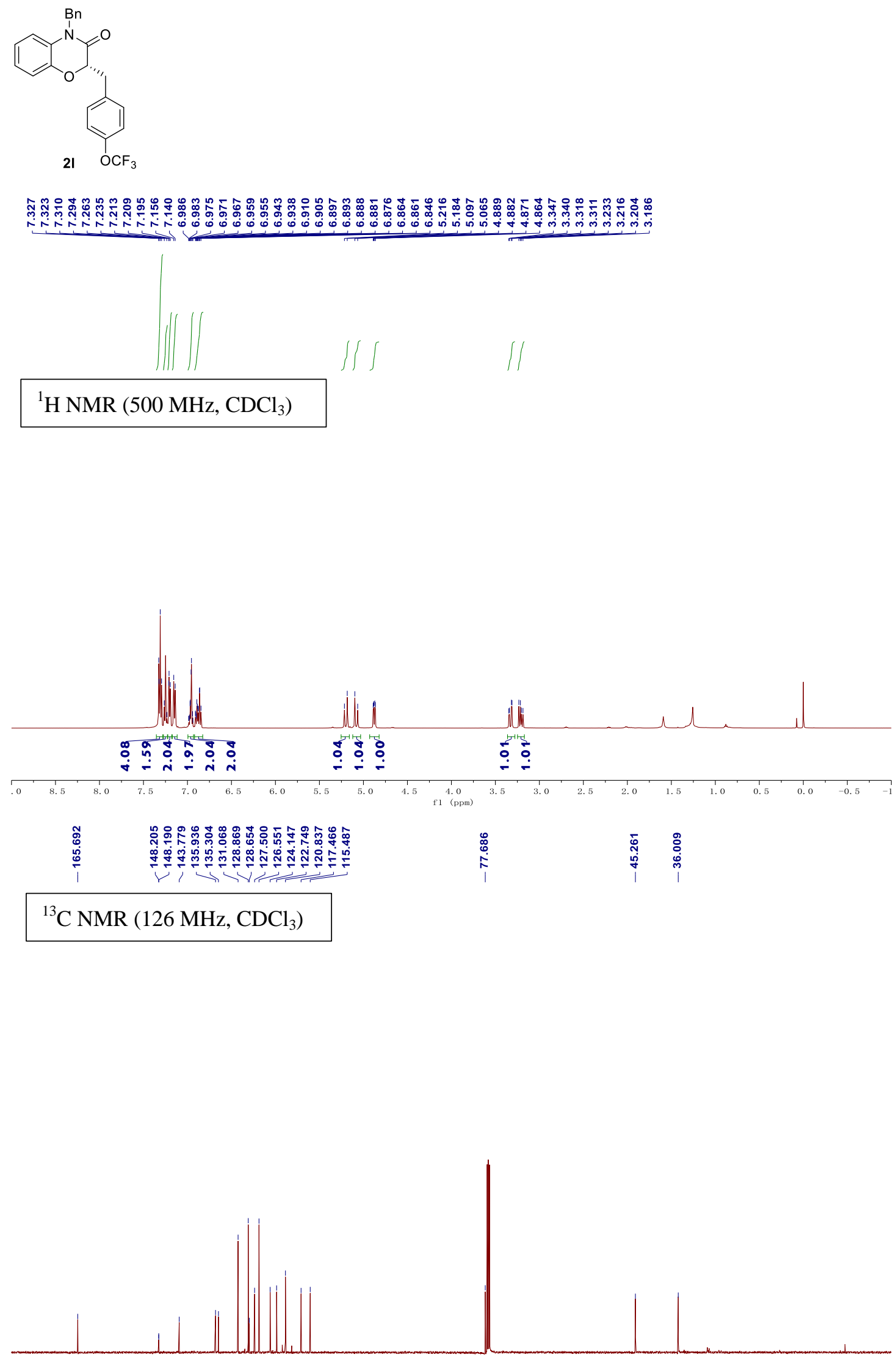

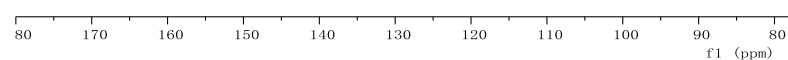




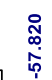

${ }^{19}$ F NMR (471 MHz, $\mathrm{CDCl}_{3}$ )

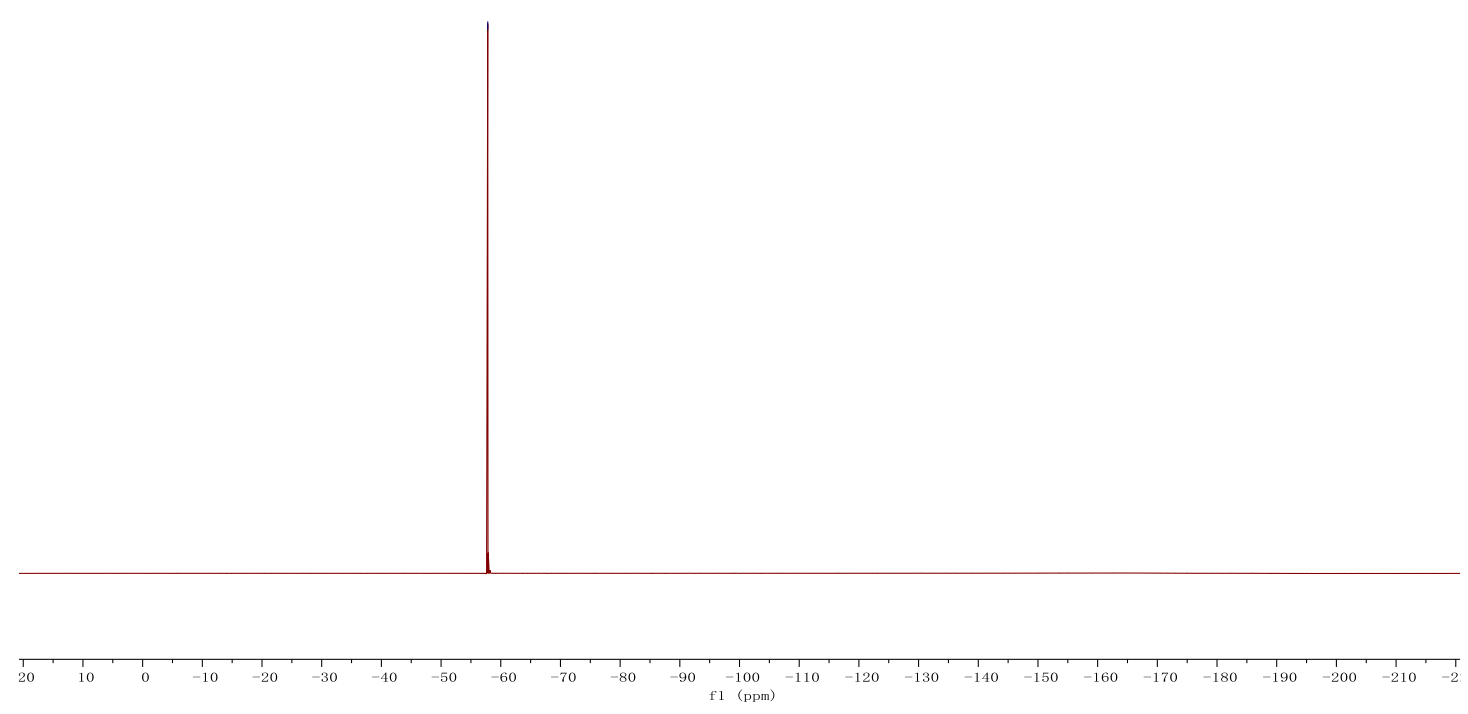




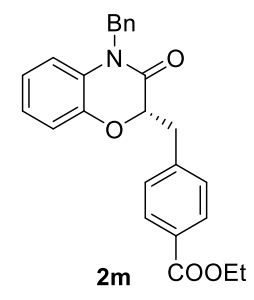

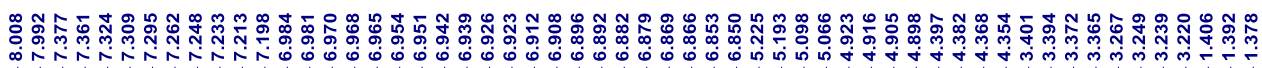

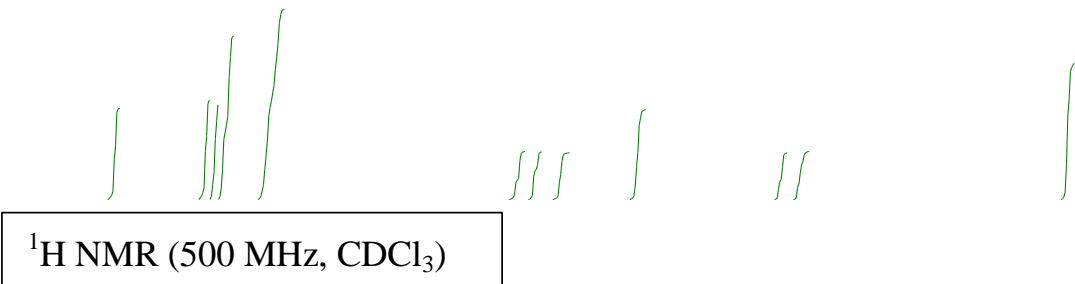

${ }^{1} \mathrm{H}$ NMR $\left(500 \mathrm{MHz}, \mathrm{CDCl}_{3}\right)$

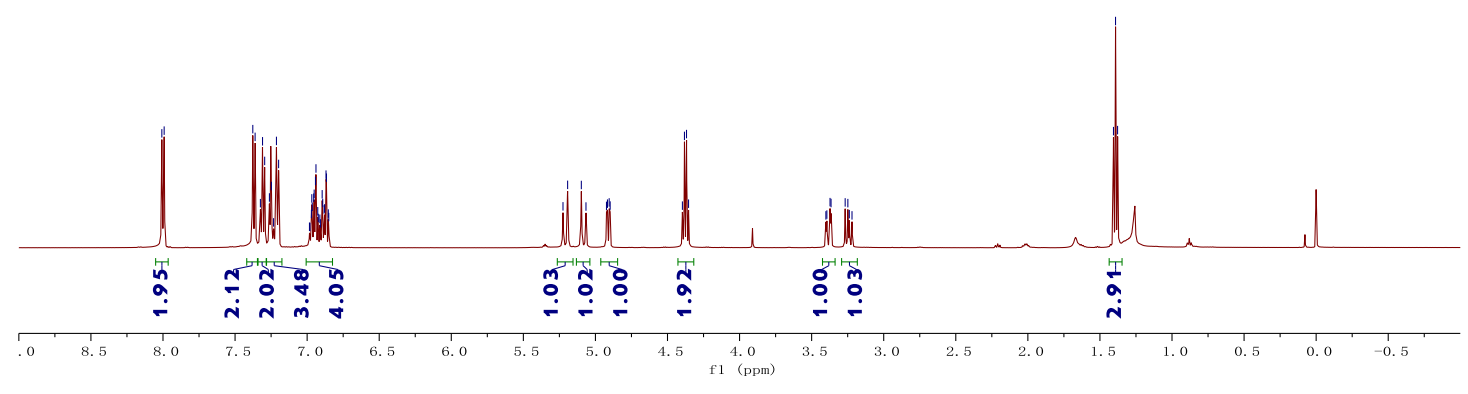

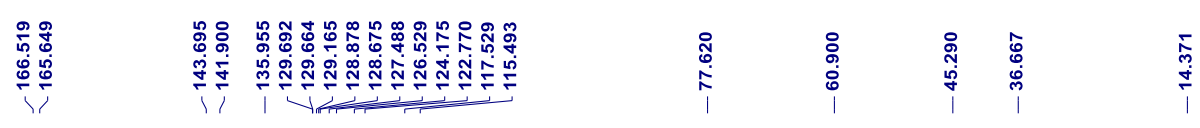

${ }^{13} \mathrm{C} \mathrm{NMR}\left(126 \mathrm{MHz}, \mathrm{CDCl}_{3}\right)$

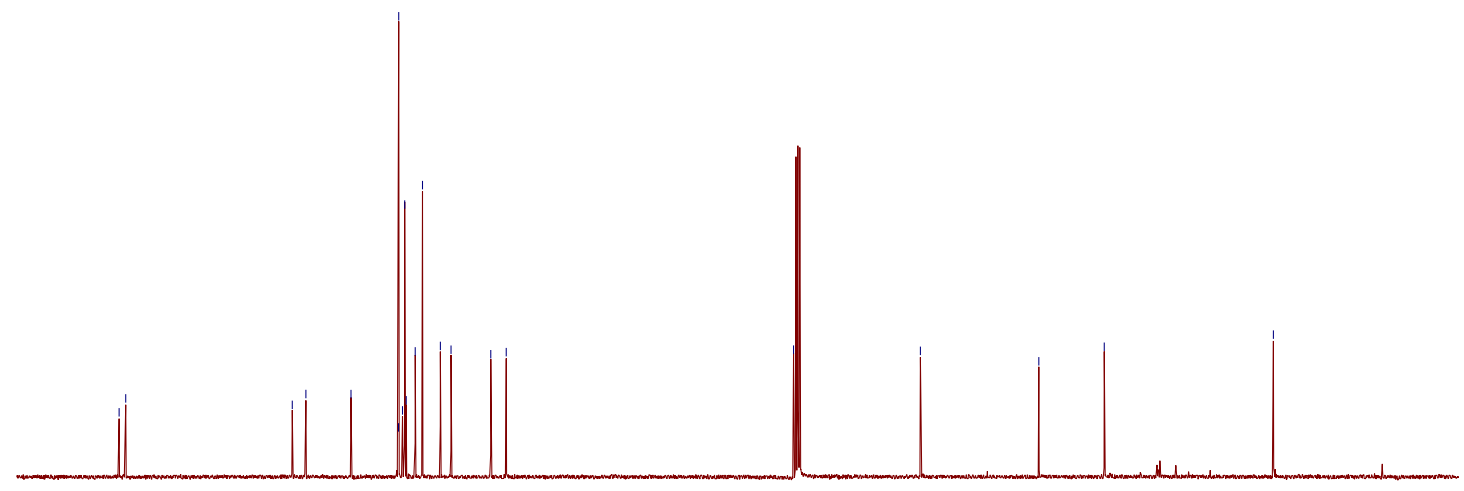

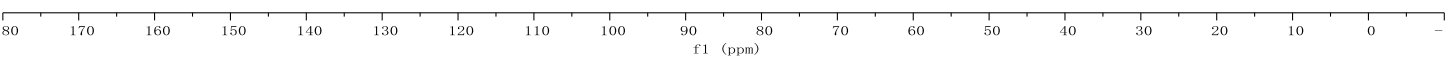



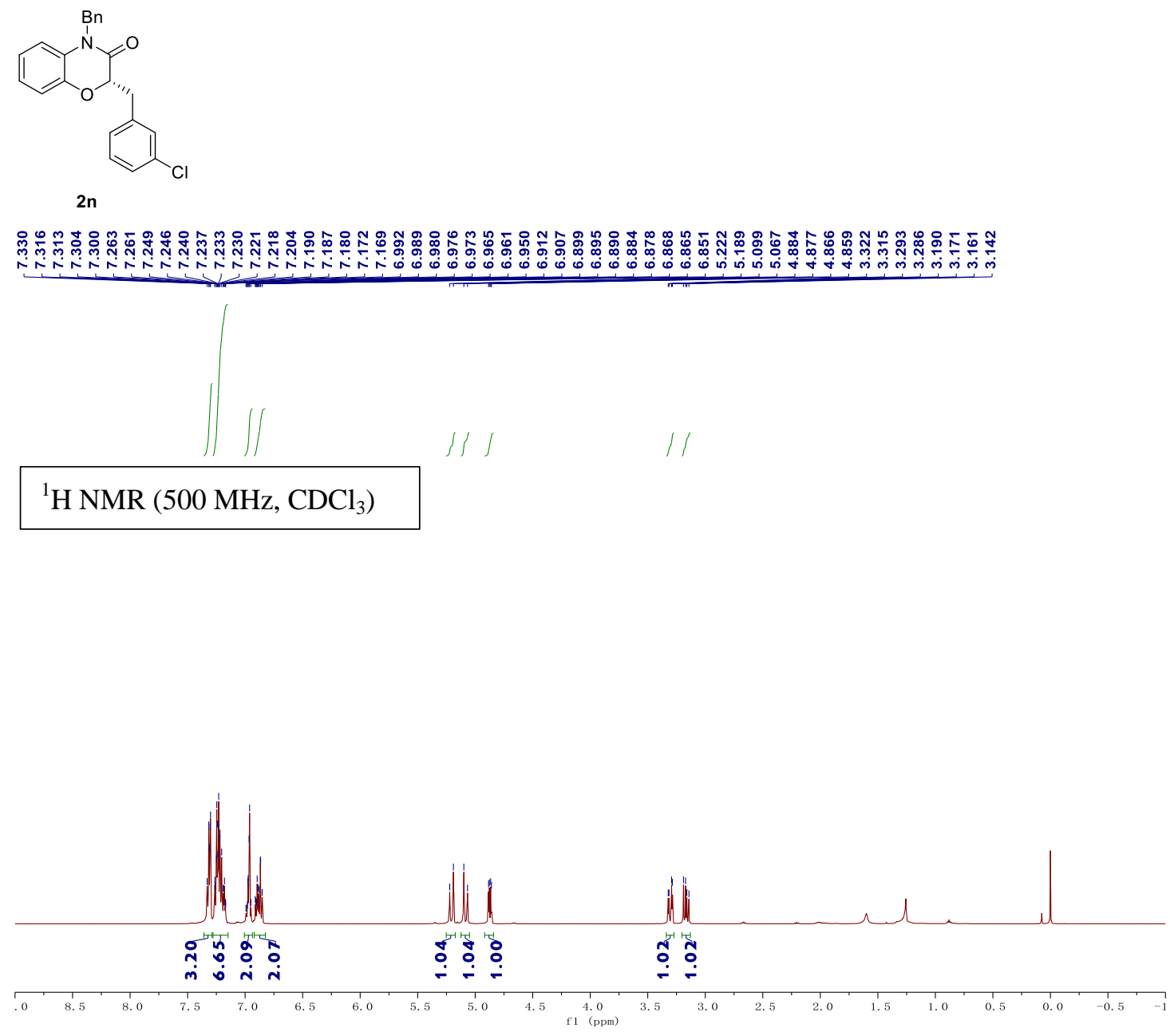

\begin{tabular}{|c|c|c|c|}
\hline $\begin{array}{l}\hat{\vdots} \\
\vdots \\
\dot{0} \\
\vdots \\
\end{array}$ & 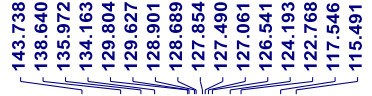 & $\begin{array}{l}\stackrel{8}{S} \\
\stackrel{5}{\hat{~}}\end{array}$ & $\begin{array}{l}\bar{p} \\
\dot{m} \\
\text { iq } \\
\text { | }\end{array}$ \\
\hline
\end{tabular}

${ }^{13} \mathrm{C}$ NMR (126 MHz, $\mathrm{CDCl}_{3}$ )
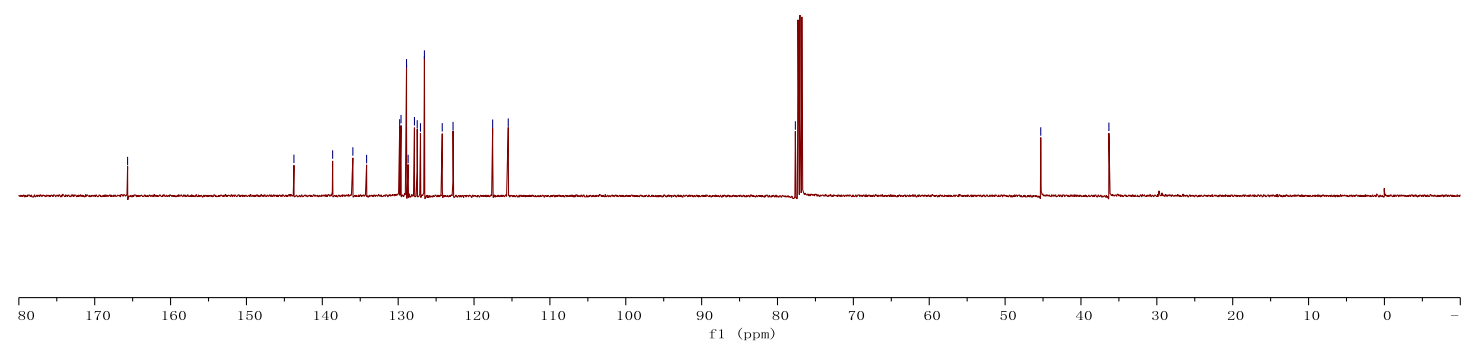

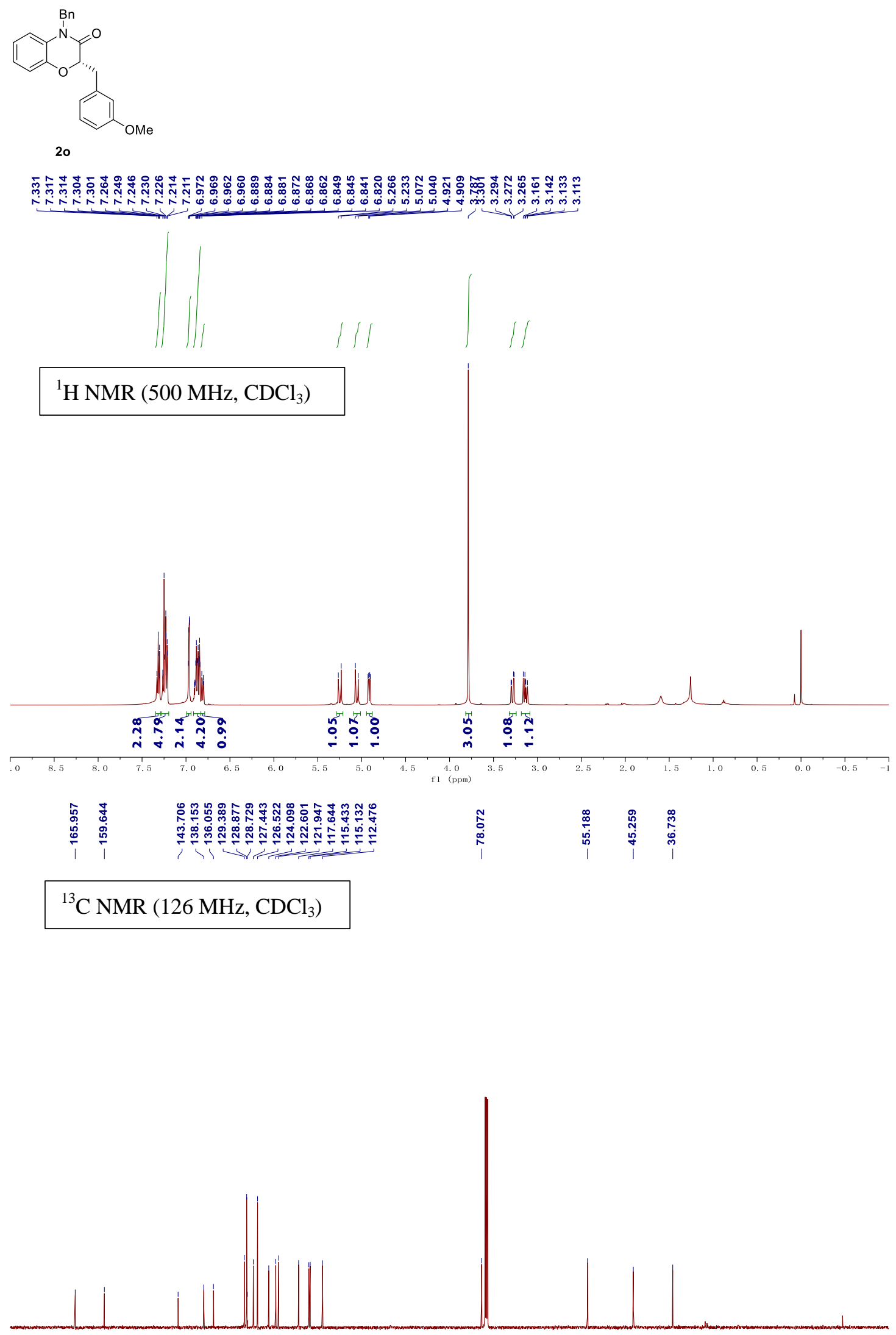


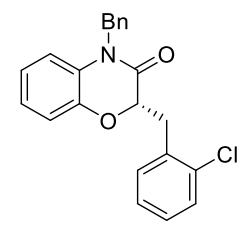

$2 p$

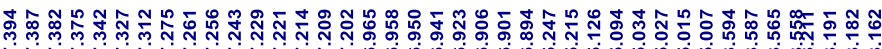 \\ 年}

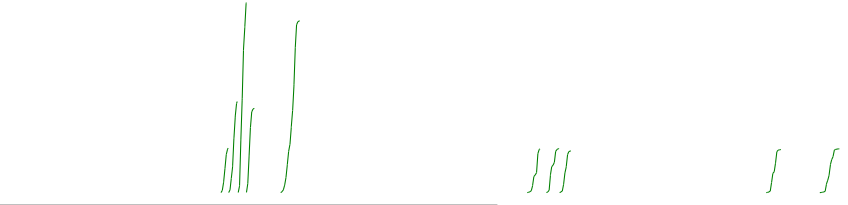

${ }^{1} \mathrm{H}$ NMR $\left(500 \mathrm{MHz}, \mathrm{CDCl}_{3}\right)$

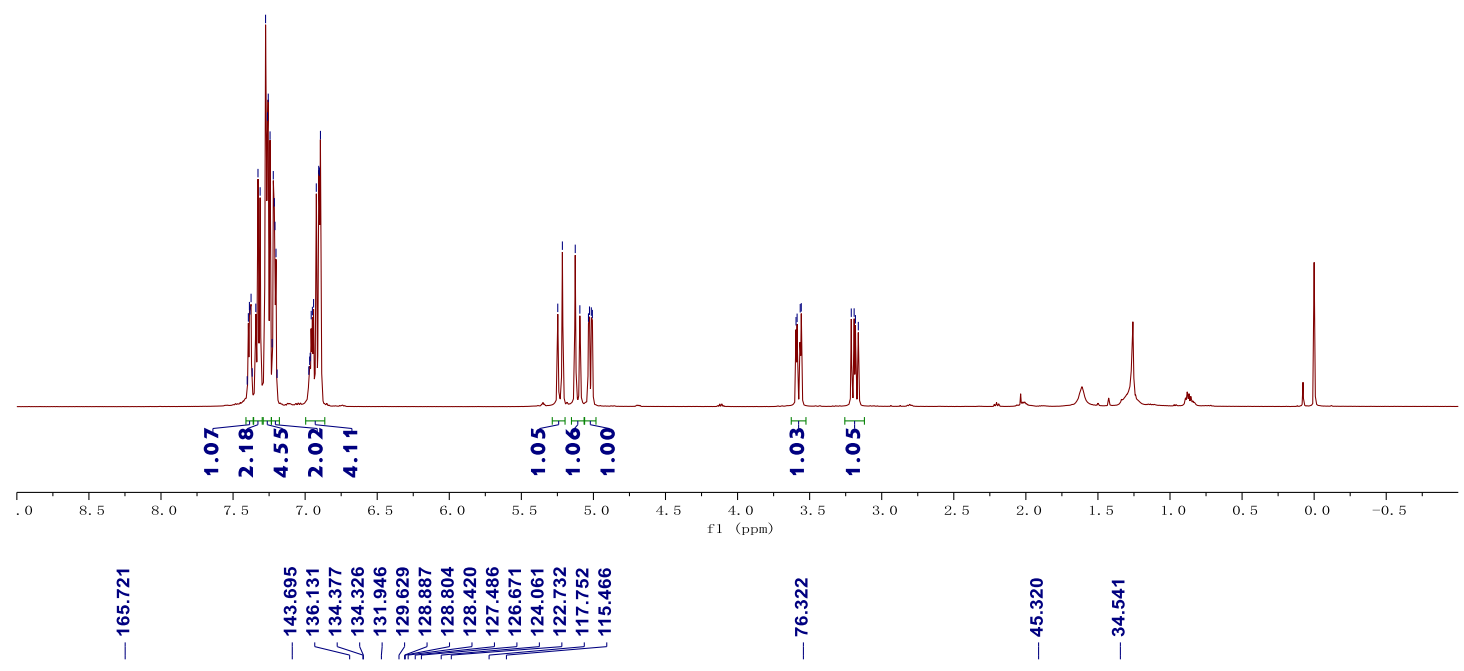

${ }^{13} \mathrm{C}$ NMR $\left(126 \mathrm{MHz}, \mathrm{CDCl}_{3}\right)$

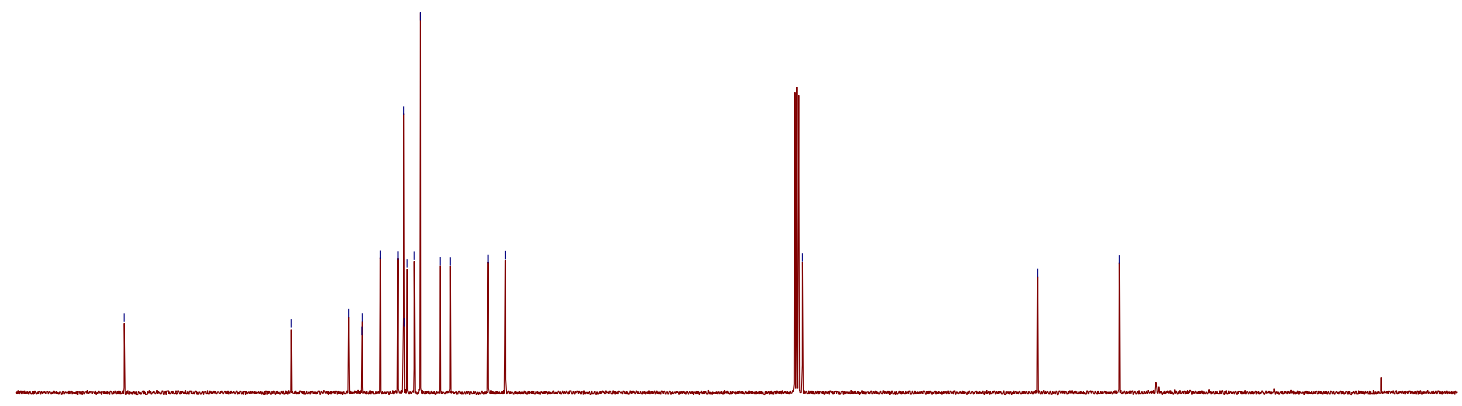

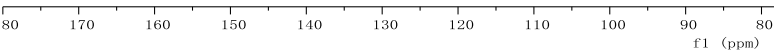




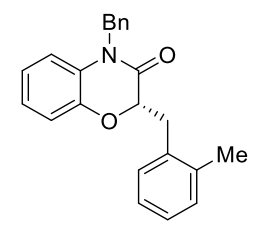

$2 q$

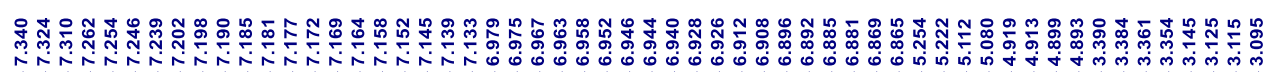

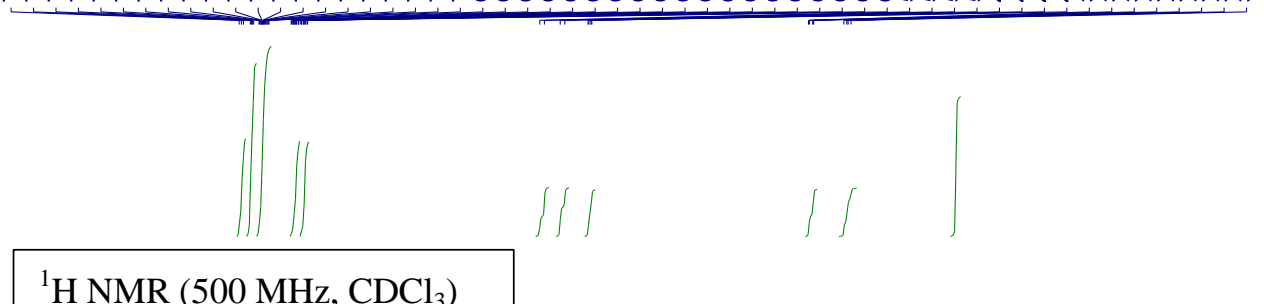

${ }^{1} \mathrm{H}$ NMR $\left(500 \mathrm{MHz}, \mathrm{CDCl}_{3}\right.$ )

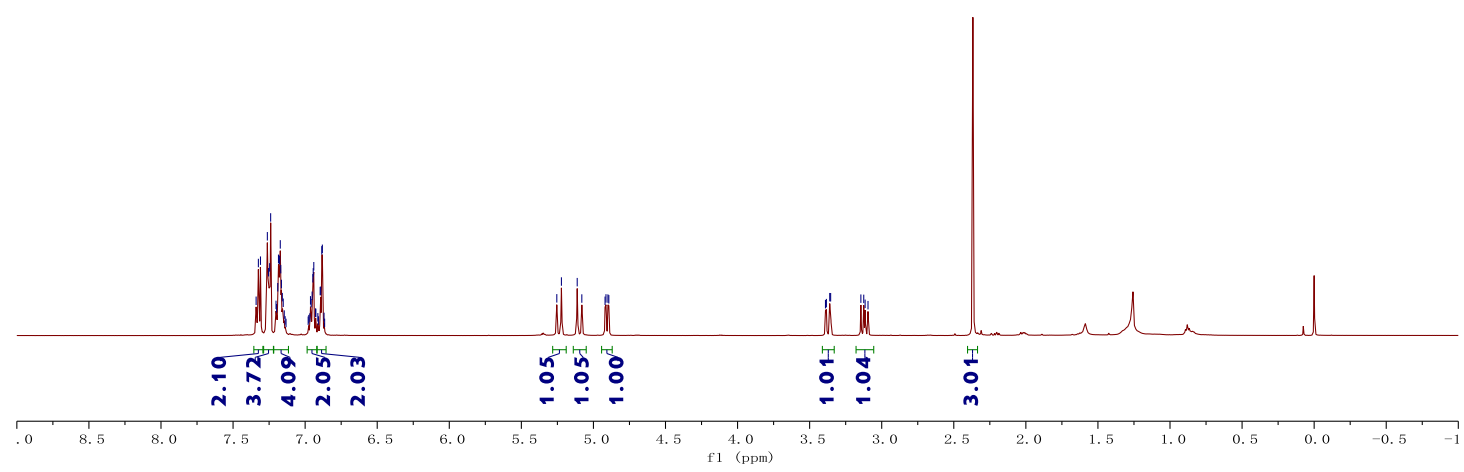

\begin{tabular}{|c|c|}
\hline & 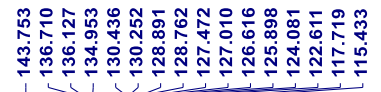 \\
\hline
\end{tabular}

${ }^{13} \mathrm{C}$ NMR $\left(126 \mathrm{MHz}, \mathrm{CDCl}_{3}\right)$

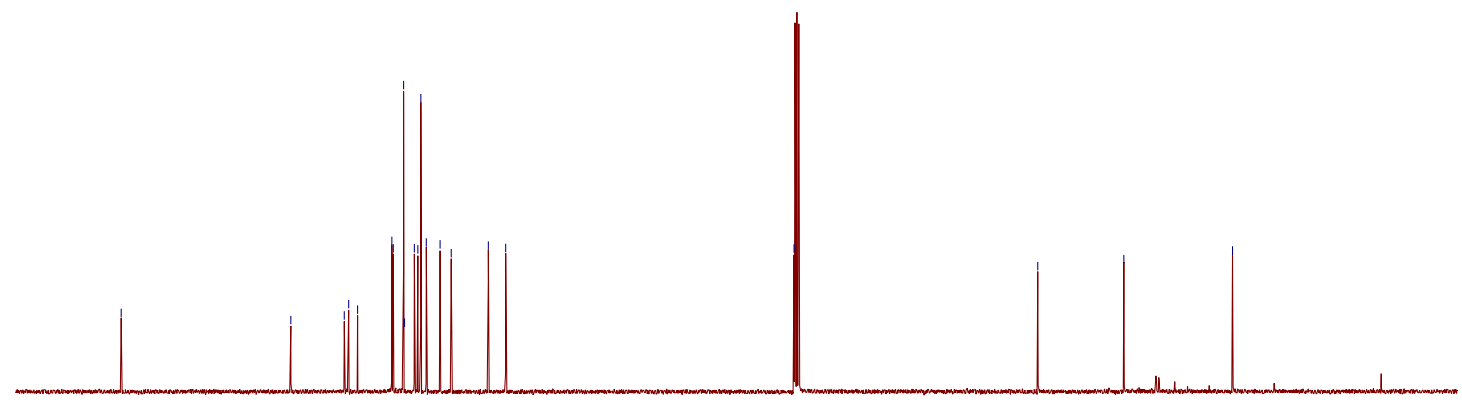

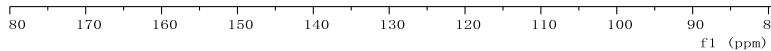




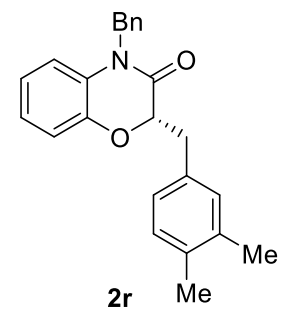

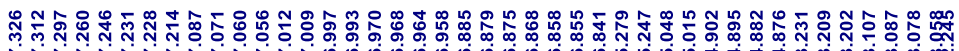

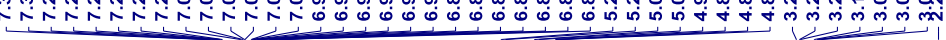
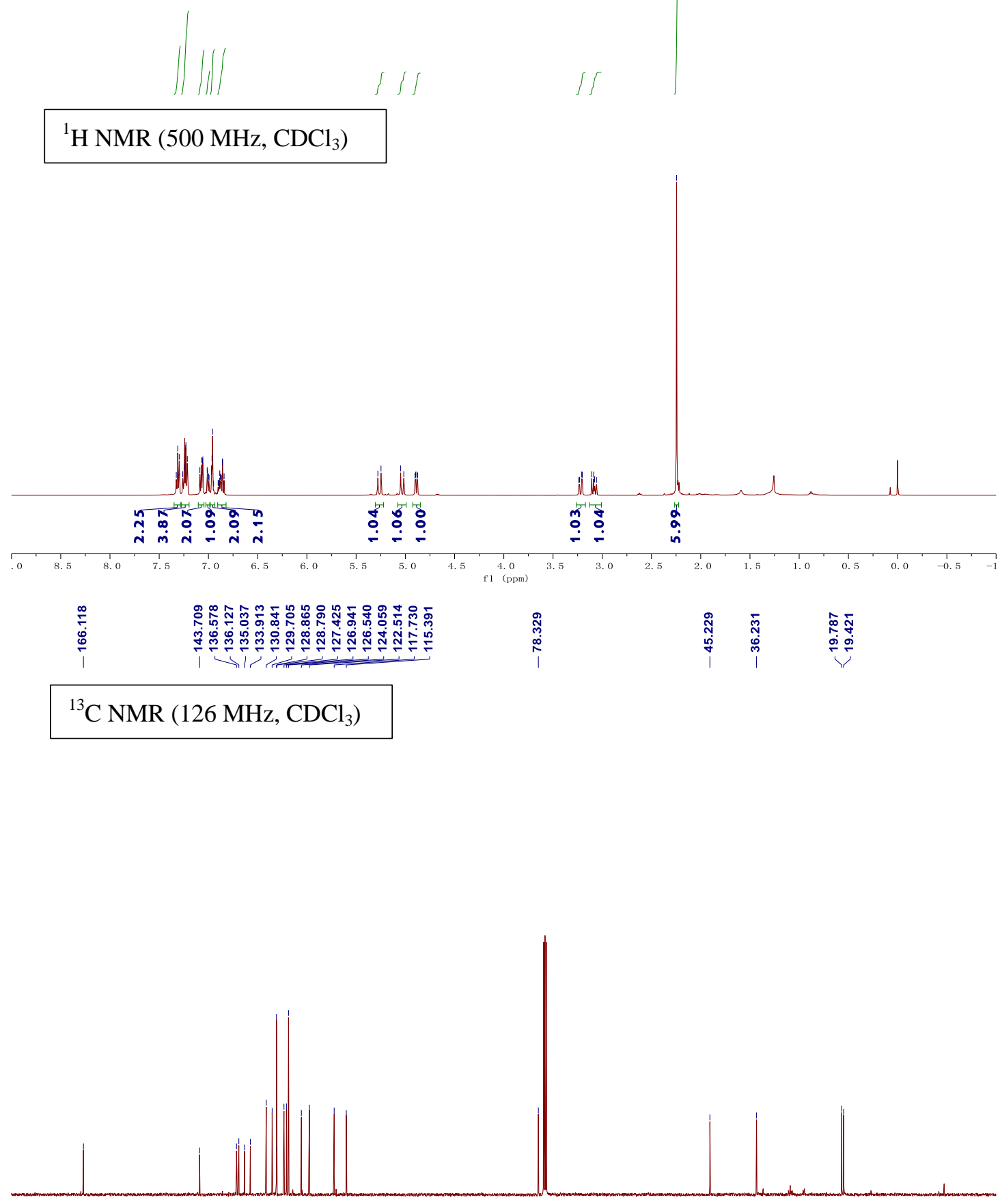

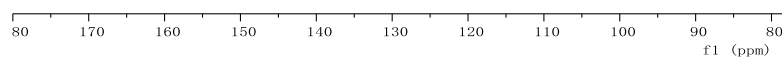




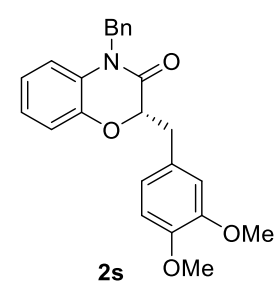

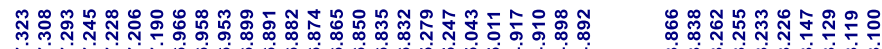

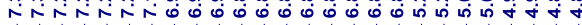

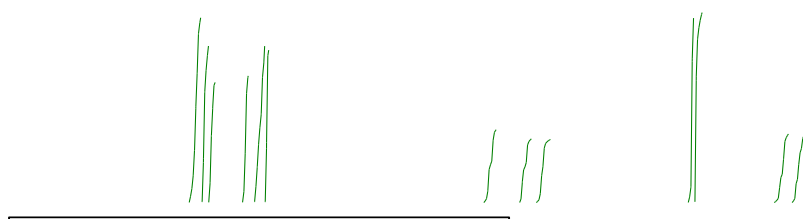

${ }^{1} \mathrm{H}$ NMR $\left(500 \mathrm{MHz}, \mathrm{CDCl}_{3}\right)$

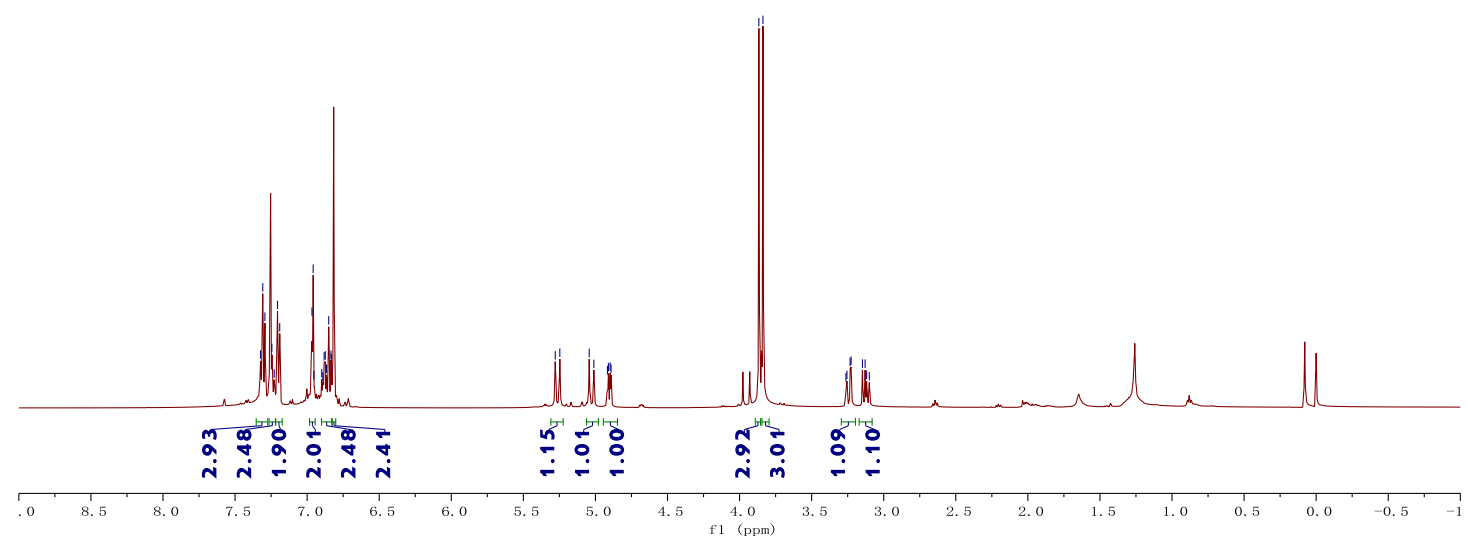

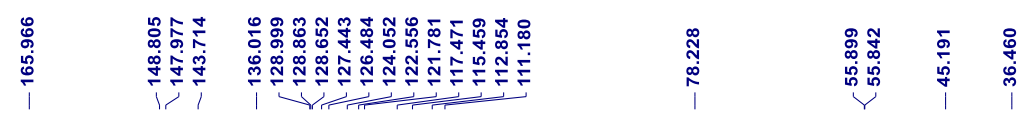

${ }^{13} \mathrm{C}$ NMR $\left(126 \mathrm{MHz}, \mathrm{CDCl}_{3}\right)$

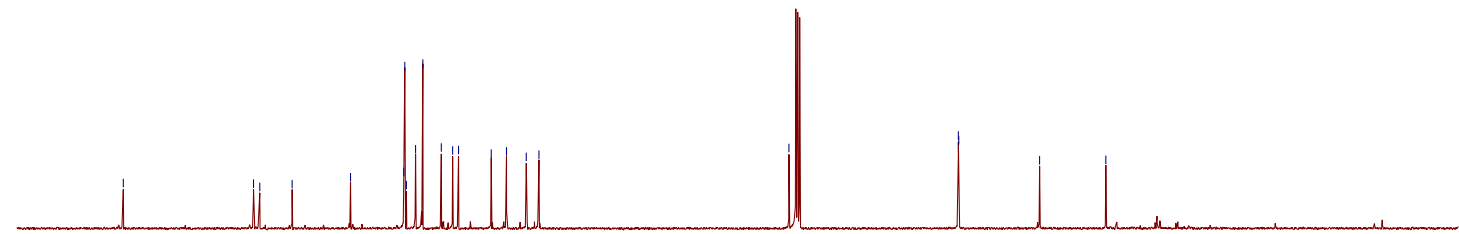

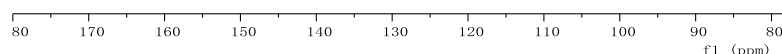




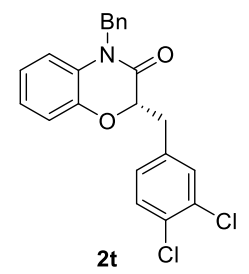

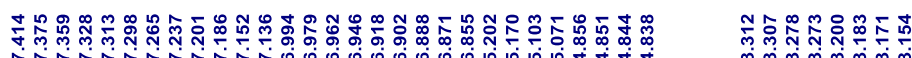

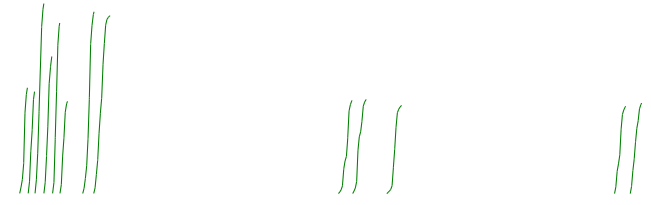

${ }^{1} \mathrm{H}$ NMR $\left(500 \mathrm{MHz}, \mathrm{CDCl}_{3}\right)$

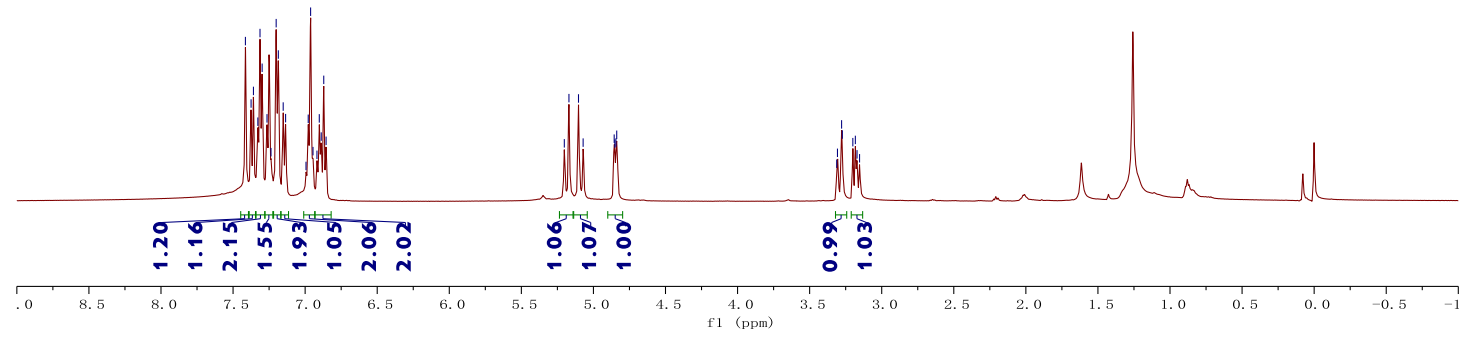

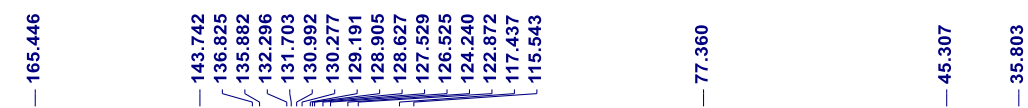

${ }^{13} \mathrm{C}$ NMR (126 MHz, $\left.\mathrm{CDCl}_{3}\right)$
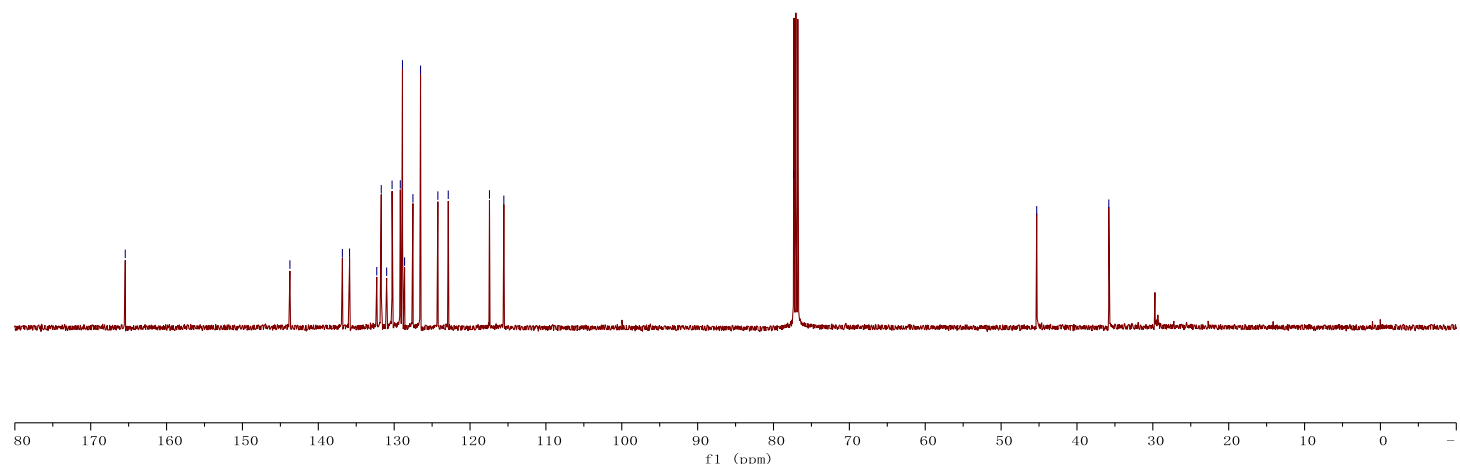


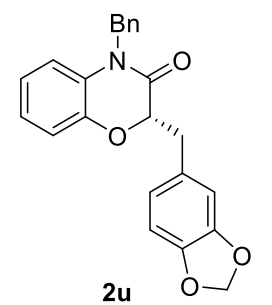

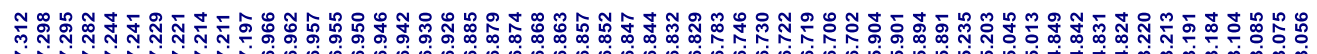

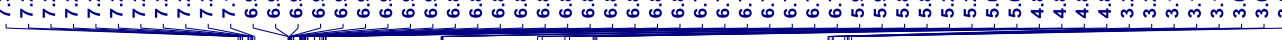

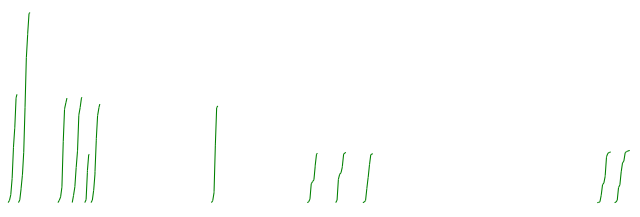

${ }^{1} \mathrm{H}$ NMR $\left(500 \mathrm{MHz}, \mathrm{CDCl}_{3}\right)$

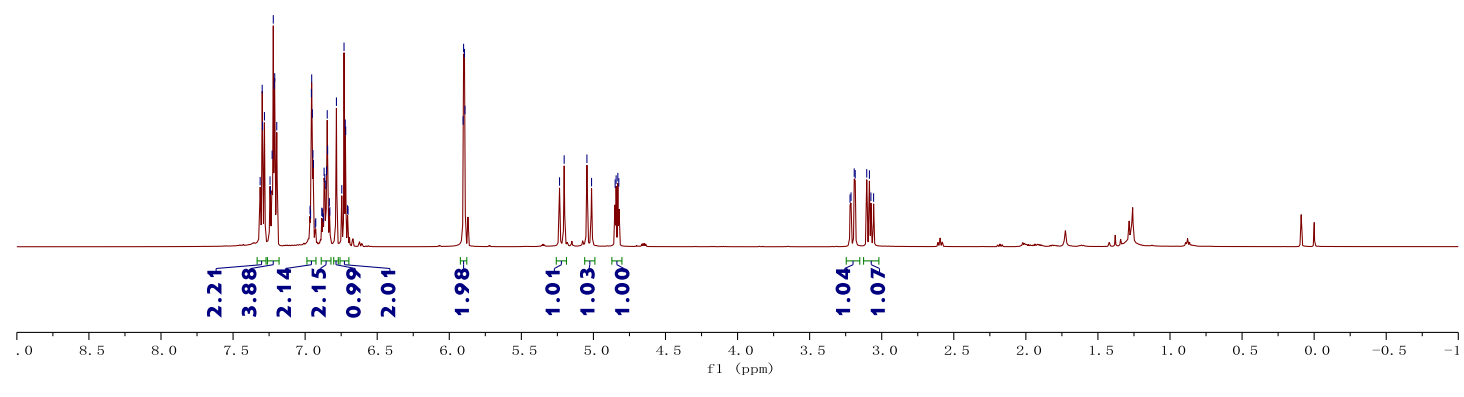

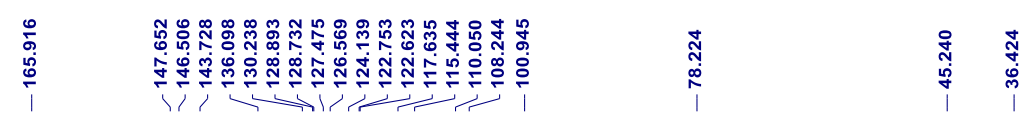

${ }^{13} \mathrm{C} \mathrm{NMR}\left(126 \mathrm{MHz}, \mathrm{CDCl}_{3}\right)$

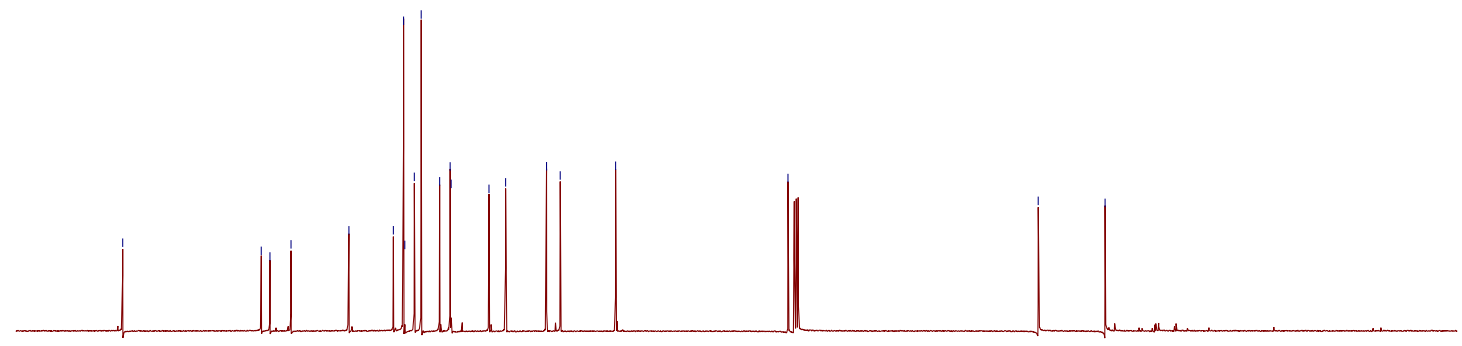

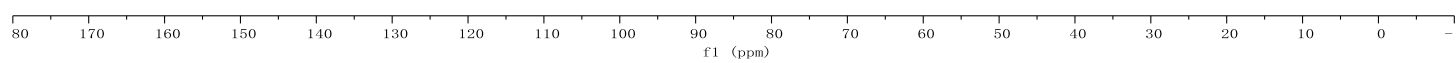




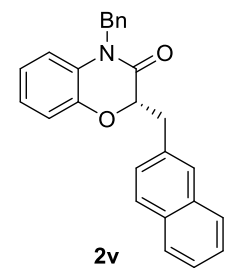

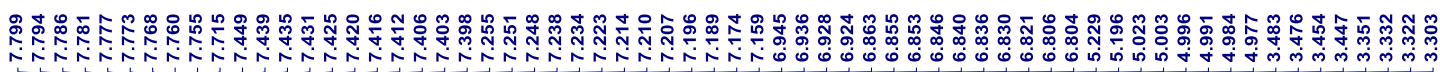

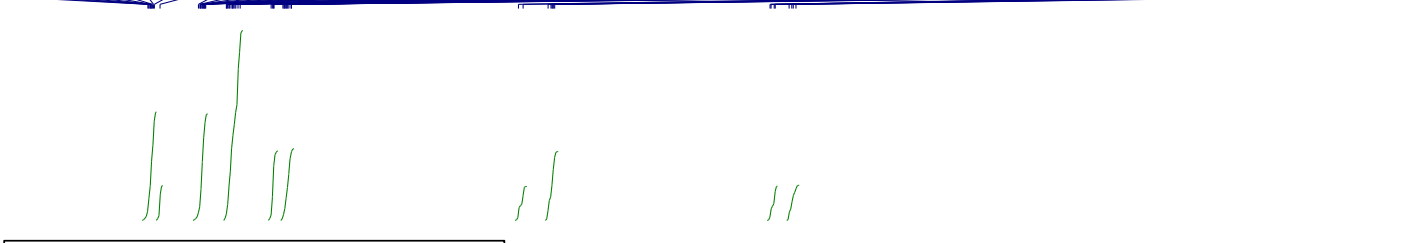

${ }^{1} \mathrm{H}$ NMR $\left(500 \mathrm{MHz}, \mathrm{CDCl}_{3}\right)$

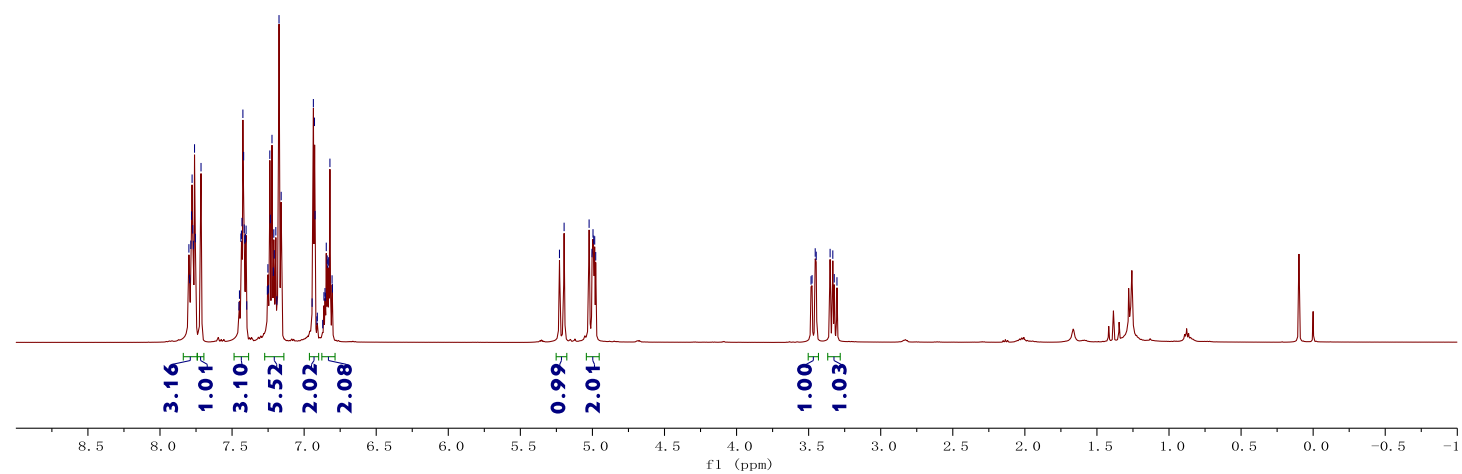

I

${ }^{13} \mathrm{C}$ NMR $\left(126 \mathrm{MHz}, \mathrm{CDCl}_{3}\right)$

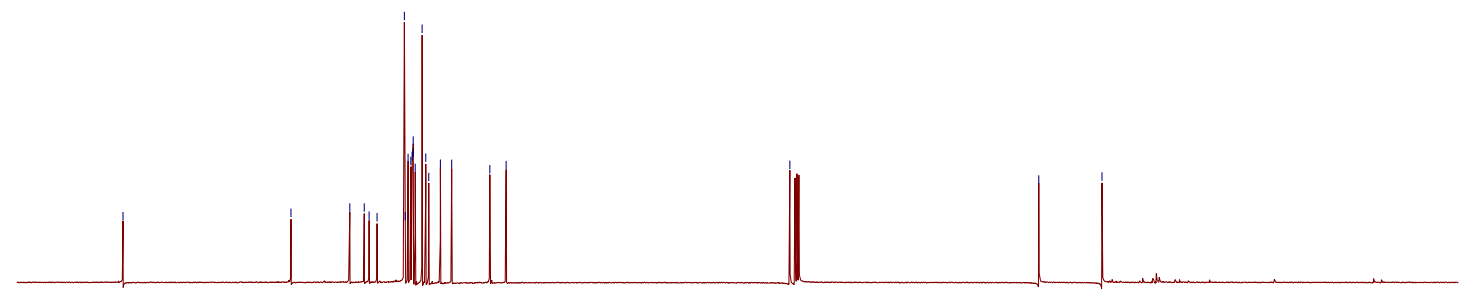

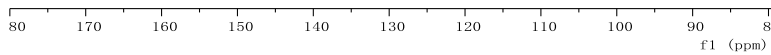




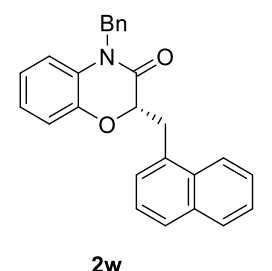

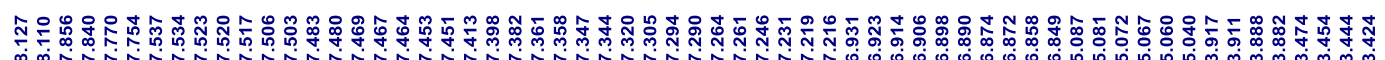

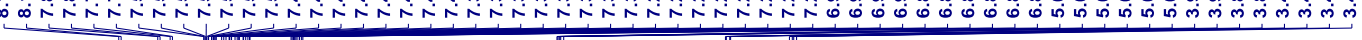
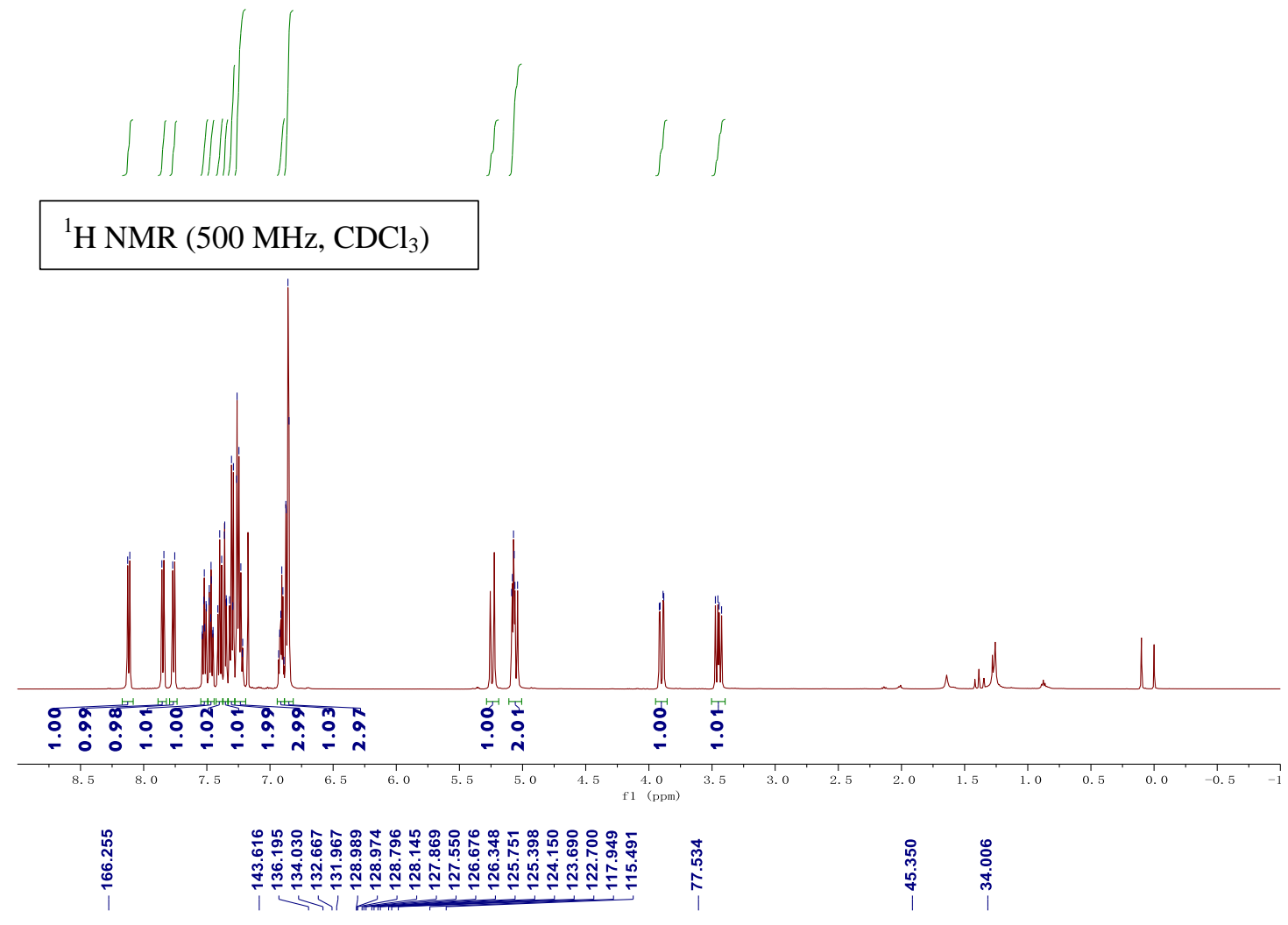

${ }^{13} \mathrm{C}$ NMR $\left(126 \mathrm{MHz}, \mathrm{CDCl}_{3}\right)$

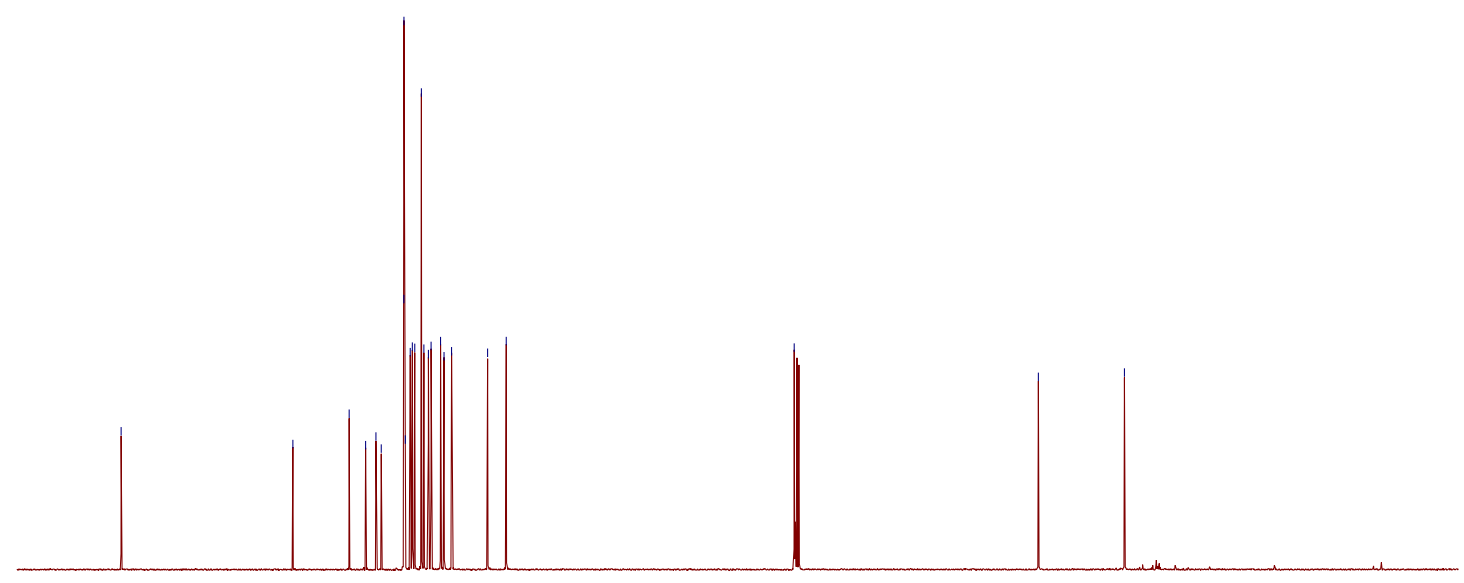

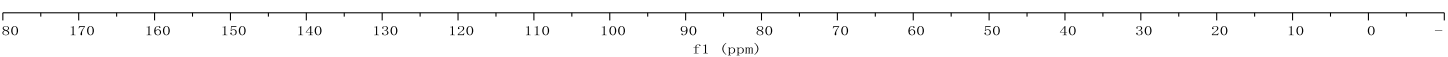




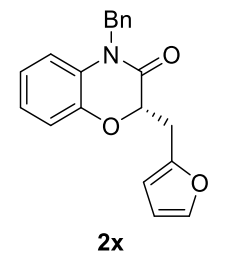

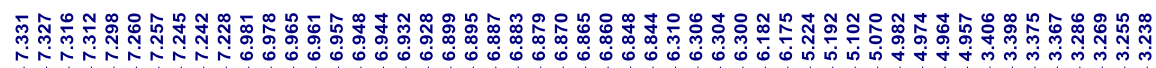

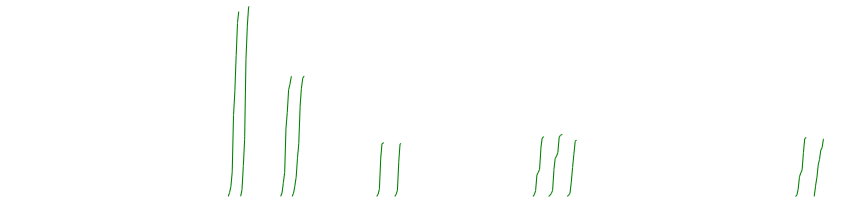

${ }^{1} \mathrm{H}$ NMR (500 MHz, $\mathrm{CDCl}_{3}$ )

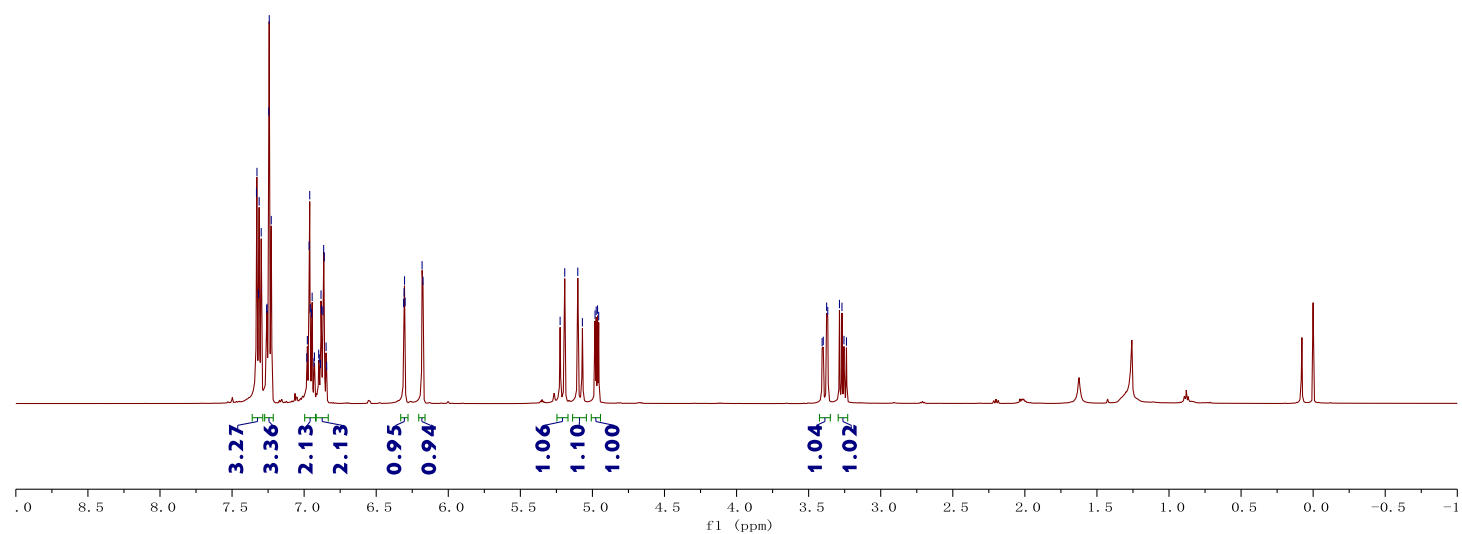

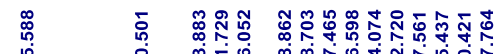

定

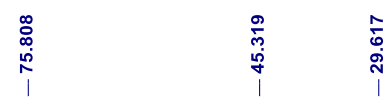

${ }^{13} \mathrm{C}$ NMR $\left(126 \mathrm{MHz}, \mathrm{CDCl}_{3}\right)$

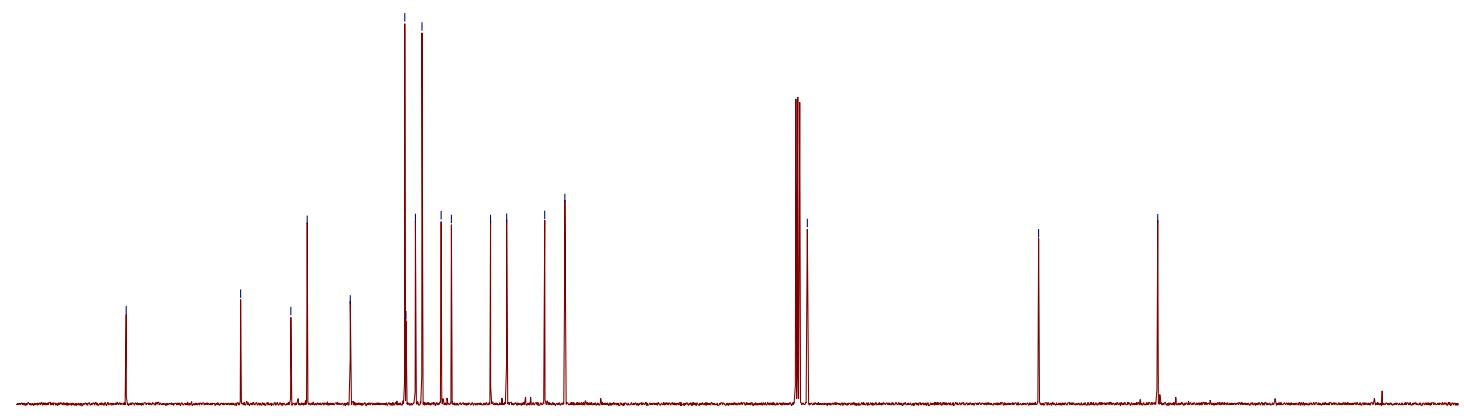

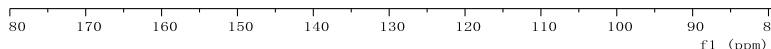




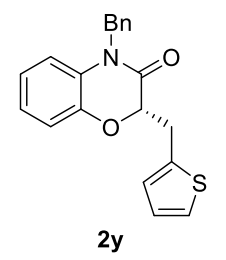

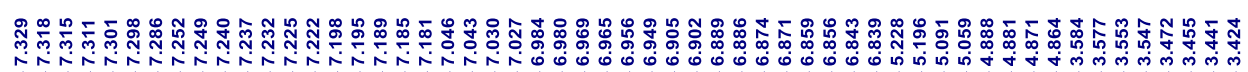

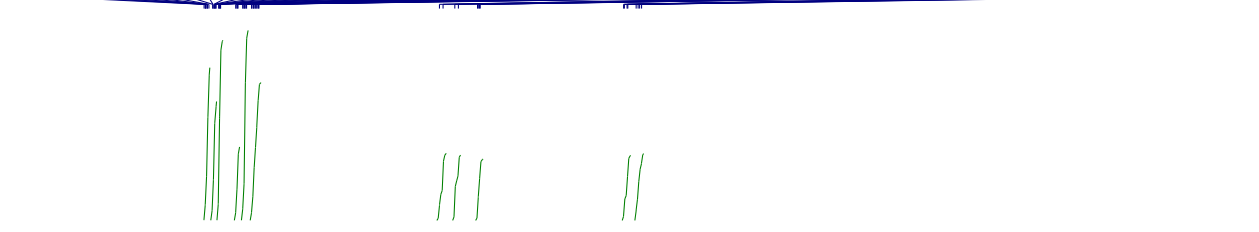

${ }^{1} \mathrm{H}$ NMR (500 MHz, $\mathrm{CDCl}_{3}$ )
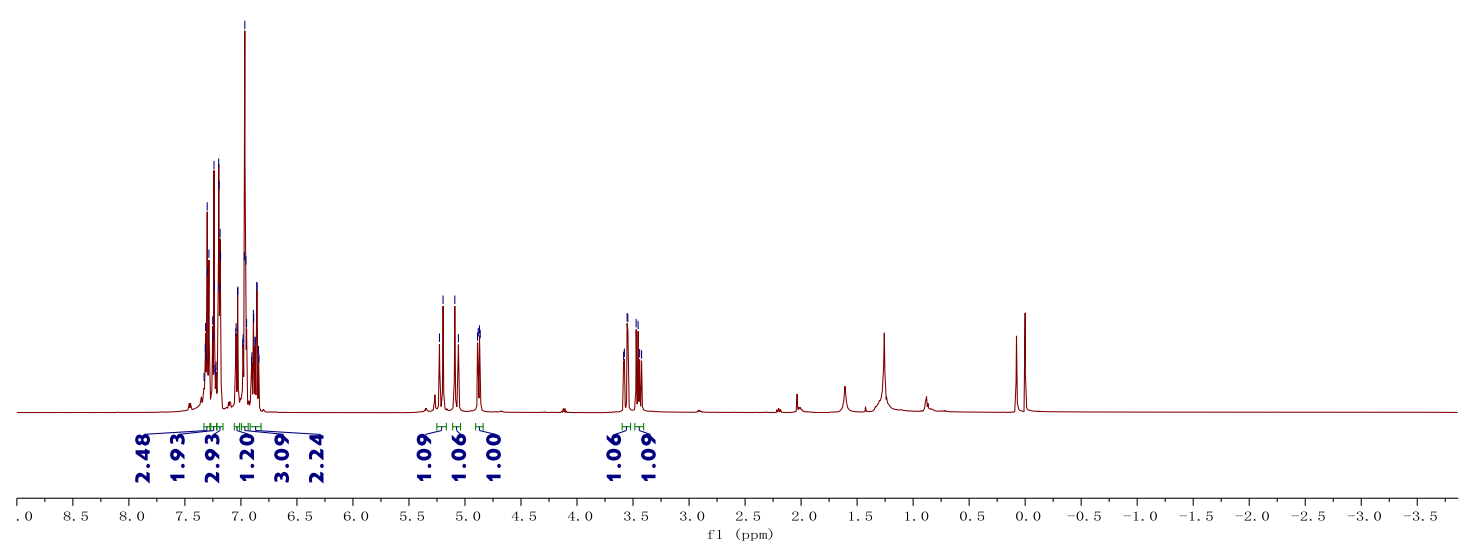

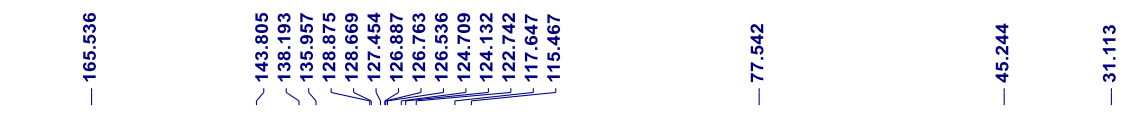

${ }^{13} \mathrm{C}$ NMR $\left(126 \mathrm{MHz}, \mathrm{CDCl}_{3}\right)$
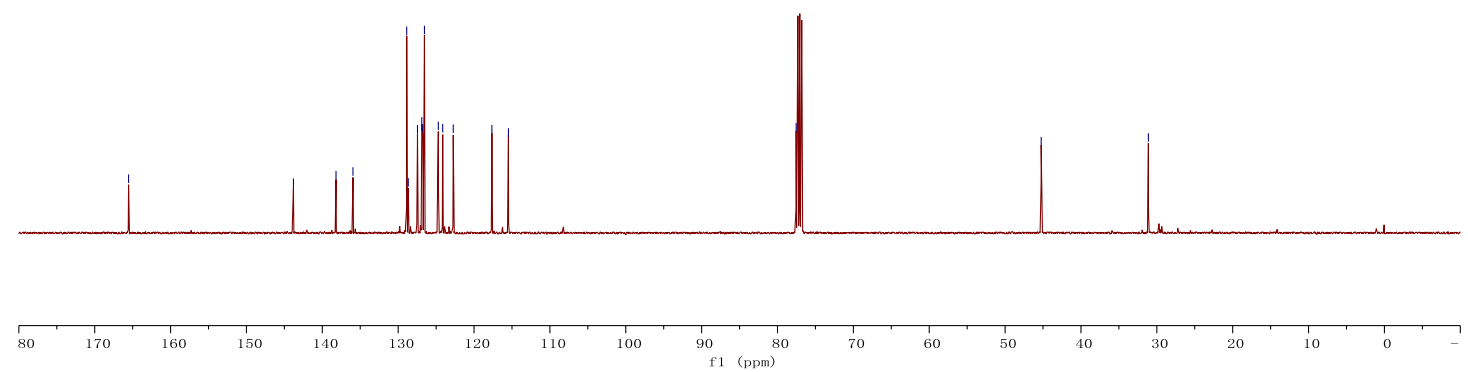


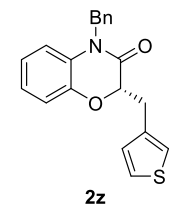

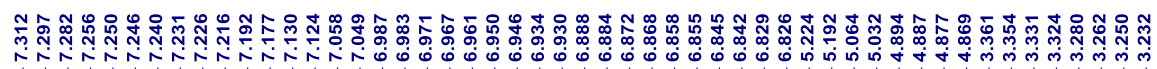

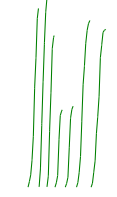

${ }^{1} \mathrm{H}$ NMR $\left(500 \mathrm{MHz}, \mathrm{CDCl}_{3}\right.$ )

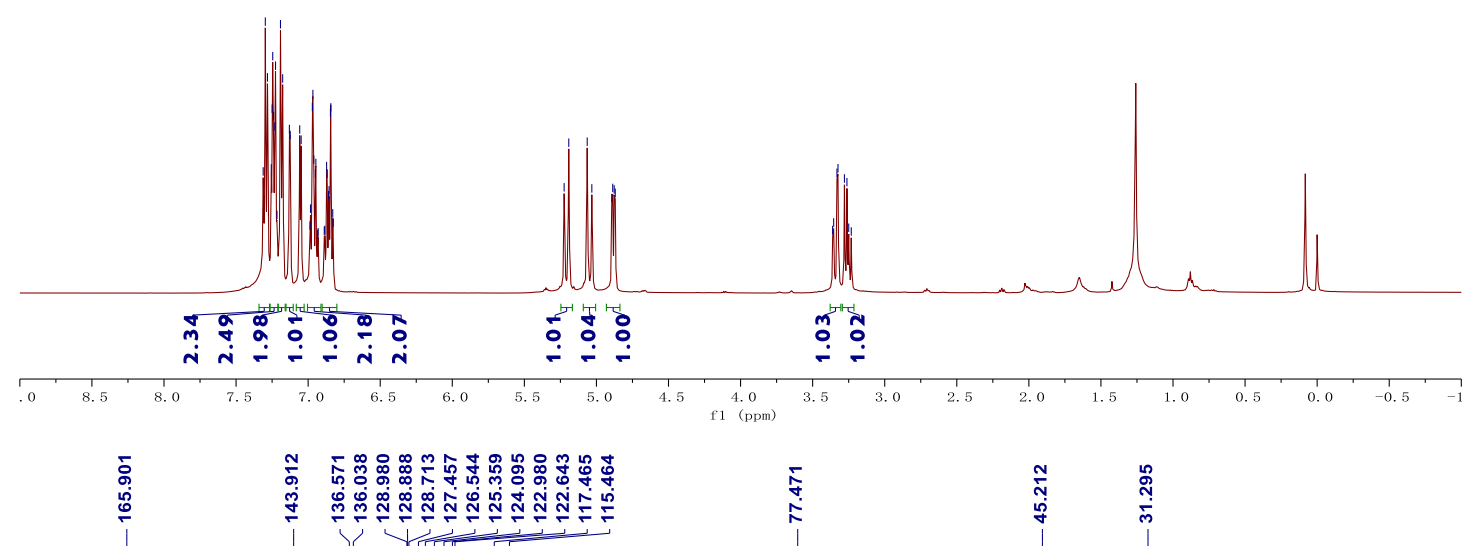

${ }^{13} \mathrm{C}$ NMR (126 MHz, $\mathrm{CDCl}_{3}$ )

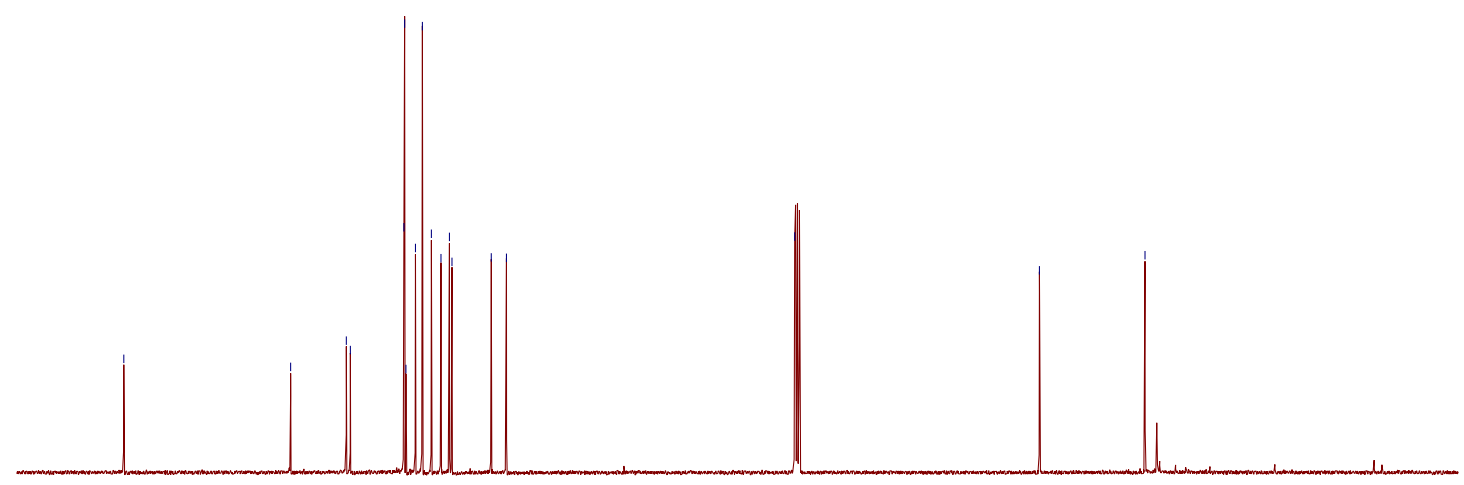




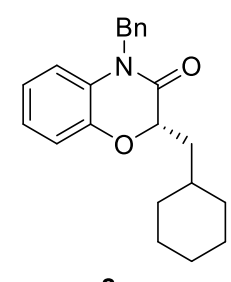

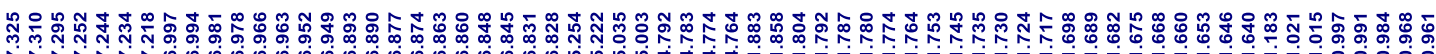
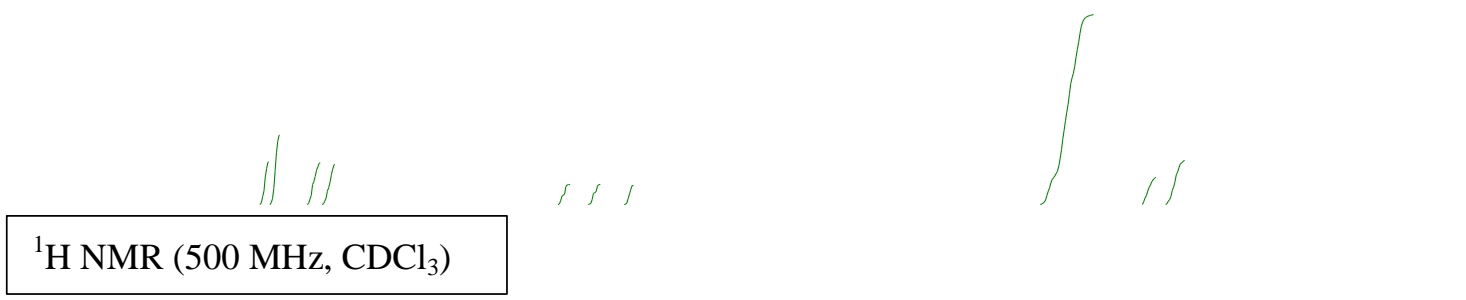

'H NMR (500 MHz, $\left.\mathrm{CDCl}_{3}\right)$
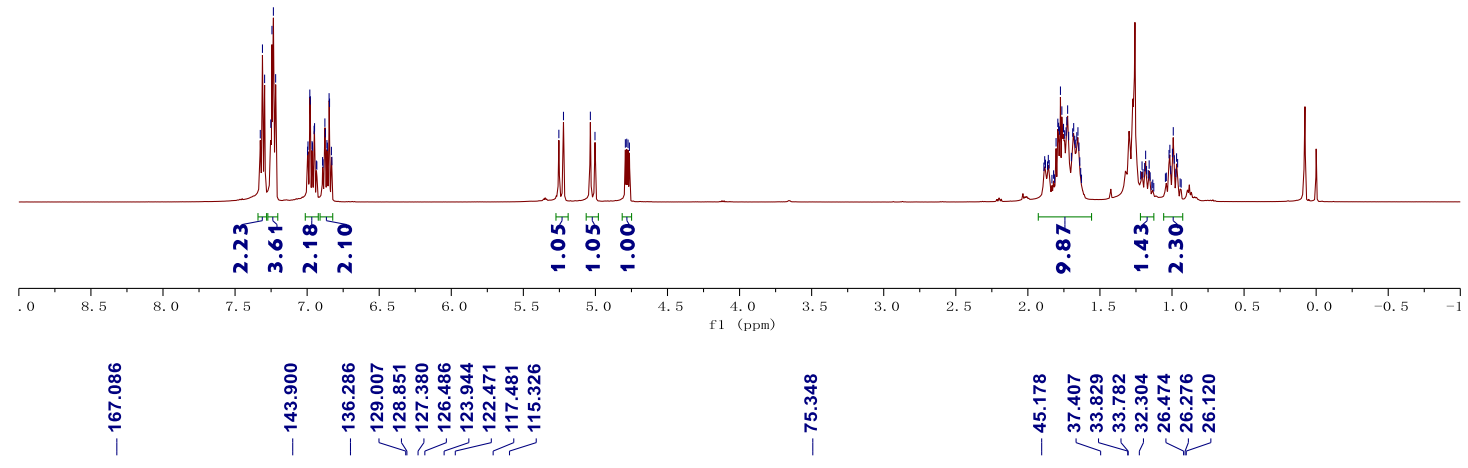

${ }^{13} \mathrm{C}$ NMR $\left(126 \mathrm{MHz}, \mathrm{CDCl}_{3}\right)$
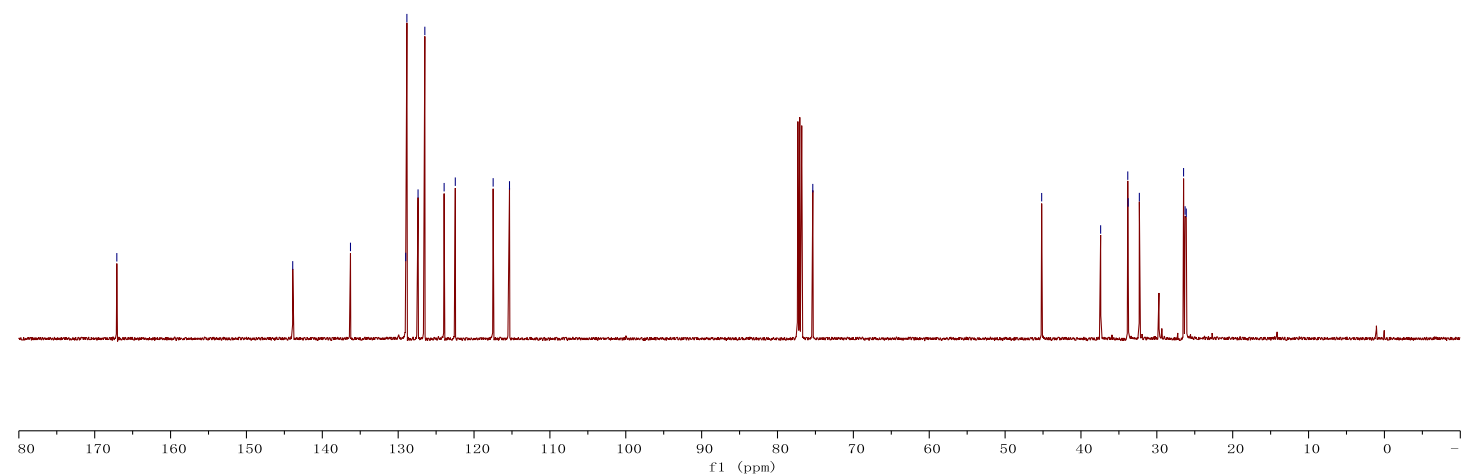

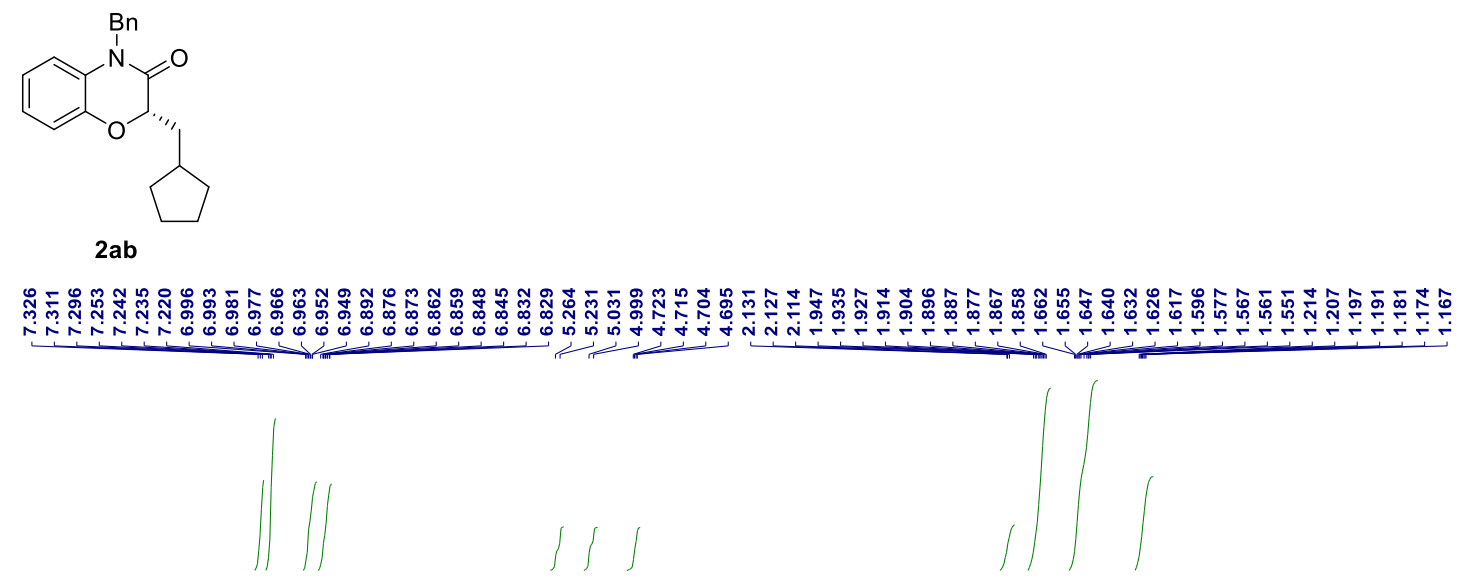

${ }^{1} \mathrm{H}$ NMR (500 MHz, $\mathrm{CDCl}_{3}$ )
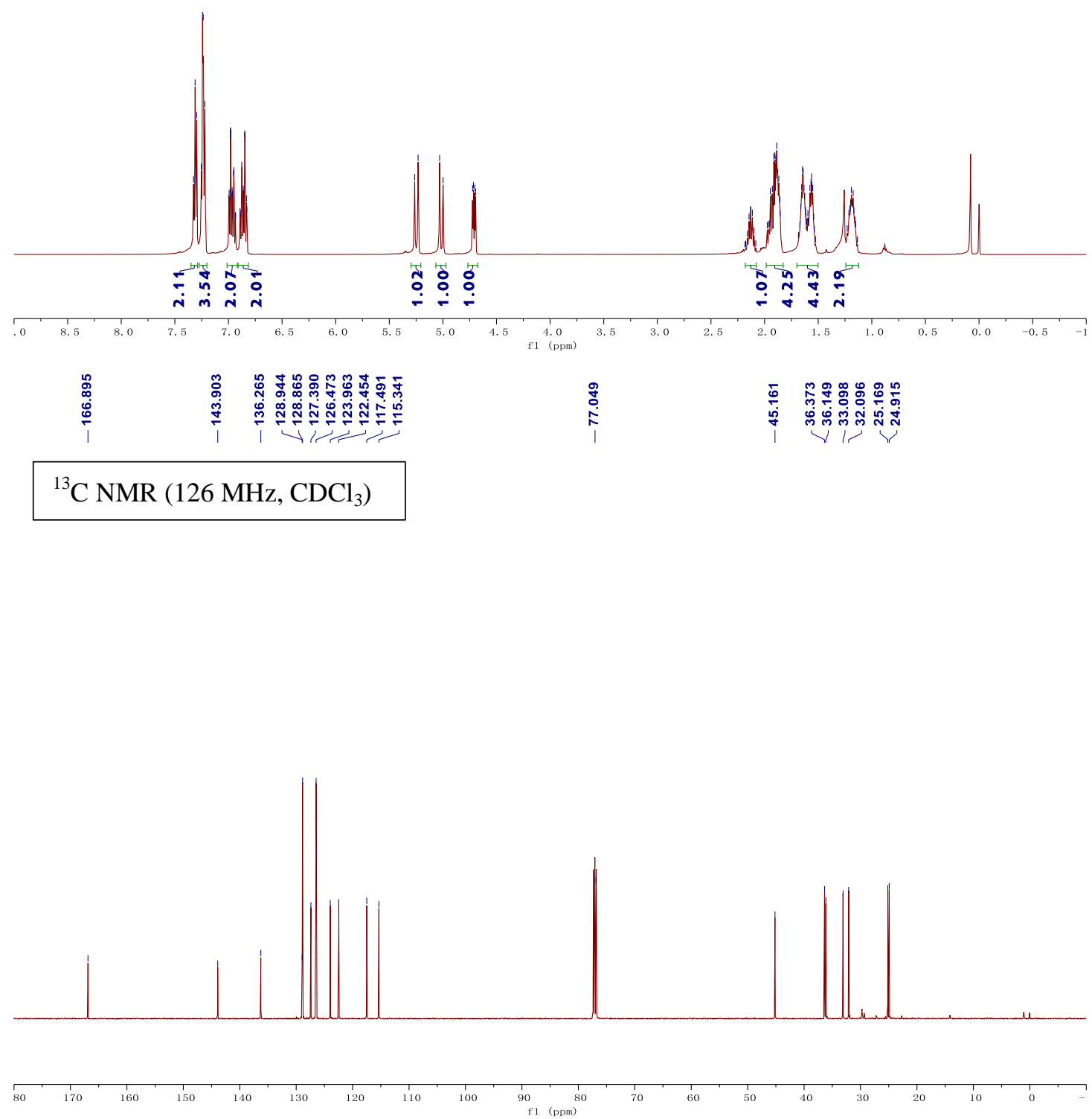


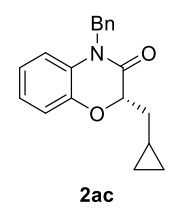

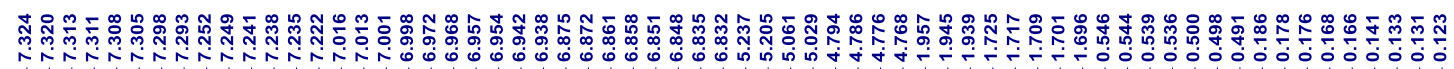

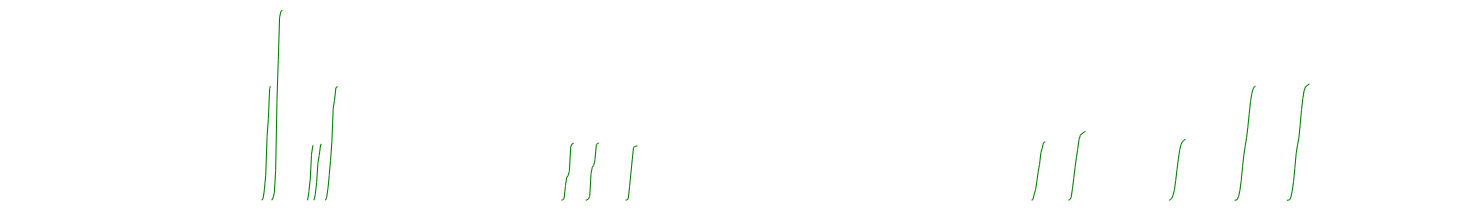

${ }^{1} \mathrm{H}$ NMR $\left(500 \mathrm{MHz}, \mathrm{CDCl}_{3}\right)$
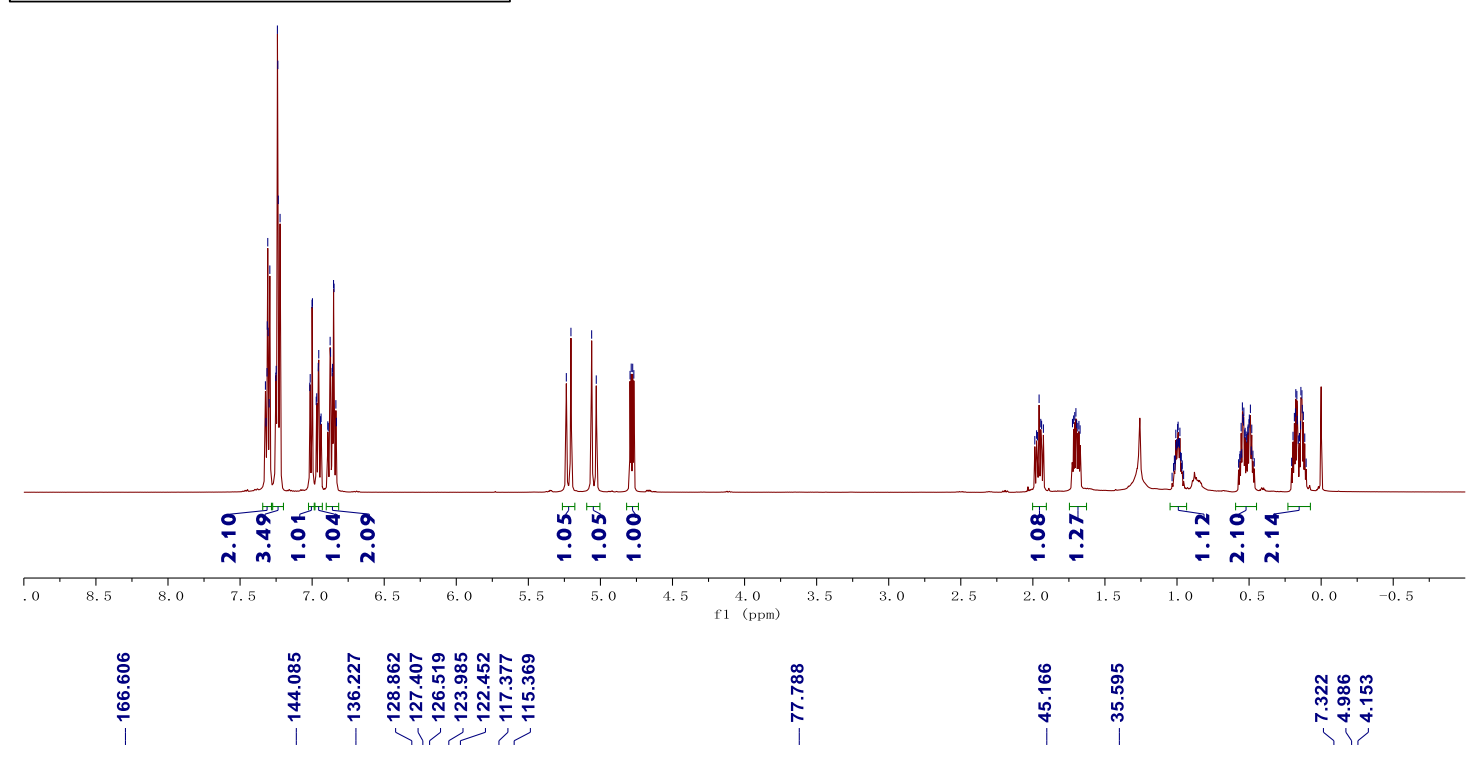

${ }^{13} \mathrm{C}$ NMR $\left(126 \mathrm{MHz}, \mathrm{CDCl}_{3}\right)$

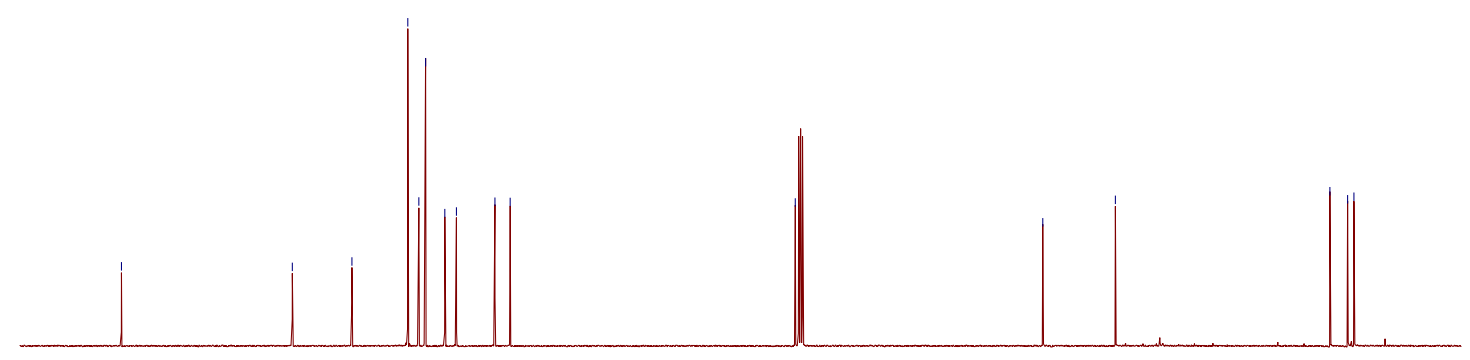




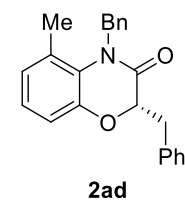

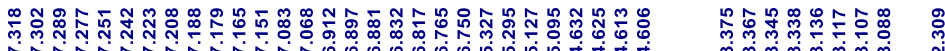

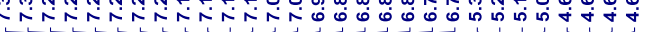

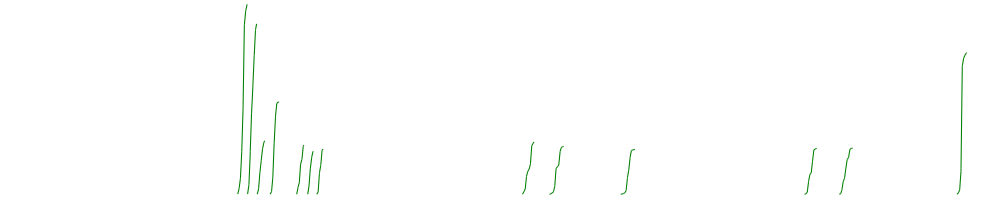

${ }^{1} \mathrm{H}$ NMR $\left(500 \mathrm{MHz}, \mathrm{CDCl}_{3}\right)$

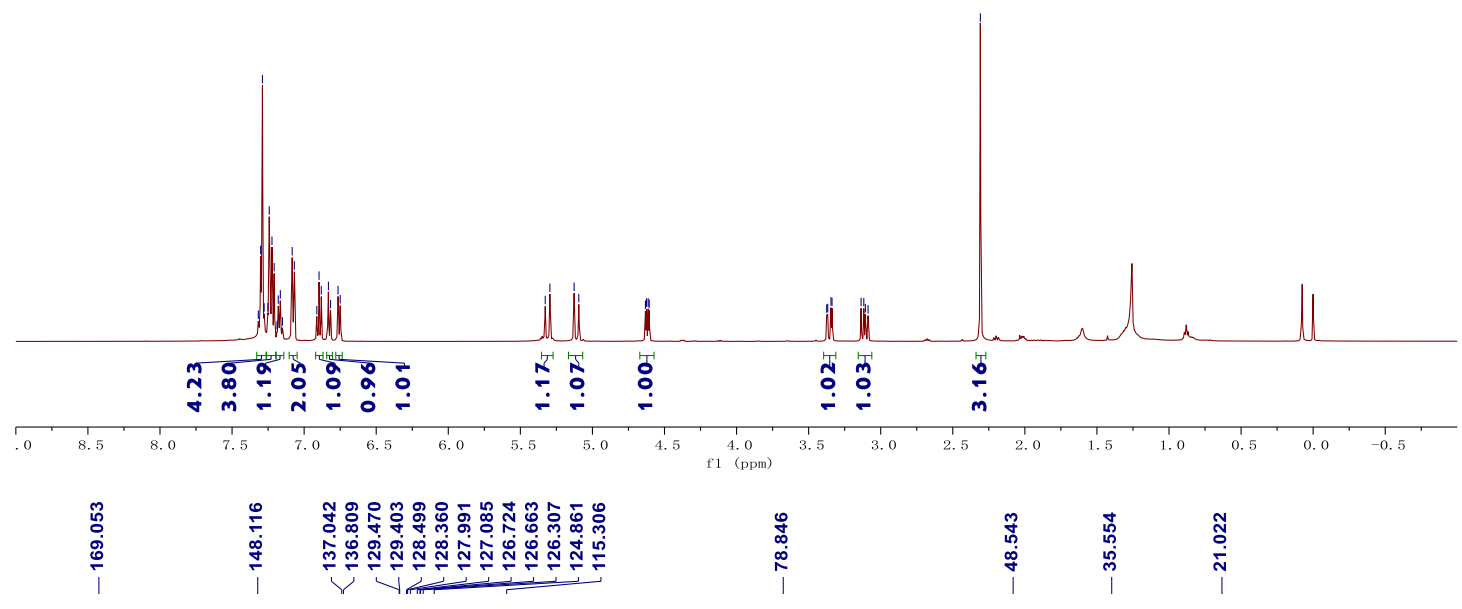

${ }^{13} \mathrm{C}$ NMR $\left(126 \mathrm{MHz}, \mathrm{CDCl}_{3}\right)$

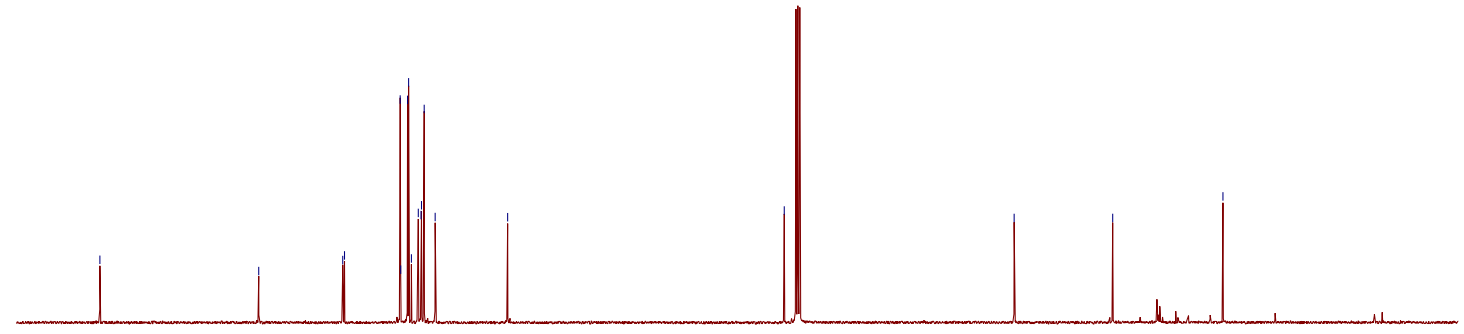




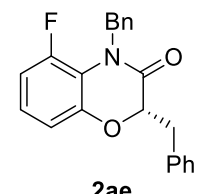

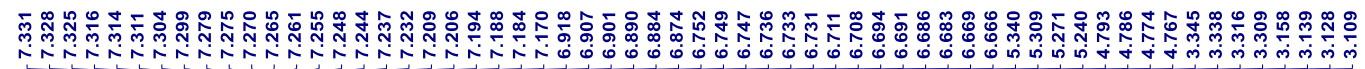
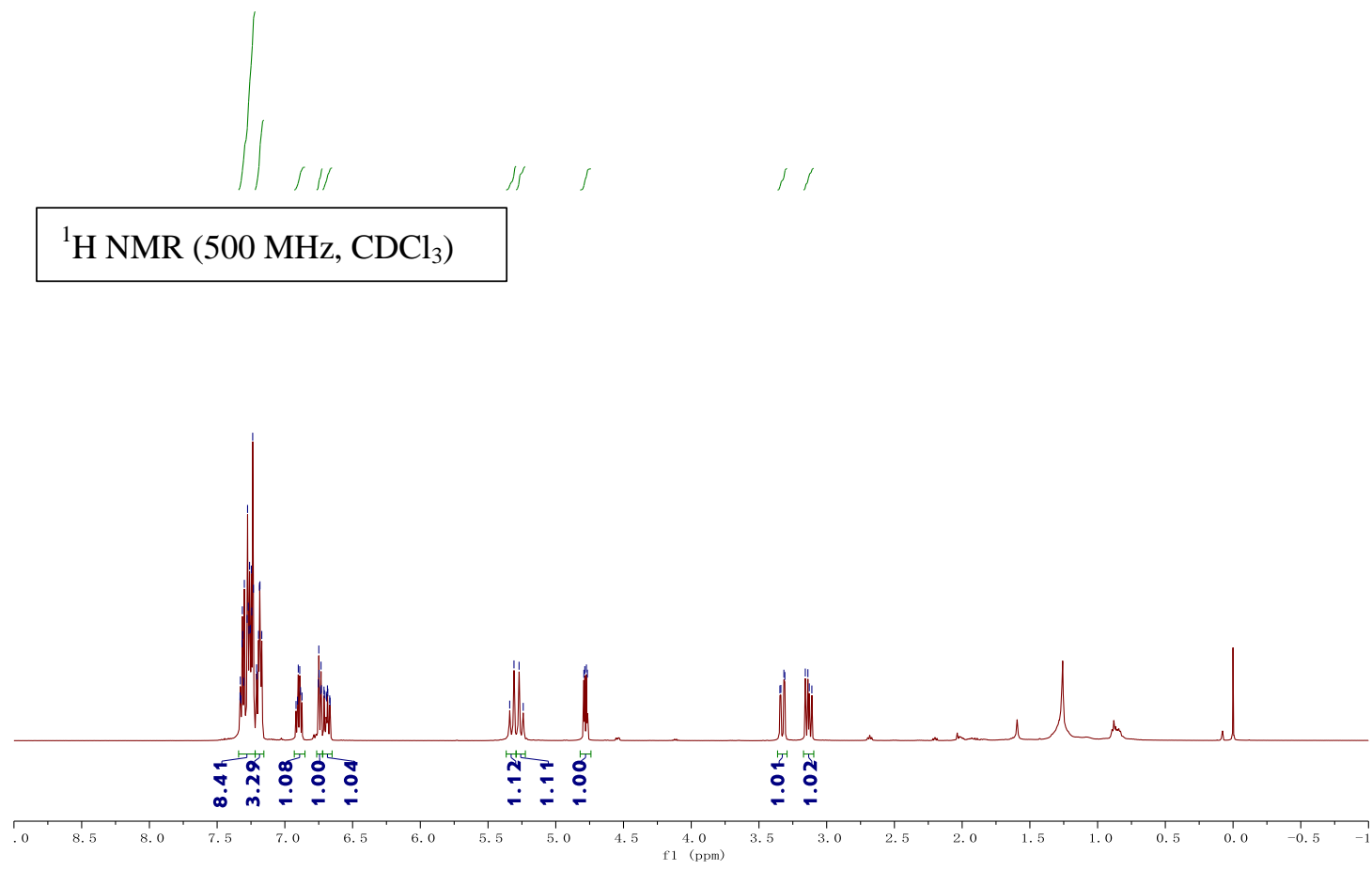

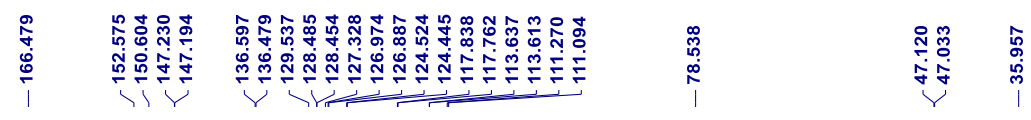

${ }^{13} \mathrm{C} \mathrm{NMR}\left(126 \mathrm{MHz}, \mathrm{CDCl}_{3}\right)$

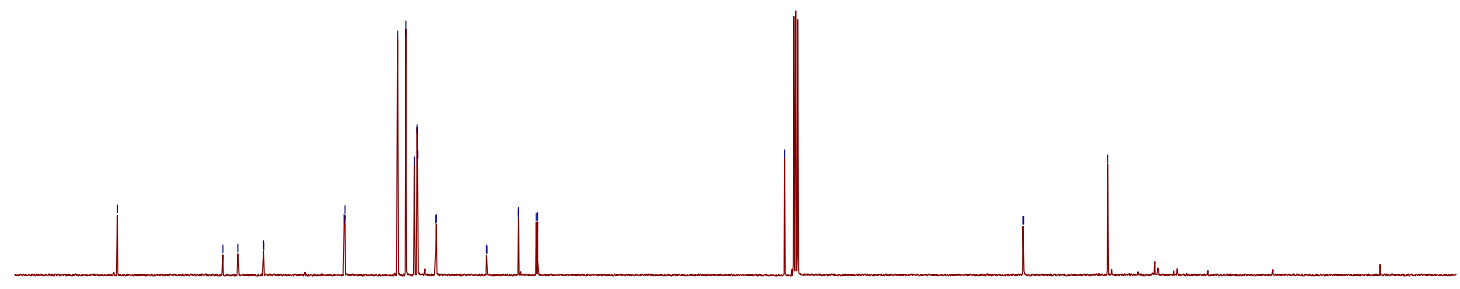

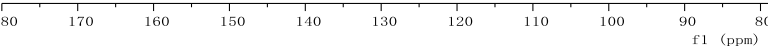


${ }^{19} \mathrm{~F}$ NMR (471 MHz, $\mathrm{CDCl}_{3}$ )

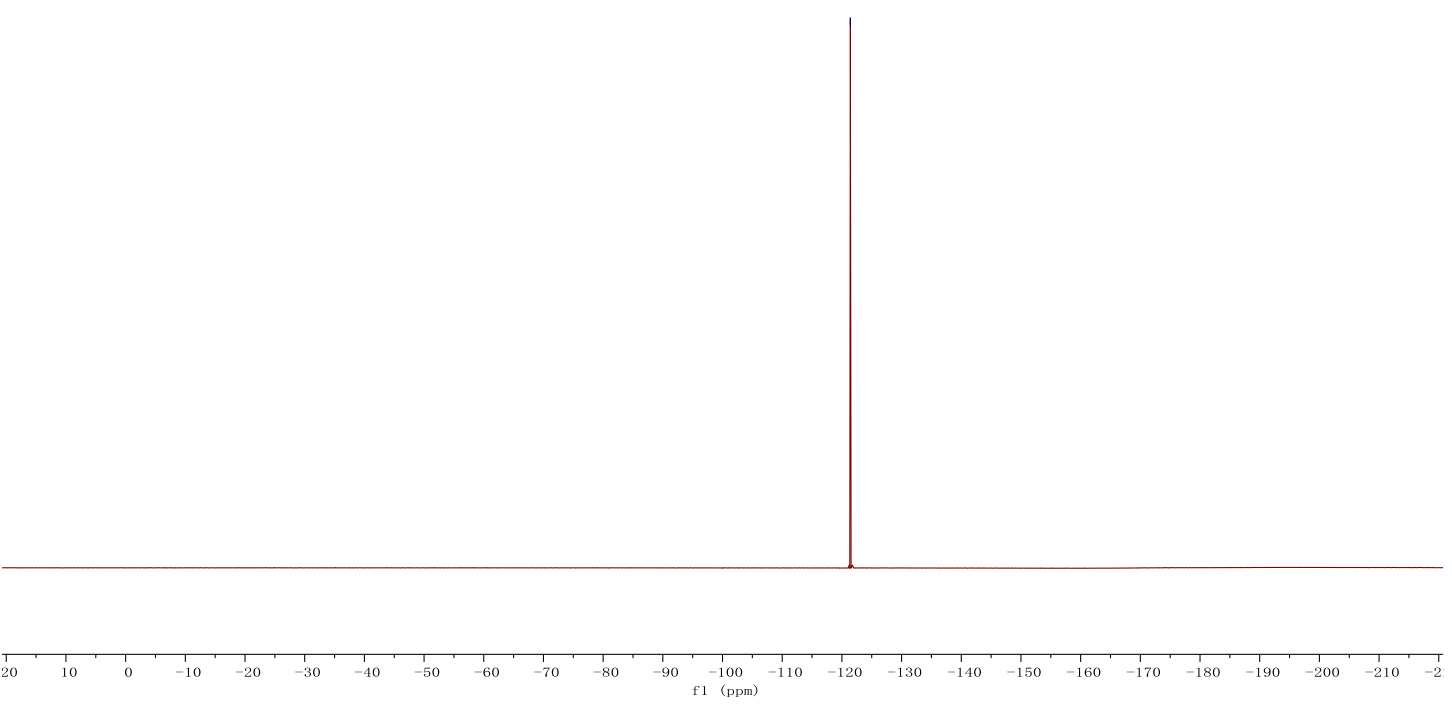


<smiles>COc1ccc2c(c1)N(Cc1ccccc1)C(=O)C(Cc1ccccc1)O2</smiles>

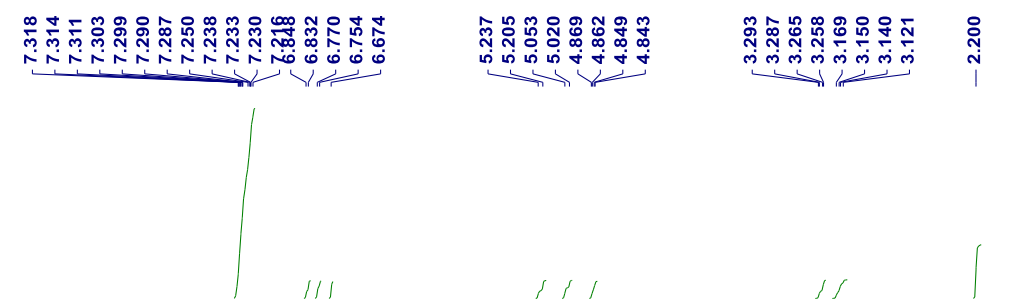

${ }^{1} \mathrm{H}$ NMR $\left(500 \mathrm{MHz}, \mathrm{CDCl}_{3}\right)$
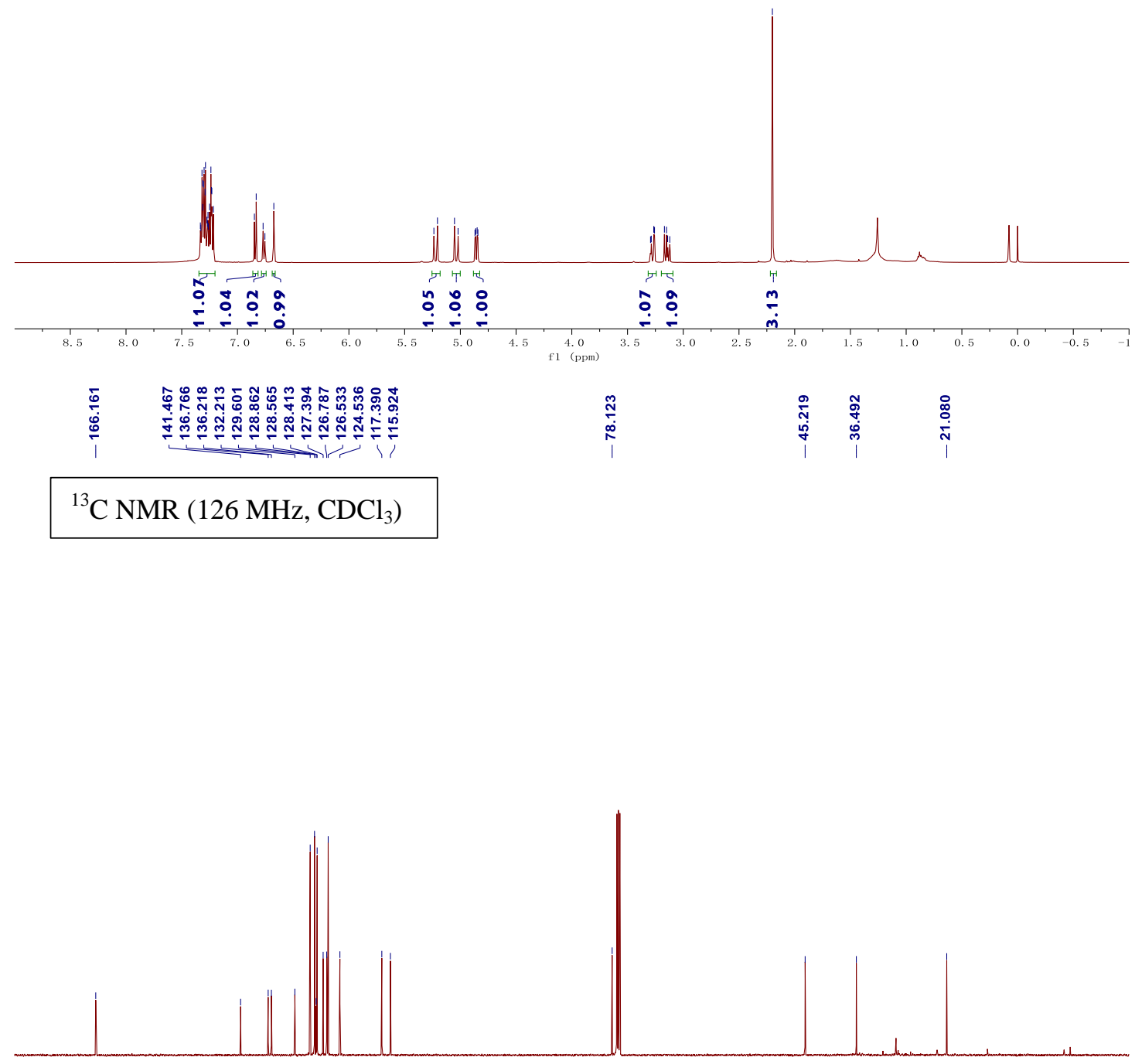

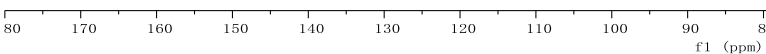



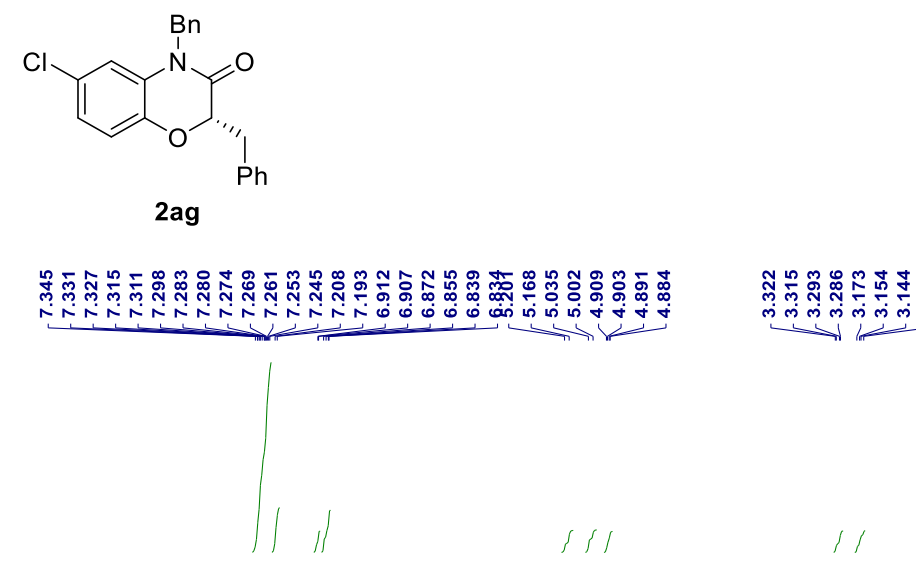

${ }^{1} \mathrm{H}$ NMR $\left(500 \mathrm{MHz}, \mathrm{CDCl}_{3}\right)$

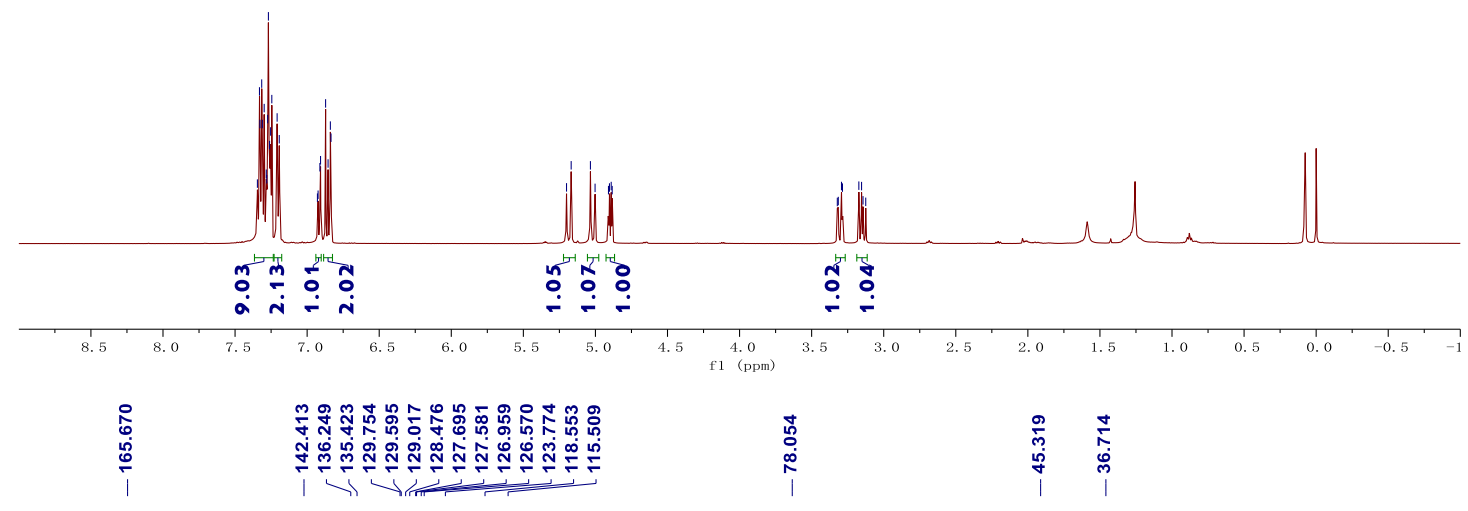

${ }^{13} \mathrm{C}$ NMR (126 MHz, $\mathrm{CDCl}_{3}$ )

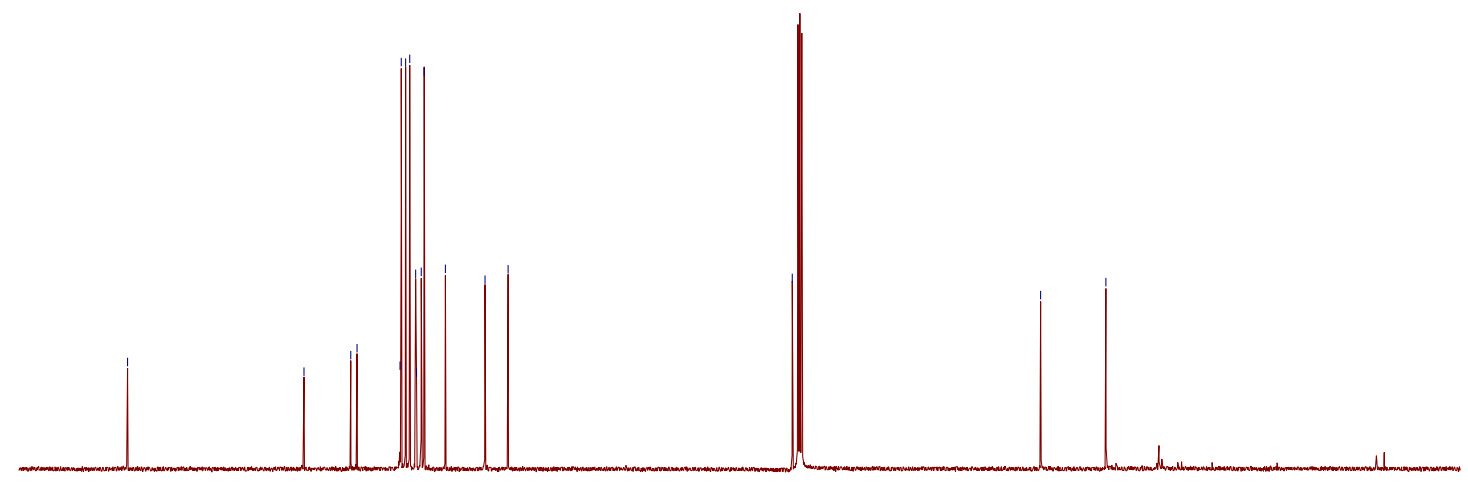




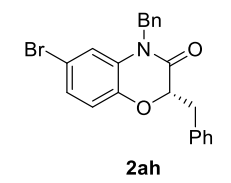

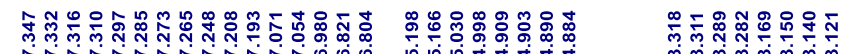

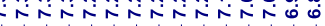

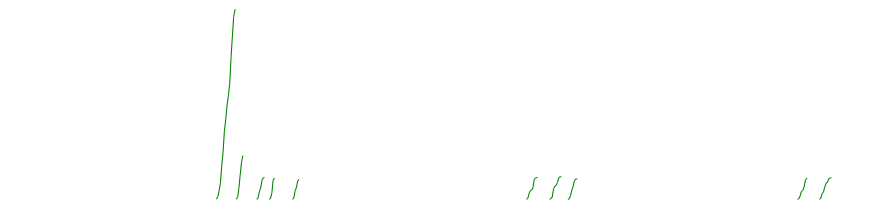

${ }^{1} \mathrm{H}$ NMR $\left(500 \mathrm{MHz}, \mathrm{CDCl}_{3}\right)$

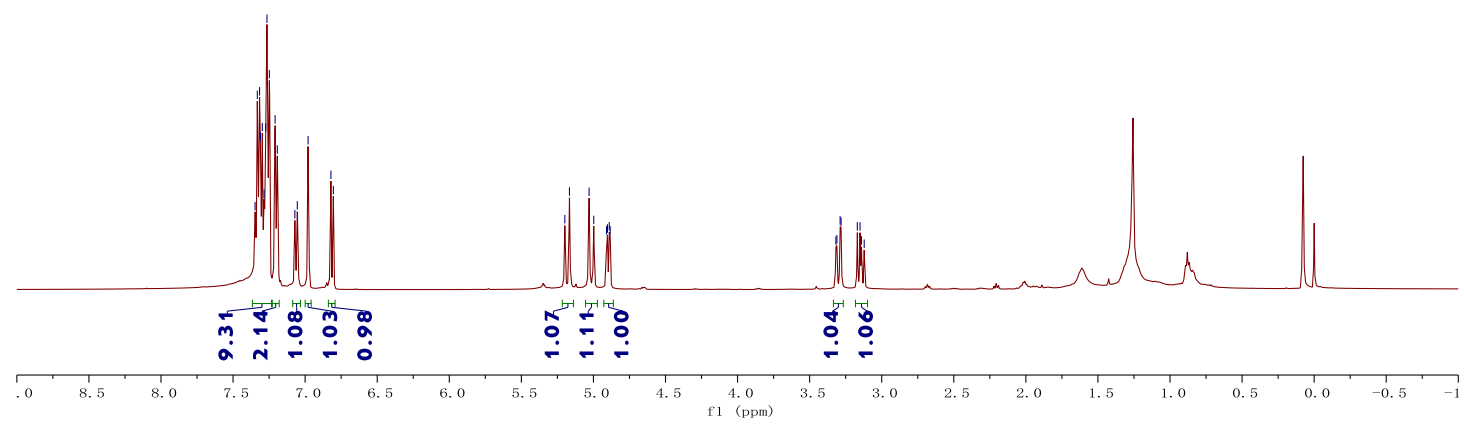

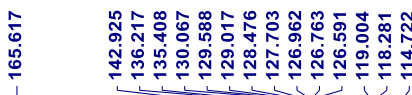

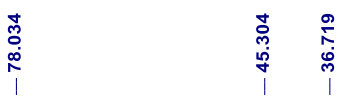

${ }^{13} \mathrm{C}$ NMR $\left(126 \mathrm{MHz}, \mathrm{CDCl}_{3}\right)$

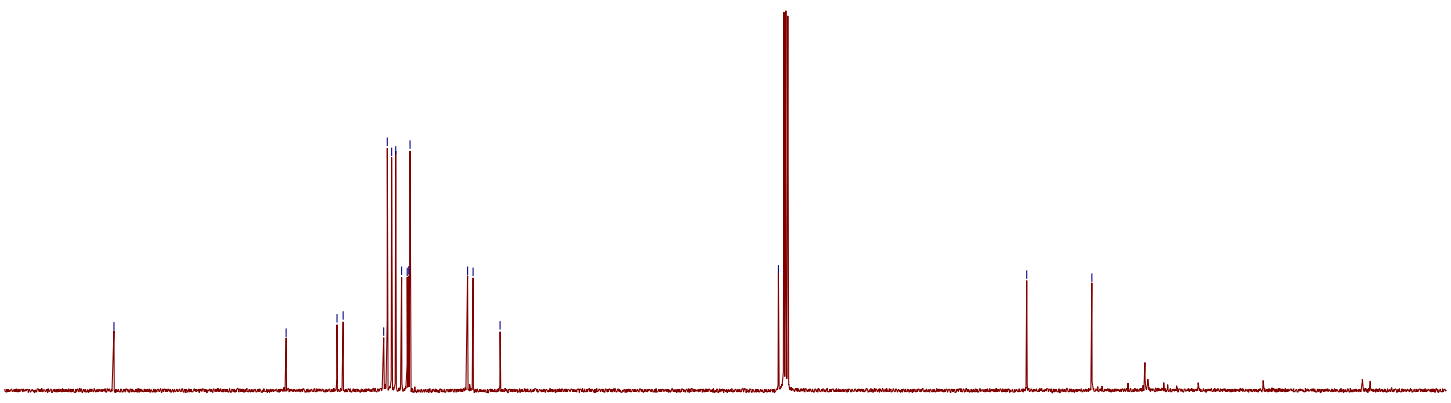

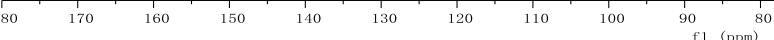




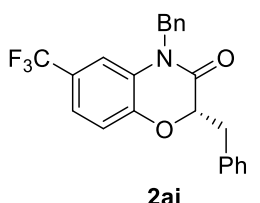

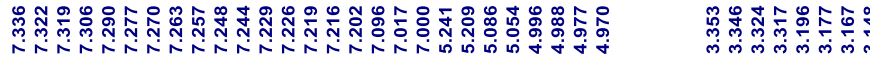

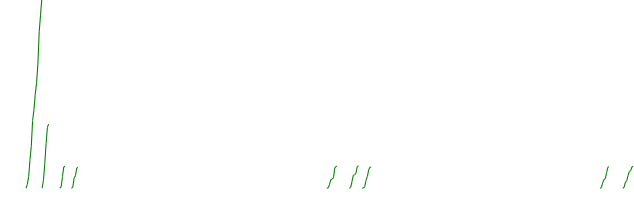

${ }^{1} \mathrm{H}$ NMR $\left(500 \mathrm{MHz}, \mathrm{CDCl}_{3}\right.$ )

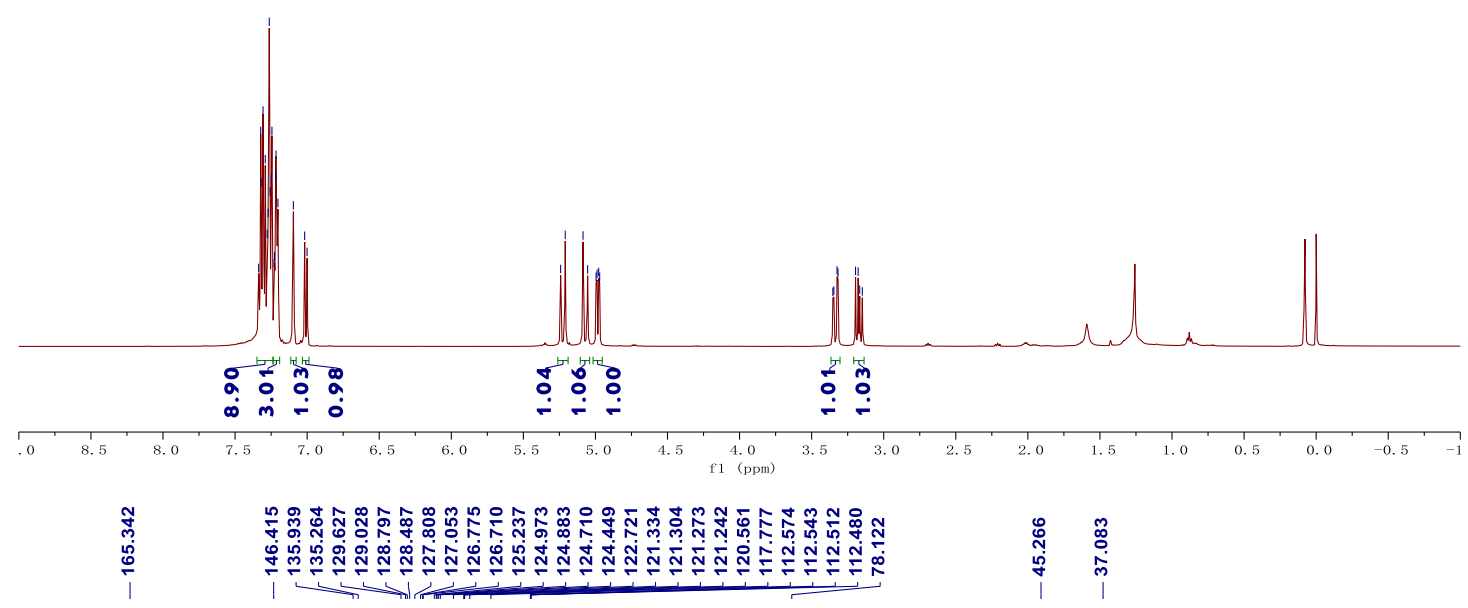

${ }^{13} \mathrm{C}$ NMR $\left(126 \mathrm{MHz}, \mathrm{CDCl}_{3}\right)$

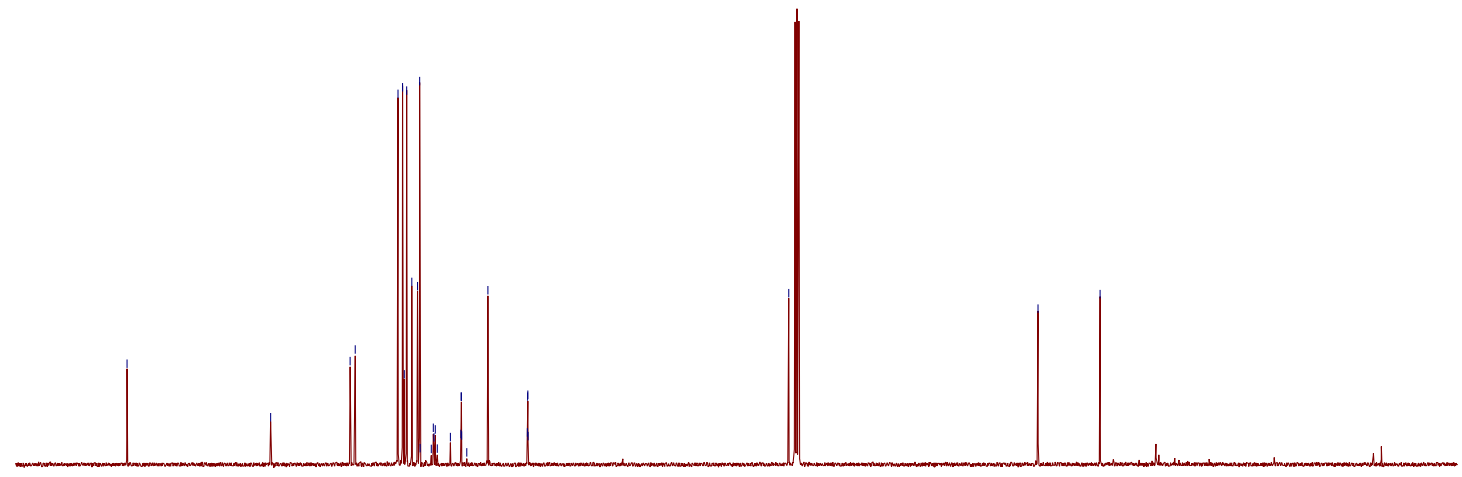




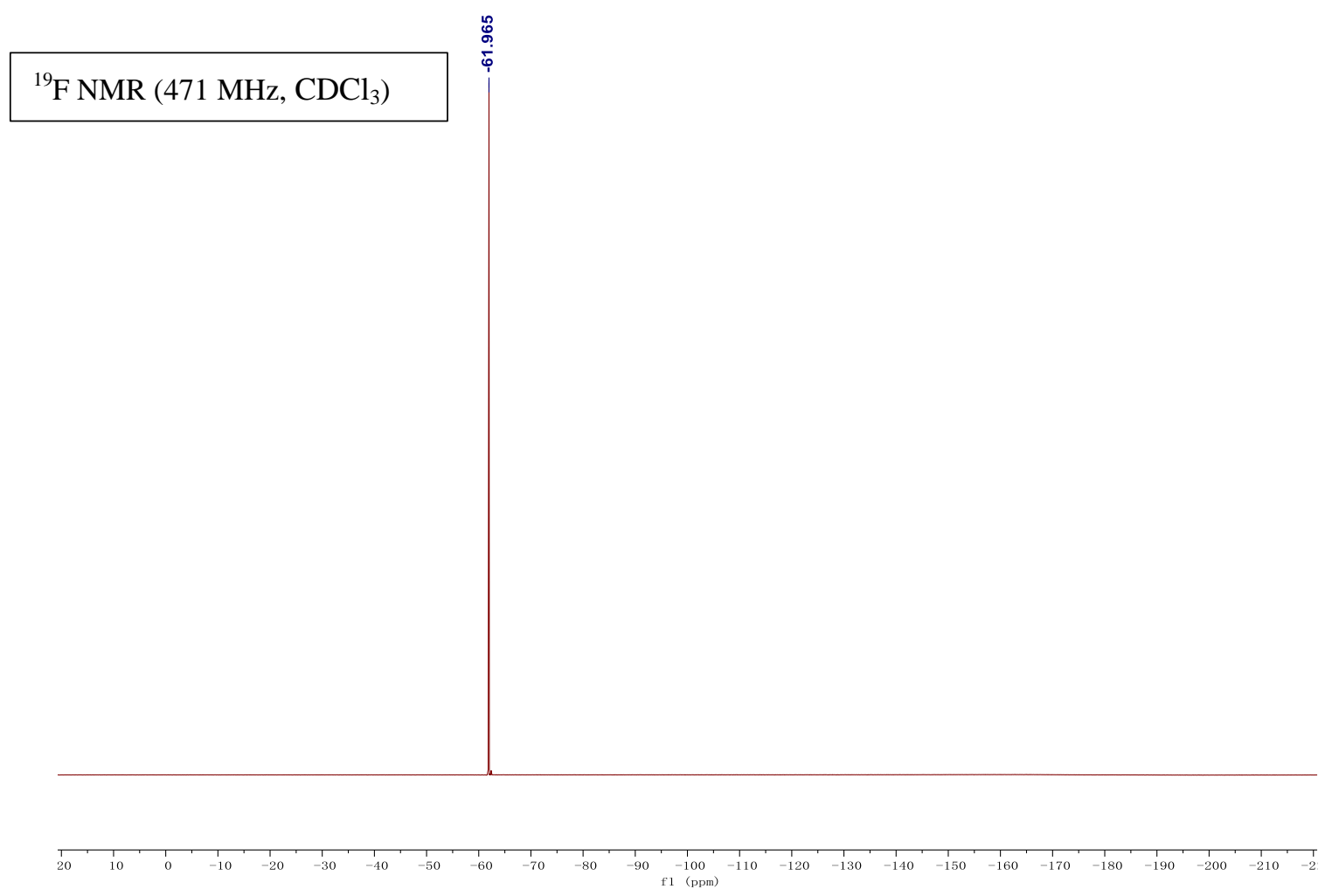




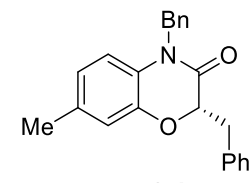

2aj

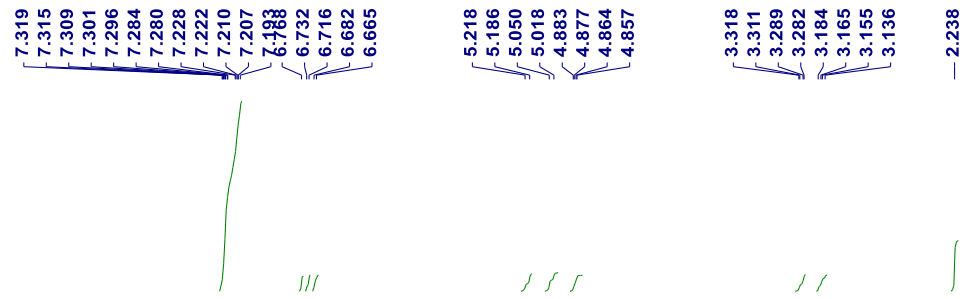

${ }^{1} \mathrm{H}$ NMR (500 MHz, $\mathrm{CDCl}_{3}$ )

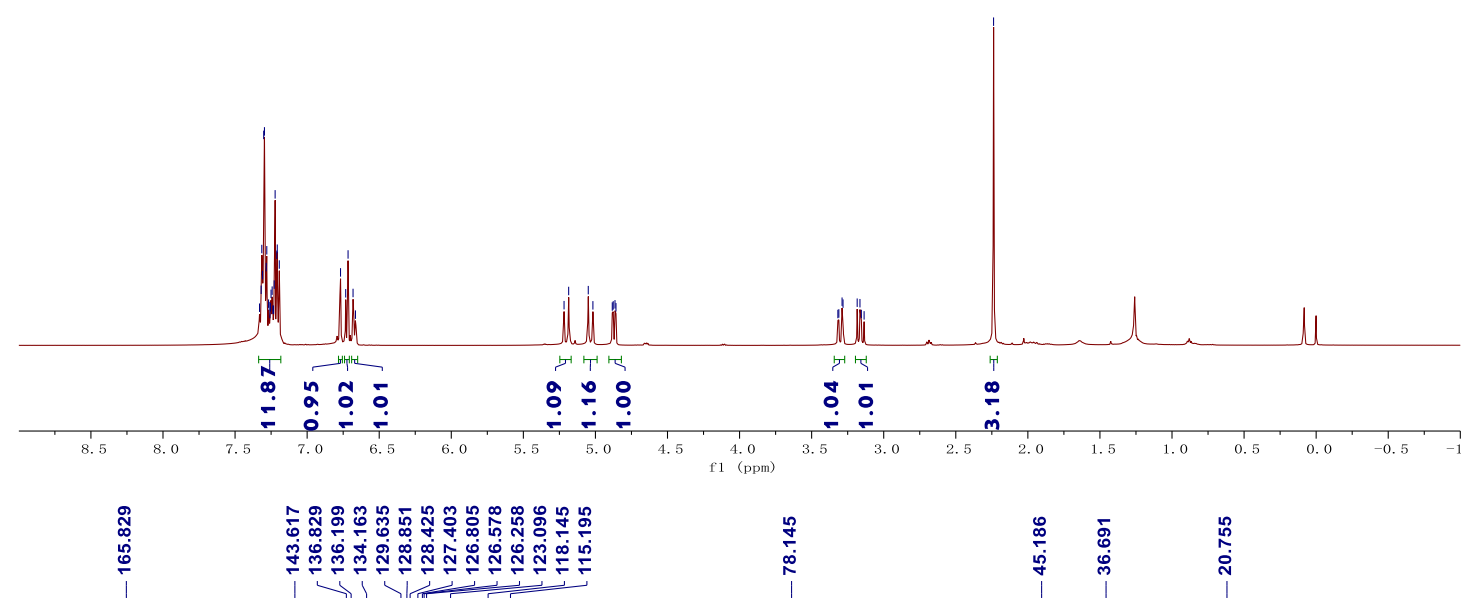

${ }^{13} \mathrm{C}$ NMR (126 MHz, $\left.\mathrm{CDCl}_{3}\right)$

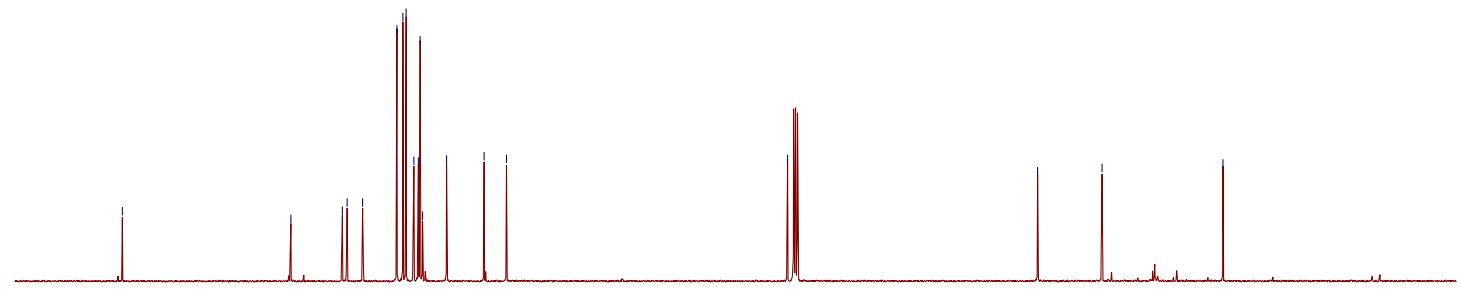


<smiles>O=C1C(Cc2ccccc2)Oc2cc(Cl)ccc2N1Cc1ccccc1</smiles>

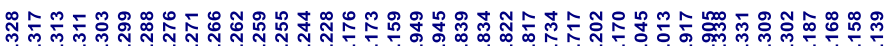

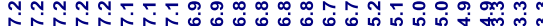

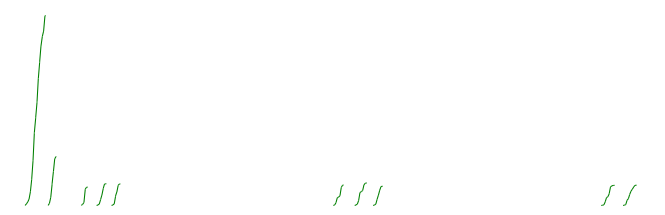

${ }^{1} \mathrm{H}$ NMR $\left(500 \mathrm{MHz}, \mathrm{CDCl}_{3}\right)$

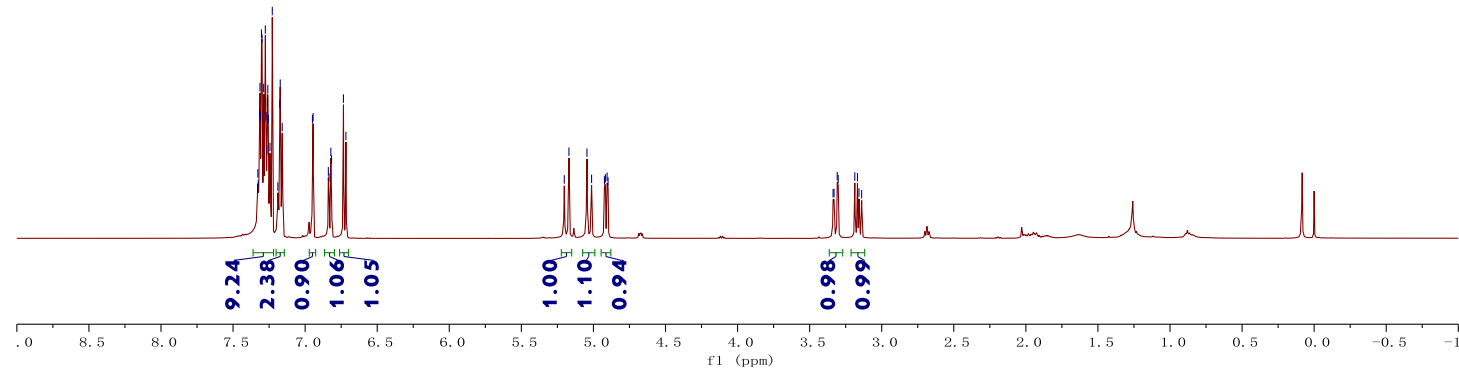

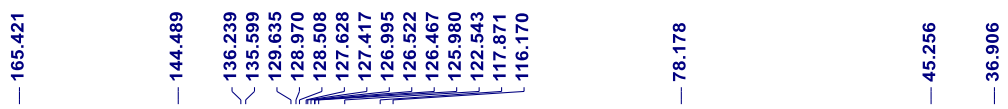

${ }^{13} \mathrm{C} \mathrm{NMR}\left(126 \mathrm{MHz}, \mathrm{CDCl}_{3}\right)$

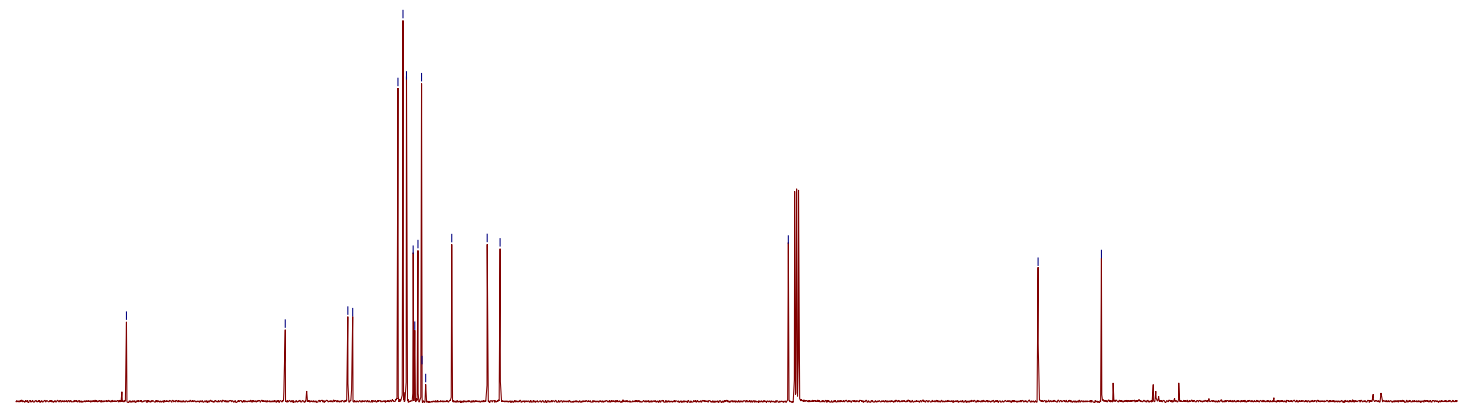

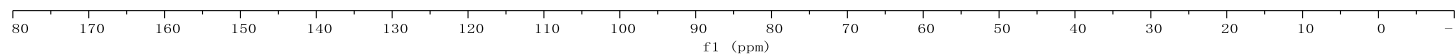




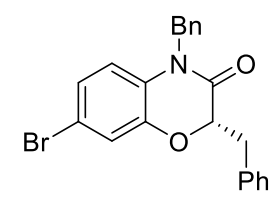

2al

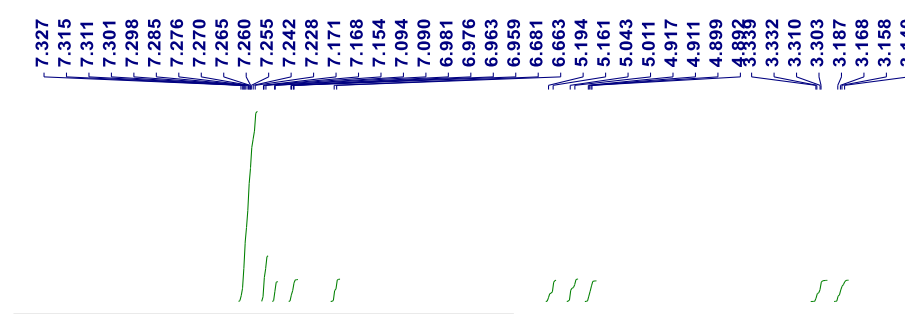

${ }^{1} \mathrm{H}$ NMR $\left(500 \mathrm{MHz}, \mathrm{CDCl}_{3}\right)$

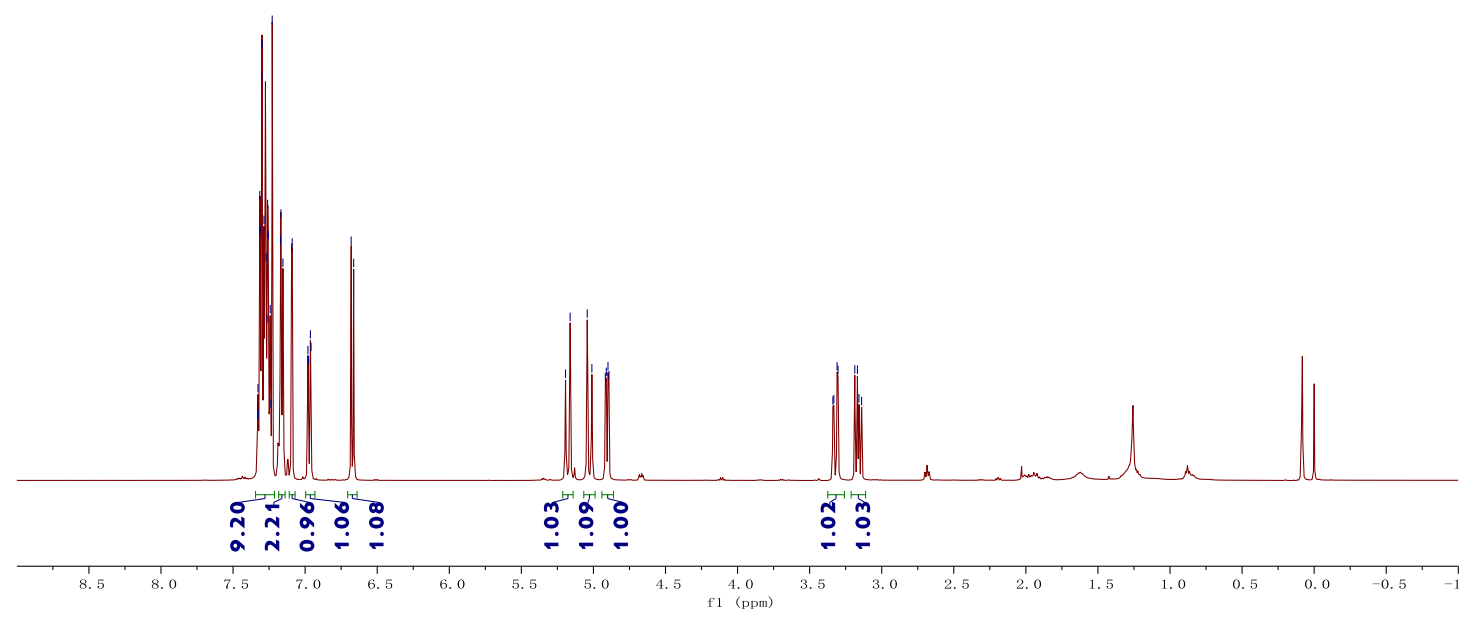

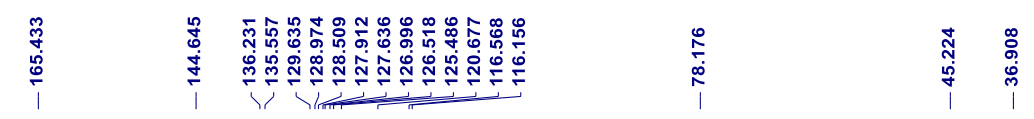

${ }^{13} \mathrm{C}$ NMR $\left(126 \mathrm{MHz}, \mathrm{CDCl}_{3}\right)$
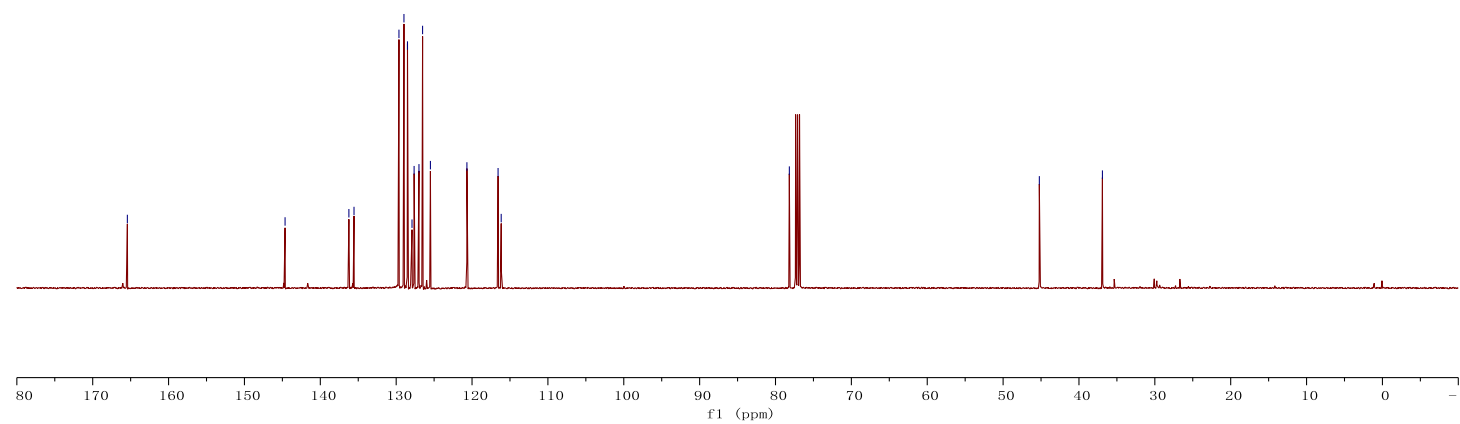

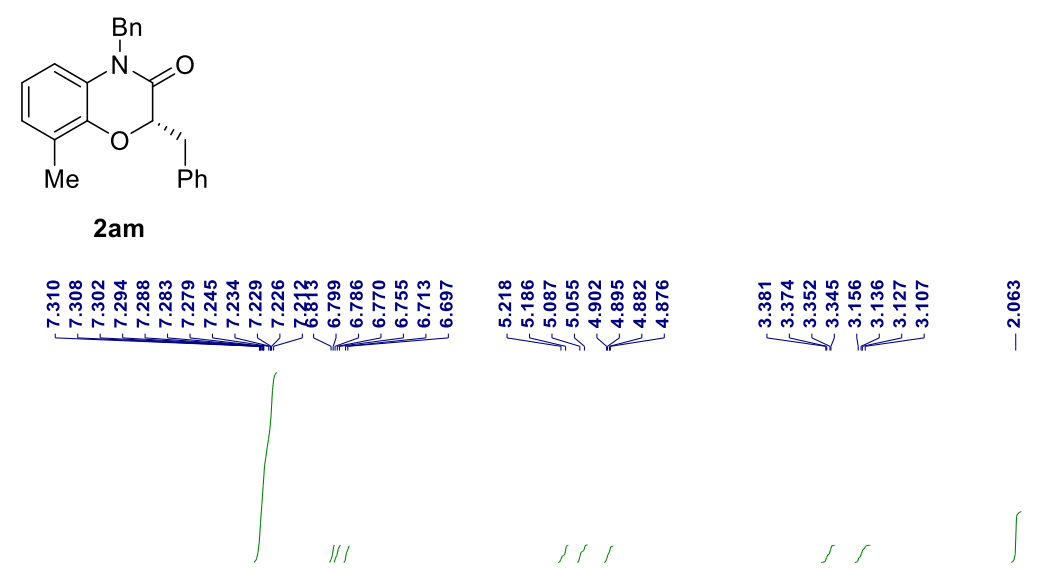

${ }^{1} \mathrm{H} \mathrm{NMR}\left(500 \mathrm{MHz}, \mathrm{CDCl}_{3}\right)$

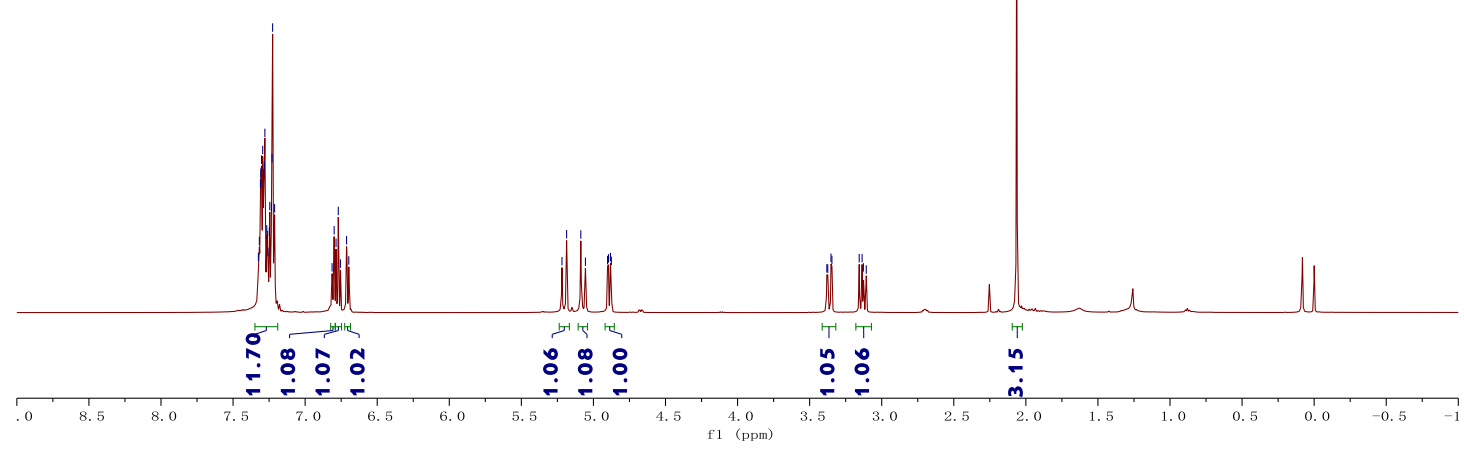

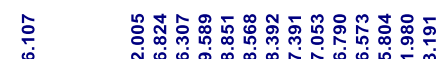

定

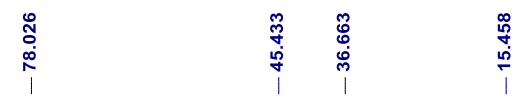

${ }^{13} \mathrm{C}$ NMR $\left(126 \mathrm{MHz}, \mathrm{CDCl}_{3}\right)$

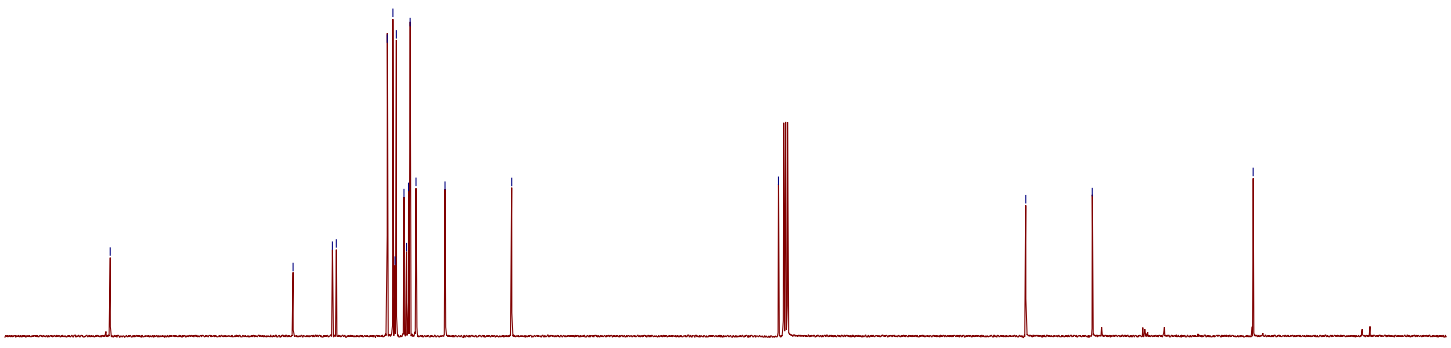

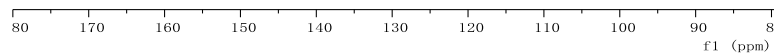




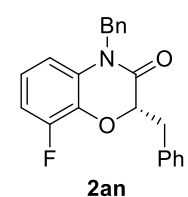

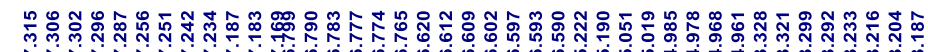

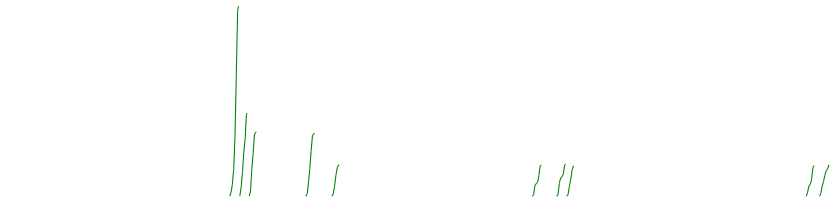

${ }^{1} \mathrm{H}$ NMR $\left(500 \mathrm{MHz}, \mathrm{CDCl}_{3}\right)$

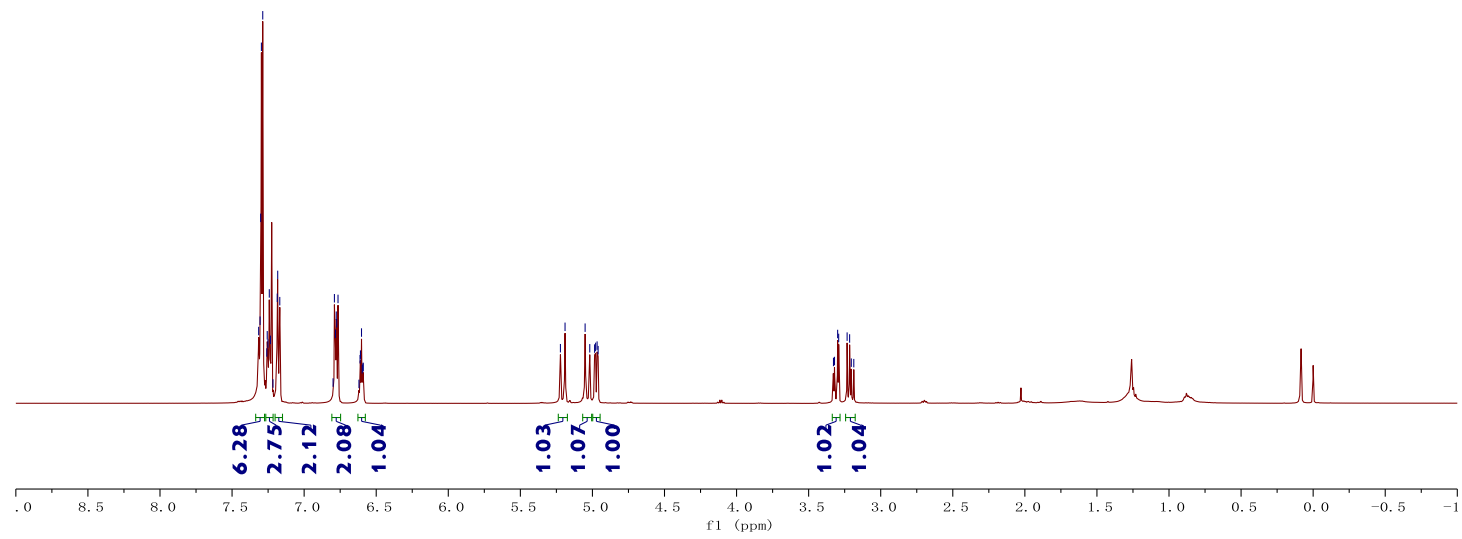

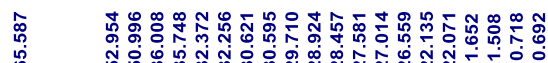

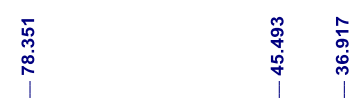

${ }^{13} \mathrm{C} \mathrm{NMR}\left(126 \mathrm{MHz}, \mathrm{CDCl}_{3}\right)$

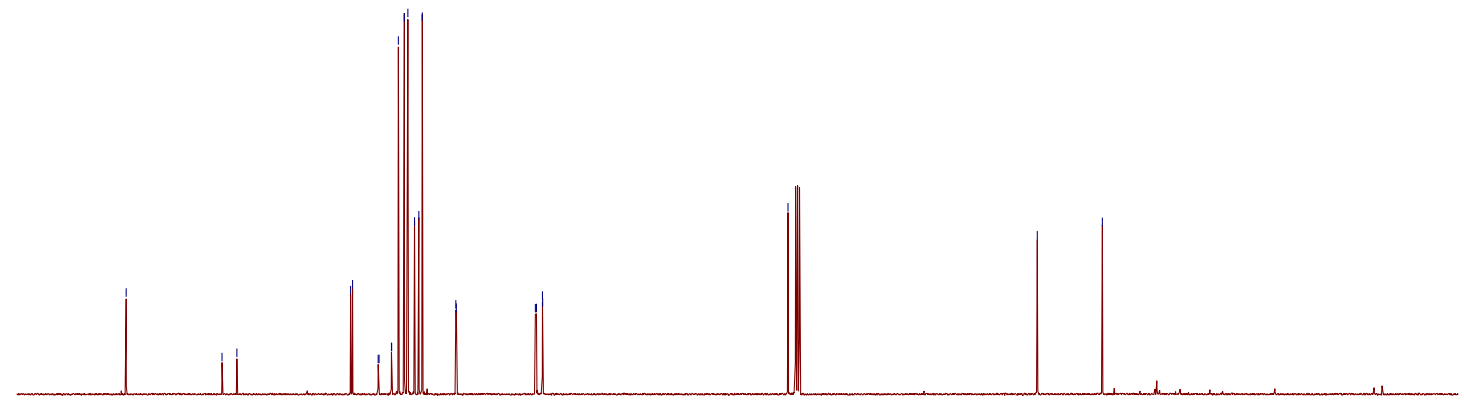

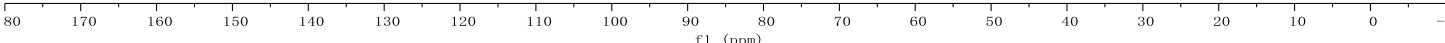


${ }^{19} \mathrm{~F}$ NMR (471 MHz, $\mathrm{CDCl}_{3}$ )

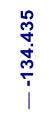

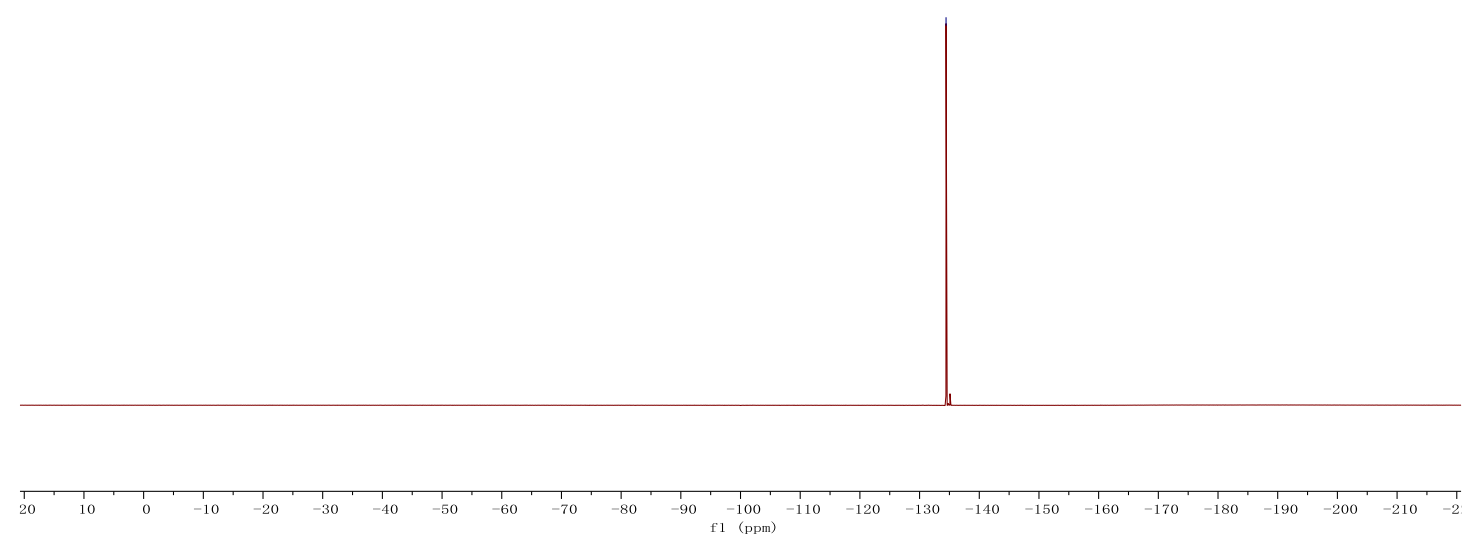




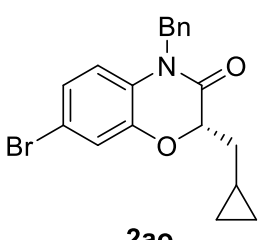

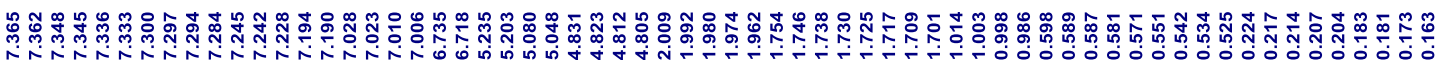

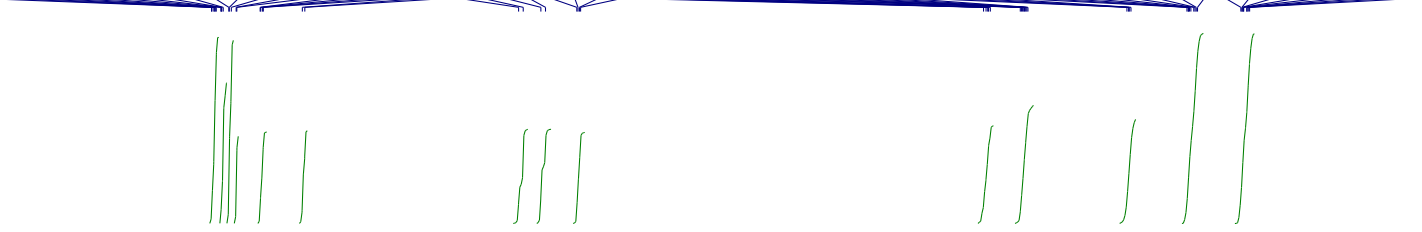

${ }^{1} \mathrm{H}$ NMR $\left(500 \mathrm{MHz}, \mathrm{CDCl}_{3}\right)$

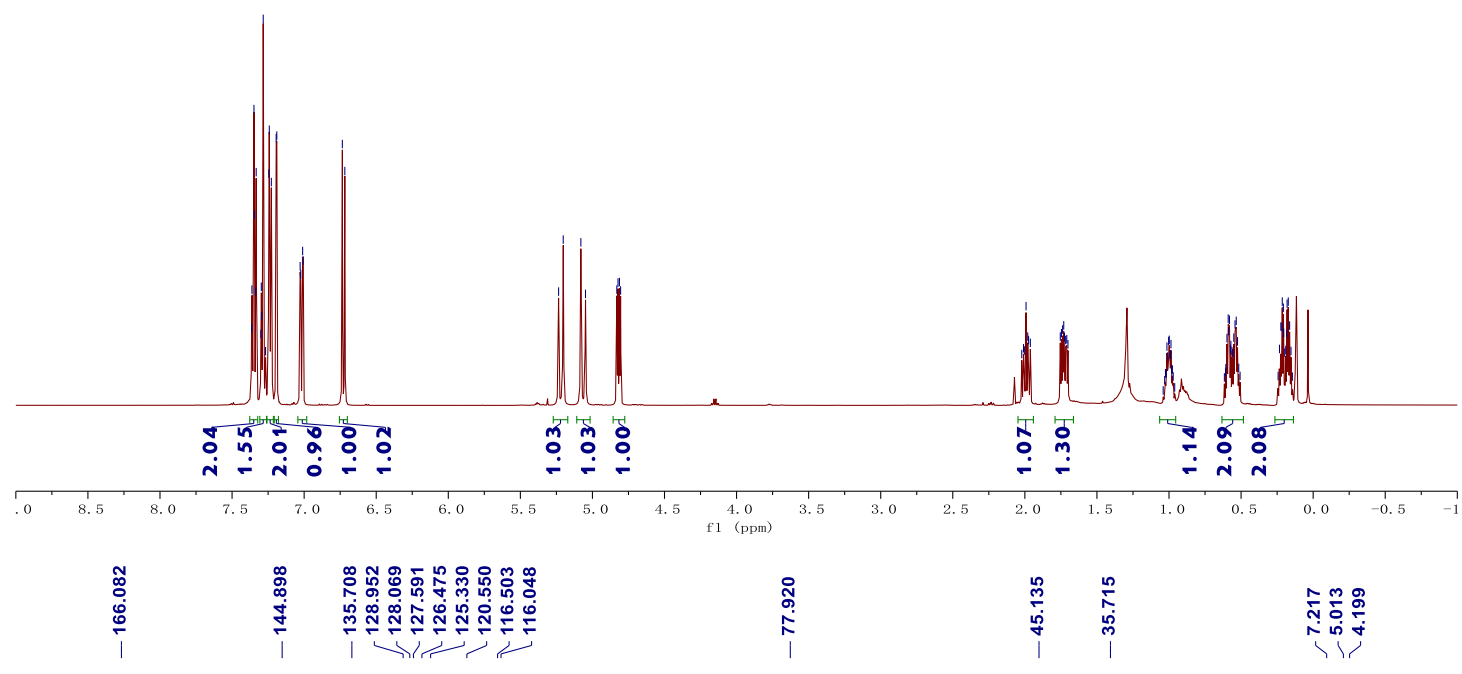

${ }^{13} \mathrm{C}$ NMR $\left(126 \mathrm{MHz}, \mathrm{CDCl}_{3}\right)$
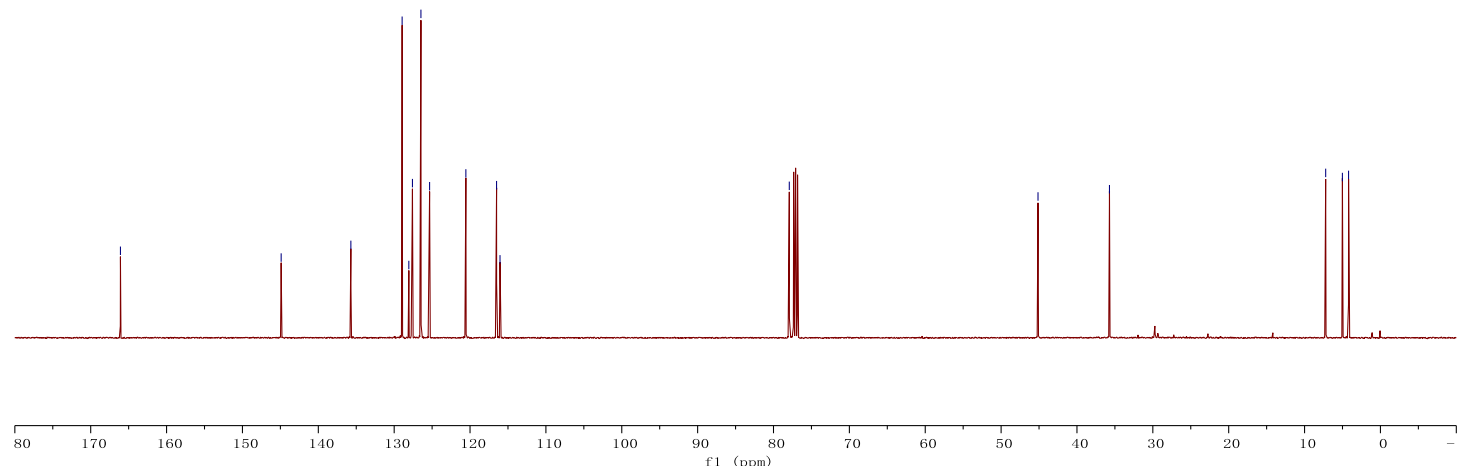


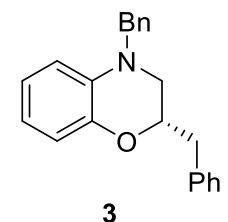

3

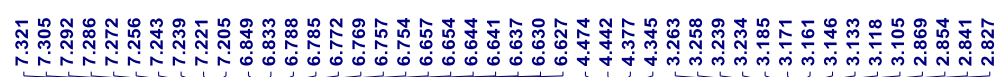

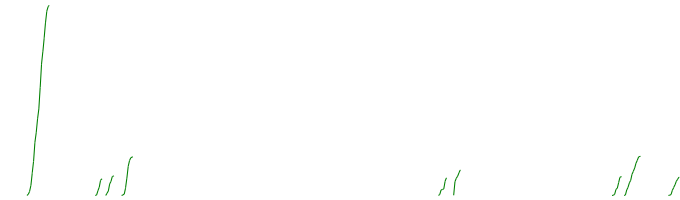

${ }^{1} \mathrm{H}$ NMR $\left(500 \mathrm{MHz}, \mathrm{CDCl}_{3}\right)$

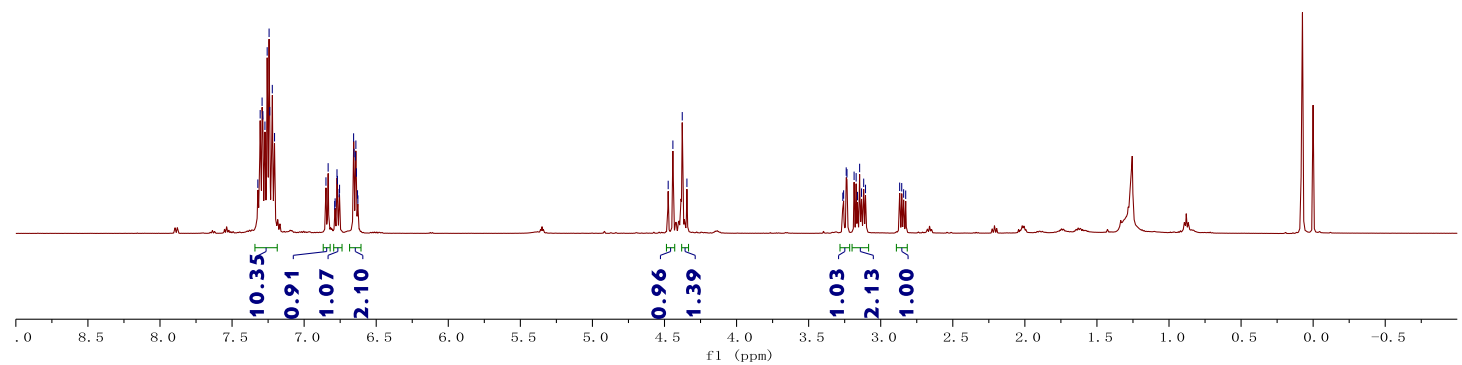

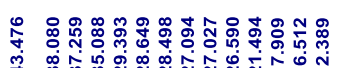

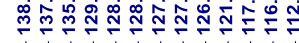

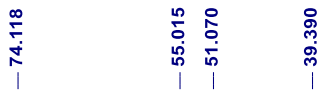

${ }^{13} \mathrm{C}$ NMR $\left(126 \mathrm{MHz}, \mathrm{CDCl}_{3}\right)$

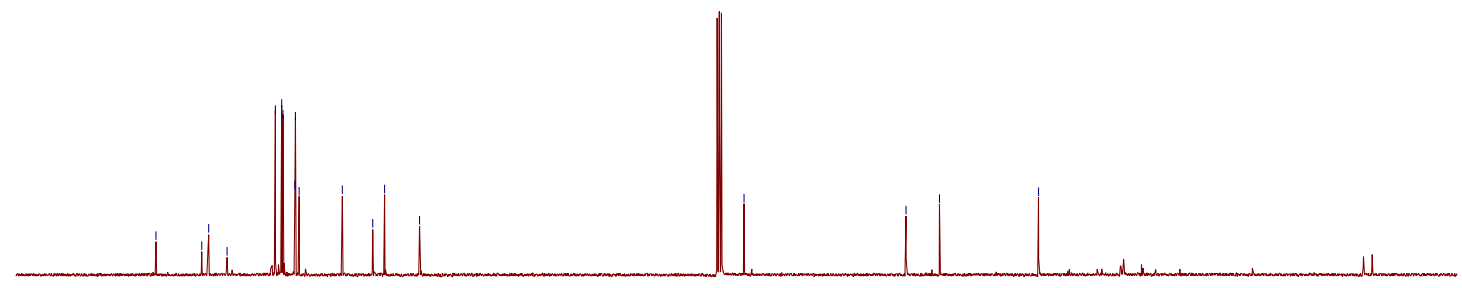

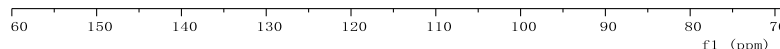




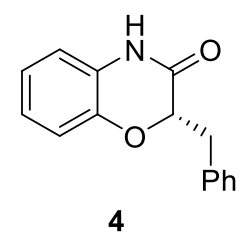

ำ

다슨

${ }^{1} \mathrm{H}$ NMR $\left(500 \mathrm{MHz}, \mathrm{CDCl}_{3}\right)$

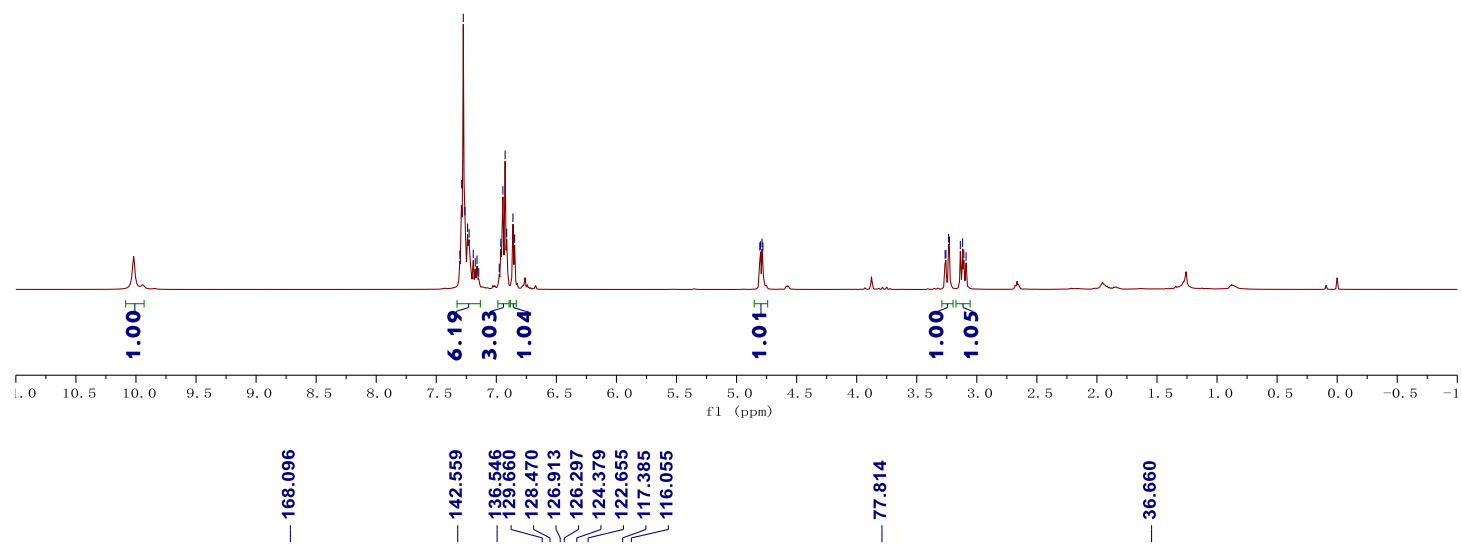

${ }^{13} \mathrm{C} \mathrm{NMR}\left(126 \mathrm{MHz}, \mathrm{CDCl}_{3}\right)$

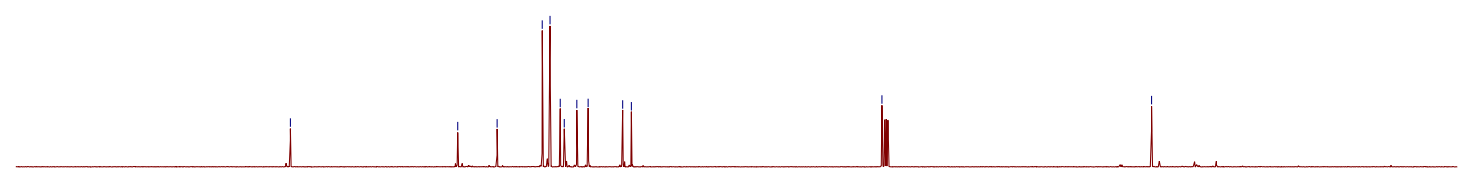

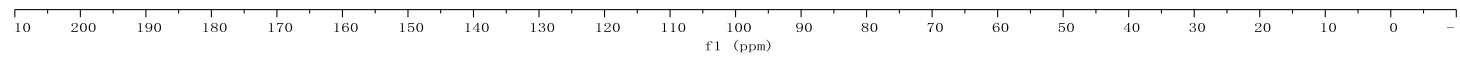



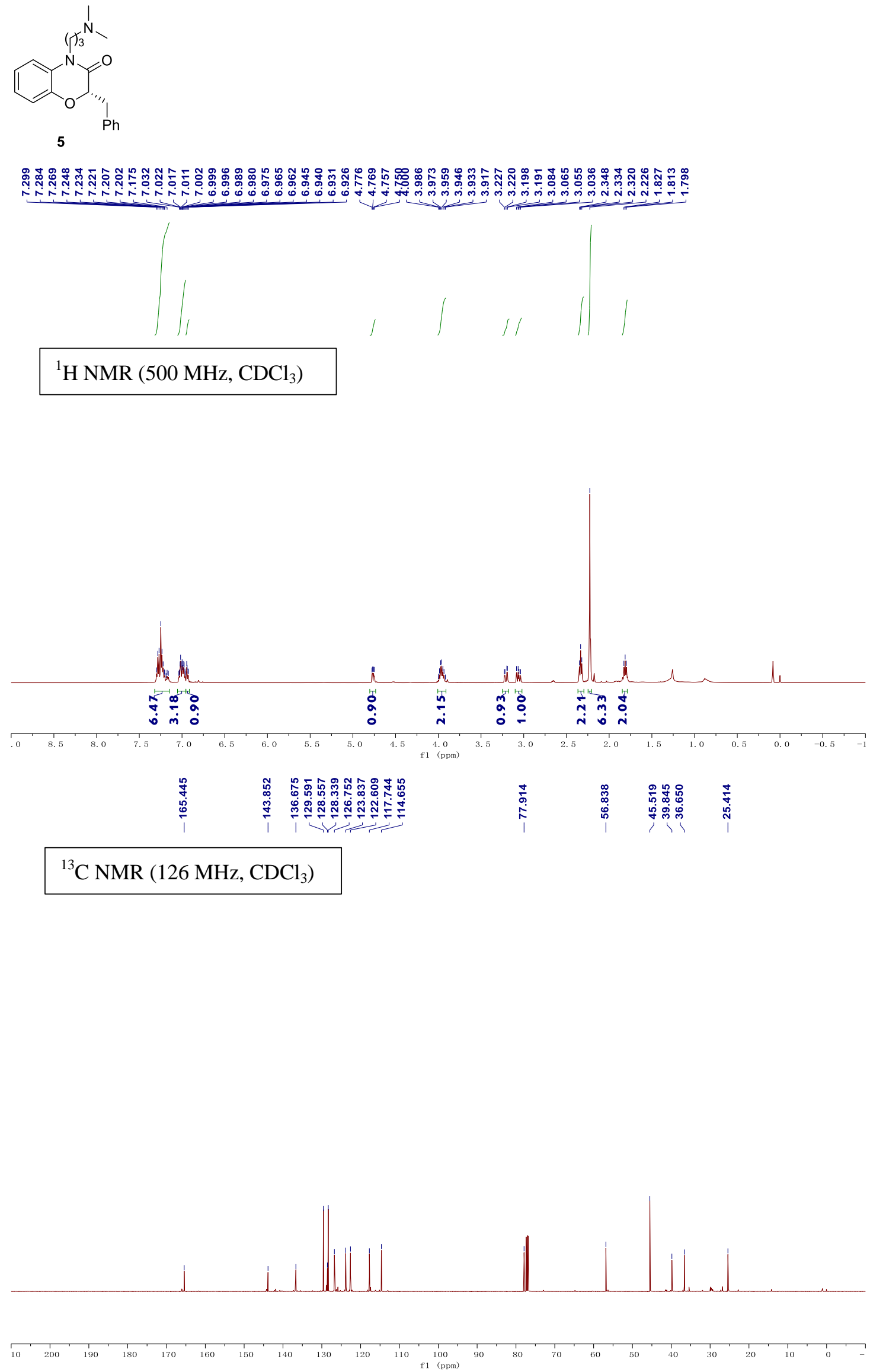
<smiles>O=C1Nc2cc(Cl)ccc2O[C@@H]1Cc1ccccc1</smiles>

6

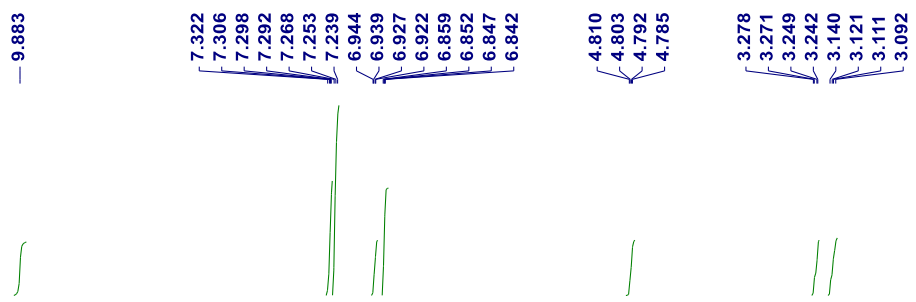

${ }^{1} \mathrm{H}$ NMR $\left(500 \mathrm{MHz}, \mathrm{CDCl}_{3}\right)$

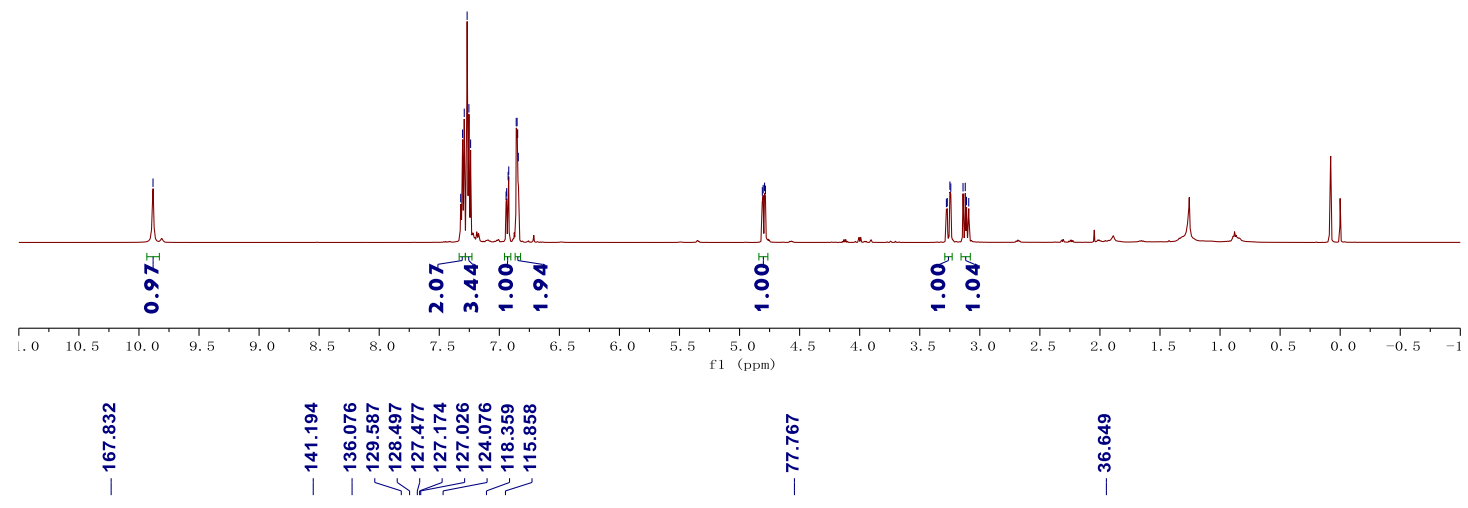

${ }^{13} \mathrm{C}$ NMR (126 MHz, $\left.\mathrm{CDCl}_{3}\right)$
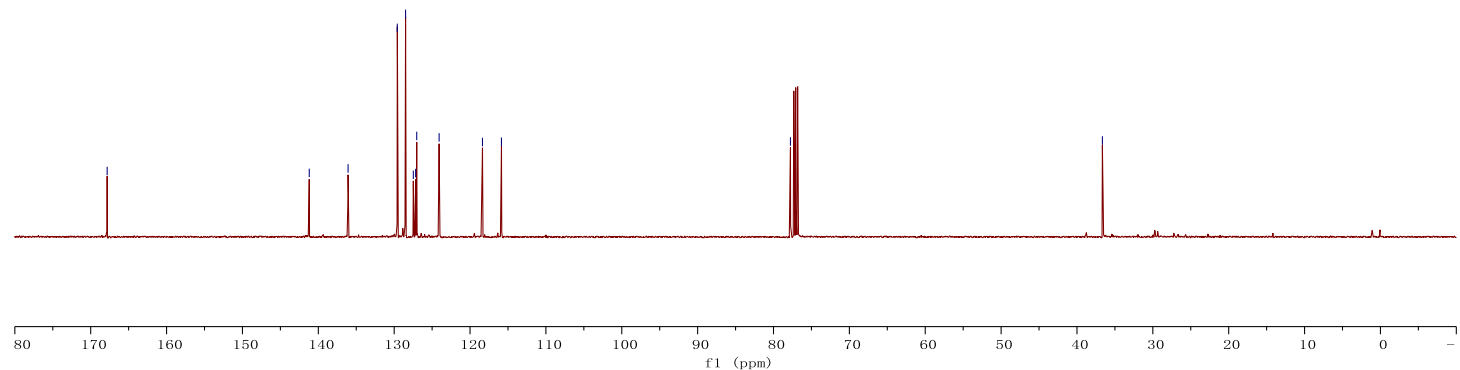
<smiles>O=C1Nc2ccc(Br)cc2O[C@@H]1Cc1ccccc1</smiles>

8

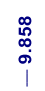

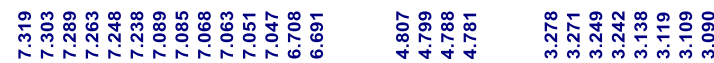

$i$ irikinisiog
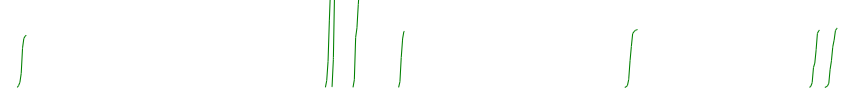

${ }^{1} \mathrm{H}$ NMR $\left(500 \mathrm{MHz}, \mathrm{CDCl}_{3}\right.$ )

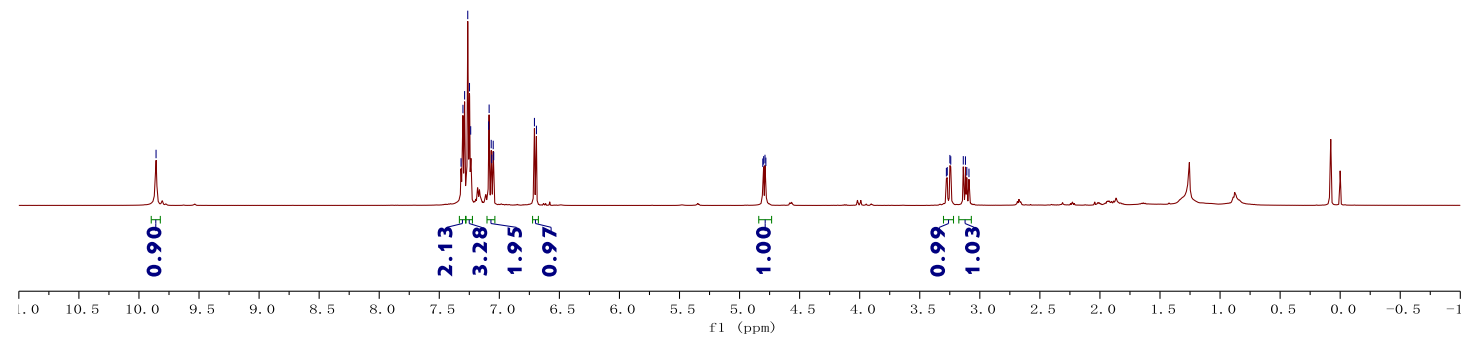

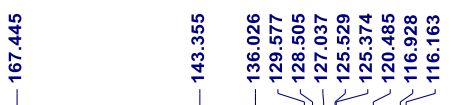

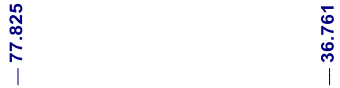

${ }^{13} \mathrm{C}$ NMR $\left(126 \mathrm{MHz}, \mathrm{CDCl}_{3}\right)$

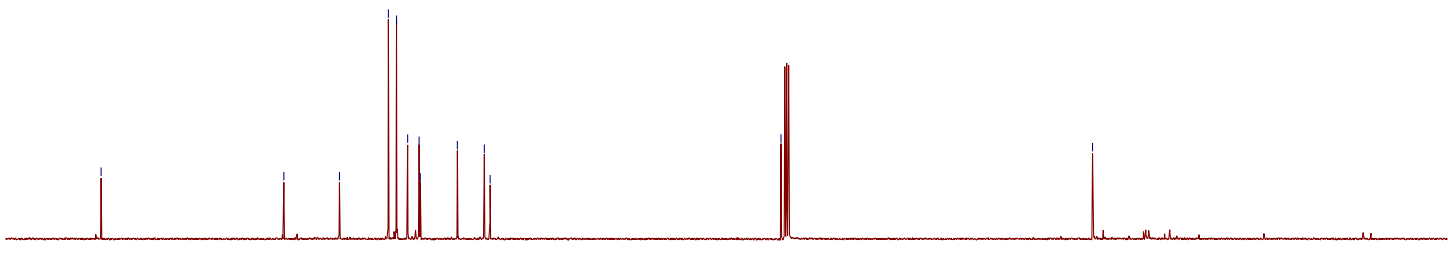

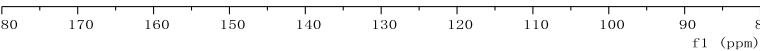




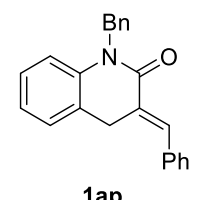

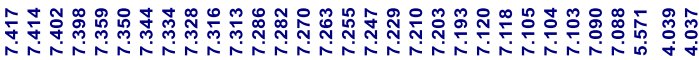

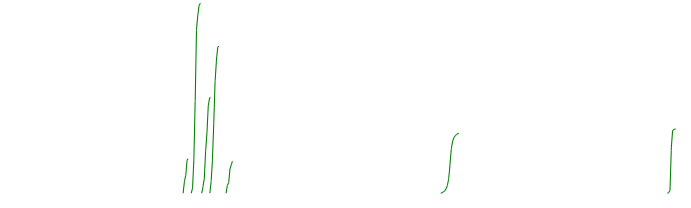

${ }^{1} \mathrm{H}$ NMR $\left(500 \mathrm{MHz}, \mathrm{CDCl}_{3}\right)$

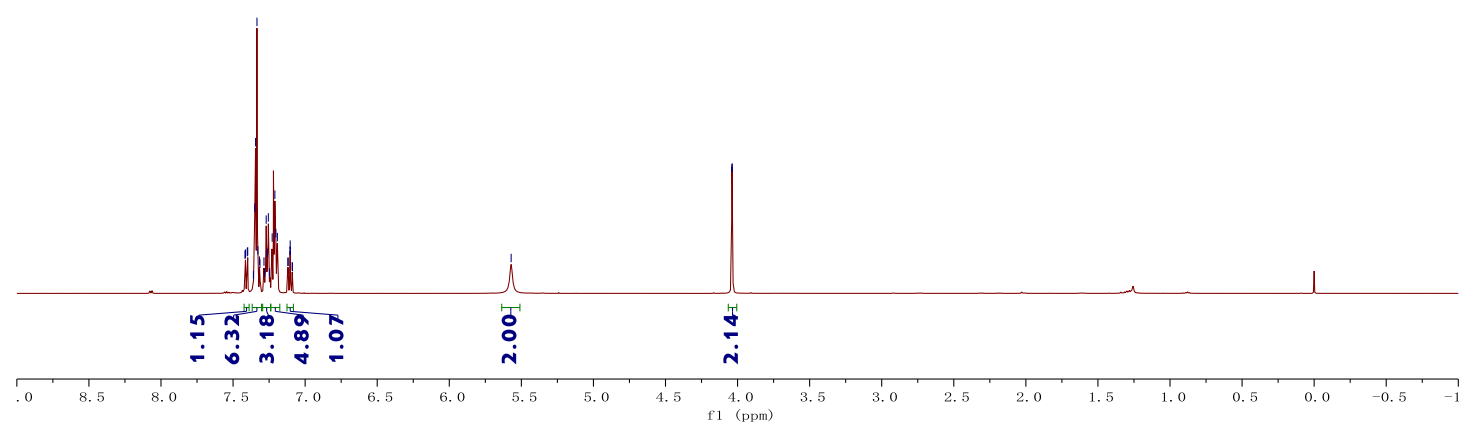

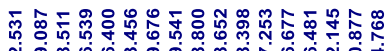

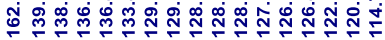

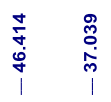

${ }^{13} \mathrm{C} \mathrm{NMR}\left(126 \mathrm{MHz}, \mathrm{CDCl}_{3}\right)$

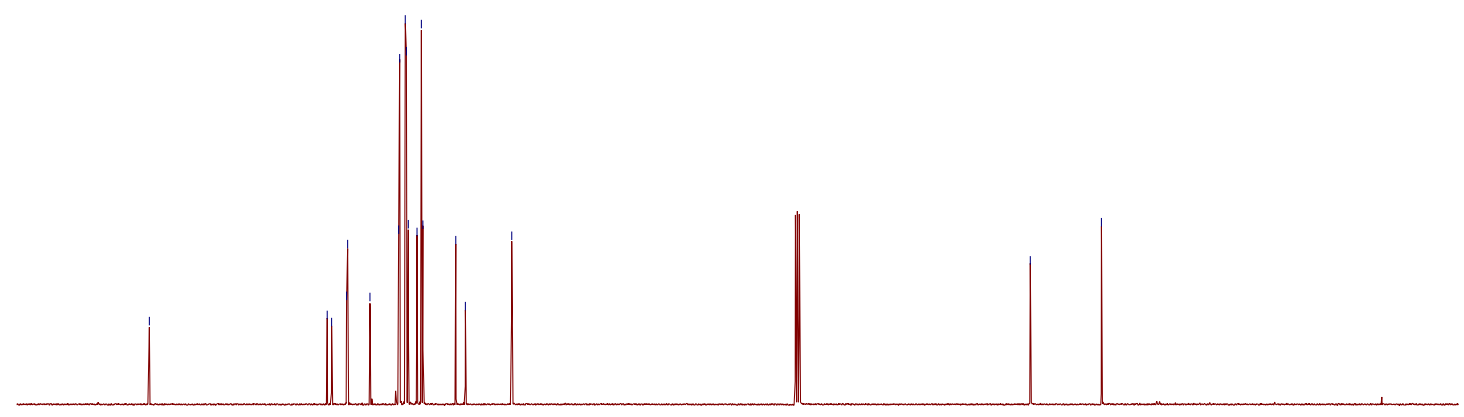

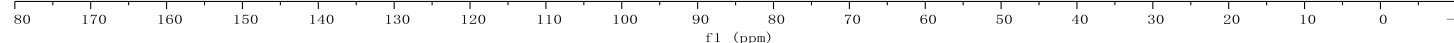




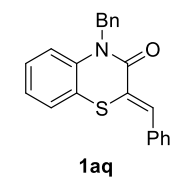

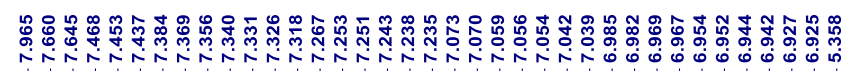

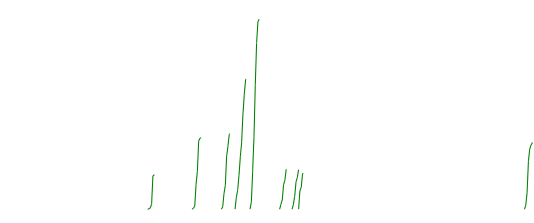

${ }^{1} \mathrm{H}$ NMR $\left(500 \mathrm{MHz}, \mathrm{CDCl}_{3}\right)$

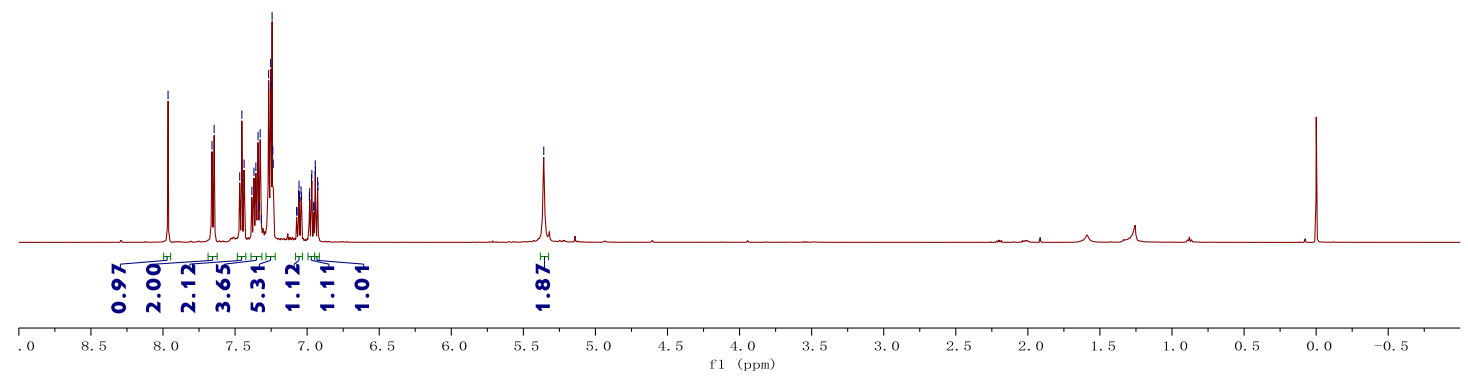

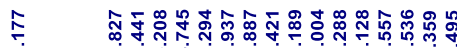

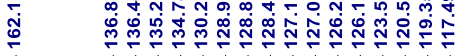

$\underset{\substack{q \\ d}}{d}$

${ }^{13} \mathrm{C}$ NMR $\left(126 \mathrm{MHz}, \mathrm{CDCl}_{3}\right)$

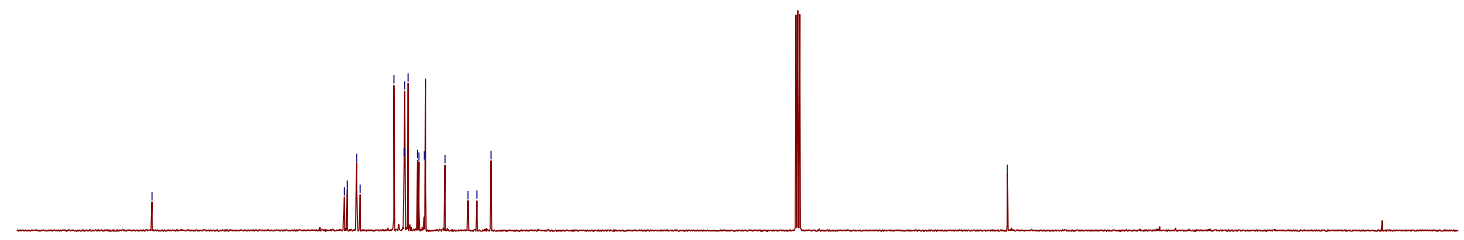

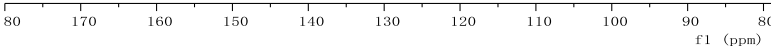




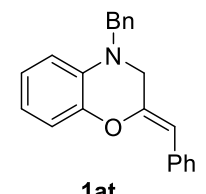

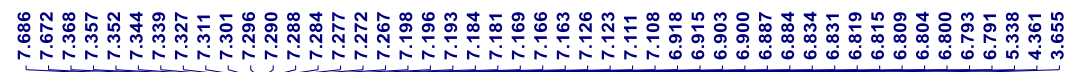

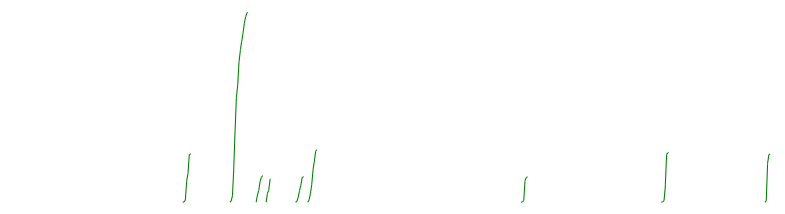

${ }^{1} \mathrm{H}$ NMR $\left(500 \mathrm{MHz}, \mathrm{CDCl}_{3}\right)$

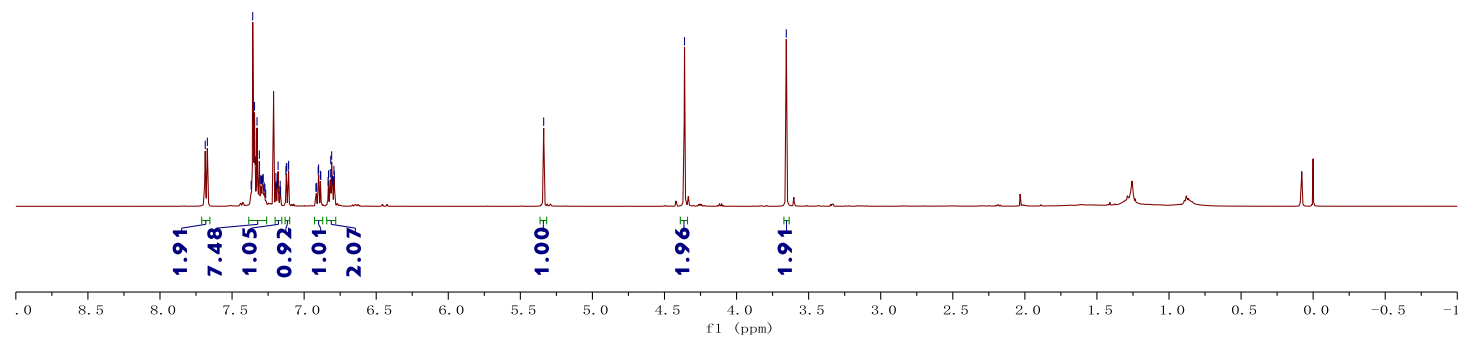

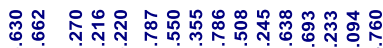

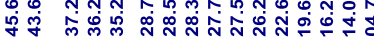

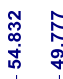

${ }^{13} \mathrm{C}$ NMR $\left(126 \mathrm{MHz}, \mathrm{CDCl}_{3}\right)$

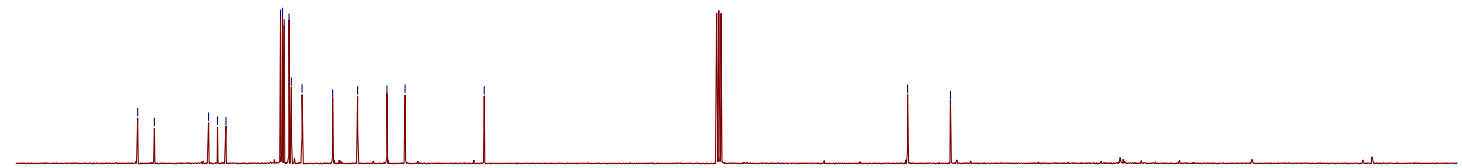

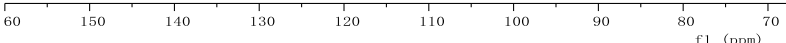




\section{HPLC Data}<smiles>O=C1C(Cc2ccccc2)Oc2ccccc2N1Cc1ccccc1</smiles>

$\mathrm{mV}$

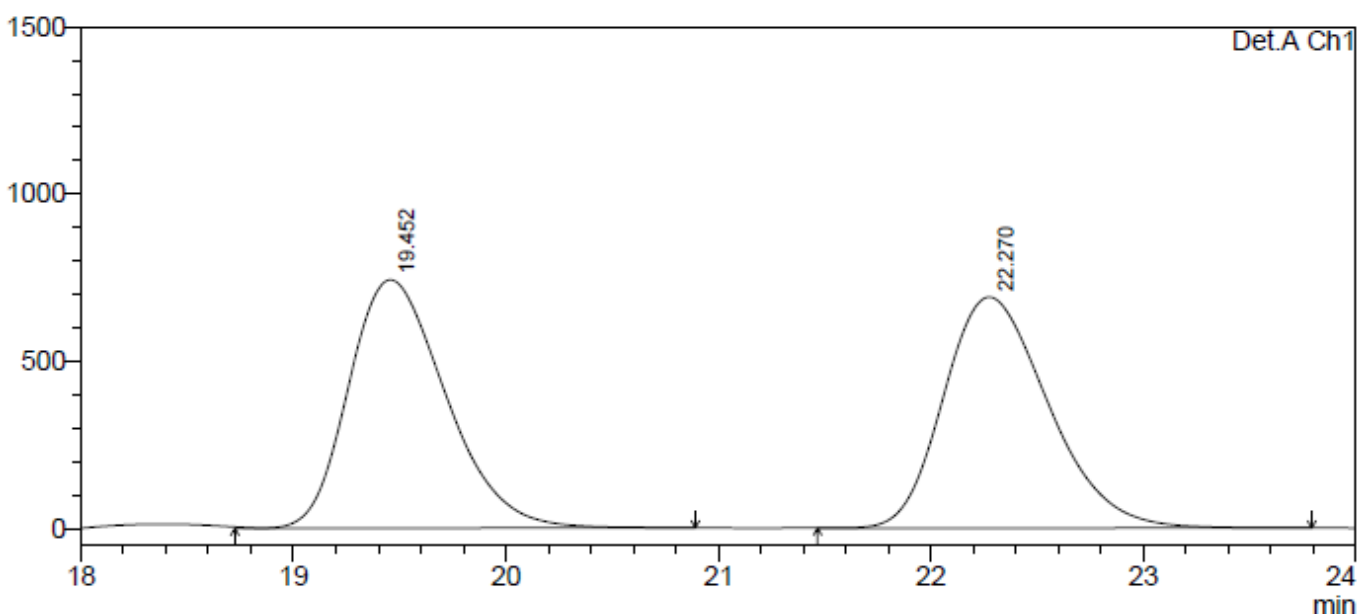

1 Det.A Ch $1 / 220 \mathrm{~nm}$

Detector A Ch1 220nm
\begin{tabular}{|r|r|r|r|r|r|}
\hline Peak\# & Ret. Time & \multicolumn{1}{|c|}{ Area } & Height & Area \% & Height \% \\
\hline 1 & 19.452 & 23553311 & 741594 & 49.904 & 51.816 \\
\hline 2 & 22.270 & 23644259 & 689614 & 50.096 & 48.184 \\
\hline Total & & 47197569 & 1431209 & 100.000 & 100.000 \\
\hline
\end{tabular}

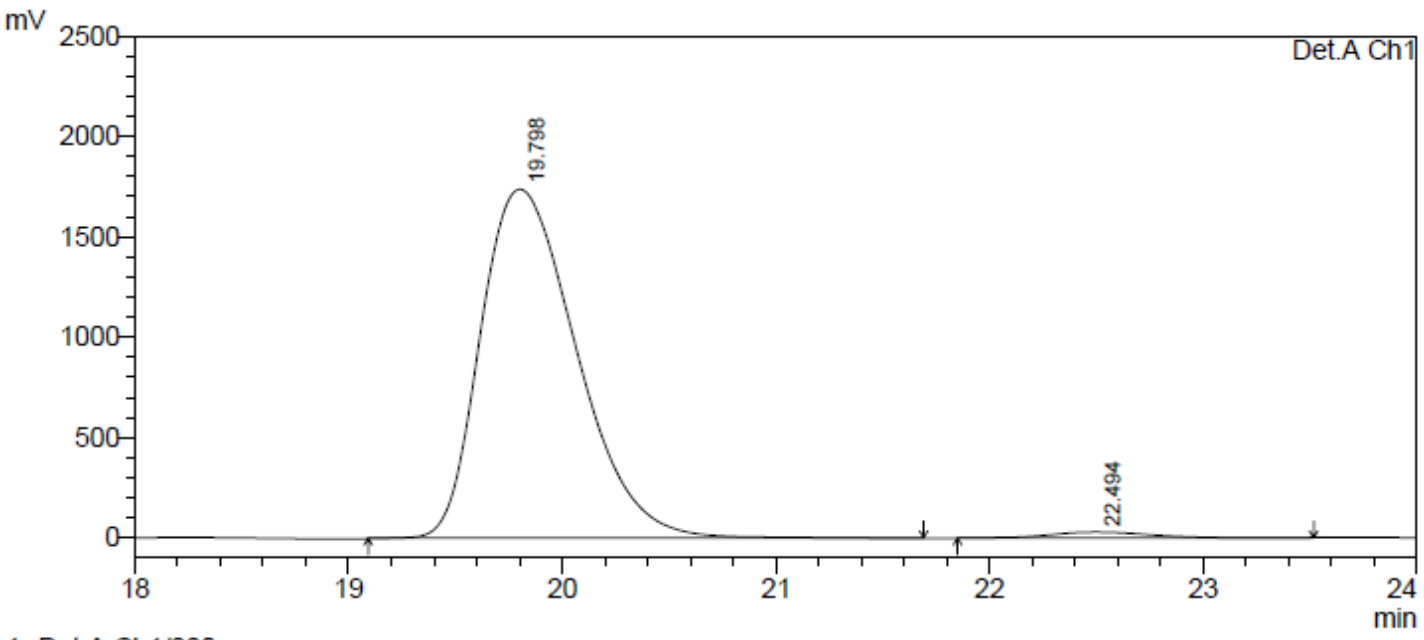

1 Det.A Ch $1 / 220 \mathrm{~nm}$

PeakTable

Detector A Ch1 220nm
\begin{tabular}{|r|r|r|r|r|r|}
\hline Peak\# & Ret. Time & \multicolumn{1}{|c|}{ Area } & Height & Area $\%$ & \multicolumn{1}{c|}{ Height $\%$} \\
\hline 1 & 19.798 & 54102217 & 1736972 & 98.333 & 98.339 \\
\hline 2 & 22.494 & 917234 & 29344 & 1.667 & 1.661 \\
\hline Total & & 55019451 & 1766316 & 100.000 & 100.000 \\
\hline
\end{tabular}


<smiles>O=C1[C@H](Cc2ccccc2)Oc2ccccc2N1c1ccccc1</smiles>

2b

$\mathrm{mV}$

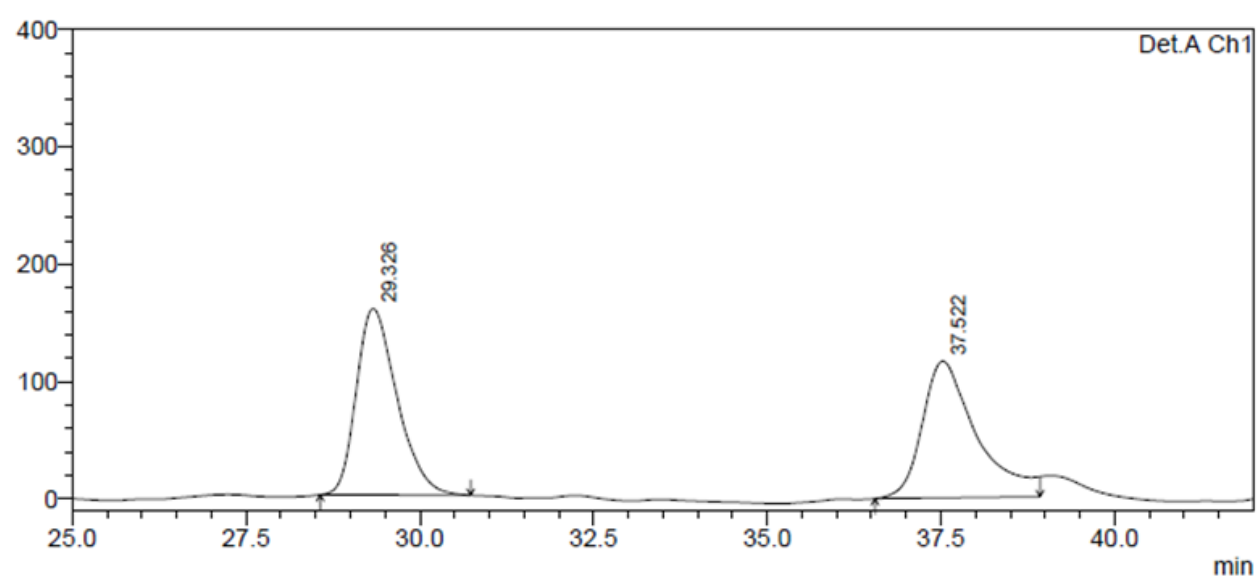

1 Det.A Ch $1 / 220 \mathrm{~nm}$

PeakTable

Detector A Ch1 $220 \mathrm{~nm}$
\begin{tabular}{|r|r|r|r|r|r|}
\hline Peak\# & Ret. Time & \multicolumn{1}{|c|}{ Area } & \multicolumn{1}{c|}{ Height } & Area $\%$ & Height $\%$ \\
\hline 1 & 29.326 & 6483071 & 158819 & 50.538 & 57.688 \\
\hline 2 & 37.522 & 6345120 & 116488 & 49.462 & 42.312 \\
\hline Total & & 12828191 & 275307 & 100.000 & 100.000 \\
\hline
\end{tabular}

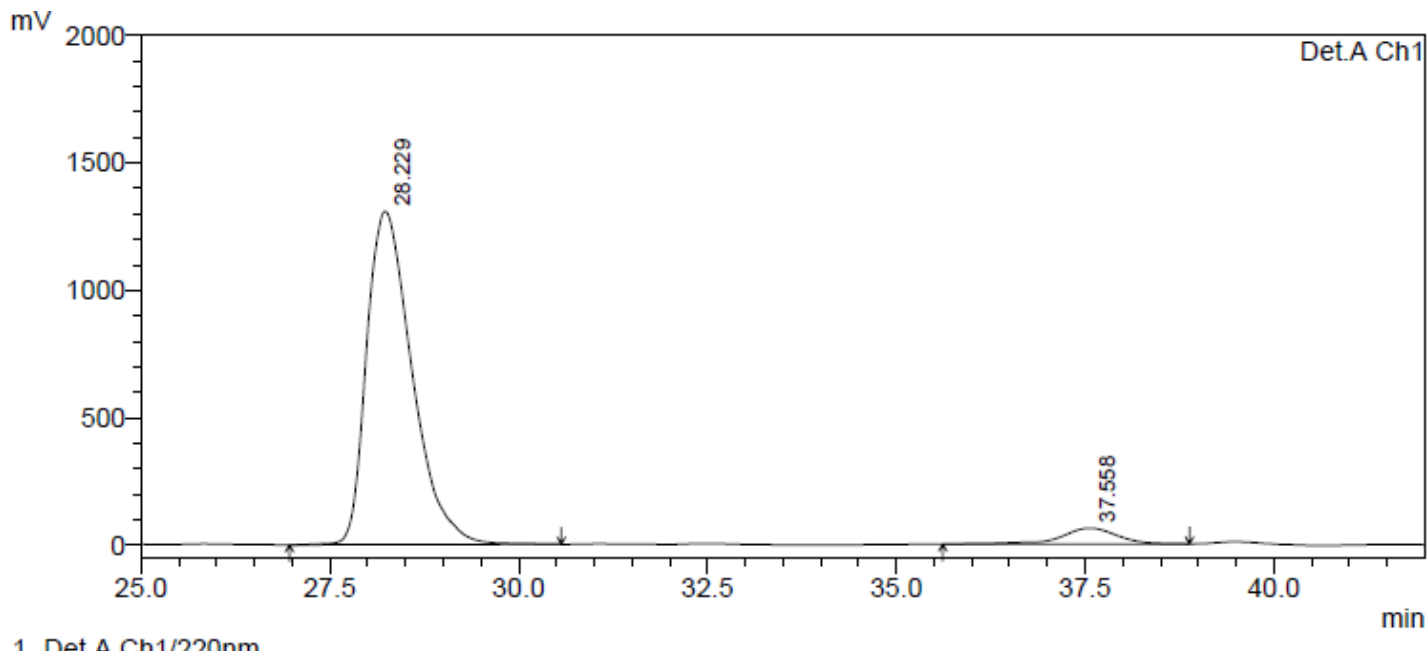

1 Det.A Ch1/220nm

PeakTable

Detector A Ch1 $220 \mathrm{~nm}$

\begin{tabular}{|r|r|r|r|r|r|}
\hline Peak\# & Ret. Time & \multicolumn{1}{c|}{ Area } & \multicolumn{1}{c|}{ Height } & Area \% & \multicolumn{1}{c|}{ Height \% } \\
\hline 1 & 28.229 & 54763184 & 1308854 & 94.453 & 95.455 \\
\hline 2 & 37.558 & 3215920 & 62315 & 5.547 & 4.545 \\
\hline Total & & 57979103 & 1371169 & 100.000 & 100.000 \\
\hline
\end{tabular}




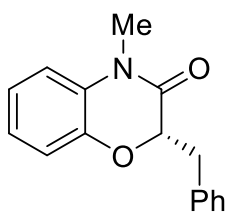

2c

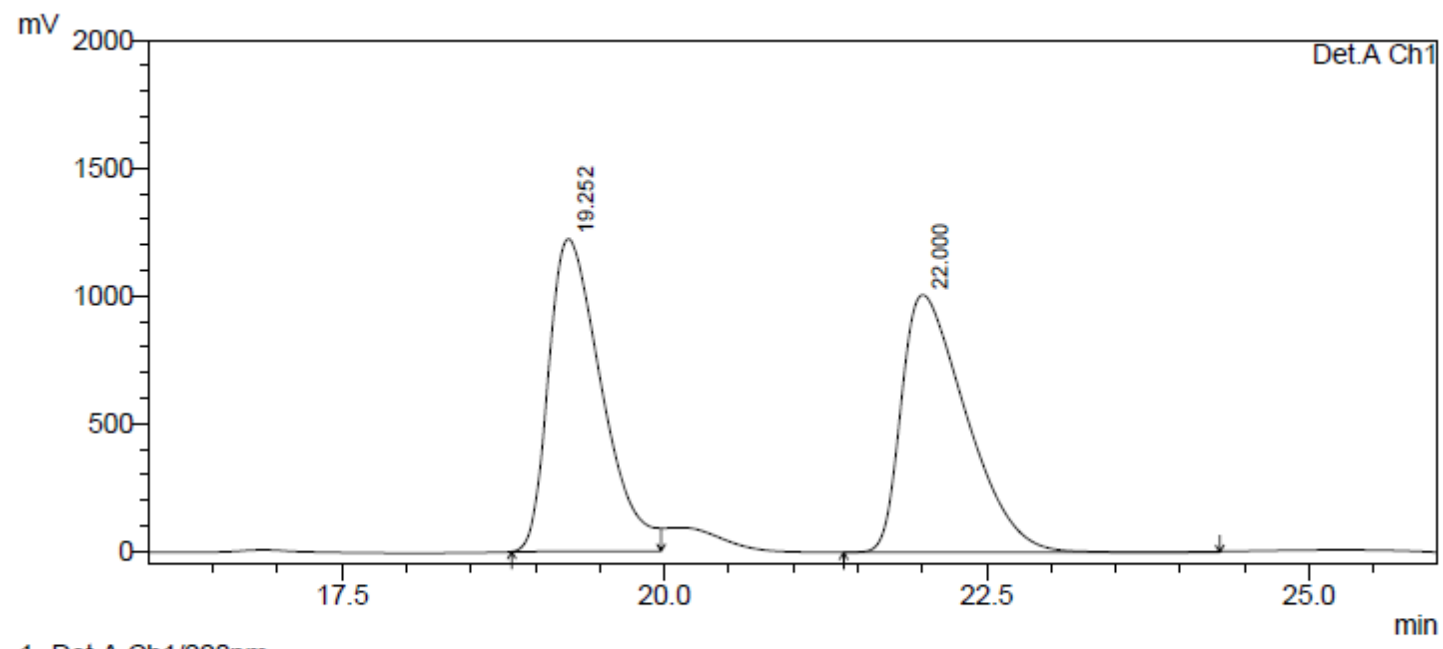

1 Det.A Ch1/220nm

PeakTable

Detector A Ch1 220nm
\begin{tabular}{|r|r|r|r|r|r|}
\hline \multicolumn{1}{|c|}{ Peak\# } & Ret. Time & Area & Height & Area $\%$ & \multicolumn{1}{c|}{ Height $\%$} \\
\hline 1 & 19.252 & 34794736 & 1222111 & 49.734 & 54.843 \\
\hline 2 & 22.000 & 35166519 & 1006252 & 50.266 & 45.157 \\
\hline Total & & 69961255 & 2228363 & 100.000 & 100.000 \\
\hline
\end{tabular}

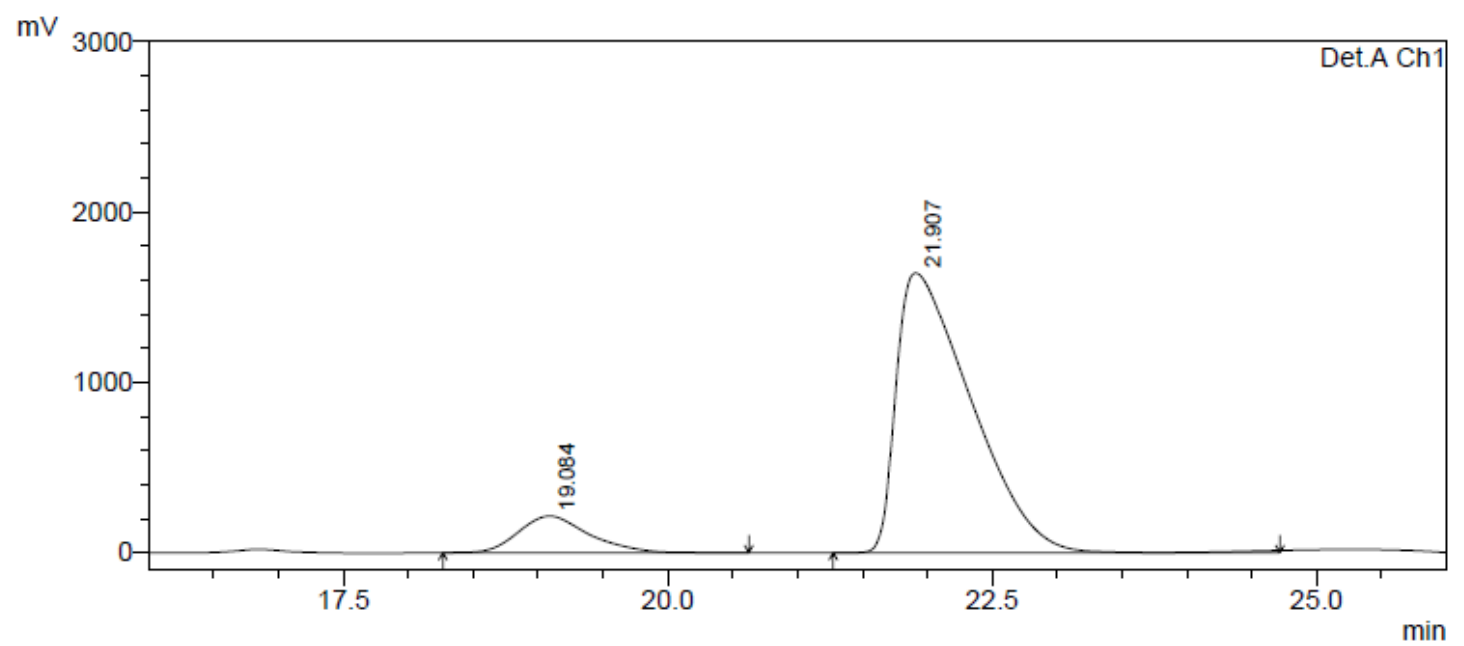

1 Det.A Ch1/220nm

PeakTable

Detector A Ch1 220nm

\begin{tabular}{|r|r|r|r|r|r|}
\hline Peak\# & Ret. Time & \multicolumn{1}{c|}{ Area } & \multicolumn{1}{c|}{ Height } & \multicolumn{1}{c|}{ Area \% } & \multicolumn{1}{c|}{ Height \% } \\
\hline 1 & 19.084 & 8046943 & 214307 & 10.914 & 11.542 \\
\hline 2 & 21.907 & 65680218 & 1642376 & 89.086 & 88.458 \\
\hline Total & & 73727161 & 1856683 & 100.000 & 100.000 \\
\hline
\end{tabular}


<smiles>Cc1ccc(C[C@@H]2Oc3ccccc3N(Cc3ccccc3)C2=O)cc1</smiles>

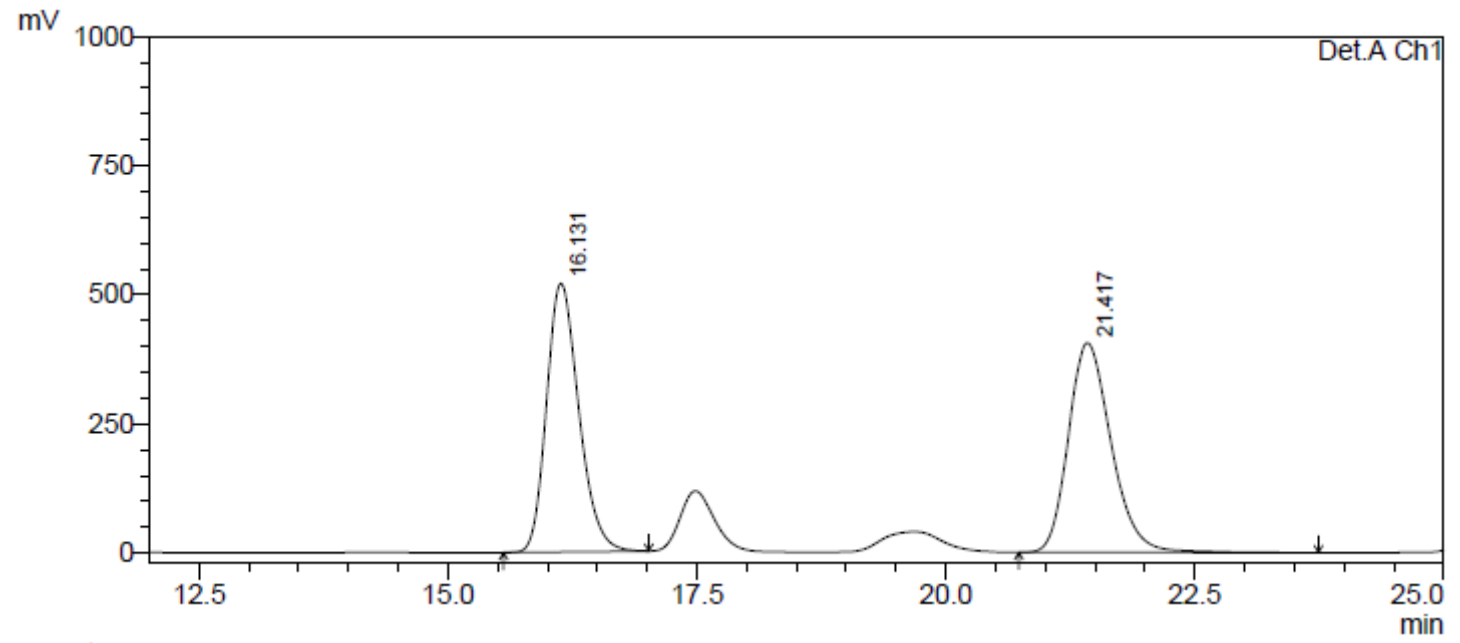

1 Det.A Ch1/220nm

Detector A Ch1 220nm
\begin{tabular}{|r|r|r|r|r|r|}
\hline Peak\# & Ret. Time & \multicolumn{1}{c|}{ Area } & \multicolumn{1}{c|}{ Height } & Area $\%$ & \multicolumn{1}{c|}{ Height $\%$} \\
\hline 1 & 16.131 & 11729192 & 519823 & 49.162 & 56.194 \\
\hline 2 & 21.417 & 12129117 & 405227 & 50.838 & 43.806 \\
\hline Total & & 23858309 & 925050 & 100.000 & 100.000 \\
\hline
\end{tabular}

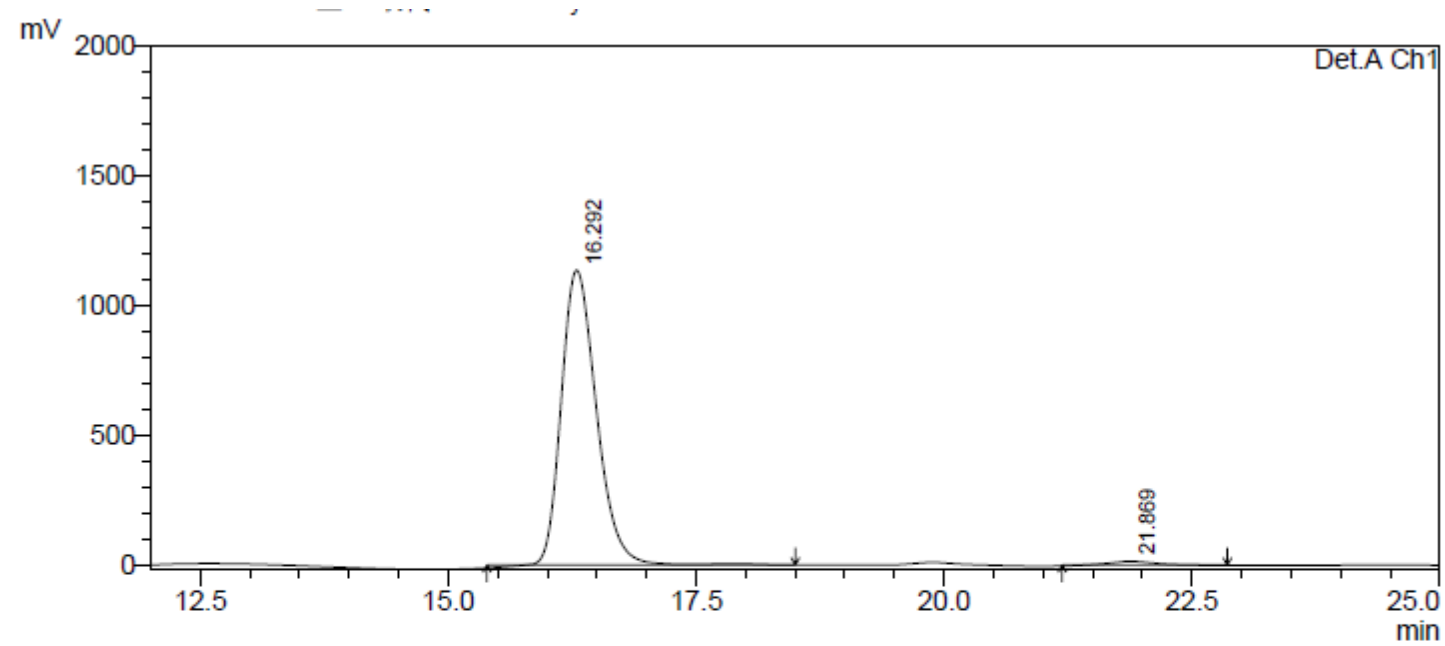

1 Det.A Ch1/220nm

PeakTable

Detector A Ch1 $220 \mathrm{~nm}$
\begin{tabular}{|r|r|r|r|r|r|}
\hline Peak\# & Ret. Time & \multicolumn{1}{|c|}{ Area } & Height & Area $\%$ & \multicolumn{1}{|c|}{ Height $\%$} \\
\hline 1 & 16.292 & 28056333 & 1134607 & 98.452 & 98.744 \\
\hline 2 & 21.869 & 441039 & 14427 & 1.548 & 1.256 \\
\hline Total & & 28497372 & 1149035 & 100.000 & 100.000 \\
\hline
\end{tabular}


<smiles>CCOc1ccc(C[C@@H]2Oc3ccccc3N(Cc3ccccc3)C2=O)cc1</smiles>

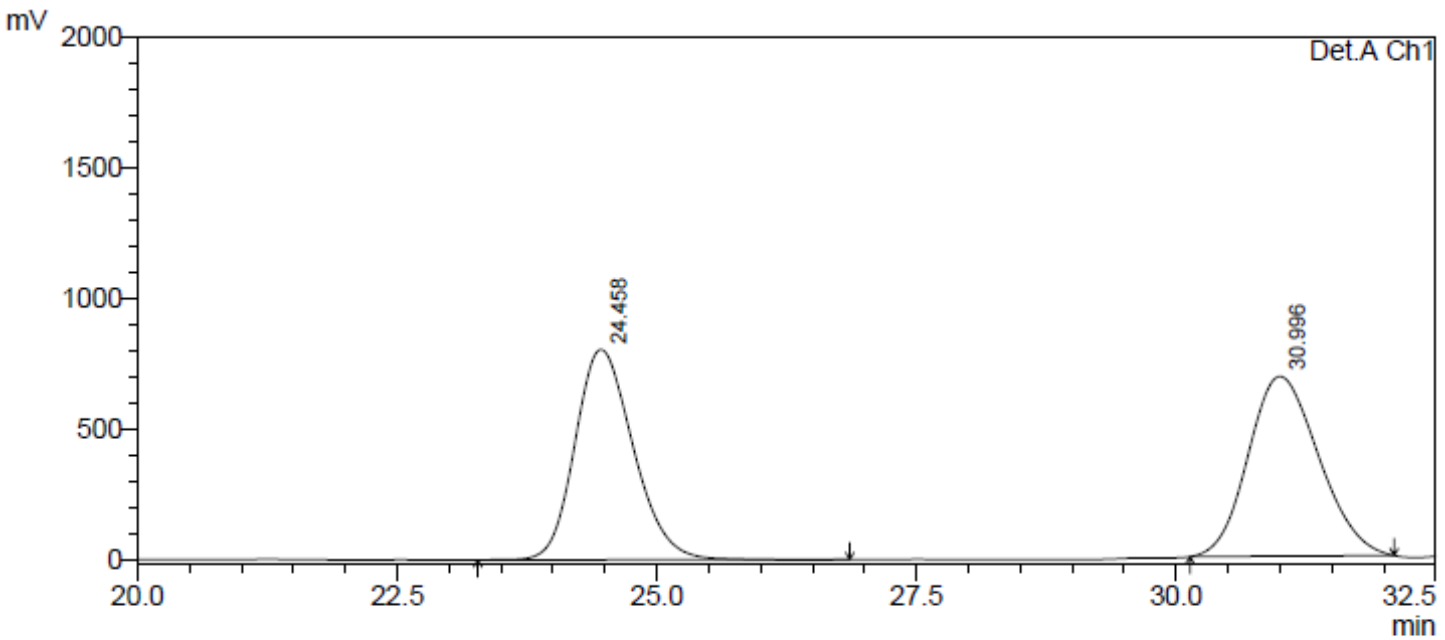

1 Det.A Ch1/220nm

PeakTable

Detector A Ch1 220nm

\begin{tabular}{|r|r|r|r|r|r|}
\hline \multicolumn{1}{|c|}{ Peak\# } & Ret. Time & \multicolumn{1}{|c|}{ Area } & \multicolumn{1}{c|}{ Height } & \multicolumn{1}{c|}{ Area \% } & \multicolumn{1}{c|}{ Height \% } \\
\hline 1 & 24.458 & 31321211 & 803629 & 49.081 & 53.898 \\
\hline 2 & 30.996 & 32493923 & 687388 & 50.919 & 46.102 \\
\hline Total & & 63815134 & 1491017 & 100.000 & 100.000 \\
\hline
\end{tabular}

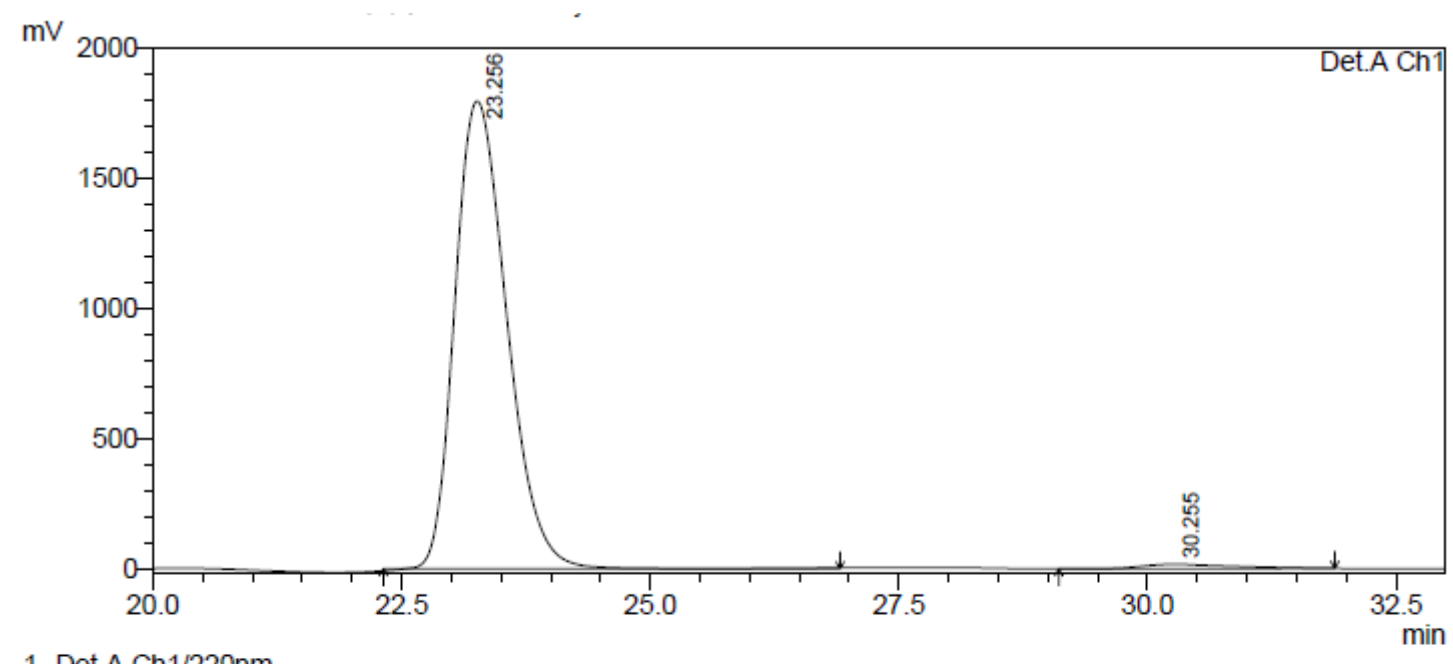

1 Det.A Ch1/220nm

PeakTable

Detector A Ch1 220nm

\begin{tabular}{|r|r|r|r|r|r|}
\hline \multicolumn{1}{|c|}{ Peak\# } & \multicolumn{1}{c|}{ Ret. Time } & \multicolumn{1}{c|}{ Area } & \multicolumn{1}{c|}{ Height } & \multicolumn{1}{c|}{ Area \% } & \multicolumn{1}{c|}{ Height \% } \\
\hline 1 & 23.256 & 65181845 & 1793913 & 98.408 & 99.056 \\
\hline 2 & 30.255 & 1054558 & 17098 & 1.592 & 0.944 \\
\hline Total & & 66236404 & 1811010 & 100.000 & 100.000 \\
\hline
\end{tabular}


<smiles>CC(C)(C)c1ccc(C[C@H]2Oc3ccccc3N(Cc3ccccc3)C2=O)cc1</smiles>

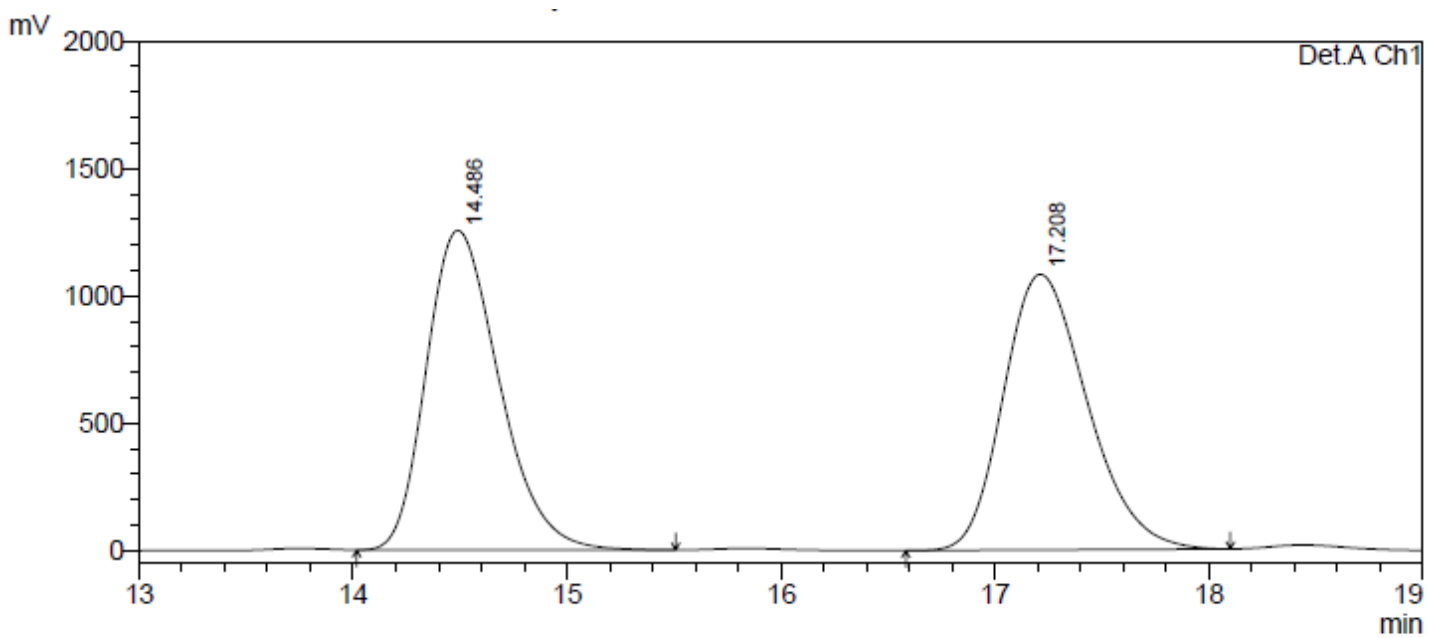

1 Det.A Ch1/220nm

Detector A Ch1 220nm
\begin{tabular}{|r|r|r|r|r|r|}
\hline Peak\# & Ret. Time & Area & Height & Area $\%$ & Height \% \\
\hline 1 & 14.486 & 29620574 & 1253525 & 50.179 & 53.696 \\
\hline 2 & 17.208 & 29408897 & 1080962 & 49.821 & 46.304 \\
\hline Total & & 59029470 & 2334487 & 100.000 & 100.000 \\
\hline
\end{tabular}

$\mathrm{mV}$

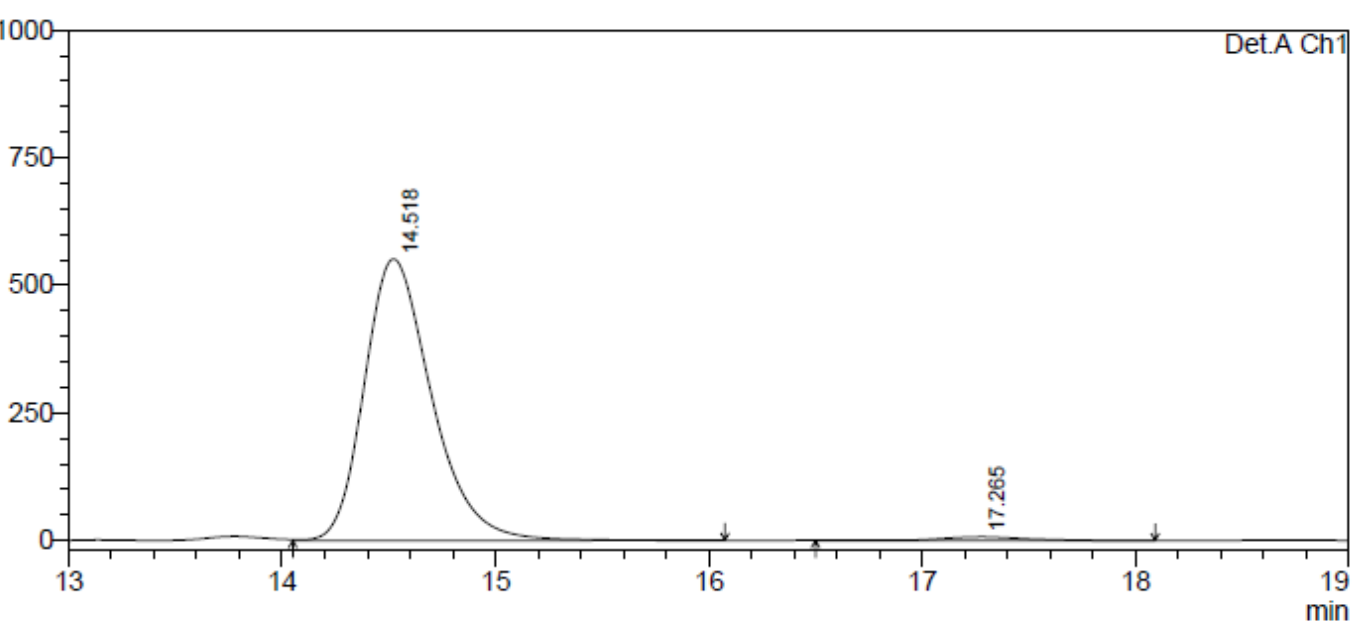

1 Det.A Ch $1 / 220 \mathrm{~nm}$

PeakTable

Detector A Ch1 $220 \mathrm{~nm}$
\begin{tabular}{|r|r|r|r|r|r|}
\hline Peak\# & Ret. Time & \multicolumn{1}{c|}{ Area } & \multicolumn{1}{c|}{ Height } & Area \% & \multicolumn{1}{c|}{ Height $\%$} \\
\hline 1 & 14.518 & 12095217 & 551482 & 98.506 & 98.750 \\
\hline 2 & 17.265 & 183406 & 6979 & 1.494 & 1.250 \\
\hline Total & & 12278623 & 558461 & 100.000 & 100.000 \\
\hline
\end{tabular}


<smiles>O=C1[C@H](Cc2ccc(F)cc2)Oc2ccccc2N1Cc1ccccc1</smiles>

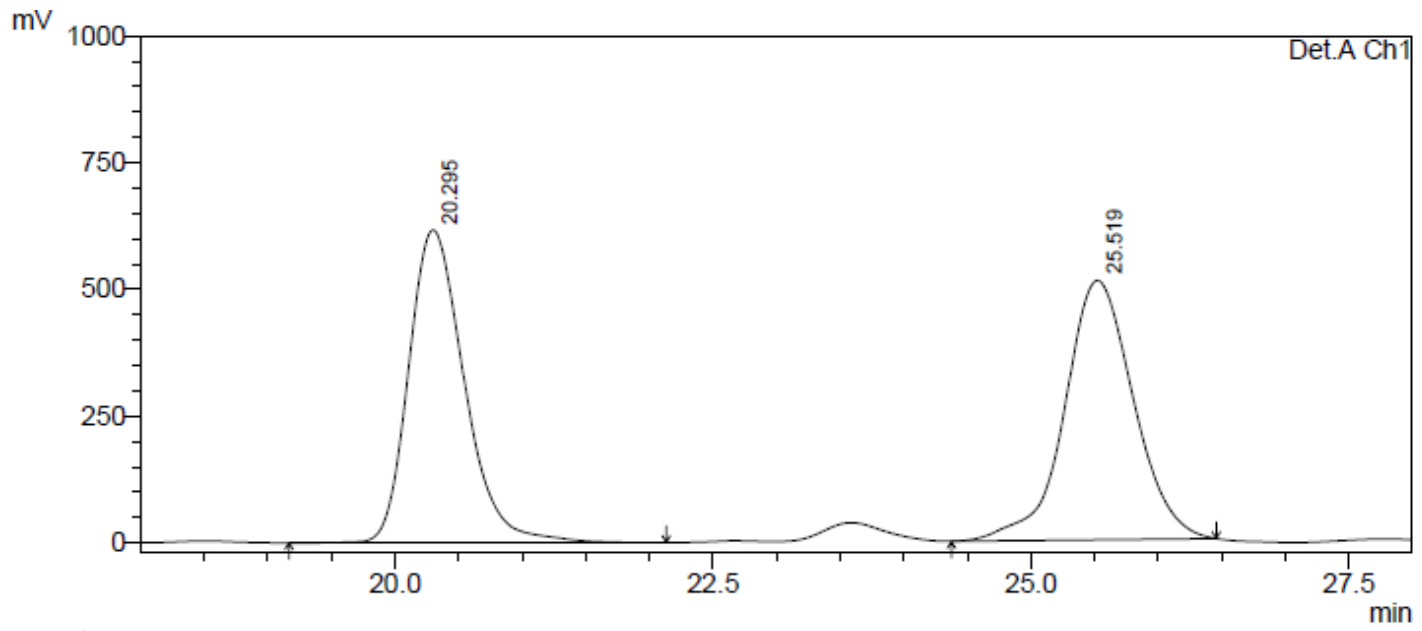

1 Det.A Ch1/220nm

Detector A Ch1 220nm
\begin{tabular}{|r|r|r|r|r|r|}
\hline Peak\# $\#$ Ret. Time & \multicolumn{1}{|c|}{ Area } & \multicolumn{1}{c|}{ Height } & Area $\%$ & \multicolumn{1}{c|}{ Height \% } \\
\hline 1 & 20.295 & 18732033 & 616367 & 49.275 & 54.643 \\
\hline 2 & 25.519 & 19283556 & 511621 & 50.725 & 45.357 \\
\hline Total & & 38015589 & 1127988 & 100.000 & 100.000 \\
\hline
\end{tabular}

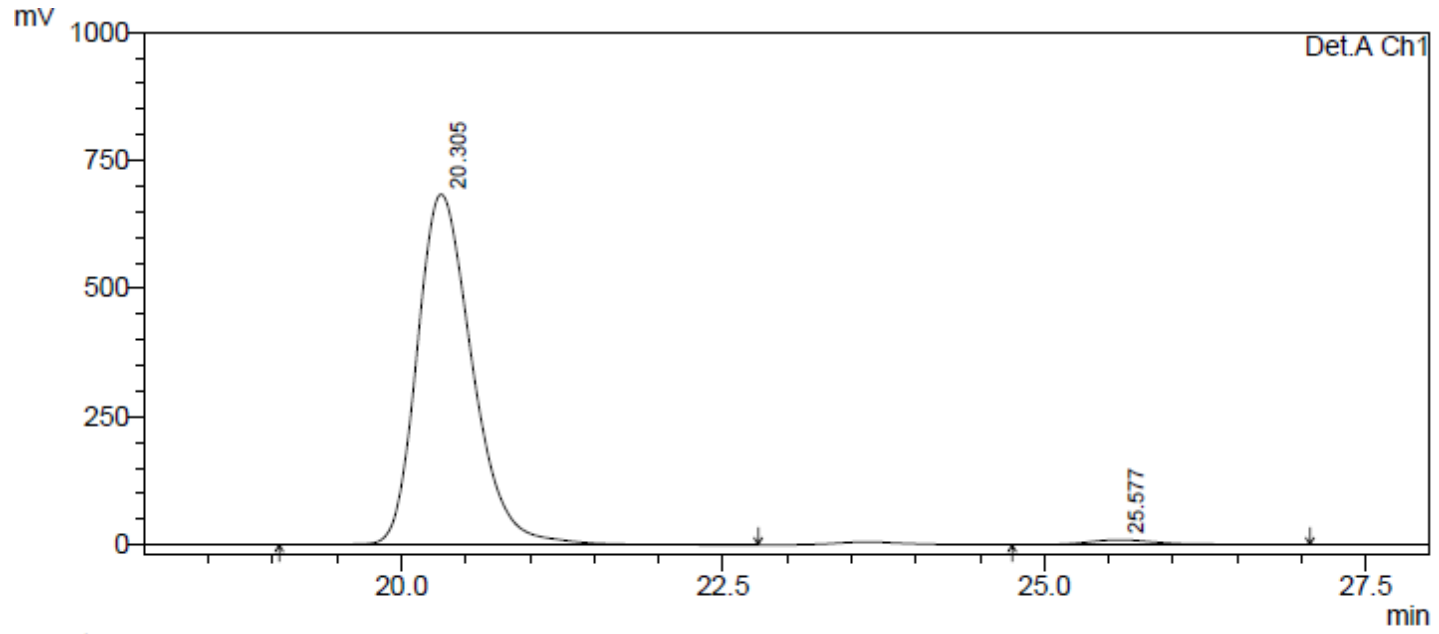

1 Det.A Ch1/220nm

PeakTable

Detector A Ch1 $220 \mathrm{~nm}$
\begin{tabular}{|r|r|r|r|r|r|}
\hline Peak\# & Ret. Time & \multicolumn{1}{c|}{ Area } & \multicolumn{1}{|c|}{ Height } & Area $\%$ & \multicolumn{1}{c|}{ Height $\%$} \\
\hline 1 & 20.305 & 20320004 & 683376 & 98.351 & 98.641 \\
\hline 2 & 25.577 & 340662 & 9415 & 1.649 & 1.359 \\
\hline Tota| & & 20660665 & 692791 & 100.000 & 100.000 \\
\hline
\end{tabular}


<smiles>O=C1[C@H](Cc2ccc(Cl)cc2)Oc2ccccc2N1Cc1ccccc1</smiles>

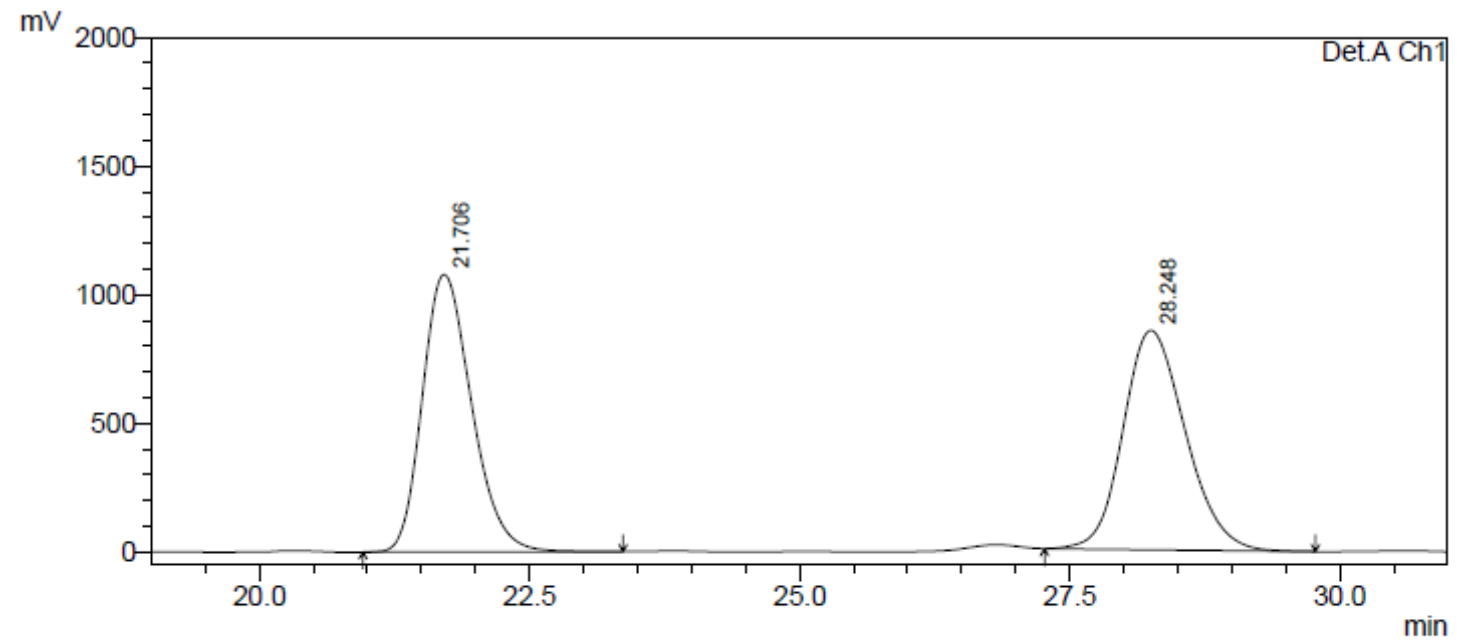

1 Det.A Ch $1 / 220 \mathrm{~nm}$

PeakTable

Detector A Ch1 220nm
\begin{tabular}{|r|r|r|r|r|r|}
\hline Peak\# $\#$ & Ret. Time & \multicolumn{1}{|c|}{ Area } & \multicolumn{1}{|c|}{ Height } & Area $\%$ & \multicolumn{1}{|c|}{ Height $\%$} \\
\hline 1 & 21.706 & 34137900 & 1078063 & 49.958 & 55.863 \\
\hline 2 & 28.248 & 34195885 & 851785 & 50.042 & 44.137 \\
\hline Total & & 68333786 & 1929848 & 100.000 & 100.000 \\
\hline
\end{tabular}

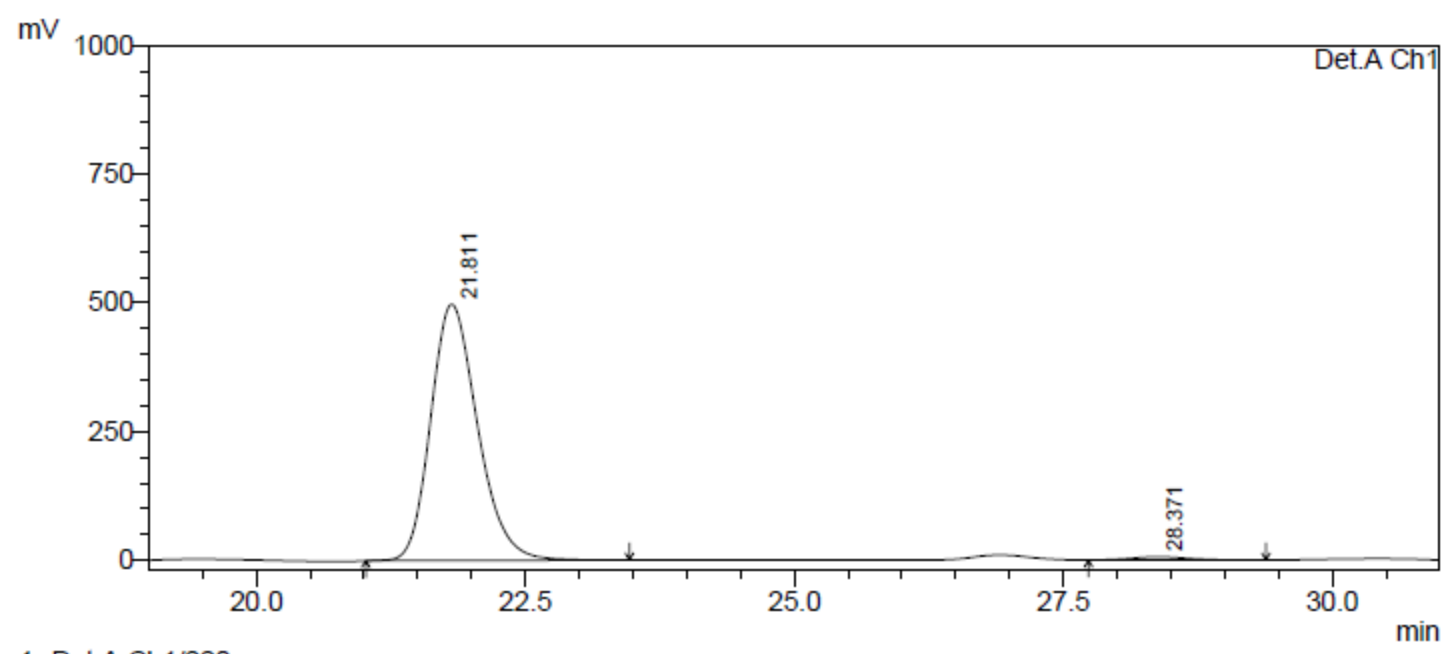

1 Det.A Ch1/220nm

PeakTable

Detector A Ch1 220nm
\begin{tabular}{|r|r|r|r|r|r|}
\hline Peak\# & Ret. Time & \multicolumn{1}{c|}{ Area } & \multicolumn{1}{c|}{ Height } & Area $\%$ & \multicolumn{1}{c|}{ Height $\%$} \\
\hline 1 & 21.811 & 15039762 & 496869 & 98.546 & 98.791 \\
\hline 2 & 28.371 & 221836 & 6080 & 1.454 & 1.209 \\
\hline Total & & 15261598 & 502949 & 100.000 & 100.000 \\
\hline
\end{tabular}


<smiles>O=C1[C@H](Cc2ccc(Br)cc2)Oc2ccccc2N1Cc1ccccc1</smiles>

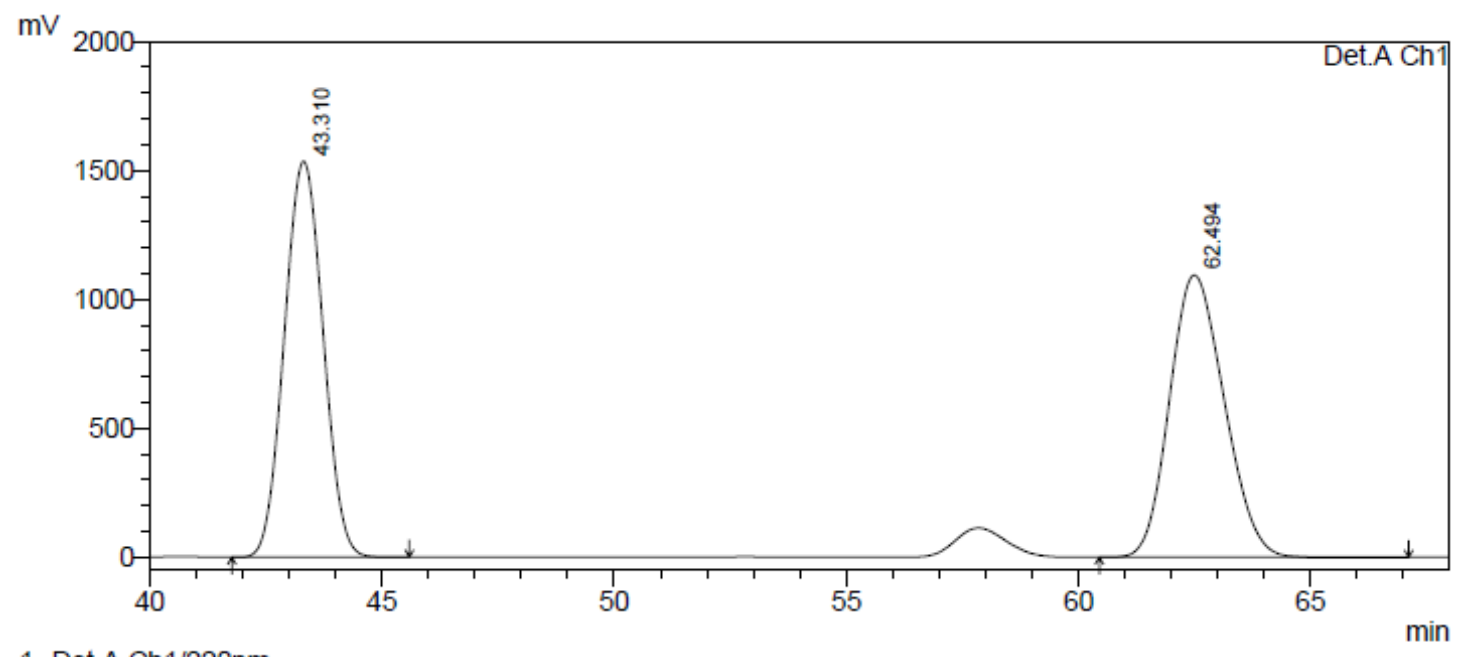

1 Det.A Ch1/220nm

PeakTable

Detector A Ch1 220nm

\begin{tabular}{|r|r|r|r|r|r|}
\hline \multicolumn{1}{|c|}{ Peak\# } & Ret. Time & \multicolumn{1}{c|}{ Area } & Height & Area \% & \multicolumn{1}{c|}{ Height \% } \\
\hline 1 & 43.310 & 89108998 & 1534642 & 49.916 & 58.389 \\
\hline 2 & 62.494 & 89407207 & 1093672 & 50.084 & 41.611 \\
\hline Total & & 178516205 & 2628314 & 100.000 & 100.000 \\
\hline
\end{tabular}

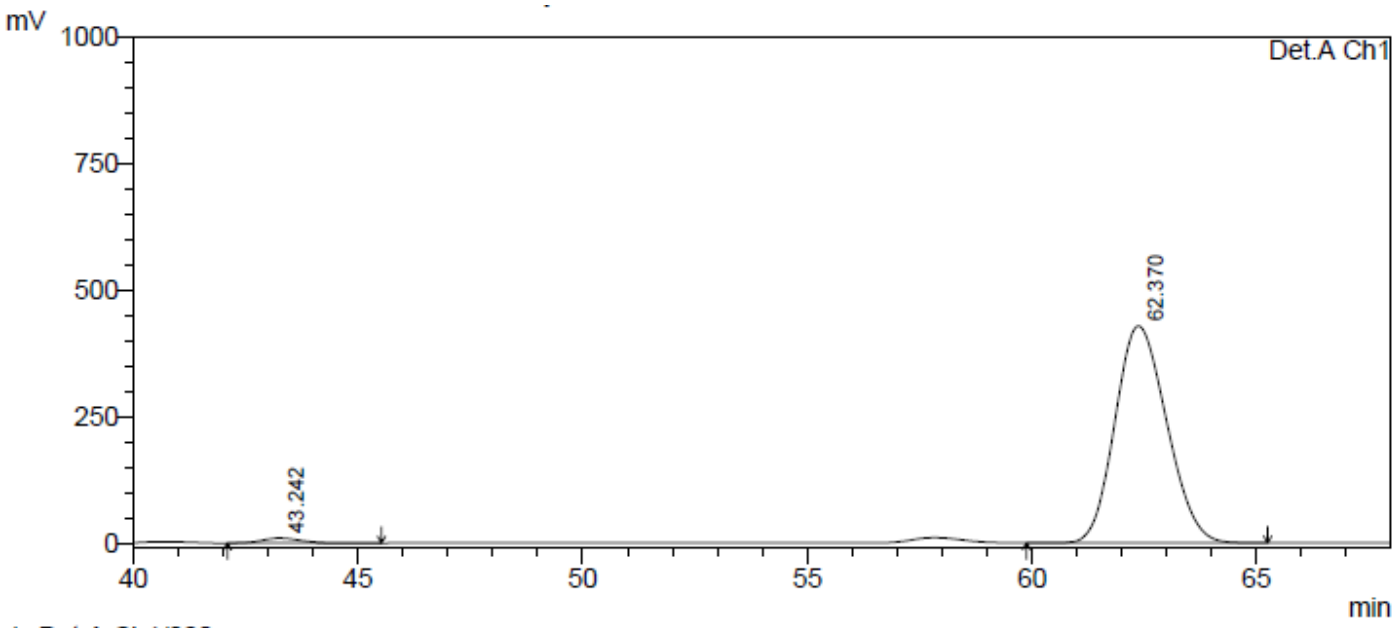

1 Det.A Ch1/220nm

PeakTable

Detector A Ch1 $220 \mathrm{~nm}$
\begin{tabular}{|r|r|r|r|r|r|}
\hline Peak\# & Ret. Time & \multicolumn{1}{c|}{ Area } & Height & Area $\%$ & Height $\%$ \\
\hline 1 & 43.242 & 573195 & 9663 & 1.645 & 2.207 \\
\hline 2 & 62.370 & 34266400 & 428192 & 98.355 & 97.793 \\
\hline Total & & 34839594 & 437855 & 100.000 & 100.000 \\
\hline
\end{tabular}


<smiles>O=C1[C@H](Cc2ccc(C(F)(F)F)cc2)Oc2ccccc2N1Cc1ccccc1</smiles>

$\mathrm{mV}$

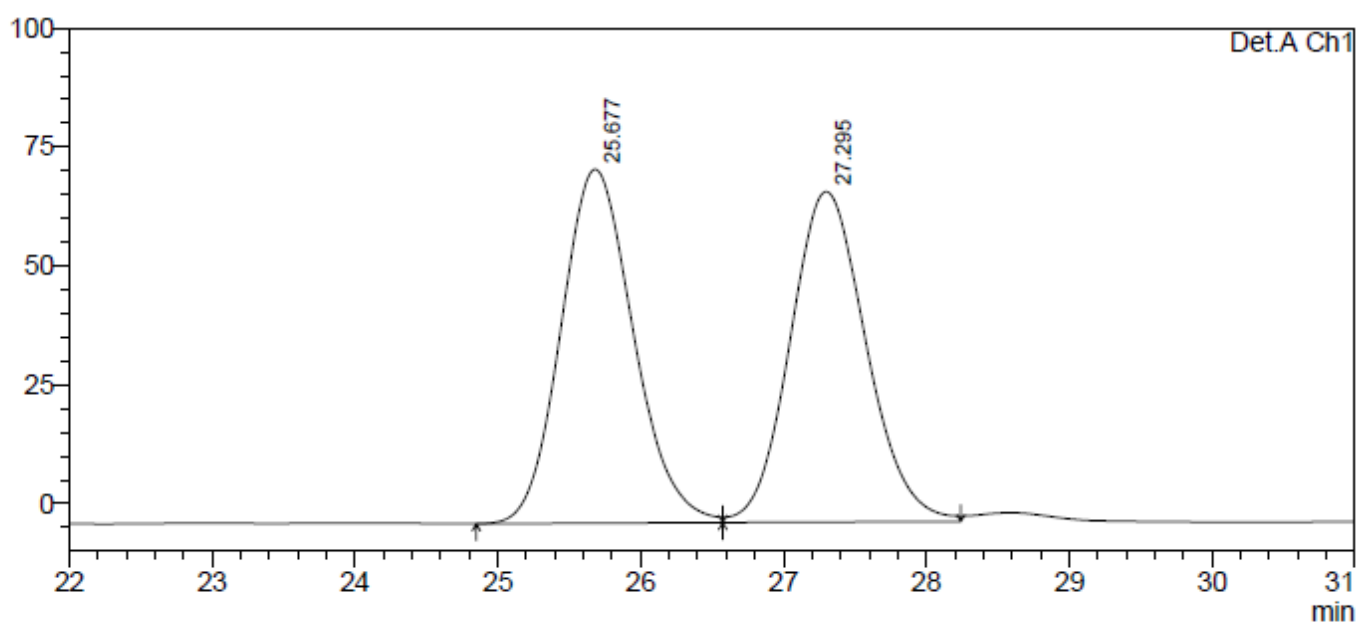

1 Det.A Ch1/220nm

Detector A Ch1 220nm
\begin{tabular}{r|r|r|r|r|r|}
\hline Peak\# & Ret. Time & \multicolumn{1}{|c|}{ Area } & Height & Area $\%$ & \multicolumn{1}{c|}{ Height \% } \\
\hline 1 & 25.677 & 2620549 & 74392 & 50.512 & 51.704 \\
\hline 2 & 27.295 & 2567407 & 69488 & 49.488 & 48.296 \\
\hline Total & & 5187956 & 143880 & 100.000 & 100.000 \\
\hline
\end{tabular}

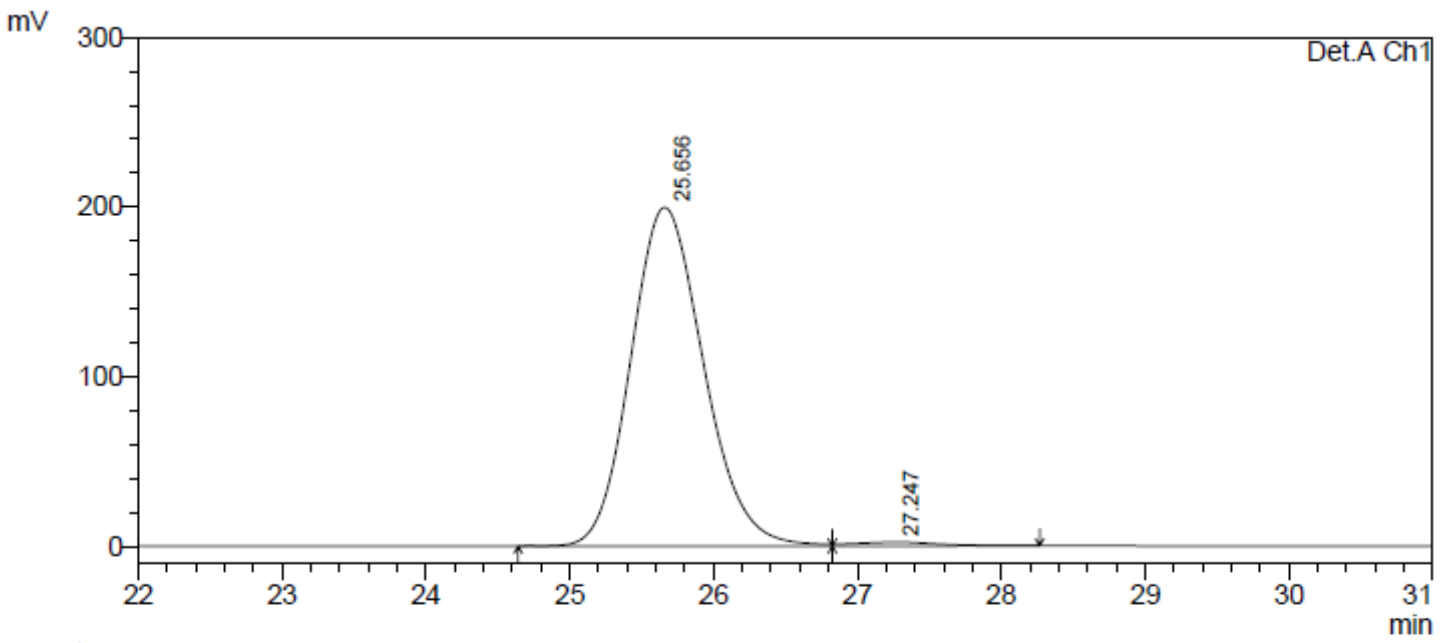

1 Det.A Ch1/220nm

PeakTable

Detector A Ch1 220nm

\begin{tabular}{|r|r|r|r|r|r|}
\hline \multicolumn{1}{|c|}{ Peak\# } & Ret. Time & \multicolumn{1}{|c|}{ Area } & \multicolumn{1}{c|}{ Height } & \multicolumn{1}{c|}{ Area \% } & \multicolumn{1}{c|}{ Height \% } \\
\hline 1 & 25.656 & 7020537 & 199302 & 98.762 & 98.897 \\
\hline 2 & 27.247 & 88004 & 2223 & 1.238 & 1.103 \\
\hline Total & & 7108541 & 201525 & 100.000 & 100.000 \\
\hline
\end{tabular}


<smiles>O=C1[C@H](Cc2ccc(OC(F)(F)F)cc2)Oc2ccccc2N1Cc1ccccc1</smiles>

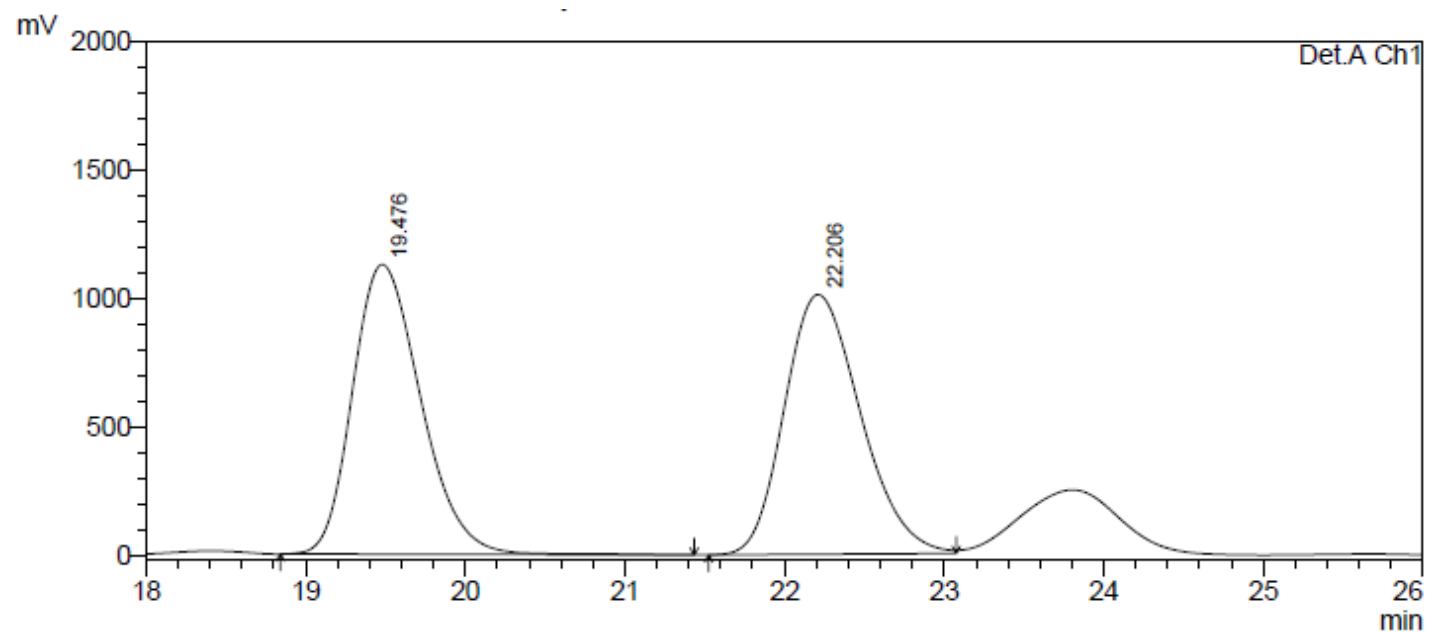

1 Det.A Ch1/220nm

PeakTable

Detector A Ch1 $220 \mathrm{~nm}$
\begin{tabular}{|r|r|r|r|r|r|}
\hline Peak\# & Ret. Time & \multicolumn{1}{l|}{ Area } & Height & Area \% & \multicolumn{1}{c|}{ Height \% } \\
\hline 1 & 19.476 & 33472154 & 1126057 & 50.060 & 52.727 \\
\hline 2 & 22.206 & 33392352 & 1009596 & 49.940 & 47.273 \\
\hline Total| & & 66864506 & 2135653 & 100.000 & 100.000 \\
\hline
\end{tabular}

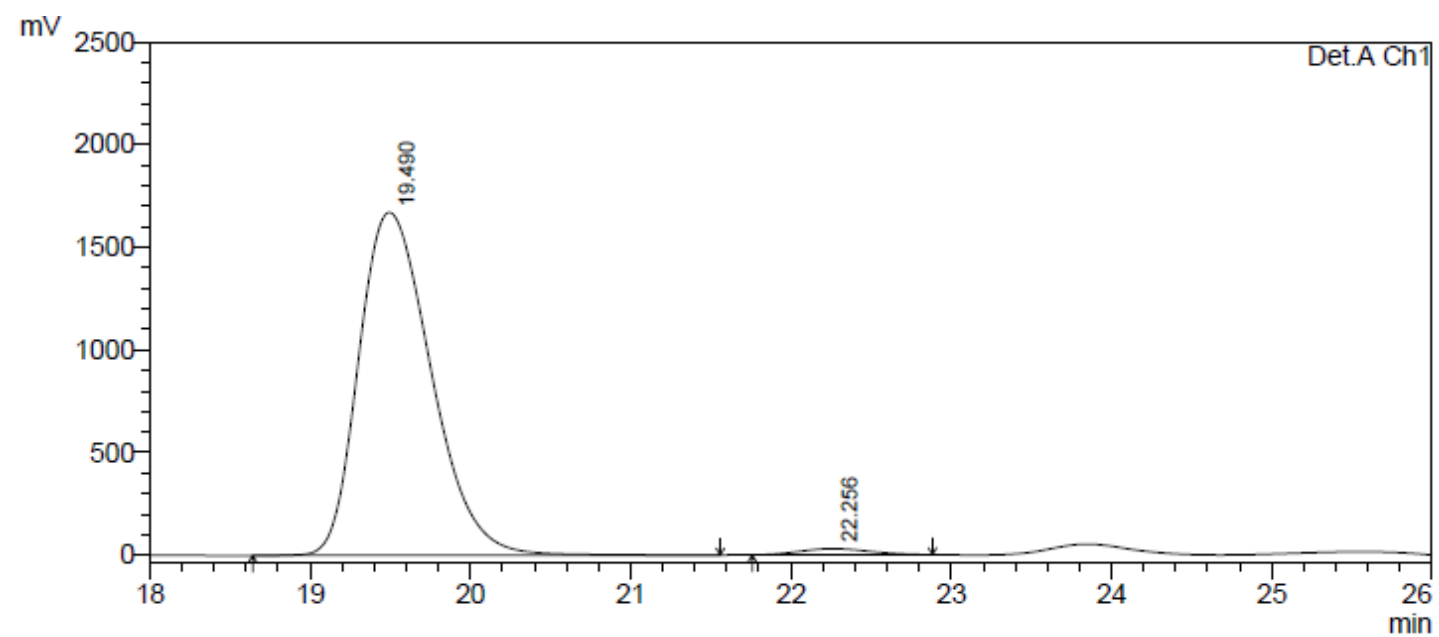

1 Det.A Ch1/220nm

PeakTable

Detector A Ch1 $220 \mathrm{~nm}$
\begin{tabular}{|r|r|r|r|r|r|}
\hline Peak\# & Ret. Time & \multicolumn{1}{|c|}{ Area } & \multicolumn{1}{c|}{ Height } & Area $\%$ & \multicolumn{1}{|c|}{ Height \% } \\
\hline 1 & 19.490 & 52282558 & 1669881 & 98.302 & 98.238 \\
\hline 2 & 22.256 & 903226 & 29956 & 1.698 & 1.762 \\
\hline Total & & 53185784 & 1699837 & 100.000 & 100.000 \\
\hline
\end{tabular}


<smiles>CCOC(=O)c1ccc(C[C@@H]2Oc3ccccc3N(Cc3ccccc3)C2=O)cc1</smiles>

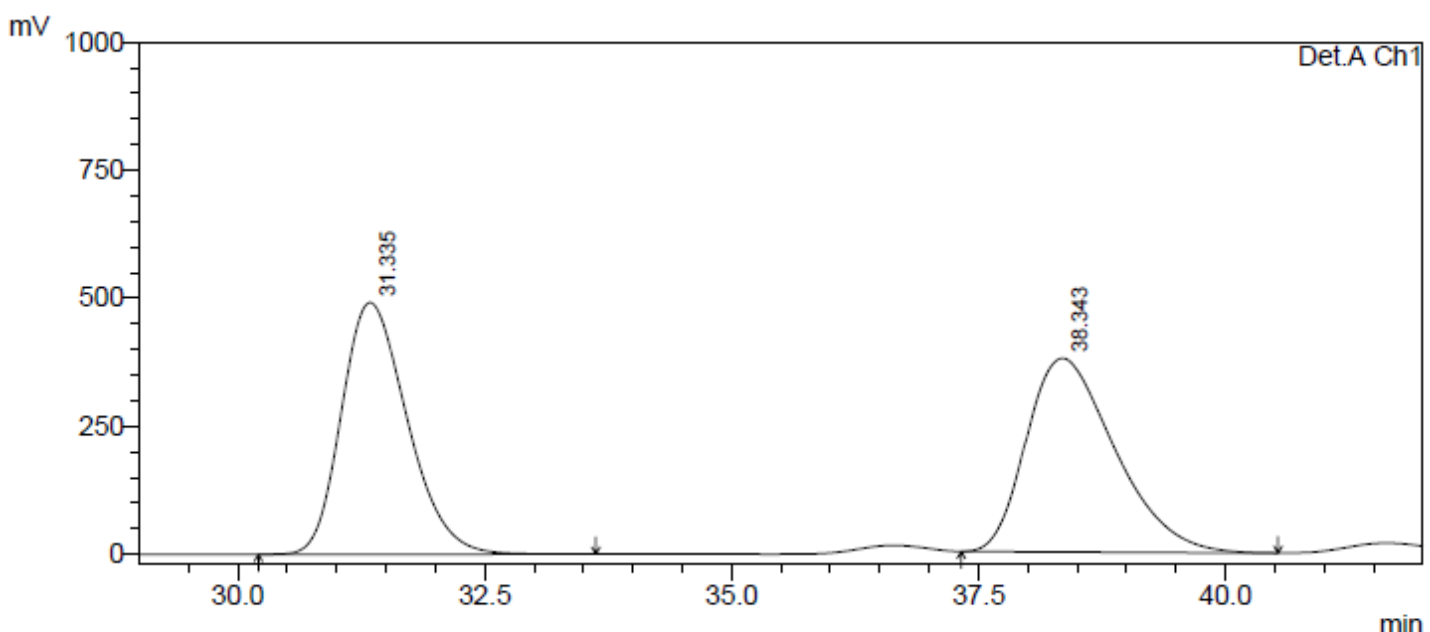

1 Det.A Ch1/220nm

PeakTable

Detector A Ch1 $220 \mathrm{~nm}$
\begin{tabular}{|r|r|r|r|r|r|}
\hline Peak\# & Ret. Time & \multicolumn{1}{l|}{ Area } & Height & Area \% & \multicolumn{1}{|l|}{ Height \% } \\
\hline 1 & 31.335 & 22758111 & 491332 & 49.087 & 56.511 \\
\hline 2 & 38.343 & 23605031 & 378119 & 50.913 & 43.489 \\
\hline Total & & 46363142 & 869452 & 100.000 & 100.000 \\
\hline
\end{tabular}

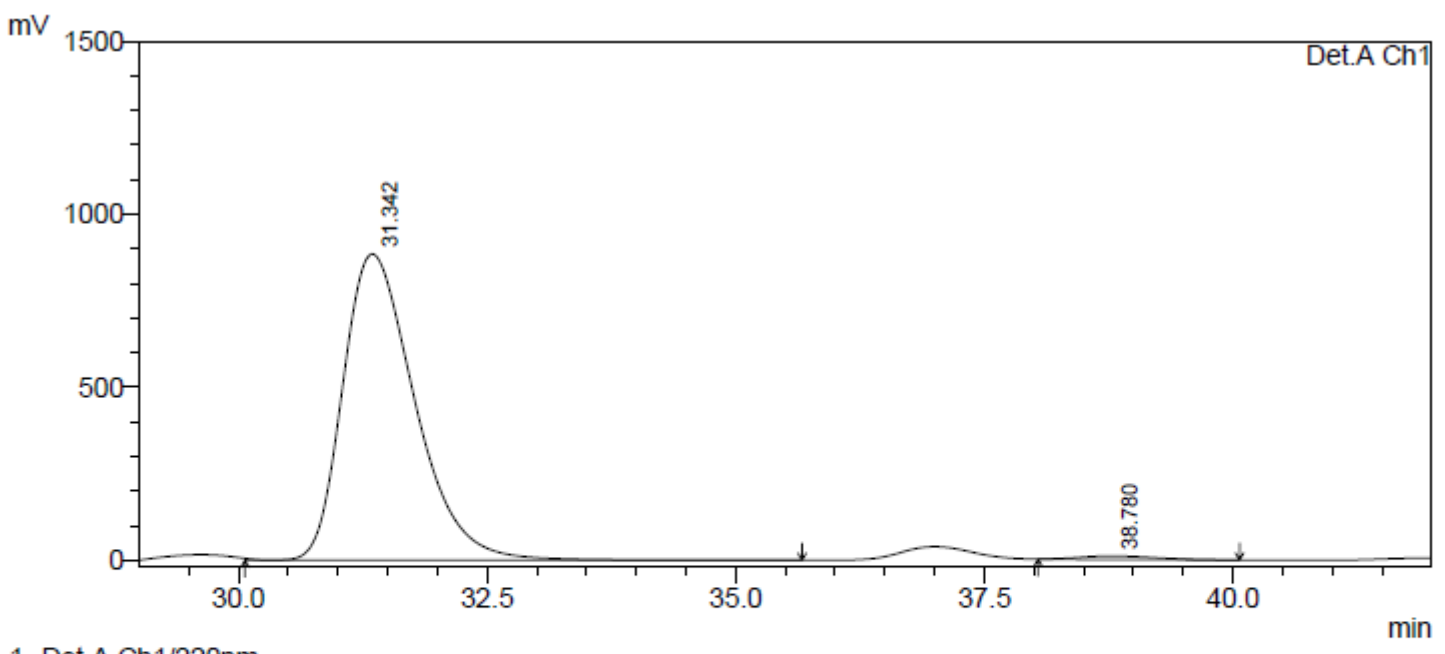

1 Det.A Ch1/220nm

PeakTable

Detector A Ch1 $220 \mathrm{~nm}$

\begin{tabular}{|r|r|r|r|r|r|}
\hline Peak\# & \multicolumn{1}{|c|}{ Ret. Time } & \multicolumn{1}{c|}{ Area } & \multicolumn{1}{c|}{ Height } & \multicolumn{1}{c|}{ Area \% } & \multicolumn{1}{c|}{ Height \% } \\
\hline 1 & 31.342 & 44544125 & 884864 & 98.848 & 98.887 \\
\hline 2 & 38.780 & 519325 & 9963 & 1.152 & 1.113 \\
\hline Total & & 45063450 & 894827 & 100.000 & 100.000 \\
\hline
\end{tabular}


<smiles>O=C1[C@H](Cc2cccc(Cl)c2)Oc2ccccc2N1Cc1ccccc1</smiles>

2n

$\mathrm{mV}$

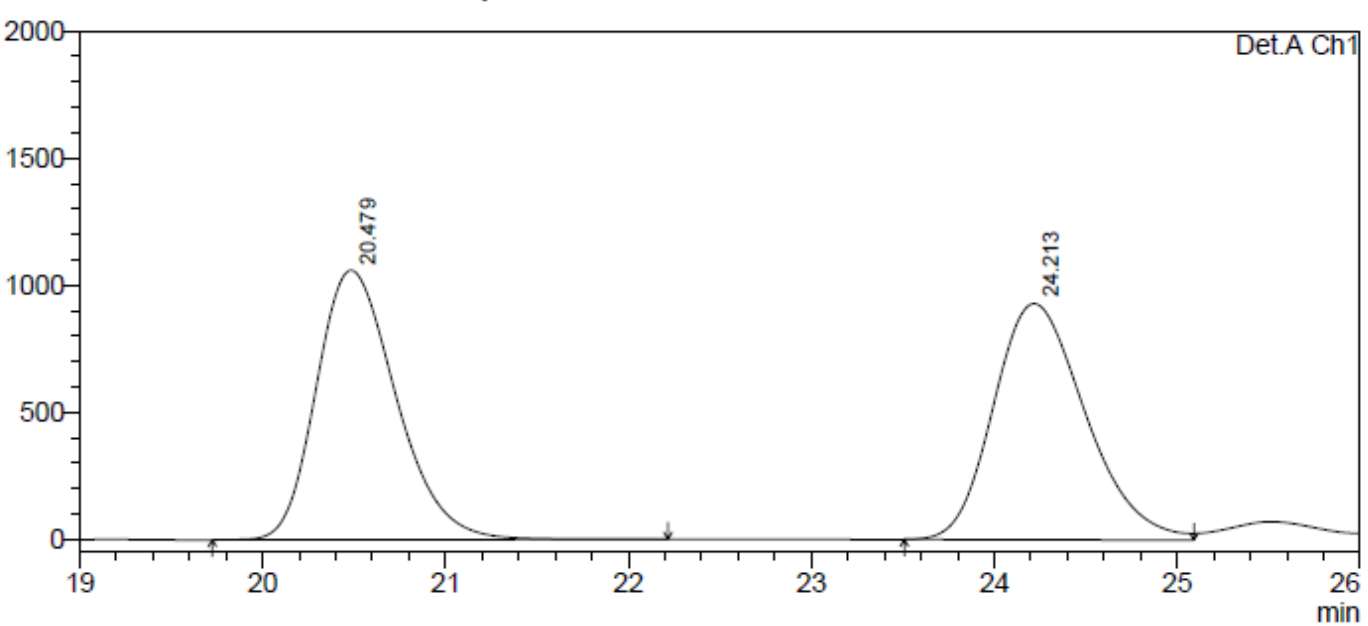

1 Det.A Ch1/220nm

PeakTable

Detector A Ch1 220nm
\begin{tabular}{|r|r|r|r|r|r|}
\hline Peak\# & Ret. Time & \multicolumn{1}{|c|}{ Area } & Height & \multicolumn{1}{c|}{ Area $\%$} & \multicolumn{1}{c|}{ Height \% } \\
\hline 1 & 20.479 & 32073227 & 1058610 & 49.665 & 53.273 \\
\hline 2 & 24.213 & 32505367 & 928528 & 50.335 & 46.727 \\
\hline Total & & 64578594 & 1987138 & 100.000 & 100.000 \\
\hline
\end{tabular}

$\mathrm{mV}$

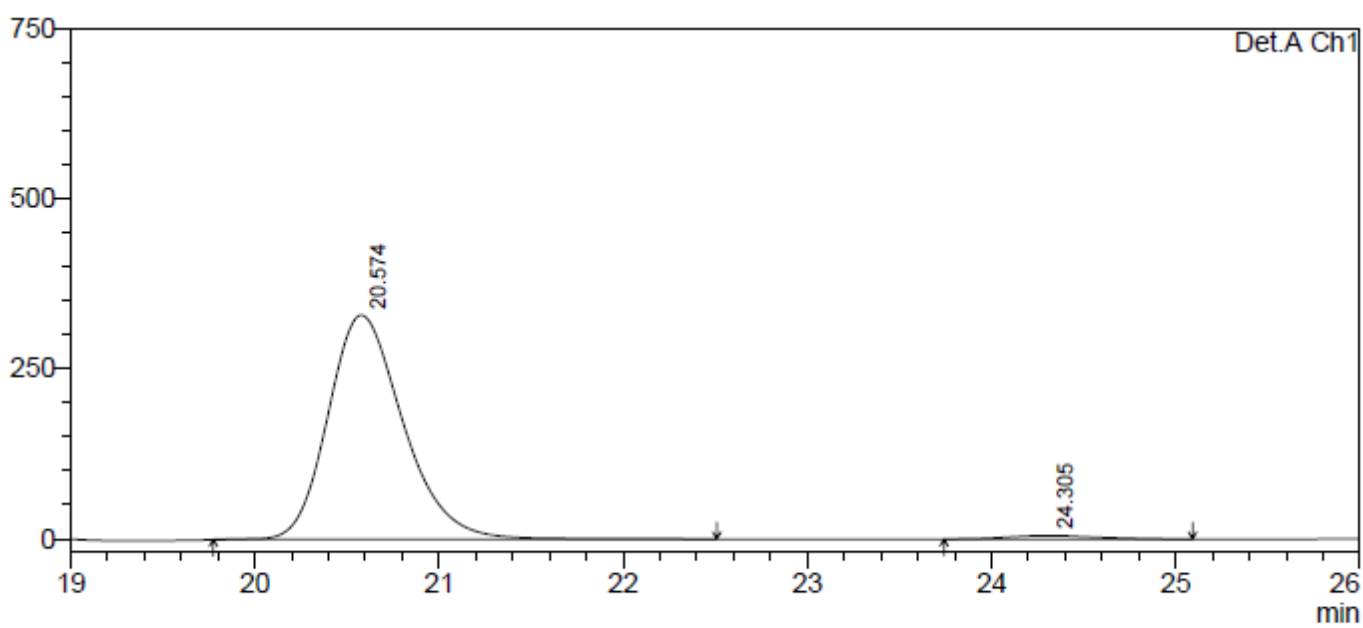

1 Det.A Ch1/220nm

PeakTable

Detector A Ch1 220nm
\begin{tabular}{|r|r|r|r|r|r|}
\hline Peak\# & Ret. Time & \multicolumn{1}{c|}{ Area } & \multicolumn{1}{c|}{ Height } & Area $\%$ & Height $\%$ \\
\hline 1 & 20.574 & 9309469 & 328919 & 98.264 & 98.460 \\
\hline 2 & 24.305 & 164452 & 5146 & 1.736 & 1.540 \\
\hline Total & & 9473921 & 334064 & 100.000 & 100.000 \\
\hline
\end{tabular}


<smiles>COc1cccc(C[C@@H]2Oc3ccccc3N(Cc3ccccc3)C2=O)c1</smiles>

20

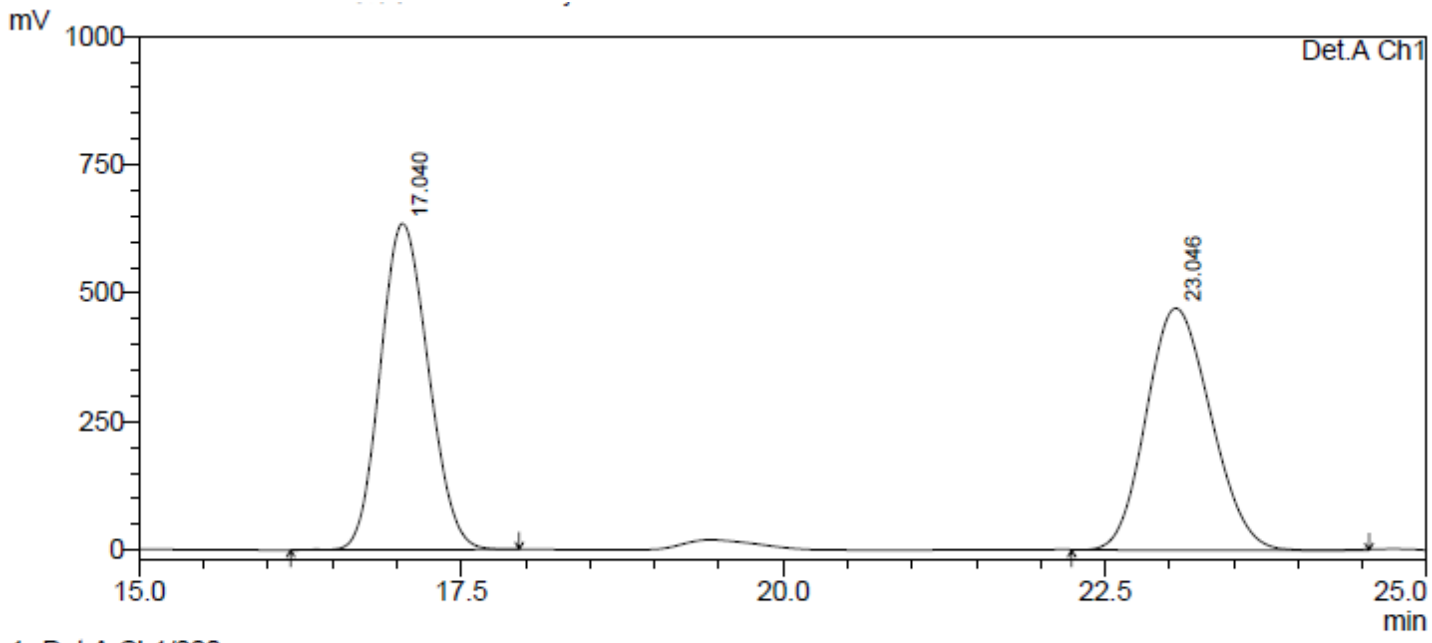

1 Det.A Ch1/220nm

PeakTable

Detector A Ch1 220nm

\begin{tabular}{|r|r|r|r|r|r|}
\hline \multicolumn{1}{|c|}{ Peak\# } & Ret. Time & \multicolumn{1}{|c|}{ Area } & \multicolumn{1}{c|}{ Height } & \multicolumn{1}{c|}{ Area \% } & \multicolumn{1}{c|}{ Height \% } \\
\hline 1 & 17.040 & 16432199 & 634768 & 50.012 & 57.423 \\
\hline 2 & 23.046 & 16424305 & 470654 & 49.988 & 42.577 \\
\hline Total & & 32856504 & 1105422 & 100.000 & 100.000 \\
\hline
\end{tabular}

$\mathrm{mV}$

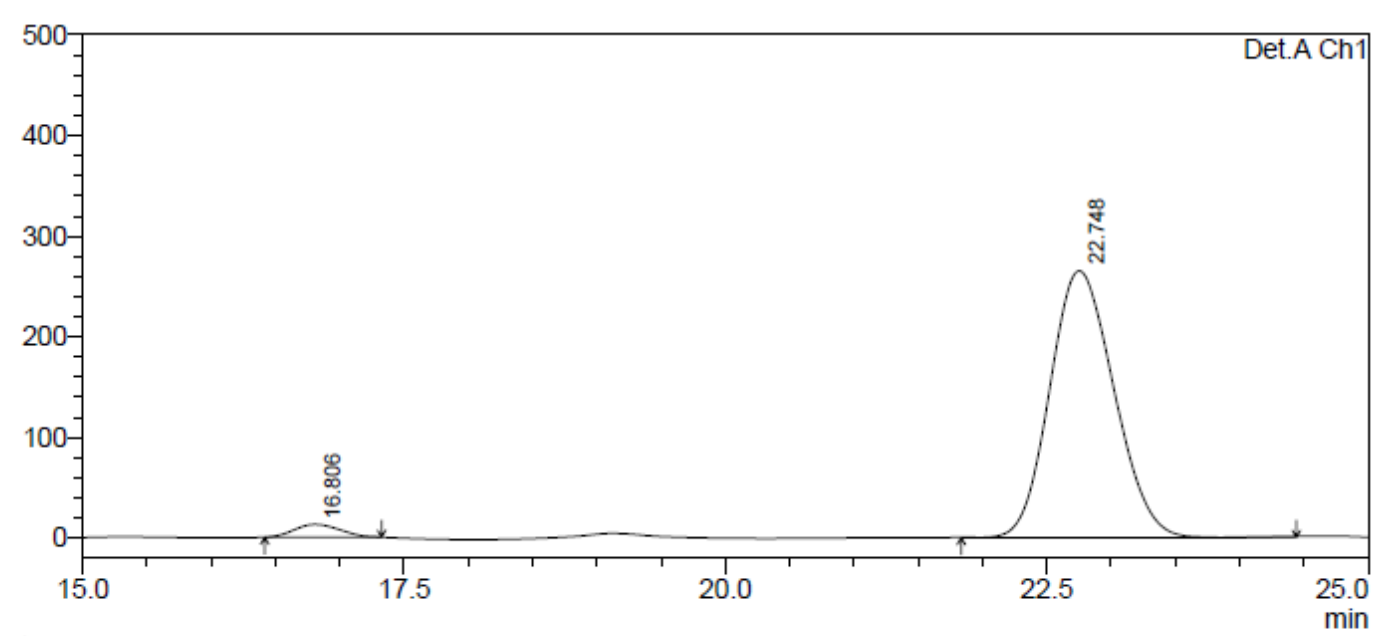

1 Det.A Ch1/220nm

PeakTable

Detector A Ch1 220nm

\begin{tabular}{|r|r|r|r|r|r|}
\hline Peak\# & Ret. Time & \multicolumn{1}{c|}{ Area } & \multicolumn{1}{c|}{ Height } & \multicolumn{1}{c|}{ Area \% } & \multicolumn{1}{c|}{ Height \% } \\
\hline 1 & 16.806 & 316253 & 12833 & 3.330 & 4.620 \\
\hline 2 & 22.748 & 9181302 & 264951 & 96.670 & 95.380 \\
\hline Total & & 9497556 & 277784 & 100.000 & 100.000 \\
\hline
\end{tabular}


<smiles>O=C1[C@H](Cc2ccccc2Cl)Oc2ccccc2N1Cc1ccccc1</smiles>

$2 p$

$\mathrm{mV}$

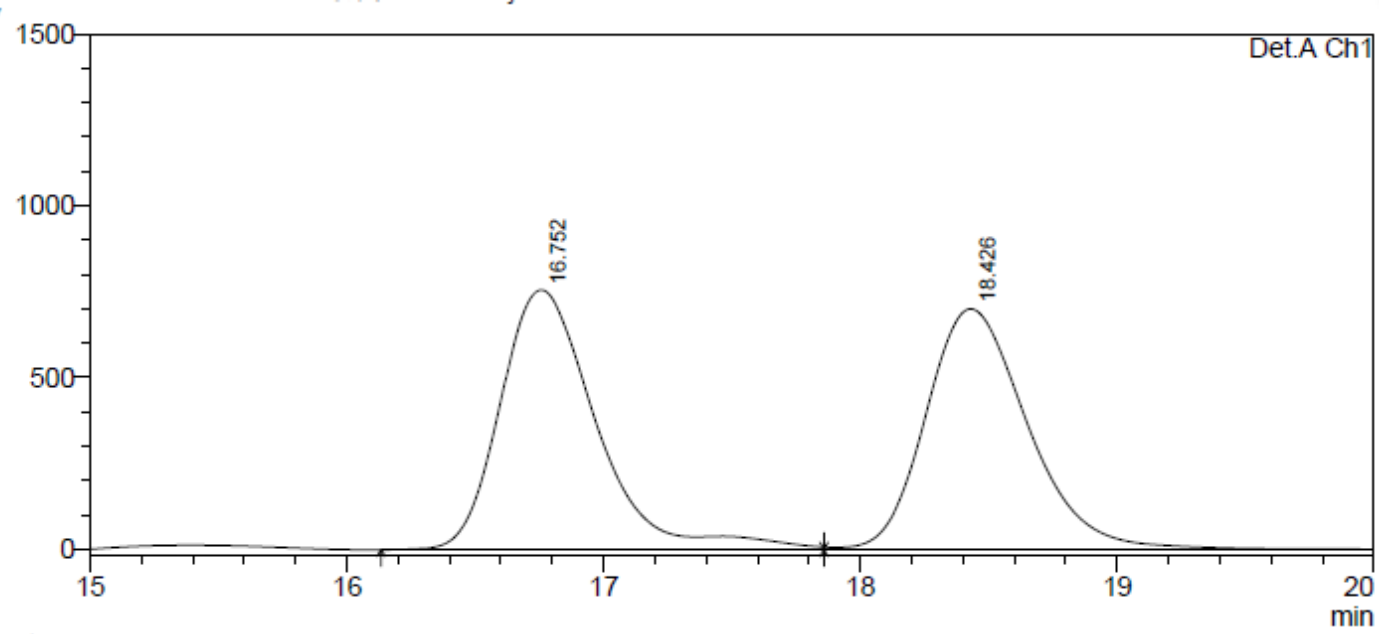

1 Det.A Ch1/220nm

PeakTable

Detector A Ch1 $220 \mathrm{~nm}$

\begin{tabular}{|r|r|r|r|r|r|}
\hline Peak\# & Ret. Time & \multicolumn{1}{c|}{ Area } & \multicolumn{1}{c|}{ Height } & Area \% & \multicolumn{1}{c|}{ Height \% } \\
\hline 1 & 16.752 & 19605820 & 754975 & 50.479 & 51.894 \\
\hline 2 & 18.426 & 19233932 & 699856 & 49.521 & 48.106 \\
\hline Total & & 38839752 & 1454830 & 100.000 & 100.000 \\
\hline
\end{tabular}

$\mathrm{mV}$

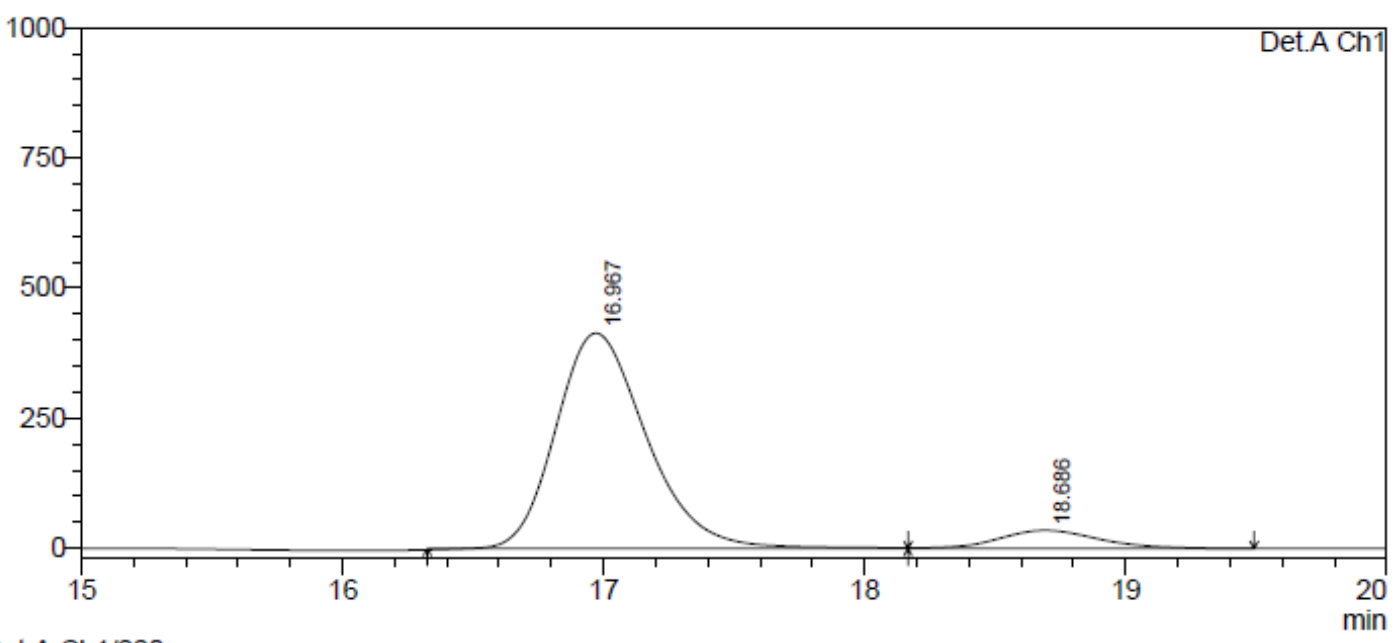

1 Det.A Ch1/220nm

PeakTable

Detector A Ch1 220nm
\begin{tabular}{|r|r|r|r|r|r|}
\hline Peak\# & Ret. Time & \multicolumn{1}{|c|}{ Area } & \multicolumn{1}{c|}{ Height } & Area $\%$ & \multicolumn{1}{c|}{ Height $\%$} \\
\hline 1 & 16.967 & 10014261 & 413661 & 91.446 & 92.193 \\
\hline 2 & 18.686 & 936773 & 35027 & 8.554 & 7.807 \\
\hline Total & & 10951034 & 448689 & 100.000 & 100.000 \\
\hline
\end{tabular}


<smiles>Cc1ccccc1C[C@H]1Oc2ccccc2N(Cc2ccccc2)C1=O</smiles>

$2 q$

$\mathrm{mV}$

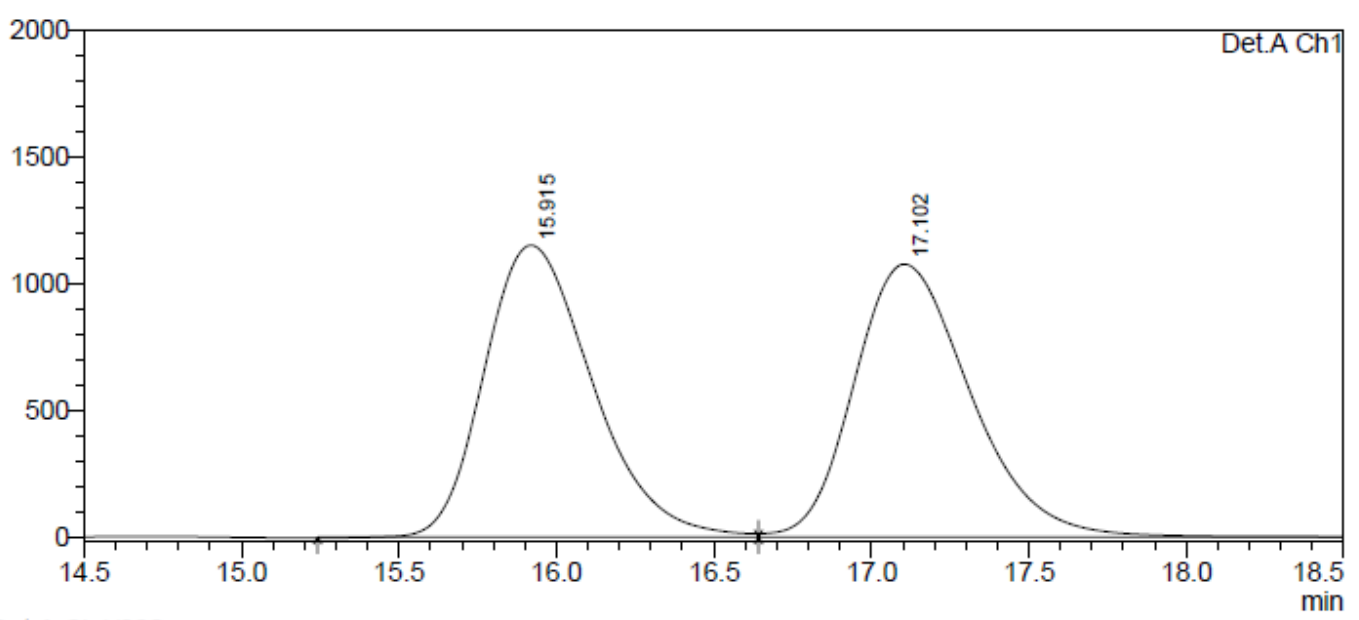

1 Det.A Ch1/220nm

PeakTable

Detector A Ch1 220nm

\begin{tabular}{|r|r|r|r|r|r|}
\hline \multicolumn{1}{|c|}{ Peak\# } & Ret. Time & \multicolumn{1}{c|}{ Area } & \multicolumn{1}{c|}{ Height } & \multicolumn{1}{c|}{ Area \% } & \multicolumn{1}{c|}{ Height \% } \\
\hline 1 & 15.915 & 27434738 & 1149785 & 49.848 & 51.684 \\
\hline 2 & 17.102 & 27602505 & 1074863 & 50.152 & 48.316 \\
\hline Total & & 55037243 & 2224648 & 100.000 & 100.000 \\
\hline
\end{tabular}

$\mathrm{mV}$

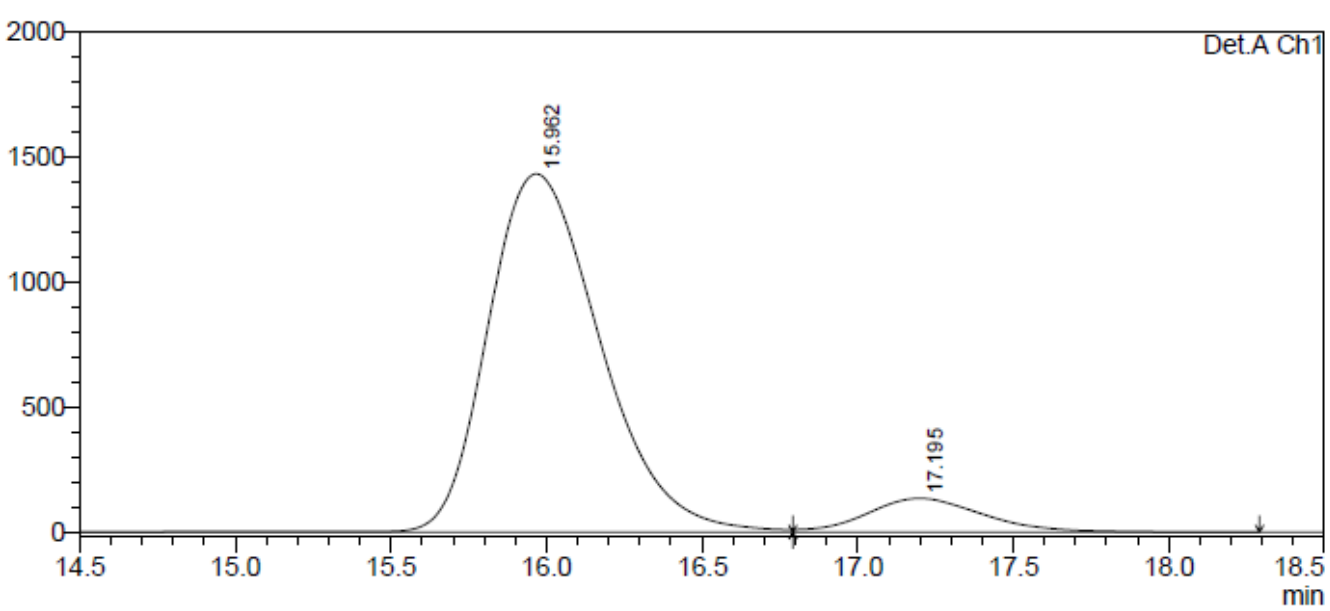

1 Det.A Ch1/220nm

PeakTable

Detector A Ch1 $220 \mathrm{~nm}$
\begin{tabular}{|r|r|r|r|r|r|}
\hline Peak\# & Ret. Time & \multicolumn{1}{|c|}{ Area } & Height & Area $\%$ & \multicolumn{1}{|c|}{ Height $\%$} \\
\hline 1 & 15.962 & 35902386 & 1429932 & 91.109 & 91.400 \\
\hline 2 & 17.195 & 3503518 & 134544 & 8.891 & 8.600 \\
\hline Total & & 39405905 & 1564475 & 100.000 & 100.000 \\
\hline
\end{tabular}




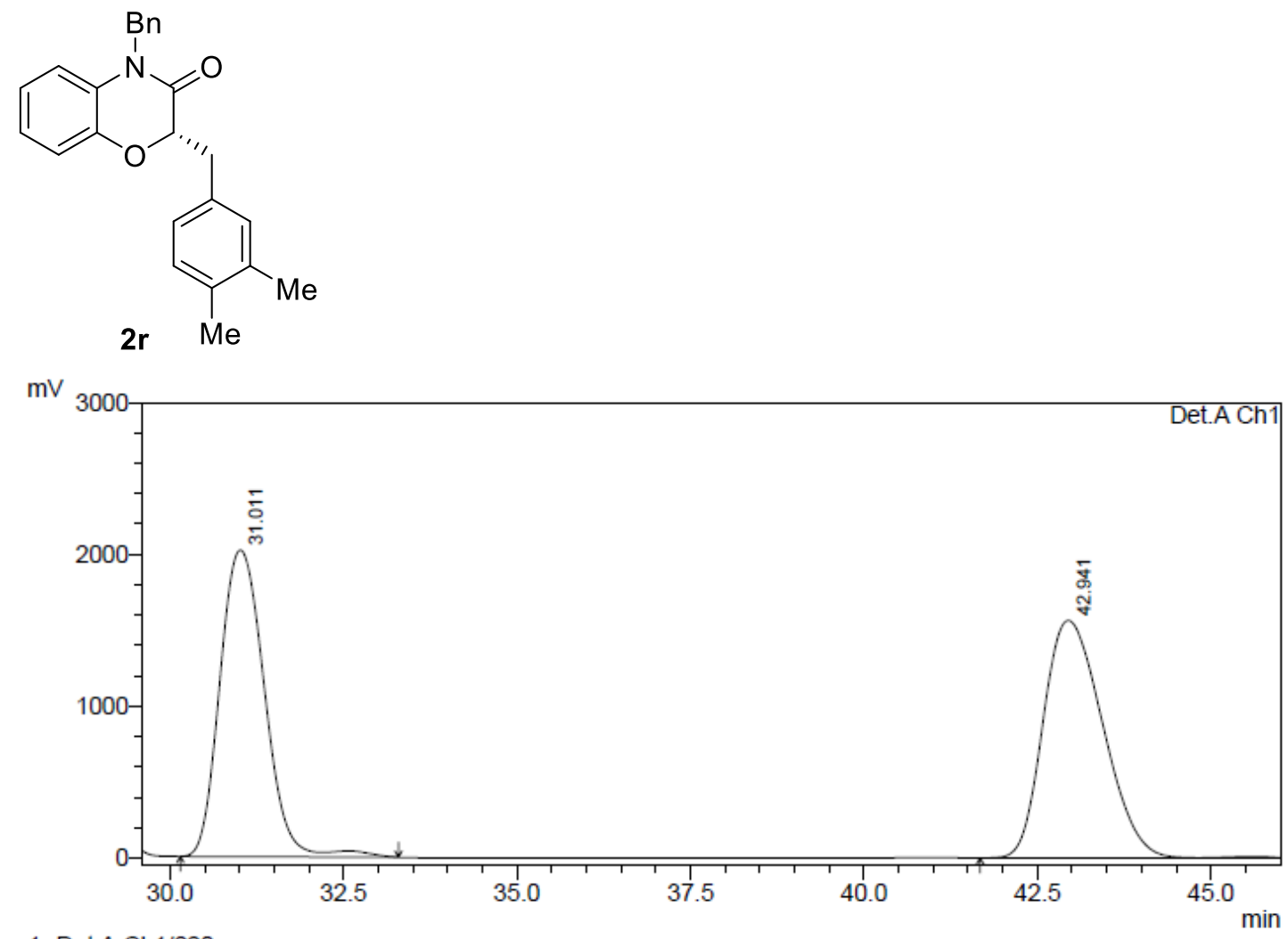

1 Det.A Ch1/220nm

PeakTable

Detector A Ch1 $220 \mathrm{~nm}$

\begin{tabular}{r|r|r|r|r|r|}
\hline \multicolumn{1}{|c|}{ Peak\# } & \multicolumn{1}{|c|}{ Ret. Time } & \multicolumn{1}{c|}{ Area } & \multicolumn{1}{c|}{ Height } & \multicolumn{1}{c|}{ Area \% } & \multicolumn{1}{c|}{ Height \% } \\
\hline 1 & 31.011 & 90569174 & 2019499 & 49.332 & 56.360 \\
\hline 2 & 42.941 & 93020535 & 1563728 & 50.668 & 43.640 \\
\hline Total & & 183589709 & 3583227 & 100.000 & 100.000 \\
\hline
\end{tabular}



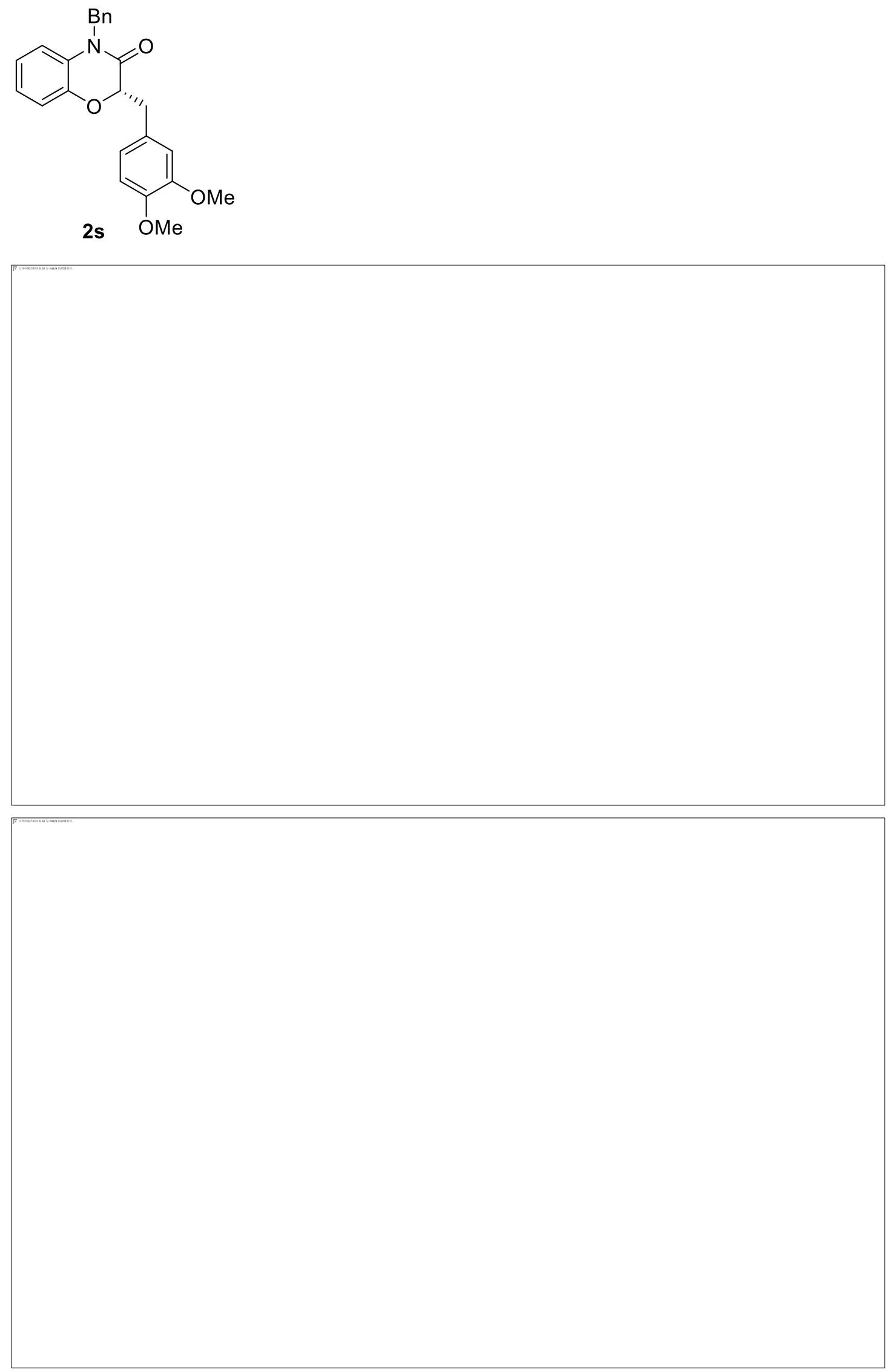

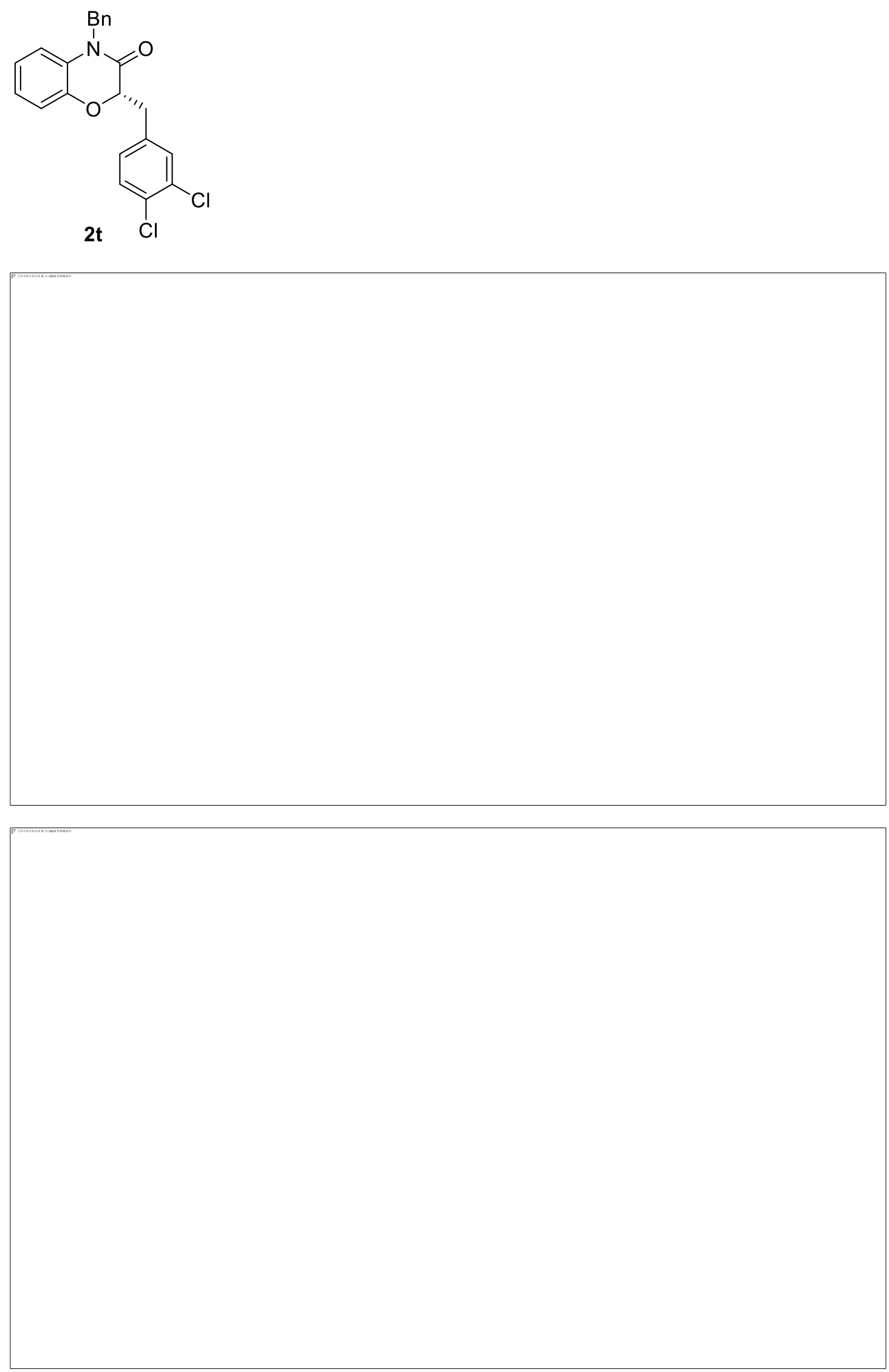

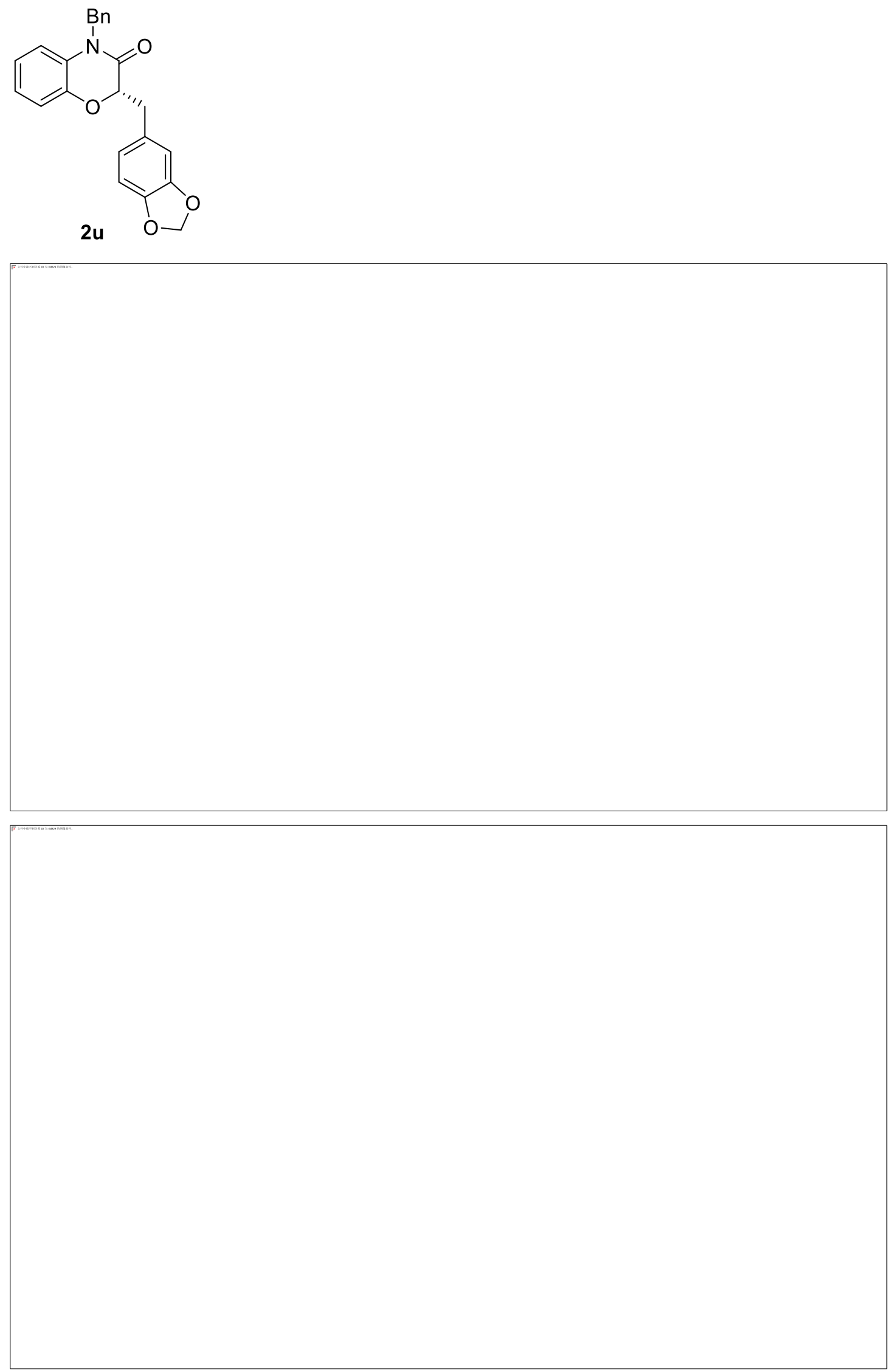

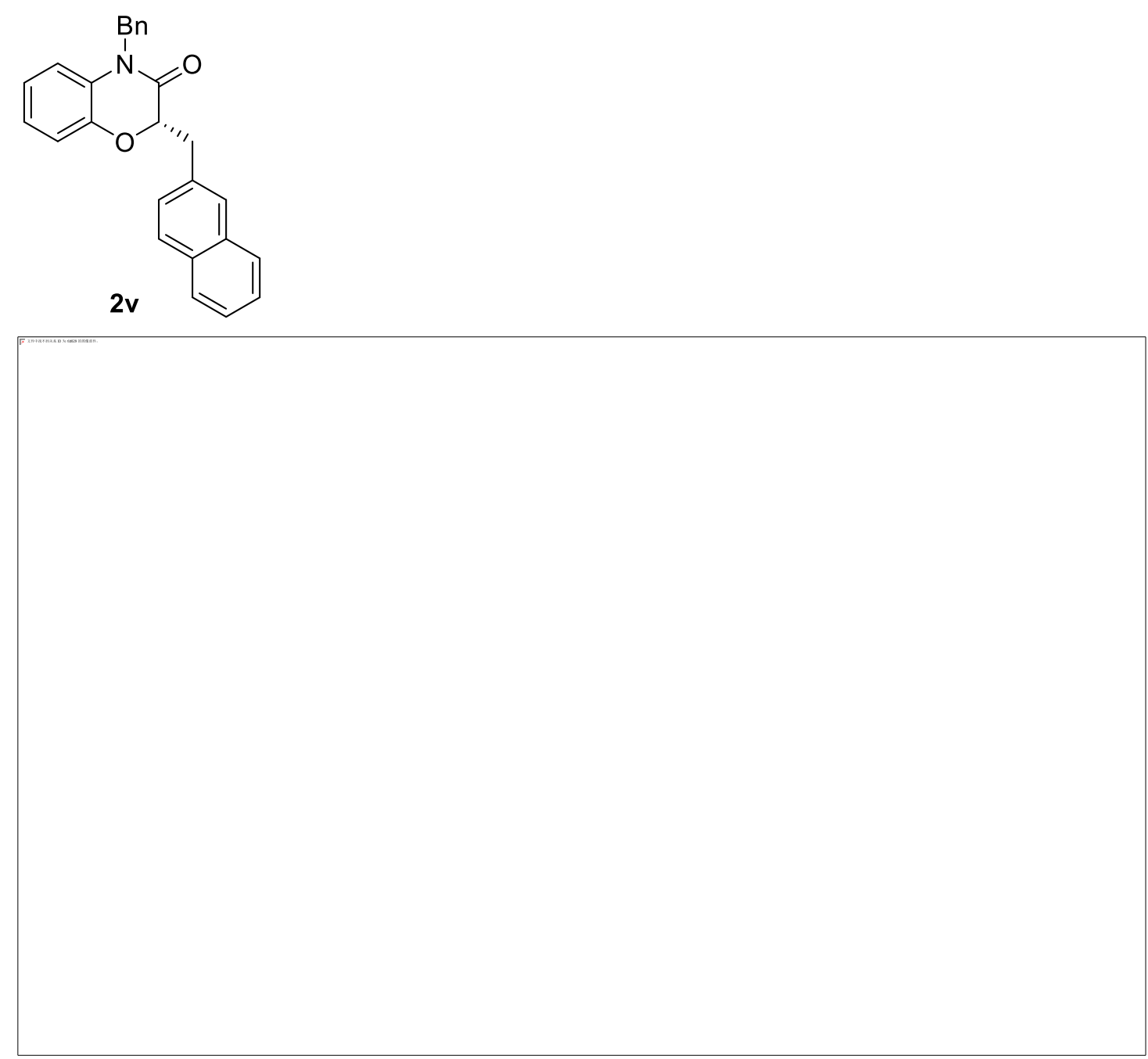


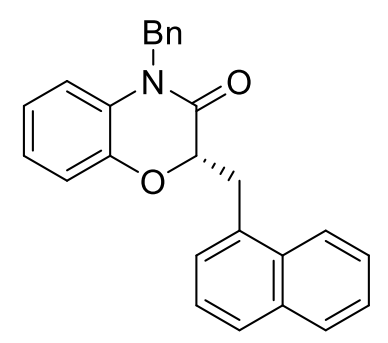

$2 w$
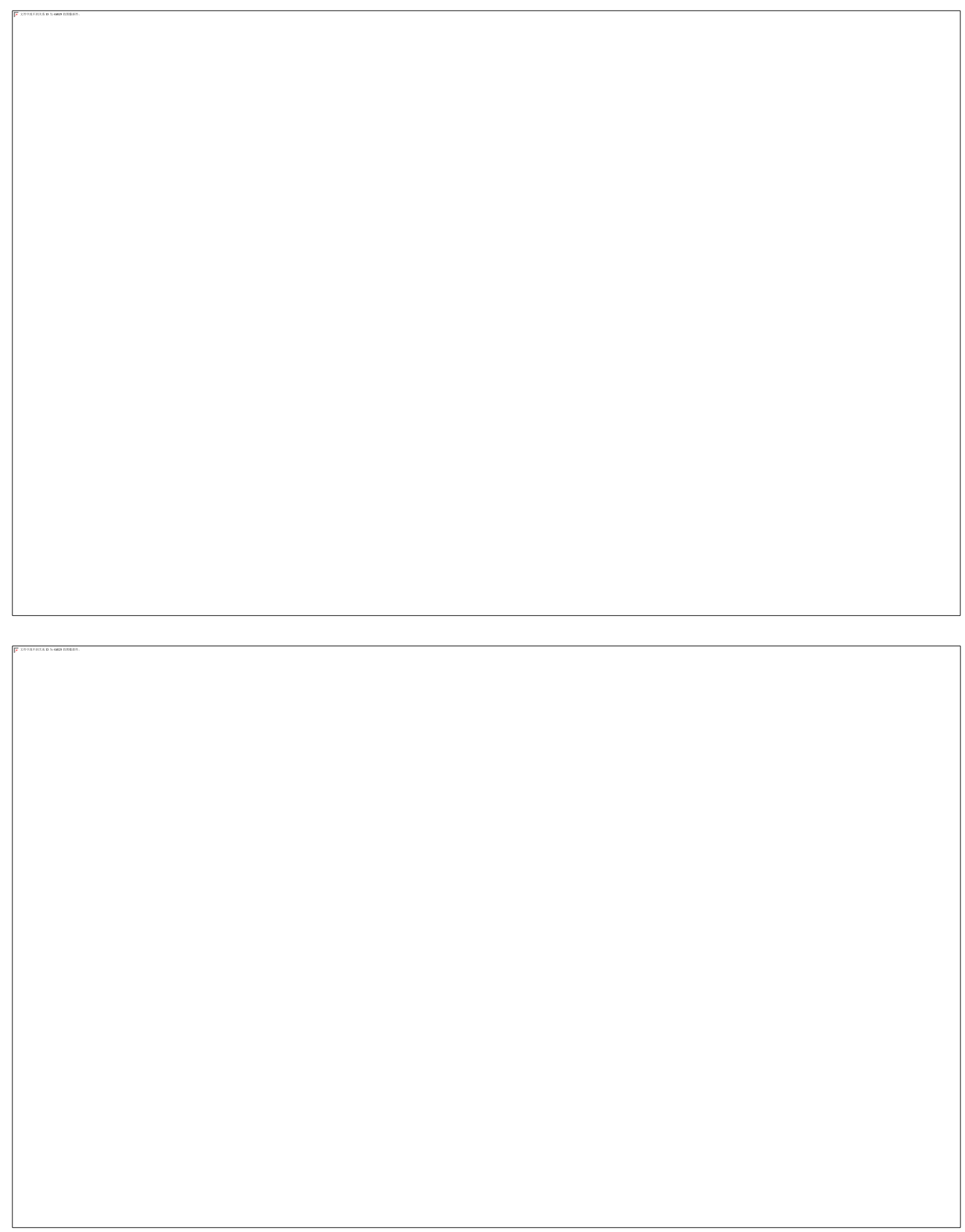

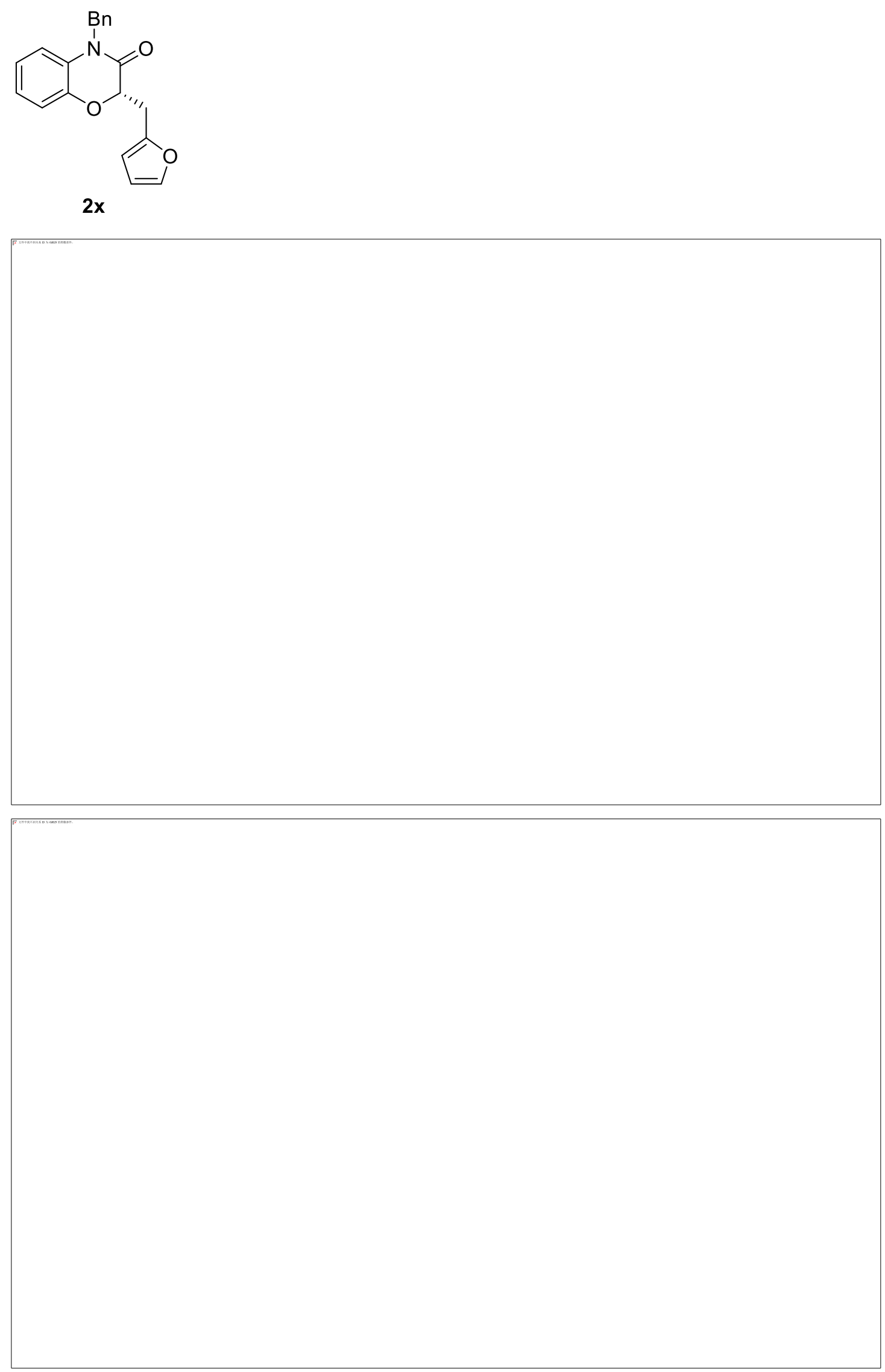


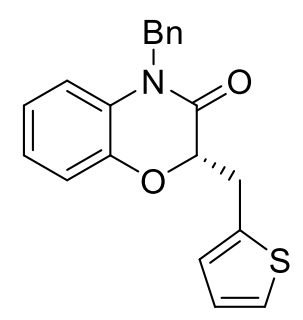

$2 y$
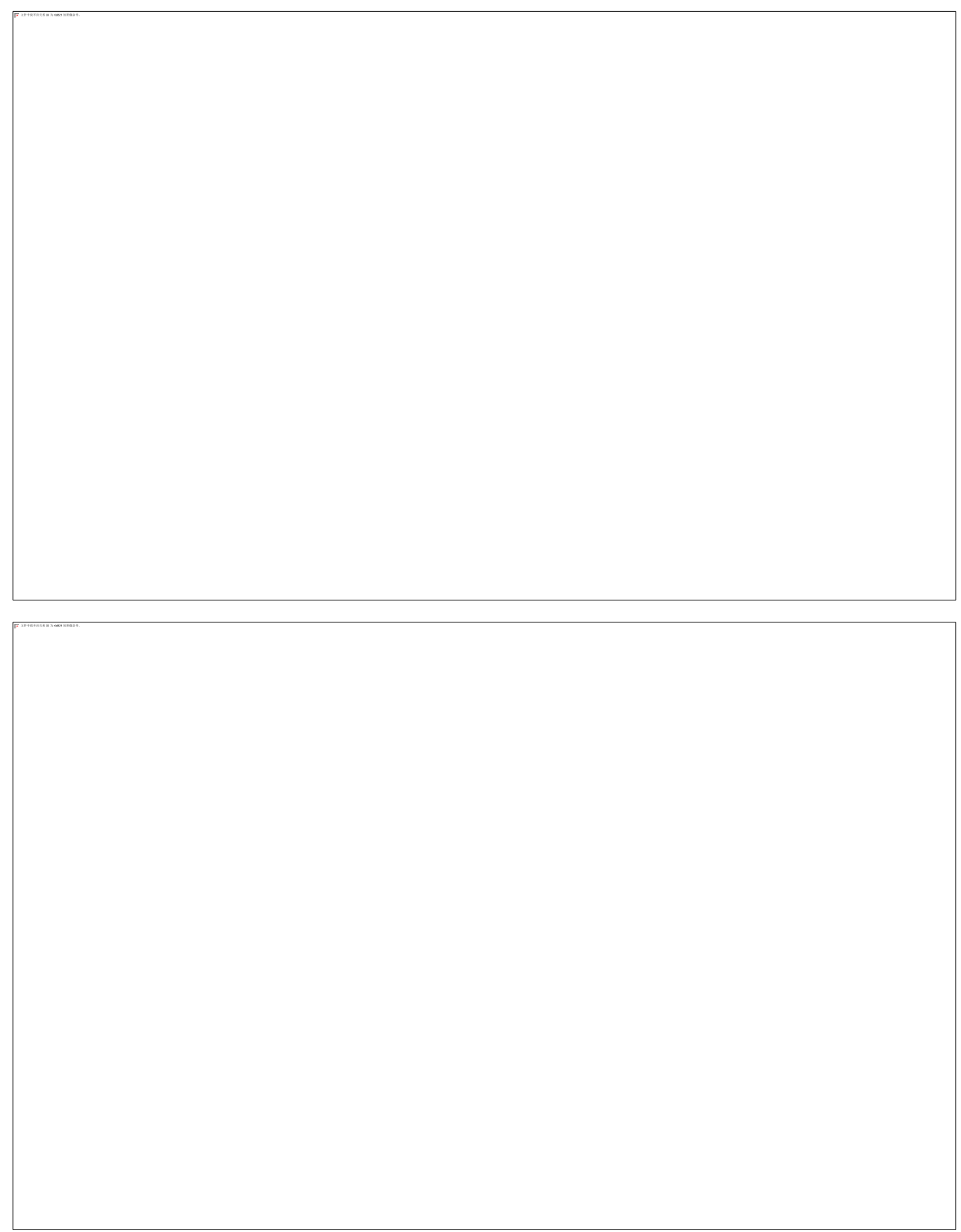

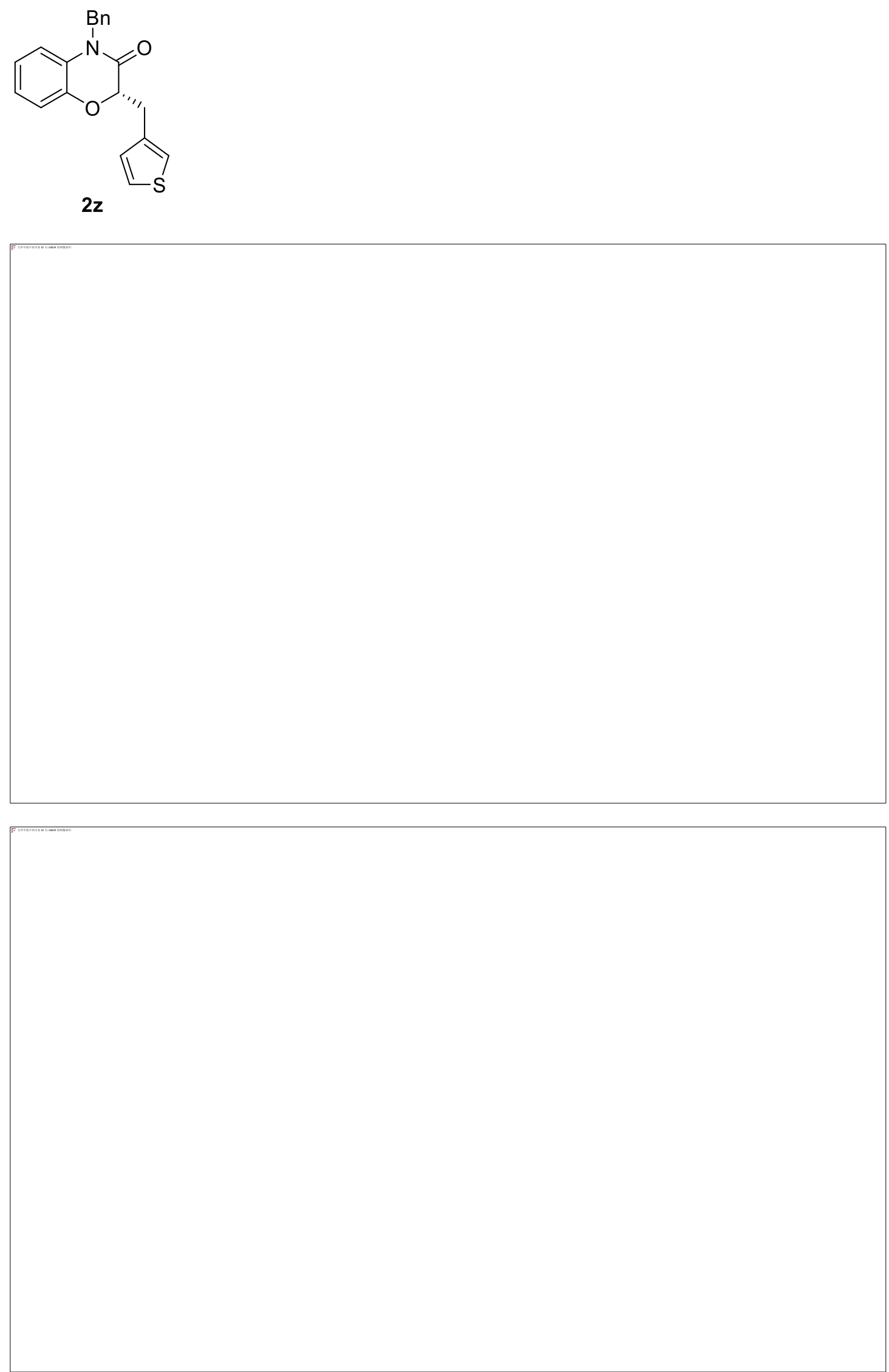


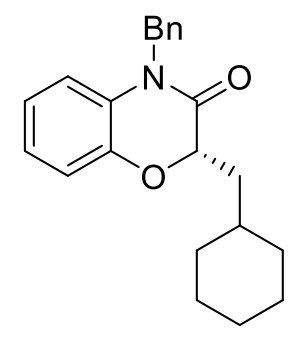

2aa
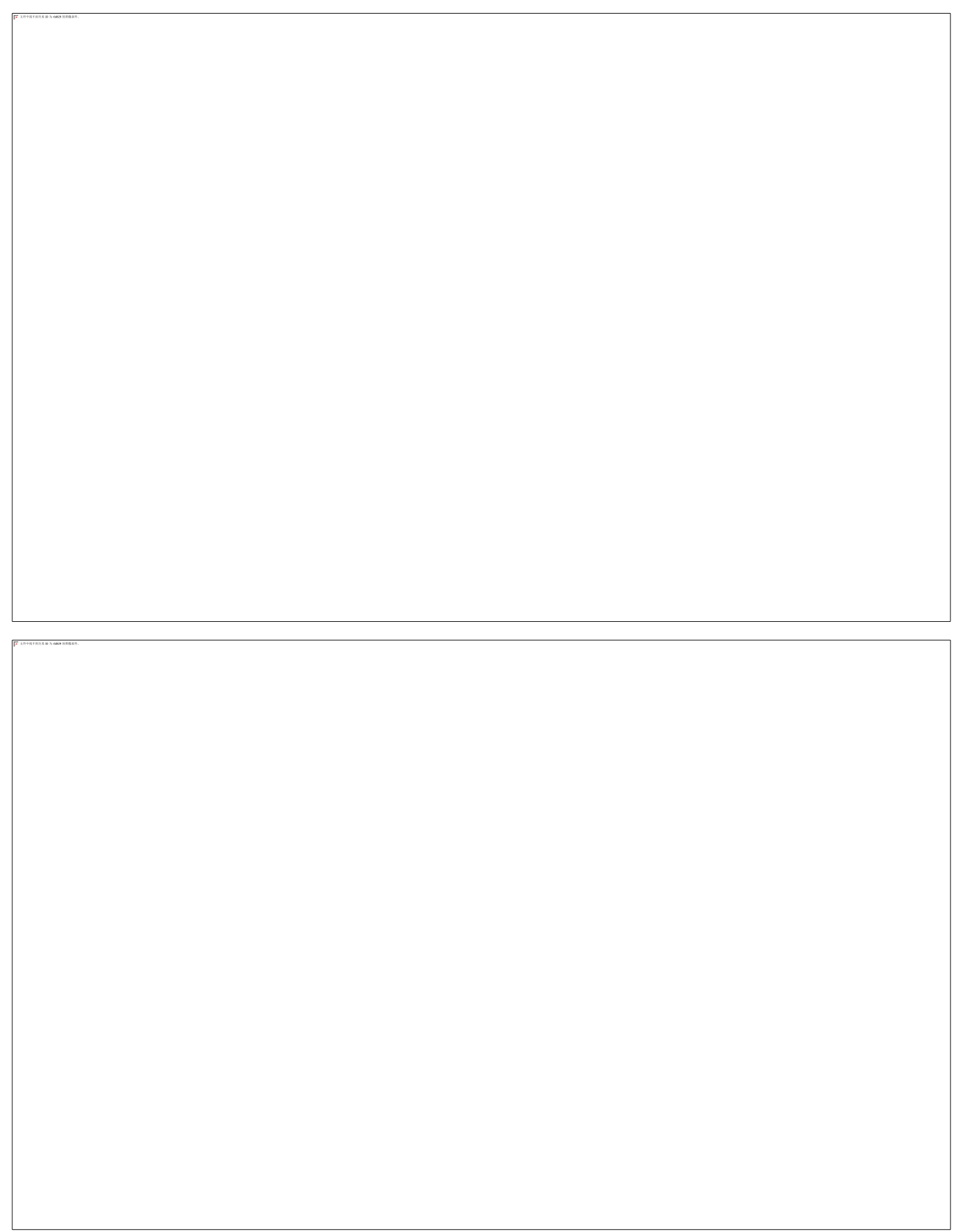


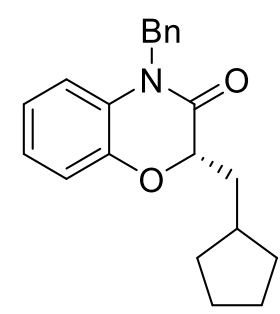

2ab
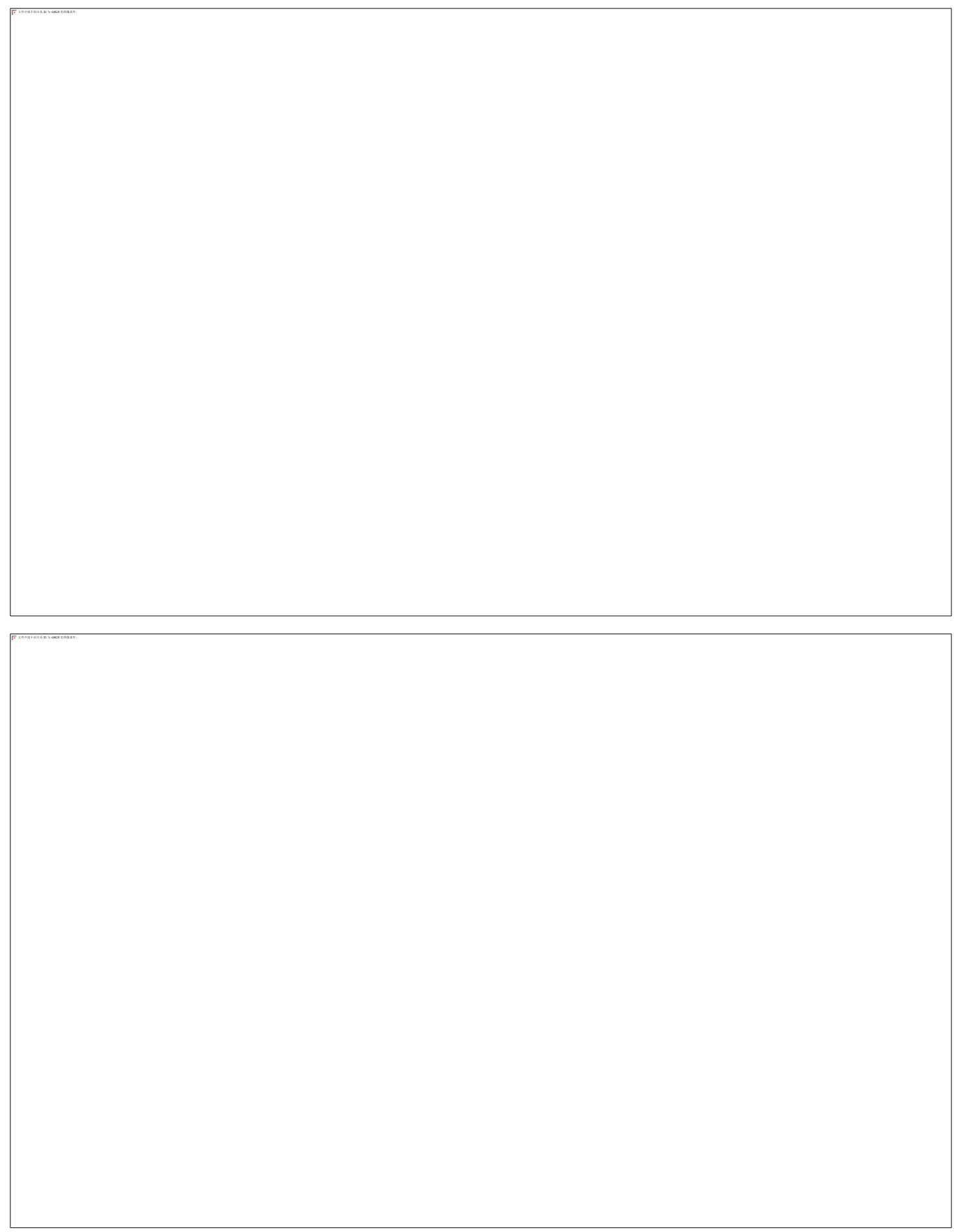


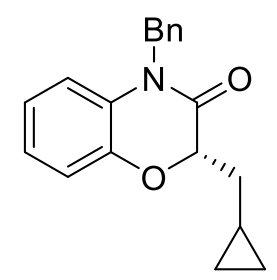

2ac
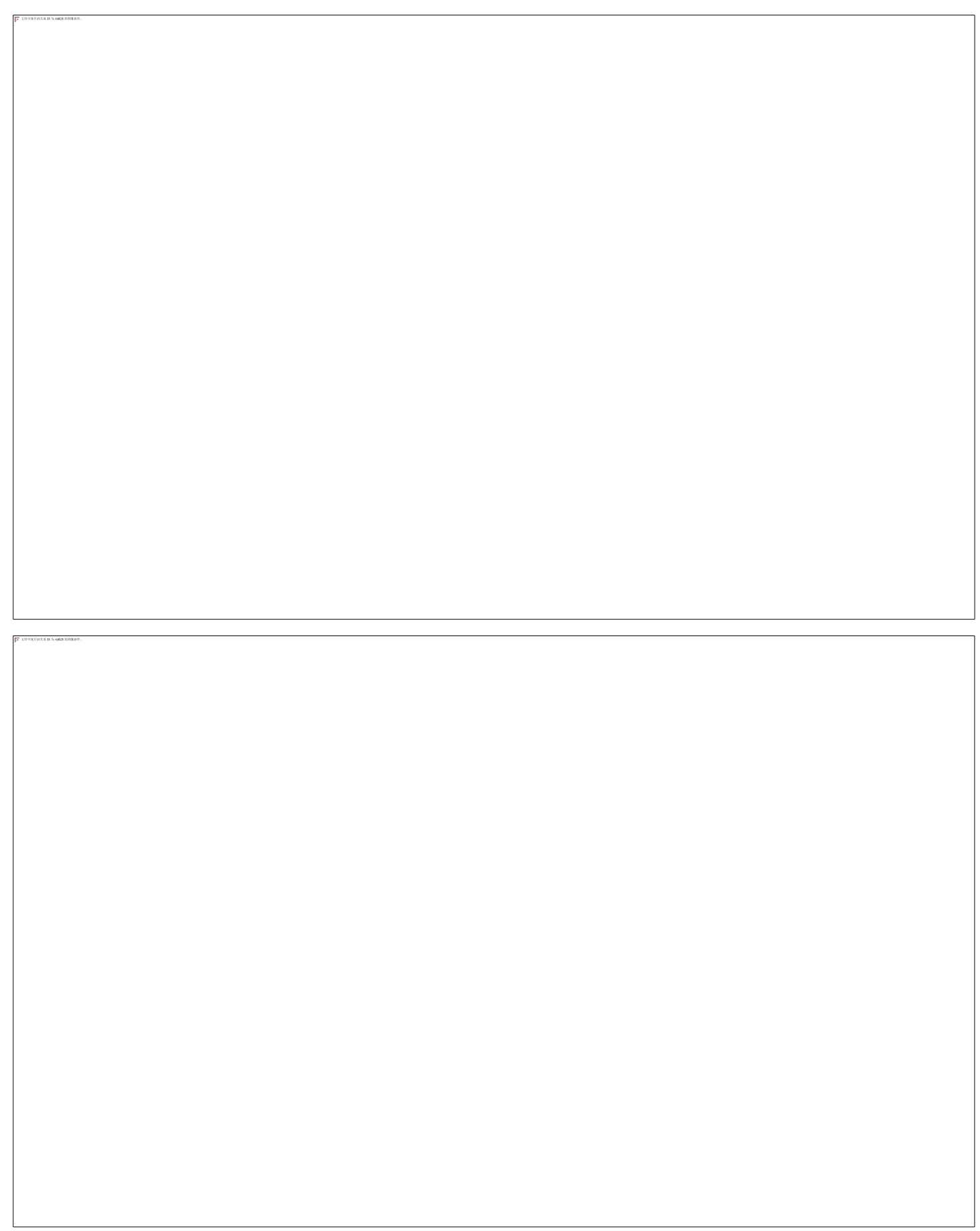


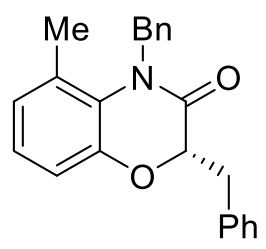

2ad
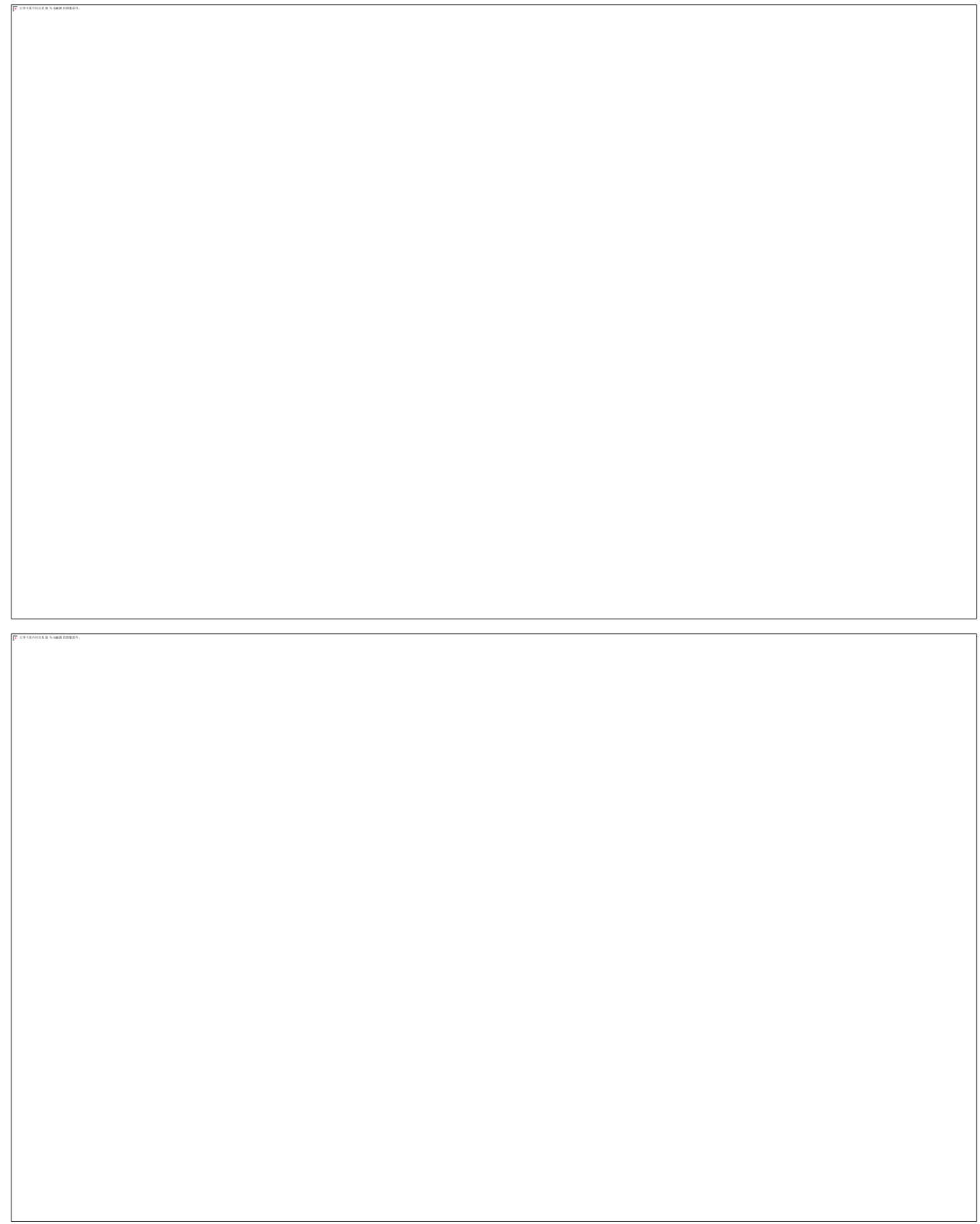


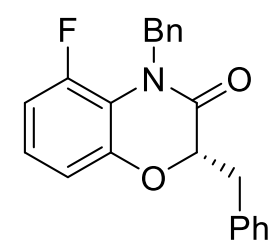

$2 a e$
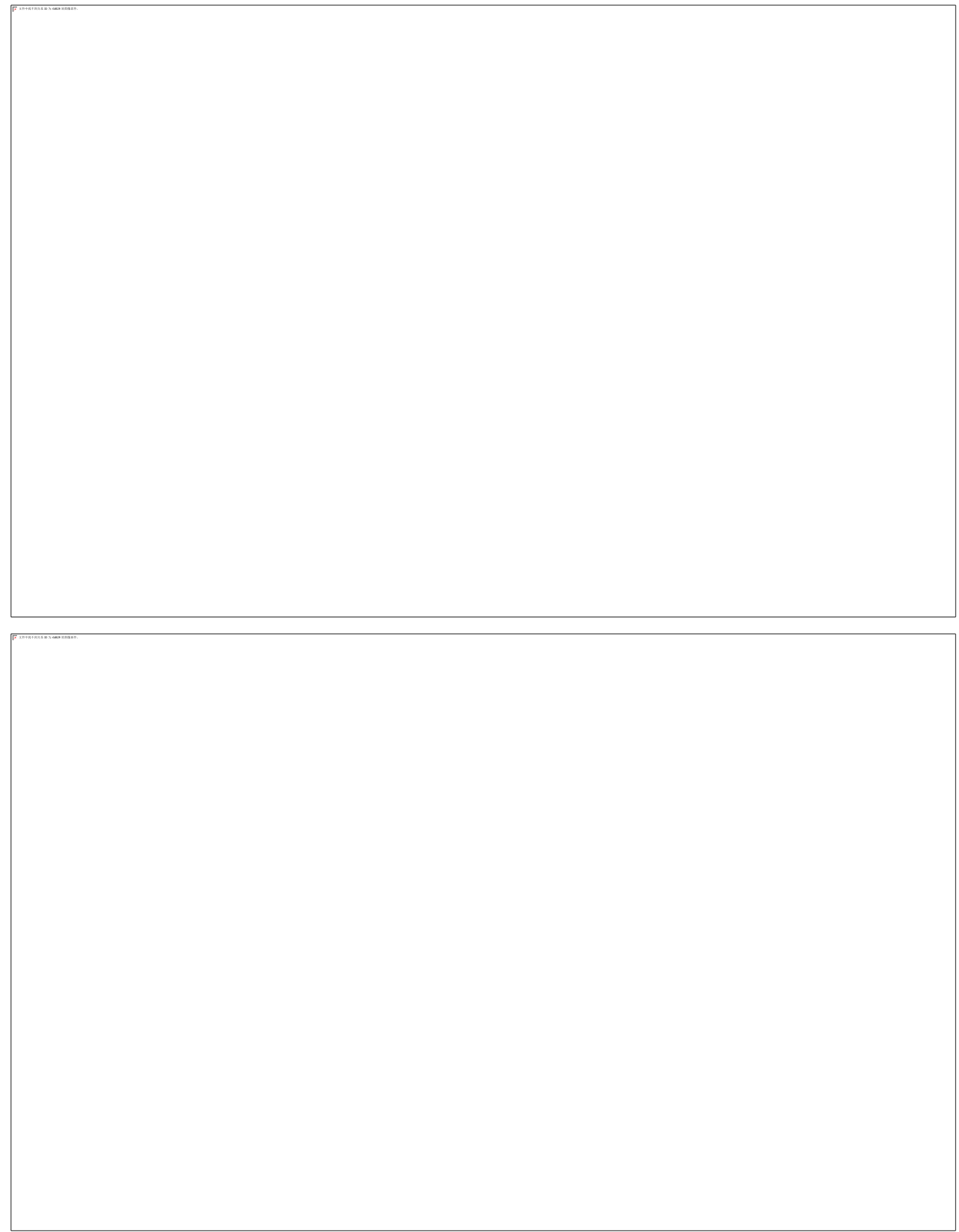


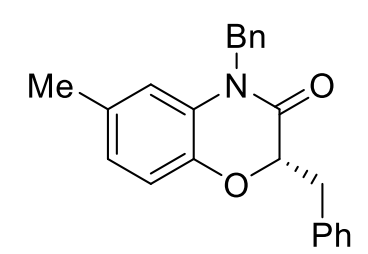

2af
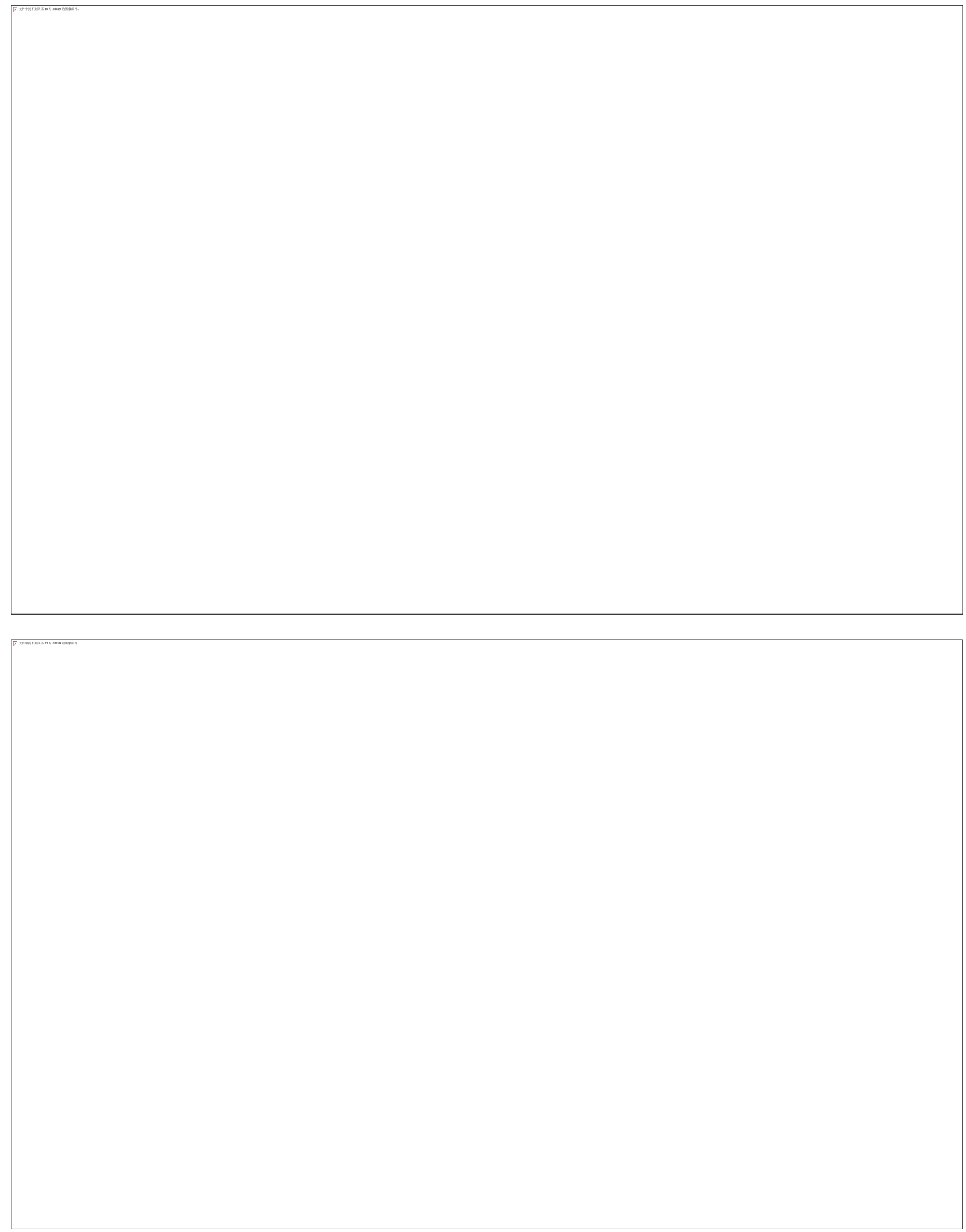


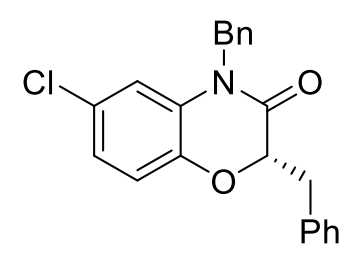

2ag
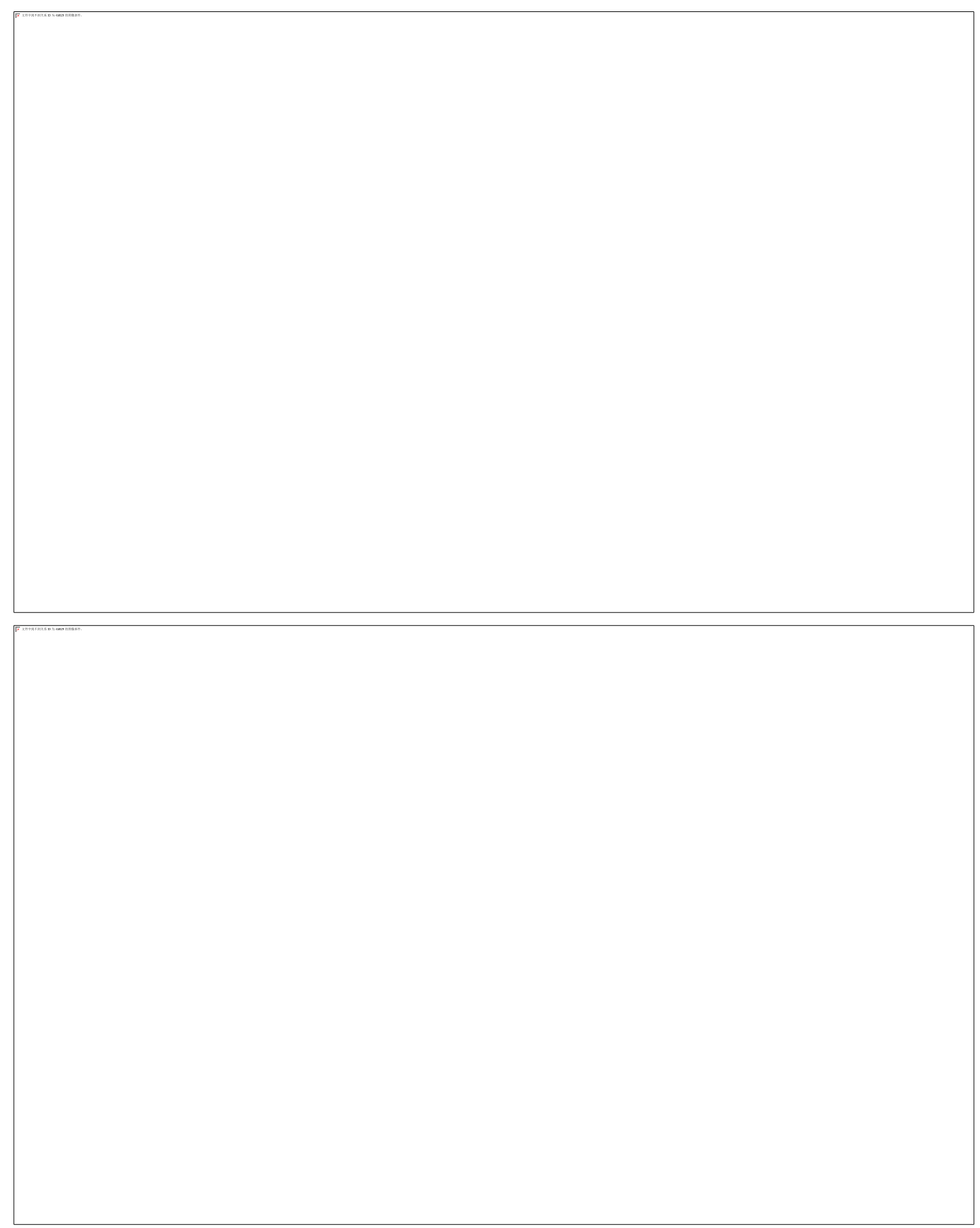


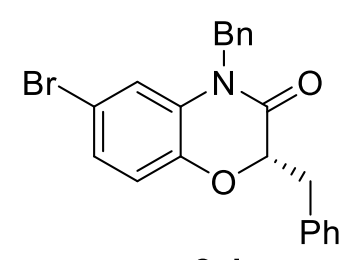

2ah
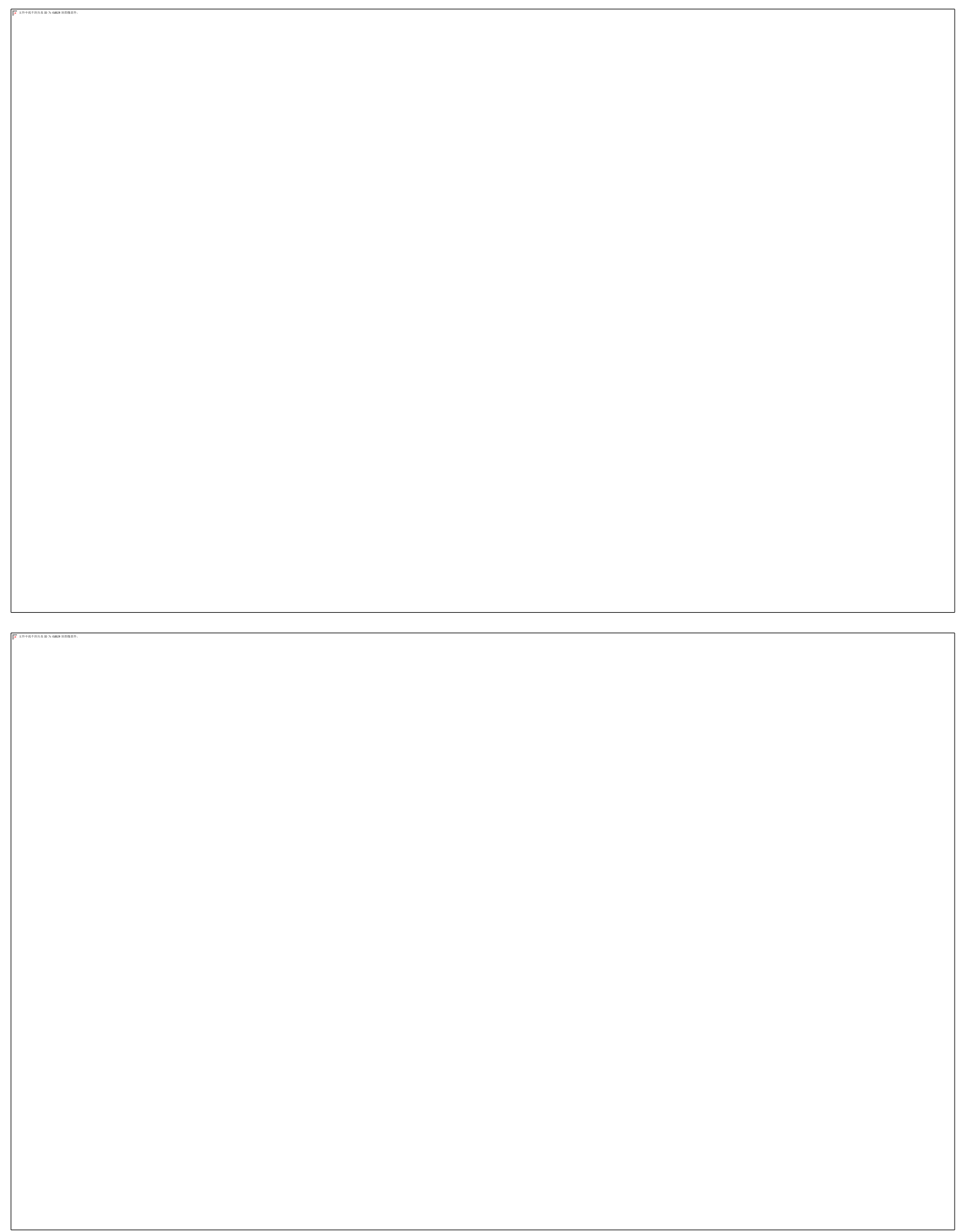

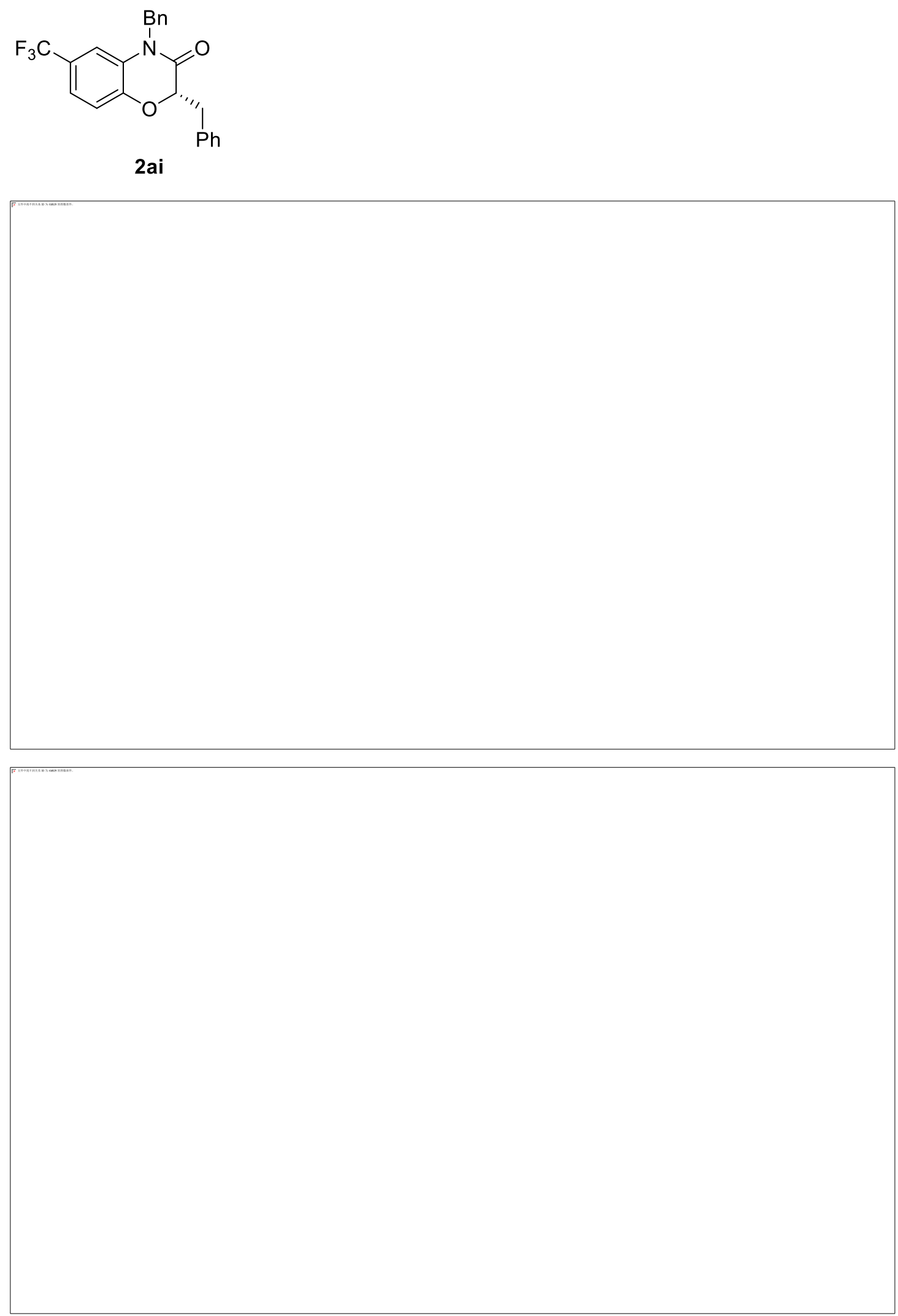


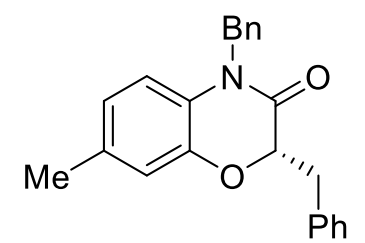

2aj
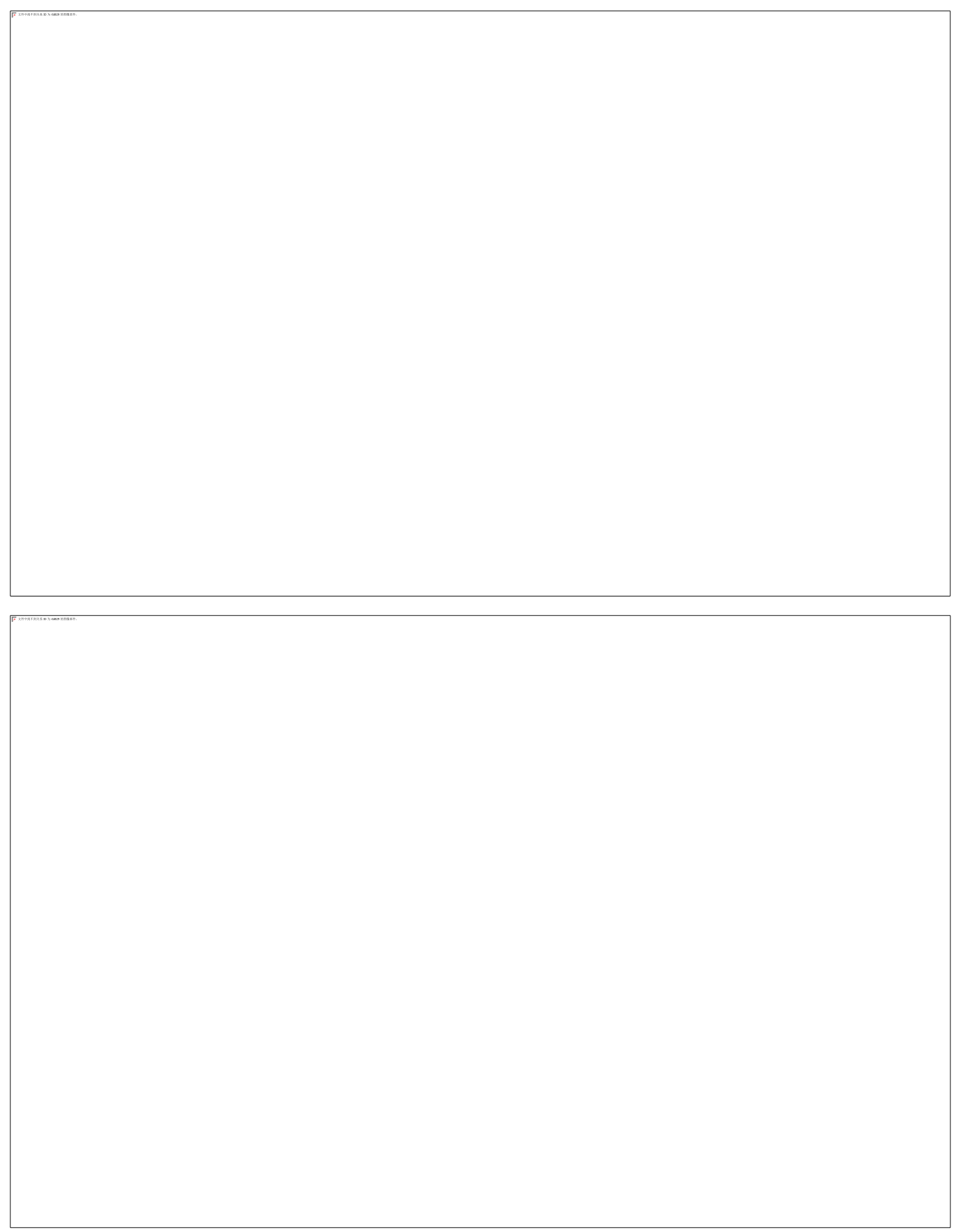

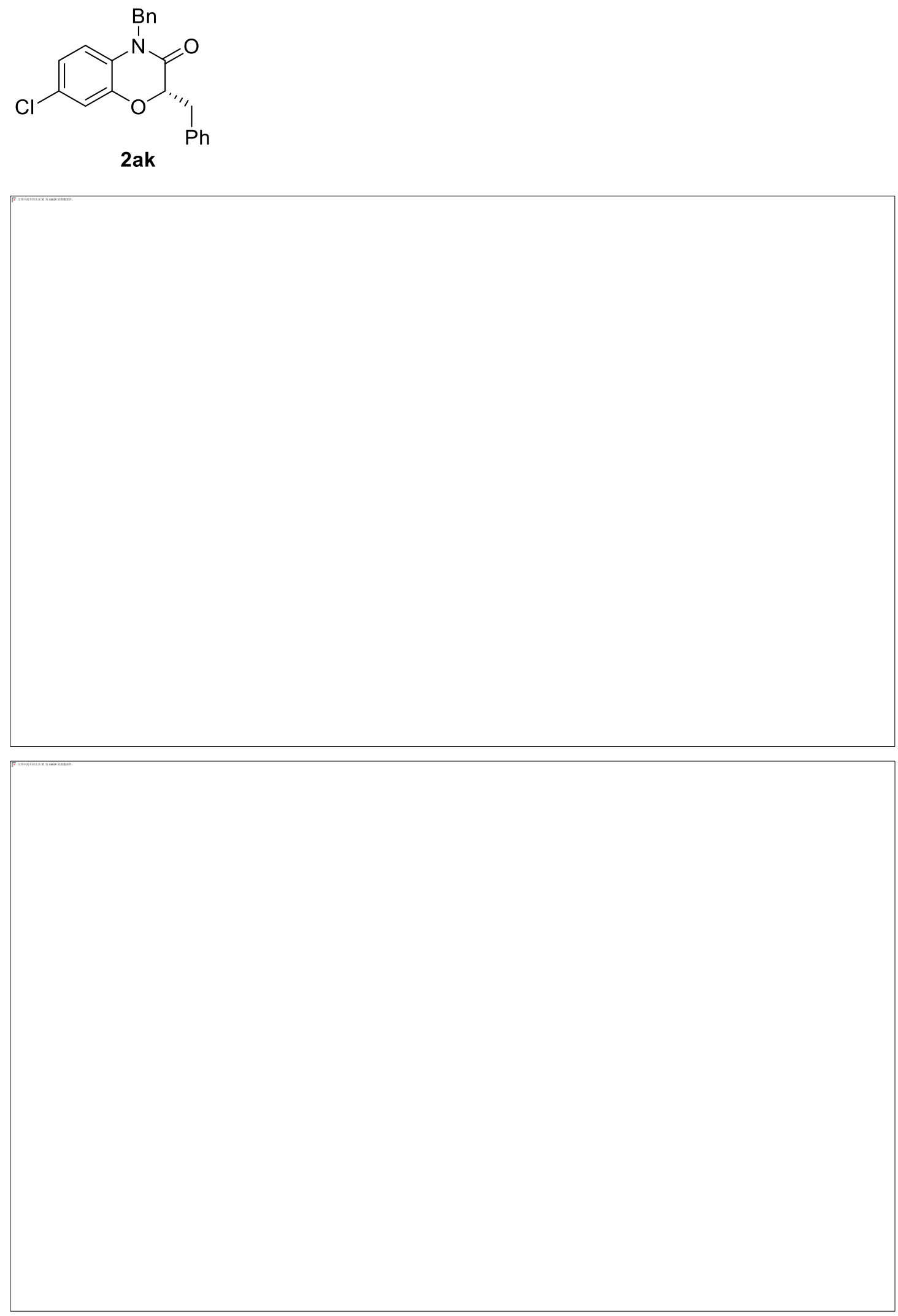

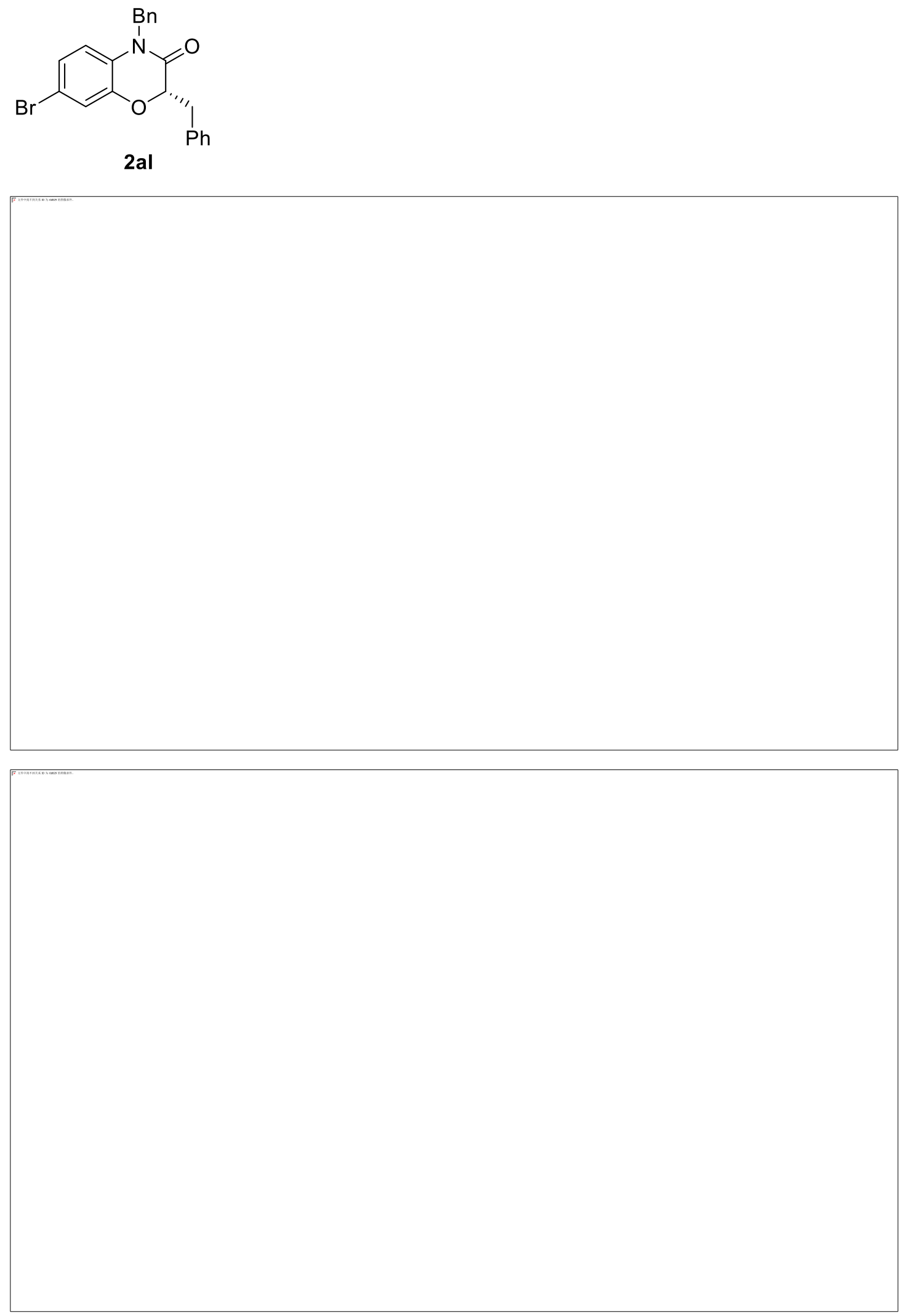

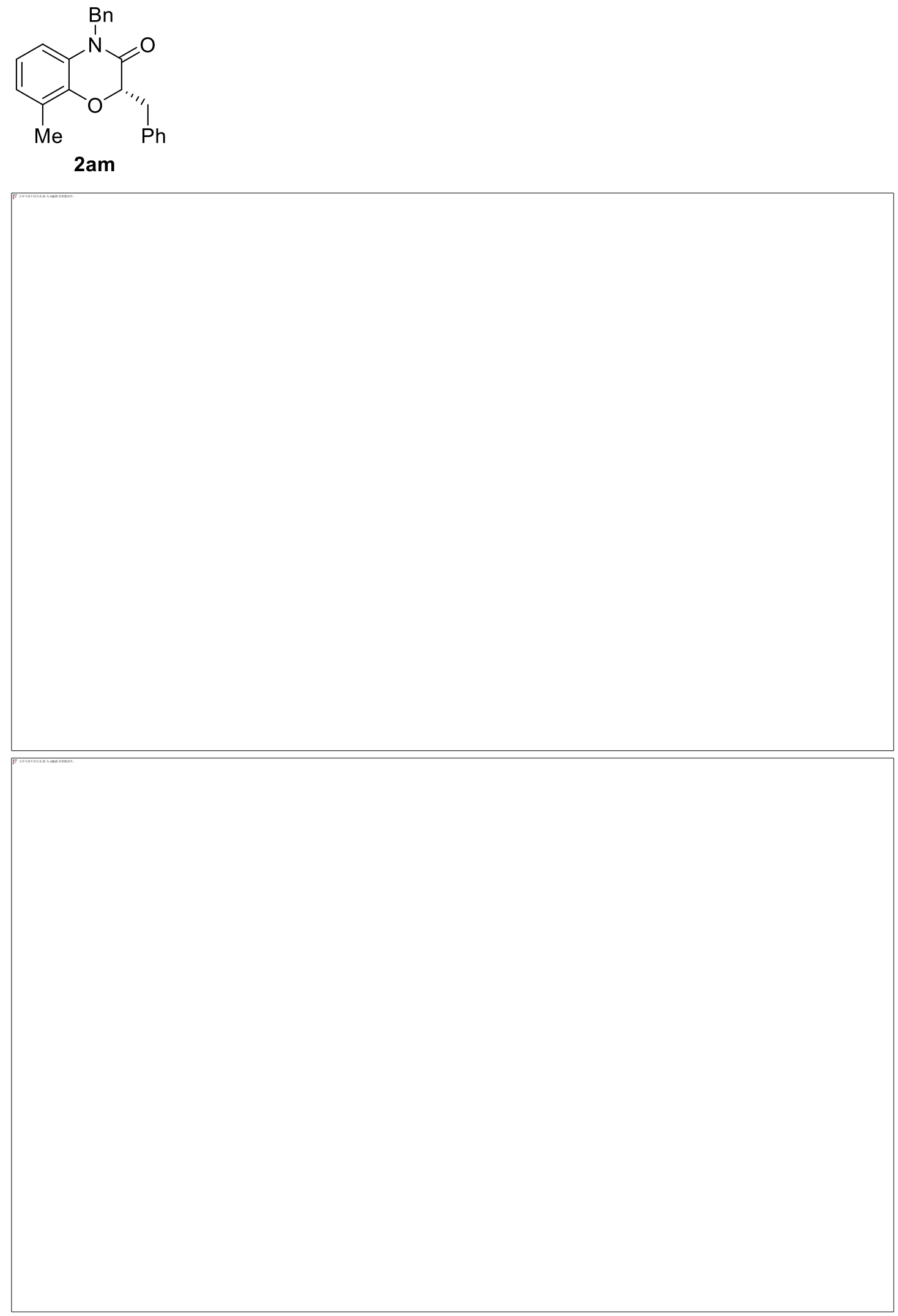

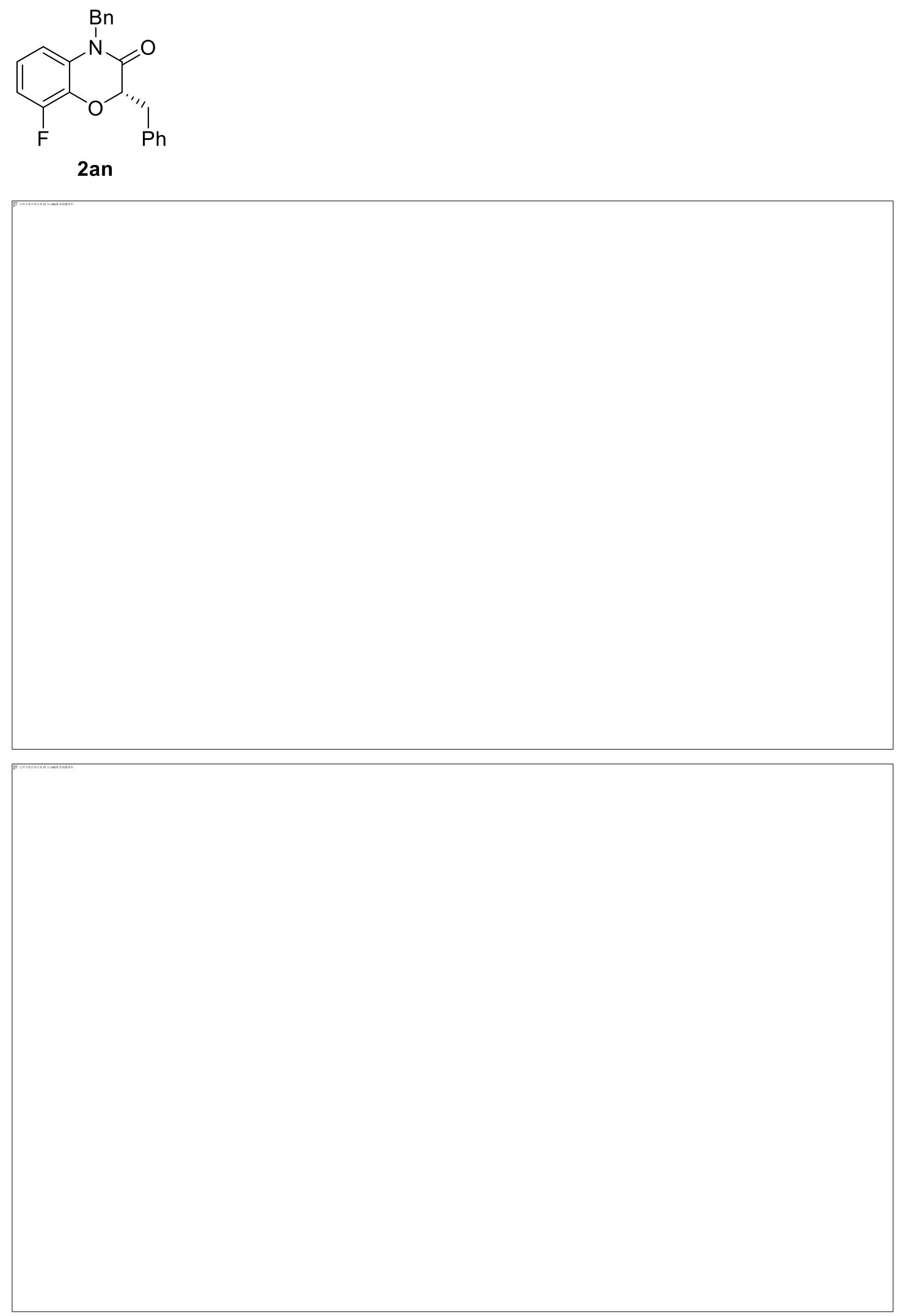


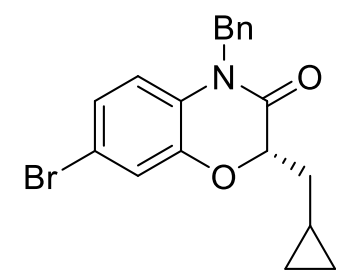

2 ao

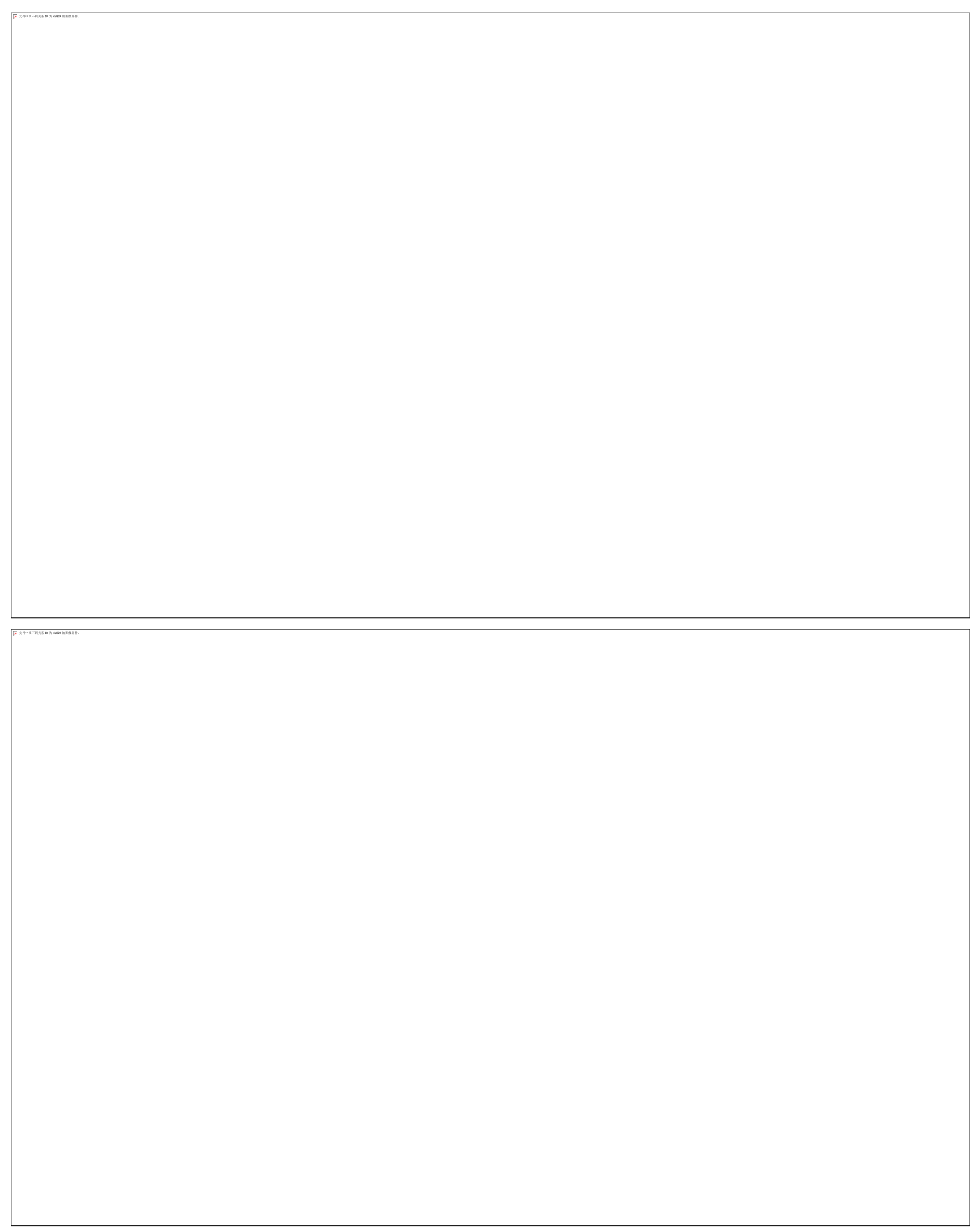



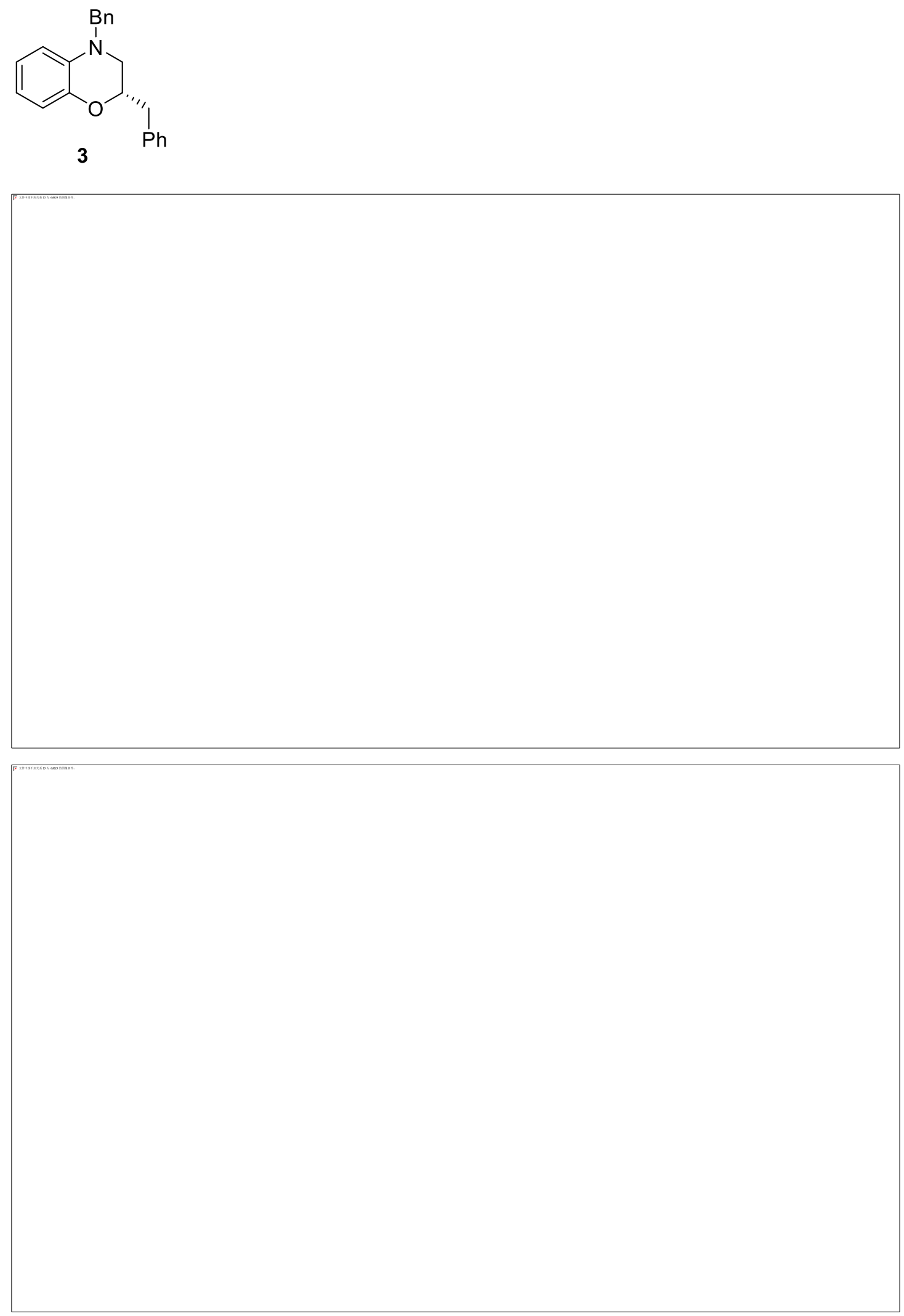

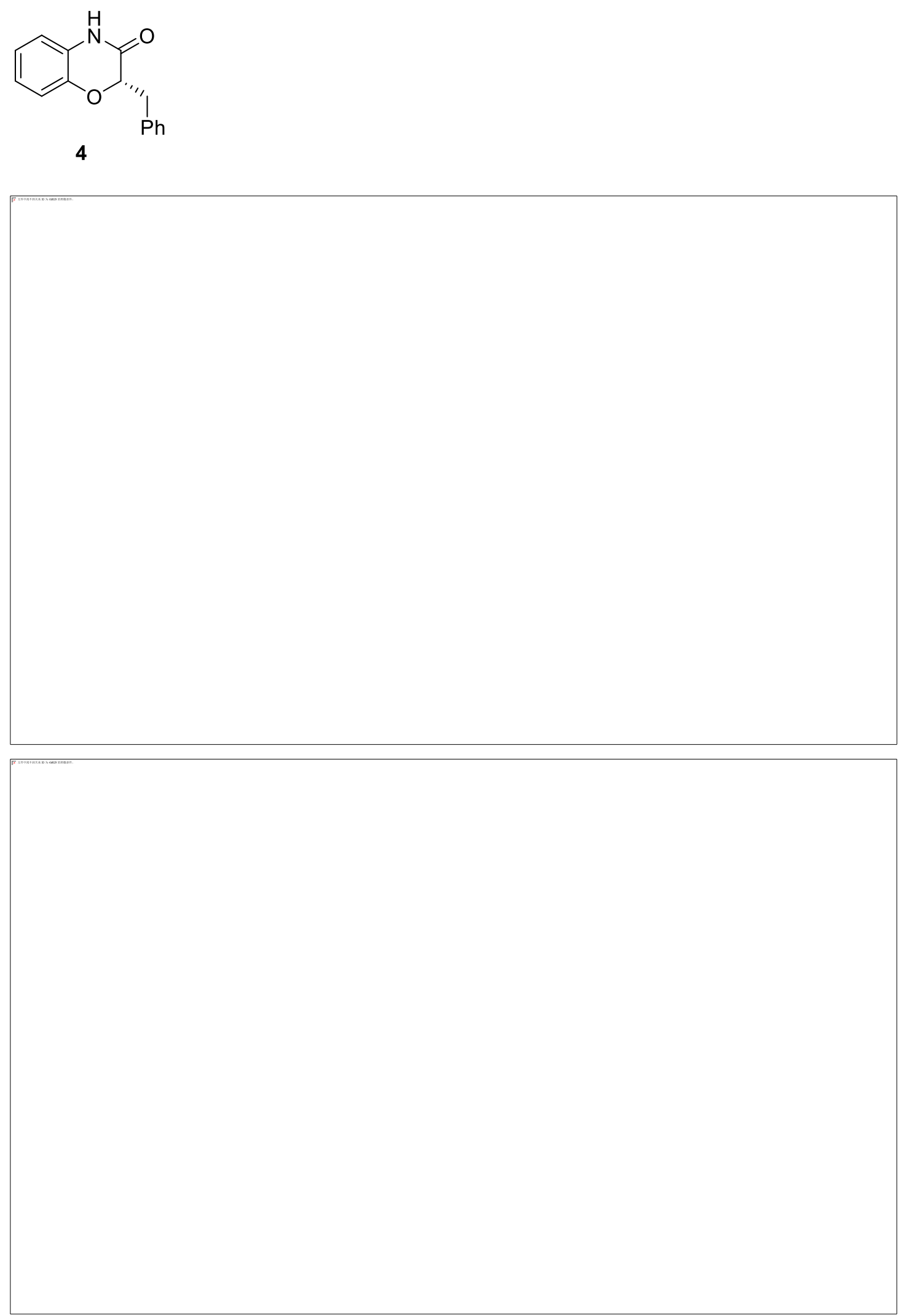


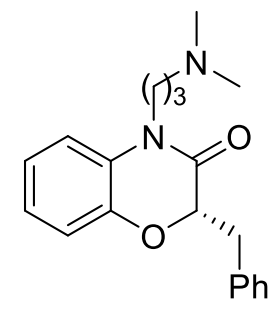

5
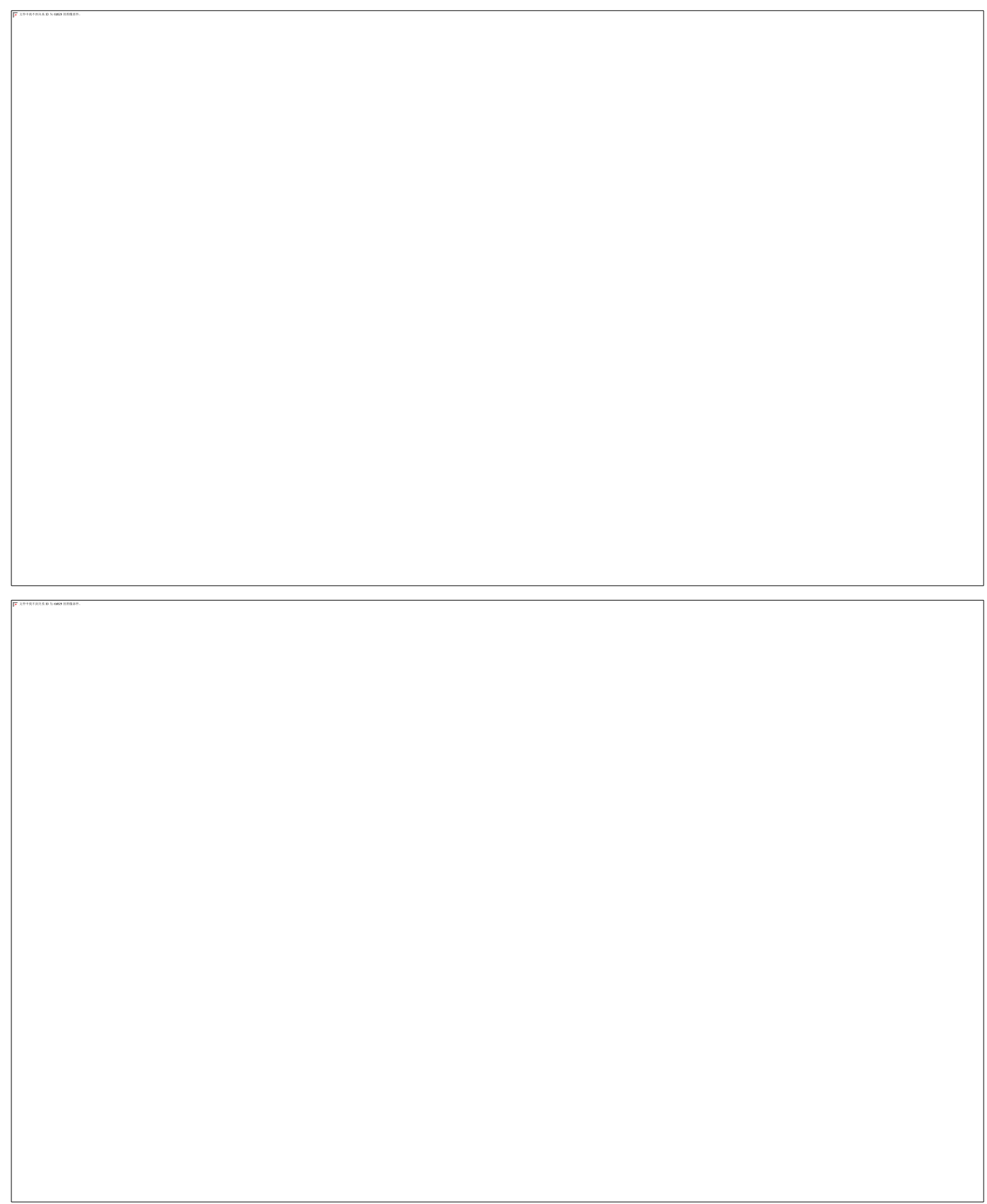


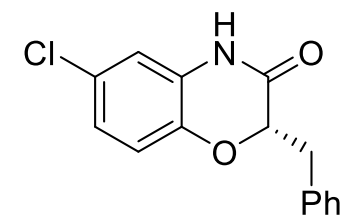

6
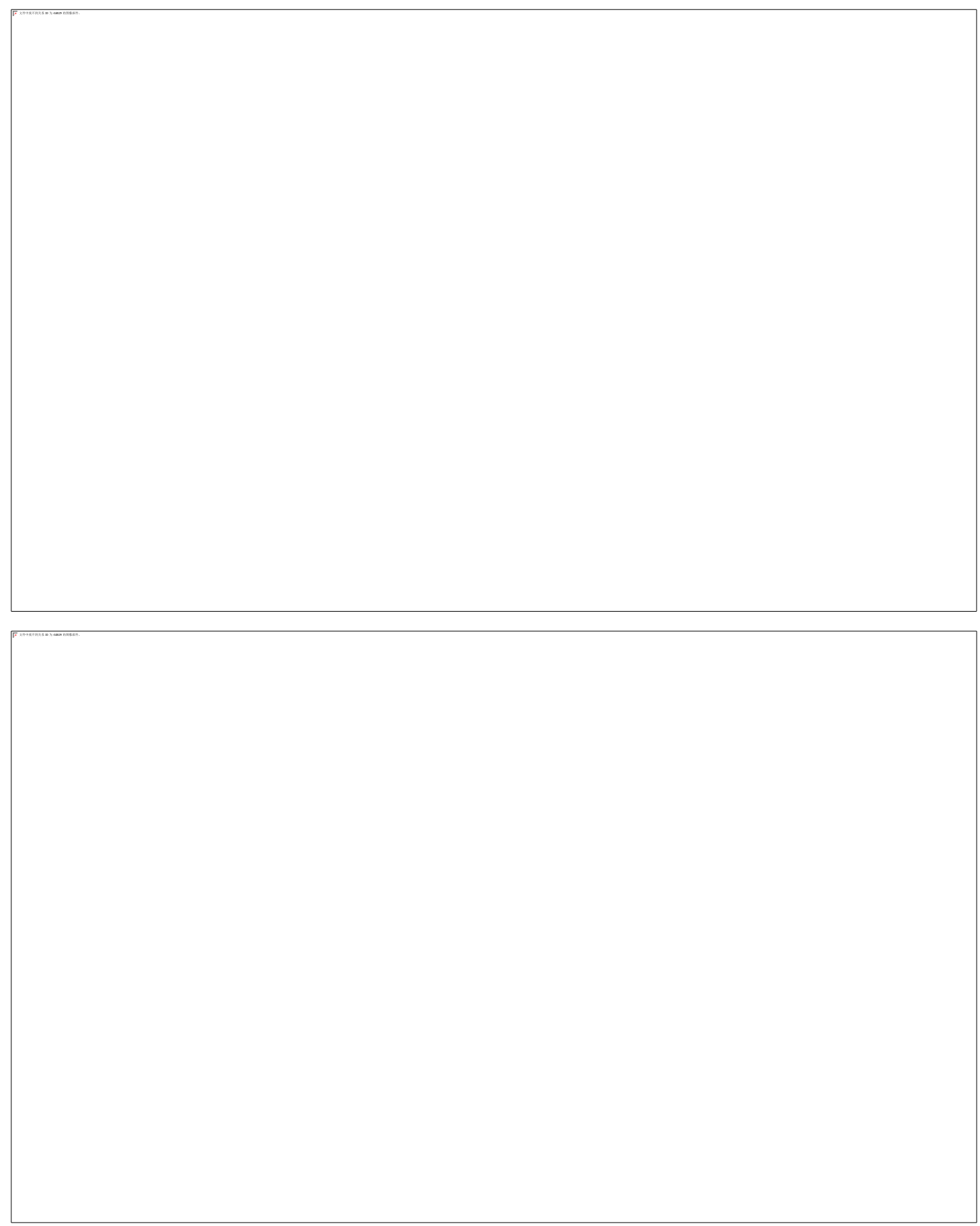


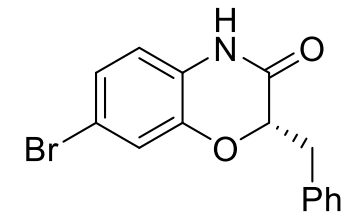

8
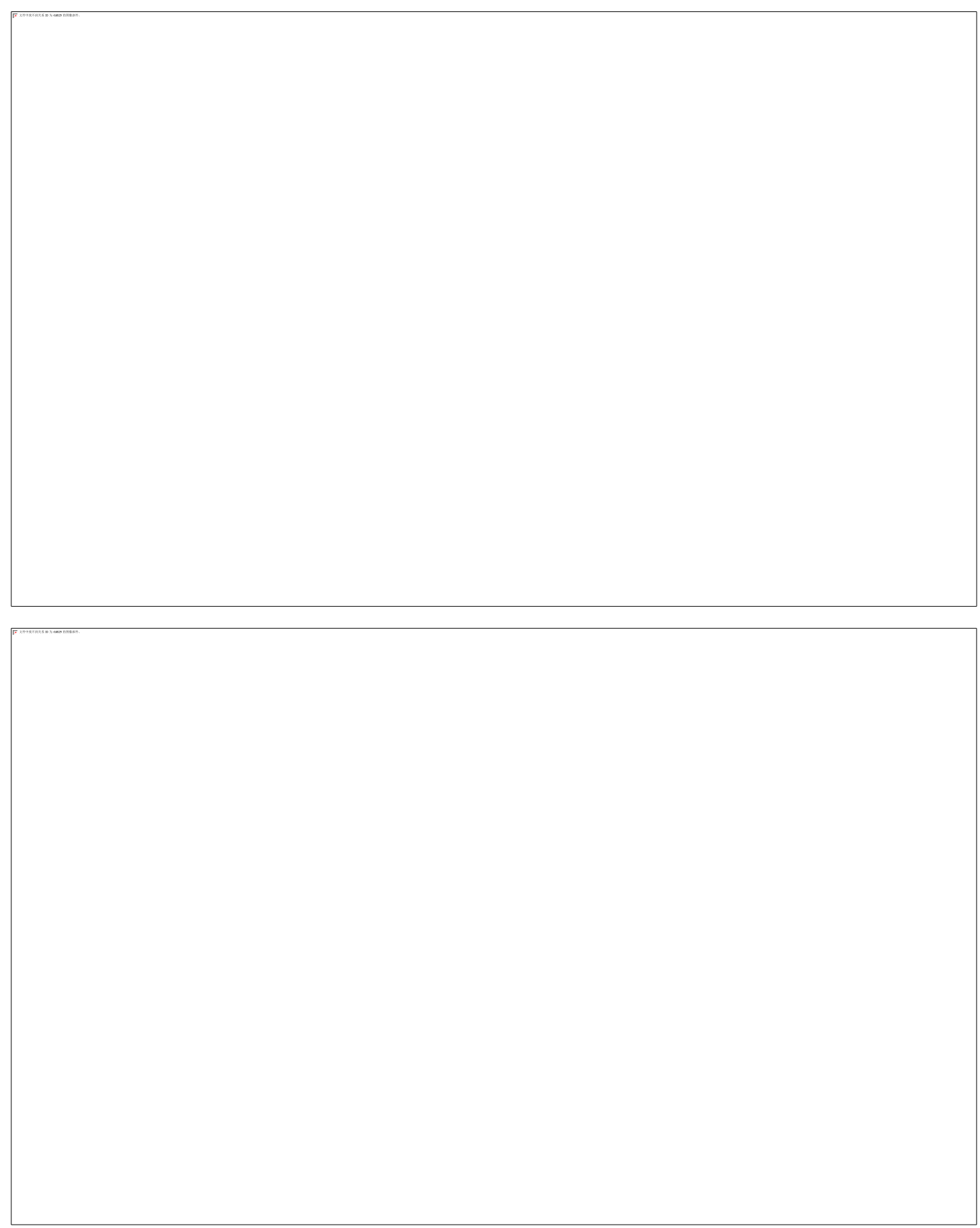

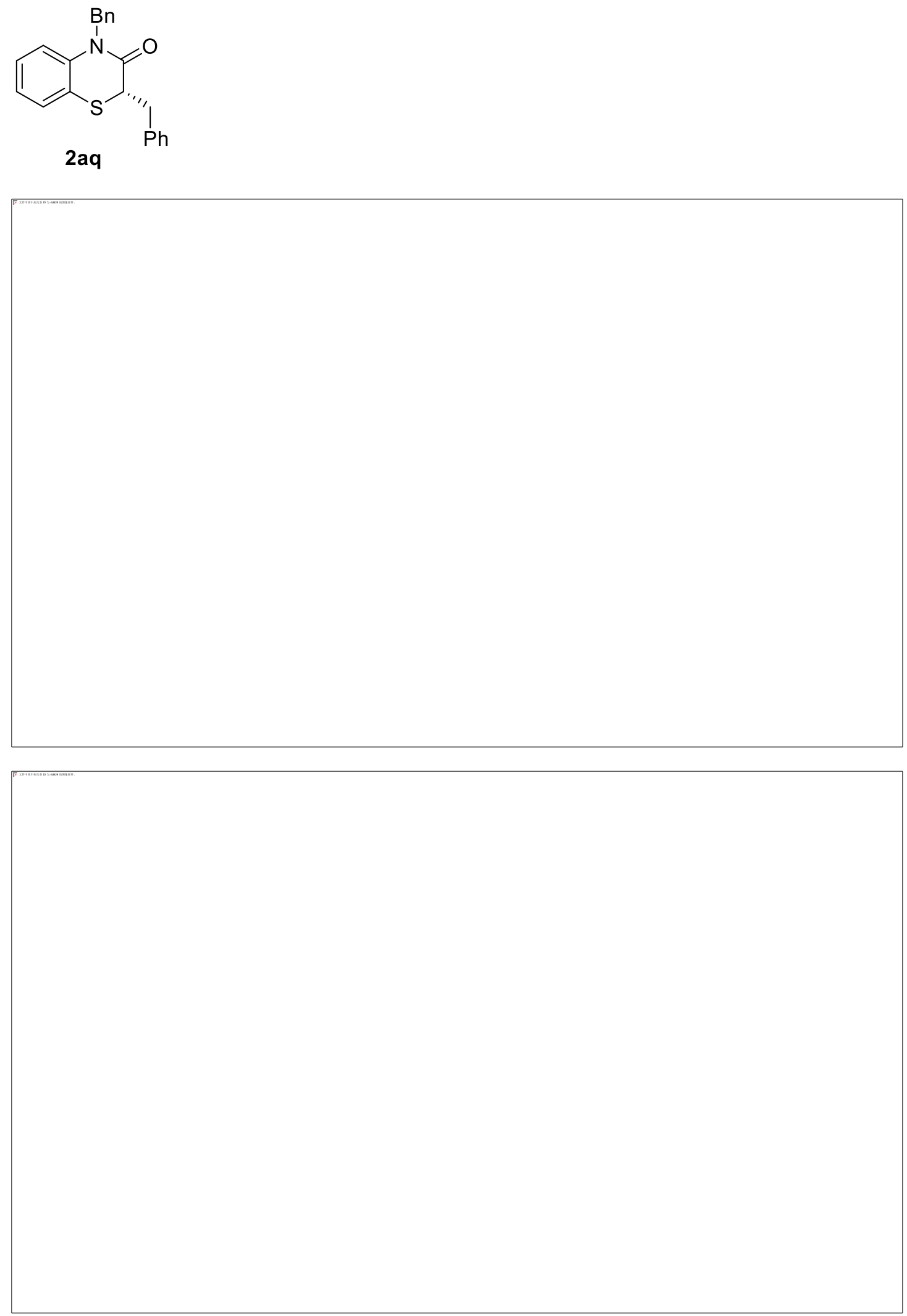

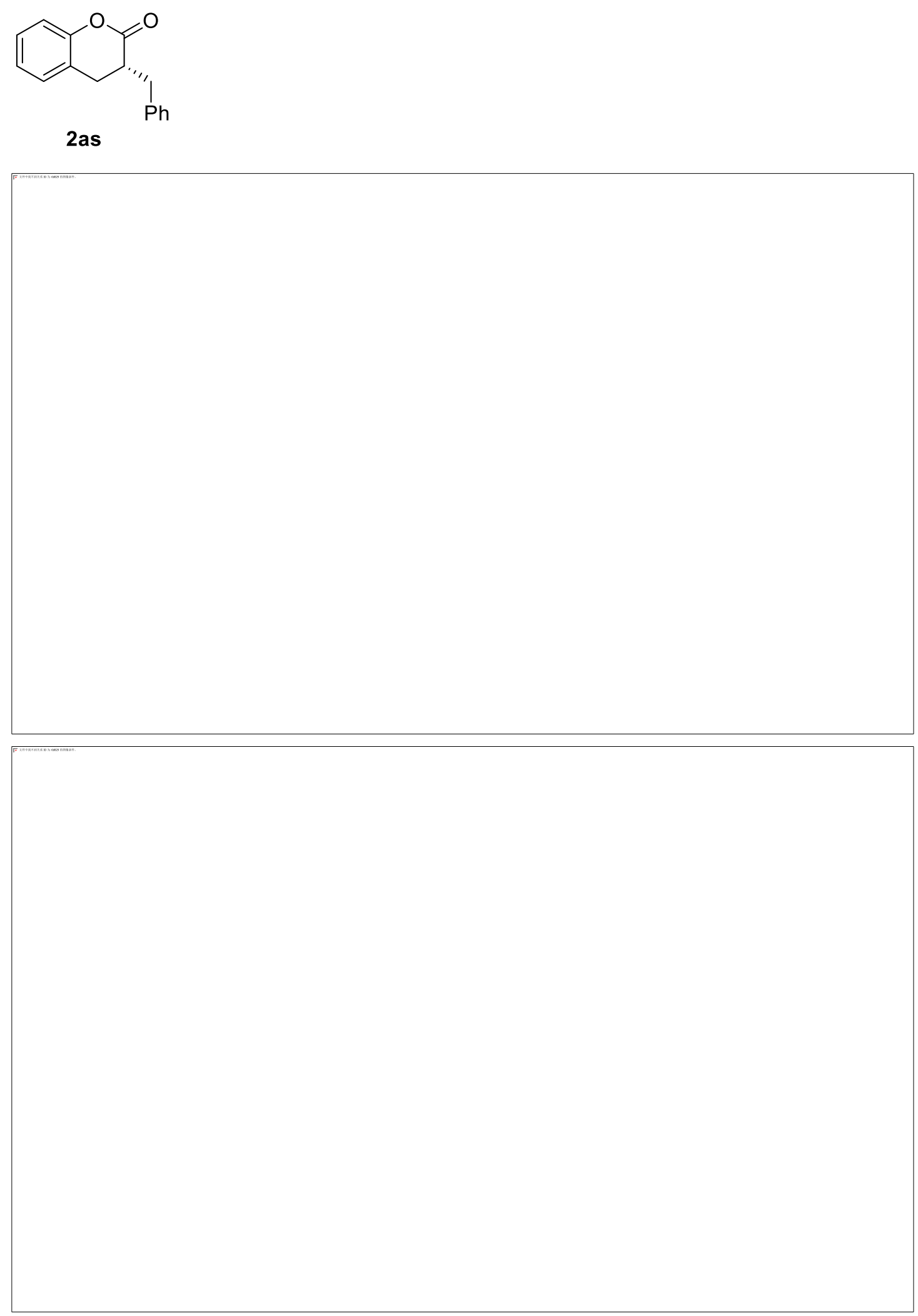


\section{X-Ray Crystal Structure Analysis of 2v}

X-Ray crystallography data for $\mathbf{2 v}$ (CCDC 2069776): A colorless crystal suitable for X-ray crystallography was obtained from a $\mathrm{n}$-hexane/dichloromethane solution at room temperature under air.

The single-crystal X-ray diffraction data were collected on a Bruker D8 VENTURE CMOS Photon II diffractometer with helios mx multilayer monochrmator $\mathrm{Cu} K \alpha$ radiation $(\lambda=1.54178$ $\AA)$ at $293 \mathrm{~K}$. Data collection, unit cell refinement and data reduction were performed using APEX3 v2019.11-0. The structure was solved by Intrinsic Phasing method and refined by full-matrix least-squares on F2 with anisotropic displacement parameters for the non-H atoms using SHELXTL program package. The hydrogen atoms on carbon were calculated in ideal positions with isotropic displacement parameters set to $1.2 \mathrm{xUeq}$ of the attached atom (1.5xUeq for methyl hydrogen atoms). The hydrogen atoms bound to nitrogen were located in a $\Delta \mathrm{F}$ map and refined with isotropic displacement parameters.

The ORTEP drawing (50\% probability for thermal ellipsoids) of $\mathbf{2 v}$ 


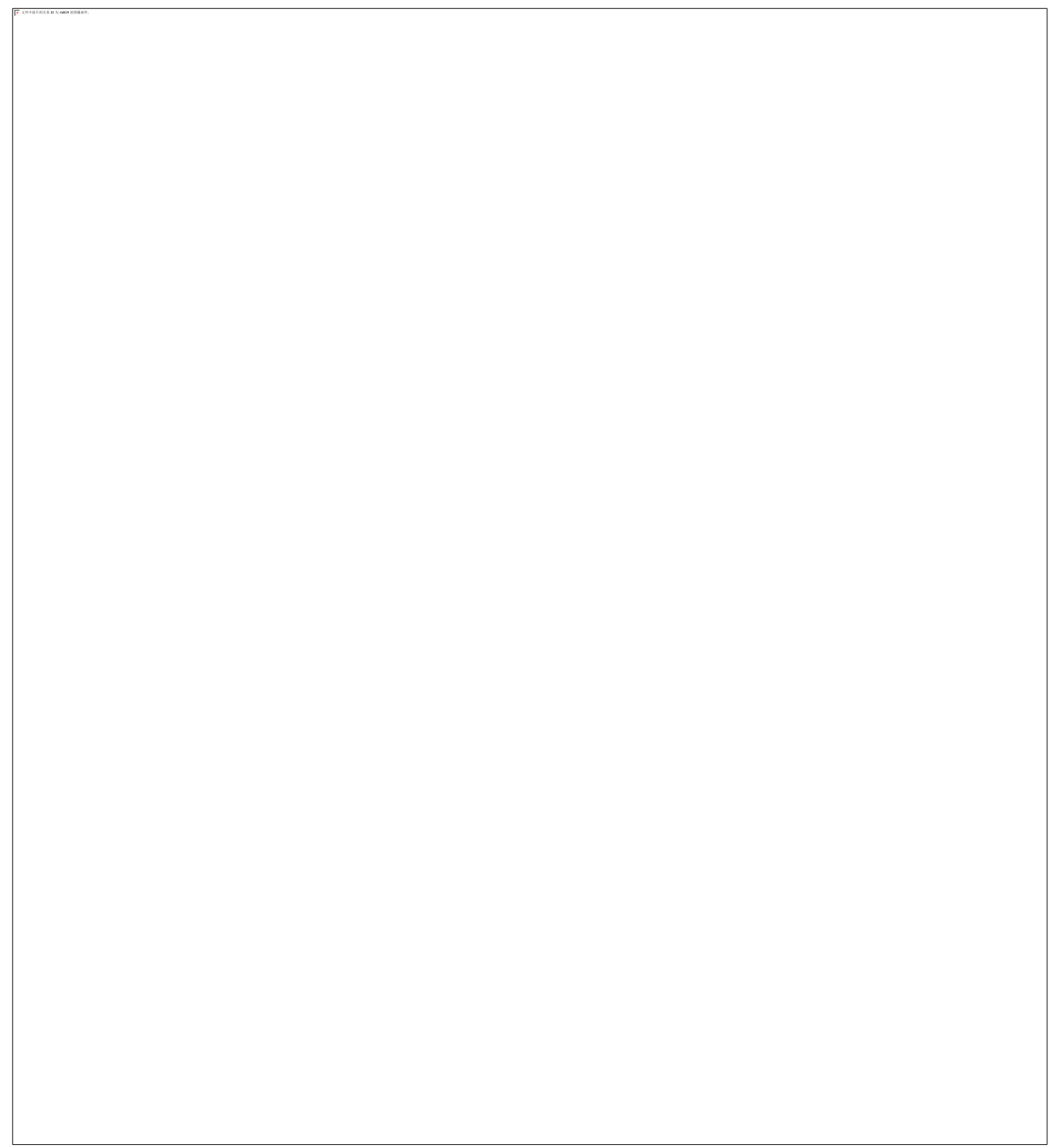

